University of Louisville

ThinkIR: The University of Louisville's Institutional Repository

Electronic Theses and Dissertations

$8-2000$

\title{
Bulk-micromachined mass airflow sensor fabrication and testing methodology for an undergraduate microfabrication course.
}

Jason B. Cole 1971-

University of Louisville

Follow this and additional works at: https://ir.library.louisville.edu/etd

\section{Recommended Citation}

Cole, Jason B. 1971-, "Bulk-micromachined mass airflow sensor fabrication and testing methodology for an undergraduate microfabrication course." (2000). Electronic Theses and Dissertations. Paper 264.

https://doi.org/10.18297/etd/264

This Master's Thesis is brought to you for free and open access by ThinkIR: The University of Louisville's Institutional Repository. It has been accepted for inclusion in Electronic Theses and Dissertations by an authorized administrator of ThinkIR: The University of Louisville's Institutional Repository. This title appears here courtesy of the author, who has retained all other copyrights. For more information, please contact thinkir@louisville.edu. 


\title{
BULK-MICROMACHINED MASS AIRFLOW SENSOR FABRICATION AND TESTING METHODOLOGY FOR AN UNDERGRADUATE MICROFABRICATION COURSE
}

\author{
By \\ Jason B. Cole \\ B.S.E.E., University of Louisville, 1997 \\ A Thesis \\ Submitted to the Faculty of the \\ University of Louisville \\ Speed Scientific School \\ as Partial Fulfillment of the Requirements \\ for the Professional Degree of \\ MASTER OF ENGINEERING \\ Department of Electrical and Computer Engineering
}

August 2000 
An electronic version of this document

may be found on the World Wide Web at:

http://mitghmr.spd.louisville.edu/research/theses 


\title{
BULK-MICROMACHINED MASS AIRFLOW SENSOR FABRICATION AND TESTING METHODOLOGY FOR AN UNDERGRADUATE MICROFABRICATION COURSE
}

Submitted by:

Jason B. Cole

\author{
A Thesis Approved on \\ Date \\ by the Following Reading and Examination Committee: \\ Dr. Kevin M. Walsh, Thesis Director \\ Dr. Robert Keyton \\ Dr. John F. Naber
}




\section{ACKNOWLEDGEMENTS}

Since early 1997, this thesis design project has been a long journey of learning that has taken me through different phases of strategic vision, design, fabrication, and testing methodologies which have challenged me to persevere, collaborate, and explore unique and creative ways to solve difficult fabrication problems despite many failures and disappointments. Perhaps the most surprising and enlightening dimension of this project has been the many diverse options generated through what I call the synergy of collaboration. In seeking to gather, assimilate, and understand innovative ideas through collaborating with others within the MicroTechnology group at Speed School, I found I was not merely limited by my own preconceived fabrication strategies and never without options and ideas I could continue to explore for this project. I am obviously deeply indebted to my thesis director, Dr. Kevin Walsh, for his expertise, strategic guidance, complete project financial support, and patience throughout this long process; and, to Mark Crain, who has offered his insight, ideas, and proactive assistance since this project's inception. I would like to thank Dr. Robert Keynton for collaboratively providing unique fabrication ideas and helping me to understand and apply fluid dynamics concepts for testing flow sensors. I was guided in circuit simulations and understanding circuit concepts to develop an effective and economical flow sensor test device by Dr. John Naber. I also want to thank Doug Hensel, Jonathan Hernandez, Stacy Miller, and Brian Reteneller for their early efforts to develop the, now extensive, Microfabrication course website, SOP's, and on-line

microfabrication course labs (at http://mitghmr.spd.louisville.edu as of Aug. 2000) as well as Don Yeager and Mike Martin for their technical advice, support, and guidance. I would like to express my gratitude to Dr. Hendersen and Jeff Simkins at the Center for Microelectronic Sensors and MEMS at the University of Cincinnati for training and permitting me to use their Logitech PM5 machine and supplies for precision lapping and polishing of borosilicate glass substrates. I would like to acknowledge the National Science Foundation for its funding of this research through a 
three-year award (the Instrumentation and Lab Improvement: Leadership in Laboratory Development program under Award \#9551869, An Advanced Undergraduate Laboratory for Microfabrication, July 1995) for the development of a laboratory-based undergraduate microfabrication course to introduce students to microelectronic fabrication principles, techniques, and processes to facilitate non-electronic interdisciplinary applications for microfabrication [1].

I want to express my appreciation for a few people who have been close to me, offered their support throughout this project, and have made a continual, incontrovertible difference in my life. First, my immediate family, Don, Sue, and Jessica have persistently and consistently offered their prayers, unconditional love, emotional, and financial support throughout my undergraduate and graduate school endeavors. I want to thank my parents for raising me with love in a supportive environment, inspiring me to ask difficult questions, and always affording me many opportunities to wonder about the world around me. A few close friends, such as Chris Lane and Chris Bajwa have distinguished themselves by demonstrating their sincere concern and compassion for my well being and giving me strength, encouragement, guidance, and support to persevere throughout this project despite many difficult obstacles. I want to thank these friends in particular for their prayers and exhortations to become a better man by growing in my faith and trusting in the character and Providence of God despite difficult circumstances. These family and friends, to their credit and my gain, have never vacillated in their support for me, and have always believed in the potential God has given me.

Finally, but not least, I want to thank God for giving me life, salvation, vital human relationships, and the opportunity, strength, and perseverance to successfully complete this research.

Who is like you, O Lord, among the gods?

Who is like you, majestic in holiness, awesome in splendor, doing wonders?

Exodus 15:11 (NRSV) 


\begin{abstract}
In July 1995, National Science Foundation Award \# 9551869 funded the development of a new inter-disciplinary microfabrication course under the primary leadership of Dr. Kevin Walsh at the University of Louisville. Along with this award, the completed construction of a new building in 1996 that contained a class 1000/100 cleanroom laboratory facilitated the development of the course. Moreover, curricula had to be completed to provide students with practical, hands-on experience in building Micro Electro-Mechanical Systems (MEMS) devices using processes and methodologies introduced in the course. Dr. Walsh wanted to include a mass airflow sensor in his portfolio of total possible devices students could build in the cleanroom lab for the course. This document describes the design of a bulk-micromachined, monolithic, mass airflow sensor with a thermally-isolated, thin-film, dielectric, microbridge/diaphragm design. In addition, several fabrication methodologies were explored, as well as a means to test and evaluate the sensors for this undergraduate class laboratory.

The mass airflow sensor architecture chosen was based upon a closed-loop-control, microelectronic thermal (hot-wire) anemometer design, which was first developed and presented by Johnson, Higashi, et. al. at Honeywell in the mid 1980s [2]. Two separate photomask sets were developed using L-Edit ${ }^{\mathrm{TM}}$ software (by Tanner Research), with each set including multiple geometric variations of a dual/triple microbridge/cantilever flow sensor structure to be suspended over a precision, anisotropically-etched pit, integrated onto a (100) silicon substrate.

Four primary structural fabrication strategies were explored to produce the thin-film material for the flow sensors: (1) RF planar magnetron sputter-deposited $1 \mu \mathrm{m}$-thick silicon nitride microbridges/cantilevers; (2) anodically-bonded-and-machined 20-30 $\mu \mathrm{m}$-thick borosilicate glass diaphragms; (3) spin-on-glass microbridges/cantilevers; and (4) low-stress, $0.5 \mu m$-thick, LPCVD silicon nitride microbridges/cantilevers. Four resistor metallizations were separately evaluated: permalloy $\left(\mathrm{Ni}_{81} \mathrm{Fe}_{19}\right)$, chromium, titanium, and platinum.
\end{abstract}


A process was developed and documented to successfully fabricate flow sensors with low stress LPCVD silicon nitride microbridges/cantilevers. DC planar magnetron sputterdeposited platinum thin-film resistors (with a $\sim 120 \mathrm{~nm}$-thick RF planar magnetron sputterdeposited chromium adhesion layer), with nominal thicknesses of $\sim 56-70 \mathrm{~nm}$, were delineated by photolithographic imaging techniques. The resistors had measured Temperature Coefficients of Resistance (TCR) in the range of $1.93-2.25 \times 10^{-3} \Omega / \Omega /{ }^{\circ} \mathrm{C}$ at $25-125^{\circ} \mathrm{C}$. Anisotropic $\mathrm{KOH}$ etching of the (100)-oriented silicon substrate was utilized to release the flow sensor microbridge/cantilever microstructures. After designing and building a flow sensor test machine capable of controlled volumetric air flow rates of up to $\sim 15 \operatorname{SLPM}(0.54 \mathrm{~m} / \mathrm{s})$, nominal sensor sensitivities $\left(\mathrm{S}_{\mathrm{V}}\right)$ of up to $0.67 \mathrm{mV} / \mathrm{SLPM}(20.4 \mathrm{mV} /(\mathrm{m} / \mathrm{s}))$ were measured. The sensitivities varied somewhat depending upon resistor values set in the flow sensor heater-driver circuit and the insertion depth of the devices within the flow channel. 


\section{TABLE OF CONTENTS}

ACPROVAL PAGE

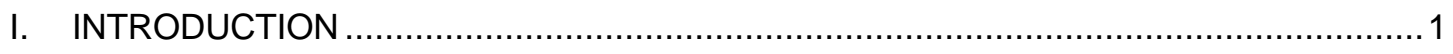

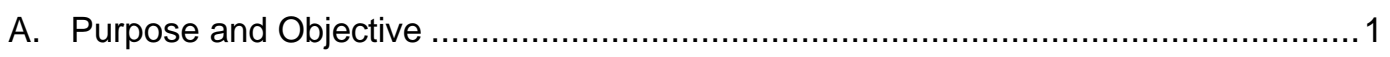

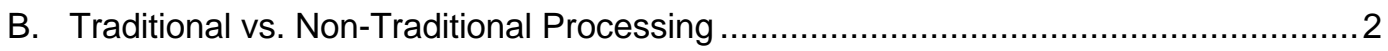

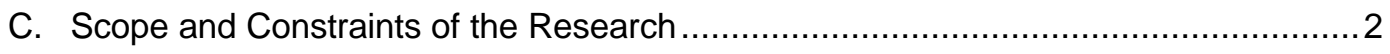

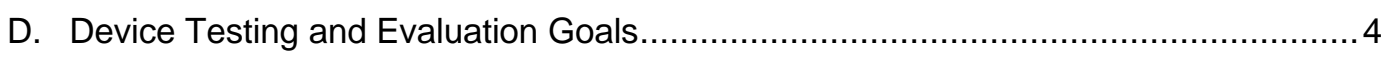

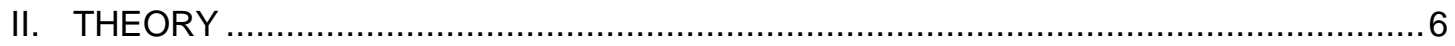

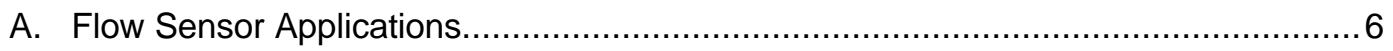

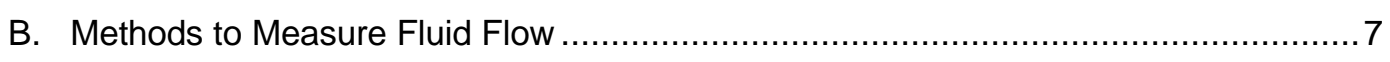

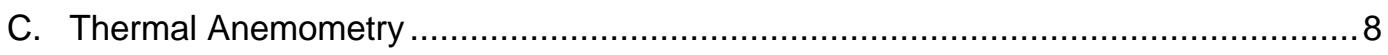

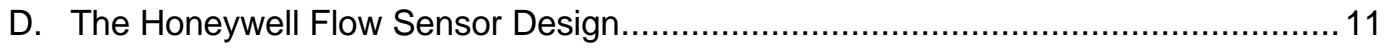

E. How the Honeywell Flow Sensor Works ................................................ 17

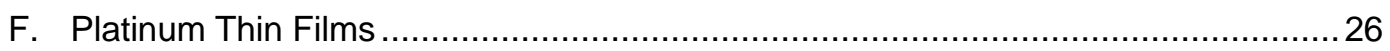

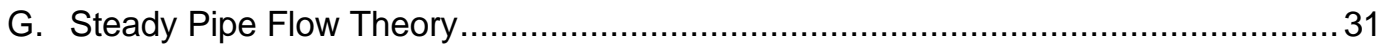

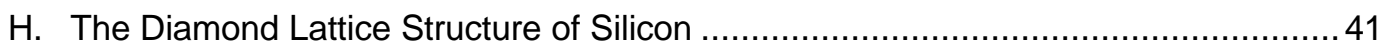

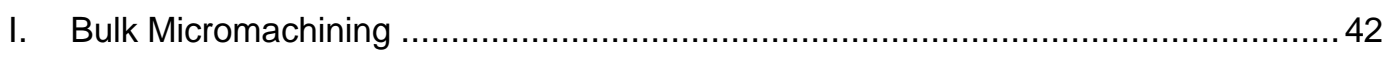

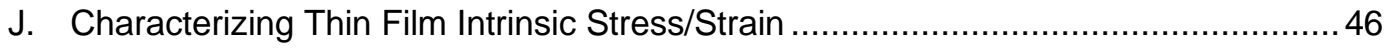

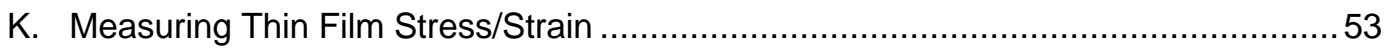




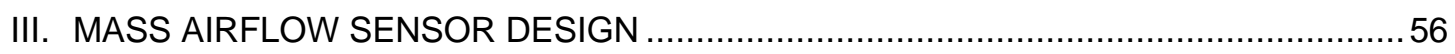

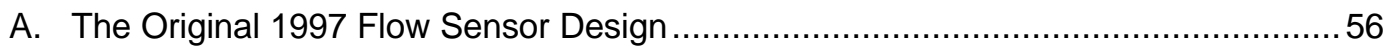

1. Photomask Design Overview..............................................................56

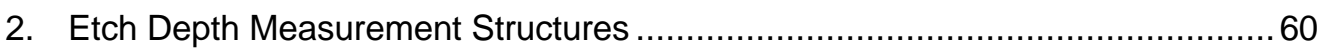

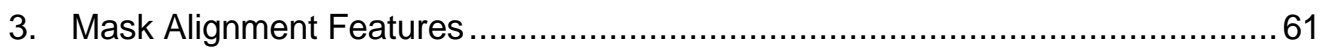

4. Sheet Resistance Measurement Structures ................................................ 63

B. The Redesigned 1998/1999 Photomask Design................................................64

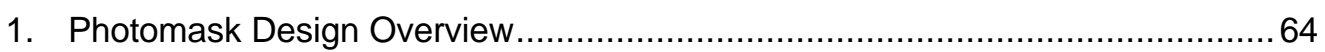

2. Etch Depth Measurement and Mask Alignment Structures.............................73

3. Sheet Resistance Measurement Structures .............................................. 75

4. New In Situ Thin Film Stress/Strain Measurement Structures ..........................76

a) Doubly Clamped Beams (Microbridges) ….......................................... 76

b) Simply-Supported Cantilevered Beams ............................................. 79

c) Archimedean Cantilever Spirals ................................................... 83

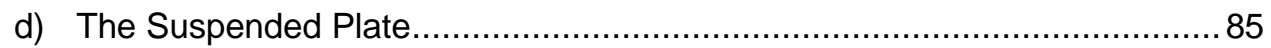

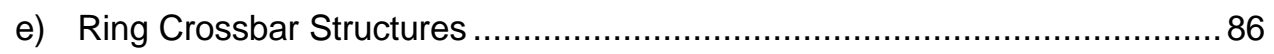

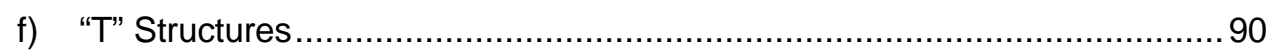

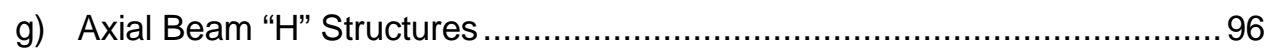

IV. SENSOR FABRICATON AND PROCESS METHODOLOGIES .................................99

A. Thin Film Structural Methods Attempted: Pre-Etched Wafer Strategies ..................99

1. Anodically-Bonded Borosilicate Glass Method ...............................................99

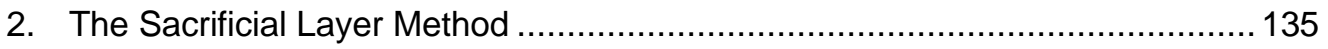

B. Thin-Film Structural Methods Attempted: Post-Etched Wafer Strategies............... 145

1. RF Planar Magnetron Sputter-Deposited Silicon Nitride Processes ................. 145

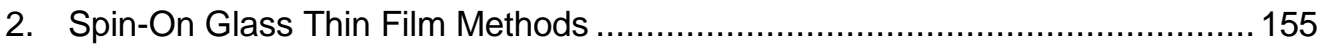

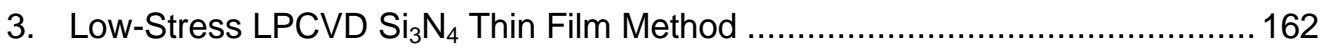

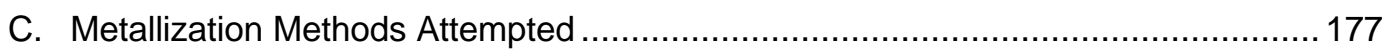

1. Bulk vs. Thin Film Electrical Properties .................................................. 177 


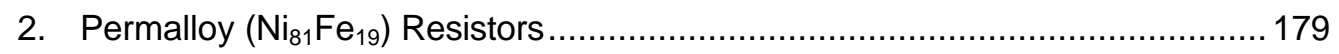

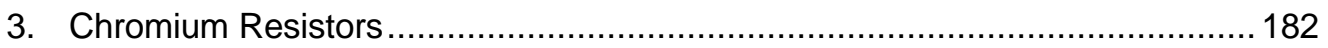

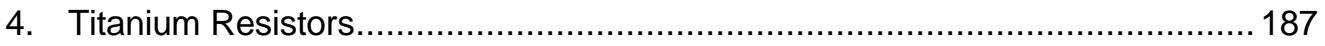

5. Gold Top Layer Metallization and RIE/KOH Masking ….............................. 190

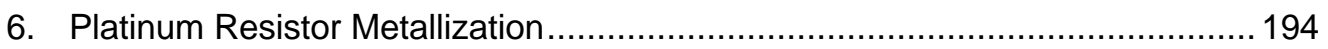

V. DEVICE TESTING: DESIGN OF THE “COLEJET 2000” MACHINE …...................... 196

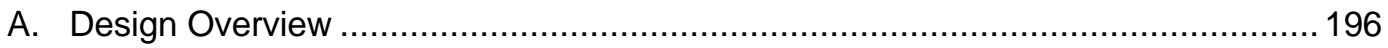

B. Straightforward Wire Bonding and Packaging Scheme .................................. 197

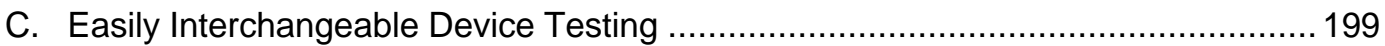

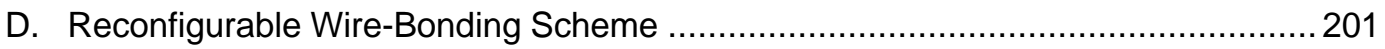

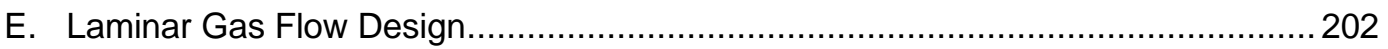

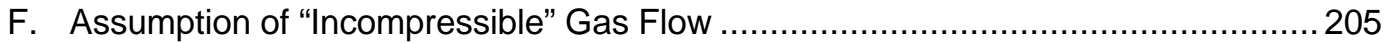

G. Measuring Actual Flow with a Calibrated Honeywell Flow Sensor ........................206

H. Precisely and Finely Controllable Gas Flow Rate ............................................209

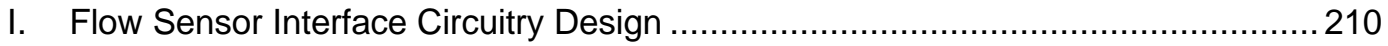

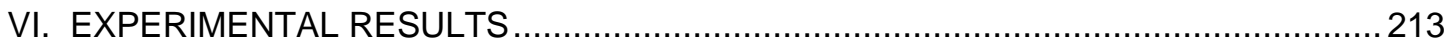

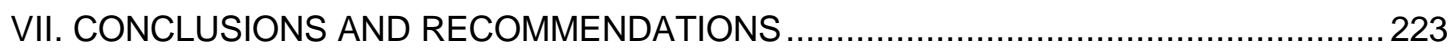

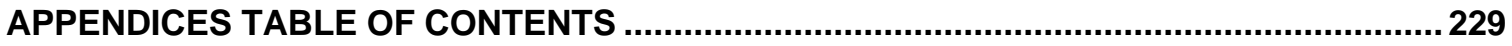

APPENDIX I................ Honeywell AWM5000 Series Flow Sensor Data Sheets .....................230

APPENDIX II............... Honeywell AWM2150V Flow Sensor Die Photos............................... 250

APPENDIX III.............. Chemical List and Compositions ................................................ 253

APPENDIX IV ............. Laboratory Apparatus Equipment Photographs ............................... 256

APPENDIX V .............. Summary of Flow Sensor Methodologies Attempted .......................... 265

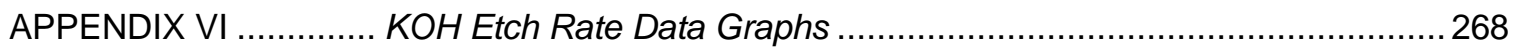

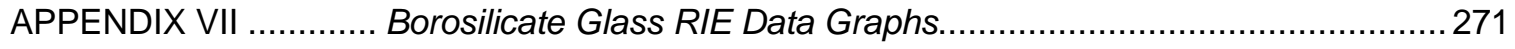

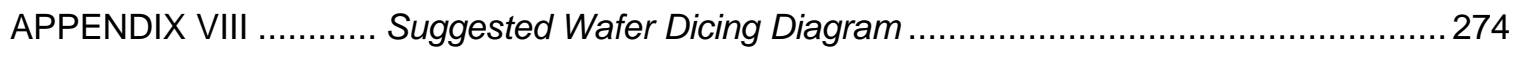

APPENDIX IX ............. Micro-Automation Dicing Saw Revised SOP .................................. 277

APPENDIX X .............. Si $i_{x} N_{y}$ Sputtering Deposition and RIE Etch Rate Data Graphs .............296 
APPENDIX XI ............ SEM Photos of Sputtered Silicon Nitride Flow Sensors .....................299

APPENDIX XII ............ Filmtronics 700 Series Spin-On Glass Data Sheet ........................... 302

APPENDIX XIII ............ Successful LPCVD $\mathrm{Si}_{3} \mathrm{~N}_{4}$ Flow Sensor Fabrication Summary ............. 305

APPENDIX XIV ........... Detailed Outline of LPCVD $\mathrm{Si}_{3} \mathrm{~N}_{4}$ Flow Sensor Fabrication .................. 311

APPENDIX XV ............ SEM Photos of Low-Stress LPCVD Si ${ }_{3} N_{4}$ Pt/Au Flow Sensors ........... 319

APPENDIX XVI ........... Air and Nitrogen Gas Properties............................................... 322

APPENDIX XVII ........... ColeJet 2000 TM Parts' Design Considerations .................................. 325

APPENDIX XVIII .......... ColeJet 2000тM Selected Parts' Data Sheets ..................................... 328

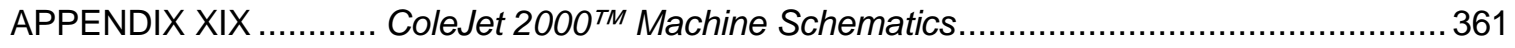

APPENDIX XX ............ ColeJet 2000TM Photographs and Descriptions ............................... 367

APPENDIX XXI ........... ColeJet 2000 ${ }^{\mathrm{TM}}$ Condensed Operating Instructions ............................ 375

APPENDIX XXII .......... 1997 Two-Layer L-Edit ${ }^{T M}$ Design File Images/Descriptions ................. 386

APPENDIX XXIII .......... 1998/99 3-Layer L-Edit ${ }^{T M}$ Design File Images/Descriptions ................ 396

VITA

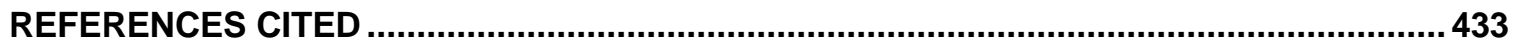




\section{NOMENCLATURE}

\section{Description of Variables}

\begin{tabular}{|c|c|c|}
\hline Variable & Symbol & Units \\
\hline Area, Cross Sectional & A & $\mathrm{m}^{2}$ \\
\hline Coefficient of Linear Thermal Expansion & $\alpha$ & $1 /{ }^{\circ} \mathrm{C}$ \\
\hline Density, Mass & $\rho$ & $\mathrm{kg} / \mathrm{m}^{3}$ \\
\hline Diameter of Circular Flow Channel & $\mathrm{D}$ & $\mathrm{m}$ \\
\hline Displacement & $\delta$ & $\mathrm{m}$ \\
\hline Entrance Length for Fully Developed Laminar Flow & $\mathrm{L}_{e}$ & $\mathrm{~m}$ \\
\hline Heat & Q & $\mathrm{J}$ \\
\hline Inlet Loss Coefficient & $\mathrm{K}_{\text {inlet }}$ & - \\
\hline Inside Diameter of Circular Flow Channel & ID & $\mathrm{mm}$ \\
\hline Length of By-Pass Circular Flow Channel & $\mathrm{L}$ & $\mathrm{m}$ \\
\hline Mass Flow Rate & $\dot{m}, Q_{m}$ & $\mathrm{~kg} \cdot \mathrm{m} / \mathrm{s}$ \\
\hline Outside Diameter of Circular Flow Channel & OD & $\mathrm{mm}$ \\
\hline Outlet Loss Coefficient & $\mathrm{K}_{\text {outlet }}$ & - \\
\hline Number of Squares & $\mathrm{N}$ & - \\
\hline Number of Squares, Platinum & $\mathrm{N}_{\mathrm{Pt}}$ & - \\
\hline Poisson's Ratio & $\mathrm{v}$ & - \\
\hline Pressure & $\mathrm{P}$ & $\mathrm{Pa}$ \\
\hline Pressure Drop Total Across Laminar Flow Bypass Channel & $\Delta \mathrm{P}_{\text {total }}$ & $\mathrm{Pa}$ \\
\hline Pressure Drop Across Laminar Flow Bypass Channel & $\Delta P_{\text {flow }}$ & $\mathrm{Pa}$ \\
\hline Resistance of Downstream Resistor & $\mathrm{R}_{\mathrm{d}}$ & $\Omega$ \\
\hline Resistance of Heater Resistor & $\mathrm{R}_{\mathrm{h}}$ & $\Omega$ \\
\hline Resistance of Substrate or Reference Resistor & $\mathrm{R}_{\mathrm{r}}$ & $\Omega$ \\
\hline Resistance of Upstream Resistor & $\mathrm{R}_{\mathrm{u}}$ & $\Omega$ \\
\hline Resistivity & $\rho_{r}$ & $\mu \Omega \cdot \mathrm{cm}$ \\
\hline Reynolds Number & $\mathrm{Re}$ & - \\
\hline Reynolds Number, Critical Value & $\operatorname{Re}_{\mathrm{cr}}$ & - \\
\hline Sensor Sensitivity (Constant Current) & $\mathrm{S}_{\mathrm{I}}$ & $\mathrm{m} \Omega / \mathrm{sccm}$ \\
\hline Sensor Sensitivity (Constant Voltage) & $S_{V}$ & $\mathrm{mV} / \mathrm{sccm}$ \\
\hline Sheet Resistance & $\mathrm{R}_{\mathrm{s}}$ & $\Omega$ /square \\
\hline Specific Heat & $C_{p}$ & $\mathrm{~J} / \mathrm{kg} \cdot \mathrm{K}$ \\
\hline Strain, Residual & $\varepsilon$ & - \\
\hline Stress, Residual (Average Residual Stress) & $\sigma\left(\sigma_{0}\right)$ & $\mathrm{Pa}$ \\
\hline Temperature & $\mathrm{T}$ & ${ }^{\circ} \mathrm{C}$ \\
\hline Temperature Coefficient of Resistance (TCR) & TCR & $\Omega / \Omega /{ }^{\circ} \mathrm{C}$ \\
\hline Thickness & $\mathrm{t}$ & $\mathrm{m}$ \\
\hline Velocity, Instantaneous & $\mathrm{V}$ & $\mathrm{m} / \mathrm{s}$ \\
\hline Velocity, Time Mean & $\bar{v}$ & $\mathrm{~m} / \mathrm{s}$ \\
\hline Viscosity, Absolute, or Dynamic, & $\mu$ & $\mathrm{kg} / \mathrm{m} \cdot \mathrm{s}$ \\
\hline Viscosity (Kinematic) & $\mathrm{v}$ & $\mathrm{m}^{2} / \mathrm{s}$ \\
\hline Volume & V & $\mathrm{m}^{3}$ \\
\hline Volumetric Flow Rate & $Q_{v}$ & $\mathrm{~m}^{3} / \mathrm{s}$ \\
\hline Young's Modulus of Elasticity & $E$ & $\mathrm{~Pa}$ \\
\hline
\end{tabular}




\section{Abbreviations}

\begin{tabular}{|c|c|}
\hline Acronym & Description \\
\hline BCC & Body Centered Cubic (lattice structure) \\
\hline $\mathrm{BOE}$ & Buffered Oxide Etch $\left(6: 1: 1 \mathrm{H}_{2} 0: \mathrm{HF}: \mathrm{NH}_{4} \mathrm{~F}\right)$ \\
\hline $\mathrm{CF}_{4}$ & Freon $^{\mathrm{TM}} 14$ \\
\hline $\mathrm{CHF}_{3}$ & Fluoroform (Freon ${ }^{\mathrm{TM}}$-23) \\
\hline CVD & Chemical Vapor Deposition \\
\hline $\mathrm{DC}$ & Direct Current \\
\hline dia & diameter \\
\hline DIP & Dual Inline (Integrated Circuit) Package \\
\hline DUT & Device Under Test \\
\hline FCC & Face Centered Cubic \\
\hline GDS-II & $\begin{array}{l}\text { A Calma binary stream file format designed for the interchange of mask } \\
\text { geometry information }\end{array}$ \\
\hline $\mathrm{H}_{2} \mathrm{O}_{2}$ & Hydrogen Peroxide \\
\hline $\mathrm{HCl}$ & Hydrochloric Acid \\
\hline $\mathrm{HF}$ & Hydrofluoric Acid \\
\hline HVAC & Heating Ventilation and Air Conditioning \\
\hline IC & Integrated Circuit \\
\hline IPC & $\begin{array}{l}\text { Independent Pressure Control (automatic pressure control feature in the } \\
\text { March RIE system) }\end{array}$ \\
\hline $\mathrm{KOH}$ & Potassium Hydroxide \\
\hline LPCVD & Low Pressure Chemical Vapor Deposition \\
\hline MEMS & Micro Electro Mechanical Systems \\
\hline MFC & Mass Flow Controller \\
\hline $\mathrm{NH}_{4} \mathrm{OH}$ & Ammonium Hydroxide \\
\hline NSF & National Science Foundation \\
\hline PECVD & Plasma Enhanced Chemical Vapor Deposition \\
\hline PTFE & Polytetrafluoroethylene (Teflon ${ }^{\mathrm{TM}}$ ) \\
\hline PVC & Polyvinylchloride \\
\hline $\mathrm{RF}$ & Radio Frequency \\
\hline RIE & Reactive lon Etching \\
\hline RIT & Rochester Institute of Technology \\
\hline RTD & Resistance Thermal Device \\
\hline $\mathrm{sccm}$ & $\begin{array}{l}\text { Standard Cubic Centimeters Per Minute (a flow measurement referenced to } \\
\text { standard conditions of } 0{ }^{\circ} \mathrm{C}, 760 \text { torr at sea level, } 50 \% \text { Relative Humidity) }\end{array}$ \\
\hline SEM & Scanning Electron Microscope \\
\hline $\mathrm{SF}_{6}$ & Sulfur Hexafluoride \\
\hline $\mathrm{Si}_{3} \mathrm{~N}_{4}$ & Stoichiometric Silicon Nitride \\
\hline $\mathrm{Si}_{X} \mathrm{~N}_{Y}$ & $\begin{array}{l}\text { Non-stoichiometric (e.g. RF planar magnetron sputter-deposited) Silicon } \\
\text { Nitride }\end{array}$ \\
\hline $\mathrm{SiO}_{2}$ & Silicon Dioxide; glass \\
\hline SLPM & Standard Liters Per Minute $\left(0^{\circ} \mathrm{C}, 760\right.$ Torr at sea level, $\left.50 \% \mathrm{RH}\right)$ \\
\hline SOG & Spin On Glass \\
\hline SOP & Standard Operating Procedure (for equipment or lab processes) \\
\hline TCR & Temperature Coefficient of Resistance \\
\hline TMAH & Tetramethylammonium Hydroxide, $\left(\mathrm{CH}_{3}\right)_{4} \mathrm{NOH}$ \\
\hline TPD & Temperature Penetration Depth \\
\hline TTV & Total Thickness Variation (e.g. across a silicon wafer substrate) \\
\hline ZIF & Zero Insertion Force \\
\hline
\end{tabular}




\section{Constants}

\begin{tabular}{|l|c|c|c|}
\hline Constant & Symbol & Value & Units \\
\hline Acceleration of Gravity & $\mathrm{g}$ & 9.80665 & $\mathrm{~m} / \mathrm{s}^{2}$ \\
\hline Density of Air $\left(\right.$ at $\left.25^{\circ} \mathrm{C}\right)$ & $\rho_{\text {air }}$ & 1.1845 & $\mathrm{~kg} / \mathrm{m}^{3}$ \\
\hline $\mathrm{Pi}$ & $\partial$ & 3.1415926 & - \\
\hline Specific Heat of Air $\left(\right.$ at $\left.25^{\circ} \mathrm{C}\right)$ & $\mathrm{C}_{\mathrm{p} \_ \text {air }}$ & 1006.3 & $\mathrm{~J} / \mathrm{kg} \cdot{ }^{\circ} \mathrm{C}$ \\
\hline Thermal Conductivity of Air $\left(\right.$ at $\left.25^{\circ} \mathrm{C}\right)$ & $\mathrm{k}_{\text {air }}$ & 0.025969 & $\mathrm{~W} / \mathrm{m} \cdot{ }^{\circ} \mathrm{C}$ \\
\hline Universal Gas Constant & $\mathrm{R}$ & 8.31451 & $\mathrm{~J} /(\mathrm{gmol} \cdot \mathrm{K})$ \\
\hline
\end{tabular}




\section{LIST OF TABLES}

TABLE

TABLE I TITLE

Flow Sensor Applications

Physical Characteristics of the Honeywell Flow Sensor

TABLE II Honeywell Flow Sensor Operating Characteristics.

Platinum TCRs Under Different Annealing Conditions Material Properties of Various MEMS Materials.

TABLE VI Synopsis of the 1997 Photomask Set Design .58

TABLE VII Summary of Flow Sensor Physical Specs in the 1997 Mask Set. 59

TABLE VIII Summary of Flow Sensor Electrical Specs for the 1997 Mask Set 60

TABLE IX Synopsis of the 1998/1999 Photomask Set Design. 69

TABLE $X$ 1998/1999 Photomask Set Flow Sensor Physical Specifications 70

TABLE XI 1998/1999 Photomask Set Flow Sensor Electrical Specifications .72

TABLE XII Summary of Borosilicate Glass Thin-Film Diaphragm Process. 101

TABLE XIII Borofloat $\AA^{\circledR}$ Glass Mechanical and Thermal Properties 107

TABLE XIV. Summary of the Experimental Sacrificial Layer Process 136

TABLE XV...... Summary of Fab. Steps for 1997 Liftoff Process, Sputtered $S_{x} N_{y}$ 147

TABLE XVI. Fab. Summary for 1998/1999 Wet Etch Process, Sputtered $\mathrm{Si}_{x} N_{y}$ 151

TABLE XVII Successful LPCVD $\mathrm{Si}_{3} \mathrm{~N}_{4}$ Flow Sensor Fabrication Summary 163

TABLE XVIII. Properties of Chromium Metal Films. 182

TABLE XIX Chromium Thin Film Annealing Experimental Results. 186

TABLE XX Properties of Bulk Titanium 187

TABLE XXI. Titanium Thin Film Annealing Experimental Results 189

TABLE XXII. Properties of Gold Metal Films (at $27^{\circ} \mathrm{C}$ ) 191

TABLE XXIII. Properties of Platinum Metal Films . 194 
TABLE XXIV .......... Design Parameters for AWM5000 Series Bypass Sensor, $21^{\circ} \mathrm{C}$ Air..........208

TABLE XXV .......... Comparison Between Various Axial and Blower Fans Evaluated ..............210

TABLE XXVI .......... Heat Transfer Measurements for Flow Sensor \#1, Die \#1 ....................... 221

TABLE XXVII ......... Heat Transfer Measurements for Flow Sensor \#1, Die \#2........................ 221

TABLE XXVIII ........ Appendix III, Laboratory Chemical Descriptions..................................... 254

TABLE XXIX .......... Appendix V, Comprehensive Fabrication Methods Explored ....................266

TABLE XXX ........... Appendix XIII, Successful LPCVD $\mathrm{Si}_{3} \mathrm{~N}_{4}$ Sensor Fab. Summary ............. 306

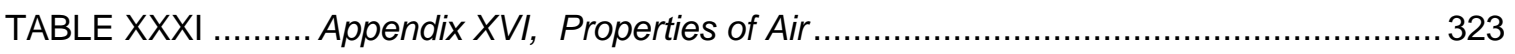

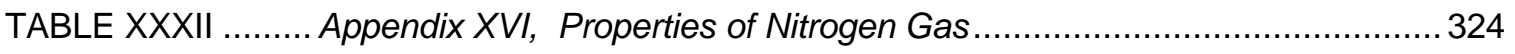

TABLE XXXIII ........ Appendix XVII, Flow Channel Design Parameter Relationships ............... 326

TABLE XXXIV ........ Appendix XVII, Design Parameters with AWM5000 Bypass Sensor......... 327 


\section{LIST OF FIGURES}

\section{FIGURE}

Figure $1 \ldots \ldots \ldots . . . A$ diagram of a common thermal anemometer arrangement........................... 8

Figure 2 .......... Possible operating modes for anemometer heaters .................................. 10

Figure $3 \ldots \ldots \ldots . . .3-\mathrm{D}$ rendering of the Honeywell double-bridge flow sensor ............................. 12

Figure 4 .......... Photograph of a commercial Honeywell AWM2150V flow sensor die ............... 12

Figure 5 .......... Cross-section of the Honeywell mass airflow sensor microstructure ................. 14

Figure 6 ...........A cut-away view of the Honeywell sensor on a (100) silicon substrate..............14

Figure 7 .......... Honeywell's year 2000 mass airflow sensor product line .............................. 17

Figure 8 ........... Honeywell's Wheatstone bridge sense-resistor configuration ......................... 18

Figure 9 .......... Temp. differences between the up and downstream sense resistors ................ 19

Figure $10 \ldots \ldots \ldots .$. The error-integrator Wheatstone half-bridge heater driver circuit..................... 19

Figure $11 \ldots \ldots . .$. Output vs. mass flow rate for non-linear TCR permalloy flow sensors ..............25

Figure $12 \ldots \ldots \ldots .$. Thin-film resistance vs. temp. characteristic for permalloy and platinum...........26

Figure 13......... Callendar-Van Dusen constants for thin-film platinum RTDs ........................ 27

Figure $14 \ldots \ldots \ldots .$. Relative resistance vs. temp. curve for a typical platinum RTD .......................28

Figure 15.........A plot of TCR vs. thin-film thickness for a platinum RTD ............................. 30

Figure 16.........A plot of TCR sensitivity versus thin-film thickness for a platinum RTD............ 30

Figure $17 \ldots \ldots \ldots$. A possible classification scheme for characterizing fluid flow ......................... 32

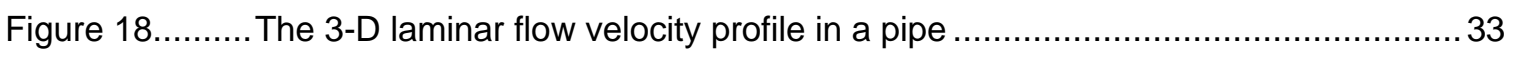

Figure $19 . \ldots \ldots \ldots .$. Velocity profile of developing laminar flow over a semi-infinite flat plate ...........37

Figure $20 \ldots \ldots \ldots$. Average velocity profile comparison for laminar and turbulent pipe flow ............37

Figure $21 \ldots \ldots \ldots$. The entrance length of a pipe in which laminar flow fully develops ................... 38

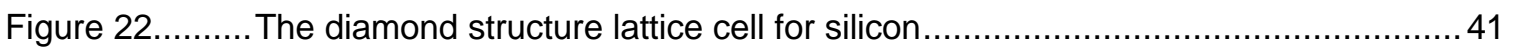

Figure 23.........A table of standard Miller Indices notation............................................. 41 
Figure $24 \ldots \ldots \ldots .$. Illustration of standard coded "flats" on 4 -inch silicon wafers ........................... 42

Figure $25 \ldots \ldots \ldots .$. Illustration of an isotropic, glass wet-etching sidewall profile ........................... 44

Figure 26......... Illustration of anisotropic wet etching of a (100) silicon substrate .................... 44

Figure $27 \ldots \ldots \ldots .$. Wafer bow due to tension or compression within a deposited thin film ............. 47

Figure $28 \ldots \ldots \ldots$. Examples of intrinsic and extrinsic residual stresses ................................. 48

Figure 29.......... Illustration of an Instron specimen for measuring Young's modulus..................51

Figure $30 \ldots \ldots \ldots . .$. A screen-capture of the 1997 L-Edit ${ }^{T M}$ photomask design file ........................56

Figure $31 \ldots \ldots \ldots$. Three pyramidal etch-depth measurement structures ...................................60

Figure $32 \ldots \ldots . . .$. How to align the metallization mask to the (110) wafer flat ............................. 62

Figure 33.......... Manual mask alignment (or registration) marks in the 1997 mask set..............62

Figure 34.........Van der Pauw test structures, with labels, in the 1997 mask set.....................63

Figure $35 \ldots \ldots \ldots . .$. A design file image of the parallel substrate resistor in the ' $98 /$ '99 mask set ......68

Figure 36..........An L-Edit ${ }^{T M}$ design file image of the 1998/1999 mask alignment area ..............73

Figure $37 \ldots \ldots \ldots .$. How to align the $1998 / 1999$ resistor metal mask to the (110) wafer flat ............74

Figure 38.......... Van der Pauw sheet resistance test structures in the '98/'99 mask set............. 75

Figure $39 . \ldots \ldots \ldots . . . A$ series of incremental-length bridges, included in the ' $98 /$ '99 mask design .......76

Figure $40 \ldots \ldots . . .$. Example of clamped-clamped beams used to find the critical buckling length ... 77

Figure $41 \ldots \ldots . .$. An array of incremental-length cantilevers, included in the ' $98 /$ '99 photomask ... 80

Figure $42 \ldots \ldots \ldots . .$. An SEM photo of buckled, sputtered $\mathrm{Si}_{x} \mathrm{~N}_{\mathrm{y}}$ thin film cantilevers ...................... 80

Figure $43 \ldots \ldots \ldots . .$. f free-body diagram of in-plane stress distribution in a deposited thin film ........81

Figure $44 \ldots \ldots \ldots$. An Archimedean cantilever spiral used to measure strain gradients in films ......84

Figure $45 \ldots \ldots . .$. Various spiral microstructures included in the 1998/1999 photomask design..... 85

Figure 46..........A suspended plate included in the 1998/1999 mask design ........................... 86

Figure $47 \ldots \ldots \ldots .$. Illustration of ring-crossbar structures used to measure tensile stress ...............87

Figure $48 \ldots \ldots \ldots .$. Photomicrographs of ring and beam strain diagnostic structures ..................... 87 
Figure $49 . \ldots \ldots . .$. Plot of deflection ratios as a function of ring radius for $10 \mu \mathrm{m}$ beam width........ 88

Figure $50 \ldots \ldots \ldots . .$. ' $98 /$ '99 mask array of ring-crossbar structures with $10 \mu m$-wide crossbars ......89

Figure $51 \ldots \ldots \ldots . .$. An SEM photo of a sputtered $\mathrm{Si}_{\mathrm{x}} \mathrm{N}_{\mathrm{y}}$ ring-and-beam test structure ....................90

Figure 52..........An '98/'99 L-Edit ${ }^{\mathrm{TM}}$ design file image of the smallest "T" shaped test structure ..91

Figure 53......... Schematic diagram of the "T" structure with dimensions nomenclature ............91

Figure 54..........Close-up ‘98/’99 design file image of "T” structure fiducial marks ....................94

Figure 55......... Geometry and mechanics illustration of "T" with out-of-plane deflection ............95

Figure 56.......... A '98/'99 L-Edit'TM image of an "H" structure for tensile stress measurement. ....97

Figure $57 \ldots \ldots \ldots .$. A top-view schematic of an "H" beam structure with dimensions .....................97

Figure $58 \ldots \ldots \ldots . .$. p photo of the hotplate setup used for anodic bonding ............................... 104

Figure 59.......... An electrical schematic of an anodic glass-to-Si wafer bonding setup ............ 105

Figure $60 \ldots \ldots . .$. Thermal expansion coefficient graph of Si and Corning 7740 Pyrex $^{\mathrm{TM}}$ glass.... 106

Figure $61 \ldots \ldots . .$. System setup for $\mathrm{KOH}$ anisotropic bulk micromachining of silicon substrates .. 110

Figure 62 ..........A photo of a pre-etched double-side-polished (100) silicon substrate ............. 112

Figure 63..........Photo showing placement of the glass/Si wafer sandwiches on the hot plate .. 113

Figure 64 ..........Photo of violet-colored corona at Si/glass interface during anodic bonding......114

Figure $65 . . . . . . . . . A$ photo of an anodically-bonded borosilicate glass/Si wafer sandwich ............115

Figure 66.......... A photo of Logitech PM5 machine during 70 RPM borosilicate glass lapping ... 116

Figure $67 \ldots \ldots \ldots .$. The glass polishing setup for the Logitech PM5 machine ............................. 118

Figure $68 \ldots \ldots \ldots .$. A photo of a polished borosilicate glass/Si wafer sandwich .......................... 119

Figure $69 . . . . . . . .$. Photo of a polished glass/Si sandwich with residual rough, frosted regions ..... 120

Figure $70 \ldots \ldots \ldots .$. Diagram illustrating sealed flow sensor diaphragm cavities ........................... 121

Figure $71 \ldots \ldots \ldots . .$. Photo of a metallized Si/glass wafer mask-alignment strategy ...................... 122

Figure $72 \ldots \ldots \ldots . .$. A photo collage of exploded 10 - $38 \mu m$-thick glass diaphragms .................... 123 
Figure 73.........A schematic, cut-away illustration showing the "double-lithography" process... 128

Figure $74 \ldots \ldots . . .$. A photo of a wire-bonded, quad-die flow sensor mounted in a DIP-24 pkg ...... 130

Figure $75 \ldots \ldots . . .$. Photos of an aluminum RIE-masking layer with poor adhesion 131

Figure $76 \ldots \ldots . . .$. Photos showing lateral damage to microbridge resistor metallization 132

Figure $77 \ldots \ldots . . .$. Photos showing imploded pad areas on a borosilicate glass/Si wafer 134

Figure 78.........Titanium-metallized glass diaphragm devices with good wire-bonds 134

Figure $79 \ldots \ldots . . .$. A polished $10-\mathrm{cm}$ (100) Si wafer with a positive photoresist sacrificial layer .... 142

Figure $80 \ldots \ldots \ldots . .$. A close-up view of positive photoresist topography in device cavities 142

Figure $81 \ldots \ldots . . .$. Two views of a polished Si wafer with surface-coating sacrificial-layer 143

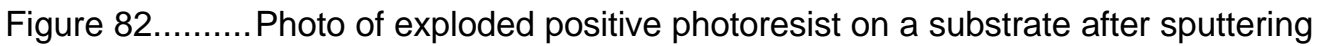
144

Figure 83..........A photo of Filmetrics-measured $\mathrm{Si}_{x} \mathrm{~N}_{\mathrm{y}}$ depths across a test silicon wafer ..... 149

Figure $84 \ldots \ldots \ldots . . . \mathrm{A}$ Wyco-Instrument image of the topography of a sputtered $\mathrm{Si}_{\mathrm{x}} \mathrm{N}_{\mathrm{y}}$ thin film 150

Figure $85 \ldots \ldots . . .$. An SEM photo of warped nitrogen-sputtered $\mathrm{Si}_{x} \mathrm{~N}_{\mathrm{y}}$ cantilevers 154

Figure $86 \ldots \ldots \ldots . .$. An image of the porous surface topography of a sputtered $\mathrm{Si}_{x} \mathrm{~N}_{\mathrm{y}}$ thin film....... 155

Figure $87 \ldots \ldots . .$. . Photo of the metered, precision dispensing tool for applying SOG ................. 156

Figure 88..........Photo of the customized Teflon ${ }^{\mathrm{TM}}$ die basket used for TMAH/KOH etching ..... 160

Figure 89..........Photo of the TMAH anisotropic (100) silicon etching system ........................161

Figure $90 \ldots \ldots \ldots$. An SEM photo montage of laterally buckled SOG microbridges .................... 162

Figure $91 \ldots \ldots . . .$. A photo of diluted, heated aqua regia set-up for delineating platinum............. 168

Figure $92 \ldots \ldots \ldots . .$. Photo of wafer PT1 after delineating the platinum metal layer ....................... 169

Figure 93.........Examples of poor positive photoresist adhesion to Pt after delineation............ 170

Figure $94 \ldots \ldots \ldots .$. Photos of over-etched $5 \mu \mathrm{m}$-line-width platinum resistors ........................... 170

Figure $95 \ldots \ldots \ldots .$. A severely cracked chromium layer on a 4-inch borosilicate glass wafer ......... 178

Figure $96 \ldots \ldots \ldots .$. . graph of sputtering parameter relationships to metal film stress .................. 179

Figure $97 \ldots \ldots . .$. Step coverage comparison for magnetron sputtering vs. evaporative sputtering.......... 183 
Figure $98 \ldots \ldots . . .$. Photos comparing a chromium layer's appearance before/after annealing ...... 186

Figure 99..........Photos comparing a titanium layer's appearance before/after annealing ......... 190

Figure $100 \ldots \ldots . .$. A top view of the ColeJet $2000^{\text {TM }}$ flow sensor testing machine........................ 196

Figure $101 \ldots \ldots . .$. A photo of the supplemental power supply in ColeJet $2000^{T M} \ldots \ldots \ldots \ldots \ldots \ldots \ldots \ldots . . . . . . . . .197$

Figure 102........ Photo of the Device Under Test insertion tube assembly with 24-pin ZIF .......200

Figure 103........ Photo of the machined 1-inch PVC "T" section where the DUT is inserted.......200

Figure $104 \ldots . . .$. Side-by-side photos showing patch wiring board pin correspondence .............201

Figure 105........Photo of the flow sensor interface/heater-driver board and patch board ..........202

Figure 106........ Photos of the laminar-flow cocktail straw section of clear PVC tubing .............204

Figure 107.......A view of the Device Under Test device insertion region inside the PVC "T" ....204

Figure 108........A plot of the resistance vs. temperature characteristic for the MA103 thermistor .........206

Figure 109........Table showing full-scale pressure drop values for Honeywell flow sensors......207

Figure 110........A photo of the commercial Honeywell AWM5104VN flow sensor....................209

Figure $111 \ldots . . .$. Graph of bridge output voltage vs. flow rate for Flow Sensor \#1, die \#1 .........215

Figure 112........Graph of bridge output voltage vs. flow rate for Flow Sensor \#1, die \#2 .........216

Figure $113 \ldots . . .$. Graph of Wheatstone bridge output voltage vs. flow rate, combined results ....216

Figure $114 \ldots \ldots .$. A graph comparing fabricated sensor output responses to Honeywell's ..........218

Figure $115 \ldots . . .$. Flow velocity vs. measured temp. difference between sense resistors in the

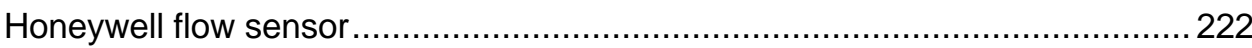




\section{INTRODUCTION}

One closed-loop differential method to measure gas flow is to monitor the temperatures of the gas upstream and downstream of a hot wire. When there is no gas stream flow, the temperature profile around the wire is symmetrical. When gas flows, the symmetry is perturbed as the upstream detector is cooled, the gas is heated by the hot wire, and the downstream detector is then, in-turn, heated. A flow rate can then be correlated to this differential change in temperature [3]. In the mid 1980s Johnson, et al. [4] at Honeywell applied common batch processing methods for precision, anisotropic, monocrystalline-silicon etching and delineation of thermally-isolated, precisely-dimensioned, dielectric-film structures containing temperaturesensitive resistors to create a hot-wire anemometer microtransducer on a silicon substrate. Based on their work, a similar microtransducer, described herein, was developed for introduction in a microfabrication laboratory course curriculum.

\section{A. Purpose and Objective}

As part of the NSF-funded (award \# 9551869) development of a new inter-disciplinary microfabrication course at the University of Louisville, this thesis document describes the vision of creating, building, and testing a bulk-micromachined, MEMS, thin-film, microbridge-based, mass airflow sensor to give undergraduate students practical, hands-on experience in learning and applying microfabrication principles and techniques to build and test a hot-wire anemometer MEMS device. The purpose of this research was to create a design for these sensors, document one or more methods to successfully fabricate them, and build a means to effectively test them. The following constraints were imposed upon the research:

1. The practical and reasonably economical process sequence to build these devices must be appropriate for a 15-week undergraduate-level microfabrication course laboratory.

2. Preference would be given to fabrication methods that require minimal or no outsourcing of processes from external vendors. 
3. The chosen fabrication methodology to build the devices need not be "traditional." A more complex, non-standard fabrication sequence might give students experience with processes not normally used to build this type of sensor, or any commercially-produced MEMS sensor.

\section{B. Traditional vs. Non-Traditional Processing}

The flow sensor fabrication process may be divided into two primary approaches, either "traditional" or "non-traditional," depending upon when anisotropic etching of the silicon substrate occurs in the process sequence. A traditional or standard approach would be one in which the dielectric thin-film layer is first deposited (by traditional means, such as RF magnetron sputtering), followed by deposition and patterning of the resistor and pad metallization layer(s), an optional second deposition for a top dielectric/passivation layer, patterning the dielectric layer(s), and then anisotropic etching of the silicon substrate. Thus, in this approach, anisotropic silicon etching would be at, or near, the end of the fabrication process sequence. Alternatively, the fabrication sequence could follow a non-traditional approach in which anisotropic silicon etching does not occur at the end of the process. While a non-traditional approach might be more challenging and time-consuming to implement, it potentially offered the distinct advantage of not requiring that the metallization and thin-film dielectric layers be resistant to degradation in an alkaline anisotropic silicon etchant, such as $\mathrm{KOH}$ or TMAH (since these layers would not be exposed to the etchant at the end of the process sequence).

\section{Scope and Constraints of the Research}

This research consisted primarily of the following problem-solving dimensions and constraints (sequential contingencies):

1. Determine the type of thermal isolation microstructure(s) to use in the flow sensor design (e.g. one or more microbridges, cantilevers, diaphragms).

2. Find an optimal dielectric thin-film to use as the thermal isolation structure for the sensors. This film should have a low thermal conductivity relative to silicon to provide high thermal decoupling. It could be sputter-deposited (in a traditional fabrication 
approach) or otherwise applied/attached to the silicon substrate. In both cases, it must be possible to achieve a controlled final design thickness for the film. If a traditional processing approach is applied, this dielectric material has to be both photolithographically patternable (by wet-etching techniques or plasma dry-etching in a Reactive Ion Etcher) and resistant to degradation in the anisotropic silicon etchant $(\mathrm{KOH}$ or TMAH). For non-standard dielectric film deposition/application methods, it may or may not need to be resistant to degradation in the silicon etchant, depending upon the process sequence.

3. Determine the optimal resistor metallization to use, with appropriate thin-film sheet resistance and TCR electrical properties, that can be sputter-deposited (i.e. with a controllable deposition thickness), and photolithographically patterned. If a singlemetallization layer is used in conjunction with a top dielectric passivation layer in a standard fabrication sequence, the metallization layer must be resistant to degradation in the anisotropic silicon etchant.

4. Determine whether or not a dielectric, passivation layer will be deposited on top of the metallization layer(s). If so, determine whether or not it will be the same material originally deposited/attached/applied. And, if deposited, it would have to be patterned to not only expose etch windows at thermal-isolation-microstructure areas in the silicon substrate but also expose bonding pad areas for etching to expose the top bonding-pad metal layer (which would have to be resistant to degradation in the anisotropic silicon etchant if a standard process sequence is utilized with one photomask for both patterning the top passivation layer and etching into the substrate to release microbridge/cantilever microstructures).

5. Determine whether a second metallization layer of gold might be needed for improved wire-bonding ohmic contact at the pads and resistance to degradation in the silicon etchant. If so, and if a top dielectric passivation layer is deposited, a design could be implemented such that only this gold layer would be exposed to the anisotropic silicon 
etchant at the bonding-pad areas, allowing for the use of a resistor metallization layer that need not be resistant to degradation in the silicon etchant.

6. Determine whether the metallization layer(s) will be patterned by either wet-etching or liftoff techniques, and with what type of photoresist (positive or negative). If wet-etching is chosen, find a documented chemistry (with known etch rate) that can be used to photolithographically delineate the desired metallization layer(s).

7. Design an appropriate photomask set with a resistor metallization layer and an optional gold layer. Specify whether the photomask(s) will be darkfield or lightfield, depending upon whether wet-etching or lift-off techniques will be used with either positive or negative photoresist to pattern the metal layer(s).

8. Design a silicon bulk-micromachining etch-window photomask to match the metallization-layer photomask(s) set. Specify a mask type (i.e. lightfield or darkfield) to be ordered depending upon whether positive or negative photoresist will be used.

9. Design and build a test apparatus for students to use to evaluate their fabricated microtransducers with controlled laminar flow across the flow sensors.

\section{Device Testing and Evaluation Goals}

The following objectives were used to develop a flow sensor test methodology and apparatus to evaluate fabricated devices:

1. Wire bonding and packaging for the microtransducers should be straightforward and uncomplicated, allowing students to learn fundamental device packaging concepts. Fabricated devices should be mounted and wire-bonded in standard Dual Inline Packages (DIPs).

2. Once packaged, the microtransducers should be easily interchangeable within a machined flow channel for expeditious testing of multiple devices.

3. The test apparatus should be reconfigurable to allow for different wire-bonding schemes (e.g. connections for the heater resistor should not be permanently assigned to specific Dual Inline Package pins). 
4. For simplified theoretical flow rate calculations, choose a gas and controlled-flow methodology such that the simplifying assumption of incompressibility may be applied to the gas stream (i.e. gas density and temperature variations can be assumed to be negligible throughout the test apparatus).

5. The gas flow rate should be precisely and finely controllable.

6. The gas flow should be laminar (or fully-developed, laminar) across the mass airflow sensor being tested.

7. A calibrated, commercially-available airflow sensor should be incorporated into the design to provide an accurate, real-time indication of the actual flow rate. 


\section{THEORY}

\section{A. Flow Sensor Applications}

Fluid flow has an important role in processes such as hydraulic machines, various manufacturing industries, and in many other areas. Most practical flows are turbulent, with turbulent processes contributing significantly to the transport of momentum, heat, and mass. Fluid friction losses, due to turbulence, can be observed as pressure losses in fluid-transport systems [5]. Flow sensors are used to measure the mass, average velocity, differential pressure, temperature, or volumetric flow of a gas or liquid. They can also be used to measure concentration changes in gas mixtures and phase changes in multi-phase flows [6]. As shown in Table I, they may be found in biomedical, environmental monitoring, automotive, and process control applications [7]. For example, Honeywell's Micro Switch division markets mass airflow sensors for applications including: HVAC damper controls, gas analyzers, gas leak detection systems, low vacuum control, process control, medical respirators, spirometers, oxygen concentrators, anesthesia control, and gas metering.

TABLE I

FLOW SENSOR APPLICATIONS

\begin{tabular}{|c|c|c|c|}
\hline Biomedical & Automotive & Environmental & Process Control \\
\hline Blood Flow & Air/Gasoline Mixture & Water Flow & $\begin{array}{c}\text { Mass-Flow } \\
\text { Controller }\end{array}$ \\
\hline $\begin{array}{c}\text { Cardiovascular } \\
\text { Flow }\end{array}$ & Exhaust Control & Wind Flow & $\begin{array}{c}\text { Liquid Cooling } \\
\text { Systems }\end{array}$ \\
\hline $\begin{array}{c}\text { Pulmonary } \\
\text { System }\end{array}$ & $\begin{array}{c}\text { Gasoline } \\
\text { Consumption }\end{array}$ & Air Conditioning & $\begin{array}{c}\text { Micro-chemical } \\
\text { Reactions }\end{array}$ \\
\hline Urinary Flow & Air Conditioning & Exhaust Control & $\begin{array}{c}\text { Chemical } \\
\text { Microanalysis } \\
\text { Systems }\end{array}$ \\
\hline
\end{tabular}




\section{B. Methods to Measure Fluid Flow}

The following are several methods or operating principles used to measure fluid flow [8]:

1. Differential-pressure sensing techniques (e.g. the orifice plate, venturi tube, Pitot tube, capacitive differential pressure sensing);

2. Mechanical sensing techniques (e.g. sensing physical deflections with rotary or spring-loaded vanes, cantilever beams, or the measurement of lift force deflection on an airfoil structure [9]);

3. Ultrasonic sensing techniques (e.g. Doppler shift, transit time, optical Doppler tomographic imaging);

4. Optical sensing techniques (laser Doppler velocimetry for single-point velocity measurements);

5. Particle Image Velocimetry (a flow-tracing technique to measure velocity fields of particle-seeded flows [10]);

6. Electromagnetic sensing techniques, (e.g. detecting the deflection of a flexible permanent-magnet beam placed perpendicular to the fluid motion [11]);

7. Oscillating-fluid sensing techniques (e.g. Karman vortex, vortex shedding or Karman street);

8. Resonant frequent shift sensing techniques (e.g. of a microresonator microbridge, used as a resonating force gauge [12]); and

9. Thermal sensing techniques (e.g. the Thomas flow meter, or hot-wire anemometer).

In the last method, mass flow can be inferred from a thermal measurement technique using thermally-isolated structures which carry heaters and basic temperature sensing elements that can be either a silicon $p-n$ junction diode (with a forward voltage vs. current characteristic that varies with temperature), a thin-film polysilicon or metal resistor (with well-defined TCR properties), or a thermocouple (or thermopile comprised of more than one thermocouple). This is one of the most mature and well understood techniques for measuring flow. To measure flow with a thermal-type of device, five different strategies or techniques can be distinguished [13]: 
1. Heat loss where a hot object is cooled by the flow (boundary-layer-flow measurement, thermal anemometers);

2. The thermo-transfer principle or calorimetric method (by which mass flow is directly calculated from the heat capacity of the flow, or the energy needed to heat up the fluid);

3. The micro thermo-transfer principle (in which a boundary layer of the fluid is heated, and the temperature rise is measured by very small devices that are close to each other in respect to the fluid channel);

4. Thermal tracing or time-of-flight measurements (in which a short-duration heat impulse is injected into the flow, and is traced for its time-of-flight from the heater to downstream detector); and

5. Thermal detection of flow oscillation caused by fluidic oscillators or vortex shedding (in which a fluidic oscillator generates differential pressure oscillations, the frequency of which is directly proportional to the flow rate $[14,15])$.

\section{Thermal Anemometry}

The most commonly used operating principle for flow sensors is a thermal (or hot-wire) anemometer (an example of which is shown in Figure 1), which is a boundary-layer type of

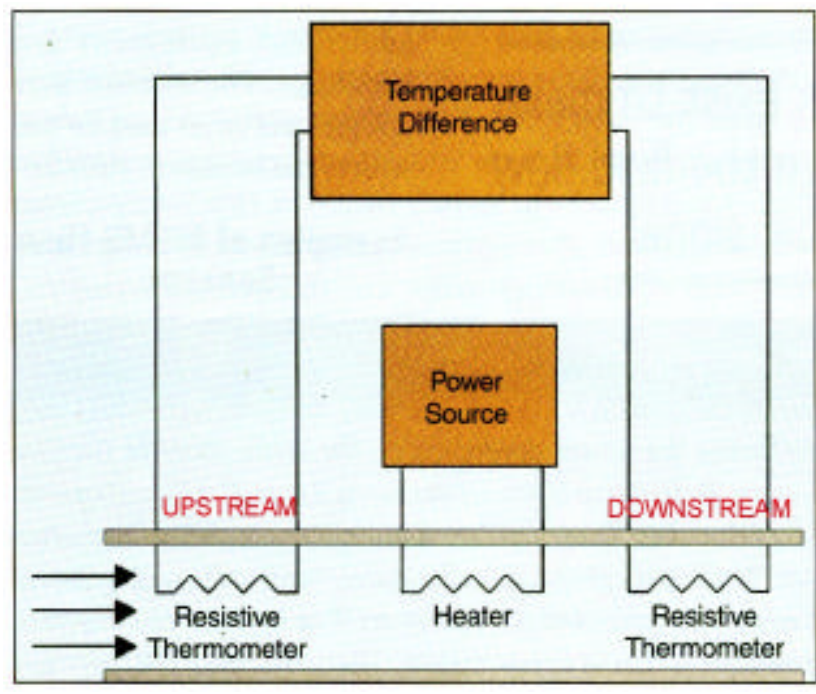

FIGURE 1 - A diagram of a common thermal anemometer arrangement. This is for a microfabricated type of sensor with upstream and downstream sensing resistors [7]. 
thermal flow sensor (strategy three in the above list) that can measure the direction of flowing mass under laminar and turbulent conditions. Thermal anemometer flow sensors monitor convective heat transfer, or total heat loss, of a heated wire or film placed into the flowing fluid. Hot-wire anemometry is a popular research tool for most turbulent air/gas flow studies offering advantages of very small sensor size, wide (one, two, or three-component) velocity measurement range, simultaneous fluid temperature measurement, good accuracy $(0.1-1 \%)$, very low signalto-noise ratios, excellent frequency response, operational simplicity, a continuous analog output signal (that can be time-domain sampled for frequency-domain analysis), and capability to provide spatial information about turbulent fluctuations in the flow. A common configuration (shown for a microfabricated thermal flow sensor in Figure 1) makes use of a central heat source and two separate temperature sensors (or thin film thermoresistors) upstream and downstream in a machined flow channel. Any change in the fluid flow condition that affects the heat transfer from the heated element will be detected virtually instantaneously [6]. With fluid flow, the temperature distribution around the heater becomes unbalanced. Heat is transferred from one sensing resistor to the other causing a change in the resistance of the thermoresistor that is detectable in a Wheatstone bridge configuration. This heat loss is dependent upon the fluid flow rate and increases proportionally with an increase in flow, depending upon the operating mode for the structure. This measurement of the thermal gradient between the temperature sensor probes is a sensitive measure for the flow.

As shown in Figure 2, there are two possible operating modes for a thermal anemometer, depending upon how the heating element is controlled. These methods are delineated as follows:

1. The anemometer heater may be driven with a constant current (or constant applied power). A constant current supply is used to drive a Wheatstone bridge configuration which has the heated sensor element in one bridge arm (thus, some of this current is used to heat this resistor). As the fluid flow rate increases, the heated sensor is cooled which causes a change in its resistance, and a corresponding imbalance in the Wheatstone bridge. This is detectable as a ratiometric change in output voltage 
across the bridge that is proportional to the flow rate. When configured in open-loop mode, the temperature of the heater wire is measured as a function of the flow rate. Since, the temperature-dependent heating resistor resistance changes with flow rate, the power delivered to the heater must be independent of the temperature. In a differential mode, a temperature difference between two specified locations can be monitored (e.g. between the heating element and an ambient reference temperature location; between an "upstream" and "downstream" detector; or between a temperature "near the heater" and an ambient substrate temperature) $[3,7]$.

2. The heater may be driven at a constant temperature above the ambient. In this closed-loop, constant-resistance mode, the heater is kept at a constant temperature above the ambient, and the power required to drive it is measured. Fluid flow causes an increase in the heating power required to drive the heater. The heater resistor resistance remains fixed, even with changes in temperature. A simple Wheatstone bridge configuration can be used in this operating mode with a temperaturecompensating (or substrate, reference) sensor in one bridge arm and a heated sensor element (the heater) located in the other bridge arm. The bridge becomes unbalanced when the heated sensor is cooled from the flow velocity of the fluid.

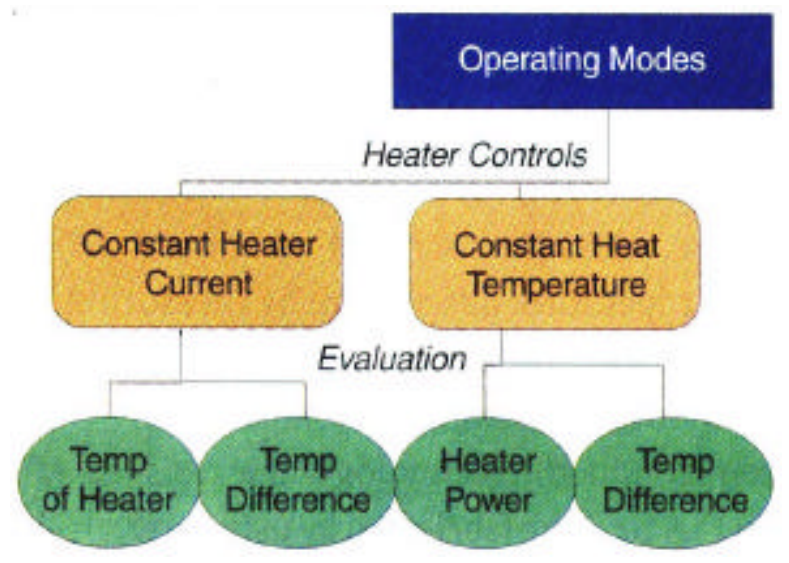

FIGURE 2 - $\quad$ Possible operating modes for anemometer heaters. Adapted from $[7,16]$.

Since measuring the temperature of the heater element is quite difficult without customized equipment, most anemometer flow sensors are designed with the structure portrayed 
in Figure 1, in which the temperature gradient between an upstream and downstream sensor element (sensing resistor) is measured. The sensing resistors can, therefore, be incorporated into a separate Wheatstone bridge configuration in which they are operated in a mode that produces a differential signal. This differential measurement technique eliminates zero-point offsets and other fluctuations due to factors such as ambient pressure and temperature changes [20].

With today's silicon micromachining tools, semiconductor and MEMS fabrication technologies, this hot-wire system can be easily miniaturized and integrated onto a silicon substrate using any temperature dependent device (such as a p-n junction diode, a thin-film polysilicon or metal resistor, or a thermocouple). Integrated silicon flow sensors offer advantages of fast response times, lower cost via batch fabrication, and integration with on-chip signal conditioning CMOS circuitry to enhance performance [17]. Significant flow sensor refinements with respect to sensitivity and power consumption can be realized, especially by using thin-film microbridges made of dielectric materials to achieve high thermal decoupling from the substrate (since the thermal conductivity of many dielectrics, such as silicon nitride, is lower than that of silicon). For example, if the anemometer's heating and temperature-sensing elements are placed on free-standing silicon nitride bridges above the substrate, power consumption is drastically reduced by thermally isolating the heating element so that most power passes into the flowing gas stream, minimizing loss due to heating of the silicon substrate. One example of a siliconnitride-microbridge-based, commercially-marketed, air-flow sensor is the device developed by Johnson, Higashi, et. al. at Honeywell in the mid 1980s [3].

\section{The Honeywell Flow Sensor Design}

A U.S. patent issued in 1985 to Higashi, Johnson, and Bohrer at Honeywell, Inc., describes a differential mass airflow sensor, shown in Figure 3, which was designed to measure either: (1) average gas velocity or volume flow rate in a flow channel; (2) dynamic differential gas pressure between channel ports across the flow sensor (where the sensor's mass flow rate output response is calibrated as a function of high flow impedance in a sufficiently small channel 
size, which results in a measurable pressure differential between inlet and outlet ports); or (3) mass flow rate through a flow channel. As shown in Figure 4, it consisted of two thermally-

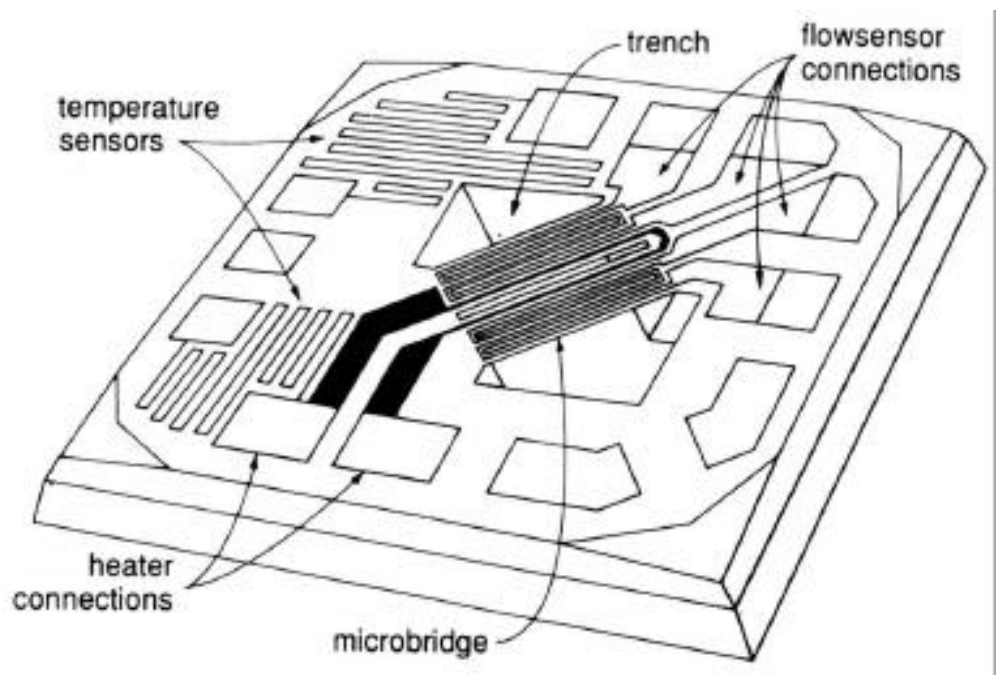

FIGURE 3 - $\quad 3-D$ rendering of the Honeywell double-bridge flow sensor [14].

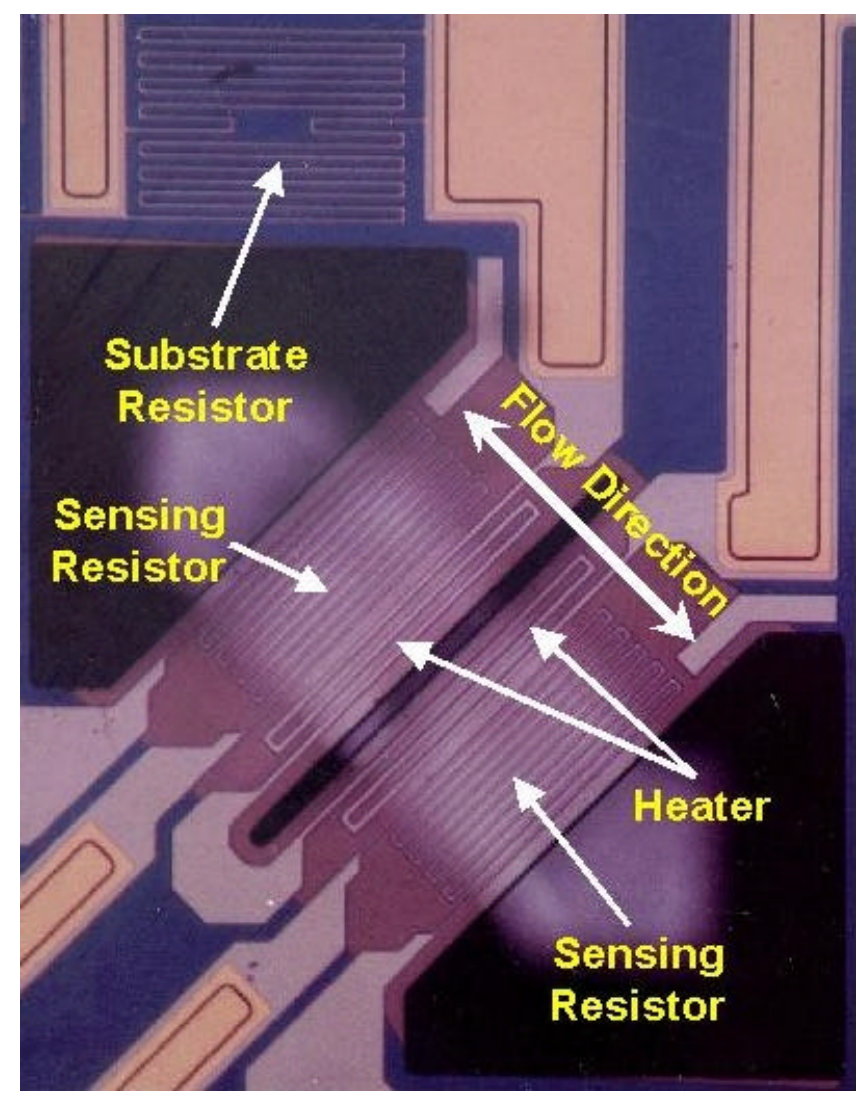

FIGURE 4 - Photograph of a commercial Honeywell AWM2150V flow sensor die. 
isolated silicon nitride membranes with a central heating, serpentine-resistor-element grid divided equally between the two bridges (or cantilevers). In addition, two identical thin-film serpentine resistor grids with relatively-large TCRs served as temperature sensors, placed symmetrically with respect to the heater on each microbridge $[3,18,19]$. Tables II and III summarize the physical and electrical/thermal properties, respectively, of this sensor design. The sensor and heater grids had $5 \mu \mathrm{m}$ line widths and spaces between lines, were made of diffused or (temperature-sensitive) thin-film platinum or permalloy $\left(\mathrm{Ni}_{80} \mathrm{Fe}_{20}\right)$, and were encapsulated in a $\sim 0.8-1.0 \mu \mathrm{m}$ thick

TABLE II

PHYSICAL CHARACTERISTICS OF THE HONEYWELL FLOW SENSOR

\begin{tabular}{|l|c|}
\hline \multicolumn{2}{|c|}{ Honeywell Dual-Bridge Flow Sensor Physical Properties [18] } \\
\hline Etch Pit Depth Below Microbridges & $125-130 \mu \mathrm{m}$ \\
\hline Microbridge Length & $254-508 \mu \mathrm{m}$ \\
\hline Individual Microbridge Width & $127-178 \mu \mathrm{m}$ \\
\hline Microbridge Thickness & $0.8-1.2 \mu \mathrm{m}$ \\
\hline Thin Film Ni ${ }_{80} F e_{20}$ Resistor Thickness & $0.08-0.16 \mu \mathrm{m}$ \\
\hline Minimum Resistor Line Width & $5 \mu \mathrm{m}$ \\
\hline Total Width of Each Serpentine Sense Resistor & $75-100 \mu \mathrm{m}$ \\
\hline Space Between Serpentine Sense and Heater Resistor & $5-25 \mu \mathrm{m}$ \\
\hline Serpentine Sense Resistor Total Length (each) & $175 \mu \mathrm{m}$ \\
\hline
\end{tabular}

TABLE III

HONEYWELL FLOW SENSOR OPERATING CHARACTERISTICS

\begin{tabular}{|l|l|}
\hline \multicolumn{2}{|c|}{ Honeywell Dual-Bridge Flow Sensor Electrical Data [18] } \\
\hline Permalloy, Niso Fe ${ }_{20}, \mathrm{TCR}$ & $\sim 4000 \mathrm{ppm} @ 0{ }^{\circ} \mathrm{C}$ \\
\hline Wheatstone Bridge Output Voltage, $V_{\text {out }}$ & $100 \mathrm{mV} @ 7.62 \mathrm{~m} / \mathrm{s}$ \\
\hline Equivalent Linearized Sensitivity (from $V_{\text {out }}$ ) Ambient & $13.12 \mathrm{mV} /(\mathrm{m} / \mathrm{s})$ \\
\hline Heater Resistor Temperature Range Above Amber & $100-400{ }^{\circ} \mathrm{C}$ \\
\hline Substrate Temperature Above Ambient (heater, $200^{\circ} \mathrm{C}$ above ambient) & $\sim 0.5^{\circ} \mathrm{C}$ \\
\hline Heater Resistor Power (heater at $200^{\circ} \mathrm{C}$ above ambient) & $10 \mathrm{~mW}$ \\
\hline Heater Resistor Current & $\sim 2-3 \mathrm{~mA}$ \\
\hline Sense Resistor Temp. at No Flow (heater at $200^{\circ} \mathrm{C}$ above ambient) & $120-140{ }^{\circ} \mathrm{C}$ \\
\hline Temperature Difference Between Sense Resistors $[20]$ & $80{ }^{\circ} \mathrm{C} @ 5 \mathrm{~m} / \mathrm{s}$ \\
\hline Probe Current (in both sense resistors at no flow conditions) & $0.1-1 \mathrm{~mA}$ \\
\hline Thermal Response Time Constant & $\sim 5 \mathrm{~ms}$. \\
\hline Thermal Efficiency of Heater Power & $15{ }^{\circ} \mathrm{C} / \mathrm{mW}$ \\
\hline Heater Resistance at $25^{\circ} \mathrm{C}$ & $600-1000 \Omega$ \\
\hline Optimal Sense Resistor Resistance (each) at $25^{\circ} \mathrm{C}$ & $700-2000 \Omega$ \\
\hline Optimal Match Between Up and Downstream Resistors & $\sim 0.1 \%$ \\
\hline
\end{tabular}


dielectric silicon nitride film, which comprised the suspended microbridges. Anisotropic etching of the silicon substrate (with $\mathrm{KOH}$ plus isopropyl alcohol) was used to create an air space pit below the microbridges that was preferably $\sim 125 \mu \mathrm{m}$ deep (as shown in Figure 5), precisely bounded on the sides by (111) silicon planes, and on the pit bottom and ends of the bridges by the (100) and other planes (as shown in Figure 6). The symmetry and effectiveness of the microbridge etched undercut was maximized by orienting the longitudinal axis of each bridge at an angle of $45^{\circ}$ with respect to the $<110>$ direction in the monocrystalline silicon substrate [4].

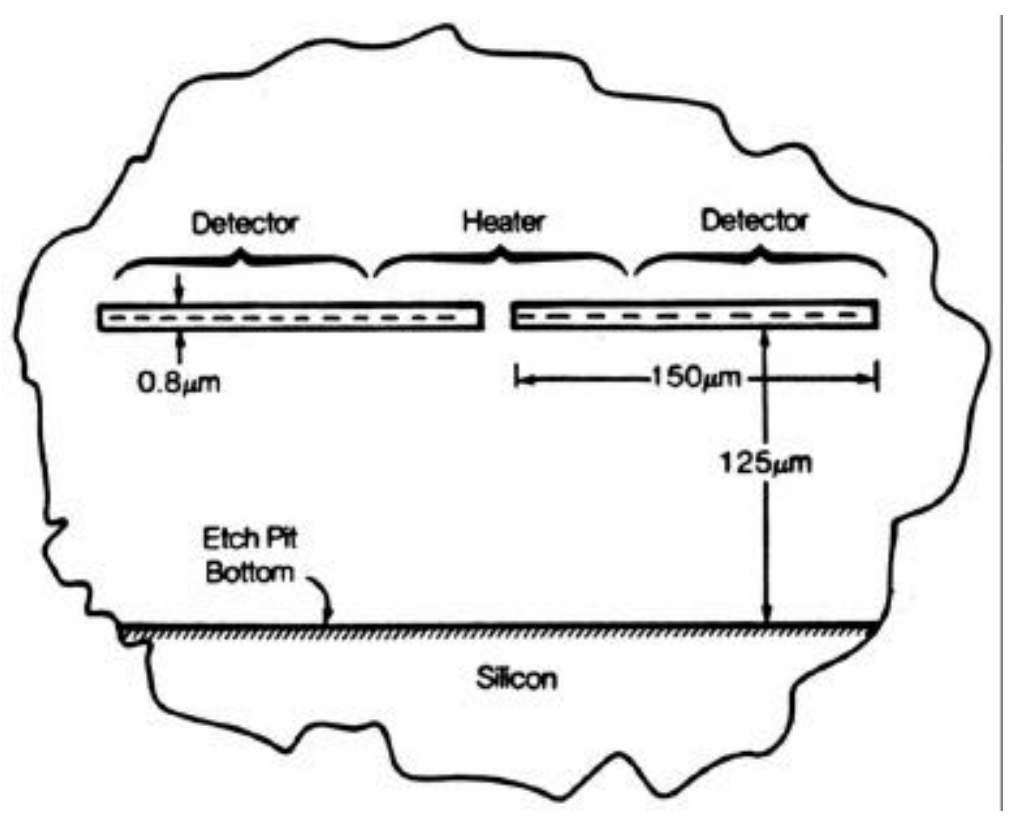

FIGURE 5 - Cross-section of the Honeywell mass airflow sensor microstructure [20].

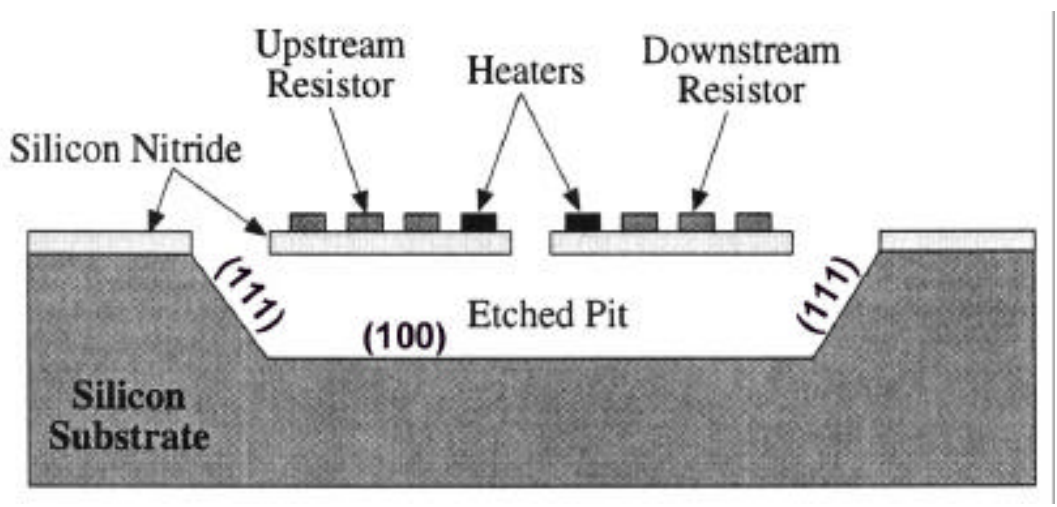

FIGURE 6 - A cut-away view of the Honeywell sensor on a (100) silicon substrate. This drawing only shows a bottom $\mathrm{Si}_{3} \mathrm{~N}_{4}$ layer and metallization layer without the top $\mathrm{Si}_{3} \mathrm{~N}_{4}$ passivation layer. [19]. 
The Honeywell flow sensor was designed to realize the following advantages from suspending the heater and sensing resistors in a silicon nitride dielectric material over an etched cavity with flow directed laterally across the dual-bridge, cantilever, membrane, or diaphragm microstructure $[18,20,21]$ :

1. Excellent thermal isolation from the silicon substrate resulted in an efficient heater and a very sensitive transducer. The sensor's air flow response was quite independent of thermal contact between the silicon substrate and the supporting $\mathrm{Si}_{3} \mathrm{~N}_{4}$ microbridges. The temperature gradient achievable between the silicon substrate and the microbridges was 100 to $200{ }^{\circ} \mathrm{C}$, with a thermal efficiency in the order of $15^{\circ} \mathrm{C} / \mathrm{mW}$ (e.g. less than 10 $\mathrm{mW}$ was required to increase the heater temperature by $\left.100^{\circ} \mathrm{C}\right)$. The thermal conductivity of $\mathrm{Si}_{3} \mathrm{~N}_{4}$ is $\sim 2.3 \mathrm{~W} / \mathrm{mK}$ compared to $150 \mathrm{~W} / \mathrm{mK}$ for silicon. Heat transfer values of $1 \times 10^{-7} \mathrm{~W} / \mathrm{mK}$ or less can be achieved in air. The thin, insulating $\mathrm{Si}_{3} \mathrm{~N}_{4}$ film contributed very little to loss of heat from the heater with nearly all the heat conducted from the heater resistor to the sense resistors through the air surrounding the heater (the thermal conductance of the $\mathrm{Si}_{3} \mathrm{~N}_{4}$ dielectric was almost negligible). Thus, compared to the internal thermal conduction in the suspended microstructure, the thermal transport of the structure to the surrounding fluid by wall heat transfer was very large. This thermallyisolated design was also responsible for achieving the large $50-80^{\circ} \mathrm{C}$ temperature differential between sense resistors at maximum flow $(5-8.6 \mathrm{~m} / \mathrm{s})$ across the microbridges.

2. The very low thermal conductivity of the $\mathrm{Si}_{3} \mathrm{~N}_{4}$ film microstructures also allowed for the design of a very compact sensor, where the sensing resistor grids could be placed immediately adjacent to the heater resistor grid (with a heater-to-sense-resistor spacing of one-to-five line widths, or $5-25 \mu \mathrm{m}$ ). Most of the heat conducted to the sensing resistors from the heater resistor passed through the surrounding air, rather than through the supporting $\mathrm{Si}_{3} \mathrm{~N}_{4}$ dielectric thin-film. The sensing resistor grids were suspended in the air space near the heater, acting as thermal probes to measure the temperature of the air near, and in the plane of, the heater resistor. 
3. The sensor had a very small heat capacity, resulting in a small time constant for the sensor's response, which was due to the exceedingly small thermal mass of the heater and sensor element microbridge structure, the thermal insulation provided by the suspended microbridges, and the surrounding air space.

4. The ambient gas stream temperature could be measured on-chip by an additional heatsunk thin-film resistor on the silicon substrate, because of the significant thermal isolation provided by the microbridges. For example, with a heater temperature of $150{ }^{\circ} \mathrm{C}$, the temperature rise detected in the substrate was $1^{\circ} \mathrm{C}$ or less with most of the thermal conduction from the heater resistor occurring through the air to the substrate. Thermal conduction through the thin dielectric film could usually be neglected.

5. Laterally-directed flow (i.e. across the suspended microstructures) allows air flow both under and over the microbridges for more efficient and complete heat transfer from the thermally-isolated heater into the air stream.

6. A more co-planar relationship between the heater and sensors was realized in this configuration (versus having separate microbridges for each of the heater and sense resistors) since each sense resistor grid was directly adjacent to, and in the same plane as, a portion of the heater resistor on the same microbridge.

7. The temperature sensitive thin film resistors and heater were largely protected from damage by impacting particles in the air stream because they were not in high velocity regions of the stream that were a substantial distance away from the neighborhood of the low velocity surface layer of the flow stream where the resistors were located (and since the flow was directed parallel to the plane of the microbridges).

Today Honeywell's Micro Switch division markets a product line of mass airflow sensors that are commercially available in a package with a flow channel including a dust filter to protect the MEMS microstructure from contamination and damage. The current (year 2000) product line is shown in Figure 7. Data sheets for the Honeywell AWM5000 series (0 - 20 SLPM) sensor product line and die photos of a AWM2150V flow sensor are included in Appendices I and II. 


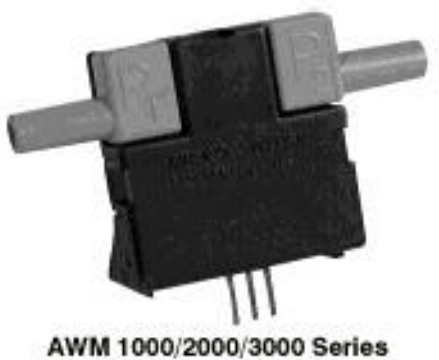

AWM 1000/2000/3000 Series

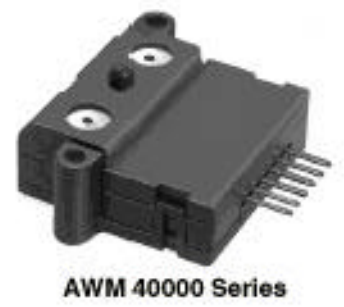

AWM 40000 Series

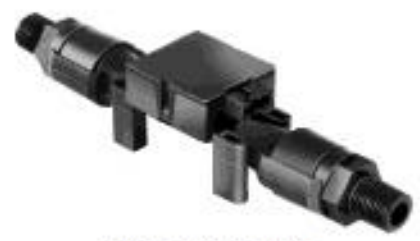

AWM 5000 Series

FIGURE 7 - $\quad$ Honeywell's year 2000 mass airflow sensor product line [22].

\section{E. How the Honeywell Flow Sensor Works}

In operation, fully-developed laminar air flow is directed laterally across the microbridges in a precisely-dimensioned, high-impedance flow channel having a $0.05 \times 0.25 \mathrm{~cm}^{2}$ cross section. Heat is transported away from the upstream sense-resistor sensor toward the heater resistor and the downstream sense resistor. The downstream sense resistor is heated to about $60-70 \%$ of the temperature elevation of the heater resistor, which is typically operated at a constant temperature of $100-200^{\circ} \mathrm{C}$ above the ambient. The resulting temperature and corresponding resistance differential between the sense resistors, when connected in a constant-voltage-mode DC Wheatstone half bridge configuration (as shown in Figure 8), yields a ratiometric bridge output voltage that is proportional to the flow velocity. The sense resistor Wheatstone bridge could also be operated in constant temperature (constant resistance), constant power, or any mode producing a differential signal. This bridge contains a sense resistor (the upstream resistor, $R_{u p}$, or downstream resistor, $\left.R_{\text {down }}\right)$ and a high-impedance resistor $\left(R_{3}\right.$ or $\left.R_{4}\right)$ in each leg, with a nulling potentiometer $\left(P_{1}\right)$ to balance the bridge at no flow. Resistors $R_{3}$ and $R_{4}$ are identical and typically $\sim 25 \mathrm{k} \Omega$. In Honeywell's commercial flow sensors, sense resistors $R_{\text {up }}$ and $R_{\text {down }}$ are typically $\sim 1.5 \mathrm{k} \Omega$ and should be ideally within $1 \%$ of each other to allow for the elimination of zero-point offsets due to variations in ambient pressure and temperature. The circuit configuration shown in Figure 8 has an optional differential instrumentation amplifier connected to the output of the sense resistor Wheatstone half-bridge to provide significant voltage amplification and introduce voltage offsets (set by resistors $R_{10}, R_{11}$, and $R_{12}$ ) to the sensor's output. The amplifier gain is adjusted with potentiometer $P_{2}$. Temperature differences between up and 


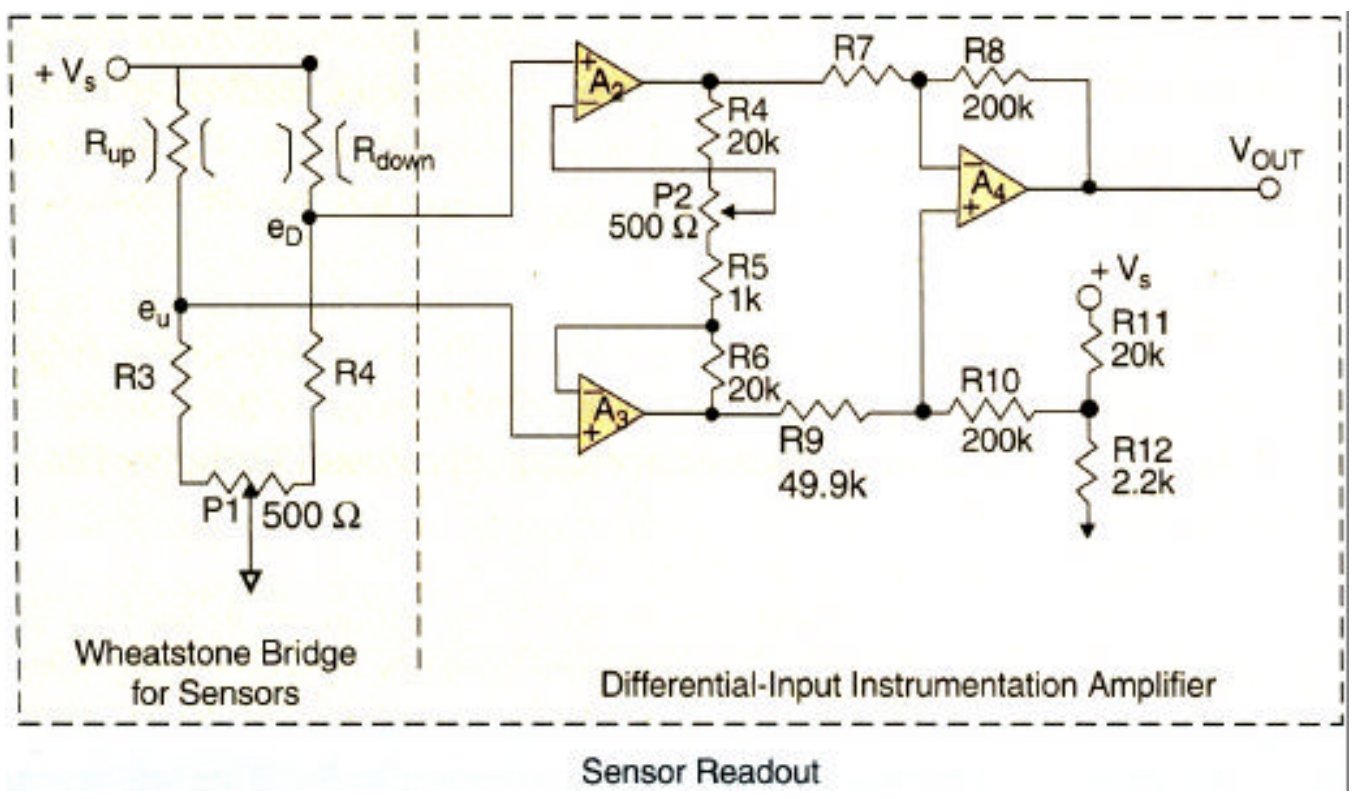

FIGURE 8 - Honeywell's Wheatstone bridge sense-resistor configuration. It included an optional external differential instrumentation amplifier, for the flow sensor, where " $R_{\text {up }}$ " is the upstream and " $R_{\text {down }}$ " is the downstream sensing resistor on the suspended microbridge. Resistors $R_{3}$ and $R_{4}$ are either laser-trimmed and located on-chip, or external with potentiometer $\mathrm{P}_{1}$, which is used to balance or null the Wheatstone bridge voltage at no flow. Note that the sensitivity of this device could be improved by making $R_{3}$ and $R_{4}$ sense resistors with an opposite TCR characteristic than $R_{\text {up }}$ and $R_{\text {down }} \mathrm{c}$.

downstream resistors achievable during air flow vary depending upon the microbridge configuration and resistor layout geometry. The 1985 Honeywell patent disclosure reported a maximum temperature difference of $50^{\circ} \mathrm{C}$ between the up and downstream resistors at a flow velocity of $\sim 8.64 \mathrm{~m} / \mathrm{s}$ (1700 ft/min), as shown in Figure 9. Other published papers describing the Honeywell device report an achieved temperature differential of $80^{\circ} \mathrm{C}$ for a flow velocity of $5 \mathrm{~m} / \mathrm{s}$ (and pressure difference of $497.7 \mathrm{~Pa}$ ).

The Honeywell sensor was designed to operate in a constant-heater-temperature mode, to both minimize errors due to flow stream ambient temperature variations, and keep the heater temperature at a constant differential above the ambient temperature (typically $160-200^{\circ} \mathrm{C}$ ), even if the ambient temperature fluctuates. This mode of operation also reduces (but does not eliminate) the effects of changing flow-stream gas composition which could alter the thermal conductance of the heater and detector resistances (thereby changing their operating 


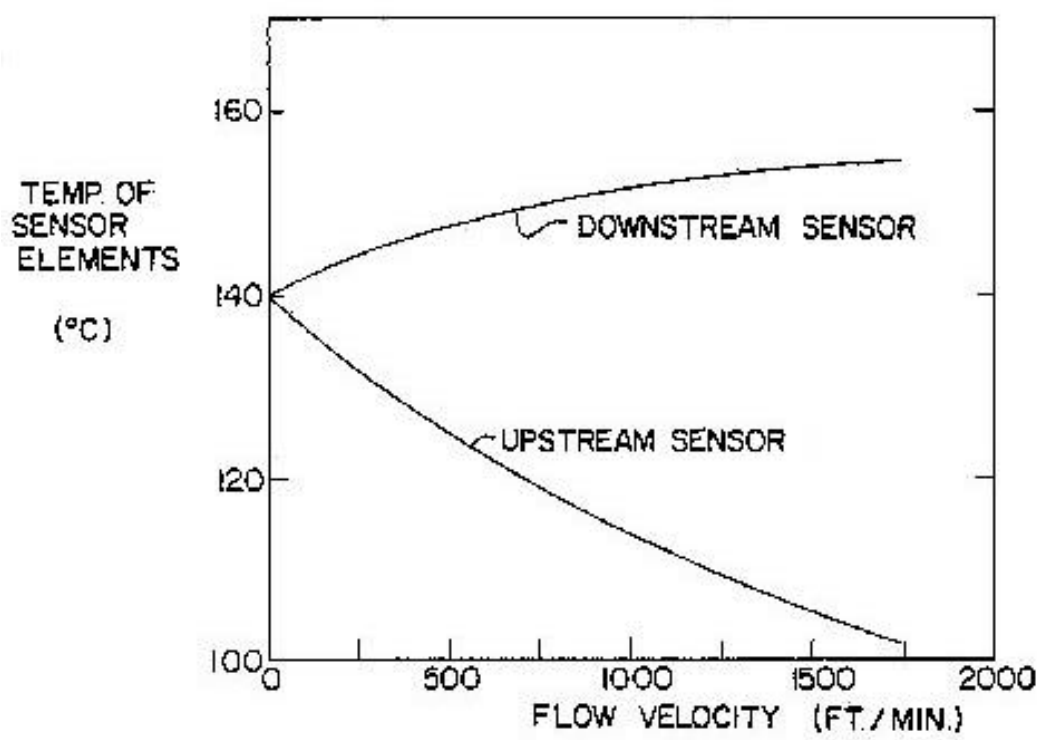

FIGURE 9 - $\quad$ Temperature differences between the up and downstream sense resistors. This was for a flow sensor operating in a constant-heater-temperature-above theambient-mode, as disclosed in U.S. Patent \# 4,501,144.

temperatures). As shown in Figure 10, a DC Wheatstone half-bridge circuit configuration was also used with the heater, $R_{h}$, substrate-temperature-sensing resistor, $R_{r}$, and non-linear $\mathrm{Ni}_{80} \mathrm{Fe}_{20}$

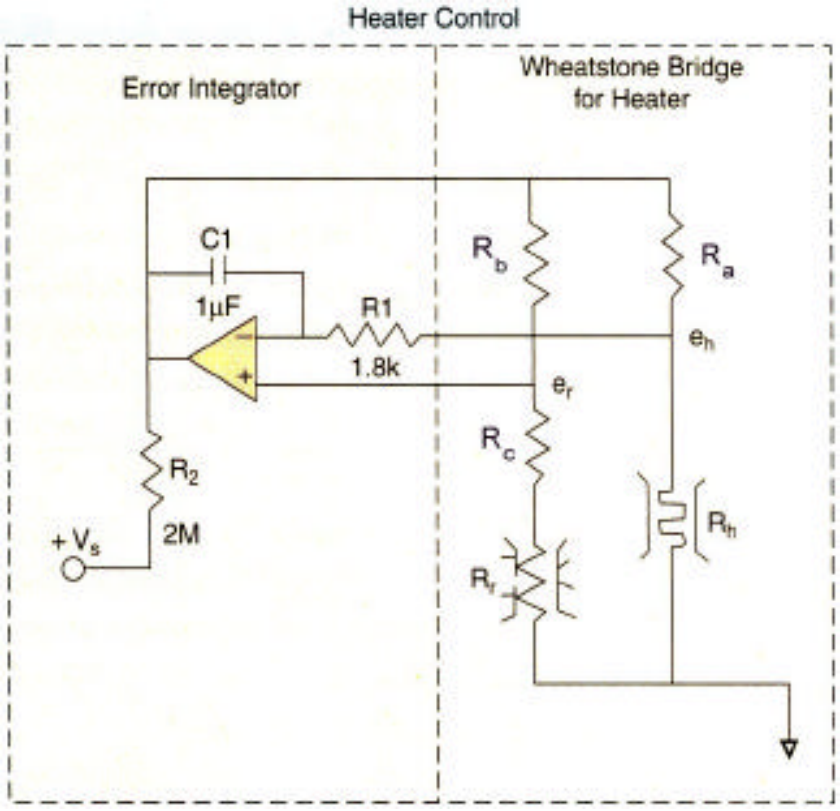

FIGURE 10 - The error-integrator Wheatstone half-bridge heater-driver circuit. In this Honeywell design, resistor $R_{r}$ is the serpentine substrate temperaturecompensation, heat-sunk, reference, sensor resistor; $R_{h}$ is the heated sensor resistor; and $R_{a}, R_{b}$, and $R_{c}$ are external resistors with values selected to compensate for the nonlinear TCR characteristic of $\mathrm{Ni}_{80} \mathrm{Fe}_{20}$ [7]. 
TCR-compensating resistors $R_{a}, R_{b}$, and $R_{c}$. This circuit has a temperature-compensating substrate resistor sensor $\left(R_{r}\right)$ in one bridge arm and a heated resistor/sensor $\left(R_{h}\right)$ located in the other bridge arm. Ambient temperature is sensed by the heat-sunk, serpentine, substrate-resistor sensor $\left(R_{r}\right)$. The substrate temperature typically remains within about one degree $(C)$ of the flow stream temperature and is a good approximation of the ambient temperature. The bridge becomes unbalanced when the heater resistor/sensor $\left(R_{h}\right)$ is cooled from the flow velocity of the fluid. The bridge output voltage is fed into an error integrator that contains a high-gain feedback amplifier. The error integrator detects any bridge unbalance, integrates voltage differences from the bridge output, and keeps the bridge balanced by varying the applied voltage potential across it, and thus the power dissipated in the heater resistor (by feeding more current into the supply voltage node of the bridge, until the bridge reaches its previous operating-point, balanced state). This compensation causes the heater resistor/sensor to be brought back to its initial temperature by increasing/decreasing current flow through the heater with increasing/decreasing thermal cooling due to air flow across it (respectively). Since the TCR characteristic of the thin-film $\mathrm{Ni}_{80} \mathrm{Fe}_{20}$ resistor metallization is nonlinear, ambient temperature changes do not produce proportional changes in the heater $\left(R_{h}\right)$ and reference resistor $\left(R_{r}\right)$ resistances, when the heater is operated at an elevated temperature. Consequently, external resistors $R_{a}, R_{b}$, and $R_{c}$ are chosen to compensate for this effect to keep the Wheatstone bridge ratios equal at a given operating temperature differential above the ambient. With proper selection of $R_{a}, R_{b}$, and $R_{c}$, the error in heater temperature can be kept to less than $1 \%$ of its differential above the ambient over an ambient temperature range of $-40^{\circ} \mathrm{C}$ to $80^{\circ} \mathrm{C}[20]$.

The responsiveness of the Honeywell flow sensor can be described in terms of the transit time for air flowing across the heated zone of the dual-bridge microstructure (which includes the serpentine detector resistors) and the related thermal diffusion time. The transit time required for a parcel of air flowing across the microstructure at a distance away equal to its width (i.e. of the heated zone of the bridges) can be comparable to the characteristic thermal diffusion time for heat transport to that distance. Hence, for that velocity, an ambient lateral thermal boundary will be located at that distance, which is comparable to the etch pit depth. Therefore, relative to the 
no-flow condition, a large increase in heat transfer will occur above the heater and detectors on the microbridges. The characteristic thermal diffusion time may be considered the transit time, which is calculated as follows:

$$
t_{c}=\frac{W_{\text {bridge }}}{\bar{v}_{\text {air }}}
$$

where $W_{\text {bridge }}$ is the width $(\mathrm{m})$ of the microbridge heated zone, and $\bar{v}_{\text {air }}$ is the average air velocity (m/s) over the bridge. For air at $25^{\circ} \mathrm{C}$ as the flowing fluid over the planar microbridge region, the thermal diffusion time is associated with the temperature penetration depth (TPD) parameter for sinusoidal temperature variation at a planar boundary surface:

$$
T P D=\sqrt{\frac{k \cdot t_{c}}{\rho \cdot c_{p}}} \quad(\mathrm{~m}),
$$

where $k$ is the thermal conductivity of air $\left(0.026 \mathrm{~W} / \mathrm{m} \cdot{ }^{\circ} \mathrm{C}\right), \rho$ is the density of air $\left(1.1845 \mathrm{~kg} / \mathrm{m}^{3}\right)$, $C_{p}$ is the specific heat of air $\left(1006.3 \mathrm{~J} / \mathrm{kg} \cdot{ }^{\circ} \mathrm{C}\right)$, and $t_{c}$ is the characteristic diffusion time in seconds. Consequently, the thermal diffusion much beyond the TPD is severely limited by the flow, which tends to remove heat as fast as it is diffused out to that distance, thus forming the ambient temperature boundary. The transit time directly upstream from detector to heater is similar, and in this case the diffusion of heat occurs directly against the flow. Therefore, the upstream detector experiences a strong cooling effect at low velocities, even though frictional drag near the supporting $\mathrm{Si}_{3} \mathrm{~N}_{4}$ film (on the microbridge surface) slightly reduces the effective flow velocity. The downstream detector tends to be slightly heated because, except for high velocities, heat transport by air flow from the heater counteracts the effect of nearby lateral thermal boundary formation [20].

A thin-film resistor's TCR is defined as: 


$$
T C R=\frac{\left(\frac{\Delta R}{R_{o}}\right)}{\Delta T} \quad\left(\Omega / \Omega /{ }^{\circ} \mathrm{C}\right),
$$

where $\Delta R$ is a proportional change in resistance, from a nominal value $R_{0}$, due to a temperature change, $\Delta T$. In the Wheatstone half-bridge shown in Figure 10, the bridge is balanced (i.e. the output voltage of the bridge or input voltage to the error integrator is zero) when:

$$
\frac{R_{b}}{R_{c}+R_{r}}=\frac{R_{a}}{R_{h}} .
$$

If the Wheatstone half-bridge is not balanced, the output voltage is:

$$
V_{\text {out }}=V_{\text {in }}\left(\left(\frac{R_{b}}{R_{b}+R_{c}+R_{r}}\right)-\left(\frac{R_{a}}{R_{a}+R_{h}}\right)\right)
$$

where $V_{\text {in }}$ is the output voltage of the error-integrator op-amp. When the heater-driver circuit is active, the heater-resistor temperature will be at a fixed differential above the ambient $\left(\Delta T_{h}\right)$, or typically $200^{\circ} \mathrm{C}$, and the substrate temperature may change (by a differential $\Delta T_{a m b}$ ) if the flow stream temperature changes from the ambient temperature. These temperature changes may be related as follows:

$$
\Delta T_{\text {total }}=\Delta T_{h}+\Delta T_{\text {amb }} .
$$

If the temperature coefficient (TCR) of the thin-film resistors is non-linear, it will not have the same magnitude in different temperature regions. Thus, the $200^{\circ} \mathrm{C}$ elevated-temperature above a $25^{\circ} \mathrm{C}$ ambient TCR of the heater resistor, or $T C R_{225}$, may not be equal to the low-temperature 
(25 ${ }^{\circ} \mathrm{C}$ ambient), temperature coefficient of the substrate reference resistor, $T C R_{25}$. Of course, if the characteristic TCR of the resistors is linear across the operating temperature range of the device $\left(-25\right.$ to $\left.+85^{\circ} \mathrm{C}\right)$ and the heater resistor $\left(175-285^{\circ} \mathrm{C}\right)$, then:

$$
T C R_{225}=T C R_{25}(\text { linear TCR })
$$

When the heater driver circuit is active, the heater-resistor resistance, $R_{h}$, will change by a differential, $\Delta R_{h}$, based on its elevated temperature $T C R_{T 2}$ at temperature $T 2$, as follows:

$$
R h_{T 2}=R h_{25}\left(T C R_{T 2} \Delta T+1\right)
$$

where $R h_{T 2}$ is the new heater-resistor resistance at temperature $T 2, R h_{25}$ is the measured heaterresistor resistance at a $25^{\circ} \mathrm{C}$ ambient, and $\Delta T=T 2-25$. The substrate reference-resistor resistance, $R_{r}$, may change based on its ambient-temperature TCR, TCR ${ }_{25}$, by a differential, $\Delta R_{r}$, if there is an ambient flow stream temperature change. For a balanced heater-driver circuit bridge configuration, Equations (3), (4), and (6) may be combined to show the effect of temperature on changes in resistance in the bridge:

$$
\frac{R_{b}}{R_{c}+R_{r}\left[T C R_{25}\left(\Delta T_{\text {amb }}+0.5\right)+1\right]}=\frac{R_{a}}{R_{h}\left[T C R_{225}\left(\Delta T_{\text {total }}\right)+1\right]},
$$

where the resistor values are all nominal values measured at $25^{\circ} \mathrm{C}$, and it is assumed that substrate heating due to the heater resistor operating at $225^{\circ} \mathrm{C}$ is $0.5^{\circ} \mathrm{C}$. With the heater resistor operated at $\Delta T_{h}{ }^{\circ} \mathrm{C}$ above an ambient flow stream temperature of $25^{\circ} \mathrm{C}$, the balanced bridge equation may be re-written as: 


$$
\frac{R_{b}}{R_{c}+R_{r}\left[T C R_{25}(0.5)+1\right]}=\frac{R_{a}}{R_{h}\left[T C R_{225}\left(\Delta T_{h}\right)+1\right]}
$$

Although the exact operating point, type of metallization(s), and corresponding TCR characteristics of the commercial Honeywell flow sensor are not publicly known, some assumptions can be made from published data sheets. It seems that Honeywell did not design its current commercially-available flow sensor products to have a perfectly balanced heater-resistor half-bridge circuit at its operating point. For example, data sheets and press releases for the AWM3303V flow sensor (with a $\pm 1000 \mathrm{sccm}$ measurement range) indicate that its thin-film heater and temperature-sensitive resistors were made of platinum, with nominal resistance values of 3,000 $\Omega$ for $R_{b}, 500 \Omega$ for $R_{a}, 1,500 \Omega$ for $R_{c}, 6,000 \Omega$ for $R_{r}$, and $600 \Omega$ for $R_{h}$ [23]. Heater resistors in Honeywell's commercial flow sensors were designed to operate at $160^{\circ} \mathrm{C}\left(\Delta T_{h}\right)$ above the ambient. Platinum thin-film resistors typically have temperature coefficients ranging from approximately $0.00375-0.00385 \Omega / \Omega /{ }^{\circ} \mathrm{C}$ [24]. At $25^{\circ} \mathrm{C}$ ambient conditions, using a "linear" TCR value of $0.0038 \Omega / \Omega /{ }^{\circ} \mathrm{C}$ for both $T C R_{25}$ and $T C R_{185}$, and using the aforementioned resistor values, the bridge ratios shown in Equation (10) become:

$$
\begin{gathered}
\frac{3000}{1500+6000[0.0038(0.5)+1]}=0.3994, \text { and } \\
\frac{500}{965 \cdot[0.0038 \cdot(160)+1]}=0.3223 .
\end{gathered}
$$

For this same configuration, if the ambient flow stream temperature increases by $50^{\circ} \mathrm{C}$ (to $75^{\circ} \mathrm{C}$ ), with the assumption of a "linear" TCR, Equation (9) can be used to find the new bridge ratios: 


$$
\frac{3000}{1500+6000[0.0038(50+0.5)+1]}=0.3468 \text {, and }
$$

$$
\frac{500}{965[0.0038(160+50)+1]}=0.2882 .
$$

Of course, if the TCR for the metallization in the Honeywell flow sensors is nonlinear (which it mostly likely is), than these ratios would be slightly different.

Permalloy $\left(\mathrm{Ni}_{80} \mathrm{Fe}_{20}\right)$, with a TCR of approximately $0.004 \Omega / \Omega /{ }^{\circ} \mathrm{C}$, has been found to be problematic as a thin-film metallization layer for this type of sensor, because of its nonlinear TCR characteristics. Illustrating this nonlinear property, the graphs shown in Figure 11 depict a thinfilm-permalloy resistor metallization Honeywell flow sensor output vs. mass flow rate for different ambient temperatures. A patent issued to Johnson, Higashi, et. al. at Honeywell, in 1988

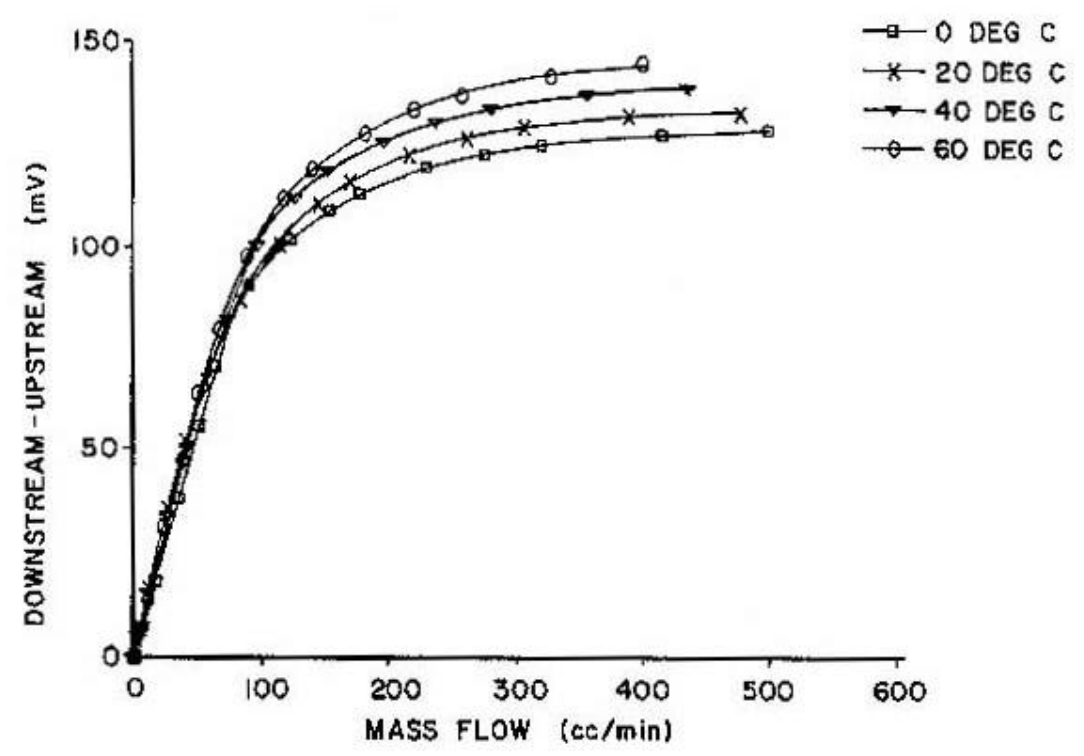

FIGURE 11 - Output vs. mass flow rate for non-linear TCR permalloy flow sensors. This type of flow sensor used permalloy $\left(\mathrm{Ni}_{80} \mathrm{Fe}_{20}\right)$ thin-film resistors with non-linear TCR properties, as described in U.S. patent \#4,739,657.

improves upon this flow sensor design to achieve a linear TCR characteristic. This patent describes the delineation of a serpentine resistor grid (for the heater and each sense resistor) that combines, in series, a small permalloy length of resistor film with a long length of platinum film 
[25]. Platinum exhibits a TCR non-linearity in one direction, and the permalloy exhibits nonlinearity in the opposite direction. Figure 12 shows the resistance vs. temperature characteristic for permalloy and platinum, with permalloy's characteristic curving up and platinum's curving down with increasing temperature.

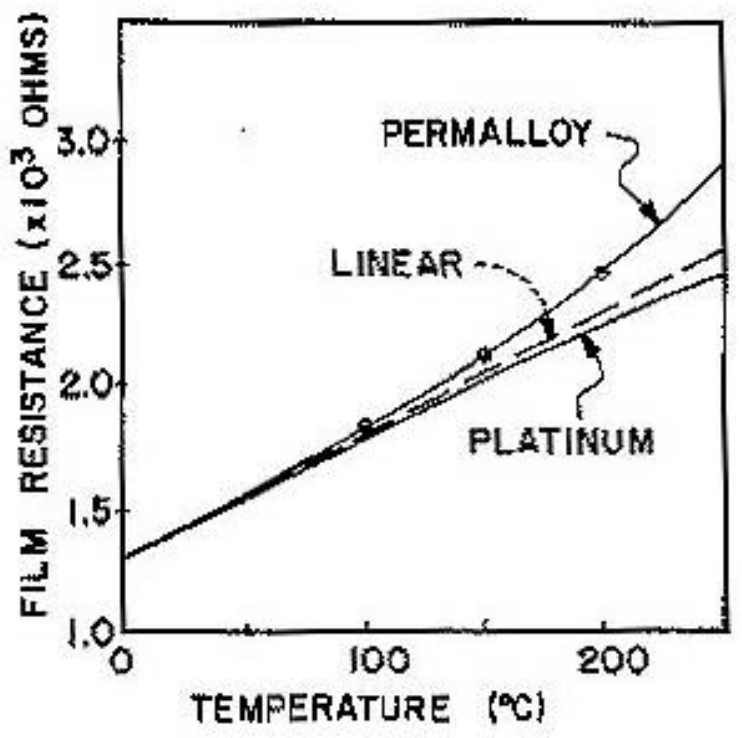

FIGURE 12 - Thin-film resistance vs. temperature characteristic for permalloy and platinum. This graph was shown in U.S. patent \#4,739,657.

\section{F. Platinum Thin Films}

Platinum is a precious metal with a very stable, and nearly linear resistance vs. temperature characteristic, which can be accurately modeled by the Callendar-Van Dusen equation [24]:

$$
R_{T}=R_{o}\left(1+A T+B T^{2}-100 C T^{3}+C T^{4}\right) \quad(\Omega)
$$

or alternatively as:

$$
R_{T}=R_{s} N_{P t}\left(1+A T+B T^{2}-100 C T^{3}+C T^{4}\right)
$$


where,

$$
R_{s}=\frac{\rho}{t} \quad(\Omega \text { /square })
$$

Sheet resistance is shown as $R_{s}$, for a thin-film platinum resistor having $N_{P t}$ number of squares, with resistivity, $\rho_{r}$, and thickness $t$. The value $R_{o}$ is the resistor's resistance at $0{ }^{\circ} \mathrm{C}$, and $R_{T}$ is the resistance at temperature $T$ (in ${ }^{\circ} \mathrm{C}$ ). Common values for constants $A, B$, and $C$, which are derived from resistance measurements at 0,100 , and $260^{\circ} \mathrm{C}$ (respectively), are shown in Figure 13. For temperatures above $0^{\circ} \mathrm{C}$, constant $C$ is equal to zero, and Equation (15) is reduced to a

CALLENDAR-VAN DUSEN CONSTANTS

\begin{tabular}{|l|c|c|}
\hline Alpha, $\alpha\left({ }^{\circ} \mathrm{C}^{-1}\right)$ & $.003750 \pm .00003$ & $.003850 \pm .0001$ \\
\hline Delta, $\delta\left({ }^{\circ} \mathrm{C}\right)$ & $1.605 \pm 0.009$ & $1.4999 \pm 0.007$ \\
\hline Beta, $\beta^{\star}\left({ }^{\circ} \mathrm{C}\right)$ & 0.16 & 0.10863 \\
\hline A $\left({ }^{\circ} \mathrm{C}^{-1}\right)$ & $3.81 \times 10^{-3}$ & $3.908 \times 10^{-3}$ \\
\hline B $\left({ }^{\circ} \mathrm{C}^{-2}\right)$ & $-6.02 \times 10^{-7}$ & $-5.775 \times 10^{-7}$ \\
\hline C $\left({ }^{\circ} \mathrm{C}^{-4}\right)^{\star}$ & $-6.0 \times 10^{-12}$ & $-4.183 \times 10^{-12}$ \\
\hline
\end{tabular}

FIGURE 13 - Callendar-Van Dusen constants for thin-film platinum RTDs. These constants are used to model their resistance vs. temperature characteristics [24].

quadratic expression which can be used to analytically model the resistance versus temperature characteristic for a platinum thin-film RTD or resistor. The Callendar-Van Dusen constants $A, B$, and $C$ are derived from $\alpha$ (a term related to the TCR of platinum), and other constants $\beta$ and $\delta$, which are obtained from actual resistance measurements, and are all related as follows:

$$
A=\alpha+\frac{\alpha \delta}{100}
$$




$$
B=\frac{-\alpha \delta}{100^{2}},
$$

$$
C_{T<0}=\frac{-\alpha \beta}{100^{4}} \quad\left(\beta \text { is a constant for } \mathrm{T}<0^{\circ} \mathrm{C}\right),
$$

$$
\alpha=\frac{R_{100}-R_{o}}{100 R_{o}} \text {, and } \delta=\frac{R_{o}(1+260 \alpha)-R_{260}}{4.16 \alpha\left(R_{o}\right)}
$$

Values $R_{100}$ and $R_{260}$ are measured resistances at 100 and $260^{\circ} \mathrm{C}$, respectively. Figure 14 shows a typical relative (i.e. normalized) resistance versus temperature plot for a typical thin-film platinum RTD.

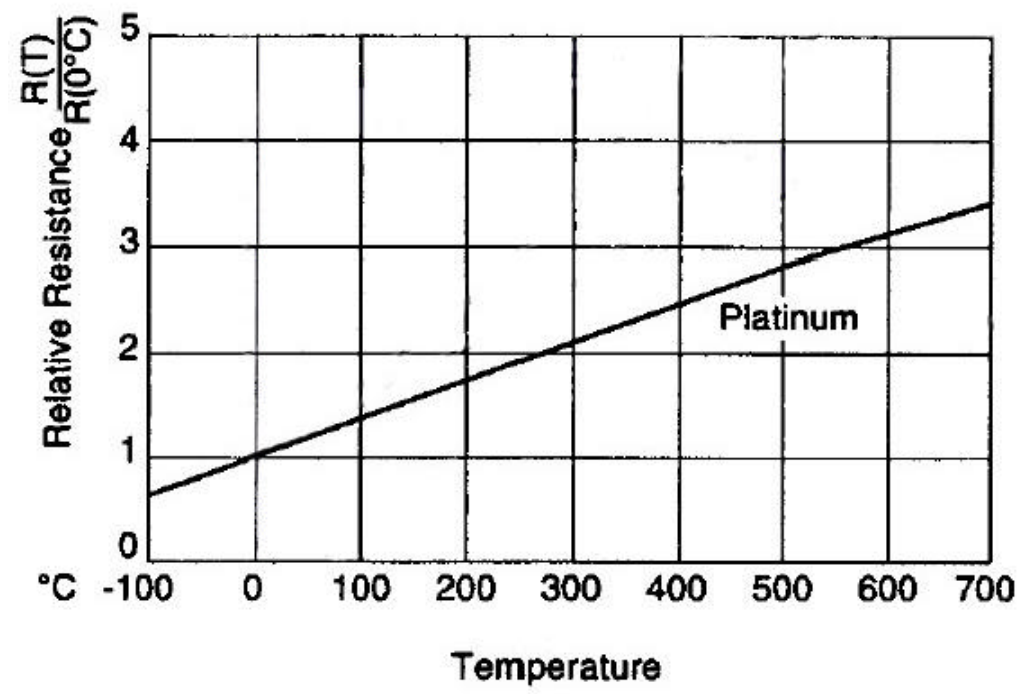

FIGURE 14 - Relative resistance versus temperature curve for a typical Platinum RTD. Adapted from [24].

It is significant to note that platinum's thin-film resistivity and TCR properties may be very different from bulk material values. Platinum thin-film resistors have temperature coefficients ranging from approximately 0.00375 to $0.00385 \Omega / \Omega /{ }^{\circ} \mathrm{C}$, which are considerably lower than the 
theoretical maximum value for pure bulk platinum: $0.0039289 \Omega / \Omega /{ }^{\circ} \mathrm{C}$. It is well known that the TCR for a pure elemental bulk metal is very sensitive to impurities, defects, and strains. Higher concentrations of impurities and defects will lower an elemental metal's TCR, which is itself a very complicated characteristic in thin films. The TCR may be affected by factors which influence overall film structure, such as the presence of impurities or defects in the microstructure; deposited grain size; grain boundary structure; grain boundary oxidation; grain shape regularity; small holes among boundaries; dislocations within polycrystal grains (the void network structure); sputtering gas atoms entrapped in the lattice during deposition; residual strains; plastic deformation (caused by tensile or compressive stress gradients); film discontinuity; film thickness; and surface roughness. In particular, a sputter-deposited platinum thin film's TCR will be affected by characteristics of the deposition process itself, surface morphology of the substrate; film thickness; residual stress caused by any mismatch in thermal expansion coefficient between it and the substrate; film discontinuity; and defects such as dislocations within polycrystal grains. The surface morphology of as-deposited platinum films is strongly influenced by the initial surface condition of the substrate. These are the main factors which result in platinum's thin-film TCR being much lower than its pure, bulk value. The control of these variables which contribute to the lowering of its TCR is very difficult; and, consequently, very few techniques are available to deposit metal films with a controlled TCR value $[26,27]$.

The thickness of a platinum thin film can also significantly affect its TCR characteristic. In its thin-film state, platinum achieves a maximum TCR with a thickness of at least $0.35 \mu \mathrm{m}$. A plot of TCR versus film thickness for a typical platinum RTD with a "bulk" TCR of $0.00388 \Omega / \Omega /{ }^{\circ} \mathrm{C}$ is shown in Figure 15. Here, "bulk" means the TCR value which is approached at a film thickness where very little change in TCR is experienced with changes in film thickness. In Figure 15, as the film thickness decreases from $4 \mu \mathrm{m}$, the TCR changes very little, until the thickness is less than $1.0 \mu \mathrm{m}$. Below $1.0 \mu \mathrm{m}$, the TCR decreases more and more rapidly with thickness. For example, at $0.2 \mu \mathrm{m}$, a $50 \%$ decrease in film thickness (to $0.1 \mu \mathrm{m}$ ) will decrease the TCR by $2.2 \%$; whereas, at $4 \mu \mathrm{m}$, a $50 \%$ decrease in film thickness will change the TCR by less than $0.1 \%$. The ratio of relative change in TCR to relative change in film thickness is a measure of TCR sensitivity 


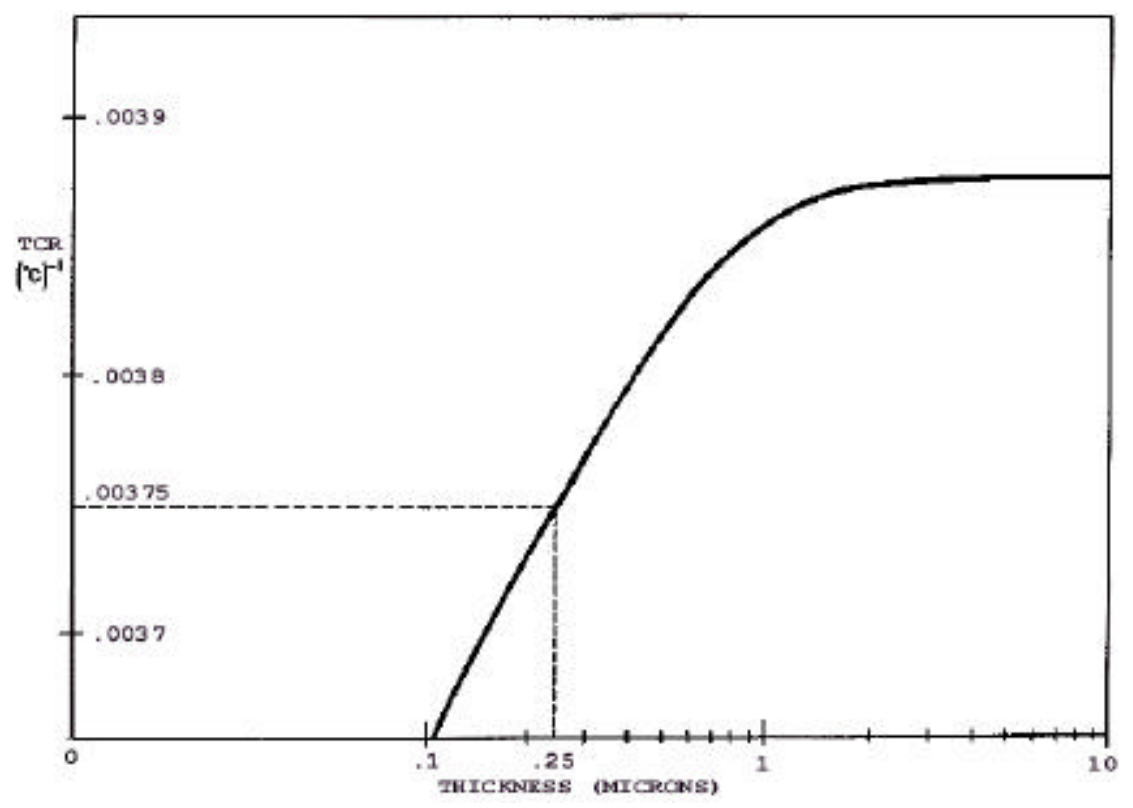

FIGURE 15 - A plot of TCR vs. thin-film thickness for a platinum RTD. These data are for a typical RTD with a "bulk" TCR of $0.00388 \Omega / \Omega /{ }^{\circ} \mathrm{C}$, showing the variation in TCR with film thickness. Sputter-deposited platinum thin films may have a different characteristic curve, where the TCR vs. thickness relationship should be experimentally determined for each unique set of sputtering parameters [27].

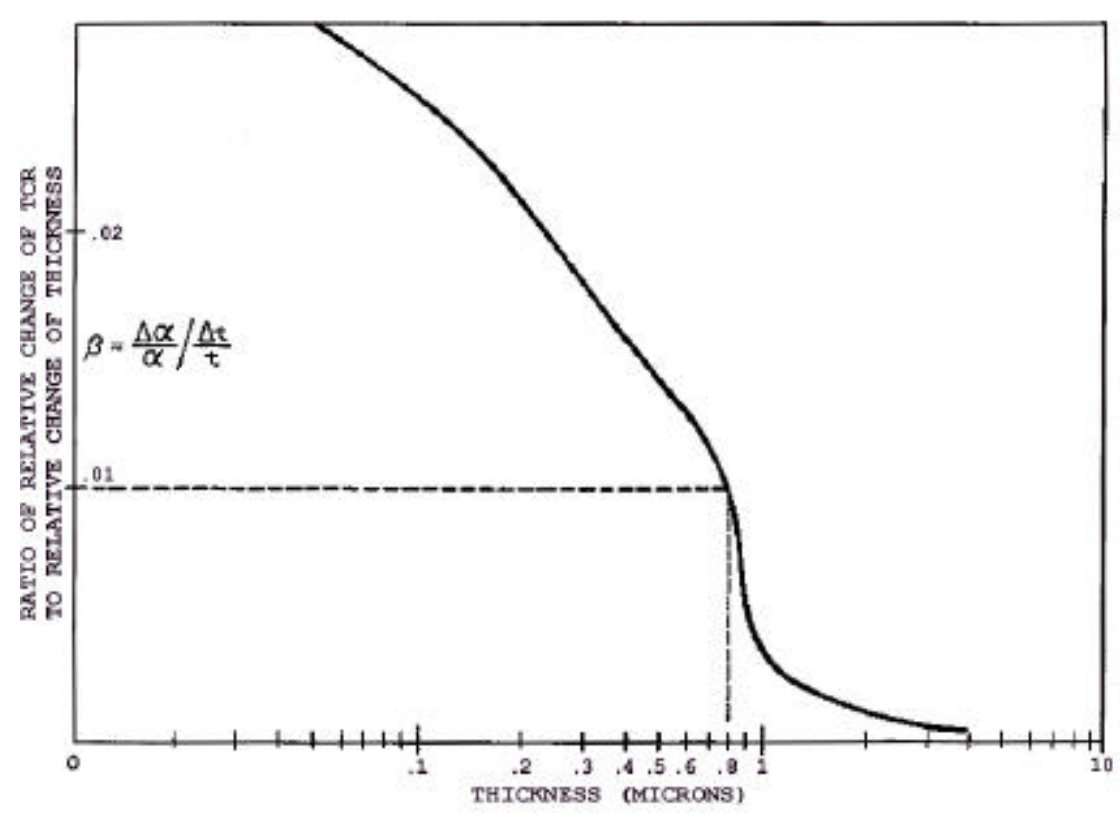

FIGURE 16 - A plot of TCR sensitivity versus thin-film thickness for a platinum RTD. Data are for a typical RTD with a "bulk" TCR of $0.00388 \Omega / \Omega /{ }^{\circ} \mathrm{C}$. Adapted from ref. [27].

to thickness, which is shown in Fig. 16 for this platinum RTD. [18,27].

RF planar magnetron sputter-deposited platinum thin films may also be thermally annealed to improve and stabilize their microstructure and, consequently, increase their TCRs. 
Annealing (or tempering) is typically done at temperatures higher than $800{ }^{\circ} \mathrm{C}$ (preferably in the range of $1000^{\circ}$ to $1200^{\circ} \mathrm{C}$ ) to achieve maximum grain growth, which is a requisite for a high TCR. Films with good microstructure can be obtained by longer heat treatments. Typical tempering heat treatments include $1000^{\circ} \mathrm{C}$ for three hours in air, or $1050{ }^{\circ} \mathrm{C}$ for two hours. Furthermore, platinum thin films which are RF planar magnetron sputter-deposited in nitrogen have been shown to exhibit better microstructure and higher TCR's than argon-sputtered films, as shown in the representative data in Table IV.

TABLE IV

PLATINUM TCR'S UNDER DIFFERENT ANNEALING CONDITIONS [26]

\begin{tabular}{|c|c|c|}
\hline \multicolumn{3}{|c|}{ Argon-Sputtered Pt Thin Film ${ }^{\star}$} \\
\hline $\begin{array}{c}1^{\mathrm{st}} \text { Heat Treatment: } \\
\text { Recrystallization }\end{array}$ & $\begin{array}{c}2^{\text {nd }} \text { Heat Treatment: } \\
\text { Annealing }\end{array}$ & Measured TCR $\left(\Omega / \Omega /{ }^{\circ} \mathrm{C}\right)$ \\
\hline $1250^{\circ} \mathrm{C}, 10$ min. & $1300^{\circ} \mathrm{C}, 15$ min. & 0.003799 \\
\hline \multicolumn{3}{|c|}{ Nitrogen-Sputtered Pt Thin Film ${ }^{\star \star}$} \\
\hline $\begin{array}{c}1^{\mathrm{st}} \text { Heat Treatment: } \\
\text { Recrystallization }\end{array}$ & $\begin{array}{c}2^{\text {nd }} \text { Heat Treatment: } \\
\text { Annealing }\end{array}$ & Measured TCR $\left(\Omega / \Omega /{ }^{\circ} \mathrm{C}\right)$ \\
\hline $1250^{\circ} \mathrm{C}, 10$ min. & $1300^{\circ} \mathrm{C}, 15$ min. & 0.003852 \\
\hline $1250^{\circ} \mathrm{C}, 5$ hours & $1250^{\circ} \mathrm{C}, 2$ hours & 0.003841 \\
\hline
\end{tabular}

Sputtered at 4 mTorr ( $<6 \times 10^{-6}$ Torr residual pressure), at $600 \mathrm{~W}(\mathrm{RF})$ for 16 minutes with a six-inch-diameter target and argon flow rate of $15 \mathrm{sccm}$ (deposition rate $>50 \mathrm{~nm} / \mathrm{min}$.).

** $1.14 \mu \mathrm{m}$-thick film deposited at $3 \mathrm{mTorr}\left(<6 \times 10^{-6}\right.$ Torr residual pressure), at $1200 \mathrm{~W}$ (RF) for 20 minutes with a six-inch-diameter target and $\mathrm{N}_{2}$ flow rate of $35 \mathrm{sccm}$.

The TCR's shown in Table IV are still lower than the pure, bulk value for platinum $\left(0.0039289 \Omega / \Omega /{ }^{\circ} \mathrm{C}\right)$. Platinum that was sputter-deposited in argon (vs. nitrogen) had a lower measured TCR of $0.003799 \Omega / \Omega /{ }^{\circ} \mathrm{C}$, which was due to its high surface roughness and high film discontinuity, compared to the nitrogen sputtered film $[26,28]$.

\section{G. Steady Pipe Flow Theory}

Heat transfer into flowing fluids is perhaps the most difficult subject in thermal sensing theory. Development of closed-form, analytical equations and/or iterative computational mathematical models for convective heat transfer in this device and flow channel geometry are beyond the scope of this research, except where applicable to design a test apparatus for laminar flow conditions. The development of an accurate analytical or computational model to relate 
monolithic hot-wire anemometer electrical parameters, such as resistor heater current, to thermodynamic or flow parameters, is left for subsequent research. Only principal parameters and equations used to develop laminar fluid flow in this thermal anemometer system will be shown.

In the case of a liquid or gas fluid, heat can be transferred by "lumps" or elements moving in the fluid. Because a mass with a certain temperature represents an amount of heat, the transport of matter at the same time constitutes a transport of heat. In the case of this hot wire anemometer system, the means of transport is forced convection, since the fluid motion is forced externally.

One way to classify fluid mechanics is on the basis of observable physical characteristics of flow fields. Although no universally accepted classification scheme exists due to the overlap encountered among various types of flow fields, one such scheme is shown in Figure 17. In

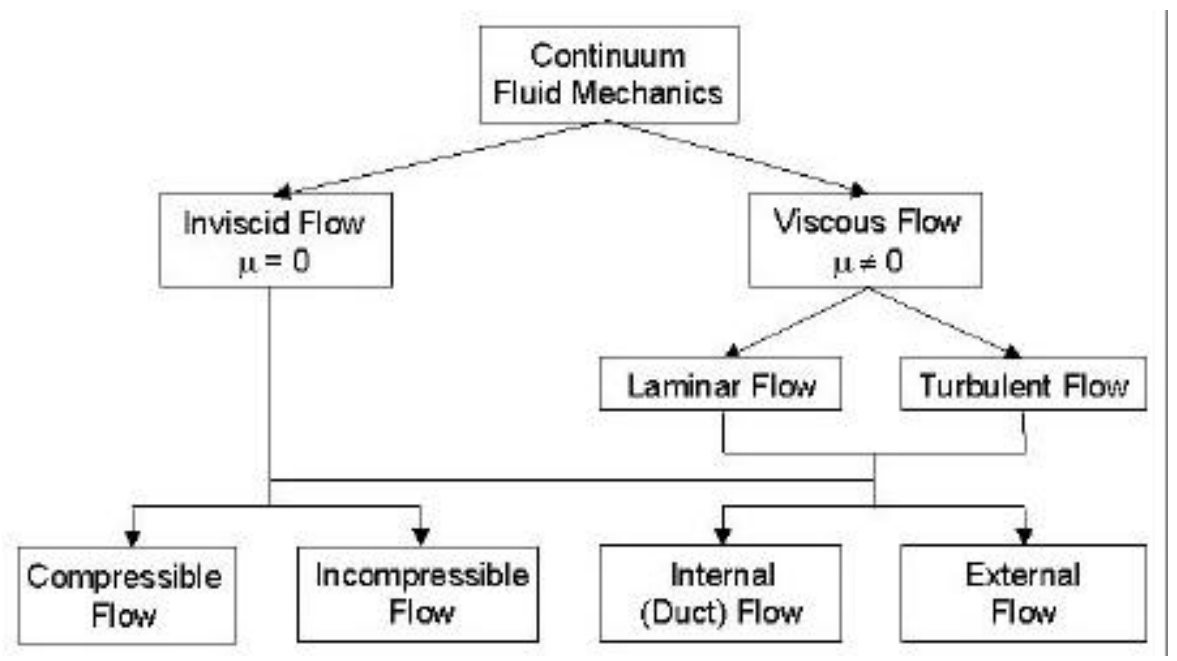

FIGURE 17 - A possible classification scheme for characterizing fluid flow. In continuum fluid mechanics, a fluid is a gas or liquid that continuously deforms under shear stress [29].

addition to this scheme, fluid flow can be classified as steady (i.e. the flow condition at any section of the system is independent of time) vs. unsteady (time-dependent) flow, boundary-layer, plane flow, unidirectional flow, Petrov flow, Poiseuille flow, etc. The main subdivision is between inviscid flow (in which the fluid viscosity, $\mu$, is assumed to be zero) and viscous flows. Even 
though fluids with zero viscosity do not exist, there are many types of fluid mechanics problems in which this simplifying assumption aids analysis and leads to meaningful results. Since all real fluids possess viscosity, characteristics of viscous flows are important.

A particularly important way to classify viscous flow regimes is based on the mode of their internal flow structure: whether they are laminar or turbulent. The conditions at which a flow is classified as laminar or turbulent are governed by many parameters, such as flow speed, the shape/size/geometry of the flow channel, and the material properties of the fluid. Laminar flow, which predominates at lower flow speeds, has a structure that is characterized by smooth fluid motion along regular, parallel, adjacent streamlines in laminae or layers. In a pipe, it is comprised of concentric cylindrical layers flowing past one another in a manner determined by the viscosity of the fluid (a velocity profile for which is shown in Figure 18). In this regime, there is no macroscopic mixing of adjacent fluid layers, and any tendencies toward instability and turbulence

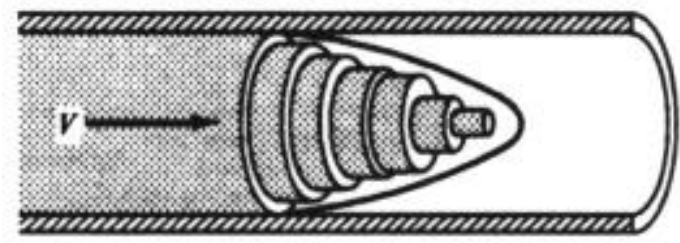

FIGURE 18 - The 3-D laminar flow velocity profile in a pipe [30].

are damped out by viscous shear forces that resist relative motion of adjacent fluid layers. For example, a thin dye injected into a laminar flow appears as a single line, with no dispersion of the dye throughout the flow, except slow scattering due to molecular motion. On the other hand, turbulent flow structure is characterized by three-dimensional, small velocity fluctuations of fluid particles (i.e. a strongly fluctuating flow component) that are transverse to the main flow direction (a violent transverse interchange of momentum [31]), superimposed in the mean flow pattern or motion, causing particles to intermingle in a very erratic and random manner. A dye filament injected into a turbulent flow disperses rapidly throughout the flow field, with the line of dye breaking up into a myriad entangled threads, due to the macroscopic mixing of particles from adjacent fluid layers. This type of flow is typically the result of instabilities in the stream occurring 
at higher flow speeds. Turbulent flow results in an increased heat transfer, as this regime intensifies the exchange of heat in the fluid.

Laminar and turbulent flows can also be distinguished by examining the output from a sensitive device at a fixed location in a pipe and immersed in the flow to measure the component of the fluid's velocity along the longitudinal axis of the pipe. For steady laminar flow, the velocity at a point remains constant with time. For turbulent flow, the velocity, if traced as a function of time, would show random fluctuations of the instantaneous velocity, $v$, about the time mean velocity, $\bar{v}$. The instantaneous velocity, $v$, may be described as the sum of the time mean velocity, $\bar{v}$, and the fluctuating velocity component, $v^{\prime}$ :

$$
v(t)=\bar{v}+v^{\prime}(t) \quad(\mathrm{m} / \mathrm{s})
$$

For steady flows, the mean velocity, $\bar{v}$, does not vary with time $[29,32]$.

Compressibility must also be considered for gas flows. Flows with negligible density variations are termed incompressible, and flows with density variations are termed compressible. For many practical cases, liquid flows are essentially incompressible. Gas flows may be considered incompressible provided the flow speeds are small relative to the speed of sound. The ratio of flow speed, $v$, to the local speed of sound, $c$, in the gas is defined as the Mach number:

$$
M \equiv \frac{v}{c}
$$

The Mach number becomes very important for high-speed flows, where density variations from pressure become significant. Gas density changes caused by dynamic compression due to motion may be neglected for engineering purposes if the local Mach number remains below about 0.3 (i.e. subsonic flow, where $M<1$ ). At $M<0.3$, changes in density are less than two percent of the mean value. Thus, subsonic gas flows with $M<0.3$ can be treated as incompressible. $A$ 
Mach number value of 0.3 in air at standard conditions corresponds to a speed of $\sim 103 \mathrm{~m} / \mathrm{s}$ (at 20 $\left.{ }^{\circ} \mathrm{C}\right)$. Furthermore, temperature changes can cause significant changes in density for a gas, even for low-speed flow. Thus, for low air flow speeds (i.e. $v<<103 \mathrm{~m} / \mathrm{s}$ ), with no air temperature change, the flow across a small thermal anemometer may be assumed to be incompressible [29]. Another way to categorize flow is by examining the extent of the flow field. Flows that are completely bounded by solid surfaces are called internal or duct flows, and external flows occur over bodies immersed in an unbounded fluid region. Internal flows may be laminar or turbulent, compressible or incompressible. For incompressible flow through a straight circular pipe, whether or not the flow is laminar or turbulent may be represented by the value of a dimensionless parameter, the Reynolds number, which indicates the relative smoothness or turbulence of the flow stream:

$$
\mathrm{Re}=\frac{\rho \bar{v} D}{\mu}=\frac{D \bar{v}}{v},
$$

where $\rho$ is the fluid's density, $D$ is the pipe diameter, $\mu$ is the absolute (or dynamic) viscosity of the fluid, $v$ is the kinematic viscosity $(v=\mu / \rho)$, and $\bar{v}$ is the average (or characteristic) flow velocity. Average flow velocity may be calculated from the volumetric flow rate, $Q_{v}$, and pipe diameter:

$$
\bar{v}=\frac{4 Q_{v}}{\pi D^{2}} \quad(\mathrm{~m} / \mathrm{s}) .
$$

The Reynolds number may also be calculated from other flow parameters:

$$
\mathrm{Re}=\frac{Q_{v} D}{v A}=\frac{4 Q_{v}}{\pi D v}=\frac{4 \dot{m}}{\pi D \mu},\left(\text { and } Q_{v}=A \bar{v}\right)
$$


where $Q_{v}$ is the volumetric flow rate, $\bar{v}$ is the average fluid velocity over the cross-sectional area, $A$, of the pipe, and $\dot{m}$ is the mass flow rate through the pipe. The Reynolds number is the ratio of inertia forces to viscous friction forces involved in the flow. If viscous forces predominate, the flow is laminar; whereas, if inertia forces dominate, the flow is turbulent [30]. Circular pipe flow is then considered laminar when:

$$
\mathrm{Re}<\mathrm{Re}_{c r}
$$

where $R e_{c r}$ is the critical value of the Reynolds number at which the transition from laminar to turbulent flow occurs. This value depends upon the cross-sectional geometry of the flow channel, the description of the flow (e.g. past an airplane wing, between rotating cylinders, with gas density changing, time-dependent flow), how $\bar{v}$ is chosen (average, maximum, far upstream), etc. For macroscopic fluidic devices such as large, straight circular tubes or ducts, laminar flow has been empirically determined to predominate for:

$$
\mathrm{Re}_{c r}<2000
$$

Others claim the transition from laminar to turbulent flow in a straight circular pipe occurs around $\operatorname{Re}_{c r} \approx 2300[29,33,34]$. For Reynolds numbers in the range of 2000 to 4000 , the flow will typically change from laminar to turbulent.

Flow in a constant-area pipe or duct is fully developed if the shape of the velocity profile is the same at all cross sections. The velocity is unidirectional and varies with distance across the pipe, but not along it. The fluid's velocity is maximum at the center of the pipe and is zero at the walls (because for viscous flow, the fluid in direct contact with a solid boundary has the same velocity as the boundary itself, with no slip at the boundary). Since the fluid velocity at the stationary, solid-surface pipe boundaries in the moving fluid is zero, but the bulk fluid is moving, velocity gradients and shear stresses must be present in the flow. The flow can be divided into 
two general regions, as shown in Figure 19. The region immediately adjacent to the solid pipewall boundary (in this case, modeled as a "semi-infinite plate"), where shear stresses are present,

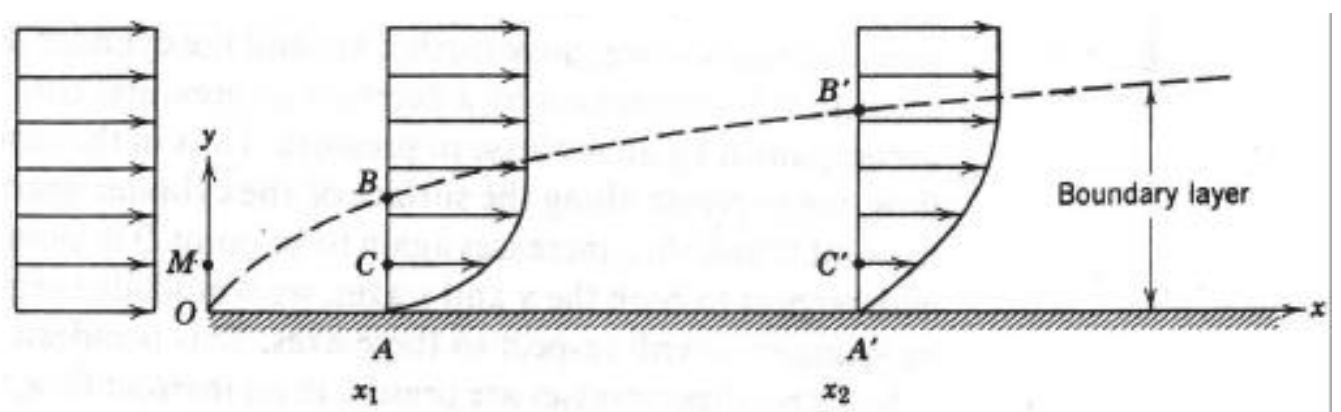

FIGURE 19 - Velocity profile of developing laminar flow over a semi-infinite flat plate [29].

is termed a boundary layer. Outside the boundary layer, the velocity gradient is zero, and hence, shear stresses are also zero. In this region, inviscid flow theory may be used to analyze the flow. A velocity profile comparison between laminar and turbulent flow in a pipe is shown in Figure 20. In turbulent flow, the velocity distribution is more uniform across the pipe diameter; whereas, in

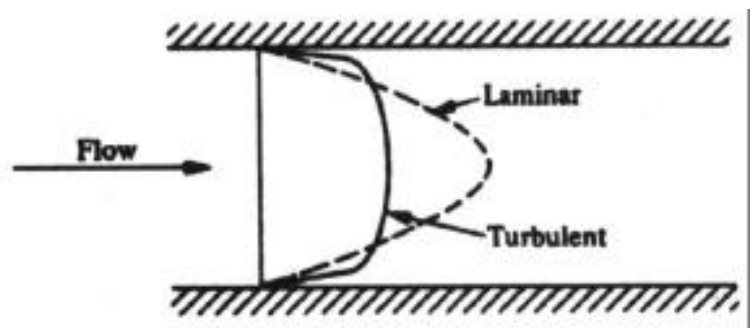

FIGURE 20 - Average velocity profile comparison for laminar and turbulent pipe flow [30].

fully-developed laminar flow, the maximum velocity occurs along the pipe axis, decreasing to zero at the walls. As shown in Figure 21, as fluid enters a pipe the velocity profile is almost uniform over the cross section. The wall shearing stress slows down the fluid near the pipe walls.

Beyond a transitional length called the entrance length, $L_{e}$, the velocity profile is fixed, with fullydeveloped laminar flow, since the boundary influence has extended to the pipe's central axis. 


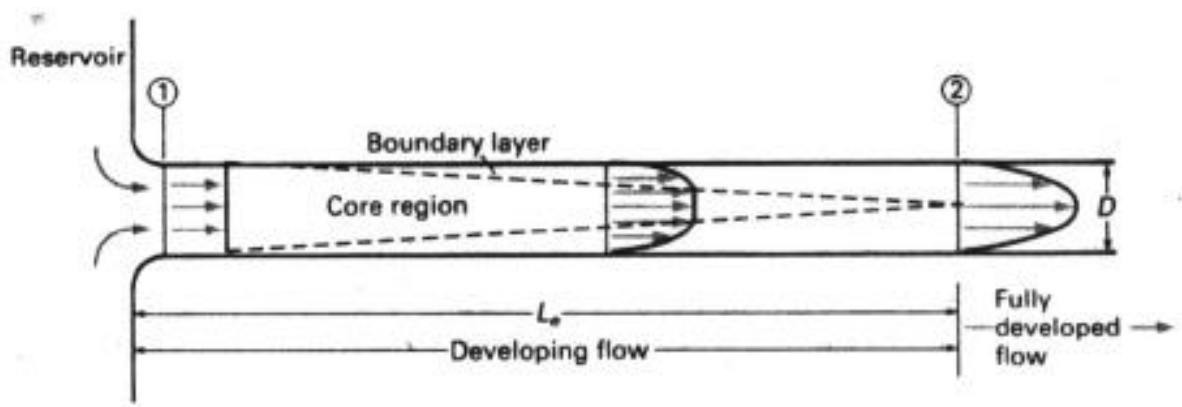

FIGURE 21 - The entrance length of a pipe in which laminar flow fully develops. In the developing flow zone, core flow is irrotational and neither laminar nor turbulent [33].

The entrance length is a function of only the Reynolds number. Many analytical and experimental investigations have shown that the following formula may be used to calculate the (minimum) entrance length needed for achieving fully-developed laminar flow in a straight circular pipe [31]:

$$
L_{e} \approx 0.058 D \operatorname{Re}_{c r} \quad(\mathrm{~m}) \text {. }
$$

In turbulent flow, the boundary layer grows more rapidly, and the entrance length is considerably shorter than given by this equation.

Note that the distinction between laminar and turbulent flow only applies to the fully developed flow condition in a pipe. In the developing zone (see Figure 21), flow is neither laminar nor turbulent. Flow in the boundary layer is laminar or turbulent. If the fully developed pipe flow is laminar, the boundary layer will also be laminar, but if the fully developed flow is turbulent, the boundary layer will be laminar near the pipe entrance, undergo a transition, and will be turbulent as it approaches the fully developed condition [33].

Finally, in order to relate pressure and velocity differentials within a steady-flow pipe system, the First Law of Thermodynamics in the form of the Bernoulli equation may be used. For a fixed mass undergoing an incremental process the first law can be stated as:

$$
d Q+d W=d E \quad(J)
$$


where $d Q$ represents an increment of heat added to the system, $d W$ an increment of work done on the system, and $d E$ an increment of the total energy of the system, which is usually comprised of the internal energy, $u$, and kinetic and potential energies (other forms of energy may also be present, such as chemical, electrical, or magnetic, but are not applicable to this flow system). For one dimensional steady flow (e.g. in a pipe), the first law may be written as:

$$
q+w_{s}=\Delta\left(u+\frac{p}{\rho}+\frac{\bar{v}^{2}}{2}+g y\right) \quad(\mathrm{J} / \mathrm{kg})
$$

where $q$ is heat, $w_{s}$ is the work done per unit mass of flowing fluid by a shaft, turbine, or pump, $p$ is the (inlet or outlet) pressure, $\rho$ is the mass density, $y$ is the (inlet or outlet) port height, and $\Delta$ represents the difference in the parenthesized quantity between the outlet and inlet of a control volume. This equation can be further simplified to:

$$
y_{1} g+\frac{\bar{v}_{1}^{2}}{2}+\frac{p_{1}}{\rho}=y_{2} g+\frac{{\overline{v_{2}}}^{2}}{2}+\frac{p_{2}}{\rho}
$$

for a control volume (or pipe) with inlet port height $y_{1}$, outlet port height $y_{2}$, inlet port average flow velocity $\overline{v_{1}}$, outlet port height $y_{2}$, outlet port average flow velocity $\overline{v_{2}}$, inlet port pressure $p_{1}$, outlet port pressure $p_{2}$, and fluid with constant density, $\rho$. This equation may only be used to represent systems with the following conditions and assumptions:

1. The flow is steady;

2. There is no friction;

3. There is no change in temperature;

4. No shaft work is done on or by the system;

5. There is no change in internal energy during the process;

6. No energy flows as heat transfer across the boundaries of the system; and

7. The fluid is incompressible (i.e. no density change across the ports).

The Bernoulli equation can be even further simplified for steady-state, horizontal pipe flow of an incompressible, frictionless fluid: 


$$
\frac{\rho \bar{v}_{1}^{2}}{2}+p_{1}=\frac{\rho{\overline{v_{2}}}^{2}}{2}+p_{2} \quad(\mathrm{~J} / \mathrm{kg})
$$

This shows that mechanical energy is conserved, and that as the cross-sectional area of the channel is increased, the velocity of the fluid decreases with a corresponding pressure increase. This equation assumes that the fluid is inviscid, with zero viscosity, although real fluids have viscous drag effects and boundary layers resisting flow at interfaces with flow channels or surfaces [34].

For steady, laminar flow of a Newtonian fluid in a horizontal pipe with circular cross section, volumetric flow rate, $Q_{v}$ (present in Equations (25) and (26) for calculating average flow velocity and Reynolds number), can be calculated from the pressure differential between pipe ports in the Hagen-Poiseuille equation:

$$
Q_{v}=\frac{\pi(\Delta p) D^{4}}{128 L \mu}=\frac{\pi\left(p_{2}-p_{1}\right) D^{4}}{128 L \mu} \quad\left(\mathrm{m}^{3} / \mathrm{s}\right)
$$

where $L$ is the pipe length, $\mu$ is the absolute viscosity of the fluid, $D$ is the diameter of the pipe, and $\Delta p$ is the pressure differential between the ports. Alternatively, the pressure differential between ends of the pipe can be written as:

$$
\Delta p=\frac{32 L \rho \bar{v}^{2}}{D \cdot \operatorname{Re}} \quad(\mathrm{Pa})
$$

where $\rho$ is the fluid density, and $\bar{v}$ is the average flow velocity (over the channel cross-section) $[30,35]$. 


\section{H. The Diamond Lattice Structure of Silicon}

Crystalline silicon forms a covalently-bonded structure. Silicon's four covalent bonds are coordinated tetrahedrally, with these tetrahedrons comprising the diamond-cubic unit cell structure, as depicted in Figure 22. This structure can also be represented as two

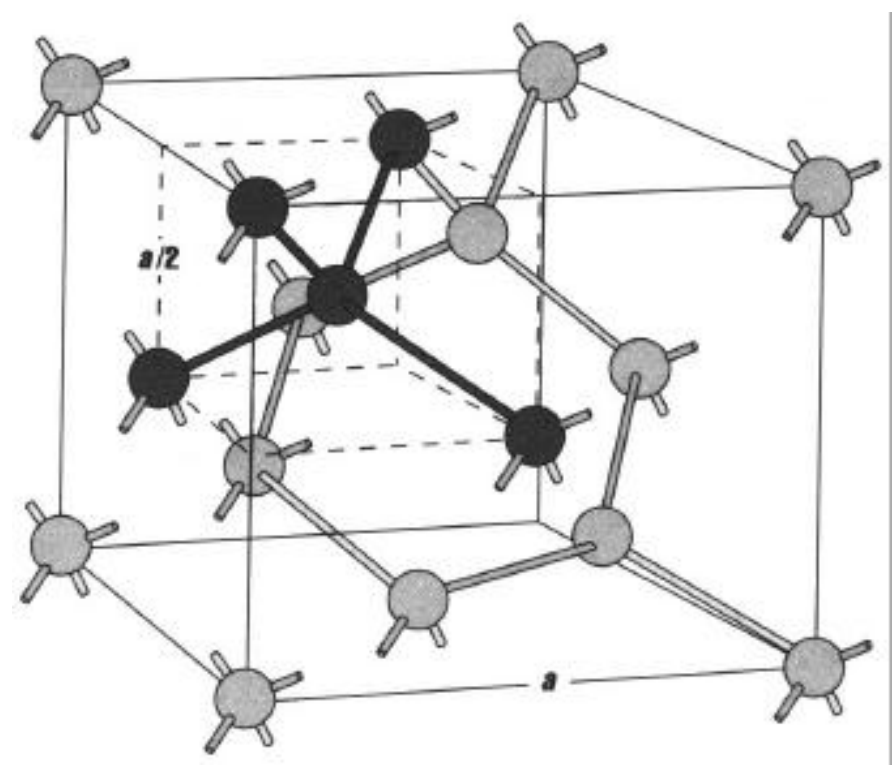

FIGURE 22 - The diamond structure lattice cell for silicon. This is also the same cell for other Group IV semiconductor elements, all with the lattice parameter a. This cell may be thought of as two interlocking Face Centered Cubic (FCC) lattices [36].

interpenetrating Face-Centered Cubic (FCC) lattices. The lattice parameter, $a$, for silicon is $5.4309 \AA$ A. The Miller indices of the main crystallographic planes in silicon are (100), (110), and (111). The $\{111\}$ planes present the highest packing density in silicon. A table, shown in Figure 23 , is included for convenience to show the definitions of unit-cell, Miller indices notation.

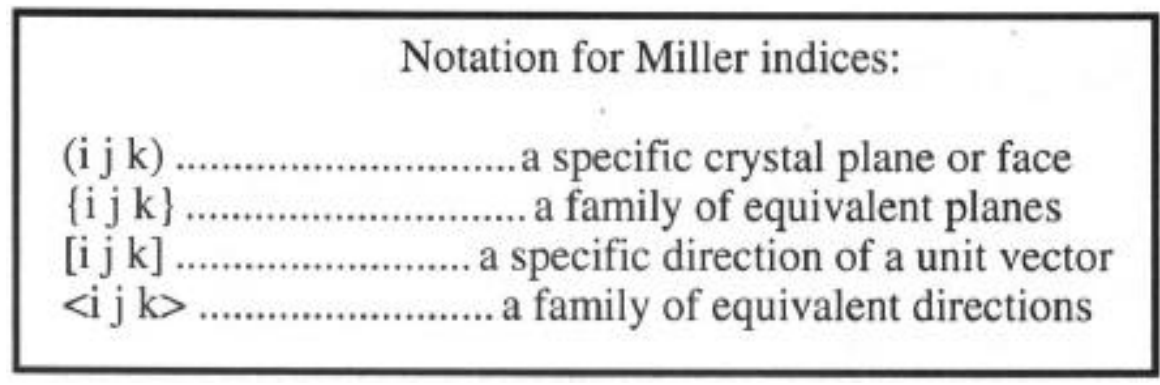

FIGURE 23 - A table of standard Miller Indices notation. These are used to indicate crystal orientations, planes, etc. [37]. 
When ordering silicon wafers, a crystallographic orientation must be specified. The most common orientations used in the IC fabrication industry are the $<100>$ and $<111>$ orientation; and, in micromachining, the $<110>$ orientation. The $<111>$ wafers are used less as they cannot be easily anisotropically etched. Silicon wafers are typically marked with coded "flats," as shown in Figure 24, to indicate the orientation of certain crystallographic planes. For example, on a $<100>$ wafer, the $<110>$ direction is specified by an orientation flat, with a precision of about $3 \%$, which varies by manufacturer [38].
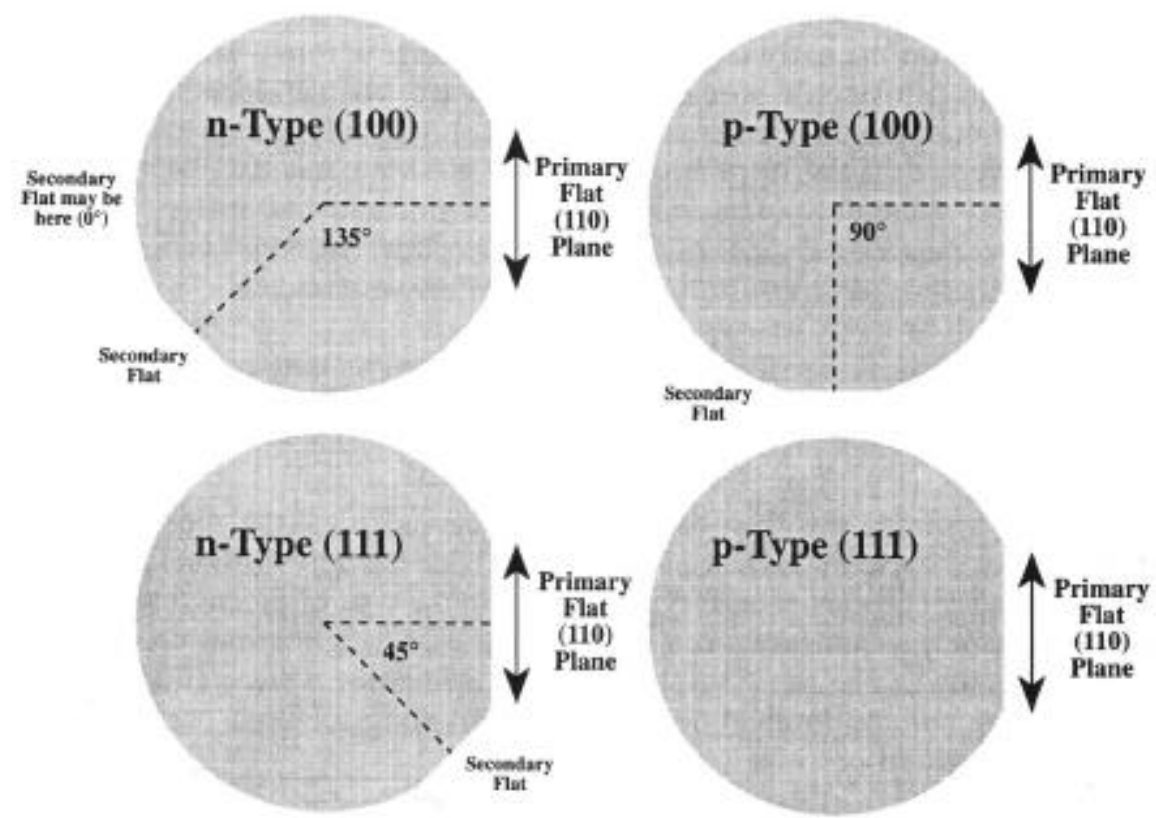

FIGURE 24 - Illustration of standard coded "flats" on 4-inch silicon wafers. These are used to help identify the substrates. The top left configuration is the most common [37].

\section{Bulk Micromachining}

Wet etching of silicon is used for cleaning, shaping, polishing, and characterizing structural and compositional features, and it generally provides a higher degree of selectivity than with traditional dry etching techniques. Etching occurs by [39]:

- Reactant transport to the surface (if it is rate-determining, than etching is diffusion-limited and can be increased by stirring); 
- $\quad$ Surface reaction (if it is the rate-determining step, etching is reaction-rate-limited and depends strongly on the etching material, solution temperature, and composition); and

- $\quad$ Reaction product transport away from the surface (if it is rate-determining, then etching is diffusion-limited and can be increased by stirring).

Many wet silicon etchants are available, with a wide variety of properties. Figures of merit for etchants include: the etch rate (including the etch-rate selectivity of various materials referenced to the etch rate of the film being patterned); the etch undercut of the photoresist (or other) masking layer; and substrate damage, which is more difficult to quantify. Etchants are fundamentally distinguished by whether their behavior tends to be isotropic or anisotropic. Etch anisotropy, which is one way to describe undercut, is defined as:

$$
A=1-\frac{R_{L}}{R_{V}},
$$

where $R_{L}$ and $R_{v}$ are the lateral and vertical etch rates, respectively. For $A=1$, a process is said to be perfectly anisotropic, where the lateral etch rate is zero. Isotropic etching occurs when $A=0$, as the lateral and vertical etch rates are identical [40].

Isotropic etchants etch in all crystallographic directions at nearly (and sometimes exactly) the same rate. They are usually acidic, and lead to rounded isotropic features in monocrystalline silicon. Agitation of the etchant can control the etch rate and geometry of resulting structures, as it helps to speed up (and increase the uniformity of) the transport of reactants and products. This is generally the only wet-etching option for glasses, for which a buffered oxide etch (BOE), containing diluted hydrofluoric acid (HF), is commonly used. Figure 25 shows the isotropic nature of $\mathrm{SiO}_{2}$ etching and undercutting of a photoresist patterning layer. Glass etch rates in $\mathrm{BOE}$ are dependent upon the density of the $\mathrm{SiO}_{2}$ film, the temperature of the etchant solution, and the type of oxide present (i.e. grown in dry oxygen, grown in oxygen with the presence of water vapor, or doped with impurities). For example, if high concentrations of boron are present in the glass, it 


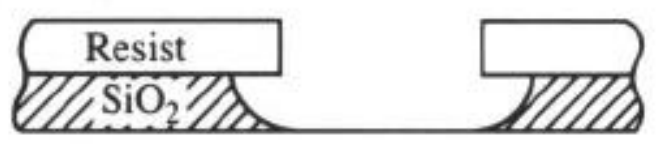

Si

FIGURE 25 - Illustration of an isotropic, glass wet-etching sidewall profile [41]

is converted into a borosilicate glass, with a reduced buffered oxide etch rate [37,41].

Anisotropic etchants are used to shape desired structures into crystalline materials, such as silicon. When properly done, anisotropic etching produces geometric shapes bounded by perfectly-defined, crystallographic planes. The most commonly used (typically alkaline, aqueous) anisotropic etchants, are $\mathrm{KOH}, \mathrm{TMAH}$, and EDP, all of which slow down markedly at the $\{111\}$ planes of silicon relative to etch rates of other planes. An illustration of typical anisotropic etching of silicon is shown in Figure 26.

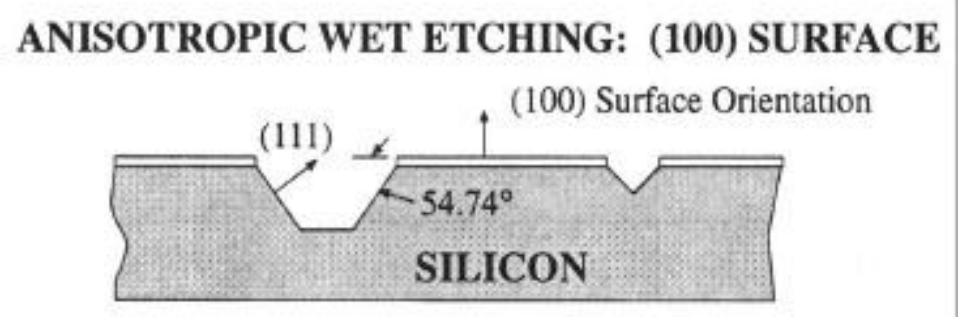

FIGURE 26 - Illustration of anisotropic wet etching of a (100) silicon substrate [37].

Aqueous $\mathrm{KOH}$ has become a major etchant used for micromachining applications, providing mirror-quality, machined surfaces, although it is incompatible with IC processes due to its potassium ions. Typical values for $\mathrm{KOH}$ relative etch rates for the three planes of interest in silicon are: the reference plane, $\{111\}=1 ;\{110\}=600$; and $\{100\}=300$ to 400 . These relative etch rates are extremely dependent upon the chemical composition, concentration, temperature, and freshness of the etchant solution. Furthermore, stirring of $\mathrm{KOH}$ solutions causes a marked increase in etch rates at low $\mathrm{KOH}$ concentrations, but has much less effect at high concentrations. The etching process at high $\mathrm{KOH}$ concentrations is primarily reaction-rate 
limited, and each crystallographic surface has its own etch rate. At lower $\mathrm{KOH}$ concentrations, the process becomes more diffusion-rate limited, and stirring has a much larger effect $[37,42]$.

There are few equations that predict aggregate $\mathrm{KOH}$ etching performance directly from user-controllable factors. However, Kendall and Shoultz have interpolated $\mathrm{KOH}$ etch rates for $\{100\}$ and $\{110\}$ wafers ( $R_{100}$ and $R_{110}$, respectively) from various data at different temperatures and $\mathrm{KOH}$ concentrations in terms of the hydration model (of Glembocki et al.) to relate them by the following equations which are accurate to about $20 \%$ over the full range of temperature and $\mathrm{KOH}$ concentrations [42]:

$$
\begin{aligned}
& R_{100}=\left[\left(2.6 \times 10^{6}\right) \cdot W^{2.5}\right] \exp \left[\frac{-\left(\frac{W}{300}+0.48\right)}{k(T+273)}\right] \quad(\mu \mathrm{m} / \mathrm{hr}) \\
& R_{110}=\left[\left(5.2 \times 10^{6}\right) \cdot W^{2.5}\right] \exp \left[\frac{-\left(\frac{W}{300}+0.48\right)}{k(T+273)}\right] \quad(\mu \mathrm{m} / \mathrm{hr}),
\end{aligned}
$$

where $W$ is the actual weight percentage of $\mathrm{KOH}$ in water (as monitored by specific-gravity measurements, not the nominal weight percentage of $\mathrm{KOH}$ pellets), $k$ is Boltzmann's constant (8.6174 $\left.\times 10^{-5} \mathrm{eV} / \mathrm{K}\right)$, and $T$ is the solution temperature in Celsius. Kendall and Shoultz also examined various published data for $\mathrm{KOH}$ etch rates of thermal oxides at various temperatures and developed an empirical relation that is purported to be about $20 \%$ accurate over a very wide range of temperatures and $\mathrm{KOH}$ concentrations: 


$$
R_{\text {SiO2 }}=2.2 \times 10^{9} W\left[\left(1.5 \times 10^{-4}\right) \cdot W^{2.15}+1\right] \exp \left[\frac{-\left(0.795+\left(6 \times 10^{-6}\right) \cdot W^{2.5}\right)}{k \cdot(T+273)}\right]
$$

where $R_{\mathrm{SIO}_{2}}$ is the $\mathrm{SiO}_{2}$ etch rate in micrometers per hour, and the other variables are the same as before [42].

The use of TMAH as an anisotropic silicon etchant is growing in popularity because it is nontoxic, IC compatible, and has very high selectivity for silicon relative to $\mathrm{SiO}_{2}$. Additionally, aluminum can be used as a masking layer if excess silicon is added to the TMAH solution (nevertheless, the aluminum still etches slightly). It has the disadvantages of etch rate reproducibility being quite difficult (in part, because solution concentrations have to be carefully maintained), and pyramidal hillocks that form during silicon etching (which is not a significant problem with $\mathrm{KOH}$ ) [42].

\section{J. Characterizing Thin Film Intrinsic Stress/Strain}

The successful fabrication of MEMS sensors made from thin films requires knowledge and control of the mechanical and material properties of the thin films utilized. The properties most needed included Young's modulus, Poisson's ratio, the internal stress (or strain) in the film, and the thermal expansion coefficient. These properties are typically anisotropic because of deposition procedures and grain growth, but generally exhibit isotropy in the plane of the substrate. Material properties of thin films are dependent upon surface preparation prior to deposition, deposition conditions, thermal annealing, and other post-deposition processing. These properties must be measured and controlled for the specific film and process of interest [43].

As shown in Figure 27, a thin film deposited on a silicon substrate can be either in tensile stress, in which the film relaxes by contracting, or in compressive stress, in which the film tends to expand parallel to the surface. The stress that develops in a film during early phases of deposition may be compressive, causing buckling and blistering, or delamination in extreme cases (e.g. with very thick films). Or, the film may be initially deposited in tensile stress, which may lead to cracking if intrinsic forces are present that are high enough to exceed the fracture 


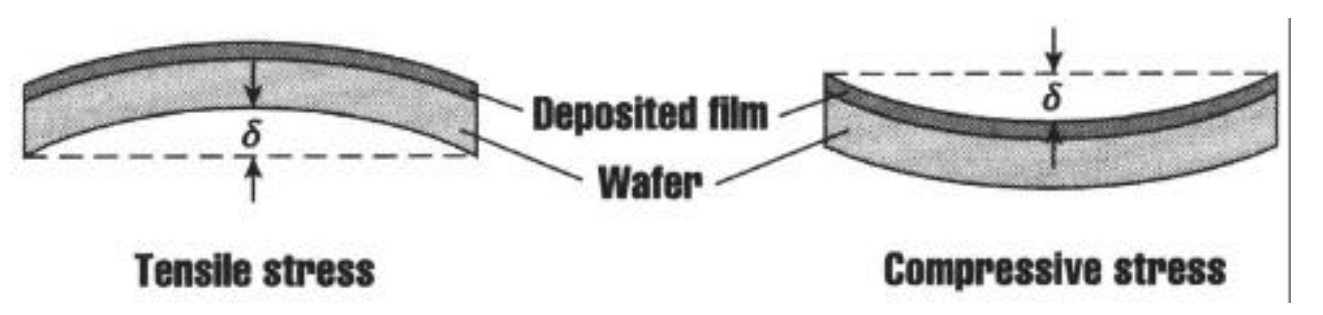

FIGURE 27 - Wafer bow due to tension or compression within a deposited thin film. This can be evaluated by measuring the corresponding change in the bow of the silicon wafer before and after film deposition [44].

limit of the film. Subsequent atomic rearrangement, either during the remainder of film deposition or with thermal annealing, can lead to further densification or expansion, decreasing remaining tensile or compressive stresses (respectively). Nitrides deposited by plasma CVD usually have intrinsic compressive stresses due to the presence of hydrogen atoms in the lattice structure. Thermal annealing can be used to drive the hydrogen atoms out, making the film highly tensile. Thermally-grown oxide $\left(\mathrm{SiO}_{2}\right)$ films are always under compressive stress. If atoms are jammed in place, such as with sputter deposition, thin films tend to be under compression. Thus, stresses present in a thin film can be due to the deposition process(es) themselves or to the postdeposition history of the film (or both) [45].

Ideally for this flow sensor, a microbridge structural thin-film is desired that has excellent adhesion, low residual stress, low pinhole density, and high chemical resistance to the anisotropic silicon etchant $(\mathrm{KOH}$ or $\mathrm{TMAH})$. The material properties of a deposited silicon nitride film will strongly depend upon the details of the deposition process and growth conditions. These properties may also be affected or changed by an optional post-deposition thermal anneal. Furthermore, although characteristics of the $\mathrm{Si}_{3} \mathrm{~N}_{4}$ bulk material may be well documented, its thinfilm form may have substantially different properties, in part because thin films generally display smaller grain sizes than bulk materials. Adhesion of $\mathrm{Si}_{3} \mathrm{~N}_{4}$ thin films is obviously very important, where cleanliness of the substrate is critically necessary for good adhesion [45].

Internal film stress is critical for all MEMS microstructures, with excessive values resulting in curling, buckling, blistering, cracking, delamination (or peeling), void formation, or long term shifts in material properties. Blistering occurs where the compressively loaded film is poorly adhered to the substrate. Cracking can occur in either tensile or compressive strain fields. 
Peeling is more likely to occur near a patterned edge of the film, at a step, or at a defect in the film, if its residual stress is too large [43]. Nearly all films have a state of residual stress due to a crystal lattice mismatch, a mismatch in thermal expansion coefficients, nonuniform plastic deformation, substitutional or interstitial impurities, and growth processes. Figure 28 lists stresscausing factors in thin films categorized as either intrinsic or extrinsic. Intrinsic, or growth, stresses are those that develop during film deposition nucleation. Extrinsic stresses are imposed by unintended external factors such as the most common type: thermal gradients. Thermally induced, mechanical strain fields arise either in a structure with dissimilar thermal expansion coefficients (such as anodically bonded glass on a silicon substrate) subjected to a uniform temperature change, or in a homogeneous material exposed to a thermal gradient. Thin film intrinsic stresses are often larger than thermal stresses because of the nonequilibrium nature

Extrinsic

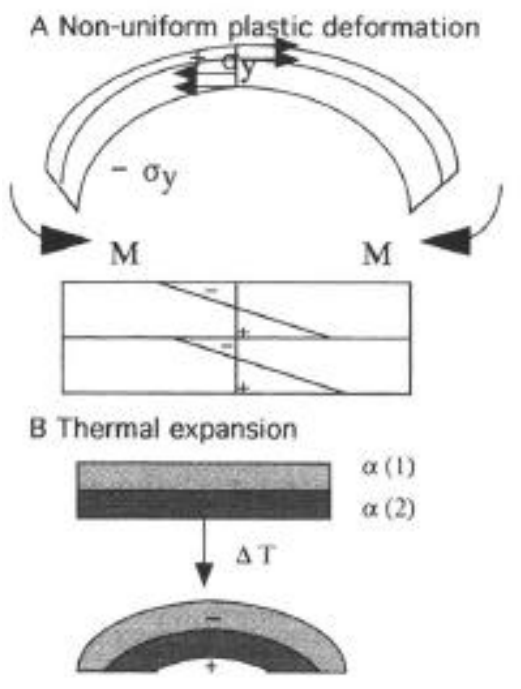

Intrinsic

C Growth

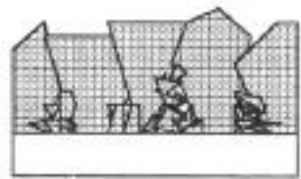

D Misfit/impurities

000000

000000

000000

- 10

둥

-

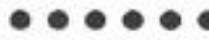

E Phase transformation

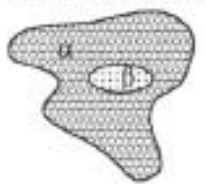

FIGURE 28 - Examples of intrinsic and extrinsic residual stresses. In (A) nonuniform plastic deformation results in residual stresses upon unloading, where $M$ is a bending moment. In (B) a thermal expansion mismatch exists between two dissimilar materials bonded together, where $\alpha$ (1) and $\alpha$ (2) are thermal expansion coefficients. In (C) growth stresses evolve during film deposition. In (D) misfit stresses exist due to mismatches in lattice parameters between an epitaxial film and the substrate and substitutional or interstitial impurities cause stresses due to local expansion or contraction associated with point defects. In (E), volume changes occurring with phase transformations cause residual stresses [45]. 
of the film deposition process itself. High stress can result in buckling and cracking of thin films. When the film deposition thickness reaches a certain value, extrinsic stresses tend to be relieved through the formation of dislocations $[45,46]$.

How to determine the internal stress in a deposited thin film is one example of a problem encountered with MEMS devices. Ideally, Young's modulus and the internal stress of a thin film could be obtained from a uniaxial tension test. When a loading force is applied to a surface, the force divided by the area over which it is applied is equal to the stress. Axial or normal stresses are produced by forces acting perpendicular to a surface. By convention, tensile stresses are positive and compressive stresses are negative. Without any externally applied stress, the total stress in a thin film is given by the sum of $\sigma_{t h}$, which is the unintended external thermal stress (due to mismatches in thermal expansion coefficients between the film and substrate, accentuated by depositions performed at elevated temperatures), and $\sigma_{\text {int }}$, which is the stress due to different intrinsic components (in part, caused by effects of grain growth and a deposition process which may lock-in atoms at lattice positions other than zero-stress positions):

$$
\sigma_{\text {tot }}=\sigma_{t h}+\sigma_{\text {int }} \quad\left(\mathrm{N} / \mathrm{m}^{2} \text { or } \mathrm{Pa}\right)
$$

The total in-plane stress in a thin film is the stress averaged across the film thickness, $t$, such that:

$$
\sigma_{\text {tot }}=\sigma_{o}=\frac{1}{t} \int \sigma_{x}(z) d z \quad\left(\mathrm{~N} / \mathrm{m}^{2} \text { or } \mathrm{Pa}\right)
$$

where $\sigma_{x}(z)$ is the magnitude of the in-plane film stress at height $z$ within the film.

With constant stress throughout the film thickness, stress components are functions of coordinates within the plane of the film itself (i.e. no stress occurs in the direction normal to the substrate). When subjected to a stress, the residual strain $(\varepsilon)$ in a thin film is related to the 
dimensional change, or consequential deformation, within its elastic limit:

$$
\varepsilon=\frac{\Delta L}{L_{o}},
$$

where $L_{o}$ is the original material length and $\Delta L$ is the change in length caused by the stress. Most materials deform linearly with loading (obeying Hooke's law). Since loading is proportional to stress and deformation is proportional to strain, stress and strain are linearly related via the elastic modulus, or Young's Modulus of Elasticity, which relates the stress due to external loads to the resulting strain (or deformation) in a material under tension or compression:

$$
E=\frac{\sigma_{o}}{\varepsilon} \quad\left(\mathrm{N} / \mathrm{m}^{2}, \text { or } \mathrm{Pa}\right)
$$

The modulus value in the plane of a thin film is generally most significant because stresses in the plane due to applied loads, thermal stresses, and intrinsic stresses are much larger in magnitude than stresses in the direction of the film thickness. The higher Young's modulus is for a given material, the stiffer it is, and the less it deforms for a given stress. It is also important to note that Young's moduli for crystalline materials is dependent upon their orientation. Furthermore, since the cross sectional area, $A$, of a specimen being uniaxially elongated, $\Delta L$, will tend to contract, a dog-bone shaped structure (called an Instron specimen) is often used to find Young's modulus. As shown in Figure 29, Young's modulus can be calculated for an Instron specimen of neck length (or gauge length of the elongation region), $L$, with neck cross sectional area, $A$, applied force, $F$, and corresponding change in Instron length, $\Delta L$ :

$$
E=\left(\frac{F}{A}\right)\left(\frac{L}{\Delta L}\right) .
$$




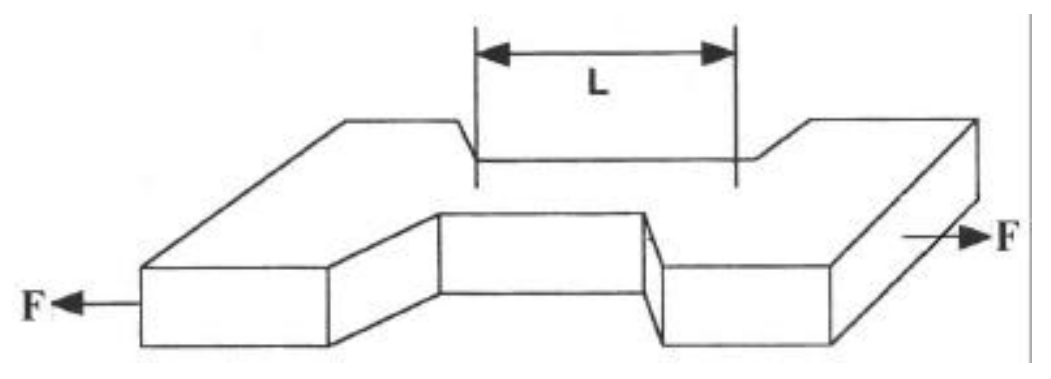

FIGURE 29 - Illustration of an Instron specimen for measuring Young's modulus.

However, making Instron specimens in MEMS thin films is a significant challenge because the film must be removed from the substrate, possibly changing the intrinsic stress state, and the process of removing the film itself may modify its properties $[43,45,47]$.

For a material that is subjected to an axial load, it may deform not only in the (longitudinal) direction of the load, but also in (transverse) directions perpendicular to the applied load. When subjected to a tensile load, the length of an object will typically increase while its girth decreases, and when compressed, its length will decrease while its girth increases. For this case, there is both an axial strain and a transverse strain. Poisson's ratio is the ratio of this transverse strain, $\varepsilon_{t}$, to the corresponding axial strain, $\varepsilon_{a}$ on a material stressed along one axis [47]:

$$
v=-\frac{\varepsilon_{t}}{\varepsilon_{a}} .
$$

Measuring Poisson's ratio for thin films has proven to be more difficult than measuring Young's modulus, as thin films tend to bend out of plane in response to in-plane shear. For the isotropic case in a thin film (where Young's modulus and Poisson's ratio for the film act independently of orientation), orthogonal strain components in the plane of the film are equal $\left(\varepsilon=\varepsilon_{x}=\varepsilon_{y}\right)$, resulting in orthogonal stress components also being equal $\left(\sigma=\sigma_{x}=\sigma_{y}\right)$. Assuming Hooke's law is valid in the plane of the film (i.e. that the stress in the elastic film is proportional to the strain responsible for it), and neglecting stresses in the direction of the thickness of the film, the in-plane 
stress (averaged across the film thickness), $\sigma$, may be related to the internal film strain, $\varepsilon$, Young's modulus $(E)$, and Poisson's ratio $(v)$ by:

$$
\sigma_{o}=\left(\frac{E}{1-v}\right) \cdot \varepsilon \quad\left(\mathrm{N} / \mathrm{m}^{2}, \text { or } \mathrm{Pa}\right)
$$

The parenthesized quantity is also called the biaxial modulus. This plane-stress calculation represents a good approximation several $(\sim 3)$ thicknesses away from the edge of the film. Inplane values of a thin film's internal stress are generally different near the film-substrate interface than at the surface, resulting in an internal moment. When a structural film with a nonzero moment is cut free, it will curl $[43,45]$.

Thermal stresses develop in thin films when high temperature deposition or annealing techniques are used, and are usually unavoidable due to the thermal expansion coefficient mismatch between the thin film and substrate. For thin films ( $1 \mu m$ thick) deposited on $\sim 550$ $\mu m$-thick silicon substrates, the substrate may be considered essentially rigid relative to the film, because it is nearly three orders of magnitude thicker than the film, and the bending stiffness is proportional to the thickness cubed. For a thin film, with coefficient of thermal expansion $\alpha_{f}$, applied strain-free, at a deposition temperature, $T_{d}$, cooled to room temperature, $T_{r}$, on a silicon substrate with coefficient of thermal expansion $\alpha_{s}$, the resulting thermal strain $\left(\varepsilon_{t h}\right)$ is [45]:

$$
\varepsilon_{t h}=\left(\alpha_{f}-\alpha_{s}\right)\left(T_{d}-T_{r}\right)
$$

as long as the coefficients of thermal expansion are temperature independent. If Young's Modulus and Poisson's ratio for the thin-film are temperature independent, the thermal stress may also be calculated [45]: 


$$
\sigma_{\text {th }}=\left(\frac{E_{\text {film }}}{1-v_{\text {film }}}\right)\left(\alpha_{f}-\alpha_{f}\right)\left(T_{d}-T_{r}\right) \quad\left(\mathrm{N} / \mathrm{m}^{2}, \text { or Pa }\right) .
$$

Intrinsic stress, $\sigma_{\text {int }}$, which reflects the internal structure of a deposited thin-film, is less clearly understood than thermal stress, and is usually dominated by thermal stress. Intrinsic stress is determined by film thickness, film deposition rate (i.e. locking-in crystallographic defects), deposition temperature, ambient pressure, method of film preparation, type of substrate used (i.e. crystallographic lattice mismatch), incorporation of impurities (e.g. sputtering gas atoms) during growth, etc. Atomic peening is one intrinsic stress causing factor (where $\sigma<0$ ), in which ion bombardment by sputtered atoms and the working gas (e.g. argon) densifies thin films, resulting in a more compressive film. In particular, magnetron-sputtered films at low working pressures $(<1 \mathrm{~Pa})$ and low temperature often exhibit compressive stress. Microvoids may also arise when byproducts during deposition (e.g. argon) escape as gases and the lateral diffusion of atoms occurs too slowly to fill all the gaps, resulting in a tensile film $(\sigma>0)$. Gas entrapment is another intrinsic, stress-causing factor. For example, hydrogen can be trapped within a thin film $\mathrm{Si}_{3} \mathrm{~N}_{4}$ lattice. Thermal annealing may remove the entrapped hydrogen, and an originally compressive $\mathrm{Si}_{3} \mathrm{~N}_{4}$ film may then become tensile if the hydrogen content is low enough [45].

Various material properties of selected MEMS materials, relevant to this flow sensor design, are shown in Table V. Clearly, the data have to be interpreted carefully because bulk and thin-film properties for the same material are different, and thin-film characteristics are very dependent upon initial substrate and exact deposition conditions (as well as the methods used to measure the in-situ properties). The reader is encouraged to refer to specific sources cited for specific details on these material property data.

\section{K. Measuring Thin Film Stress/Strain}

Various methods exist to measure the magnitude and homogeneity of residual stresses in thin films. The most common macroscopic method for measuring stresses in a deposited thin film is to quantify, optically, the bending of the substrate (illustrated in Figure 27) before and after 
TABLE V

MATERIAL PROPERTIES* OF VARIOUS MEMS MATERIALS $[48,49,50]$

\begin{tabular}{|c|c|c|c|c|c|c|}
\hline \multirow{2}{*}{ Material } & \multirow{2}{*}{$\begin{array}{c}\text { Coefficient } \\
\text { of Thermal } \\
\text { Expansion } \\
\left(10^{-6} / \mathrm{K}\right)\end{array}$} & \multirow{2}{*}{$\begin{array}{l}\text { Young's } \\
\text { Modulus } \\
(\mathrm{GPa})\end{array}$} & $\begin{array}{c}\text { Internal } \\
\text { Stress } \\
(\mathrm{MPa})\end{array}$ & \multirow{2}{*}{$\begin{array}{c}\text { Thermal } \\
\text { Conductivity } \\
(\mathrm{W} / \mathrm{m} \cdot \mathrm{K})\end{array}$} & \multirow{2}{*}{$\begin{array}{c}\text { Yield } \\
\text { Strength } \\
\text { (MPa) }\end{array}$} & \multirow{2}{*}{$\begin{array}{l}\text { Density } \\
\left(\mathrm{g} / \mathrm{cm}^{3}\right)\end{array}$} \\
\hline & & & $\begin{array}{l}\text { Limiting } \\
\text { Strain } \\
\left(10^{-3}\right)\end{array}$ & & & \\
\hline \multirow[t]{2}{*}{$\mathrm{Si}$} & \multirow{2}{*}{$2.3-4.3(\mathrm{~B})$} & \multirow{2}{*}{$62-202(B)$} & - & \multirow{2}{*}{$149-170(\mathrm{~B})$} & \multirow{2}{*}{$7000(B)$} & \multirow{2}{*}{$\begin{array}{c}2.3-2.42 \\
(B)\end{array}$} \\
\hline & & & $\frac{-}{200}$ & & & \\
\hline \multirow{2}{*}{$\mathrm{SiO}_{2}$} & \multirow{2}{*}{$\begin{array}{c}0.4(F) \\
0.43-0.59 \\
(B)\end{array}$} & \multirow{2}{*}{$\begin{array}{l}92(\mathrm{~S}) \\
70-73 \text { (B) } \\
67-69 \text { (dry) } \\
46-68 \text { (wet) }\end{array}$} & $\begin{array}{l}-320(\mathrm{~L}) \\
-300(\mathrm{~N}) \\
-250(B)\end{array}$ & \multirow{2}{*}{$\begin{array}{c}1.1(\mathrm{~F}) \\
0.59-1.8(\mathrm{~B})\end{array}$} & \multirow{2}{*}{$8400(B)$} & \multirow{2}{*}{$\begin{array}{c}2.3 \text { (plasma) } \\
2.2(\mathrm{~F}) \\
2.198-2.66 \\
\text { (B) }\end{array}$} \\
\hline & & & $112(F)$ & & & \\
\hline \multirow[t]{2}{*}{$\mathrm{Si}_{3} \mathrm{~N}_{4}$} & \multirow[t]{2}{*}{$\begin{array}{c}2.11-3.66 \\
(B)\end{array}$} & \multirow{2}{*}{$\begin{array}{l}385(\mathrm{~B}) \\
380(\mathrm{~F}) \\
290(\mathrm{~L}) \\
210(\mathrm{P}) \\
146(\mathrm{CVD}) \\
104-156(\mathrm{~S})\end{array}$} & $\begin{array}{c}+1000- \\
+1440(\mathrm{~L}) \\
-200- \\
+500(\mathrm{P})\end{array}$ & \multirow[t]{2}{*}{$18.5-19(\mathrm{~B})$} & \multirow[t]{2}{*}{$1400(B)$} & \multirow{2}{*}{$\begin{array}{c}3.44 \\
3.1(\mathrm{~S}) \\
3.1(\mathrm{~B}) \\
2.9-3.1(\mathrm{~L}) \\
2.4-2.8(\mathrm{P})\end{array}$} \\
\hline & & & $37(F)$ & & & \\
\hline \multirow{2}{*}{$\mathrm{Al}$} & \multirow{2}{*}{$\begin{array}{c}23.2-33.8 \\
(\mathrm{~B}) \\
23.0(\mathrm{~F}) \\
\end{array}$} & \multirow{2}{*}{$\begin{array}{c}\text { 47-74.1 (F) } \\
70(\mathrm{~B})\end{array}$} & - & \multirow{2}{*}{$234-237(\mathrm{~B})$} & \multirow{2}{*}{$\begin{array}{l}170(\mathrm{~B}) \\
124(\mathrm{~F})\end{array}$} & \multirow{2}{*}{$\begin{array}{c}2.692-2.70 \\
\text { (B) }\end{array}$} \\
\hline & & & $2(F)$ & & & \\
\hline $\mathrm{Cr}$ & $6.5(\mathrm{~B})$ & $140-215(F)$ & $\frac{-}{2(F)}$ & 90.3-91.3 (B) & - & $\begin{array}{c}7.2(\mathrm{~F}) \\
7.19(\mathrm{~B})\end{array}$ \\
\hline \multirow[b]{2}{*}{ A } & \multirow{2}{*}{$\begin{array}{c}14.1-16.5 \\
(B) \\
14.3(F)\end{array}$} & \multirow{2}{*}{$80(F)$} & - & \multirow{2}{*}{$\begin{array}{c}315.5-318 \\
\text { (B) }\end{array}$} & \multirow[b]{2}{*}{ - } & \multirow{2}{*}{$\begin{array}{c}19.28-19.4 \\
\text { (B) }\end{array}$} \\
\hline & & & - & & & \\
\hline \multirow[t]{2}{*}{$\mathrm{Pt}$} & $\begin{array}{c}8.9-10.2 \\
\text { (B) }\end{array}$ & $\begin{array}{l}170(F) \\
147(B)\end{array}$ & $\begin{array}{c}+185-+955 \\
(F)\end{array}$ & \multirow[t]{2}{*}{$71.5-73(\mathrm{~B})$} & - & $21.44(\mathrm{~B})$ \\
\hline & & & $1(\mathrm{~F})$ & & & \\
\hline
\end{tabular}

${ }^{*}(B)=$ bulk material, $(F)=$ film, $(L)=L P C V D$ film, $(N)=$ native oxide,

$(P)=$ PECVD film, $(S)=$ sputtered film.

the film deposition. A film with tensile stress will bend and render the surface concave, and a compressive film will render the surface convex. Stress values are obtained by measuring macroscopic changes in the bow or radius of curvature of the substrate (by measuring the deflection in the center of the wafer). This method assumes the film is homogeneous with uniform thickness, and that the stress is constant through its thickness. However, residual stress can vary considerably through the thickness of a deposited thin film. While CVD processes usually deposit uniform thin films, properties of sputter-deposited films vary considerably (in composition, uniformity, and thickness) across the substrate. Moreover, this wafer-curvature-measurement 
method does not necessarily measure local stress in the thin film, since wafer deformations are induced macroscopically [45].

Microscale methods also exist for measuring thin film stresses, making use of a variety of methods, such as measuring the deflection of thin-film structures freed by a sacrificial layer removal, external mechanical probing of free regions of the film, measuring the deflection of pressurized membranes, and measuring resonant frequencies. Local stress measurements can be made using in situ surface, or bulk, micromachined structures (such as strain gauges) made directly out of the thin film of interest itself. These structures can also be used to measure strain variations across wafer surfaces to identify sources of strain nonuniformity. Structures such as cantilever beams, clamped-clamped beams, ring crossbars, vernier gauges, and lateral resonators have been developed to measure thin film intrinsic mechanical properties. One technique for obtaining biaxial measurements of stress in low-modulus, tensile materials (such as polyimides) is the blister test, where a diaphragm of the thin film material is created on a silicon substrate, one side is pressurized, and the corresponding deflection is measured. The residual stress and biaxial modulus (E) can then be obtained. The mechanical response of cantilevers, for example, is affected by residual film stress. If the residual stress varies in the direction of film growth (i.e. a strain nonuniformity), the resulting intrinsic bending moment will warp or curl cantilever structures that have been released from the substrate. Nonuniform residual stress gradients can also be formed in thin films, leading to internal bending moments that cause the films to deform (or curl) when released (converting the stress gradient into a strain gradient). If present, such gradients are usually seen as upward or downward curvature of released microstructures. Cantilever Archimedean spirals have been developed as test structures for measuring the average values of such gradients. An array of doubly-supported beams, with identical cross sections but incrementally-increasing lengths, can also be fabricated. Below a critical length, some beams will be perfectly straight and free of bowing, and above this cutoff point, the rest will be buckled. Thus, a strain-related threshold may be revealed, leading to the accurate and simple measurement of compressive strain in a MEMS thin film $[45,51,52]$. 


\section{MASS AIRFLOW SENSOR DESIGN}

\section{A. The Original 1997 Flow Sensor Design}

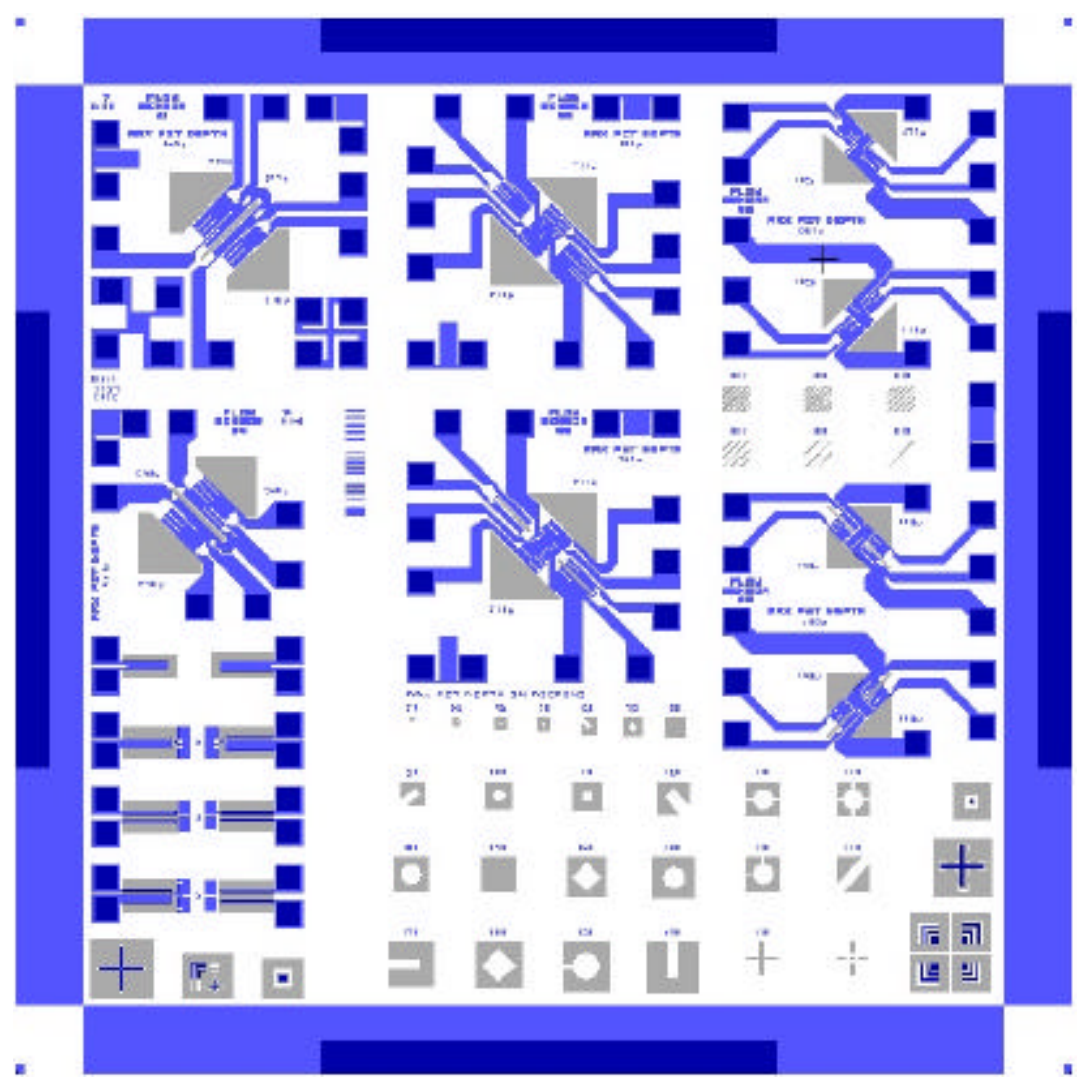

FIGURE 30 - $\quad$ A screen-capture of the 1997 L-Edit TM photomask design file. Blue regions represent metallization areas, and gray regions represent etch windows in the $\mathrm{RIE} / \mathrm{KOH}$ mask.

\section{Photomask Design Overview}

In preparation for the first undergraduate microfabrication course offering at the University of Louisville in the Fall of 1997, a first flow sensor design (shown in Figure 30) was developed based largely upon the previously-articulated Honeywell flow sensor. The fabrication process sequence was to include a single sputter-deposited metallization layer of permalloy, patterned using the lift-off technique, sandwiched between two $\sim 0.5 \mu \mathrm{m}$-thick RF planar magnetron sputter-deposited silicon nitride layers, all on a (100) two-inch-diameter silicon 
substrate. A two-layer photomask set was designed using Tanner's L-Edit ${ }^{\mathrm{TM}}$ software with a single metallization layer and substrate $(\mathrm{RIE}$ and $\mathrm{KOH})$ etch-window masking layer. The $\mathrm{Si}_{x} \mathrm{~N}_{\mathrm{y}}$ layer was to be patterned using the etch-window photomask, and an RIE system (this same mask was used to delineate windows for anisotropic etching into the silicon substrate). Both masks were designed to be used with positive photoresist patterning techniques. Detailed images from the design file for this mask set are shown and described in Appendix XXII, in which the resistor metallization layer is shown in blue, and the RIE/KOH etch-window layer is shown in gray. The L-Edit ${ }^{\mathrm{TM}}$ design file was translated into a two-layer GDS-II format file, which was sent to the Microelectronic Engineering group at RIT for mask creation (Microelectronic Engineering Mask Shop; ATTN: Suraj Bhaskaran, c/o Lynn Fuller; 82 Lomb Memorial Drive; Rochester, NY 14623; 716-475-5843), where the following additional specifications and clarifications were used to write the two masks (in addition to specifying which GDS II layers were in the design):

1. As many design replications as possible should be fit onto each 5-inch chrome mask.

2. The minimum feature size in the design file is $5 \mu \mathrm{m}$.

3. The design is square for both masks: $5930 \mu \mathrm{m}$ wide by $5930 \mu \mathrm{m}$ long.

4. Both photomasks should be darkfield masks (with digitized areas in the design file being transparent).

5. The "street width" should be zero, with no spacing between replications in the design.

6. There should be zero rotation so that the perimeter edges of the design are placed on the photomask parallel to the plate edges.

7. Since the design file has text, the design should be properly mirrored so that text is readable when it is processed onto a silicon wafer.

8. The photomasks will be used in a $1 X$ Kasper contact mask aligner system.

The photomask design file (shown in Appendix XXII) included eight separate flow sensor device geometries, mask alignment/registration structures, Van der Pauw sheet resistance measurement structures, novel etch depth estimation/measurement structures, and other test structures for etching evaluation and cantilever deflection studies within a $5930 \mu m^{2}$ area. A general synopsis and comparison of the different flow sensor design geometries is included in 
Table VI. The design included two dual-microbridge sensors (\#1 and \#4), significantly based upon the original Honeywell flow sensor design; two large, single microbridges (\#2 and \#5); and two sensors (\#3 and \#6) with two small single microbridges, each. The small, single-microbridge sensors (\#3 and \#6) were designed to operate with a flow stream directed longitudinally, along the main axis of each bridge. This configuration offered the advantage of a continuous surface over which air can flow that will not cause any minor flow turbulence, but has the disadvantage of

TABLE VI

SYNOPSIS OF THE 1997 PHOTOMASK SET DESIGN

\begin{tabular}{|c|c|c|c|}
\hline $\begin{array}{c}\text { Flow Sensor } \\
\text { Number }\end{array}$ & $\begin{array}{c}\text { Sensor } \\
\text { Structural Design }\end{array}$ & $\begin{array}{c}\text { Slotted } \\
\text { Bridge(s)? }\end{array}$ & $\begin{array}{c}\text { Design } \\
\text { Flow Stream } \\
\text { Direction }\end{array}$ \\
\hline 1 & Dual Microbridge & No & Lateral \\
\hline 2 & Single Microbridge & No & Lateral \& Longitudinal \\
\hline 3 & Two Single Microbridges & No & Longitudinal \\
\hline 4 & Dual Microbridges & No & Lateral \\
\hline 5 & Single Microbridge & Yes & Lateral \& Longitudinal \\
\hline 6 & Two Single Microbridges & Yes & Longitudinal \\
\hline
\end{tabular}

relatively little air flow under the microbridges. The dual microbridge design was for flow streams directed laterally across each bridge structure. The large single microbridge sensors (\#2 and \#5) were designed to sense flow directed either or both, laterally across, or longitudinally over, the microbridge. Flow sensor designs \#5 and \#6 included bridges with "slots" to facilitate faster etching and undercutting of the bridge structures in the anisotropic silicon-substrate etchant, $\mathrm{KOH}$. The nominal street width for dicing apart the replicated die areas (with eight flow sensors each in $5930 \mu \mathrm{m}^{2}$ ) was $760 \mu \mathrm{m}$, with a $380 \mu \mathrm{m}$-wide etch-window portion inside each street for etching trenches in the silicon substrate between replicated die. The square bonding pad (etchwindow-opening) area was 130 by $130 \mu \mathrm{m}$ at each pad, with a $10 \mu \mathrm{m}$-wide overlap for metallization (i.e. the resistor metallization at each pad extended $10 \mu \mathrm{m}$ out beyond each proximal pad-etch-window-opening edge). Heater-resistor nominal design line-widths were $10 \mu \mathrm{m}$, with sense resistor line-widths of $5 \mu \mathrm{m}$. Table VII shows a more detailed comparison of 
TABLE VII

SUMMARY OF FLOW SENSOR PHYSICAL SPECIFICATIONS IN THE 1997 MASK SET

\begin{tabular}{|c|c|c|c|c|c|}
\hline $\begin{array}{l}\text { Flow } \\
\text { Sensor }\end{array}$ & $\begin{array}{c}\text { Design } \\
\text { Length } \mathbf{x} \text { Width } \\
\text { of (each) } \\
\text { Microbridge } \\
(\mu \mathrm{m})\end{array}$ & $\begin{array}{c}\text { Design } \\
\text { Length } \mathbf{x} \text { Width } \\
\text { of Each Sense } \\
\text { Resistor }(\mu \mathrm{m})\end{array}$ & $\begin{array}{l}\text { Minimum } \\
\text { Bridge } \\
\text { Edge } \\
\text { Tolerance } \\
\quad(\mu \mathrm{m})\end{array}$ & $\begin{array}{c}\text { Bridge } \\
\text { Etch- } \\
\text { Window } \\
\text { Slot } \\
\text { Width } \\
(\mu \mathrm{m})\end{array}$ & $\begin{array}{c}\text { Minimum } \\
\text { Spacing } \\
\text { Between } \\
\text { Heater \& } \\
\text { Nearest } \\
\text { Sense } \\
\text { Resistor }(\mu \mathrm{m})\end{array}$ \\
\hline 1 & $395 \times 171$ & $254 \times 101$ & 10 & 20 & 20 \\
\hline 2 & $542 \times 298$ & $\begin{array}{c}215 \times 103 \text { (long.) } \\
169 \times 79 \text { (lat.) }\end{array}$ & $\sim 10$ & $\mathrm{n} / \mathrm{a}$ & $\mathrm{n} / \mathrm{a}$ \\
\hline 3a (top) & $385 \times 181$ & $115 \times 81$ & $\sim 10$ & $\mathrm{n} / \mathrm{a}$ & $\mathrm{n} / \mathrm{a}$ \\
\hline $3 b$ & $385 \times 181$ & $116 \times 81$ & 9 & $\mathrm{n} / \mathrm{a}$ & $\mathrm{n} / \mathrm{a}$ \\
\hline 4 & $395 \times 171$ & $254 \times 101$ & 10 & 20 & 20 \\
\hline 5 & $542 \times 298$ & $\begin{array}{r}242 \times 103 \text { (long.) } \\
169 \times 79 \text { (lat.) }\end{array}$ & $\sim 10$ & 11 & 11 \\
\hline $6 a$ (top) & $320 \times 181$ & $115 \times 69$ & 8 & 11 & 11 \\
\hline $6 b$ & $320 \times 181$ & $115 \times 123$ & $\sim 8$ & 11 & 11 \\
\hline
\end{tabular}

the different bridge and sense resistor geometries in the 1997 flow sensor photomask set. The bridge edge tolerance is the minimum distance between the proximal edge of the sense or heater resistor metallization on a given bridge, and the bridge edge itself. This tolerance is a measure of allowable photomask $x$-y alignment error. It was soon discovered after using this mask set that these tolerances of $8-10 \mu \mathrm{m}$ should have been designed larger to allow for greater mask misalignments and consequently improved device-yield when using the $1 \mathrm{X}$ Kasper contact mask aligner (with an estimated, nominal x-y optical alignment error of $~ 2-3 \mu \mathrm{m}$ ).

The electrical specifications, in terms of the number of squares for various resistors, for the 1997 flow sensor design file are given in Table VIII. Note that the upstream $\left(R_{u}\right)$ and downstream $\left(R_{d}\right)$ sense resistors were symmetric. Designed line widths for all (upstream and downstream) sense resistors and substrate temperature-sensing serpentine resistors were $5 \mu \mathrm{m}$, and line widths for all heater resistors were $10 \mu \mathrm{m}$. The number of squares specified for each resistor does not include bonding and wide-interconnect routing areas, and are approximations (e.g. corners within resistors count as 0.56 squares) [53]. 
TABLE VIII

SUMMARY OF FLOW SENSOR ELECTRICAL SPECIFICATIONS FOR THE 1997 MASK SET

\begin{tabular}{|c|c|c|c|}
\hline Flow Sensor & $\begin{array}{l}\text { Heater Resistor } \\
\text { \# of Squares } \\
(10 \mu \mathrm{m} \text { line widths) }\end{array}$ & $\begin{array}{c}\text { Upstream or } \\
\text { Downstream Resistor } \\
\text { \# of Squares } \\
\text { (5 } 5 \mathrm{~m} \text { line widths) }\end{array}$ & $\begin{array}{c}\text { Substrate } \\
\text { Resistor } \\
\text { \# of Squares } \\
(5 \mu \mathrm{m} \text { line widths) }\end{array}$ \\
\hline 1 & 203.5 & 520.5 & 519.3 \\
\hline 2 & 96.4 & $\begin{array}{c}440 \text { (long.) } \\
278.2 \text { (lateral) }\end{array}$ & $\begin{array}{c}519.2 \text { (bottom left) } \\
450.4 \text { (top right) }\end{array}$ \\
\hline $3 a$ (top) & 75.7 & 194.2 & \multirow{2}{*}{450.4 (bottom right) } \\
\hline 3b (bottom) & 75.7 & 194.2 & \\
\hline 4 & 185.7 & 520.5 & 424 (top left) \\
\hline 5 & 96.4 & $\begin{array}{l}368.2 \text { (long.) } \\
278.2 \text { (lateral) }\end{array}$ & $\begin{array}{c}519.2 \text { (bottom left) } \\
450.4 \text { (top right) }\end{array}$ \\
\hline $6 a$ (top) & 75.7 & 248.1 & \multirow{2}{*}{450.4 (top right) } \\
\hline 6b (bottom) & 75.7 & 248.1 & \\
\hline
\end{tabular}

\section{Etch Depth Measurement Structures}

170

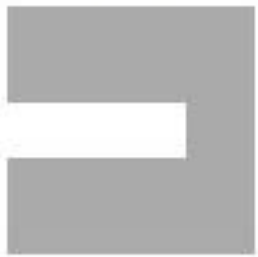

I 日

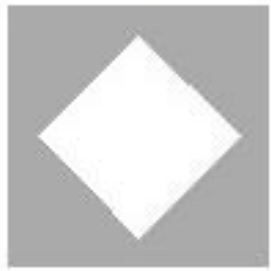

\section{0}

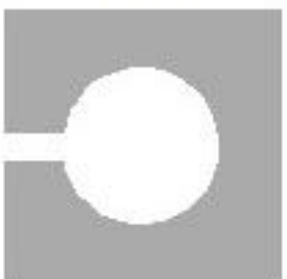

FIGURE 31 - Three pyramidal etch-depth measurement structures. These preciselydimensioned square "windows" were designed to anisotropically etch down to the maximum pyramidal etch depths written above each window.

As shown in Appendix XXII, this mask set design also included a region of novel anisotropic (100) silicon substrate etch-depth measurement structures in the etch-window photomask layer (which is shown in gray in the figures). Three of these structures are shown in Figure 31. There were a total of 19 such square windows, with each precisely dimensioned to show etch depths into the (100) silicon substrate from 20 to $200 \mu \mathrm{m}$ in increments of $10 \mu \mathrm{m}$. These square etch-window structures were designed to help monitor the progress of anisotropic silicon etching. 
Each structure was square with its corresponding maximum pyramidal etch depth shown as a (metallization) label above it. Figure 26 shows that there is a $54.74^{\circ}$ angle of inclination between the $\{111\}$ and $\{100\}$ planes in a (100) silicon substrate. It can then be shown, geometrically, that for a square, etch-window opening aligned paralled to the top of the (100) silicon substrate, with side length, $s$, aligned parallel to the [110] wafer flat, the maximum pyramidal etch depth, $d$, into the silicon substrate, bounded on the four sloping sides by $\{111\}$ and other crystal surfaces is:

$$
s=\frac{2 d}{\tan \left(54.74^{\circ}\right)} \approx \frac{2 d}{\sqrt{2}}=d \sqrt{2} \quad(\mu m)
$$

This equation was used to calculate the side-edge design length, $s$, for each etch-depthmeasurement square, for a given desired maximum etch depth, $d$.

\section{Mask Alignment Features}

The fabrication process included aligning the first (resistor metallization) photomask to the (100) silicon substrate by aligning the primary wafer flat edge parallel to any proximal edge of the rectangles that comprised the streets between replications, as shown in Figure 32 . The primary wafer flat, and the selected rectangle edge would then both be parallel to the $\{110\}$ planes in the wafer so that the microbridge longitudinal axes would be rotated $45^{\circ}$ with respect to the $<110>$ crystallographic direction in the silicon substrate. Since both photomasks were darkfield masks (digitized areas shown in the design layout were transparent with all other areas opaque), these large rectangular areas comprising the streets (or within the streets, in the case of street etch-window rectangles) between die replications were also created to allow users to see through the photomask to align to features on the wafer. Five manual mask-alignment markings, shown grouped together in Figure 33, were derived from various sources [54] and included in the 1997 mask set. The alignment structures shown in Figure 33 represent perfectly aligned structures, with gray regions (present only on the etch-window photomask) being aligned to blue 


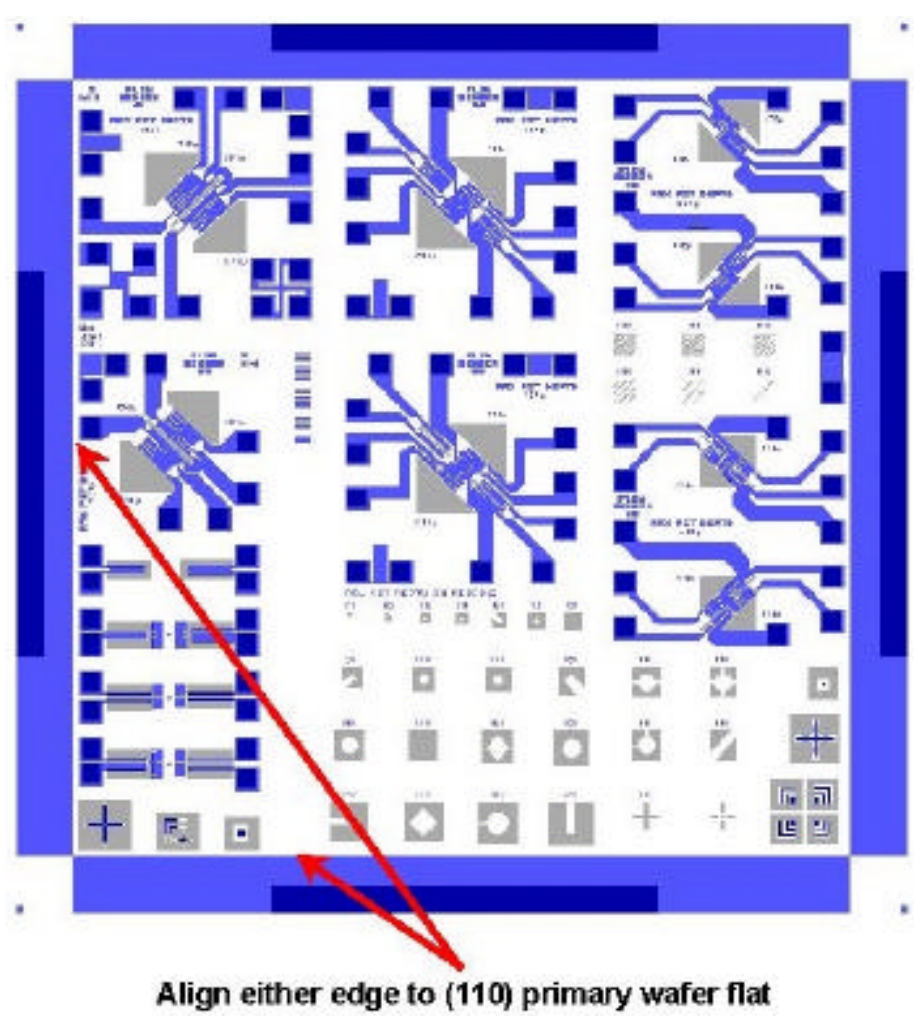

FIGURE 32 - How to align the metallization mask to the (110) wafer flat. This individual die replication shows which edges of the first (metallization) photomask are aligned to the (110) primary wafer flat on a (100) $n$ or $p$-type silicon substrate.

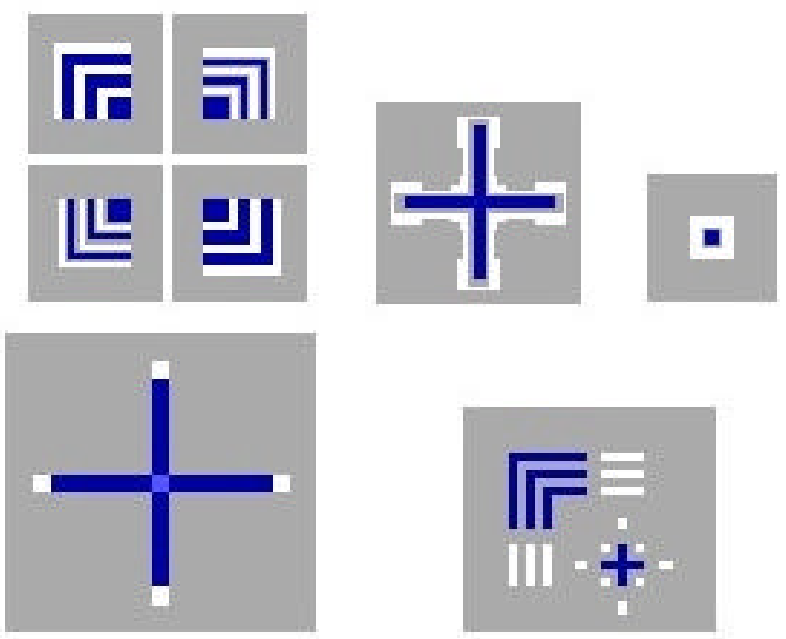

FIGURE 33 - Manual mask alignment (or registration) marks in the 1997 mask set.

metallization regions already patterned on (and aligned with respect to) the (100) silicon substrate. The alignment marking shown at the top, center in Figure 33 was found to be the most helpful for alignment after much experience was gained in using this mask set. 


\section{Sheet Resistance Measurement Structures}

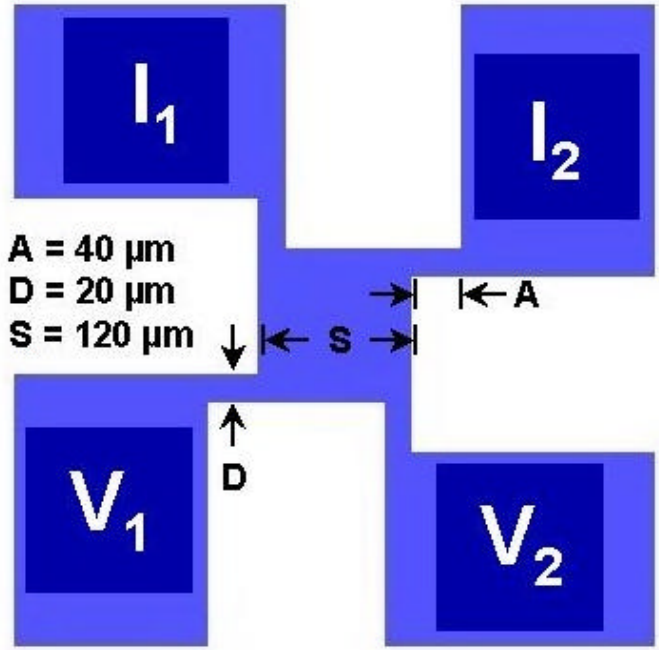

Offset Quadrate Cross

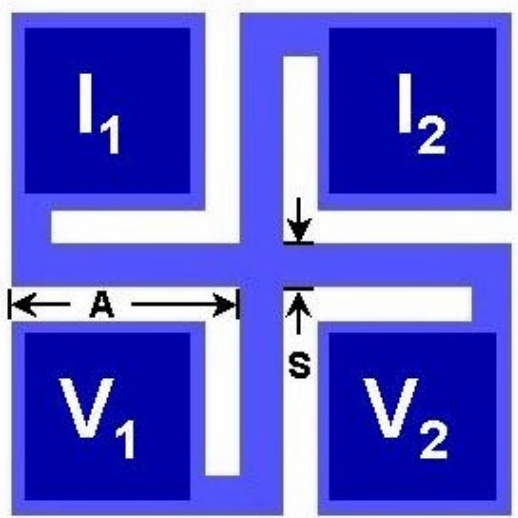

$$
A=180 \mu \mathrm{m}
$$$$
\mathrm{S}=30 \mu \mathrm{m}
$$

Greek Cross

FIGURE 34 - Van der Pauw test structures, with labels, in the 1997 mask set. These were designed to measure sheet resistance of the thin-film metallization layer. A current is injected through terminals $I_{1}$ and $I_{2}$, and the subsequent voltage drop is measured between terminals $V_{1}$ and $V_{2}$.

Finally, this mask set included a Greek cross and offset quadrate cross, four-terminal Van der Pauw structures for measuring sheet resistance of the thin-film metallization layer. These structures are shown in Figure 34. A probe station, constant-current source, and voltmeter were used to inject a known current $\left(I_{12}\right)$ into terminals $I_{1}$ and $I_{2}$, and measure the corresponding, subsequent voltage drop $\left(\mathrm{V}_{12}\right)$ between terminals $\mathrm{V}_{1}$ and $\mathrm{V}_{2}$ on either Van der Pauw structure. The formula for calculating the sheet resistance for either Van der Pauw structure is [55]:

$$
R_{s}=\frac{\left(\frac{\pi}{\ln 2}\right)\left(\frac{V_{12}}{l_{12}}\right)}{1-E} \quad(\Omega \text { /square })
$$

where $E$ is the normalized sheet resistance error due to the width and length of the contact arms for the quadrate cross (or contact arm length for the Greek cross), $V_{12}$ is the voltage across 
terminals $V_{1}$ and $V_{2}$, and $I_{12}$ is the current through terminals $I_{1}$ and $I_{2}$. Since, for the offset quadrate cross structure, $\mathrm{E}<<0.001$, and for the Greek cross structure the error is at most $3.8 \mathrm{x}$ $10^{-17}, E$ can be assumed to be zero, and the Van der Pauw equation can be reduced to:

$$
R_{s}=\left(\frac{\pi}{\ln 2}\right)\left(\frac{V_{12}}{l_{12}}\right)=\frac{\rho_{r}}{t} \quad(\Omega \text { /square })
$$

where $\rho_{r}$ is the resistivity of the thin film, with thickness $t$. This is the equation to use for these sheet resistance measurement structures [55].

\section{B. The Redesigned 1998/1999 Photomask Design}

\section{Photomask Design Overview}

Significant problems with successfully fabricating flow sensors from the 1997 photomask set led to the development of a completely redesigned and improved three-layer mask set. The original photomask set was redesigned, principally for the following reasons:

1. An additional photomask was needed for an inert gold metallization layer to protect exposed pad (resistor metallization) regions from degradation in the anisotropic, alkaline silicon etchant, $\mathrm{KOH}$;

2. A resistor metallization mask design was needed that would allow for patterning of positive photoresist to delineate resistor metallization by wet etching, instead of liftoff, which produced poor results in 1997 fabrication attempts;

3. More experimental geometries of anemometer sensors, including cantilevers, could be added to the design;

4. Larger tolerances were needed between proximal edges of resistor metallization on microbridges and the edges of the bridges themselves, to provide a greater margin for mask-to-substrate misalignments in the Kasper aligner; 
5. Thin-film, suspended microstructures could be added to the design (in the Etch Window Level 3 photomask) to permit the in-situ analysis/evaluation of residual stress/strain properties of sputtered silicon nitride thin films.

The Honeywell flow sensor geometry was again used as the basis of this design, but the fabrication process sequence was changed by adding a second metallization layer of gold, and by replacing the liftoff technique with isotropic wet-etching as the method to pattern all metallization layers. The processing sequence was again to include a sputter-deposited layer of (subsequently wet-etched) resistor and gold metallization, sandwiched between two $\sim 0.5 \mu \mathrm{m}$ thick RF planar magnetron sputter-deposited silicon nitride layers, on a (100) silicon substrate. Thus, the microbridges were again designed to be comprised of silicon nitride. All three photomasks were developed to be used with positive photoresist (wet-etching or RIE) patterning techniques. As in the 1997 design, the Etch Window Level 3 photomask was developed to be used for both Reactive lon Etching through the silicon nitride layer and anisotropic etching of the (100) silicon substrate to suspend the microbridges.

As with the first mask set, this design was created with Tanner's L-Edit ${ }^{\mathrm{TM}}$ software, converted into a three-layer GDS-II format file, and then electronically transmitted to the aforementioned RIT mask shop for photomask production. Design specifications were identical to those of the first mask set creation, except for the following clarifications:

1. The design file size is rectangular: $22,967 \mu \mathrm{m}$ wide by $11,500 \mu \mathrm{m}$.

2. The specified-layer resistor and gold metallization photomasks should be lightfield masks, and the etch window mask should be darkfield.

Images of devices, features, and regions within this mask set are shown and described, in depth, in Appendix XXIII, where three layers are visible: a resistor metallization layer shown in blue, a gold layer shown in light green, and an RIE/KOH etch window masking layer shown in gray. The replicated die size was significantly larger, and rectangular instead of square, occupying $22.967 \times 11.5 \mathrm{~mm}$. Seven and one-half of the original die would fit within one of the replicated die in this new design. The new die design included 20 flow sensor geometries, two regions of mask alignment/registration structures, Van der Pauw sheet resistance measurement 
structures, etch depth estimation/measurement structures, and seven different types of new insitu stress/strain evaluation microstructures. Research showed that the inclusion of various geometries and designs of unique, in-situ, residual stress/strain evaluation structures might be helpful, because attempts to fabricate flow sensors in 1997 failed, in part, due to intrinsic compressive strain and porosity in the RF planar magnetron sputtered thin film $\mathrm{Si}_{x} \mathrm{~N}_{\mathrm{y}}$ layer comprising the microbridges (making this structural layer both vulnerable to $\mathrm{KOH}$ degradation and structural failure due to cracking). These stress/strain evaluation structures could then be used to discover a suitable sputtering recipe that deposits a more stoichiometric $\mathrm{Si}_{x} \mathrm{~N}_{\mathrm{y}}$ layer, that is preferably under residual tensile stress. Since these stress/strain evaluation structures would occupy significant space in the design (nearly four times the size of the 1997 flow sensor photomask die replication), 16 more flow sensor geometries (for a total of 20 designs) with new structural variations were added to fill up and make the replicated die size rectangular. Thus, one half of the total die area was designated for 20 different flow sensor layouts, and the other half used for the newly-included stress/strain evaluation microstructures.

As mentioned, new structural variations based upon the original Honeywell flow sensor were added to this design. In addition to the standard dual and single (doubly-supported) microbridge flow sensors, new experimental cantilever and mixed cantilever/microbridge sensors were developed and incorporated. The inclusion of cantilever-designed sensors was obviously more risky considering previously deposited $\mathrm{Si}_{x} \mathrm{~N}_{y}$ thin films were under compression and, if not improved (to be under tension), would cause these structures to buckle up into the flow stream or down into etched pit cavities.

Flow sensor fabrication attempts in 1997 with the original mask set showed damage to, or complete destruction of, resistor metallization at exposed bonding pad areas due to degradation from exposure to elevated temperature $\mathrm{KOH}$. An exposed pad metallization layer was needed that would provide protection against degradation in the silicon etchant. Consequently, since gold is inert in highly alkaline solutions such as $\mathrm{KOH}$ and $\mathrm{TMAH}$, a gold metallization mask layer was added to the design. Gold routing was also added to other traces to decrease their total resistance (so that the overall resistance of a given resistor would approach the theoretical 
value obtained from the designed number of squares, sheet resistance and metallization thickness measurements). Mask alignment markings were upgraded to include the addition of the new gold mask layer in the design and the need to align this layer with existing structures.

The nominal street width for dicing apart replicated $22.967 \times 11.5 \mathrm{~mm}$ die areas (i.e. the spacing of periphery pad area resistor metallization between adjacent devices) in this new design was the same as in the 1997 mask set: $380 \mu \mathrm{m}$. All streets between individual flow sensors and streets around the perimeter of the in-situ stress/strain structure die area included a narrow 50 $\mu m$-wide etch window street that allowed for etching $35 \mu m$-deep shallow trenches into the silicon substrate to facilitate wafer cleaving or dicing along those streets. The square bonding pad (etch-window opening) area was increased to $150 \times 150 \mu \mathrm{m}$ for all devices.

The overlap of gold metallization with the pad etch-window opening (i.e. the distance the gold layer extended out beyond each proximal edge of the square etch-window opening at each pad), and the overlap of the resistor metallization with the gold metallization layer at the pads was increased to $20 \mu \mathrm{m}$ each. For all flow sensors, heater resistor design line-widths were again 10 $\mu \mathrm{m}$, and sense resistor design line-widths were $5 \mu \mathrm{m}$.

Three different, reconfigured substrate resistor layouts were included in this design. One very long serpentine resistor, $5 \mu \mathrm{m}$ wide, with 1966 squares was placed with some flow sensors. And two different "parallel" substrate resistors (one of which is shown in Figure 35), one with a 5 $\mu m$ line width, and the other with a $10 \mu m$ line width, were placed with other sensors. Each of these "parallel" substrate resistors had a 250, 500, and 750-square resistor all connected in parallel (allowing for seven possible resistance values), with routing designed such that one or more resistors could be removed by scratching through metallization traces, as shown in Figure 35. At the time this design was created, it was believed that this configuration would allow for greater flexibility in choosing the desired substrate resistor resistance, but in practice (depending upon the actual metallization layer's physical properties and thickness) it was discovered that it was not easy to scratch through the resistor traces (under a microscope).

Several other improvements were made to this design over the original photomask 


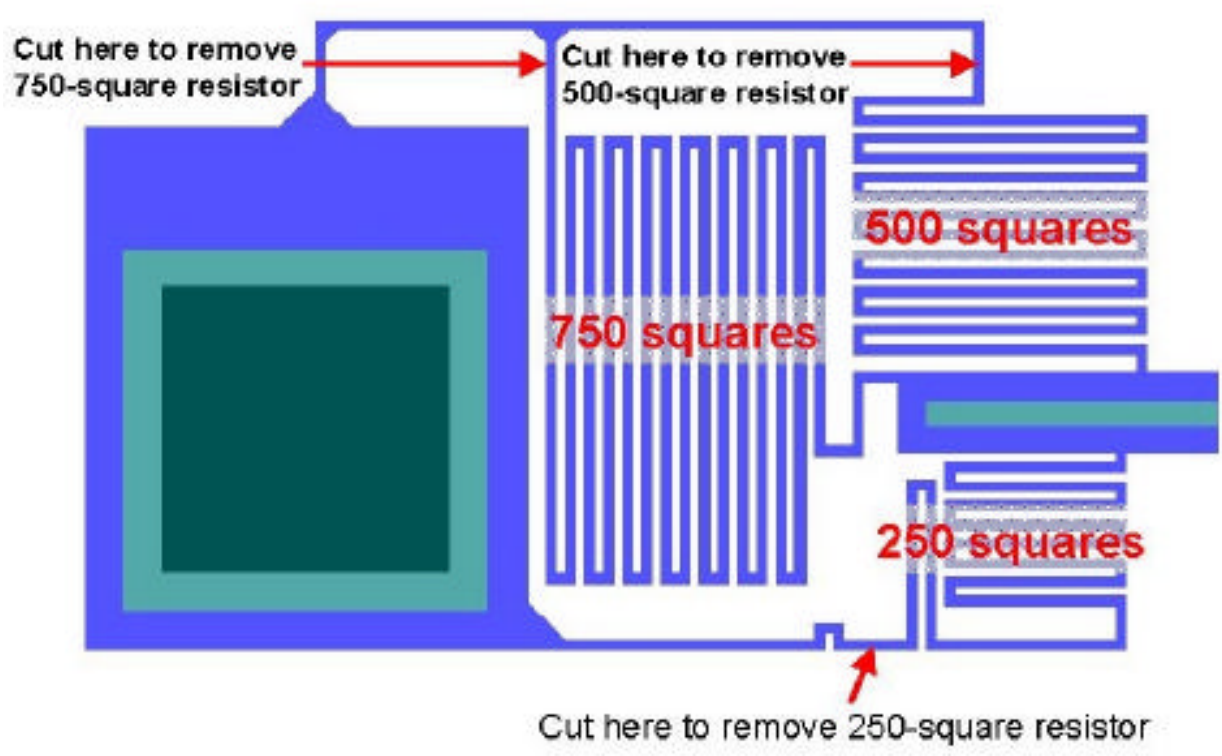

FIGURE 35 - A design file image of the parallel substrate resistor in the '98/'99 mask set. Resistor locations are shown with recommended areas to scratch through metallization traces to customize the total combined resistance.

layout. Larger "bridge tolerances" (at least 100\% larger) were provided between proximal edges of resistor metallization on bridges and bridge edges themselves, to allow for larger mask-tomask misalignment tolerances. Additional metallization structural support was added to the substrate attachment regions on some microbridges. Where applicable, etch-window slots on microbridges were widened to facilitate faster undercutting of bridges and cantilevers in the anisotropic silicon etchant. All text character and number sizes (appearing as metallization on the substrate) were increased for easier readability, and large flow sensor device numbers were added to facilitate easier, unaided (unmagnified) visual identification during dicing, packaging, etc., of individual sensors. On some flow sensors, four sense-resistor bonding pad areas were reduced to three by combining two of the nodes into one common node; and similarly, on some devices, a substrate-and-heater resistor common-bonding-pad node was provided.

Table IX provides a summary comparison of the different structural designs and flow stream directions for sensors in the redesigned 1998/1999 mask set, and Table X, shows a more detailed comparison of the different bridge and sense resistor geometries in this design. In Table $\mathrm{X}$, the specified microbridge or cantilever lengths are approximations, because the actual length depends upon how far the anisotropic silicon etchant undercuts the bridges, how long the 
TABLE IX

SYNOPSIS OF THE 1998/1999 PHOTOMASK SET DESIGN

\begin{tabular}{|c|c|c|c|c|c|}
\hline $\begin{array}{c}\text { Flow } \\
\text { Sensor } \\
\text { Number }\end{array}$ & $\begin{array}{l}\text { Flow Sensor } \\
\text { Structural Design }\end{array}$ & $\begin{array}{c}\text { Slotted } \\
\text { Bridge(s)? }\end{array}$ & $\begin{array}{c}\text { Sense- } \\
\text { Resistor } \\
\text { Common } \\
\text { Node? }\end{array}$ & $\begin{array}{l}\text { Design } \\
\text { Flow Stream } \\
\text { Direction }\end{array}$ & $\begin{array}{c}\text { Substrate } \\
\text { Resistor(s)* }\end{array}$ \\
\hline 1 & Dual Microbridge & No & Yes & Lateral & $\begin{array}{l}\mathrm{R}_{\mathrm{p} 5} \\
\mathrm{R}_{\mathrm{alt}}\end{array}$ \\
\hline 2 & Dual Microbridge & No & No & Lateral & $\begin{array}{l}\mathrm{R}_{\mathrm{p} 5} \\
\mathrm{R}_{\mathrm{alt}}\end{array}$ \\
\hline 3 & Dual Microbridge & No & No & Lateral & $\begin{array}{l}\mathrm{R}_{\mathrm{p} 10} \\
\mathrm{R}_{\mathrm{alt}}\end{array}$ \\
\hline 4 & Dual Microbridge & No & Yes & Lateral & $\begin{array}{l}\mathrm{R}_{\mathrm{p} 10} \\
\mathrm{R}_{\mathrm{alt}}\end{array}$ \\
\hline 5 & $\begin{array}{c}\text { Two Single } \\
\text { Microbridges }\end{array}$ & Yes & No & Longitudinal & $\mathrm{R}_{\mathrm{p} 5}$ \\
\hline 6 & $\begin{array}{c}\text { Two Single } \\
\text { Microbridges }\end{array}$ & Yes & No & Longitudinal & $\mathrm{R}_{\mathrm{p} 5}$ \\
\hline 7 & $\begin{array}{l}\text { Two Single } \\
\text { Microbridges }\end{array}$ & Yes & No & Longitudinal & $\mathrm{R}_{\mathrm{p} 5}$ \\
\hline 8 & $\begin{array}{c}\text { Two Single } \\
\text { Microbridges }\end{array}$ & Yes & No & Longitudinal & $\mathrm{R}_{\mathrm{p} 5}$ \\
\hline 9 & $\begin{array}{c}\text { Two Single } \\
\text { Microbridges }\end{array}$ & No & No & Longitudinal & $\mathrm{R}_{\mathrm{p} 5}$ \\
\hline 10 & Single Microbridge & Yes & No & Lat. \& Long. & $\mathrm{R}_{\mathrm{p} 5}$ \\
\hline 11 & Dual Cantilevers & No & No & Lateral & $\begin{array}{l}\mathrm{R}_{\mathrm{p5}} \\
\mathrm{R}_{\mathrm{p} 10}\end{array}$ \\
\hline 12 & Dual Cantilevers & No & No & Lateral & $\begin{array}{l}R_{p 5} \\
R_{p 10}\end{array}$ \\
\hline 13 & Dual Cantilevers & No & Yes & Lateral & $\mathrm{R}_{\mathrm{p} 5}$ \\
\hline 14 & $\begin{array}{l}\text { Dual Merged } \\
\text { Cantilevers }\end{array}$ & No & No & Lateral & $\begin{array}{l}\mathrm{R}_{\mathrm{p} 5} \\
\mathrm{R}_{\mathrm{p} 10}\end{array}$ \\
\hline 15 & $\begin{array}{c}\text { Dual Merged } \\
\text { Cantilevers }\end{array}$ & No & $\mathrm{Yes}^{\star *}$ & Lateral & $\begin{array}{l}\mathrm{R}_{\mathrm{p5}} \\
\mathrm{R}_{\mathrm{p} 10}\end{array}$ \\
\hline 16 & Dual Cantilevers & No & No & Lateral & $\mathrm{R}_{\mathrm{p} 5}$ \\
\hline 17 & Dual Microbridge & No & Yes & Lateral & $\begin{array}{l}\mathrm{R}_{\mathrm{p5}} \\
\mathrm{R}_{\mathrm{p} 10}\end{array}$ \\
\hline 18 & $\begin{array}{c}\text { Dual Cantilevers } \\
\text { with One Merged } \\
\text { Cantilever }\end{array}$ & No & No & Lateral & $\mathrm{R}_{\mathrm{p} 10}$ \\
\hline 19 & $\begin{array}{l}\text { Dual Cantilever with } \\
\text { One Microbridge }\end{array}$ & No & No & Lateral & $\mathrm{R}_{\mathrm{p} 10}$ \\
\hline 20 & Dual Microbridge & No & No & Lateral & $\begin{array}{l}\mathrm{R}_{\mathrm{p} 5} \\
\mathrm{R}_{\mathrm{p} 10} \\
\end{array}$ \\
\hline
\end{tabular}

* $R_{p 5}$ and $R_{p 10}$ are the $5 \mu \mathrm{m}$ and $10 \mu \mathrm{m}$ line-width "parallel" substrate resistors, and $R_{a l t}$ is the long serpentine substrate resistor.

** Flow Sensor \#15 was erroneously designed such that the common sense resistor node is inaccessible for external wire-bonding, making this device unusable. 
TABLE $X$

1998/1999 PHOTOMASK SET FLOW SENSOR PHYSICAL SPECIFICATIONS

\begin{tabular}{|c|c|c|c|c|c|}
\hline $\begin{array}{l}\text { Flow } \\
\text { Sensor }\end{array}$ & $\begin{array}{c}\text { Design } \\
\text { Length } \mathbf{x} \text { Width } \\
\text { of (each) } \\
\text { Microbridge/ } \\
\text { Cantilever } \\
(\mu \mathrm{m})\end{array}$ & $\begin{array}{c}\text { Design } \\
\text { Length } \mathbf{x} \text { Width } \\
\text { of Each Sense } \\
\text { Resistor }(\mu \mathrm{m})\end{array}$ & $\begin{array}{l}\text { Minimum } \\
\text { Bridge } \\
\text { Edge } \\
\text { Tolerance } \\
(\mu \mathrm{m})\end{array}$ & $\begin{array}{c}\text { Bridge } \\
\text { Etch- } \\
\text { Window } \\
\text { Slot } \\
\text { Width* } \\
(\mu \mathrm{m})\end{array}$ & $\begin{array}{c}\text { Minimum } \\
\text { Spacing } \\
\text { Between } \\
\text { Heater \& } \\
\text { Nearest Sense } \\
\text { Resistor }(\mu \mathrm{m})\end{array}$ \\
\hline 1 & $434 \times 191$ & $254 \times 101$ & 20 & 20 & 10 \\
\hline 2 & $434 \times 191$ & $254 \times 101$ & 20 & 20 & 10 \\
\hline 3 & $477 \times 202$ & $254 \times 101$ & 25 & 40 & 10 \\
\hline 4 & $477 \times 202$ & $254 \times 101$ & 25 & 40 & 10 \\
\hline $\begin{array}{l}5 a \text { (top) } \\
5 b\end{array}$ & $\begin{array}{l}366 \times 208 \\
351 \times 215\end{array}$ & $\begin{array}{l}195 \times 126 \\
194 \times 133\end{array}$ & $\begin{array}{l}19 \\
19\end{array}$ & $\begin{array}{l}20 \\
20\end{array}$ & $\begin{array}{l}24 \\
38\end{array}$ \\
\hline $\begin{array}{l}6 a \text { (top) } \\
6 b\end{array}$ & $\begin{array}{l}355 \times 211 \\
351 \times 215\end{array}$ & $\begin{array}{l}196 \times 126 \\
194 \times 133\end{array}$ & $\begin{array}{l}20 \\
20\end{array}$ & $\begin{array}{l}11 \\
20\end{array}$ & $\begin{array}{l}18 \\
17\end{array}$ \\
\hline $\begin{array}{l}7 a \text { (top) } \\
7 b\end{array}$ & $\begin{array}{l}345 \times 221 \\
337 \times 229\end{array}$ & $\begin{array}{l}196 \times 150 \\
197 \times 158\end{array}$ & $\begin{array}{l}20 \\
20\end{array}$ & $\begin{array}{l}11 \\
20\end{array}$ & $\begin{array}{l}18 \\
16\end{array}$ \\
\hline $\begin{array}{l}8 a \text { (top) } \\
8 b\end{array}$ & $\begin{array}{l}495 \times 206 \\
495 \times 190\end{array}$ & $\begin{array}{l}207 \times 129 \\
207 \times 108\end{array}$ & $\begin{array}{l}20 \\
20\end{array}$ & $\begin{array}{l}14 \\
14\end{array}$ & $\begin{array}{l}16 \\
16\end{array}$ \\
\hline $\begin{array}{l}9 a \text { (top) } \\
9 b\end{array}$ & $\begin{array}{l}495 \times 175 \\
495 \times 168 \\
\end{array}$ & $\begin{array}{c}120 \times 100 \\
120 \times 79\end{array}$ & $\begin{array}{l}20 \\
20\end{array}$ & $\mathrm{n} / \mathrm{a}$ & $\begin{array}{l}22 \\
19 \\
\end{array}$ \\
\hline 10 & $615 \times 380$ & $\begin{array}{c}309 \times 133 \text { (long.) } \\
169 \times 100 \text { (lat.) }\end{array}$ & 20 & 20 & 20 \\
\hline 11 & $373 \times 216$ & $254 \times 100$ & 20 & 40 & 15 \\
\hline 12 & $362 \times 185$ & $254 \times 100$ & 20 & 40 & 15 \\
\hline 13 & $349 \times 187$ & $254 \times 100$ & 25 & 20 & 11 \\
\hline 14 & $403 \times 192$ & $254 \times 100$ & 20 & 40 & 11 \\
\hline 15 & $409 \times 192$ & $254 \times 90$ & 20 & 40 & 10 \\
\hline 16 & $352 \times 185$ & $235 \times 101$ & 20 & 40 & 15 \\
\hline 17 & $293 \times 201$ & $127 \times 100$ & 25 & 30 & 10 \\
\hline 18 & $296 \times 140^{\star *}$ & $254 \times 100$ & 19 & 20 & 61 \\
\hline 19 & $301 \times 143^{* *}$ & $251 \times 100$ & 20 & 29 & 70 \\
\hline 20 & $390 \times 185$ & $254 \times 100$ & 20 & 40 & 15 \\
\hline
\end{tabular}

* This is either the etch-window slot width between microbridges in a dual microbridge design or the slot width inside each microbridge in a "Two Single Microbridge" design.

** The dimensions of each sense resistor cantilever. 
devices are left in the etchant, etc. The "Minimum Bridge Edge Tolerance" shown in Table X is the minimum designed spacing between proximal resistor metallization edges and bridge edges. Unlike the previous mask set design, sensors with the "Two Single Microbridge" structural designs (sensor \#5, \#6, \#7, \#8, and \#9) have a common heater connection for both devices (i.e. both heater resistors are powered simultaneously), and five devices (\#1, \#4, \#13, \#15, and \#17) have a sense resistor common node (three bonding pad areas for two sense resistors). In all but seven sensors (\#11, \#12,\#13,\#14,\#15,\#16, and \#20), the substrate temperature reference resistors share a common node with a heater resistor node. Two flow sensors have heater resistors placed on either a separate microbridge (in \#19) or on a "merged cantilever" (in \#18), which is new to this design (and essentially a separate microbridge). These devices present difficulties in measuring heater resistor temperatures, because the heater resistors are not placed adjacent to one or more sense resistors on the same microbridge, and would likely have poorer response characteristics because the heater-to-sense-resistor spacing is comparatively larger than with other sensors. Compared to the 1997 layout, there is one fewer large, single microbridge, with both laterally and longitudinally placed sense resistors. Device \#16 is unique in that it has separate heaters placed on each of the cantilever structures. Due to an oversight, flow sensor \#15 was designed incorrectly with a sense resistor common node that is not connected to a wire-bonding pad, making this device unusable because these resistors could not be wired into separate Wheatstone bridge branches.

The electrical specifications, in terms of the number of "squares" for various resistors for the 1998/1999 photomask design are presented in Table XI. Note that again for this mask set, the upstream $\left(R_{u}\right)$ and downstream $\left(R_{d}\right)$ resistors are symmetrical with equal resistances. The number of design squares for each resistor in Table $\mathrm{XI}$ is an approximation that does not include bonding pad and wide-interconnect routing areas. 
TABLE XI

1998/1999 PHOTOMASK SET FLOW SENSOR ELECTRICAL SPECIFICATIONS

\begin{tabular}{|c|c|c|c|}
\hline Flow Sensor & $\begin{array}{l}\text { Heater Resistor } \\
\text { \# of Squares } \\
(10 \mu \mathrm{m} \text { line widths) }\end{array}$ & $\begin{array}{c}\text { Upstream or } \\
\text { Downstream Resistor } \\
\text { \# of Squares } \\
(5 \mu \mathrm{m} \text { line widths })\end{array}$ & 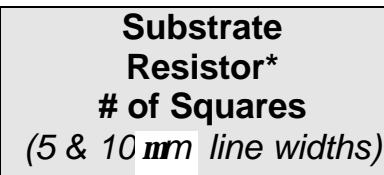 \\
\hline 1 & 193.5 & 520.5 & $\begin{array}{l}\mathrm{R}_{\mathrm{p} 5}=250 / 500 / 750 \\
\mathrm{R}_{\mathrm{alt}}=1966\end{array}$ \\
\hline 2 & 193.5 & 520.5 & $\begin{array}{l}\mathrm{R}_{\mathrm{p} 5}=250 / 500 / 750 \\
\mathrm{R}_{\mathrm{alt}}=1966\end{array}$ \\
\hline 3 & 193.5 & 520.5 & $\begin{array}{l}\mathrm{R}_{\mathrm{p} 10}=250 / 500 / 750 \\
\mathrm{R}_{\mathrm{alt}}=1966\end{array}$ \\
\hline 4 & 193.5 & 520.5 & $\begin{array}{l}R_{p 10}=250 / 500 / 750 \\
R_{\text {alt }}=1966\end{array}$ \\
\hline $5 a$ (top) & 110.6 & 249.6 & $\mathrm{R}_{\mathrm{p} 5}=250 / 500 / 750$ \\
\hline $5 b$ (bottom) & 94.1 & 249.8 & $R_{p 5}=250 / 500 / 750$ \\
\hline $6 a$ (top) & 90.6 & 247.5 & $\mathrm{R}_{\mathrm{p} 5}=250 / 500 / 750$ \\
\hline 6b (bottom) & 93.7 & 250 & $R_{p 5}=250 / 500 / 750$ \\
\hline $7 a$ (top) & 122.8 & 294 & $R_{p 5}=250 / 500 / 750$ \\
\hline 7b (bottom) & 131.9 & 299 & $R_{p 5}=250 / 500 / 750$ \\
\hline $8 a$ (top) & 167 & 375.7 & $R_{p 5}=250 / 500 / 750$ \\
\hline $8 b$ (bottom) & 77.3 & 318.5 & $R_{p 5}=250 / 500 / 750$ \\
\hline 9a (top) & 146.4 & 271.8 & $R_{p 5}=250 / 500 / 750$ \\
\hline $9 b$ (bottom) & 67 & 231 & $R_{p 5}=250 / 500 / 750$ \\
\hline 10 & 104.1 & $\begin{array}{c}480 \text { (long.) } \\
346.7 \text { (lateral) }\end{array}$ & $R_{p 5}=250 / 500 / 750$ \\
\hline 11 & 252.9 & 520.5 & $\begin{array}{l}R_{p 5}=250 / 500 / 750 \\
R_{p 10}=250 / 500 / 750\end{array}$ \\
\hline 12 & 148.6 & 516.5 & $\begin{array}{l}\mathrm{R}_{\mathrm{p} 5}=250 / 500 / 750 \\
\mathrm{R}_{\mathrm{p} 10}=250 / 500 / 750\end{array}$ \\
\hline 13 & 147.6 & 516.5 & $R_{p 5}=250 / 500 / 750$ \\
\hline 14 & 179.7 & 520.5 & $\begin{array}{l}R_{p 5}=250 / 500 / 750 \\
R_{p 10}=250 / 500 / 750\end{array}$ \\
\hline 15 & 184.5 & 465.8 & $\begin{array}{l}\mathrm{R}_{\mathrm{p} 5}=250 / 500 / 750 \\
\mathrm{R}_{\mathrm{p} 10}=250 / 500 / 750\end{array}$ \\
\hline 16 & 73.7 (each heater) & 487.7 & $R_{p 5}=250 / 500 / 750$ \\
\hline 17 & 109.5 & 265.5 & $\begin{array}{l}\mathrm{R}_{\mathrm{p} 5}=250 / 500 / 750 \\
\mathrm{R}_{\mathrm{p} 10}=250 / 500 / 750\end{array}$ \\
\hline 18 & 136.4 & 517.1 & $\mathrm{R}_{\mathrm{p} 10}=250 / 500 / 750$ \\
\hline 19 & 143.6 & 521.3 & $R_{p 10}=250 / 500 / 750$ \\
\hline 20 & 148.6 & 516.5 & $\begin{array}{l}\mathrm{R}_{\mathrm{p} 5}=250 / 500 / 750 \\
\mathrm{R}_{\mathrm{p} 10}=250 / 500 / 750\end{array}$ \\
\hline
\end{tabular}

${ }^{*} R_{p 5}$ and $R_{p 10}$ are the $5 \mu \mathrm{m}$ and $10 \mu \mathrm{m}$ line-width "parallel" substrate resistors, and $R_{a l t}$ is the long serpentine substrate resistor. 


\section{Etch Depth Measurement and Mask Alignment Structures}

\section{PLIGNMENT PRER}
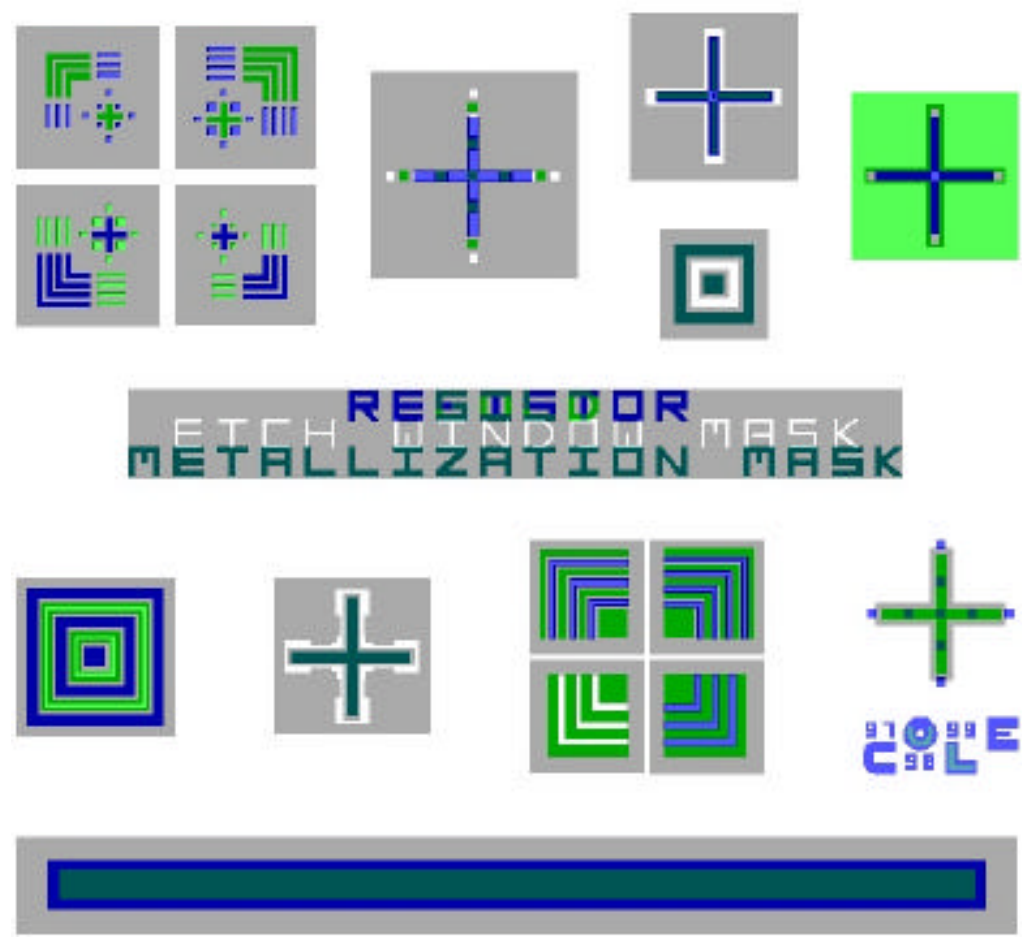

FIGURE 36 - An L-EditTM design file image of the 1998/1999 mask alignment area. This was one of two very similar areas present within each $22.967 \times 11.5 \mathrm{~mm}$ die space.

This mask set included the same region of 19 square etch-depth measurement structures (three of which are shown in Figure 31) to allow for monitoring progress of the (100) silicon anisotropic etching progress. Each window was precisely dimensioned to show pyramidal etch depths from 20 to $200 \mu \mathrm{m}$, in increments of $10 \mu \mathrm{m}$.

Two mask alignment areas, one of which is shown in Figure 36, were included within each replicated die of this mask design. The alignment methodology for this photomask set was similar to the 1997 mask set, but it was significantly easier to perform because both the resistor and gold metallization photomasks were lightfield masks (in which only digitized features in the design file, such as those shown in Figure 36, were opaque in the mask). The first resistor metallization photomask was aligned to the (100) silicon substrate by positioning the (110) primary wafer flat edge parallel to any proximal edge of a rectangle included within each "Alignment Area" region or to any straight line comprised of a row of pads within the design, as 


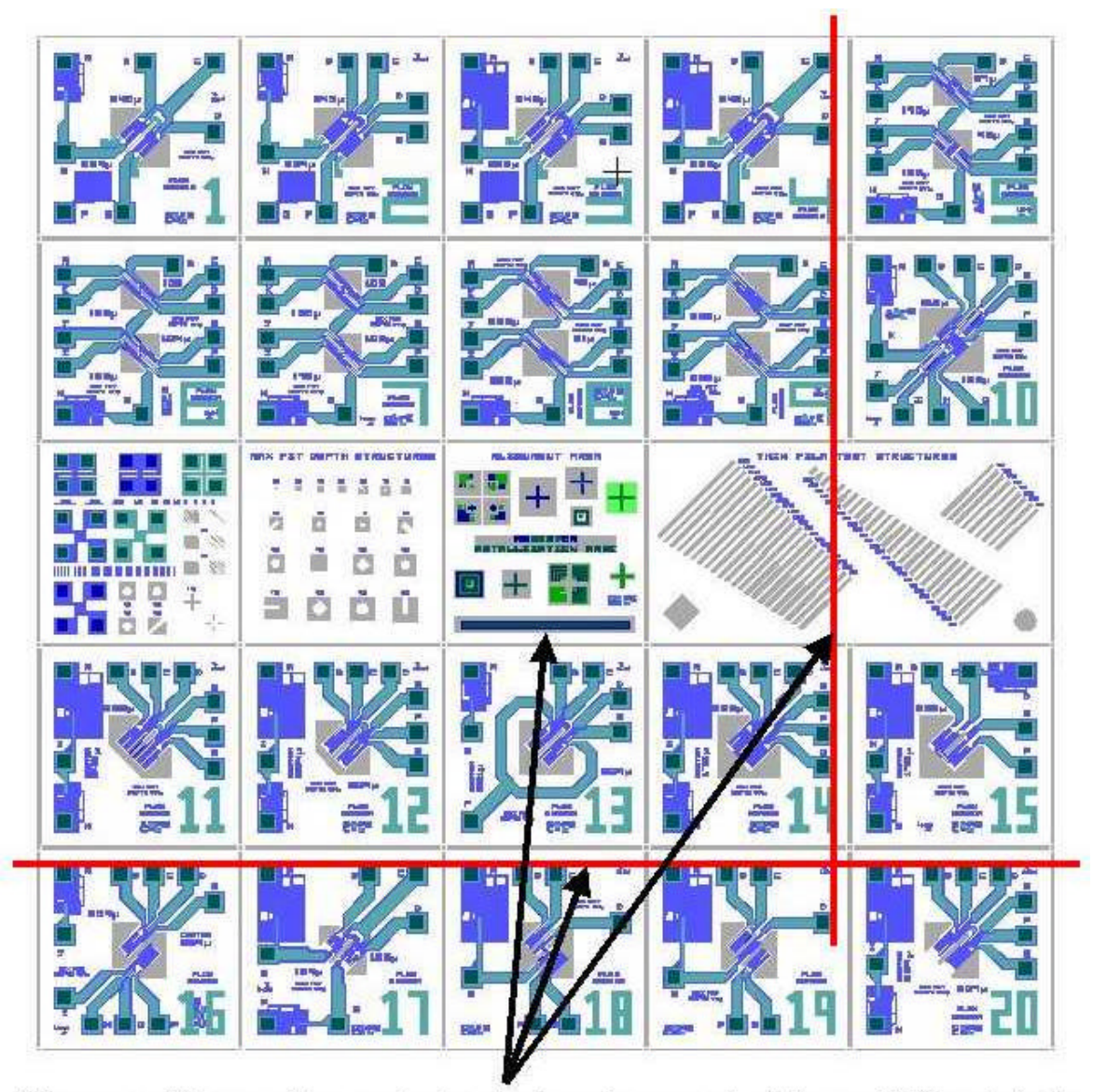

Align any of these orthogonal edges to the primary wafer flat on a (100) substrate

FIGURE 37 - How to align the 1998/1999 resistor metal mask to the (110) wafer flat. Arrows point to which edges in the device area may be used for parallel alignment to the (110) primary wafer flat on a (100) $n$ or p-type silicon substrate.

shown in Figure 37. The primary wafer flat and the selected edge, or row of pads, would then both be parallel to the $\{110\}$ planes in the wafer so that the microbridge longitudinal axes would be rotated $45^{\circ}$ with respect to the $<110>$ crystallographic direction in the silicon substrate. The Gold Metal Level 2 photomask was aligned to metallized (resistor metal) features already patterned by the first photomask within mask alignment areas on the silicon substrate. The $\mathrm{RIE} / \mathrm{KOH}$ etch-window (darkfield) photomask was aligned to both resistor and gold metallization features already patterned on the substrate within the mask alignment areas. 


\section{Sheet Resistance Measurement Structures}

As in the 1997 mask set, this design included both a Greek cross and offset quadrate cross four-terminal Van der Pauw structures for measuring sheet resistances of the thin film
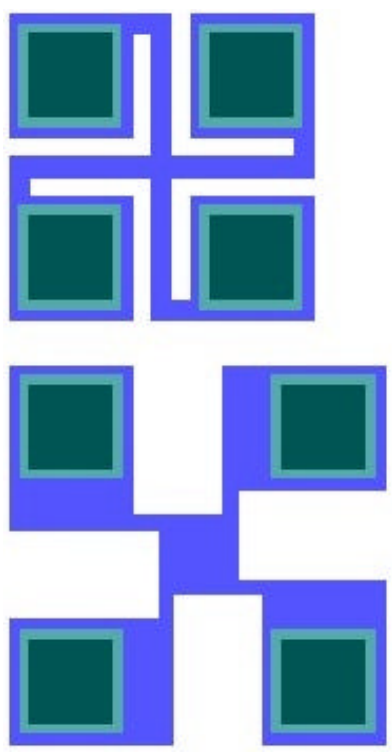
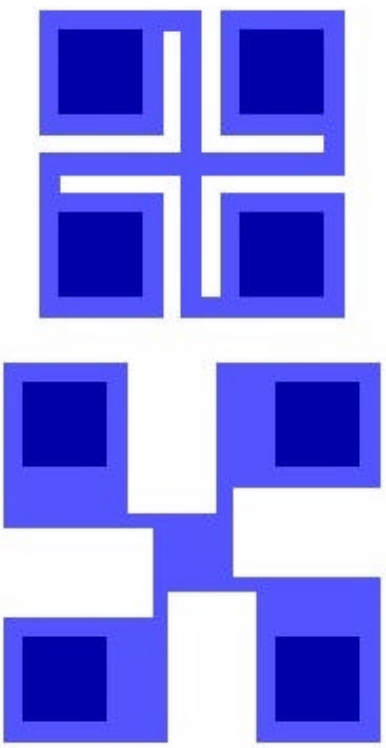
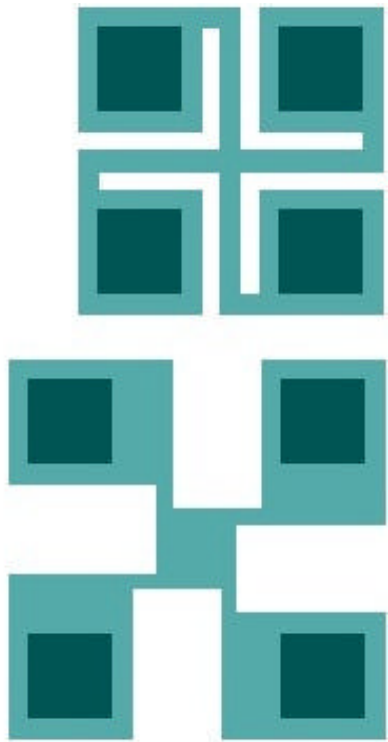

FIGURE 38 - Van der Pauw sheet resistance test structures in the '98/'99 mask set. The Greek Crosses are in the top row, and the Offset Quadrate Cross are in the bottom row. The far left column of structures are comprised of resistor metallization and gold pad areas. Structures in the middle column have only resistor metallization, and on the right are structures with both resistor and gold metallization over the entire Van der Pauw area. These structures have the same dimensions for $A, D$, and $S$ as those in the 1997 mask set (shown in Figure 34).

resistor and gold metallization layers. These structures are grouped together and shown in

Figure 38, and have the same dimensions for $A, D$, and $S$ as the structures in the 1997 mask set. Three different Greek cross and offset quadrate cross variations (each) were included: one with only resistor metallization, one with resistor metallization and gold bonding-pad area metallization, and one with both metallizations together. Designing gold-only Van der Pauw structures was not practical, as these would not survive processing, intact, because gold has poor adhesion to silicon substrates without an adhesion layer (that is typically chromium or titanium). Obtaining sheet resistance measurements with these structures uses the same process described for the 1997 mask set (using a probe station, constant current source, and voltmeter). Refer to Figure 34 and Equation (51) to learn how to use these structures. 


\section{New In Situ Thin Film Stress/Strain Measurement Structures}

Seven different types of unique, in-situ, residual strain diagnostic structures were included in this mask set, because attempts to fabricate flow sensors in 1997 failed, in part, due to intrinsic compressive strain and porosity in the RF planar magnetron sputter-deposited thin film $\mathrm{Si}_{x} \mathrm{~N}_{\mathrm{y}}$ layer comprising the microbridges. Research showed that these new structures could be used to measure residual compressive or tensile strain fields (in deposited, thin silicon nitride films) while ultimately developing a suitable sputtering recipe to deposit a lower-stress $\mathrm{Si}_{x} \mathrm{~N}_{\mathrm{y}}$ layer, that is preferably under low residual tensile stress.

\section{a. Doubly Clamped Beams (or Microbridges)}

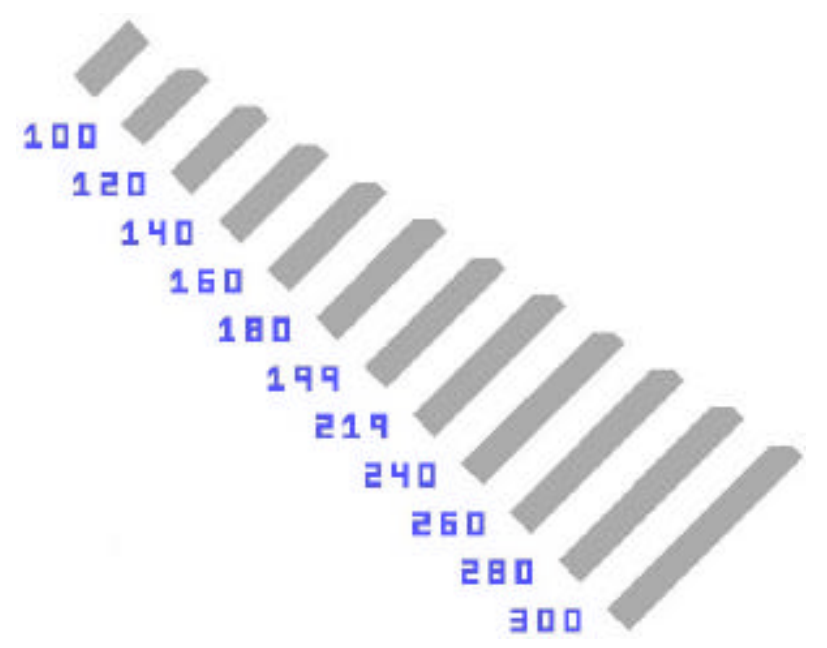

FIGURE 39 - A series of incremental-length bridges, included in the ' $98 /$ '99 mask design. These were designed to deduce residual compressive stress in the $\mathrm{Si}_{3} \mathrm{~N}_{4}$ thin film. Gray areas are openings where RIE/KOH chemistries will etch through the nitride film and anisotropically into the substrate. The blue (resistor metallization) numbers indicate the design length of each bridge, in microns.

For a thin film wherein the stress is uniform in the direction perpendicular to the substrate surface, any free regions of the film will relax into a non-stressed state, with an accompanying dimensional change. This photomask design includes rows of $50-\mu \mathrm{m}$ wide clamped-clamped beams (a portion of which is shown in Figure 39), from 100 to $1201 \mu \mathrm{m}$ long with incrementally increasing lengths (in $20 \mu \mathrm{m}$ steps) that can be used to determine the critical buckling load and, consequently, compute the residual compressive stress in a silicon nitride thin-film. These structures measure localized strain fields based on the tendency of a slender member to buckle 
when a compressive load acting on its ends exceeds the value of the critical buckling strain, $\varepsilon_{C R}$. Once these silicon nitride beams are suspended from the substrate by anisotropic etching, the Euler critical buckling strain can be computed after determining which beams have buckled at a given stress level $[51,56]$ :

$$
\varepsilon_{C R}=\frac{(\pi \cdot t)^{2}}{K\left(L_{C R}\right)^{2}},
$$

where $t$ is the beam thickness, $L_{C R}$ is the critical length of the beam, and $K$ is a constant related to the geometrical boundary conditions. A doubly-clamped beam will buckle if its length exceeds the critical length, $L_{C R}[43,56]$ :

$$
L_{C R}=\frac{\pi \cdot t}{\sqrt{3 \varepsilon_{C R}}} \quad(\mathrm{~m})
$$

An example showing buckling for polysilicon beams with residual compressive stress is shown in Figure 40. The critical strain is, thus, deduced by determining the smallest geometry for which buckling occurs. For surface, or bulk-micromachined bridges, the values for $K$ lie between those of a simply-supported beam $(\mathrm{K}=12)$ and those of a doubly-clamped beam $(\mathrm{K}=3)$. The constant $K$

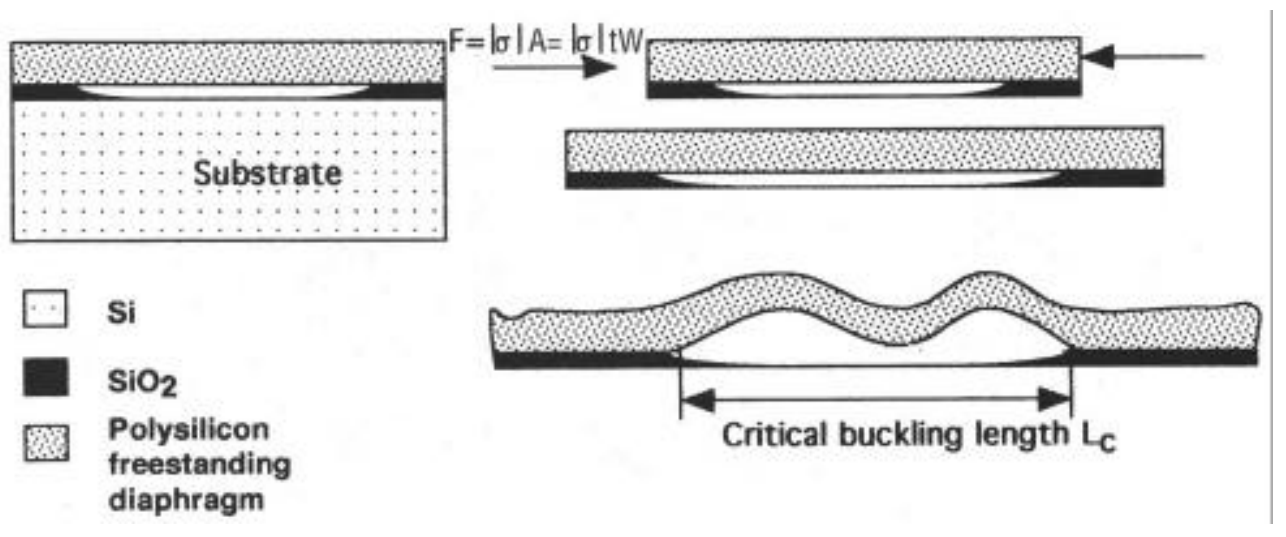

FIGURE 40 - Example of clamped-clamped beams used to find the critical buckling length. This enables measurement of residual stress in a thin film layer [45]. 
and Young's modulus, $E$, need to be determined in order to obtain the stress from this estimated strain [see Equation (43)]. Hence, for a doubly-clamped beam array, the Euler critical buckling strain can be computed from $[52,56]$ :

$$
\varepsilon_{C R}=\frac{(\pi \cdot t)^{2}}{3\left(L_{C R}\right)^{2}}
$$

Combining Equations (43) and (54), the beam buckles when its compressive, in-plane, average residual stress, $\sigma_{o}$, is:

$$
\sigma_{o}=-\frac{E(\pi \cdot t)^{2}}{3 L_{C R}{ }^{2}} \quad\left(\mathrm{~N} / \mathrm{m}^{2} \text { or } \mathrm{Pa}\right)
$$

The negative sign indicates the stress is compressive. The buckling for a rectangular doublysupported beam, made of a uniform, isotropic thin film, can therefore be computed from a function of beam geometry only and not material parameters, assuming each cross section of the beam supports a constant and uniformly-distributed axial load [52]. The upper and lower bounds for the residual strain in a compressively-stressed nitride film can be obtained by fabricating an array of doubly-clamped bridge structures of varying lengths and observing the point at which the critical buckling strain is exceeded (e.g. observing beam deformation with an interference contrast microscope). Note, however, that this simpler Euler method does not account for any additional effects such as internal moments resulting from residual stress gradients in the thin film $[45,56]$. These expressions are only valid for films in which the residual stresses do not vary through the thickness of the film (i.e. the strain field is constant over beam cross sections), because a residual strain gradient introduces an eccentricity to the axial load that will tend to alter the elastic stability of the beam [56]. 


\section{b. Simply-Supported Cantilevered Beams}

A uniform stress gradient in the thin film (i.e. where the stress is uniform in the direction perpendicular to the substrate surface), can be observed in free regions of the film that relax into a non-stressed state with an accompanying dimensional change. Direct measurement of the strain relaxation can be achieved by measuring the contraction or elongation of a thin cantilever beam after release from the substrate. A simple thin-film cantilever, with initial length $L_{o}$, under uniform stress when released from the substrate, will change in length by an amount $\Delta L$, that can be potentially measured relative to fixed verniers or fiducial marks patterned on the substrate. If the length of a cantilever is measured before $\left(L_{0}\right)$ and after release $(L)$ from the silicon substrate, the change in length of the beam $(\Delta L)$ divided by its length before release is equal to the residual stress $\left(\sigma_{o}\right)$ to Young's modulus (E) ratio [by combining Equations (42) and (43)]:

$$
\frac{\Delta L}{L_{o}}=\frac{L_{o}-L}{L_{o}}=\frac{\sigma_{o}}{E} .
$$

Note that cantilever beams provide the value of $\sigma_{o} / E$ only at one point on the stress-strain curve for the material (i.e. at the residual strain) [57].

In reality, in-plane stresses typically vary through the thickness of a thin film, especially if it has been subjected to substantial post-deposition processing. The magnitude of an intrinsic strain nonuniformity (or internal stress gradient) in a silicon nitride thin film can, therefore, be deduced by measuring the smallest geometry for which buckling occurs in an array of cantilevers with incrementally increasing lengths. The magnitude of the deflections can be measured with either an SEM or a phase-contrast microscope to estimate the magnitude of the internal stress field. A portion of the cantilever array included in this mask design and developed to reveal strain gradients, is shown in Figure 41, and an SEM photograph of fabricated RF planar magnetron sputter-deposited $\mathrm{Si}_{x} \mathrm{~N}_{\mathrm{y}}$ thin film cantilevers (under compression) is shown in Figure 42. The total array consists of $50-\mu \mathrm{m}$ wide cantilevers ranging from 100 to $1201 \mu \mathrm{m}$ in length in increments of 


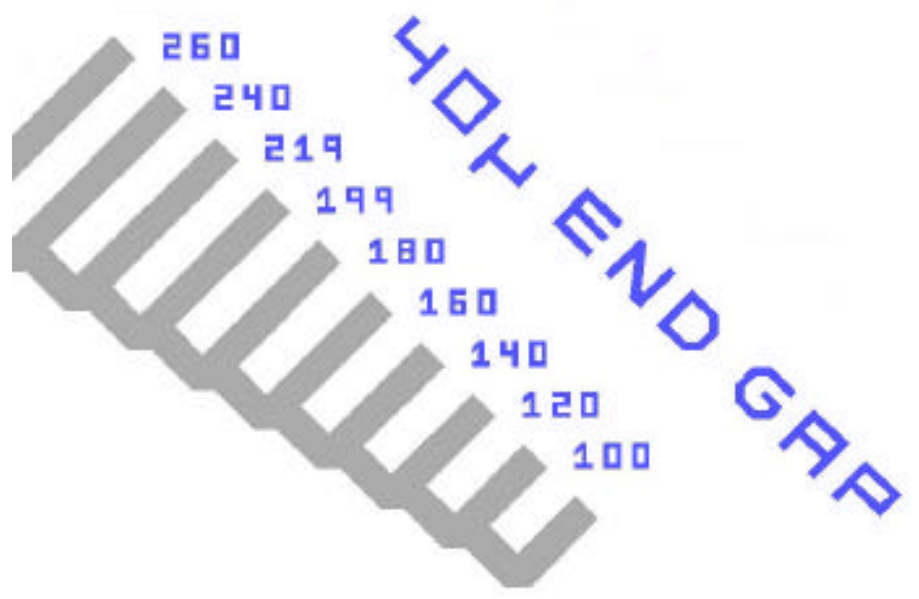

FIGURE 41 - An array of incremental-length cantilevers, included in the '98/'99 photomask. These were for deducing residual compressive stress (or strain nonuniformity) in the $\mathrm{Si}_{x} \mathrm{~N}_{\mathrm{y}}$ thin film. Gray areas are openings where RIE/KOH chemistries can be used to etch through the nitride film and anisotropically into the substrate. Numbers near the cantilevers refer to their design lengths. The " $40 \mu$ end gap" label refers to the spacing at the end of the cantilevers.

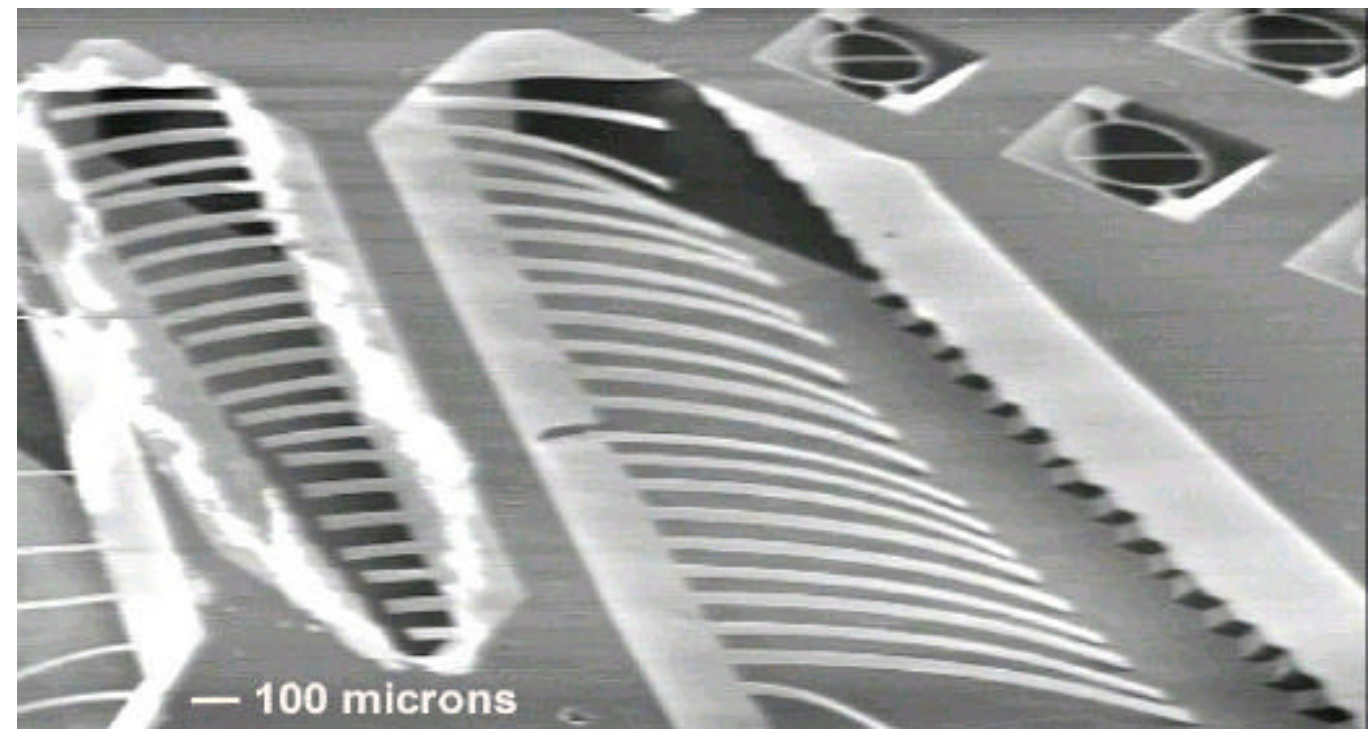

FIGURE 42 - $\quad$ An SEM photo of buckled, sputtered $\mathrm{Si}_{x} \mathrm{~N}_{\mathrm{y}}$ thin film cantilevers. These were RF planar magnetron sputter-deposited and etched using the 1998/1999 mask set. The intrinsic downward bending was caused by strains within the film due to compositional or density gradients through the film thickness, or other variations in deposition conditions with time.

approx. $20 \mu \mathrm{m}$.

A free body diagram for a non-uniform stress distribution (or strain gradient) in a deposited thin film is shown in Figure 43. For a residual stress gradient that remains constant 


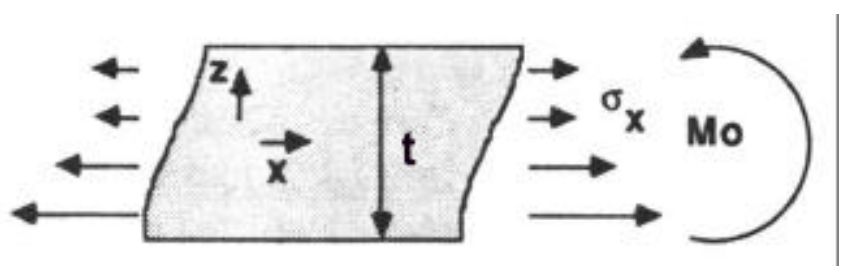

FIGURE 43 - A free-body diagram of in-plane stress distribution in a deposited thin film [43].

along the length of a cantilever beam (in the $x$ direction), the vertical deflection, $\delta(x)$, at a position $x$ along the length of the cantilever can be described by $[51,56]$ :

$$
\frac{\delta(x)}{x}=K+\frac{\left(1-v^{2}\right)}{2 E \cdot I}(M \cdot x)
$$

where $K$ is a constant that depends upon boundary conditions at the support end (where $\mathrm{x}=0$ ), $E$ is Young's modulus, $v$ is Poisson's ratio, $I$ is the moment of inertia of the cantilever about the $y$ axis, and $M$ is the internal bending moment. For a beam of rectangular cross section with width $W$ and constant thickness $t$, the moment of inertia is [51,57]:

$$
I=\frac{W t^{3}}{12} \quad\left(\mathrm{~m}^{4}\right)
$$

The internal bending moment of the cantilever beam per unit width about the $y$ axis causing its deflection is a result of pre-release residual stress and is equal to $[43,56]$ :

$$
M_{o}=\int_{-t / 2}^{t / 2} \sigma_{x}(z) z d z \quad(\mathrm{~N} \cdot \mathrm{m})
$$

where $\sigma_{x}(z)$ is the magnitude of the in-plane film residual stress at a distance $z$ (i.e. as a function of thickness) from the center of the film, which has total thickness $t$. The net bending moment could also be calculated as follows [51]: 


$$
M=W \int_{0}^{t} \sigma_{x}(z)\left(z-\frac{t}{2}\right) d z, \quad(\mathrm{~N} \cdot \mathrm{m})
$$

where $\sigma_{x}(z)$ is the stress at height $z$ above the bottom of the film (where $z=0$ ) and $W$ is the width of the cantilever in which the moment is present. The moment is zero unless $\sigma_{x}(z)$ varies with height $z$. If it exists, this effective bending moment will cause a bulk micromachined cantilever to bend out of the plane of the substrate, once it is released. If the nonuniform strain gradient, $\tilde{A}$, is linear (as a function of height) in the thin film cantilevers, resulting in a uniformly varying stress, $\sigma_{x}(z)=E \cdot \Gamma \cdot z$, Equation (59) or (60) may be reduced to [45]:

$$
M=\frac{W \cdot E \cdot \Gamma \cdot t^{3}}{12}
$$

or solving for the strain gradient [45],

$$
\Gamma=\frac{12 M}{E W t^{3}}=\frac{M}{E \cdot I}
$$

where $I$ is the moment of inertia for a rectangular cross section. Note that if the strain field is uniform, $\Gamma=0$. From beam theory, the measured corresponding vertical deflection, $z$, of the cantilever's endpoint for a cantilever with a strain gradient and an applied end moment can be calculated from [45]:

$$
z=\frac{\Gamma L^{2}}{2}
$$

After release, the internal stress gradients in the thin film cantilever relax, and it will curl with a radius of curvature equal to $[43,45,56]$ : 


$$
R=\frac{E t^{3}}{12 M_{0}}
$$

From Equation (52), for a simply-supported cantilever rectangular beam of constant height, $t$, the Euler critical buckling strain can be computed from $[45,51,56]$ :

$$
\varepsilon_{C R}=\frac{(\pi \cdot t)^{2}}{12\left(L_{C R}\right)^{2}}
$$

where $L_{C R}$ is the critical length of the beam where buckling first occurs in the array. Thus, buckling for a rectangular cantilever, made of a uniform isotropic thin film, can be computed from a function of beam geometry only and not material parameters, assuming each cross section of the beam supports a constant and uniformly distributed axial load [52].

\section{c. Archimedean Cantilever Spirals}

An Archimedean spiral microstructure can also be used for the characterization of residual uniform strain gradients (nonuniform strain fields) in thin films $[45,51,58]$. Spiral microstructures fabricated from thin-film materials having a residual strain that varies through the thickness of the film (i.e. a gradient of in-plane residual strain), but does not vary in the plane of the spiral surface, are observed to deform by winding up and curling (contracting or expanding) upon release from the substrate. The resultant deformation of a freed spiral provides three different response variables, which can be directly related to the residual strain gradient, and measured by (e.g.) optical micrographs or other methods: the angular endpoint rotation (or windup); the change in, or contraction of, lateral size (projected onto the plane of the substrate); and the out-of-plane height of the spiral's free end. The advantage of this type of spiral structure is that the film's strain gradient can be estimated from a single data point, and both end rotation and lateral contraction do not require the use of an SEM or phase contrast microscope to obtain the data. The angle of rotation and lateral contraction of a released spiral can be predicted as a function of the average strain gradient in the thin film through fourth-order Runge-Kutta numerical 
methods. Note that if the strain field is uniform within the thin-film spiral, there will be no rotation of its free end, once released from the substrate.

Released and deformed spirals are characterized by contractions in spiral diameter, rotations of their inner ends, and curling either up or down in height. The algebraic sign of the height, or elevation of the highest end of the spiral above the substrate, is determined by the algebraic sign of the uniform strain gradient, $\Gamma$. As shown in Figure 44, if the strain gradient is positive (increasingly tensile with increasing height above the substrate), the spiral will assume an open bowl shape, in that a spiral anchored at its center will spring upwards, rotate, and contract (i.e. tending to curl a cantilever upwards). Conversely, if the stress gradient is negative (increasingly compressive with increasing height), the spiral will assume a dome shape, in that a spiral anchored at its outmost end will spring upwards, rotate, and contract. Theoretically, positive

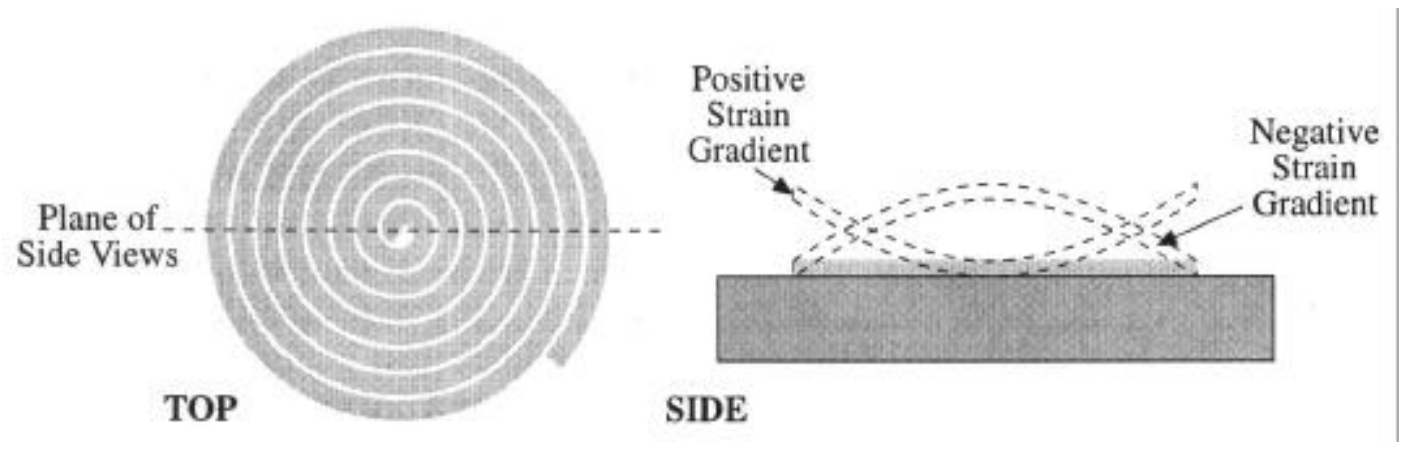

FIGURE 44 - An Archimedean cantilever spiral used to measure strain gradients in films. Relaxed states of the spiral are shown at the right for positive and negative strain gradients, viewed as slices through the spiral along the plane indicated at the left [51].

and negative strain gradients produce spirals with mirror symmetry. Numerical methods are required to solve the differential equations relating the three-dimensional internal moments caused by the strain field $[45,51,56]$.

Spiral microstructures included within this mask design are shown, grouped together, in Figure 45. Note that to cover stress gradients of both polarities, test spirals should have been designed and included with anchors to the substrate at both the center and outermost turn so that their relaxed states are above the substrate. This design only includes spirals with attachment 


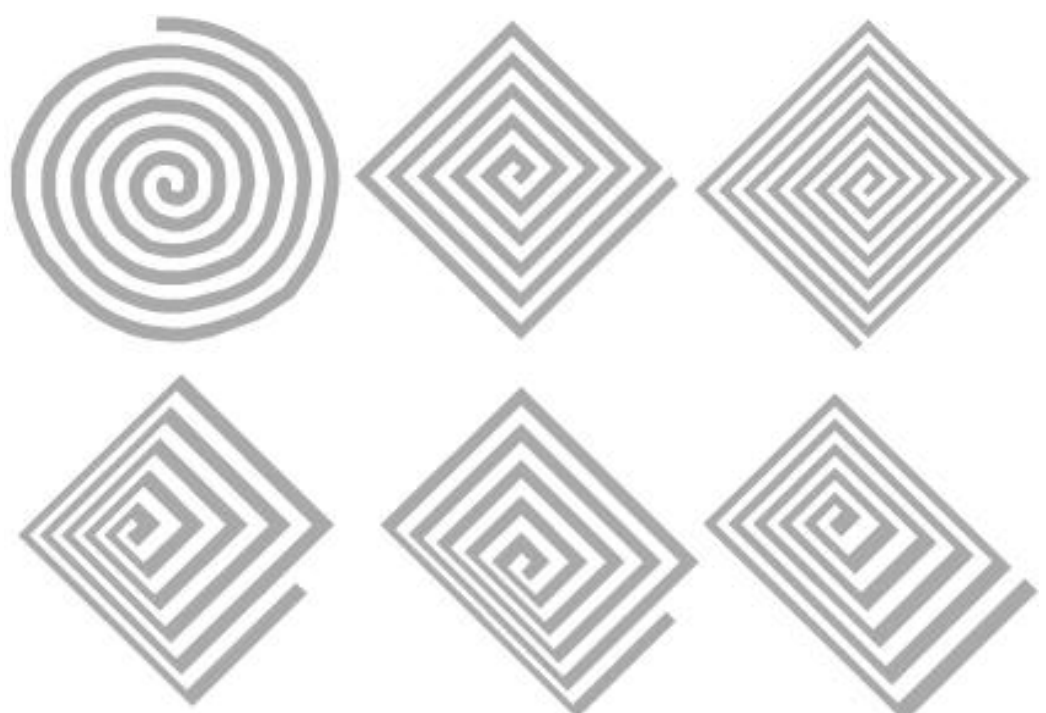

FIGURE 45 - Various spiral microstructures included in the 1998/1999 photomask design. These were developed to reveal residual strain gradients in the $\mathrm{Si}_{x} \mathrm{~N}_{\mathrm{y}}$ thin film.

points at their outmost ends (not in the center). The minimum design width for these spirals was $\sim 9 \mu \mathrm{m}$. In all attempts to fabricate flow sensors thus far, none of these spiral microstructures have survived fabrication processing intact. In theory, if these structures do survive fabrication, they can be used to determine the uniform strain gradient magnitude by measuring contractions in diameter, rotations of their inner ends, and final curling height of the free end to compare to numerical simulation results (which must be developed). This type of detailed and tedious analysis is left to the reader, if desired. Otherwise, these spirals provide convenient indicators of the presence (and perhaps the algebraic sign) of significant potential residual stress gradients extant in the thin film layer (e.g. $\mathrm{Si}_{x} \mathrm{~N}_{\mathrm{y}}$ ) used for the flow sensor microbridges and cantilevers [58].

\section{d. The Suspended Plate}

As shown in Figure 46, a suspended thin-film plate, held in place by four supporting arms, was included in this mask design. Any stress induced shrinkage of the four attachment arms (each $40 \mu \mathrm{m}$ wide), acting as two pairs of couples, will rotate the square plate in the center. As of this writing, no suspended/rotated plate structures have survived fabrication attempts intact. Typically one or more supporting arms fracture, collapsing the entire structure. If any survive fabrication, these plates can only be used to indicate the presence of residual stress in the thin film [57]. 


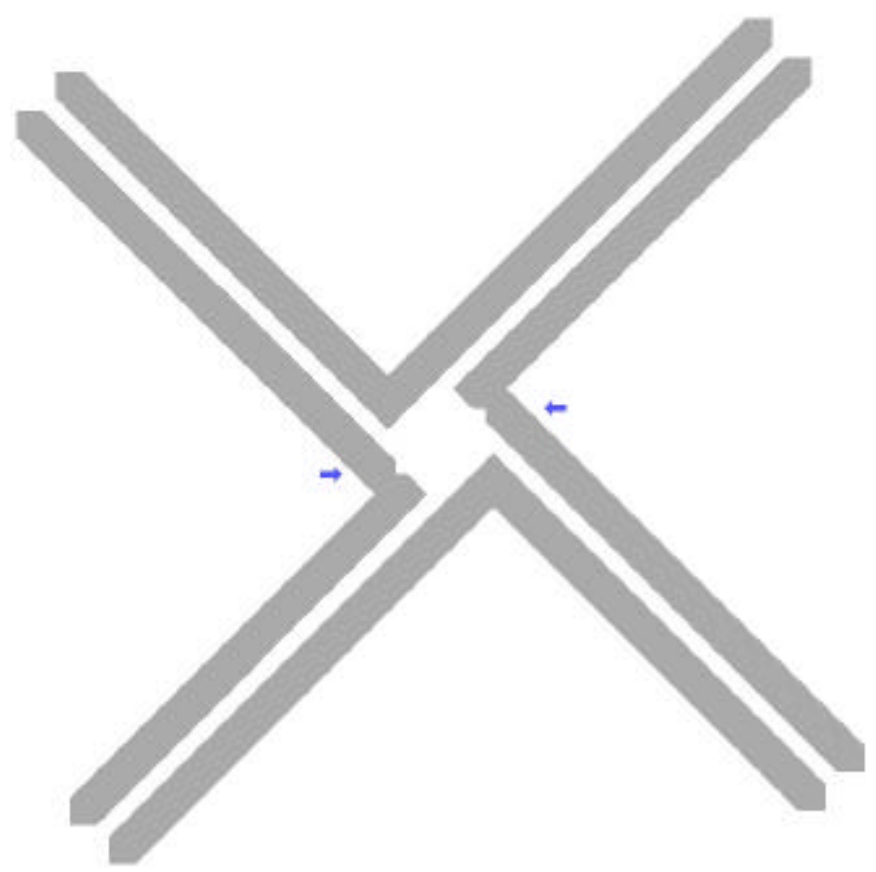

FIGURE 46 - A suspended plate included in the 1998/1999 mask design. This was to indicate the presence or residual stress in the thin film layer by rotating. Grey areas are etch windows for the anisotropic silicon etchant. As shown, before release from the substrate, the metallization arrows are designed to be aligned horizontally with the triangular extensions protruding from the plate.

\section{e. Ring Crossbar Structures}

As shown in Figure 47, residual tensile strain fields in a thin film layer can be measured by a series of rings with changing radii constrained to the substrate at two points on a diameter and spanned orthogonally by a clamped-clamped beam $[45,56,59,60,61]$. Once suspended from the substrate so that the structure is supported at the ends of two supporting members, a ring that is clamped rigidly only at two points along a diameter will contract significantly (as the thin film relaxes to relieve residual tensile stress) along a spanning beam diameter that is perpendicular to the support diameter, if the thin-film ring material is under tensile strain. Thus, as a result of the tensile load to the ring at its two opposing anchor points, the ring will tend to contract radially at the point where it joins the internal crossbar, causing the spanning cross-beam to contract (as it is placed in a compressive load), which, if this is large enough, will cause the beam to buckle. Large geometries become unstable, resulting in buckling of the beam(s), which can be seen, for example, under a phase contrast microscope. The critical buckling length can be found from 


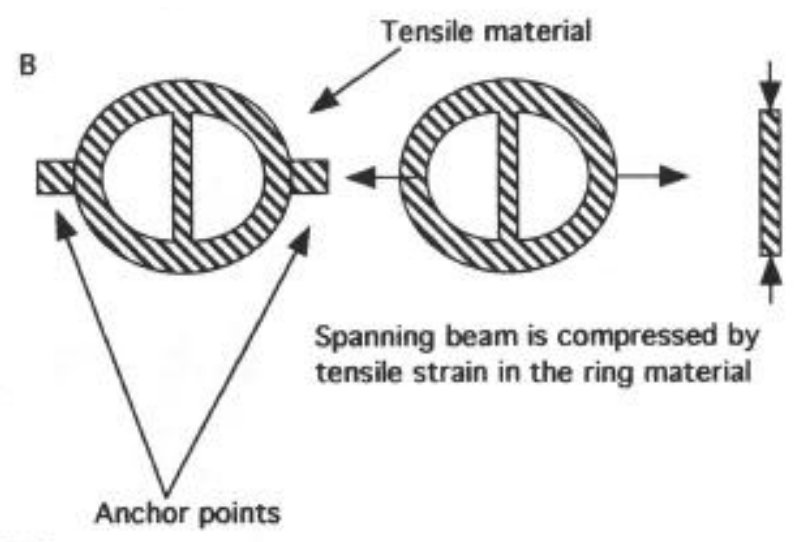

W silicon nitride thin film

FIGURE 47 - Illustration of ring-crossbar structures used to measure tensile stress. An array of these structures can be used to measure tensile stress through buckling induced in the crossbar of the ring structure [45].

an array of these ring crossbar structures (as shown in Figure 48), where observation of the critical geometry at which buckling first occurs can lead to a determination of the average strain in the film $[45,59]$.

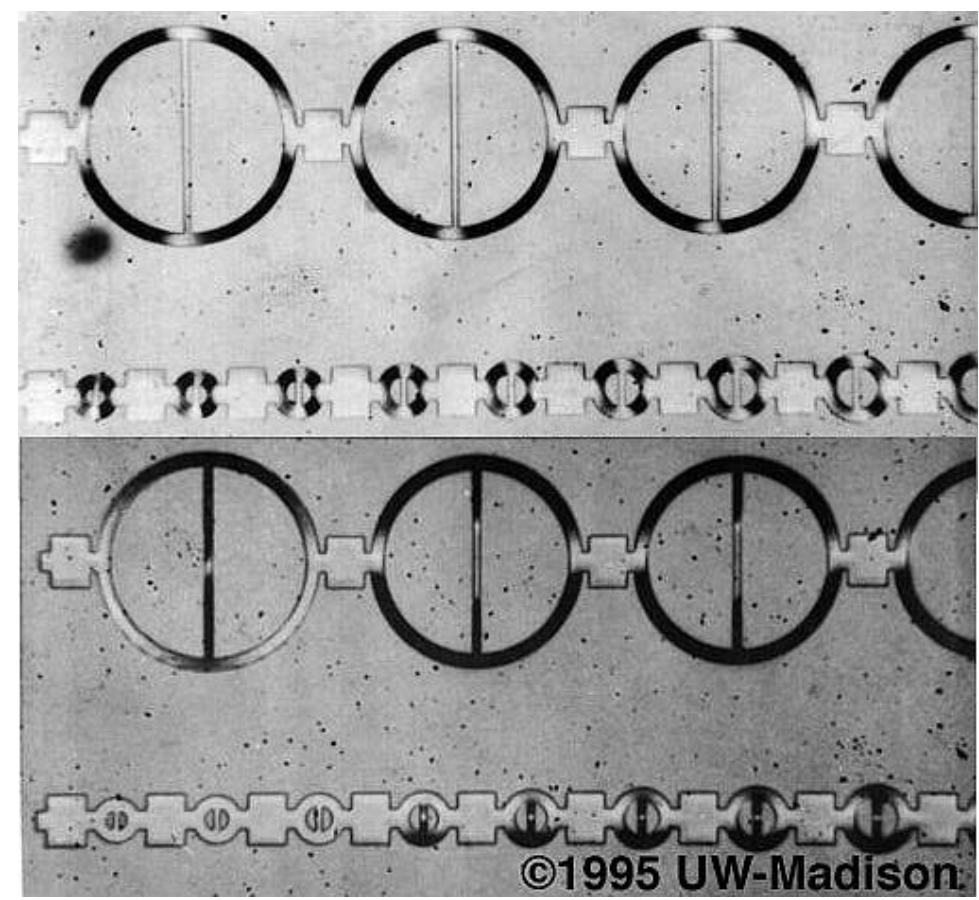

FIGURE 48 - Photomicrographs of ring and beam strain diagnostic structures. These were fabricated from polysilicon films under compression and tension. In the top photo, tight cross members and deformed rings indicate large compressive residual strain. In the bottom photo, buckled center beams and deformed rings for critical geometries and larger reveal a high tensile strain field in the film [60]. 
A series of thin film ring-crossbar structures with incrementally increasing radii (but with constant beam and ring widths) accomplishes for tensile strain what a series of clamped-clamped beams of incrementally-increasing lengths accomplishes for compressive, residual strains. For a ring structure under a tensile load, the radial contraction (at the point where the ring joins the crossbar) will introduce compressive stress in the crossbar that is related to the magnitude of the average residual strain in the thin film by the following expression $[56,61]$ :

$$
\varepsilon_{\text {crossbar }}=g\left(R_{C R}\right) \cdot \varepsilon_{\text {film }},
$$

where $g\left(R_{C R}\right)$ is the deflection ratio of two points on the ring $90^{\circ}$ apart, which is a function of the inner and outer ring diameter, and is determined by the specific geometry of the ring and beam structure. In Figure 49, the deflection ratio is plotted for different ring thicknesses and a crossbar beam width of $10 \mu \mathrm{m}$. Since the function $g\left(R_{C R}\right)$ is negative, the tensile field $\boldsymbol{\varepsilon}_{\text {film }}^{C R}$ is positive.

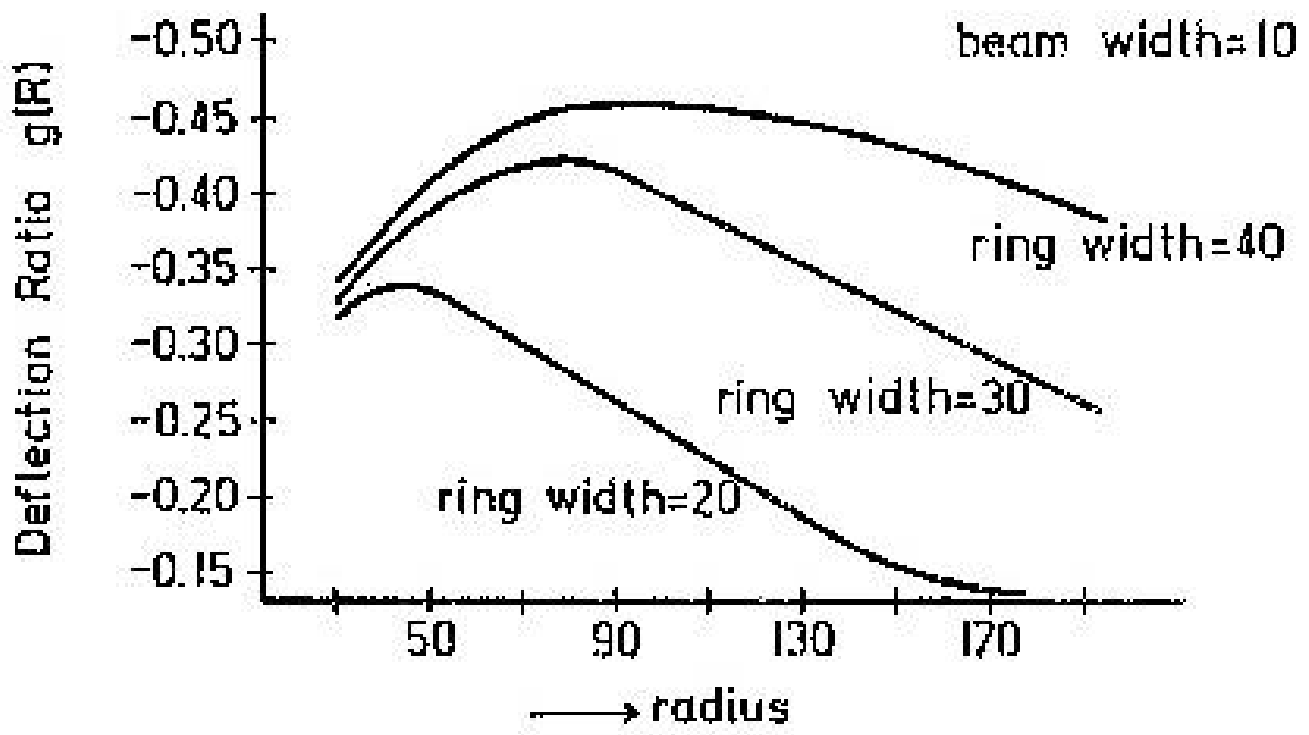

FIGURE 49 - Plot of deflection ratios as a function of ring radius for $10 \mu \mathrm{m}$ beam width. This is for ring-crossbar structures with crossbar beam widths of $10 \mu \mathrm{m}$. Ring radii and widths are in $\mu \mathrm{m}$. For the 1998/1999 mask set, the ring-crossbar structures all have ring widths of $20 \mu \mathrm{m}$. One array has a crossbar beam width of $10 \mu \mathrm{m}$, and another array has a beam width of $20 \mu \mathrm{m}$ [61]. 
The critical value of the average residual strain in the film required to buckle the crossbar is given by $[51,56,61]$ :

$$
\varepsilon_{\text {film }}^{C R}=\frac{-(\pi \cdot t)^{2}}{12\left(R_{C R}\right)^{2} g\left(R_{C R}\right)}, \quad g\left(R_{C R}\right) \leq 0.918 \quad\left(\mathrm{~N} / \mathrm{m}^{2} \text { or } \mathrm{Pa}\right) .
$$

where $R_{C R}$ is the ring radius where buckling is first observed. Combining Equation (67) with Equation (43), buckling in these structures occurs when the residual stress exceeds:

$$
\sigma_{o}=\frac{(\pi \cdot t)^{2} E}{12\left(R_{C R}\right)^{2} g\left(R_{C R}\right)}, \quad g\left(R_{C R}\right) \leq 0.918 \quad\left(\mathrm{~N} / \mathrm{m}^{2} \text { or } \mathrm{Pa}\right)
$$

which is only useful if Young's Modulus, $E$, is known for the thin film $[51,56,61,62]$.

Two separate arrays of these ring-crossbar structures were included in this photomask design: one with a $10 \mu \mathrm{m}$-wide crossbar and another with a $20 \mu \mathrm{m}$-wide crossbar. The $10 \mu \mathrm{m}$ wide crossbar array is shown in Figure 50. Each array consisted of 45 ring-crossbar structures

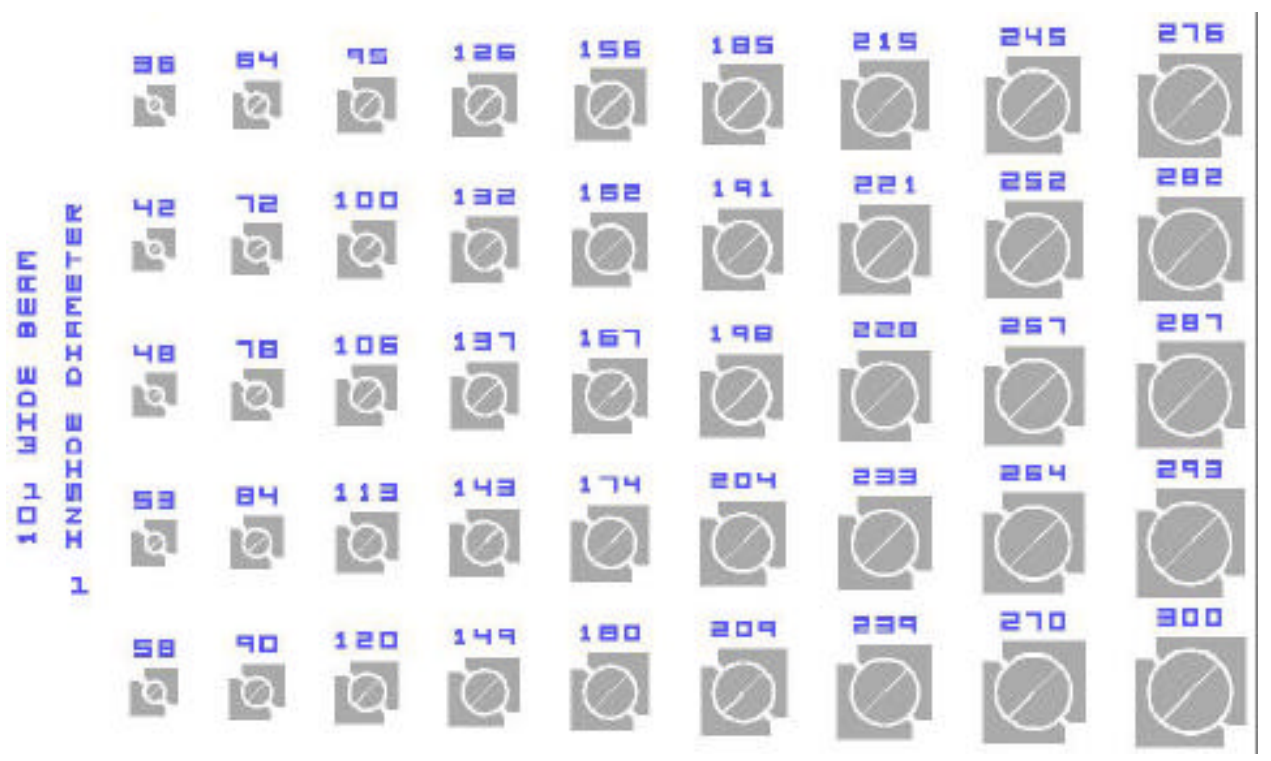

FIGURE 50 - A ' $98 /$ '99 mask array of ring-crossbar structures with $10 \mu \mathrm{m}$-wide crossbars. These were included for the measurement of residual tensile stress. 
with 36 to $300 \mu \mathrm{m}$ crossbar lengths (i.e. ring inside-diameters), increasing in approximately $30 \mu \mathrm{m}$ increments. The width of the rings were all designed to be approximately $20 \mu \mathrm{m}$. A closeup SEM photo of one of these structures, fabricated with an RF planar magnetron sputterdeposited $\mathrm{Si}_{x} \mathrm{~N}_{y}$ thin film, is shown in Figure 51. Once suspended from the substrate, the approximate value of the residual film stress can be estimated by observing which length beams have buckled, typically with optical microscopy methods.

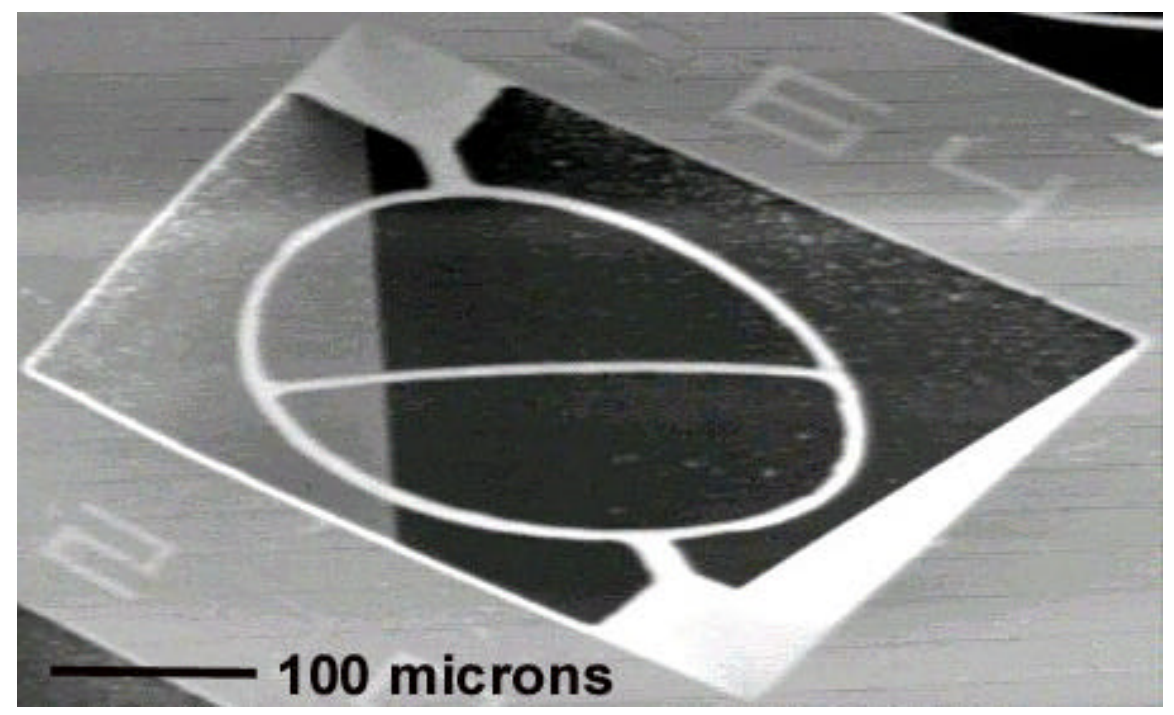

FIGURE 51 - An SEM photo of a sputtered $\mathrm{Si}_{x} \mathrm{~N}_{y}$ ring-and-beam test structure. The $\mathrm{Si}_{x} \mathrm{~N}_{\mathrm{y}}$ thin film was RF planar magnetron sputter-deposited, and was under compression warping the ring downwards. For this structure, the crossbar's design length was $264 \mu \mathrm{m}$. The design width of the ring itself was $20 \mu \mathrm{m}$.

\section{f. "T" Structures}

Three "T" shaped structures were included in this photomask design, one of which is shown in Figure 52, to measure residual stress non-uniformity in the thin film. Geometrical parameters for these structures were selected to provide measurable deflections over a broad range of possible expected residual stress values for a deposited $\sim 1 \mu m \mathrm{Si}_{x} \mathrm{~N}_{\mathrm{y}}$ thin film, while allowing for placing them within a reasonable photomask die-size area. A diagram showing the geometrical relationships for this structure is shown in Figure 53. The fixed-end, transverse beam is loaded in a bending mode by the residual tensile stress in the stem of the "T," which results in large deflections at small strains. The deflection $(\delta)$ at the center of the crossbow is 


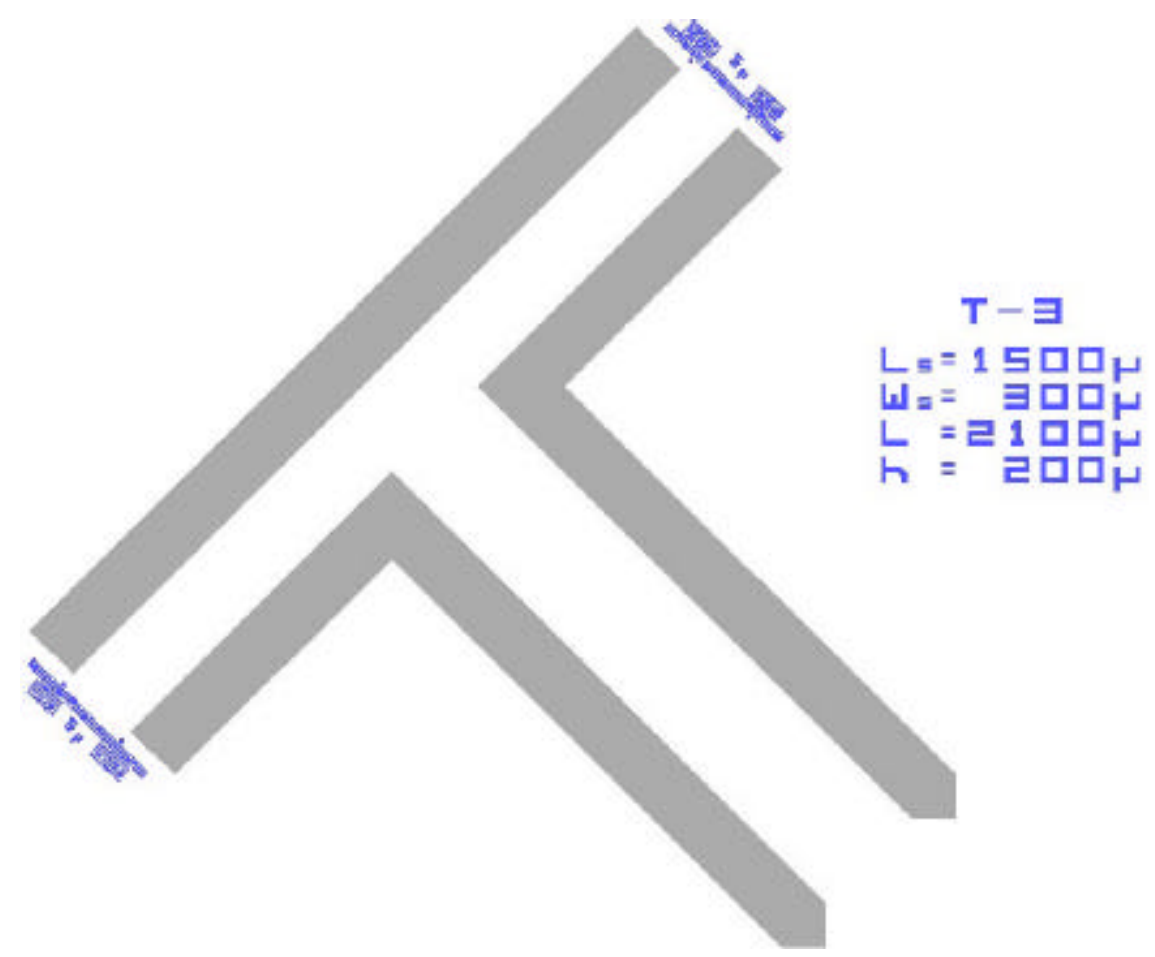

FIGURE 52 - An '98/'99 L-EditTM design file image of the smallest "T" shaped test structure. This was designed to measure residual tensile stress in the thin film. To suspend this " $T$ " from the substrate, anisotropic etching must occur through the substrate, due to the size of these structures. Verniers at the lower left and top right of the structure (with $\sim 5 \mu \mathrm{m}$ resolution, allow for measuring the deflection, $\delta$, of the structure. The definitions of the geometrical parameters, $L_{s}, W_{s}, L$, and $h$ are shown in Figure 53.

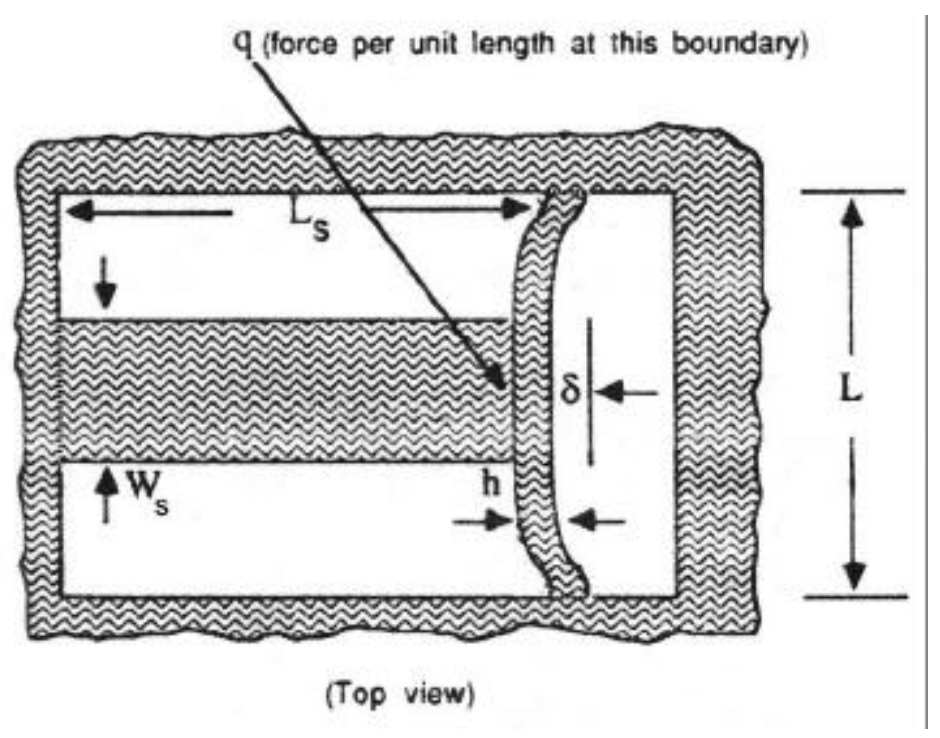

FIGURE 53 - Schematic diagram of the "T" structure with dimensions' nomenclature. This is shown after release from the substrate, with deflection, $\delta$, at the center of the crossbow due to residual tensile stress in the stem of the "T" [57]. 
proportional to the residual stress over Young's modulus ratio i.e. residual strain). Once suspended from the substrate, if the behavior of the thin-film material is linear-elastic, large deflections in the structure can be achieved at small residual strains.

With the dimensions of the structure properly selected such that the center deflection, $\delta$, of the fixed-end beam is always less than $30 \%$ of the beam width, $h$, the center deflection can be determined by small-deflection (linear-elastic, beam-bending) theory, and the membrane stresses created as a result of the stretching of the middle plane of the beam can be neglected. For small deflections, the effect of residual tensile stress in the fixed-end beam may be neglected. Mehregany, et al., found these two assumptions to be valid for a polyimide thin film $[57,63]$. The "T" structure is modeled as a fixed-end beam with uniform loading in the center region by the residual stress in the stem of the "T." When the structure is released from the substrate, the force per unit length, $q$, on the beam can be found in terms of the center deflection, $\delta$, after release, which can be found from superposition of the deflections due to bending and shear (as shear forces aid deflection). The load force, $q$, on the beam that is generated by stress-induced shrinkage of the stem is given by [57]:

$$
q=t \cdot\left(\sigma_{o}-\frac{E \delta}{L_{s}}\right)
$$

where $t$ is the uniform film thickness, $E$ is Young's Modulus, $\sigma_{o}$ is the average residual tensile stress, and $L_{s}$ is the stem's initial length (neglecting residual strain, a second-order correction). In finding $q$, it is modeled as uniform across the width of the stem, $W$. This is, however, not the case, due to the curvature in the beam (once released). But, the length of the stem is very large compared to the variation in shrinkage across the width, $W$. The radio of the stress to Young's Modulus is, thus, related to the center deflection, $\delta$, and geometrical dimensional parameters of the "T" $[57,63]$ : 


$$
\frac{\sigma_{o}}{E}=\delta\left(\frac{1}{L_{s}}+\frac{16 h^{3}}{W\left[L^{3}-L W^{2}+\frac{W^{3}}{2}\right]}\right)
$$

This equation includes the contribution due to the bending moment only, while neglecting the contribution due to shear forces, which is a close approximation if the fixed-end beam is made slender (a large ratio of the beam length, $L$, to beam width, $h$ ). For a beam length-to-width ratio of 10 or more, the shear component is less than $10 \%$, which is the case for the "T" structure shown in Figure 52, where this ratio is 10.5 . Thus, this equation can be used to calculate the residual stress to Young's Modulus ratio for slender, fixed-end beam "T" structures under residual tensile stress.

The contribution to deflection due to shear forces is not negligible for stocky fixed-end beams (or for the "T" structures with the largest beam length to width ratio in this design), where the ratio of stress to Young's Modulus is related to the center deflection, $\delta$, geometrical dimensional parameters, and Poisson's ratio, $v$ as follows [57]:

$$
\delta=\left(\frac{\sigma_{o}}{E}-\frac{\delta}{L_{s}}\right)\left[\frac{W}{16 h^{3}}\left(L^{3}-L W^{2}+\frac{W^{3}}{2}\right)+\frac{3 W(1+v)}{4 h}\left(L-\frac{W}{2}\right)\right] \quad(\mathrm{m}) .
$$

Thus, measurement of a suspended "T" structure's center deflection in conjunction with this equation yields an expression with two unknowns: $\sigma_{o} / E$ and $i$. With at least two of these "T" structures (three are included in the 1998/1999 photomask design), it is possible to generate several expressions with these two algebraic unknowns. A least-squares fit of the data to this equation can then be used to solve for $\sigma_{o} / E$ and $i$. Although this photomask design only includes slender, fixed-end-beam "T" structures, this equation is more accurate, and can be used to calculate the residual stress to Young's Modulus ratio and Poisson's ratio of the thin film. 
This photomask design includes vernier scales (or fiducial marks) at both ends of the slender fixed-end beams in the three "T" structures for measuring center deflections. A close-up of these vernier markings is shown in Figure 54. Two distinct problems arise in using these fiducial marks to measure center deflections. First, the design dimensions of, and spacing between these marks will not be exactly $5 \mu \mathrm{m}$ due to wet etchant undercutting of the patterned (flow sensor resistor) metallization layer used to create the marks. Secondly, it is quite difficult to use high-magnification optical microscopy techniques to measure center deflections of the "T's"

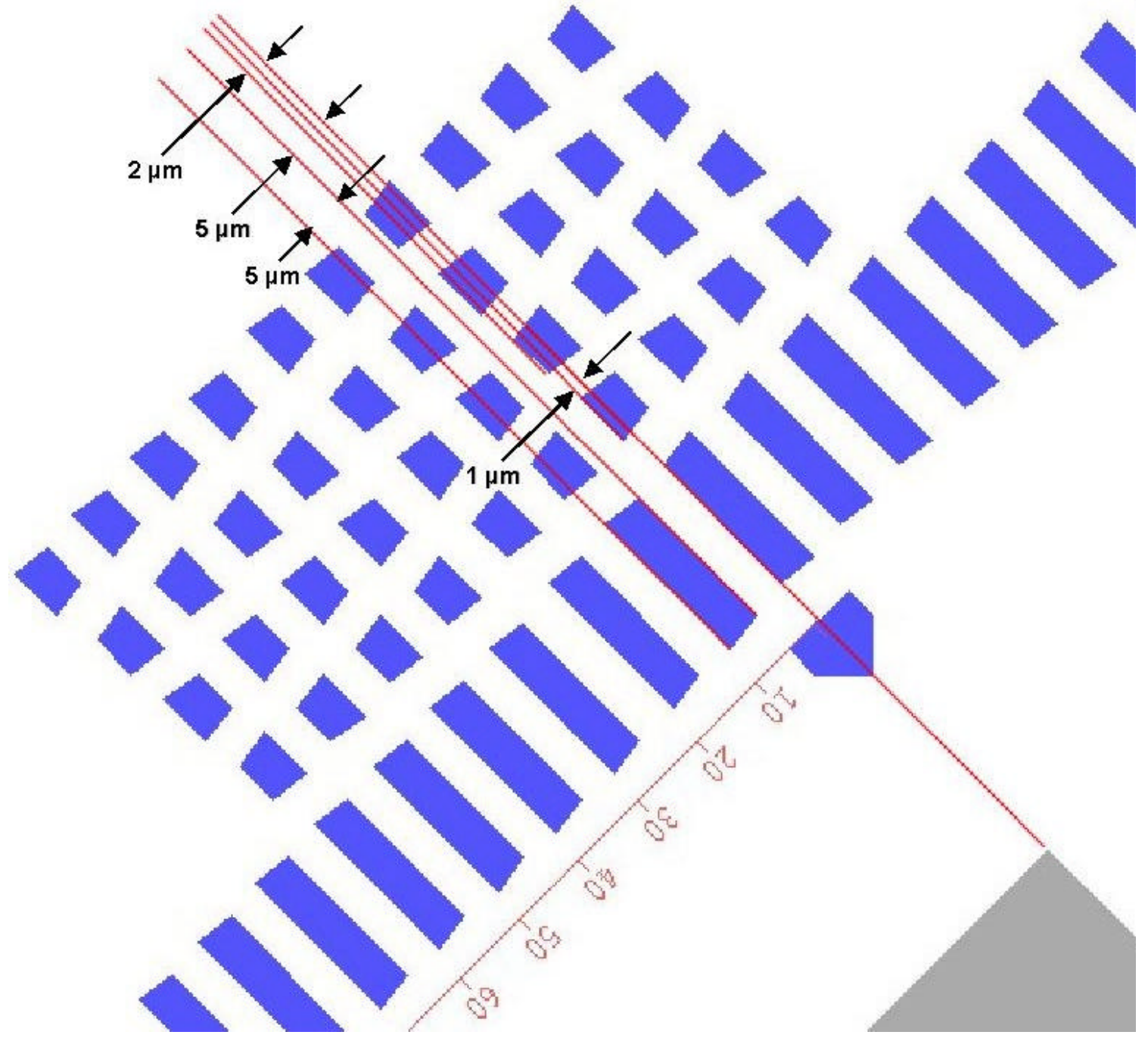

FIGURE 54 - Close-up '98/'99 design file image of "T" structure fiducial marks. The vernier scale spacing and dimensions were designed to measure center deflection of the "T" structure. The metallization traces shown in blue will typically not be the exact design-width of $5 \mu \mathrm{m}$ (nor will the spacing between them be exactly $5 \mu \mathrm{m}$ ), due to wet-etching undercut of the photoresist layer used to pattern these fiducial marks. 
with accuracies much better than $\pm 3 \mu \mathrm{m}$, because the entire fixed end beam and fiducial marks are not all in view at the same time under higher magnifications where the accuracy of deflection measurements increases. This $\pm 3 \mu \mathrm{m}$ uncertainty can result in extractions of Poisson's ratio that are meaningless (where $v>0.5$ ). Thus, in some cases, the measurement error in $\delta$ is not small enough to permit accurate extraction of Poisson's ratio. Although to suspend these large "T" structures, the substrate has to be completely etched through, these structures are reasonably accurate for determining the thin film's residual stress to Young's Modulus ratio at small strains. To extract Poisson's ratio, the center deflection measurements have to be accurate to fractions of a micrometer [57].

These "T" structures can also be used to evaluate non-uniformities in the thin film's residual stress, which (if present) will result in out-of-plane deflections of the structure. A stress non-uniformity in the thin film is analogous to a uniform stress and bending moment and is used to model the out-of-plane deflections. The bending moment and the minimum film thickness required for planarity of prototype thin-film sensors can be calculated. A schematic of the "T" structure geometry and associated mechanics is shown in Figure 55. The loading column of the

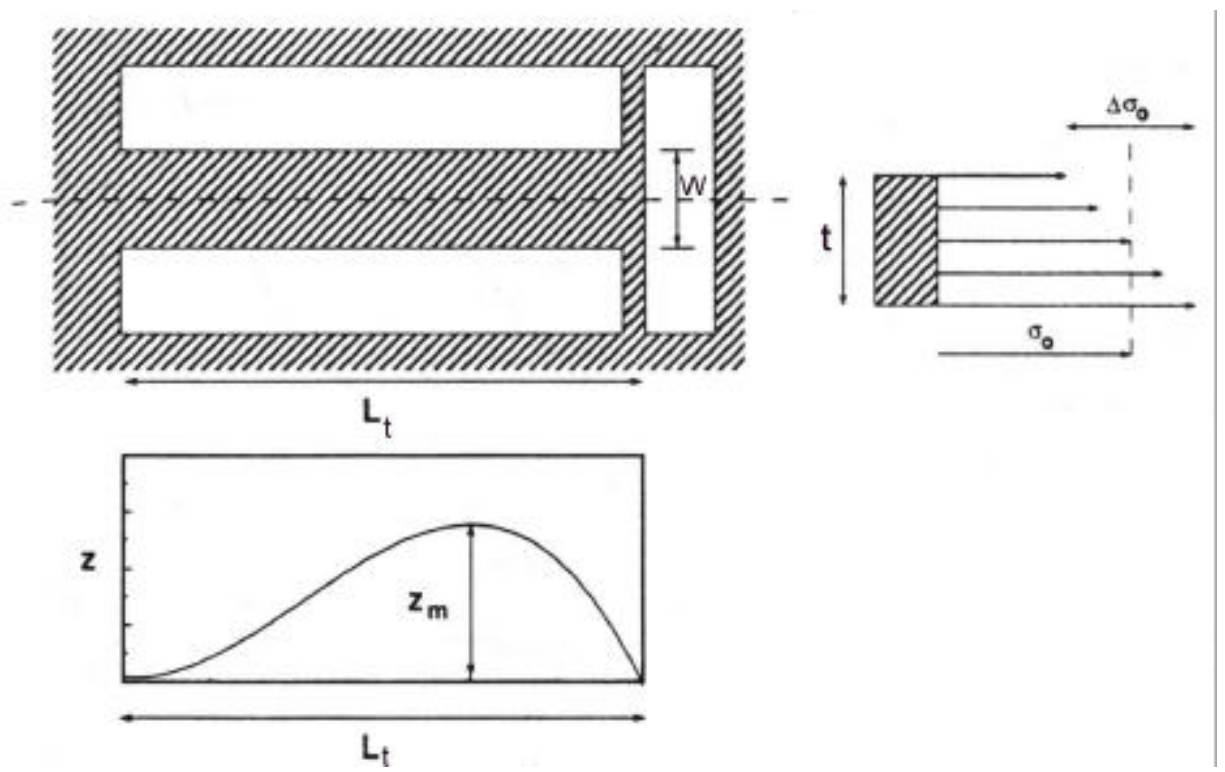

FIGURE 55 - Geometry and mechanics illustration of "T" with out-of-plane deflection. The total length is $L_{t}\left(L_{t}=L_{s}+h\right)$ and stem width is $W$. The average residual stress, $\sigma_{o}$, in the thin film is nonuniform, varying by $\Delta \sigma_{o}$, across the film thickness, $t$, causing the structure to deflect out of the plane of the substrate by $z_{m}$ [64]. 
"T" will deflect out of the plane of the wafer and is assumed to have a fixed support at one end and a roller support at the other end. With these assumptions the deflection curve (shown in Figure 55) for the column is given by [64]:

$$
\frac{z}{L_{t}}=\frac{3 L_{t} M_{f}}{E W \cdot t^{3}}\left(\frac{x}{L_{t}}-1\right)\left(\frac{x}{L_{t}}\right)^{2} \text {, and } M_{f}=\frac{W \cdot t^{2}\left(\Delta \sigma_{o}\right)}{12}
$$

where $M_{f}$ is the bending moment in the film, $L_{t}$ is the total length of the "T", $W$ is the stem width, and $t$ is the film thickness. As shown in Figure 54, the deflection curve has a maximum deflection, $z_{m}$, at $x / L_{t}=2 / 3$. Substituting this value and the expression for $M_{f}$ into the deflection curve equation, and rearranging, the variation in stress (residual strain) with the depth of the film can be calculated from a measurement of $z_{m}[64]$ :

$$
\frac{\Delta \sigma_{o}}{E}=\frac{27 t \cdot Z_{m}}{\left(L_{t}\right)^{2}}
$$

where $E$ is Young's Modulus.

\section{g. Axial Beam "H" Structures}

Five "H" shaped beam structures, with various geometries, were included in this photomask design, a design for one of which is shown in Figure 56, to measure residual tensile stress in the thin film. Geometrical parameters for these structures were chosen to allow for placing them within a reasonable photomask replicated-die-size area and provide measurable deflections for a broad range of expected residual stress values in a deposited $\sim 1 \mu m \mathrm{Si}_{\mathrm{x}} \mathrm{N}_{\mathrm{y}}$ thin film. A diagram showing the geometrical relationships for this structure (corresponding to metallization nomenclature shown in Figure 56) is shown in Figure 57.

Once the "H" structure is released, residual tensile stress will cause an axial shrinkage of the wide part of the beam and a corresponding axial extension of the narrower necks (placing 


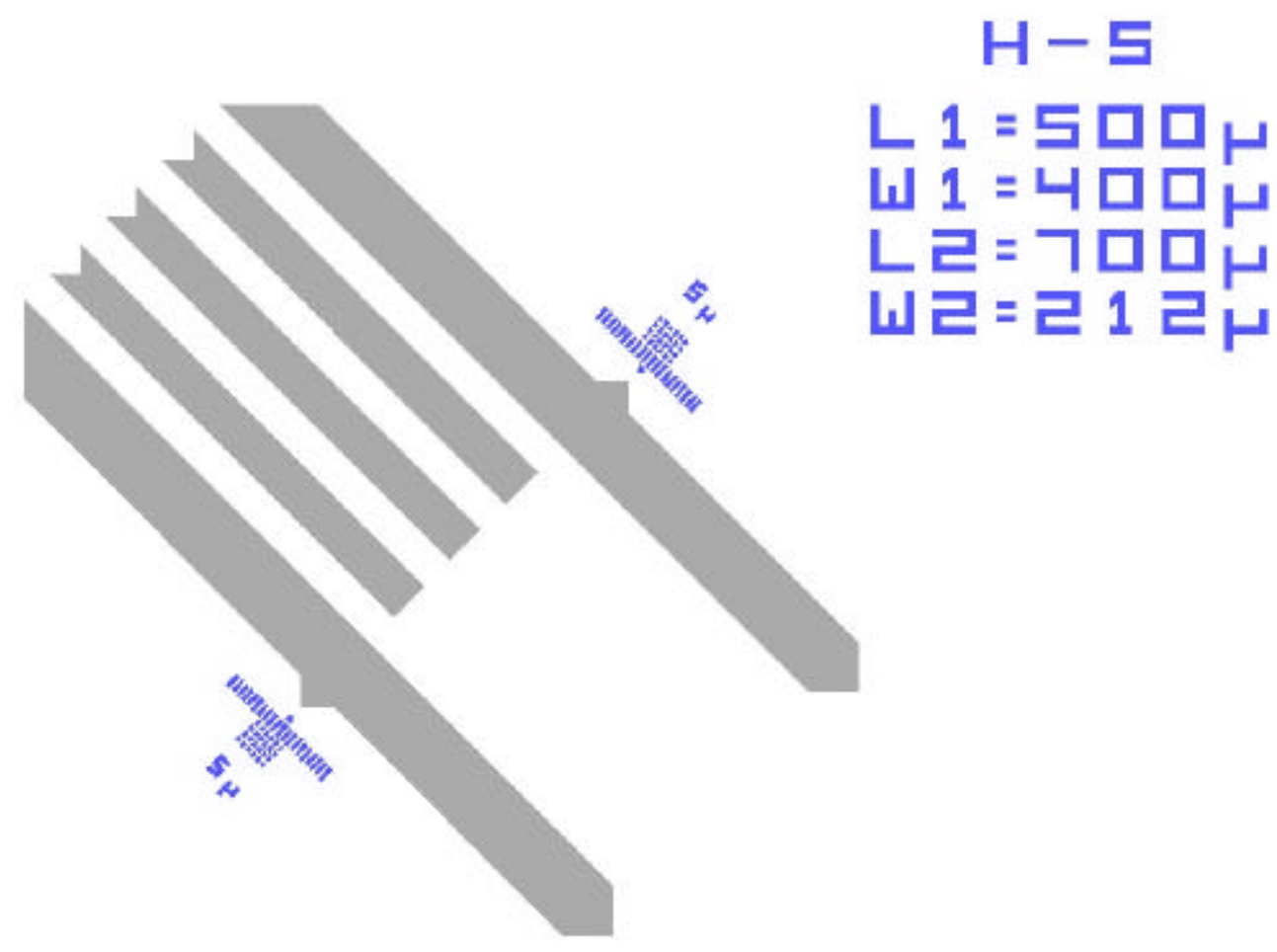

FIGURE 56 - A '98/'99 L-EditTM image of an "H" structure for tensile stress measurement. This was one of five " $\mathrm{H}$ " beam test structures included in the photomask design for measuring residual tensile stress in the deposited thin-film layer. Verniers on each side of the structure can be used to measure the deflection of the line where the thinner necks attach to the wide suspended strip. Refer to Figure 57 to match parameters shown at the top right to the physical geometry of the structure.

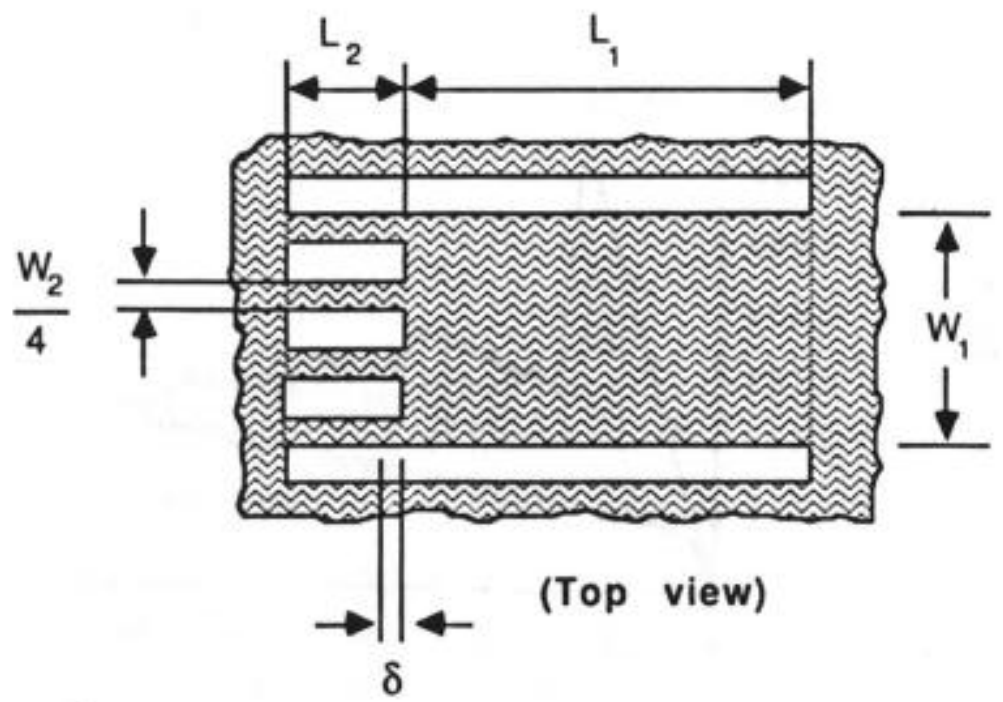

FIGURE 57 - A top-view schematic of an "H" beam structure with dimensions. It has already been released from the substrate, with the wide strip pulling on the thinner strips, resulting in a deflection at the boundary, $\delta$, due to the film's residual tensile stress. Note that $W_{2}$ is the total combined width of the narrow strips [65]. 
them under an increasing tensile stress once released). Thus, the wide suspended strip (with width $W_{1}$ ) will pull on the thinner necks (with total width $W_{2}$ ), resulting in a deflection, $\delta$, at the boundary from its original mask position toward the right (in Figure 57) into its final position. By varying the geometry, structures can be created in which the residual strain in the thinner sections is very small, and others in which the ultimate strain (in the thin necks) of the film is exceeded and the structure deflects, partially separating from the substrate. Since the residual stress does not change with strain, and since there are "H" structures with different geometries within this design, the in-plane stress-strain curve (normalized by the residual stress) can be measured in addition to the yield and ultimate strains. Thus, Young's Modulus can be studied as a function of strain. For the case when the ultimate strain in the thin necks is not exceeded (for sufficiently small structures), the in-plane stress-strain behavior of the thin film can be modeled by using axial-beam, linear-elastic theory. The beam structure is analyzed by the stiffness method, assuming a uniform stress distribution across the thickness of the thin film. The displacement, $\delta$, of the boundary after release can be related to the residual (tensile) stress to Young's Modulus ratio as follows $[57,63,65]$ :

$$
\frac{\sigma_{o}}{E}=\delta\left[\frac{W_{1} L_{2}+W_{2} L_{1}}{L_{1} L_{2}\left(W_{1}-W_{2}\right)}\right]
$$

where $L_{1}$ and $L_{2}$ denote initial lengths of the members, neglecting the residual strain (a secondorder correction). The strain in the narrow necks can also be calculated [57]:

$$
\varepsilon_{2}=\frac{\sigma_{o}}{E}\left(1+\frac{\delta}{L_{2}}\right)+\frac{\delta}{L_{2}}
$$




\section{SENSOR FABRICATION AND PROCESS METHODOLOGIES}

A list of all chemicals and photographs of all laboratory equipment utilized for these experimental processes are included in Appendices III and IV, respectively. A summary of all attempted flow sensor fabrication methodologies and their outcomes is included in Appendix V. The methods are grouped by the experimental, thin-film microbridge/cantilever structural material utilized and how the silicon substrate was anisotropically etched: at the beginning, or at the end of the fabrication process.

\section{A. Thin Film Structural Methods Attempted: Pre-Etched Wafer Strategies}

\section{Anodically-Bonded Borosilicate Glass Method}

Attempts in 1997 to successfully fabricate flow sensors with RF planar magnetron sputter-deposited silicon nitride thin films resulted in two primary structural problems which required the exploration of some uncommon, yet economical, techniques for fabricating the thinfilm layer of the flow sensors. First, it was discovered that RF planar magnetron sputtered silicon nitride films had significant cracking, buckling/warping, porosity/pinholes, and possible residual strain gradients through the film thickness, once released from the substrate by the anisotropic silicon etchant, $\mathrm{KOH}$. Warped microbridges tended to be damaged or cracked (or perhaps potentially structurally unstable within a flow stream), possibly allowing $\mathrm{KOH}$ to come into contact with and degrade the resistor metallization layer sandwiched between $\mathrm{Si}_{x} \mathrm{~N}_{\mathrm{y}}$ layers on each bridge. Warped structures would also induce stresses in, and change the electrical properties of, the thin film resistor metallization layer, potentially causing greater undesired mismatches between upstream and downstream resistors. The second dilemma was related to the first, in that the sputtered $\mathrm{Si}_{x} \mathrm{~N}_{\mathrm{y}}$ thin film structural layer was susceptible to unacceptable degradation in the alkaline silicon etchant, $\mathrm{KOH}$. Thus, a thin-film microbridge/cantilever material was needed that could be deposited or applied (with a controllable thickness) with minimal or no residual 
compressive stress, and a thin film or "non-traditional" process methodology was needed to reduce the structural layer's susceptibility to degradation in $\mathrm{KOH}$.

Table XII shows a summary of one of the more creative and unique strategies for fabricating the thin-film flow sensor microbridge/cantilever structural layer: using borosilicate glass, anodically-bonded to a pre-etched (100) silicon substrate, thinned by wet-etching and machining techniques, and then patterned to form the flow sensor microbridges/cantilevers. If this method could be proven feasible, it was believed that the glass layer would provide a suitable underlayer for resistor metallization, with little or no residual compressive stress. A major disadvantage of this method was the potential difficulty in controlling the final thin-film glass thickness, as it would not be deposited, but instead machined into its desired thickness. The use of a thermally-grown oxide for the thin-film layer was also considered (because it allows for much greater control and precision over the final film thickness), but ultimately rejected, because it would not solve the buckling problem. Thermally-grown oxide layers are commonly under residual compressive stress (with a deposition compressive stress of $300 \mathrm{MPa}$ [66]), typically resulting in buckled/warped microbridges and cantilevers.

Anodic bonding, also known as field-assisted thermal bonding, or electrostatic bonding, was pioneered by Wallis and Pommerantz in 1969, and is commonly used to join glass to silicon by applying a potential difference across an elevated-temperature, silicon-glass structure $[67,68,69,70,71]$. The resulting bond is hermetic and irreversible, with a bond strength exceeding that of the substrate. This type of bonding is restricted to electrically conducting glasses with a thermal expansion coefficient close to that of the semiconductor wafer substrate. A bond can be established between silicon and a sodium-rich glass, such as Pyrex ${ }^{\mathrm{TM}}$ (Corning 7740), or a boron-doped, borosilicate glass. Bonding is done in atmosphere, on a hotplate at temperatures between 180 and $500{ }^{\circ} \mathrm{C}$ with a constant DC potential difference of 200 to $1000 \mathrm{~V}$ applied across the wafer and glass combination (e.g. at $400{ }^{\circ} \mathrm{C}$ and $600-1000 \mathrm{~V}$ for Pyrex ${ }^{\mathrm{TM}} 7740$ borosilicate glass), depending upon the glass thickness. Since it is a relatively low temperature process, both the glass and silicon substrate remain rigid during bonding. For a good bond to form, the contacting surfaces to be bonded must be in intimate contact, must be flat (with a surface 
TABLE XII

SUMMARY OF BOROSILICATE GLASS THIN-FILM DIAPHRAGM PROCESS

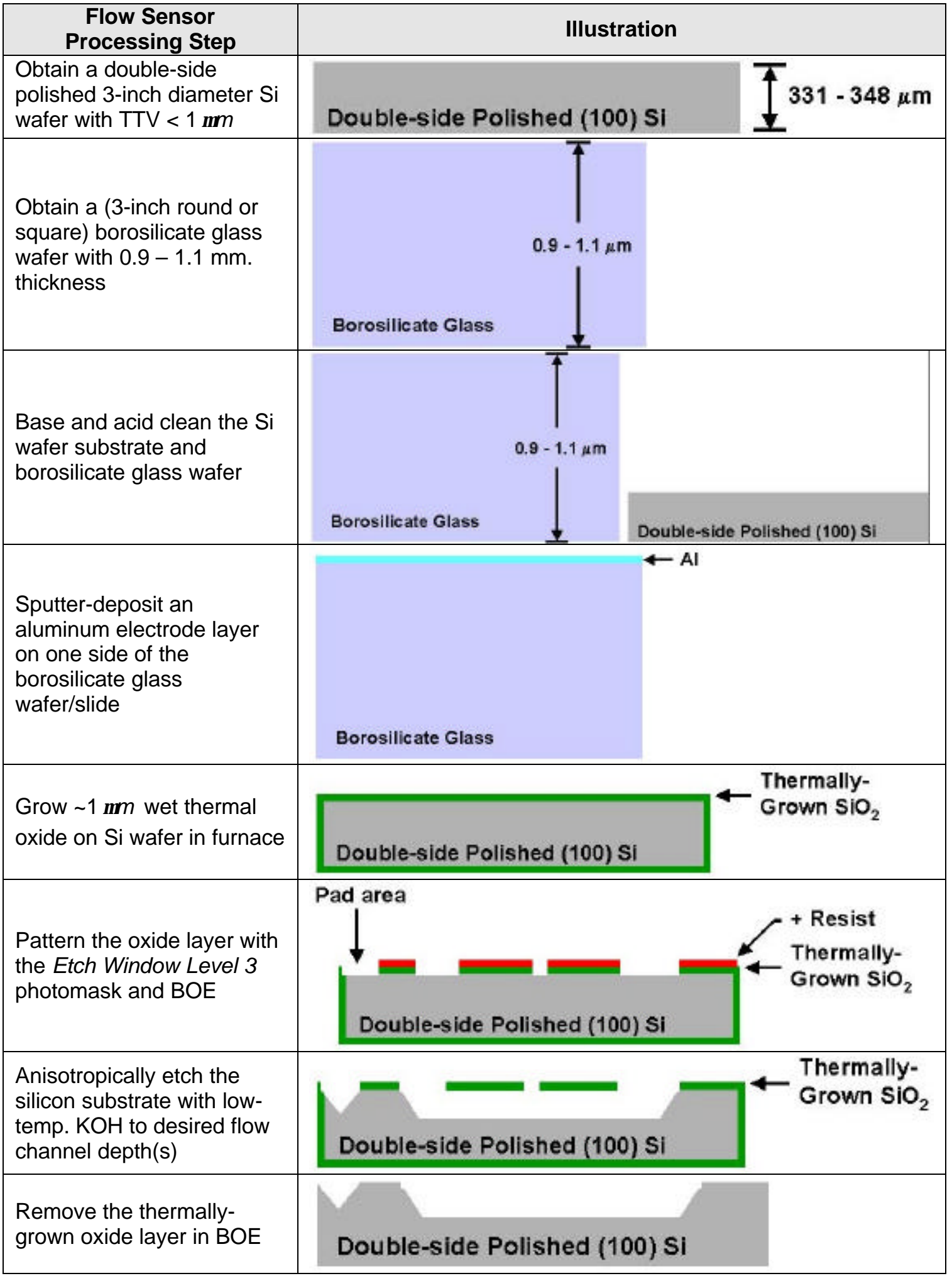




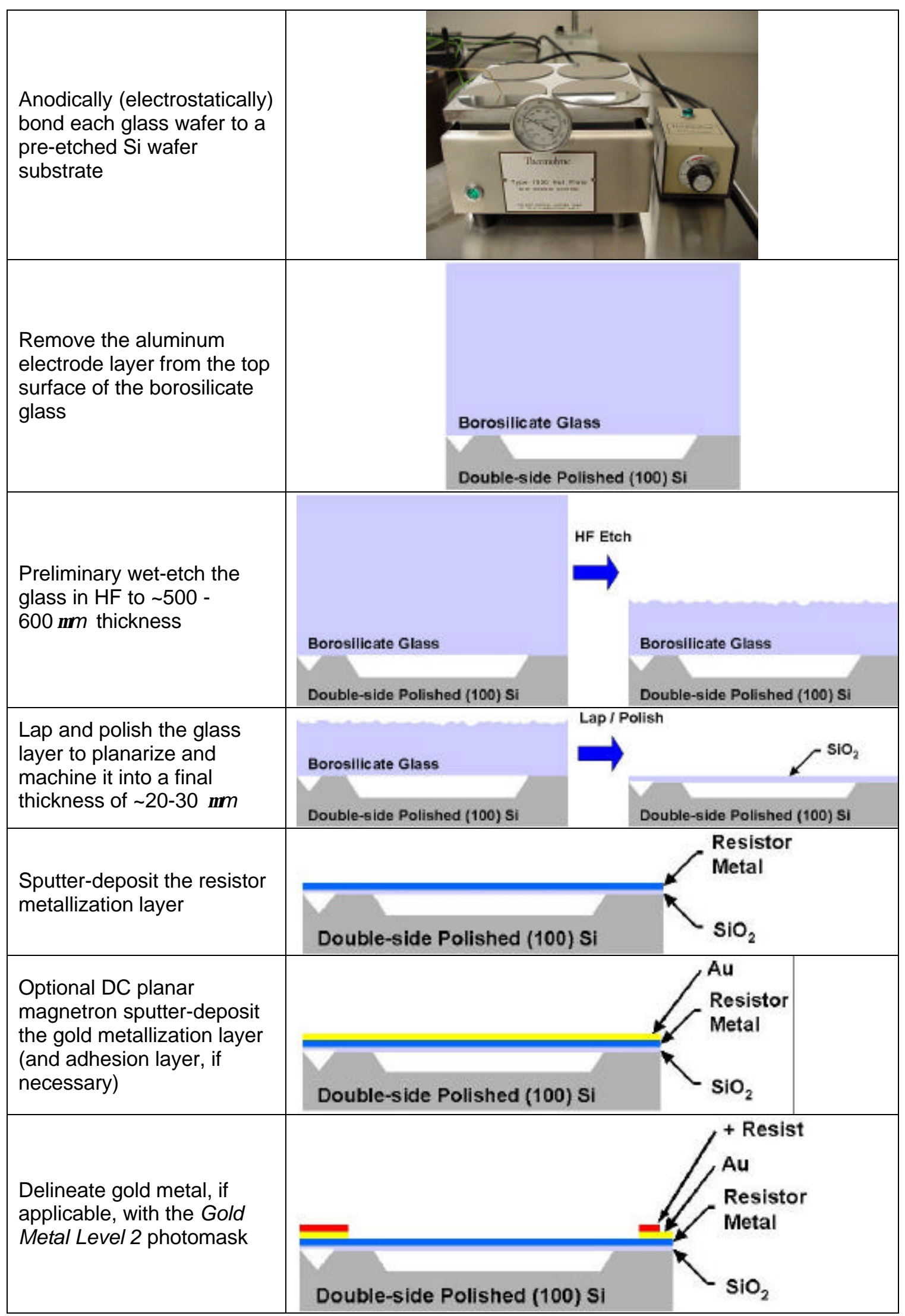




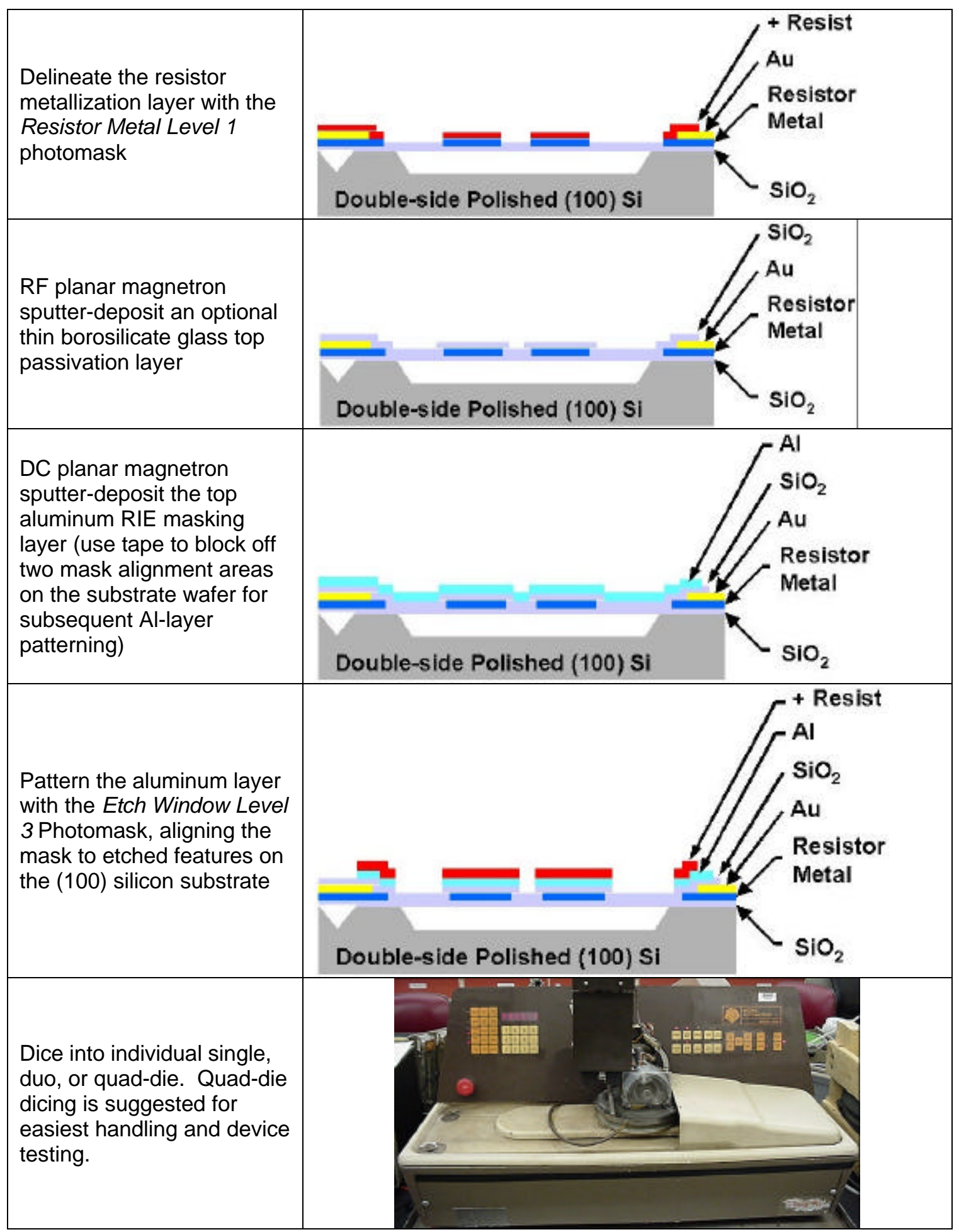




\begin{tabular}{|l|l|}
\hline $\begin{array}{l}\mathrm{RIE} \text { or wet-etch through } \\
20-30 \mu \mathrm{m} \text { thick glass to } \\
\text { create microbridge/ } \\
\text { cantilever flow sensors and } \\
\text { remove the top aluminum } \\
\text { layer(s) }\end{array}$ \\
$\begin{array}{l}\text { Wire-bonding, packaging, } \\
\text { and flow sensor testing. } \\
\text { Note that with this mask } \\
\text { set, cavities will exist under } \\
\text { each pad area. }\end{array}$
\end{tabular}

roughness less than $1 \mu \mathrm{m} \mathrm{rms}$ ), free of particulates, and free of organic residues. Because of the strong fields at the interface, good quality bonding is possible for substrates of poor interface planarity. Any oxide on the silicon surface must also be less than $200 \mathrm{~nm}$ thick. Generally, the glass slide or plate is placed on top of a silicon wafer substrate. As shown in Figures 58 and 59, a pin-point electrical probe-tip cathode makes contact to the top surface of the glass, holding it at a constant negative bias with respect to the electrically-grounded and heated silicon substrate, which is the anode. Temperature and applied voltage are typically kept constant throughout the bonding process. Although the dominant bonding mechanism is not yet clearly known,

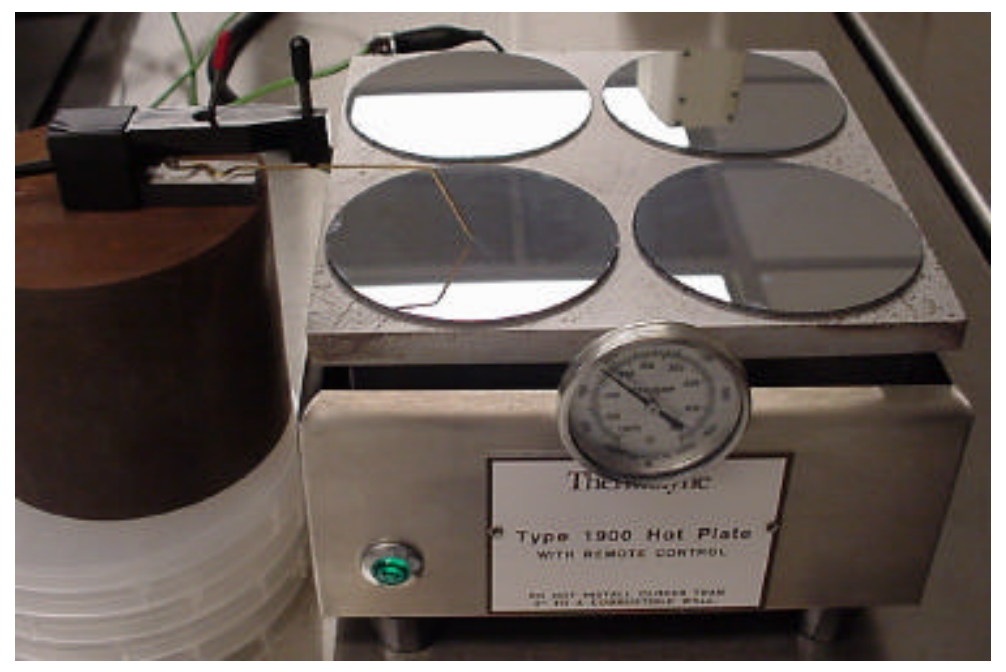

FIGURE 58 - A photo of the hotplate setup used for anodic bonding. Borosilicate glass was bonded to three-inch, double-side-polished, (100) silicon wafer substrates. 


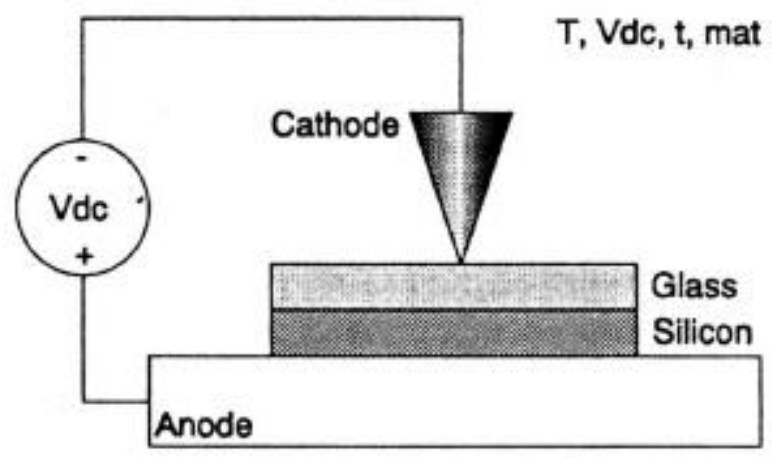

FIGURE 59 - An electrical schematic of an anodic glass-to-Si wafer-bonding setup. Control parameters include temperature, $T$, bias voltage $V_{d c}$, time for bonding $t$, and materials (e.g. silicon, $\mathrm{SiO}_{2}$ ) [67].

electrochemical, electrostatic, and thermal mechanisms have been suggested. As soon as the voltage is applied to the elevated-temperature conductive solid electrolyte glass, a current peak occurs, indicating the drift of mobile positive ions (sodium or boron) to the cathode, leading to the buildup of a space-charge depletion region adjacent to the glass-silicon interface. Most of the voltage drop is situated across the space-charge region, resulting in a high electric field strength between the glass and silicon (pulling the wafers into close contact). Covalent bonds between surface atoms of the wafers form as a result of the elevated temperatures. The bonding process can be observed through the glass, as a front of interference fringe patterns slowly spreads out from the spot below the cathode tip while the color of the bonded regions appears darker than the unbonded silicon/glass interface. The process can also be monitored by measuring the current flow, for constant applied DC voltage. Bonding is completed when the entire interface between silicon wafer and glass shows a uniform gray color (with a few residual, persistent air pockets), with no further "bubbling" under the glass, and current flow is nil. Although the anodic bonding process is generally slow, requiring a high voltage bias to bond areas that are far removed from the small cathode point, it may be expedited through using a metallization electrode layer deposited/patterned on top of the glass (e.g. at NEC, a Ti mesh bias electrode was deposited on top, completing bonding at $400{ }^{\circ} \mathrm{C}$ and $600 \mathrm{~V}$ in less than $5 \mathrm{~min}$., compared to over an hour without the mesh) $[67,68,69,70,71]$. 
In summary, the following requirements must be met for anodic bonding between silicon and glass to occur [71]:

1. The glass must be slightly conductive;

2. The temperature must stay well below the softening point of the glass;

3. The metal (probe tip) must not inject charge carriers into the glass;

4. The surface roughness of the silicon and glass must be smaller than $1 \mu \mathrm{m} \mathrm{rms;}$

5. The surfaces must make intimate contact

6. The surfaces must be clean, free of particulates (e.g. dust), and organic residues;

7. Any oxide on the silicon substrate must be thinner than $200 \mathrm{~nm}$; and

8. The thermal expansion coefficients of the bonded materials must match within the range of anodic bonding temperatures (e.g. to avoid cracking during cooling).

For this experimental flow sensor process methodology, it was decided that a round borosilicate glass plate would be utilized, with a thermal expansion coefficient closely matching that of the supporting (100) silicon substrate within the temperature range of anodic bonding. Monocrystalline silicon has coefficients of linear thermal expansion that are a function of direction with respect to the $\{100\},\{110\}$, and $\{111\}$ crystal planes. A mismatch in coefficients could cause both thermally induced and built-in residual mechanical stress in the glass. A graph comparing the coefficients for silicon and 7740 glass is shown in Figure 60. Since, the thermal properties of the materials begin to seriously deviate above $450{ }^{\circ} \mathrm{C}$, the process should be limited to $450{ }^{\circ} \mathrm{C}$. Three-inch and four-inch diameter, round $1.1 \mathrm{~mm}$-thick Schott BOROFLOAT® borosilicate glass

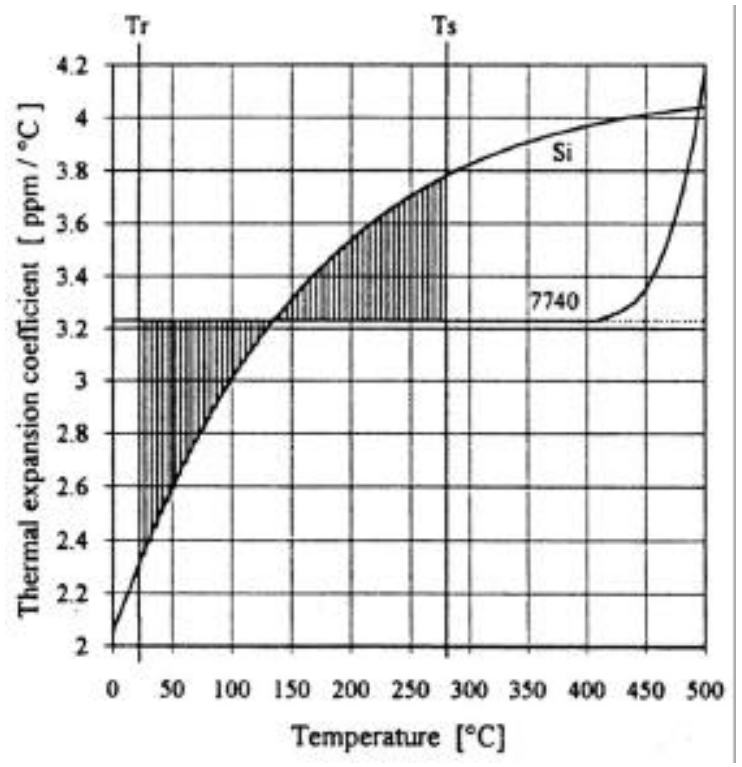

FIGURE 60 - Thermal expansion coefficient graph of Si and Corning 7740 Pyrex ${ }^{\mathrm{TM}}$ glass. Room temperature is indicated by $T_{r}$, and $T_{s}$ is a sample selected bond-seal temperature [67]. 
wafers (US $\$ 9.00$ and $\$ 9.40$ ea., respectively), were obtained from Cincinnati Gasket $\AA$ (40 Illinois Ave.; Cincinnati, $\mathrm{OH} 45215 ; 513-761-3458)$ for use in this process. Additionally, a four-inch diameter, 0.25 -inch thick borosilicate sputtering target was obtained from the same vendor. Table XIII shows the mechanical and thermal properties of this glass. Note that the bending strength is influenced by the surface conditions of the glass, the rate at which the load is applied, and the area of the glass placed under the load. Since wafer curvature measurements have indicated that 7740 glass/Si sandwiches will transition from concave (silicon under compression) to convex (under tension) around a anodic bonding seal temperature of $315^{\circ} \mathrm{C}$, a higher hotplate temperature is preferred, and $450{ }^{\circ} \mathrm{C}$ was selected. Thus, since tensile stress is preferred for the substrate and glass sandwich, a safety margin toward higher bonding temperatures was used $[67,72]$.

For this processing methodology, it was important to control the final thickness of the borosilicate glass, flow sensor microbridge/cantilevers. Since the purchased borosilicate glass wafers had a large initial thickness tolerance $( \pm 100 \mu \mathrm{m})$, silicon substrates were needed with very

TABLE XIII

BOROFLOAT® GLASS MECHANICAL AND THERMAL PROPERTIES [73,74]

\begin{tabular}{|c|c|c|}
\hline Bulk Property & \multicolumn{2}{|c|}{ Value } \\
\hline Sheet Thickness Tolerance for $1.1 \mathrm{~mm}$ Thick Glass & \multicolumn{2}{|c|}{ $\pm 0.1 \mathrm{~mm}$} \\
\hline Density at $25^{\circ} \mathrm{C}$ & \multicolumn{2}{|c|}{$2.22 \mathrm{~g} / \mathrm{cm}^{3}$} \\
\hline Young's Modulus of Elasticity at $25^{\circ} \mathrm{C}$ & \multicolumn{2}{|c|}{$63 \mathrm{GPa}$} \\
\hline Poisson's Ratio, v & \multicolumn{2}{|c|}{0.20} \\
\hline Bending Strength & \multicolumn{2}{|c|}{$25 \mathrm{~N} / \mathrm{mm}^{2}$} \\
\hline Linear Thermal Expansion Coefficient $\left(20-300^{\circ} \mathrm{C}\right)$ & \multicolumn{2}{|c|}{$3.25 \times 10^{-6} \mathrm{~K}^{-1}$} \\
\hline \multirow{4}{*}{ Thermal Conductivity } & $10^{\circ} \mathrm{C}$ & $1.11 \mathrm{~W} / \mathrm{m} \cdot \mathrm{K}$ \\
\hline & $50^{\circ} \mathrm{C}$ & $1.17 \mathrm{~W} / \mathrm{m} \cdot \mathrm{K}$ \\
\hline & $90^{\circ} \mathrm{C}$ & $1.22 \mathrm{~W} / \mathrm{m} \cdot \mathrm{K}$ \\
\hline & $190^{\circ} \mathrm{C}$ & $1.31 \mathrm{~W} / \mathrm{m} \cdot \mathrm{K}$ \\
\hline Specific Heat Capacity & \multicolumn{2}{|c|}{$0.83 \mathrm{~kJ} / \mathrm{kg} \cdot \mathrm{K}$} \\
\hline Annealing Point $\left(10^{13} \mathrm{dPa} \cdot \mathrm{s}\right)$ & \multicolumn{2}{|c|}{$560^{\circ} \mathrm{C}$} \\
\hline Softening Point $\left(10^{7.6} \mathrm{dPa} \cdot \mathrm{s}\right)$ & \multicolumn{2}{|c|}{$815^{\circ} \mathrm{C}$} \\
\hline Maximum Long-Term Operating Temperature & \multicolumn{2}{|c|}{$450{ }^{\circ} \mathrm{C}$} \\
\hline
\end{tabular}


precisely known thicknesses to minimize errors in measuring final glass thicknesses (in the glass/silicon sandwich) due to silicon substrate thickness uncertainty. Consequently, three-inch diameter double-side polished, (100), two to six ohm-centimeter resistance, n-type (phosphorus doped) silicon wafers were obtained with a TTV less than $1 \mu \mathrm{m}$ across each wafer. These substrates were purchased from Pure Sil Inc. (One Silicon Way; Bradford, PA 16701; 814-3623586) at a price of US $\$ 32.50$ each. Total thicknesses of the wafers varied, but were in the range of $333-353 \mu \mathrm{m}$. Digital-micrometer-measured total thickness variations (TTV) for individual wafers were $\sim 1-3 \mu \mathrm{m}$.

The fabrication process began by thermal oxidation of the double-side-polished wafers (which were initially scribed with nomenclature to track them) to create an $\mathrm{SiO}_{2}$ layer that could be subsequently patterned for $\mathrm{KOH}$ anisotropic pre-etching of the substrates. Various wet (and one dry) oxidations were performed in a three-zone $1200^{\circ} \mathrm{C}$ Lindberg Model 55122 Moldatherm Hinged Tube Furnace to achieve a target thermally-grown oxide thickness of approximately $1.0 \mu \mathrm{m}$. A typical wet oxidation process was run at $1100^{\circ} \mathrm{C}$ for 130 min., passing high purity oxygen at a flow rate of $\sim 1000 \mathrm{~mL} / \mathrm{min}$ through a distilled-water bubbler operating at $90-95^{\circ} \mathrm{C}$, resulting in oxide thicknesses of $0.870-0.907 \mu \mathrm{m}$. Oxide thickness variations seemed to largely depend upon the geometric placement of wafers within the wafer boat, spacing between the wafers, etc. Most wafers had a thicker $\mathrm{SiO}_{2}$ layer in their center regions, than along their outer edge peripheries. Resulting oxide thicknesses were measured with the Filmetrics F20 Thin-Film Measurement System (shown with all other apparatus used, in Appendix IV), which determines single, double, or triple-layer, thin-film thicknesses (for $10 \mathrm{~nm}$ to $20 \mu \mathrm{m}$-thick films) and wavelength-dependent optical constants by analyzing the sample's reflectivity at 512 wavelengths (400 $-850 \mathrm{~nm}$ ), and comparing the results to theoretical mathematical models. This PC-based spectrometer system had a typical accuracy of $1 \%$, with best accuracy for thermally grown oxides (compared to other thin films) [75].

Next, the thermally-grown oxide layer was patterned to prepare for anisotropic $\mathrm{KOH}$ etching of the (100) double-side-polished (100) silicon substrates. A typical process started by 
dehydrate-baking a wafer in atmosphere, at $200^{\circ} \mathrm{C}$ for $\sim 30$ minutes in a Lindberg/Blue $\mathrm{M}$ laboratory vacuum oven. The backside of each wafer was first coated with photoresist to protect the oxide layer from etching, by spinning on primer and then photoresist. After allowing the wafer to cool to ambient temperature, HMDS primer was spun onto the wafer's backside using a Solitec Model 5100 Manual, Single-Head Wafer/Substrate Spinner. Primer spin parameters typically included a ramp value of 200 , a 500 RPM spread for five seconds, and a 4000 RPM spin for 15 20 seconds. Shipley 1813 positive photoresist was then spun on at the same parameters. The wafer was then soft-baked in atmosphere on a hot plate at $50{ }^{\circ} \mathrm{C}$ for 5 to 15 minutes. Next photoresist was applied to, and patterned on, the front side of the wafer. HMDS primer was spun onto the front side with the same aforementioned spin parameters. Then, Shipley 1813 or 1827 photoresist was spun-on with a ramp value of 100, a 500 RPM spread for five seconds, and a 4000 RPM spin for $15-20$ seconds. The wafer was then soft-baked in a Lindberg/Blue M laboratory vacuum oven at $90-95^{\circ} \mathrm{C}$ for 20 - 30 minutes with forced air convection. The Kasper 2001 contact mask wafer aligner system was then used to pattern photoresist on the wafer's front side with the Etch Window Level 3 lightfield photomask (in the 1998/1999 mask set). In the Kasper system, the (110) primary wafer flat edge was aligned parallel to any proximal edge of a rectangle within each "Mask Alignment Area" or to any straight line comprised of a row of pads within the photomask (as shown in Figure 37). Once the silicon wafer was in intimate contact with the photomask, the front-side photoresist was then exposed for 15 seconds to $436 \mathrm{~nm}$ wavelength UV light at $6.4 \mathrm{~mW} / \mathrm{cm}^{2}$ intensity through the Etch Window Level 3 mask. The frontside photoresist was then developed for approximately 50 seconds in Microposit MF-319 developer, rinsed in high-purity DI water for two to five minutes, and blow-dried with high-purity nitrogen. After verifying complete development of the photoresist under a microscope, the wafer was hard-baked in a Lindberg/Blue M laboratory vacuum oven at $95-100{ }^{\circ} \mathrm{C}$ for at least 30 minutes in atmosphere. The wafer was then immersed in room temperature, fresh buffered oxide etch (BOE) solution, in which the typical etch rate is $1160-2000 \AA / \min$ (at $22{ }^{\circ} \mathrm{C}$ ). Measured etch rates were $560-900 \AA / \mathrm{min}$, depending upon the age and agitation of the solution. The wafer was removed from the solution once a pattern became clearly apparent in the oxide, or 
inspection of the substrate under a microscope showed that the oxide had been etched through, to the silicon substrate. The wafer was thoroughly rinsed in DI water, and photoresist was removed from both sides with acetone.

The patterned-oxide wafer was then anisotropically etched in aqueous $45 \%$ wt. potassium hydroxide solution. Experimentally-measured, aggregate, median etch rates for silicon and thermally-grown $\mathrm{SiO}_{2}$ (based on data from processing 20 three and four-inch diameter wafers) are graphically shown in Appendix VI. Since $\mathrm{KOH}$ etches $\mathrm{SiO}_{2}$, an appropriate $\mathrm{KOH}$ solution temperature was chosen (at room temperature, $22^{\circ} \mathrm{C}, 43,50$, or $52^{\circ} \mathrm{C}$ ) to etch to an approximate target depth of $\sim 170$ - $195 \mu \mathrm{m}$ into the silicon substrate without etching away the entire $\mathrm{SiO}_{2}$ thin-film layer. The typical $\mathrm{KOH}$ etching system setup is shown in Figure 61. Fresh $\mathrm{KOH}$ solution was used, cool tap water was flowed through the reflux condenser, and the hotplate was programmed with the desired solution temperature and stir rate before any silicon wafers were immersed. Each wafer was briefly immersed in 40:1 HF dip to remove any residual native

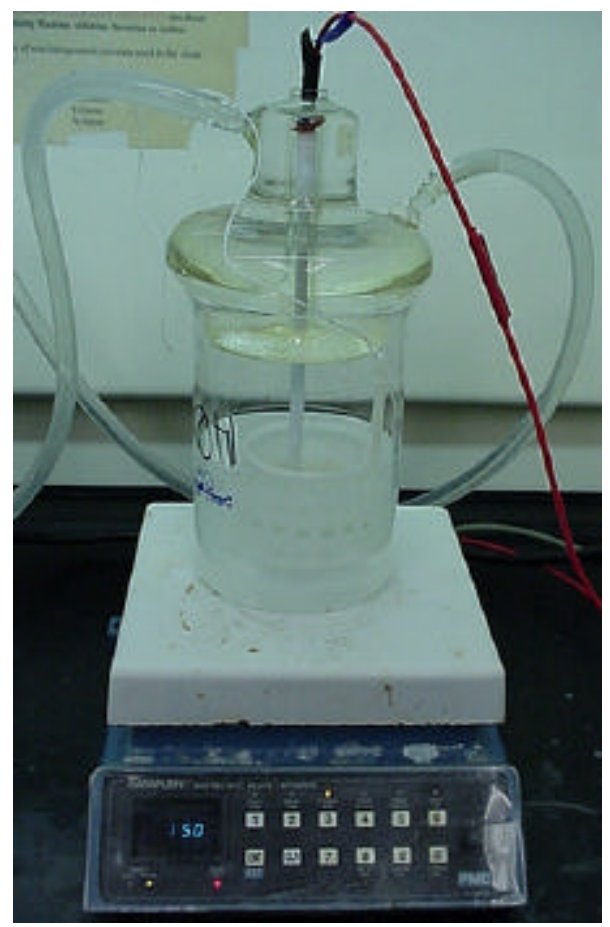

FIGURE 61 - System setup for $\mathrm{KOH}$ anisotropic bulk micromachining of silicon substrates. It included a closed-loop-control Dataplate ${ }^{\mathrm{TM}}$ Series 720 programmable digital hotplate with Teflon ${ }^{\mathrm{TM}}$ thermometer probe feedback and rpm-controlled magnetic stir bar, a quartz beaker, a water-cooled reflux condenser, and 45 wt.\% aqueous $\mathrm{KOH}$ solution. 
oxide from patterned, exposed silicon surfaces before immediate, subsequent immersion in $\mathrm{KOH}$ solution. Silicon substrate etch rates in $\mathrm{KOH}$ were affected by the stir rate, solution $\mathrm{pH}$, concentration, temperature variations (which were typically $\pm 2^{\circ} \mathrm{C}$ ), solution age, and the presence of impurities or contaminants in the solution. Problems experienced during this stage of wafer processing included one wafer with some thermal oxide areas completely etched away in $\mathrm{KOH}$, and etched pit regions that were misaligned by $5-10^{\circ}$ from the $<100>$ direction on some substrates.

Silicon substrate etch depths were measured by examining, under a microscope, substrate regions where square, etch-depth-measurement structures (i.e. square openings) were patterned. Since there was not yet any metallization patterned on the wafers, geometries in these regions had to be compared to the L-Edit ${ }^{T M}$ design file to see which structures corresponded to which maximum pyramidal etch depths (the photomask set was designed assuming anisotropic etching of the substrate would occur last in the fabrication process sequence: i.e. a more traditional process). Structures which had etched to a maximum depth and where etching had essentially stopped at the $\{111\}$ silicon crystallographic planes had a pyramidal-shaped etch pit, ending in a point. A (100) horizontal plane (parallel to the surface of the wafer) could be seen at the bottom of square structure openings which had not yet etched completely down to their maximum designed depth. The largest square opening which had a fully-etched pyramidalshaped depth (down to a point) was taken as the approximate (minimum) etch depth achieved.

After anisotropic etching of the silicon substrate, the residual $\mathrm{SiO}_{2}$ layer on the wafer was removed by immersion in BOE solution. The wafer was then thoroughly rinsed in DI water. A photo of a sample, resulting pre-etched (100) wafer substrate is shown in Figure 62. A portion of these pre-etched wafers was allocated for the anodically-bonded glass process and another portion allocated for experimental sacrificial layer processes (described later).

In preparation for anodic bonding, pre-etched three-inch diameter silicon wafers and borosilicate glass plates/wafers were first base and acid cleaned. Most of the glass wafers were wiped with an acetone-soaked swab before cleaning to remove fingerprints and other particulates. Base cleaning was accomplished by mixing $\mathrm{DI}$ water and $\mathrm{NH}_{4} \mathrm{OH}$ in a 5:1 ratio in a beaker; 


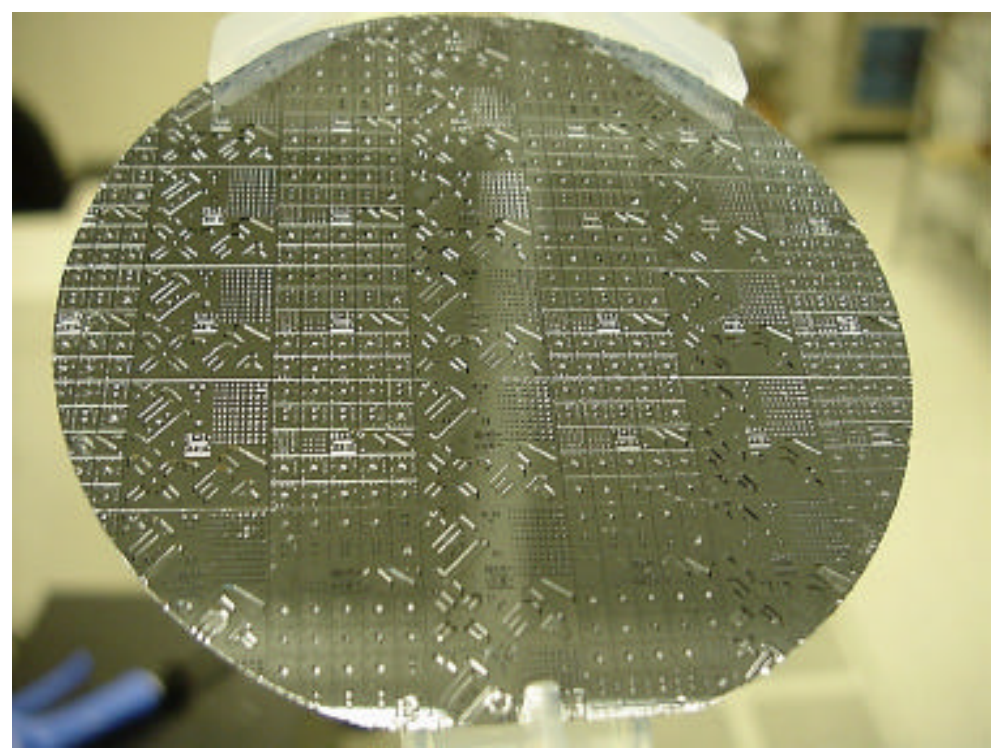

FIGURE 62 - $\quad$ A photo of a pre-etched double-side-polished (100) silicon substrate. This threeinch diameter wafer shows the Etch Window Level 3 photomask pattern.

heating the mixture to $70^{\circ} \mathrm{C}\left( \pm 5^{\circ} \mathrm{C}\right)$ on a hotplate; removing the beaker; adding the same volumetric quantity of fresh $\mathrm{H}_{2} \mathrm{O}_{2}$ as $\mathrm{NH}_{4} \mathrm{OH}$ in the mix; waiting for the solution to bubble vigorously; and then soaking the silicon wafer(s) for $\sim 15$ minutes in the mix, with periodic agitation. The wafers were then rinsed under running DI water for 3-5 minutes. Acid cleaning was then performed by mixing $\mathrm{DI}$ water and $\mathrm{HCl}$ in a $4: 1$ ratio in a beaker; heating the mixture to $70{ }^{\circ} \mathrm{C}( \pm 5$ ${ }^{\circ} \mathrm{C}$ ) on a hotplate; removing the beaker; adding the same volumetric quantity of fresh $\mathrm{H}_{2} \mathrm{O}_{2}$ as $\mathrm{HCl}$ in the mix; waiting for the solution to bubble vigorously; and then soaking the silicon wafer(s) for $\sim 15$ minutes in the mix, with periodic agitation. The wafers were then rinsed under running DI water for 3-5 minutes and subsequently blow-dried with high-purity nitrogen. Prior to anodic bonding, the thicknesses of the silicon wafer substrates and borosilicate glass wafers were carefully measured with a Mitutoyo digital micrometer and recorded to facilitate precision etching and machining of the bonded glass plates into their final desired thicknesses after anodic bonding.

A thin aluminum metallization layer was then deposited on one side of most of the cleaned borosilicate glass wafers to provide an electrode that was anticipated to expedite the anodic bonding process. An aluminum-silicon-copper alloy (98.5\% Al, $1 \% \mathrm{Si}$, and $0.5 \% \mathrm{Cu})$ was RF planar magnetron sputter-deposited in argon onto the glass wafers using the Technics Model 
4604 Manual Control Sputtering System. The initial pump-down base pressure achieved was $\sim 1.6 \times 10^{-5}$ mTorr. The RF power was set at 300 Watts, and the argon sputtering pressure maintained at $\sim 10$ mTorr, for 2 - 4 minute sputtering depositions. The maximum electrode metallization layer target depth was about $0.11 \mu \mathrm{m}$. One wafer had a target depth of $\sim 550 \AA$, in which the aluminum-alloy layer was translucent.

The next step was to anodically bond each cleaned $1.1 \mathrm{~mm}$-thick borosilicate glass wafer to a pre-etched and cleaned double-side-polished (100) silicon substrate. Where applicable, silicon wafers that had just been cleaned were first dehydrate-baked at $200{ }^{\circ} \mathrm{C}$ for at least 20 minutes to avoid trapping residual moisture inside etched cavities during bonding. No more than four pre-etched silicon wafers were placed with the etched cavities face-up on the Thermolyne Type 1900 hot plate. As shown in Figure 63, clean borosilicate glass wafers were then placed on top of the silicon wafers, with those having a sputter-deposited thin-film aluminum alloy electrode layer, facing up. The probe tip was then carefully positioned to contact the center of one of the wafers. The hotplate base (anode) was then connected to the positive DC output of the Glassman High Voltage Model ER3R100 power supply, and the probe tip connected to the power supply ground. Great care was exercised to insure that these high-voltage electrical connections were sufficiently isolated from each other and from any contact with the conductive stainless steal table on which the setup was placed. The hot plate remote control was then turned on (to a setting just

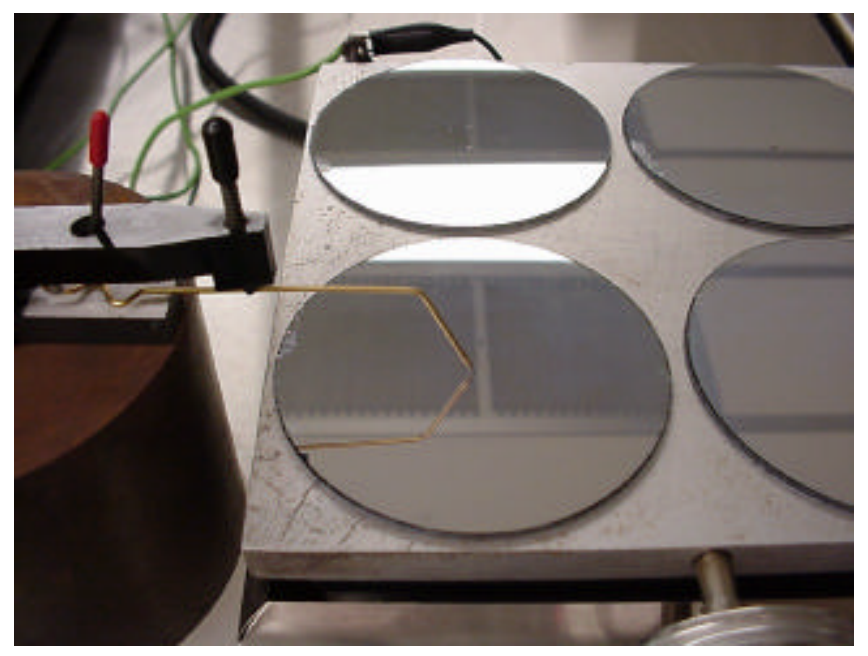

FIGURE 63 - Photo showing placement of the glass/Si wafer sandwiches on the hot plate. A sputter-deposited aluminum alloy electrode layer is shown, face-up. 
within the "red" marked region) to slowly heat up the glass/Si wafer sandwiches to a target temperature of $\sim 450^{\circ} \mathrm{C}$ (indicated by a dial thermometer inserted into the hot plate base, as shown in Figure 58). Once the target temperature was reached, the power supply was switched on, the output voltage adjusted to $\sim 1 \mathrm{kV}$, and the output turned on. The output current was carefully monitored, and if needed, the output voltage was adjusted to achieve maximum current flow. If the output voltage was adjusted too high, arcing occurred at the probe tip or across the sandwich (as shown in Figure 64); the power supply had to be switched off; the probe tip had to be re-positioned on the aluminum-alloy electrode surface; and the power supply was switched back on to continue bonding. Once the output current decreased to a nominal (near-zero), steady-state value, the anodic bonding process was assumed to be completed (but nonetheless more time than this was allowed as a safety margin). For aluminum-alloy-coated glass wafers, anodic bonding was completed in well under five minutes, but for glass wafers that did not have this electrode layer, bonding took 10-11 minutes to complete (and the probe tip had to be periodically moved around on the glass surface to eliminate residual "pockets" or gaps under the glass). Once all of the wafer sandwiches on the hot plate were bonded, the power supply and hot plate were turned off, and the wafers were allowed to slowly air cool. If any of the wafer sandwiches were removed from the hot plate and allowed to rapidly air cool, the glass would crack (which happened in one case). Once at room temperature (several hours later), the glass/Si sandwiches were immersed in aluminum etch solution to remove the aluminum-alloy electrodes, and then rinsed in DI water. Once the aluminum layer was removed from the top of

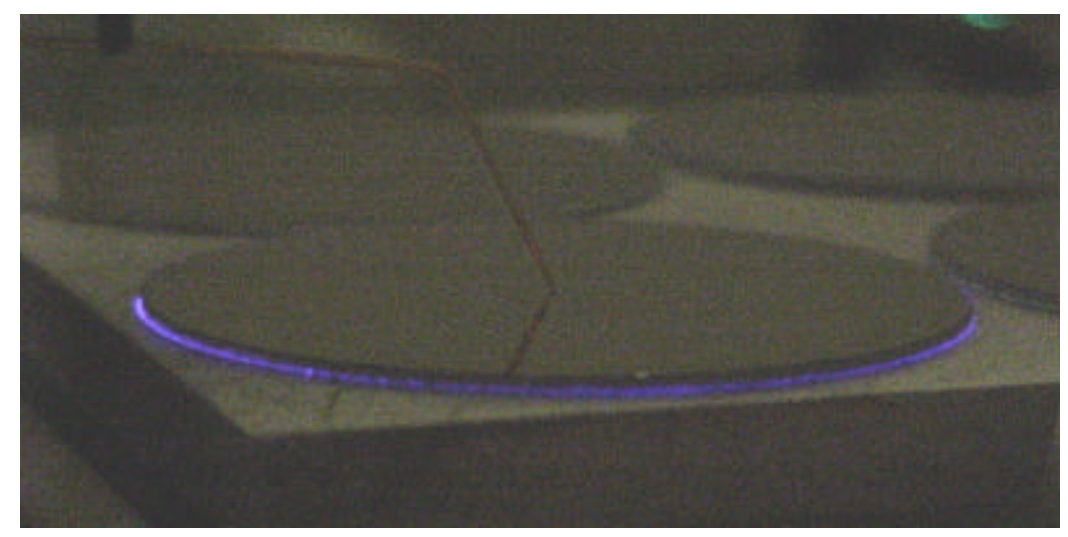

FIGURE 64 - Photo of violet-colored corona at Si/glass interface during anodic bonding. 
the glass, it was quite apparent that the glass-to-silicon interface bond was of much better observable quality than that typically achieved without the presence of an electrode layer, because there were few, if any, air pockets, or residual non-bonded/discolored, bubbled areas under the glass. A photo of one of the bonded glass/Si wafer sandwiches is shown in Figure 65.

The next step was to initially wet-etch most of the borosilicate glass layer on each wafer sandwich down to a few hundred micrometers prior to machining and planarizing the glass down to its final thickness. First the glass/Si wafer sandwich (outer-edge) thicknesses were measured with a digital micrometer. Measured variations in total thickness across each wafer sandwich ranged from $5 \mu \mathrm{m}$ to $21 \mu \mathrm{m}$.

A concentrated HF wet-etch bath at room temperature was set up in a plastic beaker on top of a hot plate with manual heating and stirring controls. A magnetic stir bar was placed in the beaker, and a stir rate of $160-200$ RPM in the HF solution was established. Note that extreme caution must be exercised in handling concentrated HF solutions, as HF is extremely toxic and potentially fatal if mishandled. One wafer was etched down to a glass thickness of $\sim 71-114 \mu \mathrm{m}$ in 210 minutes (at room temperature), and other wafers were etched down to glass thicknesses of $\sim 556-617 \mu \mathrm{m}$ in 83 minutes (at $5.0-7.5 \mu \mathrm{m} / \mathrm{min}$ etch rates, which is slower than that for non-boron-doped glass). After HF wet etching, the typical variation in micrometer-measured glass/Si sandwich total thickness of each wafer ranged from $34 \mu \mathrm{m}$ to $57 \mu \mathrm{m}$, which indicated a

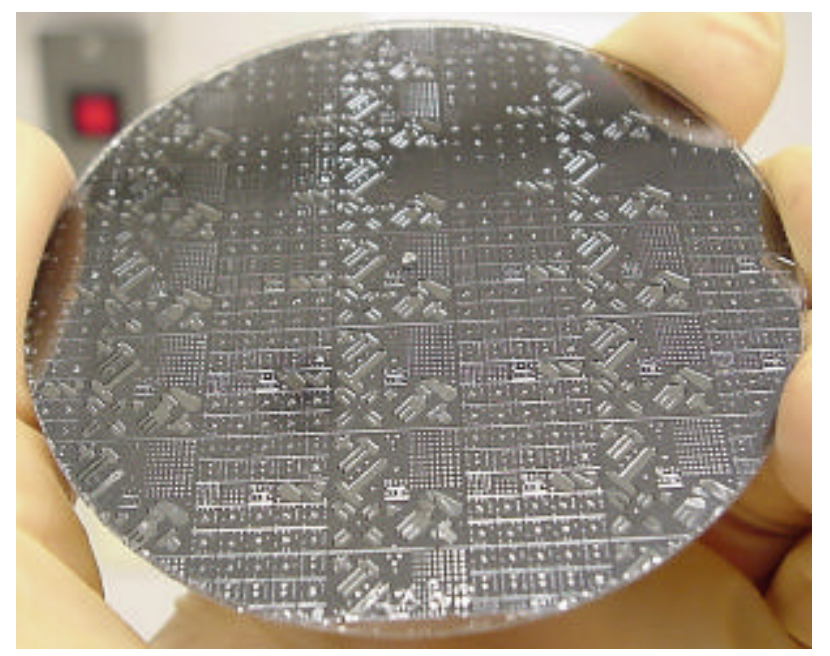

FIGURE 65 - A photo of an anodically-bonded borosilicate glass/Si wafer sandwich. 
significantly increased glass surface topography roughness.

Next, the remaining $71-617 \mu m$-thick glass layer on five of the three-inch-diameter glass/Si wafers was planarized and machined to its final target thickness of $30-50 \mu \mathrm{m}$ for the flow sensor microbridge/cantilever structures. To accomplish this, training for, and access to, a Logitech PM5 digitally-programmable lapping and polishing machine was graciously provided (gratis) by the University of Cincinnati to machine and polish these glass/Si wafer sandwiches. The PM5 setup for lapping, or the initial planarization and bulk removal of glass, is shown in Figure 66. The abrasive grit utilized was $9 \mu \mathrm{m}$ calcined aluminum oxide, or alumina, powder (sold by Logitech Ltd.; Glasgow, Scotland, UK). Approximately ten parts DI water were mixed, by volume, with one part $9 \mu \mathrm{m}$ alumina grit powder into the slurry tank to create the abrasive slurry mix. The slurry was homogenized by vigorous shaking of the tank, and its dispensing valve was adjusted to permit an approximate one-drip-per-second flow rate onto the rotating, serrated castiron lapping wheel/plate which was first "conditioned" prior to actual lapping. During "conditioning," a cast-iron conditioning ring was positioned on top of the wheel, the wheel's rotational speed was slowly increased while dripping slurry onto it, rust and particulates were

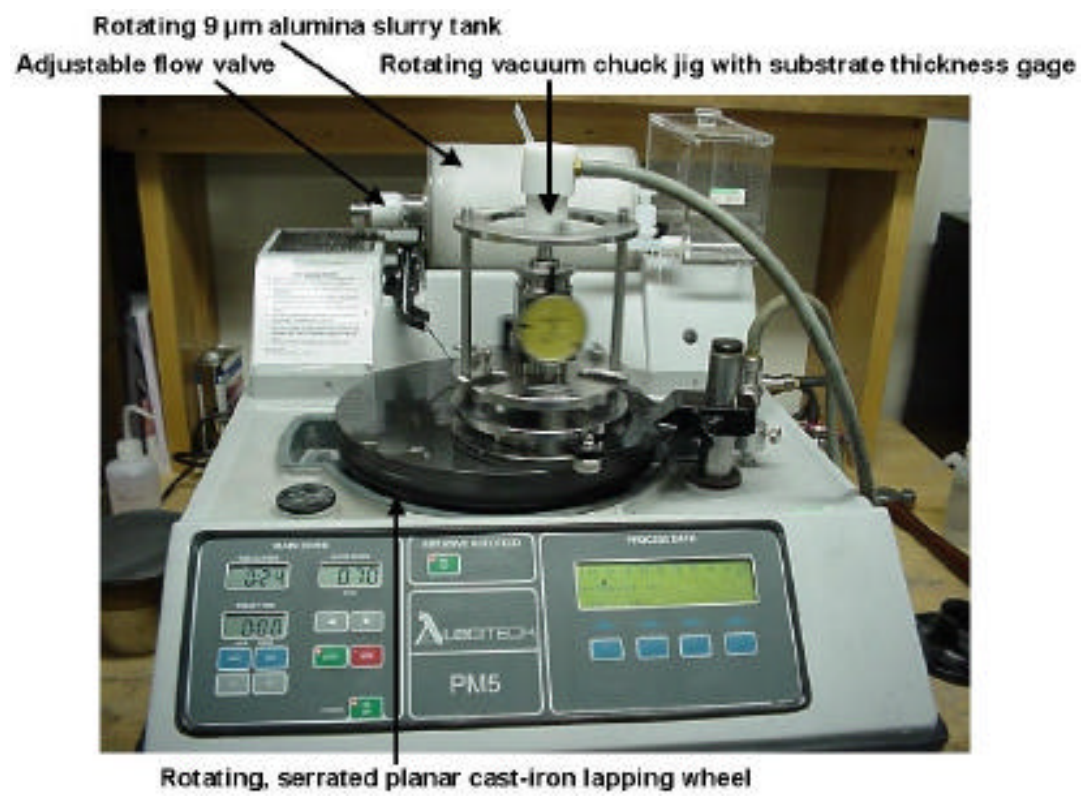

FIGURE 66 - A photo of Logitech PM5 machine during 70 RPM borosilicate glass lapping. Periodically, the machine was stopped and the substrate thickness dial gauge checked to see how much glass had been removed. 
removed, and it was machined back into a near-planar condition (if it was not originally in that state). Each glass/Si wafer sandwich was individually lapped by first vacuum-affixing it to the cleaned four-inch vacuum chuck at the bottom of the rotating substrate-holder pressure jig (shown blurred in Figure 66 because it was rotating). The wafer sandwich was either directly vacuum-affixed to the chuck, or affixed by wax to a planar glass substrate, which was then held in place by vacuum. For three-inch-diameter glass/Si wafer sandwiches held directly in place by vacuum against the chuck, since the vacuum chuck had a larger diameter, a blue plastic adhesive film (the same one used for holding die together during wafer dicing procedures) was cut out in a ring shape (with inside circumference matching the shape of the wafer sandwich) to cover up exposed vacuum chuck rings outside the area of the wafer sandwich. After conditioning the cast-iron lapping wheel, the pressure jig, with the wafer sandwich to be lapped vacuumattached to its bottom, was then set in place on top of the wheel. The substrate thickness dial gauge (with minimum resolution of $2 \mu \mathrm{m}$ ) on the pressure jig was then set to zero to measure planar bulk removal of glass from each sandwich. The spring-loaded applied weight (or pressure) in the pressure jig was adjusted to its maximum value ( $3 \mathrm{~kg}$ mass applied). To begin lapping, dispensing of the $9 \mu \mathrm{m}$ abrasive slurry was turned on ( $1-3$ drips/sec.), and the lapping wheel was then run at various RPMs, typically 70 . Measured average bulk borosilicate glass removal rates were $2.7-5.5 \mu \mathrm{m} / \mathrm{min}$ to achieve final target glass thicknesses of $40-50 \mu \mathrm{m}$. In general, bulk glass removal rates could be increased by using higher grit sizes, applying more weight on top of the rotating substrate-holder jig (if possible), by increasing the angular speed of the lapping wheel, or by increasing the slurry drip rate. Glass/Si wafer sandwiches that originally had a sputter-deposited, aluminum-alloy electrode layer on the glass to facilitate anodic bonding generally had lower bulk removal rates, possibly because some material properties of the glass changed due to excessive bonding times (because the spreading of the interference fringe pattern under the glass during bonding could not be observed through the aluminum-alloy electrode to determine when bonding was completed). Even at five minutes, the actual anodic bonding time may have been closer to one minute. There was even a harsher, louder grinding noise associated with lapping the glass in these wafer sandwiches, compared to others which did 
not have the aluminum-alloy electrode during anodic bonding. The top glass surface on each glass/Si wafer sandwich was frosted by the lapping process. After lapping, the PM5 was shut off, the vacuum was shut off, and each wafer was carefully slid (or very gently pried) from the pressure jig vacuum chuck to remove it. Several wafers were fractured while trying to hastily remove them.

After lapping was finished, the PM5 machine was configured for polishing the top glass surface of each glass/Si wafer sandwich to reduce the surface roughness and overall topographical variation. The polishing setup utilized is shown in Figure 67. The lapping wheel was removed and replaced with a wheel on which a black, adhesive-backed polishing pad had been affixed. The polishing wheel, pressure jig, and vacuum chuck area were all thoroughly rinsed with DI water to remove alumina grit that could potentially cross-contaminate the polishing pad. Premixed "Polishing Suspension Type SF1" colloidal silica (sold by Logitech Ltd.; Glasgow, Scotland, UK) was added to the polishing solution tank, and the tank outlet valve adjusted to achieve a flow rate of approximately one drip per second onto the pad. After a glass/Si wafer sandwich (and any necessary blue plastic film) was attached to the vacuum chuck, the pressure jig (with pressure spring adjusted for maximum force at $3 \mathrm{~kg}$ ) was placed on top of the polishing pad, and its rotation was started. For some wafers, "sweeping arm mode" was utilized in which

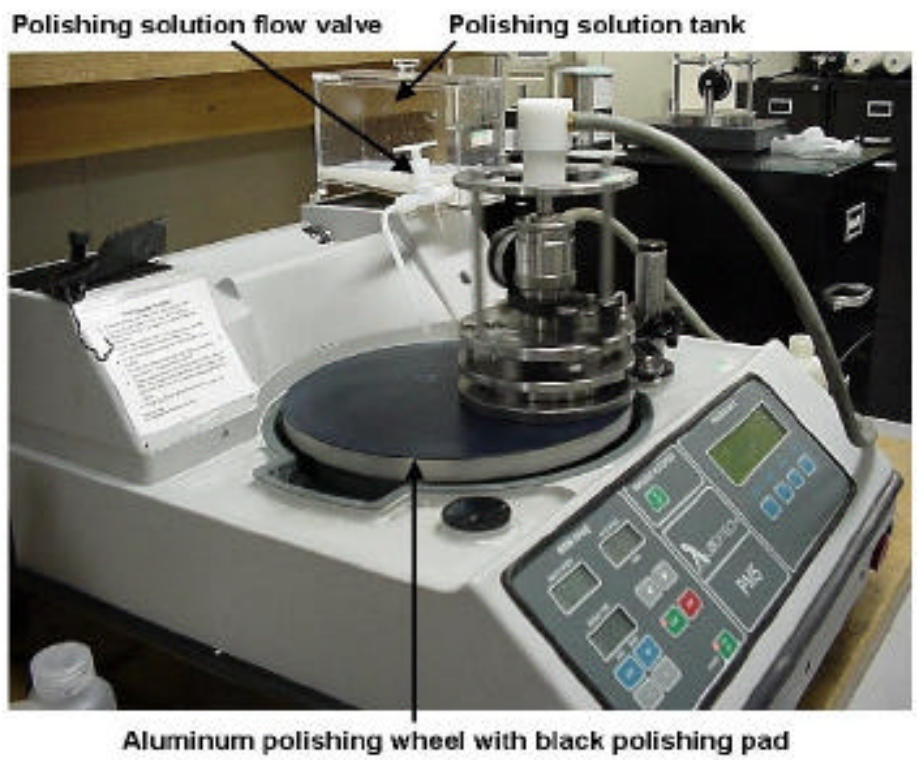

FIGURE 67 - The glass polishing setup for the Logitech PM5 machine. 
the PM5 moved the pressure jig back and forth across the polishing pad (to achieve better substrate contact with the silica). Total polishing times were $25-60$ minutes at 70 RPM with a colloidal silica flow rate of 1-2 drips per second. Polishing was stopped for each wafer sandwich when the top glass surface appeared to be uniformly translucent. However, in most cases a few patches of frosted glass remained, due to the wafers having particulates or wax residue trapped under them and not being completely and uniformly pressed flat against the vacuum chuck during polishing (frosted areas were "valleys" in the glass). Estimated final glass thicknesses for glass/Si wafer sandwiches polished only with the PM5 machine were $7-38 \mu \mathrm{m}$ (or 14.5-29 $\mu \mathrm{m}$ median thickness range using median values of measured glass thickness ranges from individual wafers). One of the polished glass/Si wafer sandwiches is shown in Figure 68.

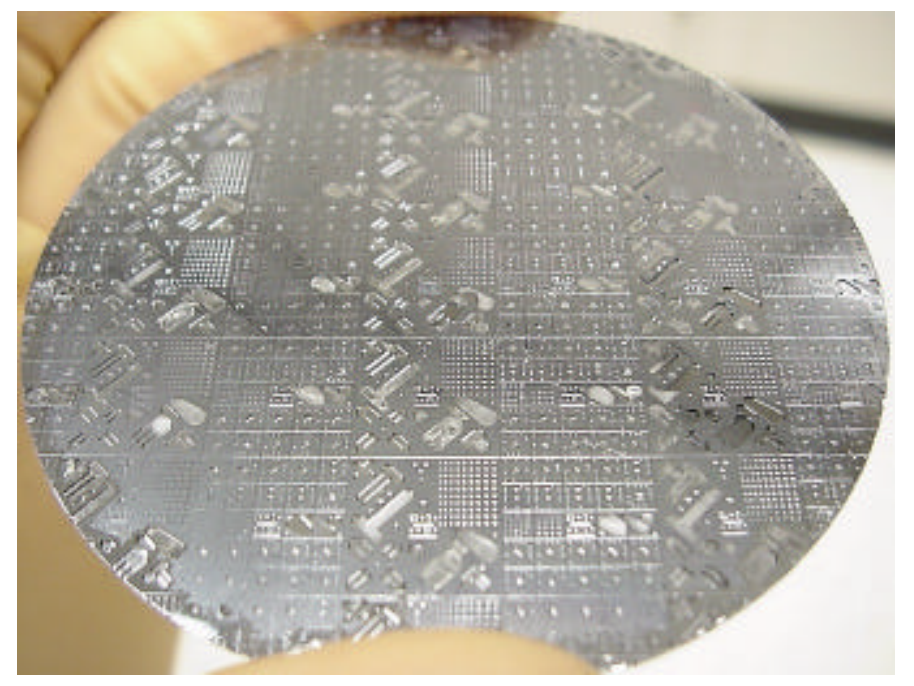

FIGURE 68 - A photo of a polished borosilicate glass/Si wafer sandwich. The measured, estimated glass thickness range for this wafer was $7-32 \mu \mathrm{m}$.

Two three-inch-diameter glass/Si wafer sandwiches that had been polished with the Logitech machine, but which still had residual frosted regions (as shown in Figure 69), were further polished with a Lapmaster Model 15 lapping and polishing machine obtained by Dr. Kevin Walsh for the University of Louisville. These frosted regions were depressed areas in the glass that could only be polished smooth by properly pressing and affixing the wafers to the chuck that sits on top of (and spins with) the polishing wheel. Unlike the PM5, the Lapmaster machine had a wax-mount chuck, which would theoretically provide for better control over how uniformly each 


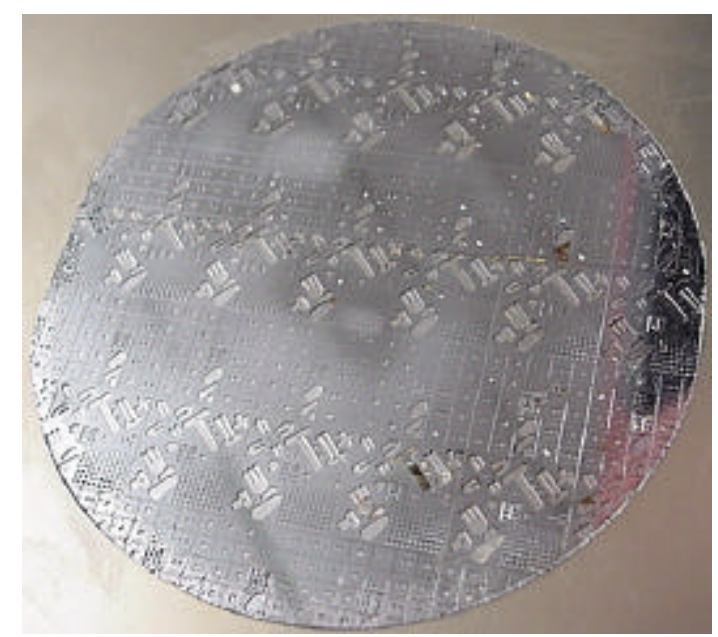

FIGURE 69 - Photo of a polished glass/Si sandwich with residual rough, frosted regions. This wafer was lapped and polished with the Logitech PM5 machine.

glass/Si wafer sandwich could be affixed and pressed onto the planar chuck. Each wafer was affixed to the chuck by heated "sticky" wax (obtained from The Kindt-Collins Co.; 12651 Elmwood Ave.; Cleveland, $\mathrm{OH} 44111 ; 216-252-4122)$. A uniform weight of 32 pounds was applied on top to press the soft wax into place. A new polishing pad, affixed to the machine's aluminum polishing wheel, was used with colloidal silica (although a mix of eight ounces of cerium oxide with each gallon of water was recommended by Lapmaster, Inc. to polish glass). The chuck (with a mounted glass/Si wafer sandwich) was placed on the polishing pad, with a total applied weight of 16 pounds. A micarta conditioning ring was utilized to more evenly spread out the silica on the pad during the polishing process, which was set at 50-60 RPM, with a silica flow rate of approximately 1-2 drips per second. The total polishing time for each wafer sandwich was 20 minutes, resulting in a final $18-33 \mu \mathrm{m}$ measured range of glass thicknesses between the two wafers. Thus, the total estimated glass thickness range for all five polished glass/Si wafer sandwiches was $7-38 \mu \mathrm{m}$ (or $14.5-31.5 \mu \mathrm{m}$ thickness range using median values of measured glass thickness ranges from individual wafers). Now that the glass layer was machined and polished to its final thickness, flow sensor microbridge/cantilever and pad area cavities in the silicon substrates were actually enclosed spaces covered with thin borosilicate glass diaphragms, as illustrated in Figure 70. Glass areas on some glass/Si sandwich wafers were ruptured by lapping and polishing, especially diaphragms previously suspended over 


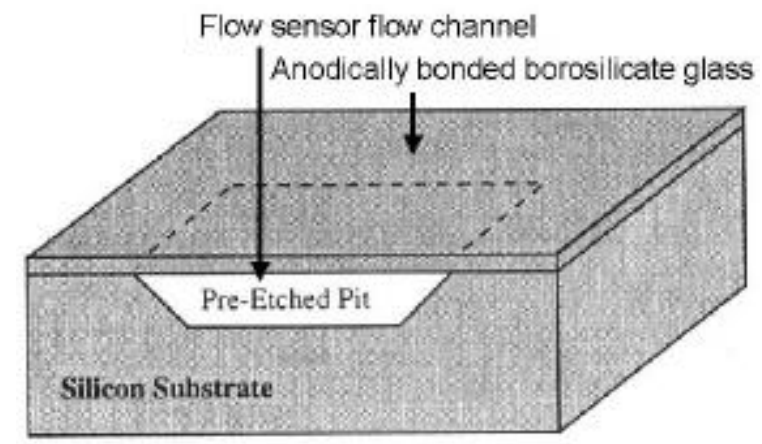

\section{SEALED CAVITY}

FIGURE 70 - Diagram illustrating sealed flow sensor diaphragm cavities. These cavities were in pre-etched substrates after anodic bonding and machining of the borosilicate glass layer. Adapted from ref. [76].

cantilever and bridge array regions, and some large " $\mathrm{T}$ " and " $\mathrm{H}$ " structure regions in the thin-film residual stress/strain test structure die areas.

The next step was to sputter-deposit the resistor metallization and (optional) top gold metallization layers on the glass surface of each wafer sandwich, and then pattern both layers. Since these wafers were pre-etched with $\mathrm{KOH}$, depositing and delineating a top gold layer metallization (which would be inert in $\mathrm{KOH}$ ), was not required, but deposited anyway for improved wire-bonding ohmic contact in flow sensor pad areas. Two of the five polished wafer sandwiches were base and acid cleaned to improve resistor metallization layer adhesion to the borosilicate glass substrates (which were contaminated after lapping and polishing). For delineating the metallization layers, in order to align the resistor and gold metallization photomasks to pre-etched features in the silicon substrates (looking though the borosilicate glass layer), a small piece of blue plastic adhesive film (the same versatile film used for wafer dicing procedures) was placed over two adjacent mask alignment areas on the top glass surface of each wafer sandwich, as shown in Figure 71. This was necessary because the photomask set was designed assuming a traditional flow sensor fabrication processing methodology would be utilized in which silicon substrate anisotropic etching would occur as the last step. Furthermore, the thin glass layer did not have a surface topography mirroring the underlying substrate topography. Due to mysterious top-layer gold adhesion problems experienced (discussed later), chromium and titanium (with a resistivity nearly four times larger than chromium) were deposited as resistor metallization 


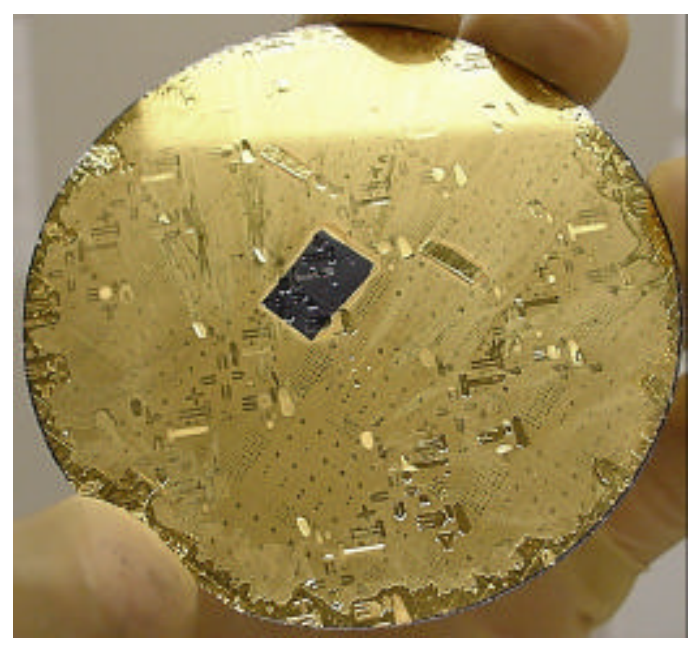

FIGURE 71 - Photo of a metallized Si/glass wafer mask-alignment strategy. An area was blocked out for resistor and gold metallization sputter-deposition by a small piece of blue plastic adhesive film. This non-metallized area was translucent, facilitating resistor and gold metallization photomask alignment to the substrate. This was also the same wafer shown in Figure 68.

layers, as they both had desired resistivity and TCR values while being excellent adhesion layers for gold. First, either chromium or titanium was RF planar magnetron sputter-deposited (at 250 Watts and 10 mTorr), and then gold was DC planar magnetron sputter-deposited (at 350 Watts and 10-15 mTorr) in each run (both in argon), without breaking vacuum in the Technics 4604 sputterer (with the chiller set to $\sim 15^{\circ} \mathrm{C}$ ). Various target depths were chosen for different flow sensor resistor total resistance values. Chromium metallization sputter-deposition target depths were $0.23,1.0$, and $1.5 \mu \mathrm{m}$, and titanium target depths were $0.1,0.16$, and $1.5 \mu \mathrm{m}$. Top-layer gold metallization sputter-deposition target depths were $0.1,0.21$, and $1.0 \mu \mathrm{m}$.

After metallization sputter deposition, two wafers had cracked, damaged, or imploded/ exploded diaphragm areas. On one wafer with $\sim 18-37 \mu \mathrm{m}$ thick diaphragms, with $1.5 \mu \mathrm{m}$ (target) thick titanium and $1.0 \mu \mathrm{m}$ (target) thick gold (which had a measured thickness of $1.8-$ $2.35 \mu \mathrm{m}$ with the Tencor profilometer), most glass diaphragm areas appeared cracked and possibly imploded. The underlying glass was fractured and damaged, most likely due to the long deposition times required to deposit the layers of titanium (150 minutes) and gold (38 minutes), and the consequential large substrate heating that likely occurred, thereby heating and expanding the air trapped in the glass diaphragm cavities, possibly stressing some diaphragms beyond their 
elastic limits. Although the diaphragms appeared imploded after sputtering, they would have actually exploded to release trapped air, with the only visible evidence sometimes being cracks around the periphery of some diaphragms. They may have appeared imploded because a partial layer of deposited metal (during sputtering) on top of the diaphragms may have kept then intact as they stretched and fractured to release trapped air in the cavities, and then imploded after the sputtering chamber was returned to atmospheric pressure as the poorly metallization-sealed cavities contained lower pressure regions. After sputtering, another wafer with $10-38 \mu \mathrm{m}$ thick diaphragms, with $1.0 \mu \mathrm{m}$ (target) thick chromium and $0.1 \mu \mathrm{m}$ (target) thick gold showed exploded diaphragm cavities (a collage of which is shown in Figure 72), possibly caused by excessive substrate heating due to the long chromium deposition (45 minutes). Thus, it is believed that long-duration metallization sputtering runs should be avoided with (near atmospheric-pressure) sealed-cavity diaphragm devices, as the cavities may rupture due to excessive plasma heating of the glass/Si wafer sandwich substrate, expansion of air inside the cavities, and consequential stressing of the thin glass diaphragms beyond their linear-elastic regions (exceeding their ultimate tensile strength).

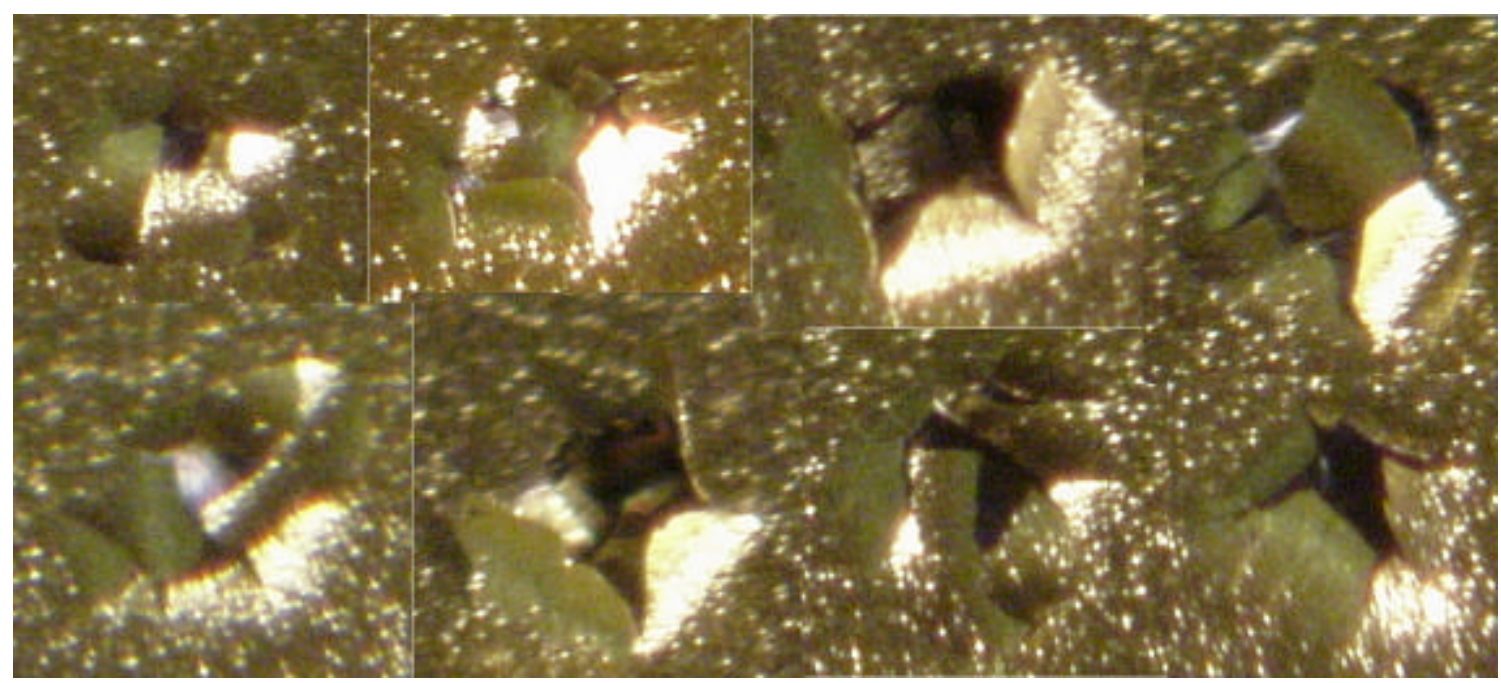

FIGURE 72 - A photo collage of exploded $10-38 \mu \mathrm{m}$-thick glass diaphragms. These were on one glass/Si sandwich wafer after sputtering $1.0 \mu \mathrm{m}$ of chromium and $0.1 \mu \mathrm{m}$ of gold. It was theorized that exceptionally long sputtering runs can excessively heat the substrate, expanding air in the cavities, and rupturing the diaphragms if the pressure exceeds the diaphragm's ultimate yield strength. Gold speckles in this photo are an indication of the surface roughness of the glass layer. 
After chromium/titanium and gold metallization sputter-deposition on the glass/Si sandwich wafers, the top gold metallization layer was first delineated. Where applicable, the wafers were first dehydration baked at $200{ }^{\circ} \mathrm{C}$ for at least 30 minutes, and allowed to cool. Wafers which were processed immediately after metallization sputtering were not baked, as the vacuum in the sputtering chamber should remove residual moisture from the wafers. Microposit HMDS primer was spun onto each wafer (with a ramp value of 900 , a 500 RPM spread for five seconds, and a 4500 RPM spin for ten seconds). Then, 1813 positive photoresist was spun onto each wafer with the same parameters. Each wafer was soft baked at $90^{\circ} \mathrm{C}$ for $20-30$ minutes with forced air convection, and then allowed to cool to the ambient. In the Kasper contact mask aligner, mask alignment structures in the Gold Metal Level 2 lightfield photomask (from the 1998/1999 mask set) were aligned to corresponding wells/cavities in mask alignment areas on each silicon wafer substrate in regions where the top glass was not metallized (due to the use of the blue adhesive film in the sputterer). Once each glass/Si sandwich wafer was in intimate contact with the photomask, the photoresist was exposed for 15 seconds to $436 \mathrm{~nm}$ wavelength UV light at $6.4 \mathrm{~mW} / \mathrm{cm}^{2}$ intensity through the Gold Metal Level 2 lightfield photomask. The photoresist was then developed for 50 seconds in MF-319 developer, rinsed in DI water for about two minutes, and blow-dried with high-purity nitrogen. After verifying proper development of the photoresist under a microscope, each wafer was hard-baked at $100{ }^{\circ} \mathrm{C}$ for 30 minutes. Each wafer was then immersed in fresh $\mathrm{KI}$ solution until the gold metallization layer was patterned, which typically occurred in under one minute. Since the solution was very opaque, and since gold would also rapidly etch under the photoresist (undercutting), it was important to periodically rinse each wafer in DI water and check the status of gold etching. Estimated $\mathrm{KI}$ gold etch rates were approximately $0.5-1.0 \mu \mathrm{m} / \mathrm{min}$ [77]. Significant gold adhesion problems (discussed in depth, later) were discovered on wafers with titanium and chromium adhesion layers. Consequently, direct rinsing of wafers under running DI water streams and blow drying with nitrogen guns were avoided, because in many cases, gold layers (e.g. pads and interconnect routings) would peel off the wafers. After patterning the gold layer on each glass/Si sandwich wafer, the remaining photoresist was stripped off with acetone, and each wafer rinsed in DI water. 
Next, the titanium or chromium resistor metallization layer was delineated on each glass/Si sandwich wafer. Each wafer was first dehydration baked at $200^{\circ} \mathrm{C}$ for at least 30 minutes, and then allowed to cool. Microposit HMDS primer was spun-on with a ramp value of 900, a 500 RPM spread for five seconds, and a 4500 RPM spin for ten seconds. Then, 1813 positive photoresist was spun-on each wafer with the same parameters. The photoresist was soft-baked at $90{ }^{\circ} \mathrm{C}$ for 20 minutes with forced air convection, and each wafer was then allowed to cool to the ambient. In the Kasper aligner, mask alignment structures in the Resistor Metal Level 1 lightfield photomask (from the 1998/1999 mask set) were aligned to gold alignment patterns in mask alignment areas on each silicon wafer substrate. Once each glass/Si sandwich wafer was in intimate contact with the photomask, the photoresist was exposed for 15 seconds to $436 \mathrm{~nm}$ wavelength UV light at $6.4 \mathrm{~mW} / \mathrm{cm}^{2}$ intensity through the Resistor Metal Level 1 photomask. The photoresist was then developed for $~ 50$ - 60 seconds in MF-319 developer, rinsed in DI water for about two minutes, and blow-dried with high-purity nitrogen. After verifying proper development of the photoresist under a microscope, each wafer was hard-baked at 100 ${ }^{\circ} \mathrm{C}$ for at least 30 minutes. Wafers with chromium resistor metallization were then immersed in chrome etch solution, with an etch rate of $\sim 550-750 \AA / \mathrm{min}$., until the thin-film chromium layer was delineated. One of the wafers had significant problems with fully-undercut $5 \mu \mathrm{m}$ and $10 \mu \mathrm{m}$ line-width resistors, because of cracks in the glass that permitted etchant undercutting of the photoresist. Two glass/Si sandwich wafers with titanium metallization were immersed in two different etching solutions: a 20:1:1 $\mathrm{H}_{2} \mathrm{O}: \mathrm{H}_{2} \mathrm{O}_{2}: \mathrm{HF}$ mix, with a resultant titanium etch rate of $\sim 1500 \AA / m i n$, and a 40:1:1 $\mathrm{H}_{2} \mathrm{O}: \mathrm{H}_{2} \mathrm{O}_{2}: \mathrm{HF}$ mix with an etch rate of $\sim 1200 \AA /$ min. The purported etch rate for the 20:1:1 $\mathrm{H}_{2} \mathrm{O}: \mathrm{H}_{2} \mathrm{O}_{2}: \mathrm{HF}$ titanium-etch mix was $8800 \AA /$ min [78]. Titanium etching proceeded very rapidly and was generally quite difficult to control without completely undercutting photoresist on $5 \mu \mathrm{m}$ line-width resistors. Adding more DI water to dilute the etching solution reduced the etch rate and generally made etching more uniformly controllable (i.e. it could be stopped as soon as metallization in the center of each wafer was fully delineated). The sputterdeposited titanium layer on each wafer was typically much thicker in the center than at the outermost edges. Consequently, if titanium etching was stopped after resistor metallization in the 
center of each wafer was delineated, $5 \mu \mathrm{m}$ line-width metallization areas around the periphery of the wafer might be etched away (fully undercutting the photoresist). In general, most $5 \mu \mathrm{m}$ linewidth titanium resistors had open circuits in them due to aggressive undercutting of photoresist by the etchant solution. Residual photoresist was then removed from each wafer with acetone and by soaking substrates in Nano-Strip® solution for approximately 15 minutes.

Most of the glass/Si sandwich wafers had undesired, residual metallization regions (errors) left on them caused by poor photoresist dispersion across each wafer during spin-on. This was due to some areas having ruptured glass diaphragms over cantilever and bridge array regions, and some large " $T$ " and " $H$ " structure regions in the thin-film, test-structure die areas, resulting in cavities that trapped photoresist. Most of these residual metallization error regions were eliminated by re-processing the wafers to apply new photoresist (with different spin parameters), re-exposing and re-delineating the resist with the gold and resistor metallization photomasks, and wet-etching of the gold or titanium/chromium layers. Correctly delineated metallization regions from the first patterning process were kept intact (under the photoresist, and protected from the wet etchants), assuming mask alignment was accurately done. This "repeatlithography" process was found to be helpful in reducing metallization delineation errors due to exposed cavities in the glass/Si sandwich wafers.

An optional, top-glass passivation layer was then sputter-deposited using the Schott borosilicate glass sputtering target on one of the four remaining glass/Si sandwich wafers which had a $0.2 \mu m$-thick chromium and $0.32-0.43 \mu \mathrm{m}$-thick, patterned gold metallization layer, respectively. This passivation layer was added in case chromium would be used as an RIE etchwindow masking layer for etching through the glass layer at the microbridges/cantilevers (because the process of patterning the top-layer chromium would also partially degrade the chromium resistors underneath, without a top glass passivation layer). An approximate RF planar magnetron deposition rate of $84 \AA$ /min for the glass was measured at an RF power setting of 350 Watts and 15 mTorr pressure in argon using the Technics sputtering system. A target depth of $\sim 0.5 \mu \mathrm{m}$ borosilicate glass was sputter-deposited onto the wafer, after achieving a base pressure of $1.5 \times 10^{-5}$ Torr. 
A thick, top aluminum layer was then sputter-deposited on each remaining glass/Si wafer sandwich to provide an etch-window mask in the March RIE system for etching through the glass to create the microbridge/cantilever flow sensor structures. On one wafer, pure aluminum was DC planar magnetron sputter-deposited in the Technics system at 350 Watts and 10 mTorr (in argon, with a chiller temperature of $15^{\circ} \mathrm{C}$, and base pressures ranging from $1.2 \times 10^{-5}$ to $3.5 \times 10^{-}$ ${ }^{5}$ Torr). The target aluminum thickness for this deposition was $1.0 \mu \mathrm{m}$. On two wafers, aluminum alloy $(98.5 \% \mathrm{Al}, 1 \% \mathrm{Si}, 0.5 \% \mathrm{Cu})$ was DC planar magnetron sputter-deposited in the Technics system at 350 Watts and $10 \mathrm{mTorr}$ (in argon, with base pressures ranging from $1.0 \times 10^{-5}$ to $1.5 \mathrm{x}$ $10^{-5}$ Torr), with target depths of $1.0 \mu \mathrm{m}$. And, on the last wafer, the aluminum alloy was RF planar magnetron sputter-deposited in the Technics system at 300 Watts and $15 \mathrm{mTorr}$ (in argon, with a base pressure of $2 \times 10^{-5}$ Torr), with a target depth of $1.0 \mu \mathrm{m}$.

The aluminum layer was then patterned to provide RIE plasma-etch chemistry windows to etch through the borosilicate glass layer on three of the four glass/Si sandwich wafers. Shipley 1813 resist was spun onto the top of the aluminum layer of each wafer and patterned by photolithographic techniques similar to those previously described for metallization layer delineation. In the Kasper aligner, mask alignment structures in the Etch Window Level 3 photomask (from the 1998/1999 mask set) were aligned to gold and resistor metallization alignment patterns and features (seen through the topography of the aluminum layer) in mask alignment areas on each silicon wafer substrate. Once each glass/Si sandwich wafer was in intimate contact with the photomask, the photoresist was exposed for 15 seconds to $436 \mathrm{~nm}$ wavelength UV light at $6.4 \mathrm{~mW} / \mathrm{cm}^{2}$ intensity through the Etch Window Level 3 photomask. The photoresist was developed, hard-baked, and the aluminum layer patterned by immersing each wafer in $40-45^{\circ} \mathrm{C}$ heated, aluminum etch solution.

The remaining fourth glass/Si sandwich wafer was processed with an experimental "double-lithography" technique to pattern an aluminum layer on top of the borosilicate glass and etch through the glass only at the microbridge/cantilever etch-window areas and protect bonding pad area metallization from RIE glass-etch chemistries. This process was utilized because the Etch Window Level 3 photomask was designed for a more traditional fabrication methodology, in 
with RIE and $\mathrm{KOH}$ etching should occur at the end of the process, and in which it was assumed a top passivation layer would be deposited and etched. This photomask had etch window regions at both the microbridge/cantilever areas and at all bonding pad areas. If it had been originally designed without square etch window regions at all bonding pads, this "double-lithography" process would not have been needed to protect pad area metallization from RIE glass-etch chemistries. This process was attempted because it was believed that exposed pad-area metallization might experience significant degradation (i.e. oxidation) in the RIE plasma while etching through exposed thick glass layers at the microbridge/cantilever areas. A schematic profile representation of this process is shown in Figure 73. This process left a layer of aluminum on top of all pad metallization areas, where gold would normally be exposed to potential degradation in the RIE plasma glass-etch chemistry. First, standard photolithographic techniques were used with Shipley 1813 positive photoresist and the Resistor Metal Level 1 photomask to delineate the previously sputter-deposited ( $1 \mu \mathrm{m}$ thick) aluminum layer in the exact pattern that mirrored the underlying resistor metallization layer (using slightly heated aluminum etch solution). After this first aluminum layer was patterned, residual photoresist was not removed, and was, thus, left on top of the aluminum layer covering both the resistor and gold metallization layers (as shown in Figure 73). Next, a second aluminum layer, with a target thickness of $0.2 \mu \mathrm{m}$ was DC planar magnetron sputter-deposited in the Technics sputtering system (in argon, at 350 Watts and $10 \mathrm{mTorr}$ ) on top of the photoresist and uncovered borosilicate glass areas. This top aluminum layer was then delineated by standard photolithographic processing, using 1813

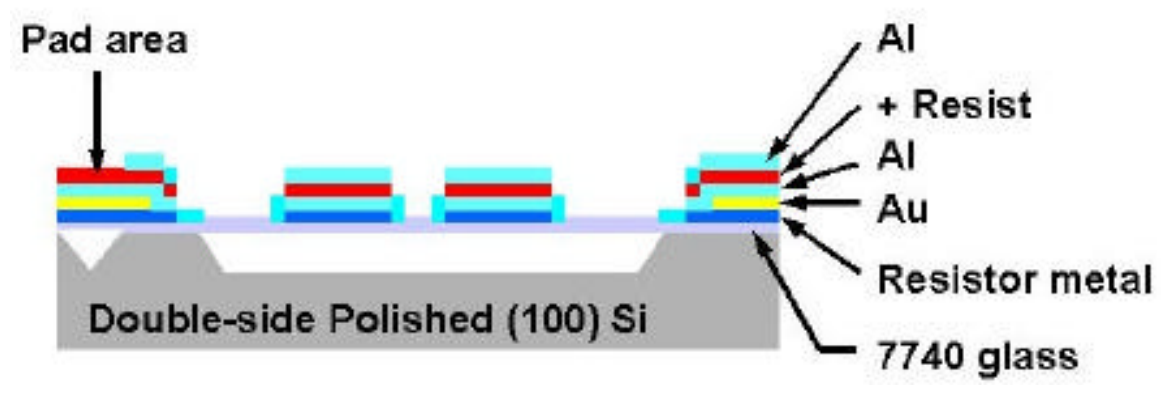

FIGURE 73 - A schematic, cut-away illustration showing the "double-lithography" process. In this process, a top aluminum layer with RIE windows was delineated only at microbridge/cantilever areas, and not at pad regions. Pad gold metal would not be exposed to RIE plasma glass-etch chemistries. 
positive photoresist, and the Etch Window Level 3 photomask in the Kasper aligner to pattern it in the same way the aluminum layer was patterned for the previous three glass/Si sandwich wafers. After etching the aluminum layer in slightly-heated aluminum etch solution, pad areas had photoresist on top of unetched aluminum, and aluminum was etched away only at flow sensor microbridge/cantilever etch window regions on top of the glass (as shown in Figure 73).

The glass/Si sandwich wafer with chromium resistor metallization and a top, thin sputterdeposited borosilicate glass passivation layer was processed to etch through the passivation layer, down to pad metallization. This was accomplished by immersing the wafer in BOE solution until top-layer-glass step height measurements (taken with the Tencor profilometer) and inspection under a microscope revealed that pad gold metallization areas were exposed.

At this point, all of the glass/Si sandwich wafers were ready for dicing apart individual flow sensor die. In order to protect the aluminum masking layer from damage (such as chipping) during dicing, a layer of Shipley 1813 or 1827 positive photoresist was spun onto the top of each wafer (at 2000 RPM for seven seconds) and hard-baked. The Micro Automation Dicing Saw, Model 1006 was utilized to dice apart the die. Since the online Standard Operating Procedure (SOP) for this machine was significantly enhanced and re-written to make it more "user-friendly," it is included in Appendix IX. For one of the glass/Si sandwich wafers, die were originally diced into individual flow sensors, but since it was difficult to handle such small die and very time consuming to dice and test them individually, a new dicing scheme was devised. This suggested quad (four flow sensors each) and duo-die (two sensor) dicing pattern is shown in Appendix VIII. Each quad or duo-die would be mounted in a DIP-24 package, as shown in Figure 74, such that the longitudinal axes of the microbridges/cantilevers were oriented transverse, or across, the flow channel stream (which was designed to be along the longitudinal axis of the DIP-24 package). In this suggested wafer dicing pattern, all but one of the duo/quad die arrangements had flow sensor microbridge/cantilever longitudinal axis orientations that were parallel to one another. For testing the sensors, the flow stream would be directed orthogonal to these axes (laterally across the microbridges/cantilevers), and longitudinally across the DIP-24 package. So, with all the flow sensors within a given duo or quad-die arranged in "parallel," any sensor in each die could be 


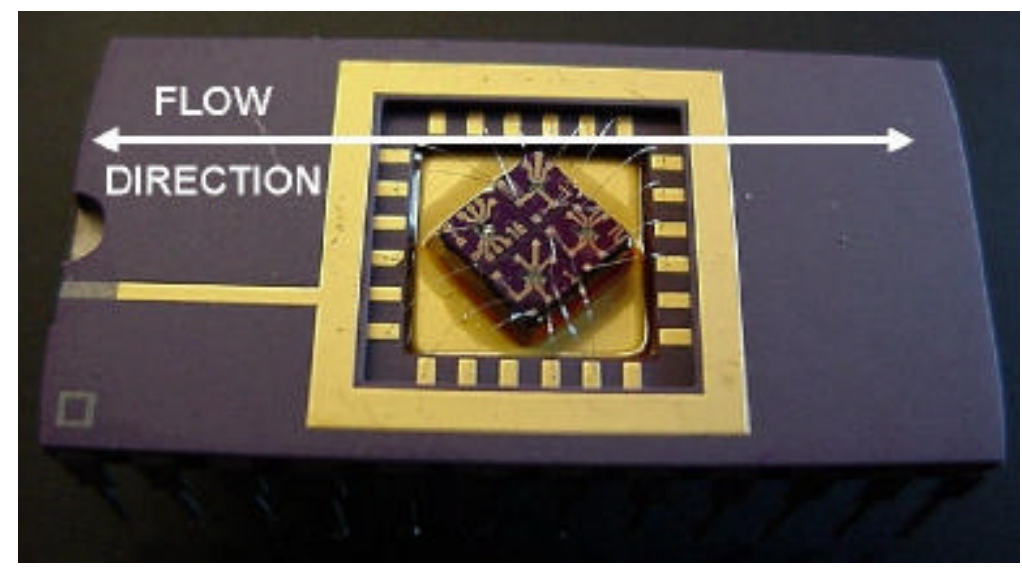

FIGURE 74 - A photo of a wire-bonded, quad-die flow sensor mounted in a DIP-24 package. Note that the quad-die is rotated $45^{\circ}$ so that the microbridge/cantilever longitudinal axes are aligned at a $90^{\circ}$ angle with respect to the longitudinal, or main, axis of (i.e. the airstream flow direction over) the DIP-24 package.

wire-bonded and tested within the same DIP-24 package (and the same geometrical arrangement within the flow channel). The dicing saw was programmed as per the SOP with the following parameters, in metric units, prior to cutting:

1. $\mathbf{1}^{\text {st }}$ Index: $2.3 \mathrm{~mm}$. (the street width between individual flow sensors);

2. $2^{\text {nd }}$ Index: $2.3 \mathrm{~mm}$;

3. Height (amount of substrate to leave undiced): 0.150 or $0.200 \mathrm{~mm}$;

4. Angle: $90^{\circ}$;

5. Speed: $1.27 \mathrm{~mm} / \mathrm{s}$ (the slowest possible speed for cutting).

Each glass/Si wafer sandwich was treated as a "round" specimen, with its maximum length or width dimension (in $\mathrm{mm}$.) entered as the Diameter parameter. The backside of each wafer was carefully affixed to blue adhesive film (while eliminating air pockets), which was cut out in the shape of the wafer, and which was used to hold die together during the dicing process. The total micrometer-measured thickness of the wafer and blue film was entered (in $\mathrm{mm}$.) as the Thickness parameter. Typically, the blue film had a measured thickness of $70 \mu \mathrm{m}$. A progressive-depth-cut distance parameter was entered (with the zero key) on the saw to allow for, usually, three passes per cut. The saw was operated in single-cut (manual) mode for each pass after the blade was aligned to cut on a given street (as per the suggested wafer dicing diagram in Appendix VIII). Although the dicing saw was found to be extremely "temperamental," and in serious need of calibration, flow sensor die were successfully diced apart. Unfortunately, after dicing, it was discovered that the aluminum RIE-masking layer adhesion to the polished borosilicate glass on 


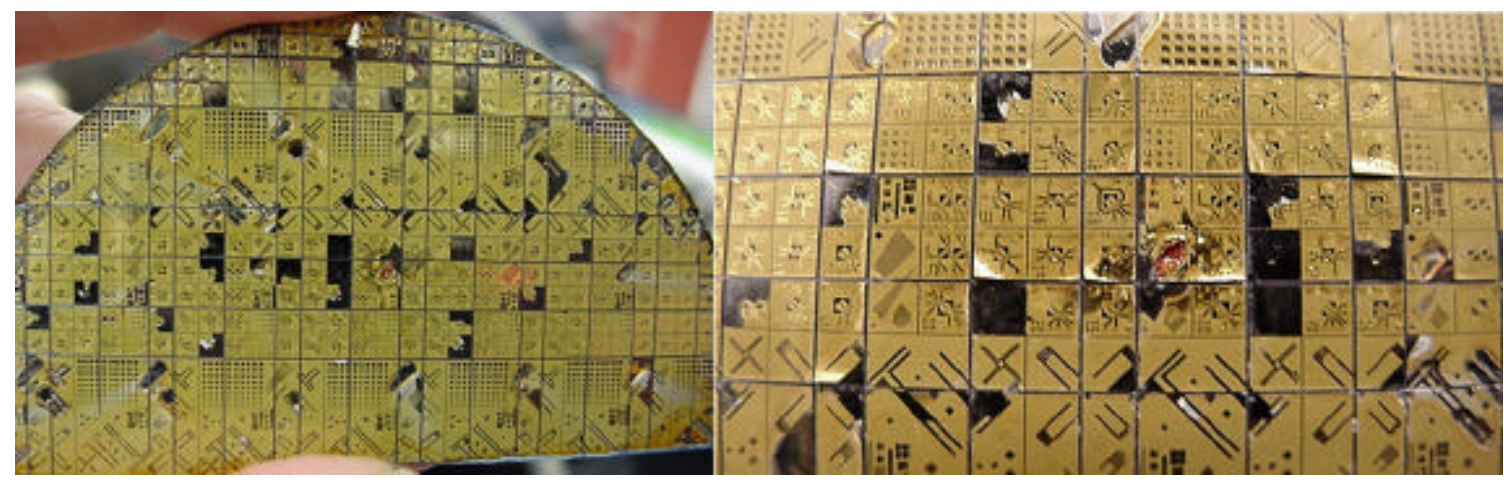

FIGURE 75 - Photos of an aluminum RIE-masking layer with poor adhesion. This problem was apparent after dicing this borosilicate glass/Si diaphragm wafer.

most wafers was marginal, at best, as shown in Figure 75. Thus, many flow sensor die no longer had a viable, intact aluminum etch-window mask for borosilicate glass etching in the RIE system. A contaminated sputtering system in need of cleaning was most likely the cause of this problem.

The next step was to attempt to use the March RIE system to anisotropically etch through the borosilicate glass layer (which had an estimated thickness of up to $38 \mu \mathrm{m}$ ) to create the microbridge/cantilever flow sensor structures. Dice with intact aluminum, etch-window masking layers were separated from each wafer, and any residual photoresist was removed with acetone. Individual quad/duo die thicknesses were then measured with a digital micrometer to estimate the total glass depth to be etched through in the RIE system. First, borosilicate glass etch rates were established in the March RIE system. The RIE chamber was initially thoroughly cleaned with methanol, isopropyl alcohol, acetone, $\mathrm{H}_{2} \mathrm{O}_{2}$, and abrasively cleaned with a Scotch-Brite $\AA$ pad to remove Teflon ${ }^{\mathrm{TM}}$ residues. After this thorough chemical and abrasive cleaning, and between individual etching runs, organic residues were cleaned from the chamber with an oxygen plasma, at maximum MFC oxygen flow, at 400 Watts (RF), for $5-15$ minutes. In some cases, this was followed by an argon plasma cleaning of the chamber (at 400 Watts, RF, for five minutes). This mechanical, chemical, and oxygen/argon-plasma-cleaning regimen was followed, because the use of $\mathrm{CF}_{4}$ or similar gases typically caused the reaction chamber and components to be coated with by-products (such as Teflons ${ }^{\mathrm{TM}}$ ), which could easily re-deposit onto surfaces being etched, potentially reducing overall etch rates [79]. Borosilicate glass wafers to use for establishing etch rates were prepared with either a masking layer of 1813 positive resist or RF planar magnetron 
sputter-deposited chromium (250 Watts, 10 mTorr in argon, with a target depth of $0.88 \mu \mathrm{m}$ ), all patterned with the Etch Window Level 3 photomask. Many different recipes for etching the glass were used, with both photoresist and chromium masking layers. Results of these test runs are shown graphically in Appendix VII. Chemistries of $\mathrm{CF}_{4}$ and $\mathrm{H}_{2}$ were utilized to etch glass with a photoresist masking layer; and chemistries with $\mathrm{CF}_{4}$ and $\mathrm{O}_{2}$ combined were used to etch glass with a chromium masking layer. In all cases, Independent Pressure Control (or automatic pressure control) was enabled to control the chamber pressure. Etch rates with $\mathrm{CF}_{4}$ and $\mathrm{O}_{2}$ chemistries were as much as 30 times faster than those achieved with $\mathrm{CF}_{4}$ and $\mathrm{H}_{2}$ mixes. Unfortunately, etch anisotropy or isotropy was not evaluated during these tests. Exposed borosilicate glass in several flow sensor quad-dice (with an aluminum masking layer) was etched in the RIE system with $\mathrm{CF}_{4}$ and $\mathrm{O}_{2}$ chemistries. These chemistries etched through the glass layer, but also etched isotropically too far into the microbridge structures, as shown in Figure 76, degrading resistor metallization on the bridges. Since the glass was up to $38 \mu \mathrm{m}$ thick, etch times were at least 40 minutes at 300 - 500 Watts to aggressively etch completely through the glass layer. This prolonged etch time (and elevated RF power) resulted in too much aluminum masking layer degradation, for example, at microbridge edges. However, if the RF power was

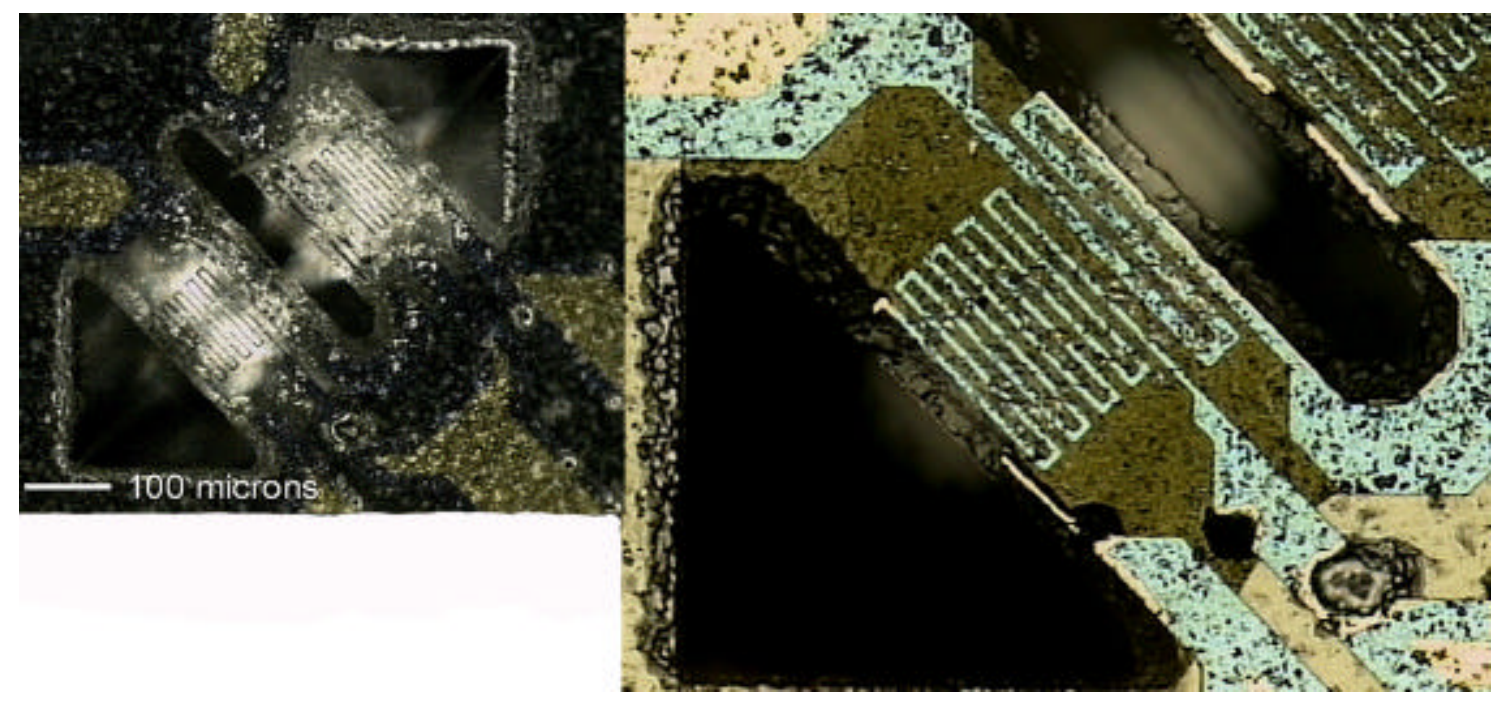

FIGURE 76 - Photos showing lateral damage to microbridge resistor metallization. These results were after reactive ion etching through the borosilicate glass (in the smallest sensor, Flow Sensor \#17). This die was etched at 300 Watts, 200 mTorr, for 43 min. with $15.375 \mathrm{sccm} \mathrm{CF}_{4}$ flow and $5 \mathrm{sccm} \mathrm{O}$ flow. 
increased to increase the etch rate, the aluminum masking layer was further damaged (and oxidized more rapidly). No successful RIE chemistries and parameters were discovered to etch completely through the (relatively thick) borosilicate glass layer without damaging underlying resistor metallization on the microbridges and cantilevers. Chemistries using positive resist as a masking layer were not used because of their impractical, required etch durations (e.g. 20 hours at $250 \AA /$ min to etch through $30 \mu m$ of glass). It is possible, however, that a chemistry could be experimentally discovered to etch through relatively thick borosilicate glass flow sensors with greater anisotropy, since RIE independent variables to control include the following:

1. The process gas selection or mix;

2. The process gas MFC flow rate(s);

3. The chamber etch pressure (using IPC mode);

4. The RF power

5. The chiller temperature (or cooling of the chamber base).

Overall, these process variables control the etch rate, selectivity, anisotropy, and uniformity of etching, making the experimental characterization of plasma etching a complicated process.

Although no microbridge/cantilever type flow sensors were successfully fabricated from the glass/Si sandwich wafers, diaphragm devices could possibly still be tested. Although it was not clear whether a diaphragm flow sensor would have desirable (linear?) characteristics, there were a few examples of similar patented devices. Higashi, et al., at Honeywell, Inc., have patented a method of fabricating a diaphragm-type airflow sensor with a sealed, etched cavity underneath. The cavity both isolates the heaters and detectors, and provides protection from contamination from foreign materials [80]. The aluminum masking layer on both a chromium and titanium resistor metallization quad glass/Si sandwich wafer die was etched away (if it had not already peeled off) in heated aluminum etch solution. The dice were rinsed and blow dried with nitrogen. Because gold adhesion was very poor on the titanium die, it was immersed in $\mathrm{KI}$ solution to remove any residual gold metallization. Each die was rotated $45^{\circ}$ and epoxy bonded into a DIP-24 package with Epo-tek 353ND epoxy, which was cured in an oven, in atmosphere, at $\sim 150^{\circ} \mathrm{C}$ for three minutes. The Kulicke and Soffa ultrasonic wedge bonder was utilized to wirebond each die to a DIP-24 package. It was quickly discovered with the chromium-metallization die that many pad areas imploded into etched pits beneath them when wire-bonding was 
attempted (note the etched cavity shown under the pad in Figure 73). A collage of damaged pads is shown in Figure 77. The pad-area glass under the metallization at each pad was actually

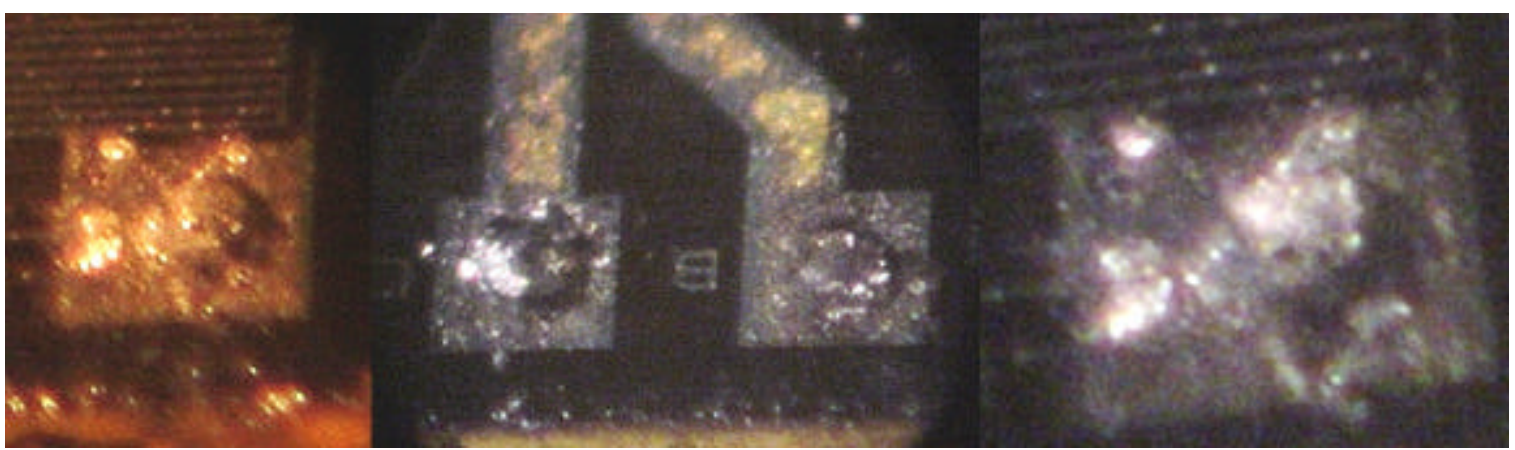

FIGURE 77 - Photos showing imploded pad areas on a borosilicate glass/Si wafer. The implosions were caused by the ultrasonic wedge bonder fracturing glass diaphragms over the pad areas (which had chromium metallization) as bonding was attempted.

a diaphragm over an etched, pyramidal cavity in the silicon substrate, as shown in Figure 73. Successful wire-bonding was achieved, however, to the titanium-metallization die, as shown in Figure 78. This was possible either because the diaphragm glass layer was thicker on this die, or because wire-bonding was done at wide metallization traces not directly over each pad (diaphragm-covered) cavity. In summary, it was generally discovered that wire-bonding to pad regions was unsuccessful on glass/Si sandwich wafer dice due to overstressing the glass

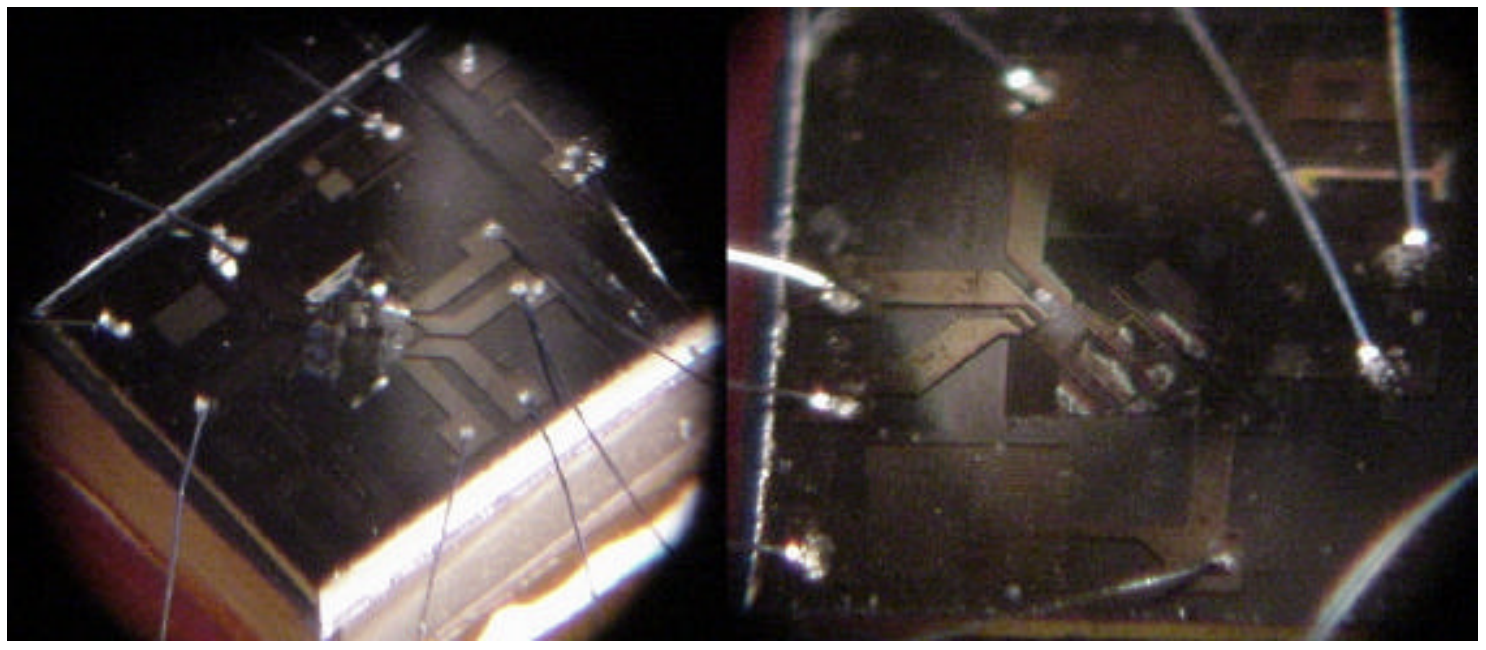

FIGURE 78 - Titanium-metallized glass diaphragm devices with good wire-bonds. 
diaphragm over sealed cavities beneath each pad when ultrasonic wire-bonding was attempted. Wire-bonding could only be successfully achieved to other metallization traces near pad regions on dice which did not have a top sputter-deposited passivation layer of borosilicate glass.

\section{The Sacrificial Layer Method}

Another unique method experimentally attempted, which was similar to surface micromachining, was to fabricate the flow sensors using a sacrificial layer that could be deposited into cavities on a pre-etched silicon substrate, and later removed to suspend the flow sensor microbridges/cantilevers. The general idea was to use a pre-etched silicon wafer substrate with microbridge/cantilever and pad area cavities; deposit a sacrificial layer into the substrate cavities; machine the top surface of the wafer smooth; deposit the microbridge/cantilever structural thin film; deposit and delineate resistor and gold pad area metallizations; pattern the thin-film structural layer with the Etch Window Level 3 photomask; and immerse the wafer in a solution to dissolve the sacrificial layer in the exposed flow sensor cavities to suspend the microbridge/cantilevers. This process is summarized schematically in Table XIV.

This fabrication process began with three separate thermal oxidation runs with 14 standard, n-type, 10-cm, round (100)-oriented, polished silicon substrates, with approximate micrometer-measured thicknesses of $465-504 \mu \mathrm{m}$ (and TTV's of $8-25 \mu \mathrm{m}$ ). The thermallygrown $\mathrm{SiO}_{2}$ layer on each wafer was to be subsequently patterned for $\mathrm{KOH}$ anisotropic preetching of the substrates. The backside of each wafer was scribed with nomenclature to track it. Each wafer was optionally base and acid cleaned prior to oxidation. Five of the wafers were wetoxidized for two hours at $1100^{\circ} \mathrm{C}$ in the Lindberg Tube Furnace, with an oxygen flow rate of $\sim 800$ $\mathrm{mL} / \mathrm{min}$, resulting in a Filmetrics-measured $\mathrm{SiO}_{2}$ thickness range of $0.6957-0.7594 \mu \mathrm{m}$. Five wafers were wet-oxidized for two hours at $1100^{\circ} \mathrm{C}$ in, with an oxygen flow rate of $\sim 827 \mathrm{~mL} / \mathrm{min}$, resulting in an $\mathrm{SiO}_{2}$ thickness range of $0.6738-0.7371 \mu \mathrm{m}$. Four wafers were wet-oxidized (although the bubbler ran dry for part of this process) for 2.85 hours at $1100^{\circ} \mathrm{C}$ with an oxygen flow rate of $\sim 2006 \mathrm{~mL} / \mathrm{min}$, resulting in a measured $\mathrm{SiO}_{2}$ thickness range of $0.8159-0.9835 \mu \mathrm{m}$. In all cases, the desired, target oxide depths were $0.9-1.0 \mu \mathrm{m}$. 
TABLE XIV

SUMMARY OF THE EXPERIMENTAL SACRIFICIAL LAYER PROCESS

\begin{tabular}{|c|c|}
\hline $\begin{array}{c}\text { Flow Sensor } \\
\text { Processing Step }\end{array}$ & Illustration \\
\hline $\begin{array}{l}\text { Obtain a } 10 \mathrm{~cm} \text {. or } 3 \text {-inch } \\
\text { (100) silicon substrate }\end{array}$ & $10 \mathrm{~cm}$ or 3 -inch $(100) \mathrm{Si}$ \\
\hline $\begin{array}{l}\text { Optional base and acid } \\
\text { clean of silicon substrate }\end{array}$ & $10 \mathrm{~cm}$ or 3 -inch $(100) \mathrm{Si}$ \\
\hline $\begin{array}{l}\text { Grow } \sim 1 \mu m \text { wet thermal } \\
\text { oxide on Si wafer in furnace }\end{array}$ & $\leftarrow \begin{array}{l}\text { Thermally- } \\
\text { Grown } \mathrm{SiO}_{2}\end{array}$ \\
\hline $\begin{array}{l}\text { Pattern the oxide layer with } \\
\text { Etch Window Level } 3 \\
\text { photomask and BOE }\end{array}$ & $10 \mathrm{~cm}$ or 3 -inch $(100) \mathrm{si}$ \\
\hline $\begin{array}{l}\text { Anisotropically etch the } \\
\text { silicon substrate with low- } \\
\text { temp. KOH to desired flow } \\
\text { channel depth(s), typically } \\
130-200 \mu m\end{array}$ & $10 \mathrm{~cm}$ or 3 -inch $(100) \mathrm{si}$ \\
\hline $\begin{array}{l}\text { Remove thermally-grown } \\
\text { oxide layer in } \mathrm{BOE}\end{array}$ & $10 \mathrm{~cm}$ or 3 -inch $(100) \mathrm{Si}$ \\
\hline $\begin{array}{l}\text { Spin-on/deposit the } \\
\text { sacrificial layer into the } \\
\text { etched cavities }\end{array}$ & $\begin{array}{l}\text { Removable sacrificial layer } \\
10 \mathrm{~cm} \text { or } 3 \text {-inch }(100) \mathrm{Si}\end{array}$ \\
\hline $\begin{array}{l}\text { Lap and polish the top } \\
\text { surface down to silicon to } \\
\text { planarize it }\end{array}$ & $10 \mathrm{~cm}$ or 3 -inch $(100) \mathrm{si}$ \\
\hline $\begin{array}{l}\text { Sputter-deposit or spin-on } \\
\text { the microbridge/ cantilever } \\
\text { thin-film structural layer }\end{array}$ & $10 \mathrm{~cm}$ or 3 -inch $(100) \mathrm{si}$ \\
\hline $\begin{array}{l}\text { Sputter-deposit the resistor } \\
\text { metallization layer }\end{array}$ & $10 \mathrm{~cm}$ or $3-i n c h(100) \mathrm{Si}$ \\
\hline
\end{tabular}




\begin{tabular}{|c|c|c|}
\hline $\begin{array}{l}\text { DC planar magnetron } \\
\text { sputter-deposit an optional } \\
\text { gold metallization layer } \\
\text { (and adhesion layer, if } \\
\text { necessary) }\end{array}$ & $10 \mathrm{~cm}$ or 3 -inch $(100) \mathrm{Si}$ & $\begin{array}{l}\text { Resistor } \\
\text { Metal } \\
\text { Thin film layer }\end{array}$ \\
\hline $\begin{array}{l}\text { Delineate the gold } \\
\text { metallization, if applicable, } \\
\text { with the Gold Metal Level } 2 \\
\text { photomask }\end{array}$ & $10 \mathrm{~cm}$ or 3 -inch $(100) \mathrm{Si}$ & $\begin{array}{l}\text { Resistor } \\
\text { Metal }\end{array}$ \\
\hline $\begin{array}{l}\text { Delineate the resistor } \\
\text { metallization with the } \\
\text { Resistor Metal Level } 1 \\
\text { photomask }\end{array}$ & $10 \mathrm{~cm}$ or 3 -inch $(100) \mathrm{Si}$ & $\begin{array}{l}\text { Resistor } \\
\text { Metal } \\
\text { Thin film layer }\end{array}$ \\
\hline $\begin{array}{l}\text { Etch through the thin-film } \\
\text { structural layer with the } \\
\text { Etch Window Level } 3 \\
\text { photomask }\end{array}$ & $10 \mathrm{~cm}$ or $3-\operatorname{inch}(100) \mathrm{Si}$ & $\begin{array}{l}\text { Resistor } \\
\text { Metal } \\
\text { Thin film layer }\end{array}$ \\
\hline $\begin{array}{l}\text { Dice into individual single, } \\
\text { duo, or quad-die }\end{array}$ & & \\
\hline $\begin{array}{l}\text { Remove the sacrificial layer } \\
\text { in the } \\
\text { microbridge/cantilever } \\
\text { cavities }\end{array}$ & $10 \mathrm{~cm}$ or 3 -inch $(100) \mathrm{Si}$ & $\begin{array}{l}\text { Resistor } \\
\text { Metal } \\
\text { Thin film layer }\end{array}$ \\
\hline $\begin{array}{l}\text { Wire-bonding, packaging, } \\
\text { and flow sensor testing }\end{array}$ & $10 \mathrm{~cm}$ or 3 -inch $(100) \mathrm{Si}$ & $\begin{array}{l}\text { Resistor } \\
\text { Metal } \\
\text { Thin film layer }\end{array}$ \\
\hline
\end{tabular}


The thermally-grown oxide layer on each wafer was then patterned to provide an etchwindow mask for anisotropic, low-temperature $\mathrm{KOH}$ etching. The backside of each oxidized wafer was first coated with photoresist to protect the oxide from etching in BOE using standard, previously described, application techniques. The photoresist was cured in atmosphere on a hotplate (typically $50{ }^{\circ} \mathrm{C}$ for $5-15$ minutes) or with forced air convection at $95^{\circ} \mathrm{C}$ for $20-30$ minutes in a Lindberg/Blue M oven. Photoresist was then spun-on and soft-baked on the frontside of each wafer using standard techniques. In the Kasper aligner, the (110) primary wafer flat edge on each substrate was aligned parallel to any proximal edge of a rectangle within any "Mask Alignment Area" or to any straight line comprised of a row of pads within the Etch Window Level 3 photomask (similar to the alignment scheme in Figure 37). Once in contact with the photomask, the photoresist on each substrate was exposed for 15 seconds to $436 \mathrm{~nm}$ wavelength UV light at $6.4 \mathrm{~mW} / \mathrm{cm}^{2}$ intensity through the Etch Window Level 3 photomask. The photoresist was developed in MF-318 developer and then hard-baked in a Lindberg/Blue M oven at $95-100{ }^{\circ} \mathrm{C}$ for at least 30 minutes in atmosphere. Each wafer was then immersed in fresh, room temperature BOE solution to pattern the front-side oxide layer. Once a pattern became visually apparent in the $\mathrm{SiO}_{2}$ layer (especially after viewing each wafer under a microscope), the wafers were rinsed in DI water, residual photoresist was removed with acetone, and rinsed again in DI. Each patterned-oxide wafer was then anisotropically etched in $45 \%$ wt. $\mathrm{KOH}$ solution. Since the relative (100) silicon-to- $\mathrm{SiO}_{2}$ etch rate increases with decreasing $\mathrm{KOH}$ solution temperature, low temperature solutions were utilized for etching. The typical $\mathrm{KOH}$ etching apparatus setup, with a closed-loop-control digital hotplate, reflux condenser, and quartz beaker is shown in Figure 61 . Two of the wafers were etched in room temperature $\left(22^{\circ} \mathrm{C}\right) \mathrm{KOH}$ solution (at a maximum stir rate of 300 RPM) for 302 hours, achieving a depth of $170-175 \mu \mathrm{m}$ in the substrates. The other two wafers were etched in $43^{\circ} \mathrm{C} \mathrm{KOH}$, at a stir rate of $300 \mathrm{RPM}$, for 50 hours, resulting in $\sim 190 \mu \mathrm{m}$ etch depths. For non-room-temperature etching, cool water was flowed through the reflux condenser. The Dataplate ${ }^{\mathrm{TM}}$ hotplate was programmed with the desired solution temperature (measured by an immersed Teflon ${ }^{\mathrm{TM}}$ shielded thermometer connected to the hotplate) and stir rate, using a magnetic Teflon ${ }^{\mathrm{TM}}$-encapsulated stir bar. As in the Anodically- 
Bonded Borosilicate Glass Method, substrate etch depths were estimated by examining etchdepth measurement structures on each wafer under a microscope. After anisotropic silicon substrate etching, the remaining $\mathrm{SiO}_{2}$ layer on each wafer was removed by immersion in room temperature BOE solution. The resulting, pre-etched wafers were rinsed in DI and dried with nitrogen. Problems experienced by this stage of wafer processing included four wafers with incorrectly marked (110) primary wafer flats (off by $45^{\circ}$ ) due to a mysterious wafer foundry error, and one wafer that was warped by scribing an identification nomenclature on its backside too deeply/vigorously. Thus, nine pre-etched $10-\mathrm{cm}$ wafers were viable for further processing.

An experimental sacrificial layer was next applied to the front-side of each wafer to fill in the etched cavities. Shipley $1813 / 1827$ positive resist, OCG negative resist, a nonphotolithographically-patternable surface coating, and a spin-on glass were all attempted as sacrificial layers. The spin-on glass and OCG negative photoresist were unsuccessfully attempted as adequate, machinable sacrificial layers, as explained below. The highly viscous negative photoresist was applied to one wafer (without spinning it on) in a thick layer and then hard-baked. Subsequent attempts to lap the negative photoresist down to the planar surface of the substrate with the Logitech PM5 machine resulted in only charring the resist with negligible reduction in overall thickness. The next sacrificial layer attempted was to apply and cure multiple layers of Allied Signal's 512 Accuglass ${ }^{\circledR}$ spin-on glass, to one pre-etched wafer. A cycle of spinning-on the glass, curing it for one minute at $80^{\circ} \mathrm{C}$ on a hotplate, curing for another minute at $150^{\circ} \mathrm{C}$ on another hotplate, and final curing at $250^{\circ} \mathrm{C}$ on another hotplate was used between applications of the glass to the wafer. If only a single SOG layer was applied (according to Allied Signal), the wafer could be cured either in an oven at $425^{\circ} \mathrm{C}$ for one hour, or on at hotplate at $250{ }^{\circ} \mathrm{C}$ for one minute. The wafer was allowed to slowly cool to the ambient temperature before spinning on each additional SOG layer. This technique failed to produce a viable sacrificial SOG layer to fill etched cavities in the wafer because the SOG film could not be applied in a sufficiently thick layer (once multiple layers had been applied and cured to the wafer) to fill them without severely cracking. According to Allied Signal the maximum possible thickness for multiple layers of this SOG without cracking was $1.5-2.5 \mu \mathrm{m}$. In etched cavities with significantly greater 
depths (of up to $\sim 190 \mu \mathrm{m}$ ), this SOG could, therefore, not be used to fill them without cracking.

Better results were achieved by experimenting with using positive photoresist and the non-patternable surface coating for potential sacrificial layers. Shipley Microposit@ FSC®-M surface coating was applied to two wafers. This material was not photolithographically patternable and was typically used to provide protection from chemicals for CMOS devices, e.g., on the backside of wafers during fabrication processes. Multiple layers of the surface coating were spun onto each wafer (at various ramp, spread, and spin parameters). After each spin-on application, the substrates were cured on a hotplate at $50-100{ }^{\circ} \mathrm{C}$ for $5-15$ minutes. This process was repeated until the surface coating appeared to completely fill up the etched cavities in the wafer (and cover the entire top surface of the wafer).

Shipley 1813 and 1827 positive photoresist was successfully applied as sacrificial layers to three pre-etched wafers. The procedure for experimentally applying positive resist was very similar to how the surface coating was applied to other wafers: by spinning-on multiple layers (at various ramp, spread, and spin speeds) and curing in-between on a hotplate at $50-100{ }^{\circ} \mathrm{C}$ for 5 - 15 minutes. However, there was a tendency for the solvent in positive resist that was spun onto a cured layer of resist to partially, or completely, redissolve the underlying resist, making this process very difficult to document and repeat. Nonetheless, the cycle of applying multiple layers of resist was repeated until etched cavities in the wafers appeared to be completely filled.

The next step was to machine, planarize, and polish the sacrificial layers (on pre-etched wafers with successfully-applied positive photoresist and surface coating) down to the top surface of each wafer substrate. The University of Cincinnati's Logitech PM5 machine was utilized to do this. The PM5 was first configured for lapping the four sacrificial-layer wafers in the same manner previously described for the Anodically Bonded Borosilicate Glass Method. These 10-cm diameter wafers were easier to affix by vacuum to the vacuum chuck because they were the same size as the chuck on the PM5. Nine micrometer alumina abrasive slurry grit was used, at a flow rate of 1-2 drips per second, with an applied spring-loaded weight of $3 \mathrm{~kg}$. In general, bulk removal and planarization of the sacrificial layer (which was initially on top of each wafer, as well as in the cavities) occurred rapidly, with the process dramatically slowing down once planarization 
and lapping of the silicon substrate started. Total lapping times varied. In one case, a positiveresist wafer was lapped at $50-70$ RPM for 23 minutes, removing $\sim 226 \mu \mathrm{m}$ of photoresist and silicon, until photoresist was machined away from the top surface, remaining only in etched cavities. One wafer with surface coating was lapped in 50 minutes. Another wafer with applied surface coating in the cavities, achieved planarity after lapping $\sim 120 \mu \mathrm{m}$ of material away at 70 RPM in just five minutes. For a few wafers, many voids (or air pockets) were visible in etched cavities after lapping. For one of the surface-coating wafers, these voids were reduced somewhat by spinning-on and curing another layer of surface coating after the first lapping procedure, and then lapping the wafer again. One additional surface coating layer was spun onto this wafer, and it was lapped again. Since several wafers were fractured into smaller pieces upon hastily attempting to remove them from the vacuum chuck assembly after lapping, these pieces had to be heated and wax-applied to a glass substrate for additional lapping or polishing. Since wax-mounting or unmounting the wafers required heating them to $\sim 85^{\circ} \mathrm{C}$, the sacrificial layer (especially positive photoresist) would also soften and bulge inside the cavities. This problem may be somewhat mitigated by very slowly heating the substrates to soften the wax.

Wafers that had been lapped were then polished using the PM5 machine to achieve a smooth, planar surface across sacrificial-layer-filled cavities. The PM5 was cleaned and configured for polishing the substrates in the same manner previously described for the Anodically Bonded Borosilicate Glass Method. Colloidal silica was again used as the polishing media, with an approximate flow rate of one drip per second. All wafers were polished at 70 RPM. Polishing times for individual wafers varied, and once finished, many wafers still had residual frosted (unpolished) regions. For wafers with a positive photoresist sacrificial layer, 11 60 minutes was required to polish them into a uniform glossy, mirrored surface. An example of the surface created by polishing a positive-resist, sacrificial-layer wafer is shown in Figure 79, and a close-up microscopic-view collage of flow sensor cavities on one of these wafers is shown in Figure 80 . The surface-coating wafer which originally took five minutes to lap required 32 minutes of polishing. The other surface-coating wafer required 91 minutes of polishing to achieve 


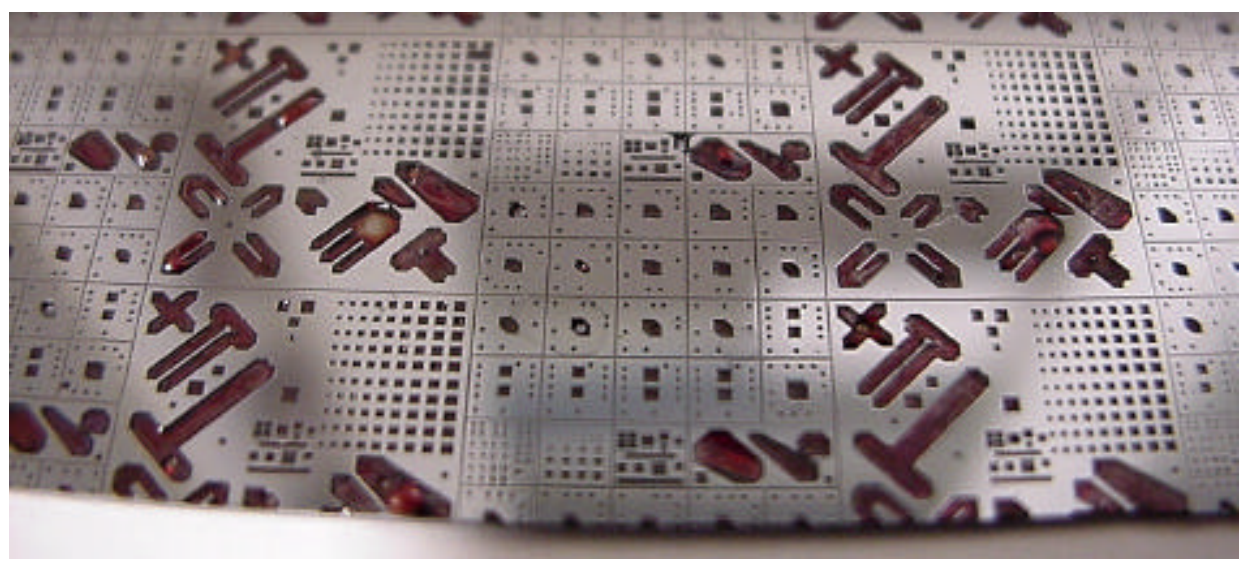

FIGURE 79 - $\quad$ A polished 10-cm (100) Si wafer with a positive photoresist sacrificial layer. The photoresist was in the flow sensor microbridge/cantilever cavities, pad area cavities, and residual stress/strain test microstructure cavities.

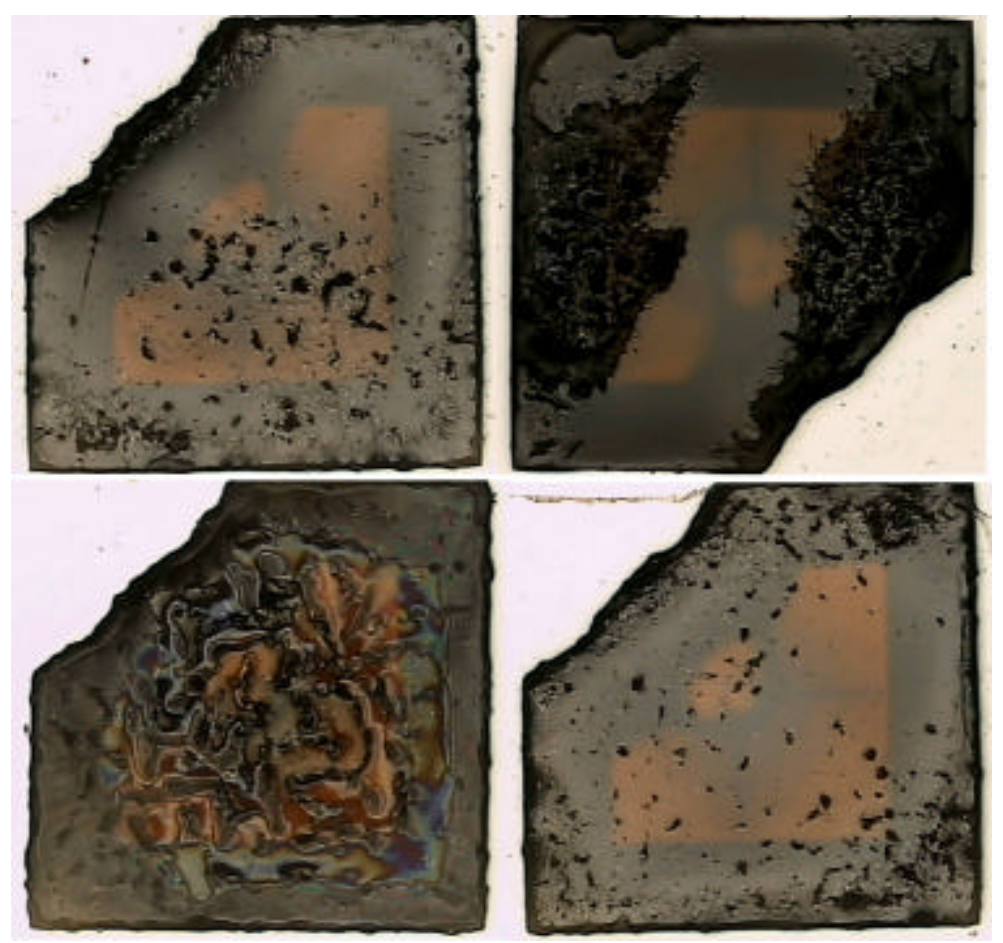

FIGURE 80 - A close-up view of positive photoresist topography in device cavities. These flow sensor cavities were in a polished $10-\mathrm{cm}(100)$ silicon wafer substrate. Note the black colored gap around the perimeter of the photoresist.

a glossy surface. Figure 81 shows both a macro and microscopic view of a surface-coating, sacrificial-layer wafer after it was polished. Examinations of these polished wafers under a microscope revealed considerable topographical variations in the filled flow sensor cavities. Almost all cavities had a narrow gap around the periphery of the sacrificial layer, which might 


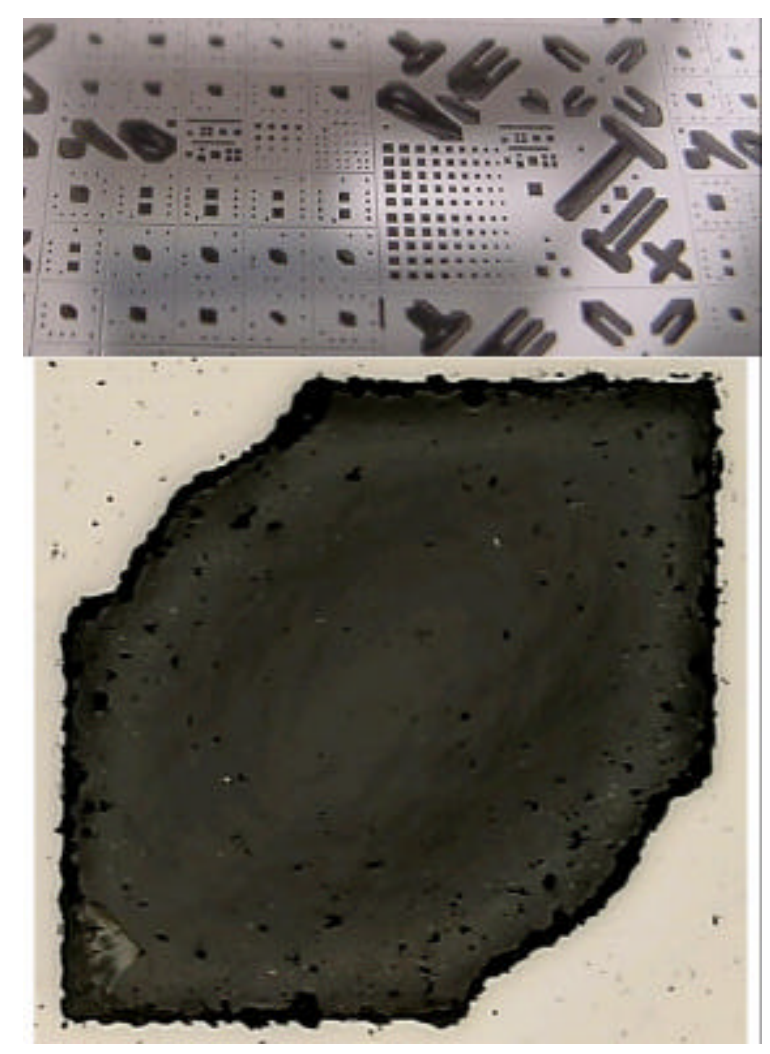

FIGURE 81 - Two views of a polished Si wafer with surface-coating sacrificial-layer. The substrate was a 10-cm (100) silicon wafer. The topography of the surfacecoating-filled flow sensor cavity shows a black colored gap around the perimeter of the photoresist, where it was not in contact with the cavity walls.

have caused subsequent problems with the structure of any deposited thin film microbridge/cantilever layer.

The next step was to apply the thin-film structural layer for the flow sensor microbridges/ cantilevers to the polished, sacrificial-layer wafers. It was quickly determined that this would not be possible with these sacrificial layers. Both the surface coating and photoresist had residual trapped air bubbles that would explode if any polished sacrificial-layer substrates were subjected to a vacuum in the Technics sputtering system (as shown in Figure 82). Hence, any sacrificial layer material used which could potentially have residual trapped air would not be viable for this process. The sputtering system (or an evaporator) would be needed to sputter-deposit any thinfilm structural layer or any metallization layers. Even if a spin-on glass could be used as the flow sensor structural layer (overcoming the need to sputter-deposit this layer), metallization layers would have to be subsequently sputter-deposited. It was further assumed that any thin spin-on 
glass structural layer applied to a polished wafer would also not survive in the sputtering system with trapped air pockets in the sacrificial-layer-filled cavities. Thus, without a means to deposit a "bubble-free" sacrificial layer in the cavities and without a means to successfully apply or deposit a thin film flow sensor structural layer on these sacrificial-layer substrates, this process was abandoned.

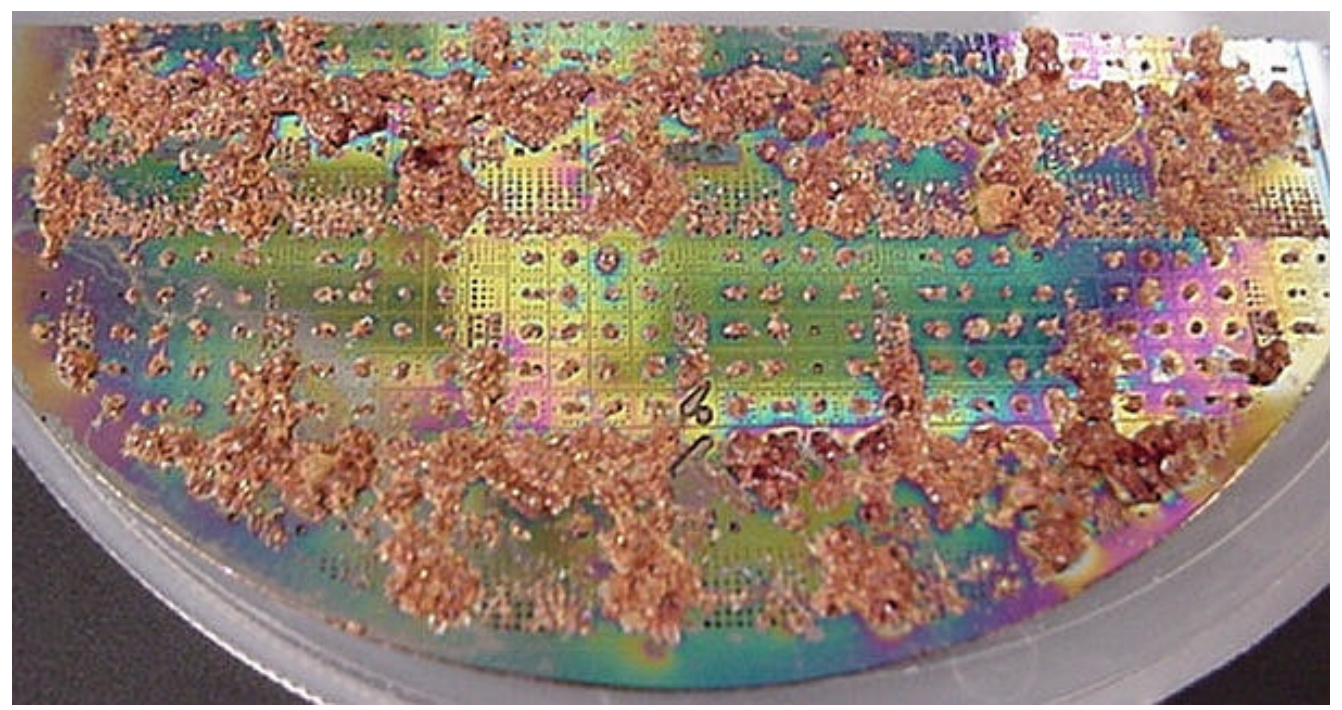

FIGURE 82 - Photo of exploded positive photoresist on a substrate after sputtering. A polished, positive photoresist sacrificial-layer, pre-etched (100) silicon wafer had photoresist explode out of the cavities once in a vacuum in the Technics sputtering system. This occurred during an attempt to sputter-deposit a borosilicate glass thin film structural layer on the wafer. 


\section{B. Thin-Film Structural Methods Attempted: Post-Etched Wafer Strategies}

\section{RF Planar Magnetron Sputter-Deposited Silicon Nitride Thin Film Processes}

The original method most extensively explored to fabricate the structure of the flow sensors was a $\sim 1 \mu \mathrm{m}$ thick RF planar magnetron sputter-deposited $\mathrm{Si}_{\mathrm{x}} \mathrm{N}_{\mathrm{y}}$ thin film (with a $\sim 0.5 \mu \mathrm{m}$ layer underneath and on top of the resistor metallization layer). This method was originally preferred because the thin-film layer could be controllably deposited and patterned without outsourcing any fabrication processes. The deposition could be done with the Technics sputtering system, where the thickness could be reasonably controlled, and the $\mathrm{Si}_{x} \mathrm{~N}_{\mathrm{y}}$ layer could be anisotropically patterned with the March RIE system. It was soon discovered, however, that sputter-deposited $\mathrm{Si}_{x} \mathrm{~N}_{y}$ thin films had several notable problems, including:

1. The stoichiometric film composition varied with sputtering gas used, sputtering pressure, and RF power setting, making film reproducibility very difficult (compounding problems associated with finding exact RIE chemistries to etch through the $\mathrm{Si}_{x} \mathrm{~N}_{y}$ thin films);

2. Sputtered $\mathrm{Si}_{x} \mathrm{~N}_{y}$ film quality was poor, as it was usually porous, allowing the anisotropic silicon etchant $(\mathrm{KOH})$ to come into contact with and degrade the resistor metallization layer;

3. Sputtered $\mathrm{Si}_{x} \mathrm{~N}_{y}$ films always contained residual compressive stresses, resulting in buckled or warped suspended microbridge and cantilever structures;

4. Residual compressive stress in the thin film often resulted in film cracking at the microbridges, allowing the anisotropic silicon etchant $(\mathrm{KOH})$ to contact and degrade the underlying resistor metallization;

5. Sandwiched buckled/warped microbridge structures with resistor metallization in the center had induced stresses in the metallization layer, which changed the electrical properties of the layer, potentially resulting in greater differences between symmetrically-designed upstream and downstream resistor resistances; 
6. Cantilever-type flow sensors with residual compressive stress in the thin film, could be warped down into etched cavities underneath them, out of the flow stream, resulting in decreased responsiveness for those devices.

The original 1997 flow sensor fabrication process, with additive metal liftoff used to pattern the resistor metallization layer is summarized in Table XV. This process used a sputtered silicon nitride sandwich (with $\sim 0.8-1.0 \mu \mathrm{m}$ total thickness) with one delineated metallization layer, which was typically chromium, in the center. Measured sputtering rates for depositing silicon nitride films are given in Appendix X. Additive metal liftoff proved to be very difficult for some processed wafers, requiring the use of an ultrasonically-agitated acetone bath, which also sometimes damaged resistor metallization layers on some wafers. The process of tearing the thin-film resistor metallization layer to delineate it during liftoff often left sharp vertical edges of metallization protruding up out of the plane of the flow sensor microbridges. These protruding edges would then be insufficiently covered by the top, sputter-deposited $\sim 0.5 \mu m \mathrm{Si}_{x} \mathrm{~N}_{y}$ thin film passivation layer, resulting in anisotropic $\mathrm{KOH}$ silicon etchant degradation of the metallization layer. It was also discovered that the single resistor and metallization layer of chromium was not resistant to degradation in $\mathrm{KOH}$, resulting in damaged or destroyed bonding-area pads. This was another reason the original mask set was re-designed to add a top-layer of gold metallization to cover bonding-pad and routing areas to provide protection against degradation in $\mathrm{KOH}$. In the 1997 process, a top layer of aluminum was sputter-deposited and delineated with the etch window photomask to provide an $\mathrm{RIE} \mathrm{Si}_{x} \mathrm{~N}_{y}$ plasma-etch-window layer to pattern the $\mathrm{Si}_{x} \mathrm{~N}_{\mathrm{y}}$ layer prior to anisotropic silicon etching in $\mathrm{KOH}$. Although many attempts were made by students in the 1997 microfabrication course lab to create viable flow sensors, none were successfully fabricated and tested from the original photomask set. Experience with argon-sputtered silicon nitride films from this process (in which it was difficult to find repeatable RIE chemistries to etch through nitride layers) also led to experimenting with the viability of nitrogen-sputtered $\mathrm{Si}_{x} \mathrm{~N}_{\mathrm{y}}$ thin films.

The original 1997 mask set was redesigned to replace positive photoresist lift-off resistor metallization delineation with wet-etching; to add a top gold layer to protect pad metallization 
TABLE XV

SUMMARY OF FABRICATION STEPS FOR THE 1997 LIFTOFF PROCESS

WITH RF MAGNETRON SPUTTER-DEPOSITED SILICON NITRIDE

\begin{tabular}{|c|c|}
\hline $\begin{array}{c}\text { Flow Sensor } \\
\text { Processing Step }\end{array}$ & Illustration \\
\hline $\begin{array}{l}\text { Obtain a } 10 \mathrm{~cm} \text {. or } 3 \text {-inch } \\
\text { (100) silicon substrate }\end{array}$ & Si wafer $(100)$ \\
\hline $\begin{array}{l}\text { Base and acid clean each } \\
\text { silicon substrate }\end{array}$ & Si wafer $(100)$ \\
\hline $\begin{array}{l}\text { RF planar magnetron } \\
\text { sputter-deposit a } \sim 0.5 \mu \mathrm{m} \\
\text { thick silicon nitride thin film } \\
\text { bottom structural layer }\end{array}$ & Si wafer $(100)$ \\
\hline $\begin{array}{l}\text { RF planar magnetron } \\
\text { sputter-deposit a } \sim 0.5 \mu \mathrm{m} \\
\text { thick silicon nitride layer on } \\
\text { the backside of the wafer to } \\
\text { protect against } \mathrm{Si} \text { etching in } \\
\mathrm{KOH}\end{array}$ & $\frac{\text { Si wafer }(100)}{\text { Packside }^{1}}$ \\
\hline $\begin{array}{l}\text { Delineate positive } \\
\text { photoresist on the } \mathrm{Si}_{x} \mathrm{~N}_{\mathrm{y}} \\
\text { layer with the resistor } \\
\text { metallization photomask }\end{array}$ & Si wafer $(100)$ \\
\hline $\begin{array}{l}\text { RF planar magnetron } \\
\text { sputter-deposit a thin film } \\
\text { chromium resistor } \\
\text { metallization layer }\end{array}$ & $\begin{array}{l}\text { Pad area } \\
\text { Si wafer (100) }\end{array}$ \\
\hline $\begin{array}{l}\text { Perform liftoff processing by } \\
\text { soaking wafer in acetone to } \\
\text { delineate chromium resistor } \\
\text { metallization (use an } \\
\text { ultrasonically-agitated } \\
\text { acetone bath if needed) }\end{array}$ & Si wafer $(100)$ \\
\hline $\begin{array}{l}\text { RF planar magnetron } \\
\text { sputter-deposit a } \sim 0.5 \mu \mathrm{m} \\
\text { thick silicon nitride thin film } \\
\text { top passivation layer }\end{array}$ & Si wafer $(100)$ \\
\hline
\end{tabular}




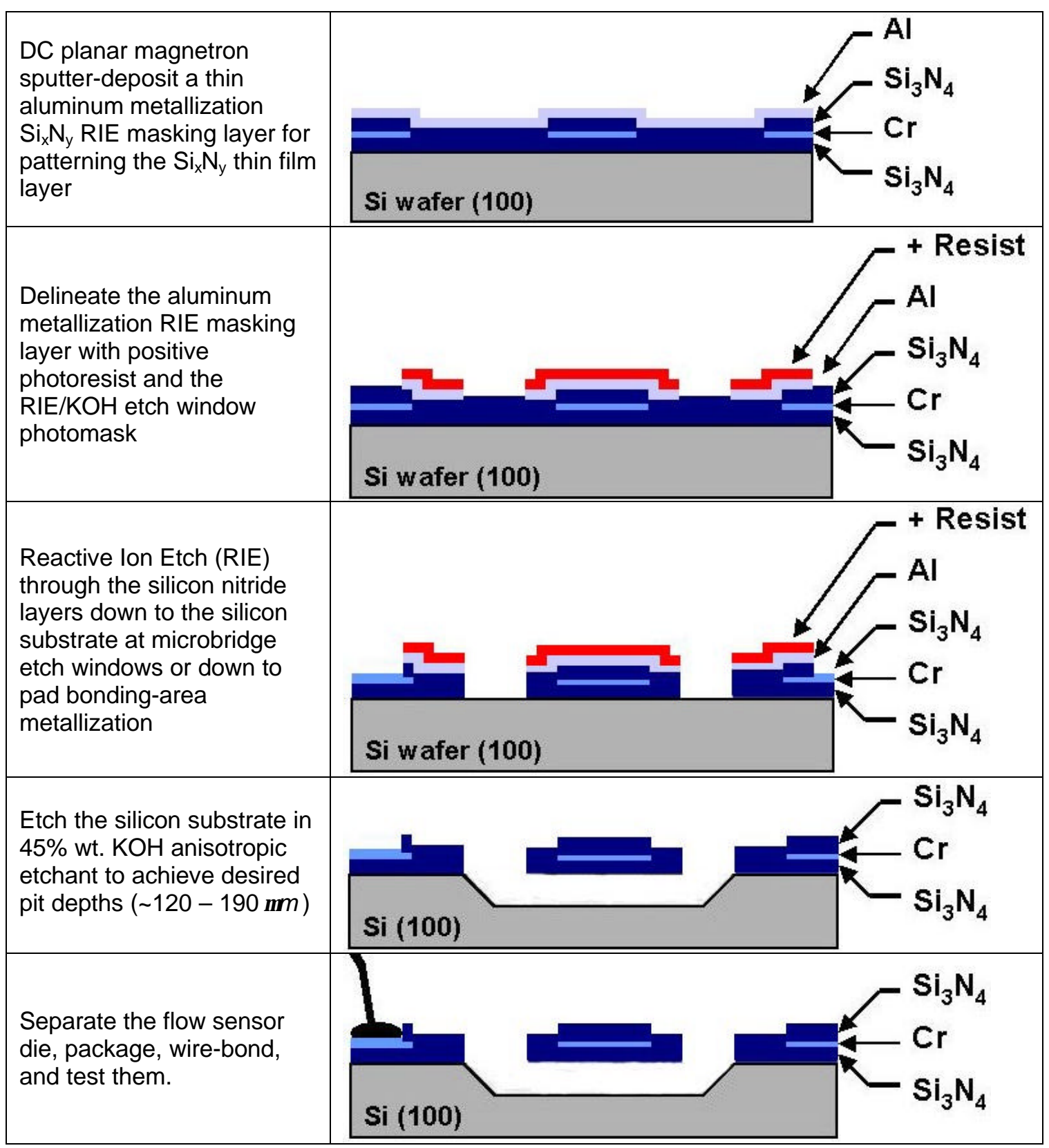

from degradation in the anisotropic silicon etchant; to improve the flow sensor geometries; and to add in-situ, thin-film, stress/strain measurement microstructures that could be utilized to potentially discover a sputtering process to deposit non-compressive $\mathrm{Si}_{x} \mathrm{~N}_{y}$ thin films. Many sputtering trials were attempted with different sputtering parameters to reduce residual compressive stress in the films. Only nitrogen-sputtered $\mathrm{Si}_{x} \mathrm{~N}_{\mathrm{y}}$ thin films were used because their stoichiometry/composition seemed to more closely match CVD $\mathrm{Si}_{3} \mathrm{~N}_{4}$ models used by the Filmetrics system for spectroscopic estimation of film depths (i.e. with less curve-fitting error than 
when measuring thicknesses of argon-sputtered $\mathrm{Si}_{x} \mathrm{~N}_{\mathrm{y}}$ films). It was believed that depositing a $\mathrm{Si}_{x} \mathrm{~N}_{\mathrm{y}}$ thin film layer with a stoichiometry closer to CVD nitride might result in more consistently reproducible nitride etching characteristics in the March RIE system. It was impossible to estimate depths of argon-sputtered $\mathrm{Si}_{x} \mathrm{~N}_{\mathrm{y}}$ thin films with the Filmetrics system, because their optical properties were significantly different from the CVD $\mathrm{Si}_{3} \mathrm{~N}_{4}$ models. Although nitrogensputtered films had different optical properties than argon-sputtered films, it was not clear how this was related to the film's stoichiometry. Regardless of whether the stoichiometry of nitrogensputtered $\mathrm{Si}_{x} \mathrm{~N}_{y}$ thin films was closer to that of CVD $\mathrm{Si}_{3} \mathrm{~N}_{4}$ films, it was easier to find consistent RIE chemistries for etching nitrogen-sputtered films than for etching argon-sputtered films.

Depth variations across each wafer were large for nitrogen sputtered nitride films, as shown in Figure 83. Figure 84 shows an image of the surface topography of one nitrogen-sputtered $\mathrm{Si}_{x} \mathrm{~N}_{\mathrm{y}}$ thin film wafer. Several nitride depositions were done at higher sputtering pressures (above $\sim 30$ mTorr) in high-purity nitrogen, as it was thought that higher sputtering pressures might result in depositing thin films under residual tensile stress. However, the results were inconclusive, because no RIE chemistries could be readily found to etch these $\mathrm{Si}_{x} \mathrm{~N}_{\mathrm{y}}$ thin films.

The redesigned 1998/1999 photomask set was utilized to successfully fabricate flow sensors with nitrogen-sputtered silicon nitride thin films and gold pad area metallization that

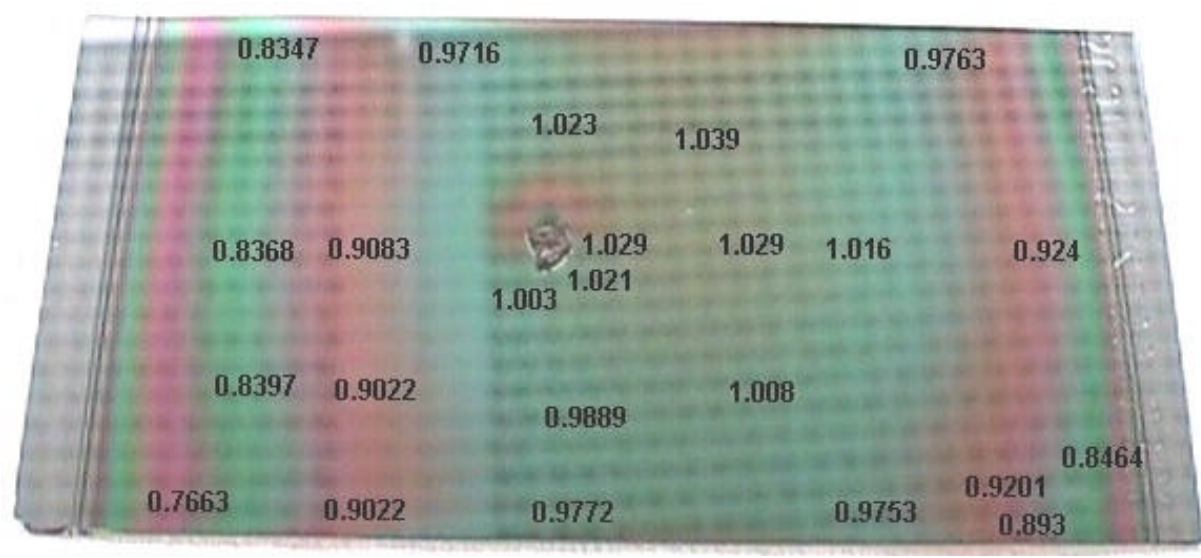

FIGURE 83 - A photo of Filmetrics-measured $\mathrm{Si}_{x} \mathrm{~N}_{\mathrm{y}}$ depths across a test silicon wafer. This nitride film was RF planar magnetron sputtered in nitrogen at 500 Watts, and 20 mTorr for 110 minutes. Its Tencor-profilometer measured thickness was $\sim 1.15$ $1.20 \mu \mathrm{m}$ in the center. This rainbow appearance was common for $\mathrm{N}_{2}$ sputtered $\mathrm{Si}_{x} \mathrm{~N}_{\mathrm{y}}$ films, but argon sputtered films had a uniform dull light-green appearance. 


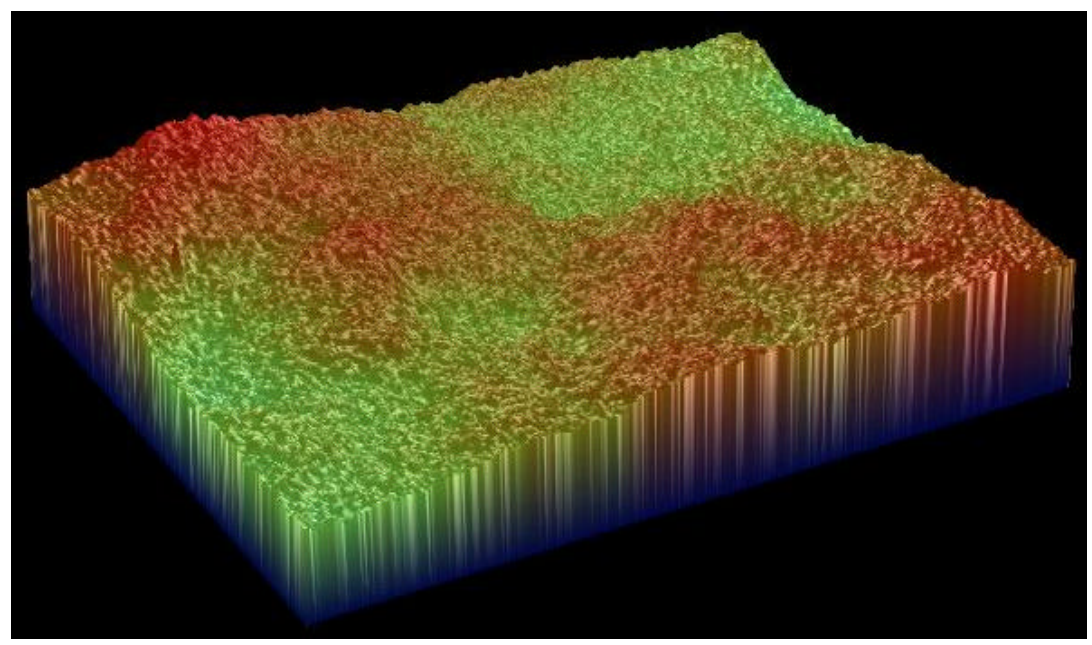

FIGURE 84 - A Wyco-Instrument image of the topography of a sputtered $\mathrm{Si}_{x} \mathrm{~N}_{\mathrm{y}}$ thin film. This film was RF planar magnetron-sputtered in nitrogen at 500 Watts, and 20 mTorr. Its Tencor-profilometer measured film thickness was $\sim 1.56 \mu \mathrm{m}$.

survived $\mathrm{KOH}$ anisotropic silicon etching mostly intact, but problems with residual compressive stresses in, and the general quality of, the nitride films still remained. The fabrication process attempted with this mask set is summarized in Table XVI. Standard photolithographic processes (similar to those previously described) were utilized to delineate metallization layers, with photomask alignments to patterned features on substrates performed as originally designed using mask alignment areas in each photomask. Chromium was selected as the resistor metallization because it provided an excellent adhesion layer for gold, and had desirable bulk resistivity and TCR values. Contiguous chromium and gold layers were deposited in the same sputtering run, where chromium was typically RF planar magnetron sputter-deposited at 250 Watts and 10 mTorr in argon, with a Tencor-profilometer-measured deposition rate of $260-280 \AA ̊$ min. Gold layers were typically DC planar magnetron sputter-deposited at 350 Watts, 10 mTorr, in argon, with a measured deposition rate of $\sim 470-620 \AA / \mathrm{min}$. The 1998/1999 process added a delineated gold metallization layer (via wet etching) to cover pad regions that were exposed to the anisotropic silicon etchant, $\mathrm{KOH}$. Additionally, an experimental top gold layer (with an underlying thin chromium adhesion layer) was added to protect the poor-quality top silicon nitride passivation layer from degradation in $\mathrm{KOH}$. This top gold layer was delineated using the Etch Window Level 3 photomask to provide a convenient inert etch-window metallization mask for both etching 
TABLE XVI

FABRICATION SUMMARY FOR THE 1998/1999 WET ETCH PROCESS

WITH RF SPUTTER-DEPOSITED SILICON NITRIDE

\begin{tabular}{|c|c|}
\hline $\begin{array}{c}\text { Flow Sensor } \\
\text { Processing Step }\end{array}$ & Illustration \\
\hline $\begin{array}{l}\text { Obtain a } 10 \mathrm{~cm} \text {. or 3-inch (100) } \\
\text { silicon substrate. }\end{array}$ & (100) Silicon wafer \\
\hline $\begin{array}{l}\text { Base and acid clean each silicon } \\
\text { substrate. }\end{array}$ & (100) Silicon wafer \\
\hline $\begin{array}{l}\text { RF planar magnetron sputter- } \\
\text { deposit a } \sim 0.4-0.9 \mu m \text { thick } \\
\text { silicon nitride thin film bottom } \\
\text { structural layer. }\end{array}$ & (100) Silicon wafer \\
\hline $\begin{array}{l}\text { RF planar magnetron sputter- } \\
\text { deposit a } \sim 0.5 \mu m \text { thick silicon } \\
\text { nitride layer on the backside of } \\
\text { the wafer to protect against } \mathrm{Si} \\
\text { etching in } \mathrm{KOH}\end{array}$ & (100) Silicon wafer \\
\hline $\begin{array}{l}\text { RF planar magnetron sputter- } \\
\text { deposit a } \sim 0.1-0.2 \mu \mathrm{m} \text { - thick } \\
\text { chromium resistor metal layer. }\end{array}$ & $\begin{array}{l}\text { Cr resistor layer } \\
\text { Sputtered } \mathrm{Si}_{x} \mathrm{~N}_{y}\end{array}$ \\
\hline $\begin{array}{l}\text { Without breaking vacuum, DC } \\
\text { planar magnetron sputter- } \\
\text { deposit a } 0.25-0.90 \mu m \text {-thick } \\
\text { gold metallization bonding-pad } \\
\text { and routing top layer. }\end{array}$ & $\begin{array}{l}\text { Au } \\
\text { Cr resistor layer } \\
\text { Sputtered } \mathrm{Si}_{x} \mathrm{~N}_{y}\end{array}$ \\
\hline $\begin{array}{l}\text { Delineate gold bonding-pad and } \\
\text { routing metallization using the } \\
\text { Gold Metal Level } 2 \text { photomask, } \\
\text { with positive photoresist and a KI } \\
\text { wet-etch bath. }\end{array}$ & $\begin{array}{l}\text { Pad area } \\
\downarrow \\
(100) \text { Silicon wafer }\end{array}$ \\
\hline $\begin{array}{l}\text { Delineate chromium resistor } \\
\text { metallization using the Resistor } \\
\text { Metal Level } 1 \text { photomask, with } \\
\text { positive photoresist and chrome } \\
\text { etch solution. }\end{array}$ & (100) Silicon wafer \\
\hline $\begin{array}{l}\text { After removing residual } \\
\text { photoresist with acetone and } \\
\text { Nano-Stripß, RF planar } \\
\text { magnetron sputter-deposit a } \\
\sim 0.4-0.9 \mu m \text {-thick silicon nitride } \\
\text { thin-film, top passivation layer. }\end{array}$ & $\begin{array}{l}\text { Pad area } \\
\downarrow \\
(100) \text { Silicon wafer }\end{array}$ \\
\hline
\end{tabular}




\begin{tabular}{|c|c|c|}
\hline $\begin{array}{l}\text { RF planar magnetron sputter- } \\
\text { deposit a } \sim 0.05 \mu m \text { - thick } \\
\text { chromium adhesion layer for } \\
\text { delineating the top gold } \\
\mathrm{RIE} / \mathrm{KOH} \text { masking layer for } \\
\text { patterning the } \mathrm{Si}_{\mathrm{x}} \mathrm{N}_{\mathrm{y}} \text { thin film } \\
\text { layer. }\end{array}$ & $\begin{array}{c}\text { Pad area } \\
\downarrow \\
\square\end{array}$ & $\begin{array}{l}-\mathrm{Cr} \text { adhesion layer } \\
\mathrm{Sputtered}_{\mathrm{Si}_{x} \mathrm{~N}_{\mathrm{y}}} \\
-\mathrm{Au} \\
-\mathrm{Cr} \text { resistor layer } \\
\text { - Sputtered } \mathrm{Si}_{x} \mathrm{~N}_{\mathrm{y}}\end{array}$ \\
\hline $\begin{array}{l}\text { Without breaking vacuum, } \mathrm{DC} \\
\text { sputter-deposit a } \sim 0.12-0.45 \\
\mu m \text {-thick, top-layer gold } \\
\mathrm{RIE} / \mathrm{KOH} \mathrm{Si}_{\mathrm{x}} \mathrm{N}_{\mathrm{y}} \text { masking layer. } \\
\text { This layer should be half the } \\
\text { thickness of the bonding pad } \\
\text { area gold metallization deposited } \\
\text { in step } 5 .\end{array}$ & $\begin{array}{l}\text { Pad area } \\
\downarrow\end{array}$ & $\begin{array}{l}\text { RIE mask layer } \\
\text { r adhesion layer } \\
\text { puttered } \mathrm{Si}_{x} \mathrm{~N}_{y} \\
\text { iu resistor layer } \\
\text { puttered } \mathrm{Si}_{x} \mathrm{~N}_{y}\end{array}$ \\
\hline $\begin{array}{l}\text { Delineate the top-layer gold } \\
\mathrm{Si}_{x} \mathrm{~N}_{\mathrm{y}} \mathrm{RIE} / \mathrm{KOH} \text { masking layer } \\
\text { using the Etch Window Level } 3 \\
\text { photomask with positive } \\
\text { photoresist and KI etch. }\end{array}$ & (100) Silicon wafer & $\begin{array}{l}\text { adhesion layer } \\
\text { uttered } \mathrm{Si}_{x} \mathrm{~N}_{y} \\
\text { resistor layer } \\
\text { uttered } \mathrm{Si}_{x} \mathrm{~N}_{y}\end{array}$ \\
\hline $\begin{array}{l}\text { Without removing the photoresist } \\
\text { from the last step, delineate the } \\
\text { chromium adhesion layer for the } \\
\text { top-layer gold } \mathrm{Si}_{\mathrm{x}} \mathrm{N}_{\mathrm{y}} \mathrm{RIE} / \mathrm{KOH} \\
\text { masking layer by immersion in } \\
\text { chrome etch solution. }\end{array}$ & (100) Silicon wafer & $\begin{array}{l}\text { adhesion layer } \\
\text { uttered } \mathrm{Si}_{x} \mathrm{~N}_{y} \\
\text { resistor layer } \\
\text { uttered } \mathrm{Si}_{x} \mathrm{~N}_{y}\end{array}$ \\
\hline $\begin{array}{l}\text { Reactive lon Etch (RIE) through } \\
\text { the silicon nitride layers down to } \\
\text { the silicon substrate at } \\
\text { microbridge etch windows and } \\
\text { down to gold-pad, bonding-area } \\
\text { metallization. }\end{array}$ & (100) Silicon wafer & $\begin{array}{l}\text { adhesion layer } \\
\text { outtered } \mathrm{Si}_{x} \mathrm{~N}_{y} \\
\text { resistor layer } \\
\text { uttered } \mathrm{Si}_{x} \mathrm{~N}_{y}\end{array}$ \\
\hline $\begin{array}{l}\text { Etch the silicon substrate in } 45 \% \\
\text { wt. } \mathrm{KOH} \text { anisotropic etchant to } \\
\text { suspend microbridges/ } \\
\text { cantilevers and achieve desired } \\
\text { pit depths }(\sim 120-190 \mu \mathrm{m}) .\end{array}$ & (100) Silicon wafer & $\begin{array}{l}\text { adhesion layer } \\
\text { uttered } \mathrm{Si}_{\mathrm{x}} \mathrm{N}_{\mathrm{y}} \\
\text { resistor layer } \\
\text { uttered } \mathrm{Si}_{\mathrm{x}} \mathrm{N}_{\mathrm{y}}\end{array}$ \\
\hline $\begin{array}{l}\text { Remove top-layer gold } \mathrm{Si}_{x} \mathrm{~N}_{\mathrm{y}} \\
\mathrm{RIE} / \mathrm{KOH} \text { masking layer by } \\
\text { immersion in } \mathrm{KI} \text { solution. } \\
\text { Warning: Over-etching will } \\
\text { remove all bonding-pad area } \\
\text { gold metallization! }\end{array}$ & (100) Silicon wafer & $\begin{array}{l}\text { adhesion layer } \\
\text { outtered } \mathrm{Si}_{x} \mathrm{~N}_{y} \\
\text { resistor layer } \\
\text { outtered } \mathrm{Si}_{x} \mathrm{~N}_{y}\end{array}$ \\
\hline
\end{tabular}




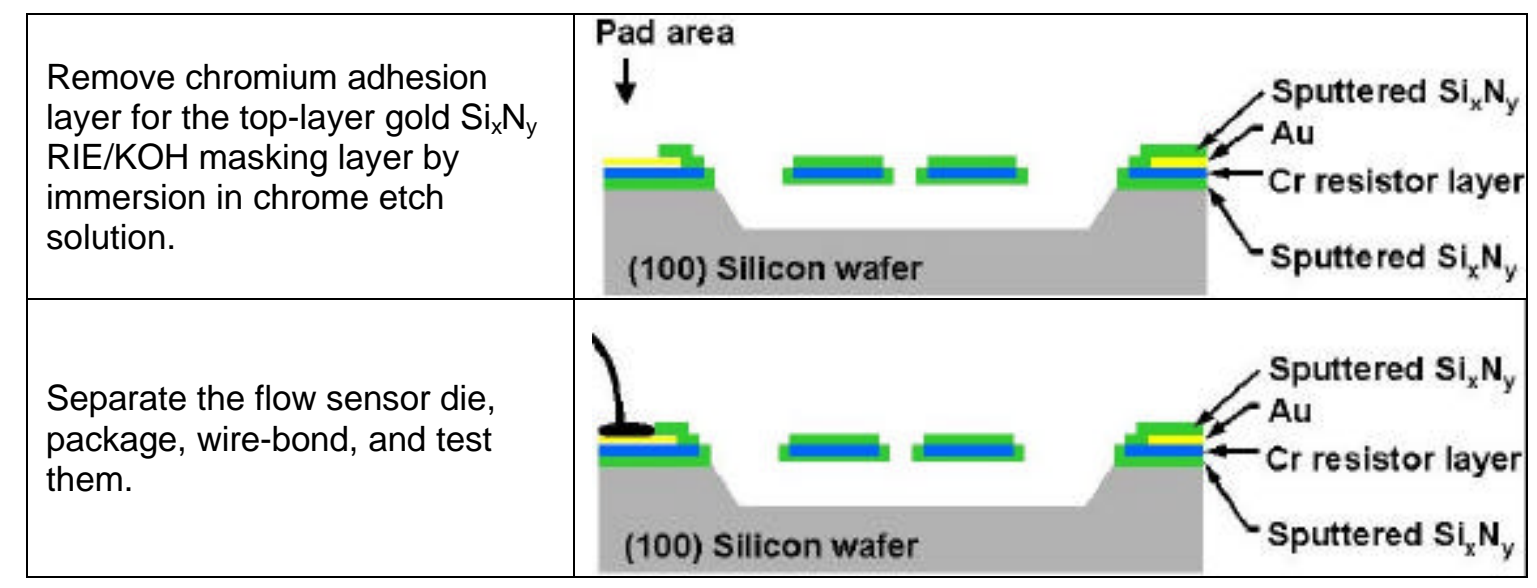

through the silicon nitride layer (down to the silicon substrate) in the RIE system, and anisotropic etching in $\mathrm{KOH}$ to suspend the microbridges/cantilevers. The major difficulty with this concept, was that the process of removing the top gold $\mathrm{RIE} / \mathrm{KOH}$ masking layer after anisotropic etching in $\mathrm{KOH}$ would also degrade or remove exposed bonding-pad-area gold metallization (since the Etch Window Level 3 photomask provided etch windows at both microbridge/cantilever areas and bonding-pad areas). Thus, as shown in Table XVI, the bonding-pad-area-and-routing gold metallization deposited in step five was deposited to be at least twice as thick as the top gold $\mathrm{RIE} / \mathrm{KOH}$ masking layer. This was simply accomplished by using the same DC planar magnetron sputtering parameters to deposit both gold layers (e.g. 350 Watts, 10 mTorr in argon), except adjusting the time of the deposition. Of course, the thickness of any sputter-deposited gold layer varied across each wafer, with the deposited topography depending, in part, on how the wafer was situated above the sputtering target in the Technics system. Controlling the exact gold-etch times in $\mathrm{KI}$ solution was difficult, because the solution etched gold vigorously, and $\mathrm{KI}$ was opaque. Since it was not instantly apparent when the top gold layer had etched away, pad area gold metallization was easily etched too thin, or etched away entirely. This was not necessarily a catastrophic problem, as it should also be noted that gold pad metallization was only required for protecting the underlying chromium resistor metallization from degradation in $\mathrm{KOH}$, but not necessary for wire-bonding and testing of fabricated devices. This process produced intact devices, but with buckled microbridges/ cantilevers, as shown in Figure 85. A collage of SEM photos of sputtered $\mathrm{Si}_{x} \mathrm{~N}_{\mathrm{y}}$ thin film flow sensors and test structures is shown in Appendix XI, 


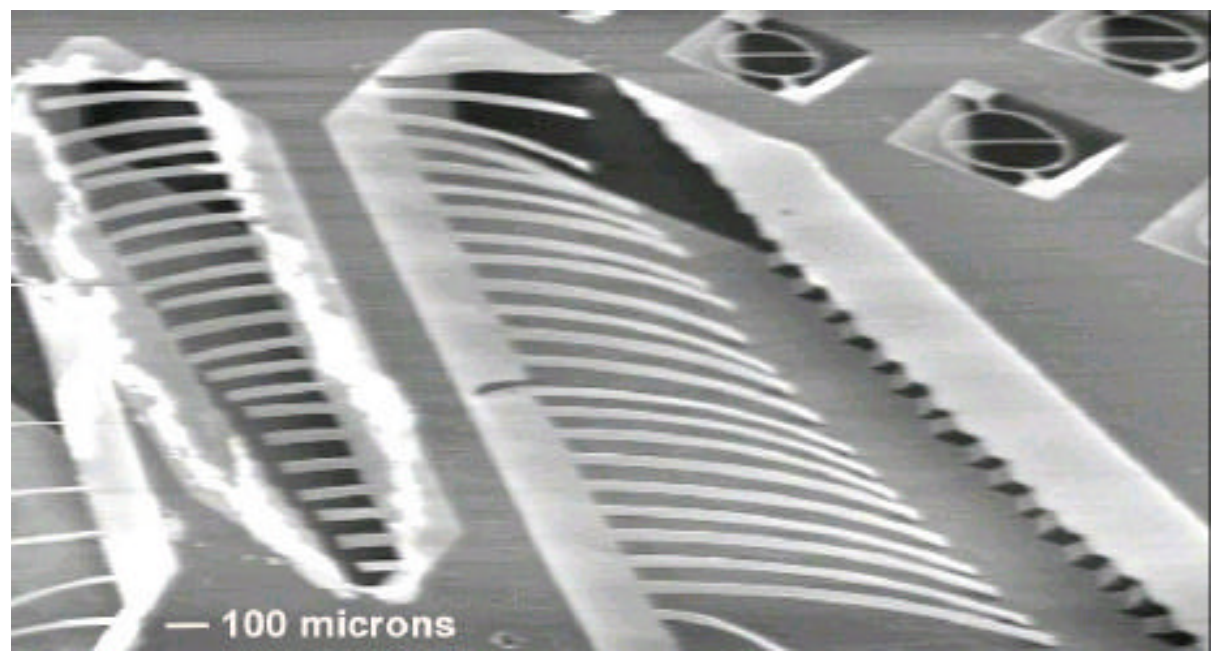

FIGURE 85 - An SEM photo of warped nitrogen-sputtered $\mathrm{Si}_{x} \mathrm{~N}_{y}$ cantilevers. The cantilevers were warped due to intrinsic residual compressive stresses in the thin film layer.

where microbridge/cantilever structures are clearly buckled, indicating the presence of residual compressive stresses within the thin film layer. No $\mathrm{Si}_{x} \mathrm{~N}_{\mathrm{y}}$ films were deposited under tension (which would have been observable by straight microbridges/cantilevers, once suspended from the substrate), and the general film quality was believed to be too unacceptably poor (cracking, porosity, and buckling) for the desired structure of flow sensor microbridges/cantilevers. The insitu stress/strain measurement structures in the 1998/1999 mask set were never utilized to estimate residual stresses in the sputtered $\mathrm{Si}_{x} \mathrm{~N}_{y}$ thin films, because it was decided that another film would be used as the structural layer for the flow sensors. Furthermore, it was also discovered that flow sensor die should not be diced apart after anisotropic silicon etching to suspend the microbridge/cantilever structures, because they were too easily damaged or destroyed by the dicing saw. Subsequent experimental processes placed the dicing procedure before $\mathrm{KOH}$ or TMAH anisotropic etching of the silicon substrates.

In both the 1997 and 1998/1999 processes, poor quality, cracking, porosity, and residual compressive stresses in RF planar magnetron sputter-deposited $\mathrm{Si}_{x} \mathrm{~N}_{\mathrm{y}}$ thin films caused resistor metallization degradation in the anisotropic silicon etchant, $\mathrm{KOH}$. An image showing the porous surface topography of an argon-sputtered nitride film is shown in Figure 86. This problem was somewhat resolved by using a gold RIE/KOH masking layer sputter-deposited on top of the the $\mathrm{Si}_{\mathrm{x}} \mathrm{N}_{\mathrm{y}}$ passivation layer. Since no experimental sputtering parameters were discovered to deposit 


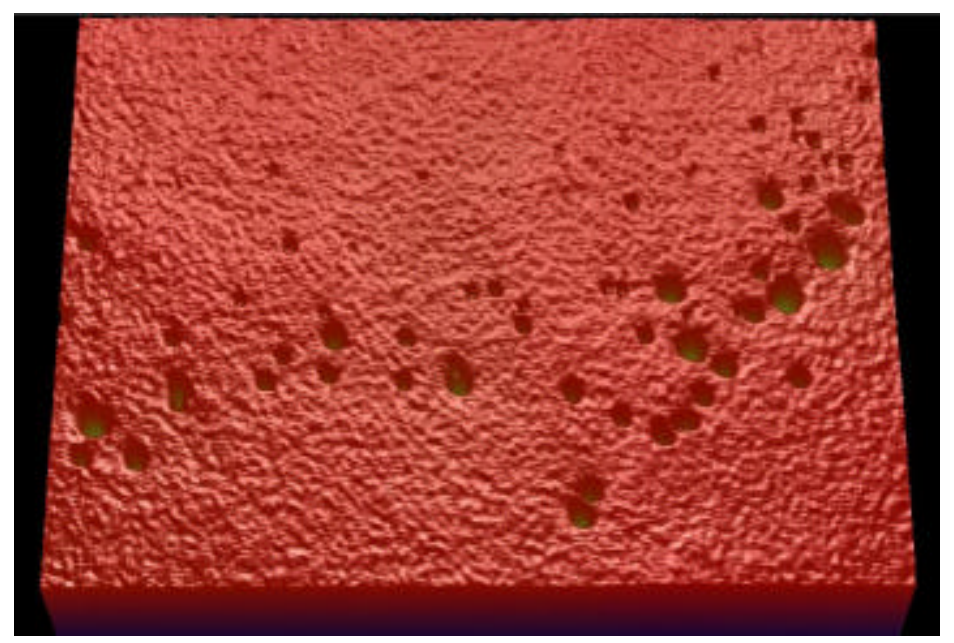

FIGURE 86 - An image of the porous surface topography of a sputtered $\mathrm{Si}_{x} \mathrm{~N}_{\mathrm{y}}$ thin film. This film was RF planar magnetron sputter-deposited in argon at 200 Watts, and 20 mTorr. Its Tencor-profilometer measured thickness was $\sim 0.84 \mu \mathrm{m}$.

thin nitride films under tension, and since it was desired to fabricate microbridges that did not have significant residual compressive stresses affecting the electrical properties of the resistor metallization layer, this process was not explored further.

\section{Spin-on Glass Thin Film Methods}

A fabrication methodology was evaluated in which a spin-on glass (SOG) was used as part of, or as the entire, experimental thin-film flow sensor microbridge/cantilever structural layer. The SOG layer was expected both to have excellent adhesion properties (to thermally-grown oxide, silicon, and itself), and to potentially have residual tensile stress once fully cured. Furthermore, once cured it was expected to be free of cracks with a very low density of pinholes and particulates. Three $10 \mathrm{~cm}$. diameter p-type (100) silicon substrates (with $450-540 \mu \mathrm{m}$ thicknesses), and three three-inch-diameter thermally-oxidized (100) substrates were processed for the SOG experiments. Depending upon the top metal masking layer selected, either TMAH, TMAH silicate $\left[\left(\mathrm{CH}_{3}\right)_{4} \mathrm{NOH} \cdot 2 \mathrm{SiO}_{2}\right]$, or $\mathrm{KOH}$ etchant was used to anisotropically etch the substrates near the end of each fabrication process to suspend the SOG microbridges and cantilevers. A Filmtronics Semiconductor Process Materials 700B spin-on glass was utilized (Filmtronics; Box 1521; Butler, PA 16003; 724-352-3790), with a precision applicator (shown in 
Figure 87) to dispense the SOG onto each substrate. From the data sheet for this glass in Appendix XII ("Silicate $\mathrm{SiO}_{2} 700$ Series Spin-On Glasses"), it was apparent that a maximum film thickness of $7000 \AA$ was theoretically achievable without film cracking using a procedure described to spin-on multiple layers of glass (each $100-300 \mathrm{~nm}$ thick) with intermediate curing steps (at 200 or $400^{\circ} \mathrm{C}$ ) between applications. In a few cases, severe cracking was observed, however, in SOG layers with total thicknesses as low as $\sim 600 \mathrm{~nm}$ (estimated using the Filmetrics

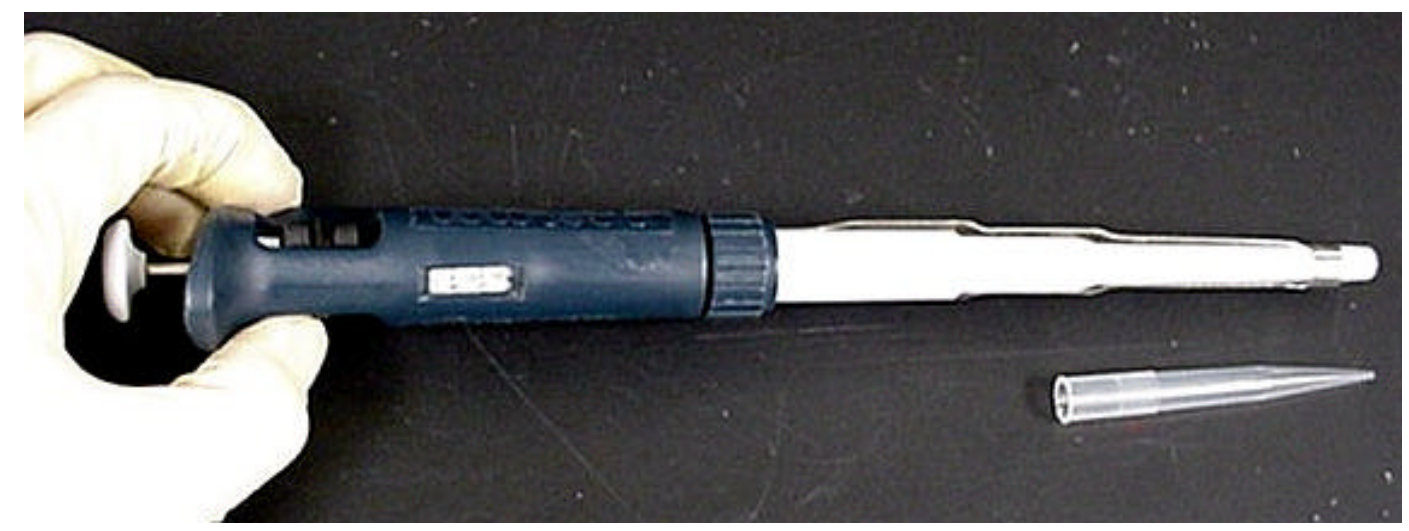

FIGURE 87 - Photo of the metered, precision dispensing tool for applying SOG. This tool was utilized to dispense SOG onto silicon substrates.

system), for glass cured in a furnace at a maximum temperature of $900{ }^{\circ} \mathrm{C}$ for one hour and allowed to slowly cool.

Various applications (i.e. spin-on parameters), thicknesses, and curing schemes for $\mathrm{SOG} / \mathrm{Cr} / \mathrm{Au} / \mathrm{SOG}$ sandwiches and thermally-grown-SiO ${ }_{2} / \mathrm{Cr} / \mathrm{Au} / \mathrm{SOG}$ sandwiches were attempted with intermediate chromium and gold metallization layers. All attempts, except one, failed to produce intact microbridge/cantilever structures. Most fabrication processes failed due to one or more of the following reasons:

1. Glass fracturing (too thick, improperly cured, etc.), sometimes combined with loss of SOG adhesion to the substrate in areas of severe cracking;

2. Particulates embedded in the SOG layers (caused by cured SOG solids falling into the liquid suspension in the $700 B$ bottle itself and appearing in the thin film during spin-on application of the SOG to the substrates with the dispensing tool); 
3. The partial melting, discoloration, or disappearance of the gold metallization layer after top-layer SOG furnace curing (due to the Cr/Au eutectic metal melting point temperature being near the SOG furnace curing temperature chosen);

4. Marginal or unacceptably poor gold adhesion for either the gold (bonding pad and routing) metallization layer or top RIE/KOH/TMAH masking layer;

5. A probable mismatch in thermal expansion coefficients between the glass and intermediate Cr/Au metallization layers, causing cracking in the top SOG layer in areas where it was cured on top of delineated metallization (for SOG/Cr/Au/SOG sandwich processes);

6. Difficulty successfully etching through all SOG layers with BOE (prior to immersing the substrates in $\mathrm{KOH}$ or TMAH to suspend the microbridges/cantilevers), especially on wafers with bottom-layer SOG that was thoroughly cured at $900{ }^{\circ} \mathrm{C}$.

In all cases, it was difficult to achieve good uniformity in the SOG thickness across each substrate, with pre-curing variations measured to be from $\sim 40-270 \mathrm{~nm}$. Since it was observed that the SOG began to rapidly cure once exposed to air, it was important to spin-on the glass quickly after applying it to each wafer.

The only successful SOG process which produced some intact microbridges began by applying 700B SOG (which had been equilibrated to room temperature overnight) to a $10 \mathrm{~cm}$. $\mathrm{p}$ type (100) silicon substrate with the tool shown in Figure 87 . A dial setting of $0.30-0.50 \mathrm{ml}$ was selected on the precision application tool. To spin-on the glass, the Solitec parameters used were a spread of 500 RPM for 0.2 seconds and a spin of 3000 RPM for 10 seconds. The substrate was then pre-baked in air on a digital hotplate at $100{ }^{\circ} \mathrm{C}$ for 13 minutes. Filmetrics system measurements indicated the initial SOG thickness was $\sim 343.9-397.7 \mathrm{~nm}$ thick. The substrate was then placed in a wafer boat and inserted into a Lindberg tube furnace with no forced air or $\mathrm{N}_{2}$ flow. The following SOG curing program was performed:

1. Ramp up from the ambient to $400^{\circ} \mathrm{C}$ at $10^{\circ} \mathrm{C} / \mathrm{min}$.;

2. Hold at $400{ }^{\circ} \mathrm{C}$ for one hour;

3. Ramp up from 400 to $900{ }^{\circ} \mathrm{C}$ at $10^{\circ} \mathrm{C} / \mathrm{min}$;

4. Hold at $900{ }^{\circ} \mathrm{C}$ for one hour;

5. Ramp down to the ambient at $5^{\circ} \mathrm{C} / \mathrm{min}$ and allow the furnace to cool overnight. 
After this cure, the SOG film was observed to be multicolored with a few embedded particulates, but it did not have noticeable cracks. The measured SOG film thickness range (using the Filmetrics system) after curing was $\sim 250.4-295.3 \mathrm{~nm}$, or about $100 \mathrm{~nm}$ less than before curing.

Next, chromium and gold metallization layers were sputter-deposited on top of the initial, cured bottom-layer spin-on glass. A chromium layer was first RF planar magnetron sputterdeposited in high-purity argon at 250 Watts and 10 mTorr, for four minutes (with the chiller at 20 ${ }^{\circ} \mathrm{C}$ ), achieving a profilometer-measured depth of $\sim 58-114 \mathrm{~nm}$. Without breaking chamber vacuum, a gold layer was then DC planar magnetron sputter-deposited in argon at 350 Watts and 10 mTorr for four minutes, achieving a measured depth of $\sim 142-165 \mathrm{~nm}$.

Using previously described standard photolithographic processing, the gold and then chromium metallization layers were delineated using the Gold Metal Level 2 and Resistor Metal Level 1 photomasks, respectively. Shipley 1813 positive photoresist was spun onto the substrate with a ramp value of 600 , a spread value at 500 RPM for five seconds, and a spin value at 4500 RPM for 10 seconds. Soft baking at $95^{\circ} \mathrm{C}$ for $20-25$ minutes preceded 15 second exposure in the Kasper contact mask aligner to $436 \mathrm{~nm}$ UV light at $6.4 \mathrm{~mW} / \mathrm{cm}^{2}$ intensity. The wafer was then immersed in MF-319 developer for $~ 50$ seconds and then rinsed. Hard baking was then performed at $95^{\circ} \mathrm{C}$ for $\sim 25$ minutes. The metallization layer was then etched in $\mathrm{KI}$ solution, if it was gold, or chrome etchant, if chromium. After chromium layer patterning, the substrate was immersed in acetone and Nano-Strip ${ }^{\circledR}$ to remove residual photoresist. The wafer was then baked at $100^{\circ} \mathrm{C}$ for approx. 10 minutes to dehydrate it prior to applying the top SOG layer.

Next, the top layer spin-on glass was applied to the substrate. The 700B SOG bottle was quickly brought up to room temperature (from $\sim 0{ }^{\circ} \mathrm{C}$ ) by placing it in a $70^{\circ} \mathrm{C}$ oven for 30 minutes and then allowed to cool for 30 minutes. The SOG was deposited on the wafer with the aforementioned application tool (using a dial setting of $0.30-0.50 \mathrm{ml}$ ) using a ramp speed of 500 RPM for 0.2 seconds and a spin speed of 6000 RPM for 20 seconds.

The top-layer SOG was then cured by again placing the wafer in a Lindberg tube furnace with no forced air or $\mathrm{N}_{2}$ flow. The furnace temperature was ramped up to $400{ }^{\circ} \mathrm{C}$ from the ambient at $2{ }^{\circ} \mathrm{C} / \mathrm{min}$, held at $400^{\circ} \mathrm{C}$ for one hour, and then ramped back down to the ambient at 
$\sim 2{ }^{\circ} \mathrm{C} / \mathrm{min}$, allowing the wafer and furnace to cool overnight. The measured SOG thickness range (obtained via the Filmetrics system) prior to curing was $656.3-734.8 \mathrm{~nm}$. After furnace curing, the measured thickness range was $586.7-650 \mathrm{~nm}$.

Chromium and gold were then sputter-deposited onto the cured, top SOG layer to provide a BOE (or RIE) etch-window masking layer, for etching through glass areas to silicon at microbridge/cantilever regions. First, the chromium layer was RF planar magnetron sputterdeposited in argon at 250 Watts and 10 mTorr for two minutes. The top gold layer was DC planar magnetron sputter-deposited in argon at 350 Watts and 10 mTorr for 2.5 minutes.

The $\mathrm{Cr} / \mathrm{Au}$ top layers were then delineated using standard photolithographic techniques. The top gold layer was first patterned with 1813 positive photoresist developed and hard baked after UV exposure through the Etch Window Level 3 photomask (and then immersed in KI solution). Then, without removing the photoresist, the underlying chromium layer was delineated by immersion in chrome etch solution. The substrate was then immersed in Nano-Strip® solution for 2-3 minutes to remove residual photoresist prior to patterning the SOG layers.

The SOG layers were then patterned to etch down to the silicon substrate at the microbridge/cantilever areas, and a protective SOG layer was applied to the wafer's backside. The wafer was immersed in BOE solution for approx. four minutes to etch through the SOG layers, and then rinsed in DI water. To protect the wafer backside from silicon etching in TMAH solution, a layer of SOG was spun onto the backside with a spread of 500 RPM for 0.5 seconds and a spin of 4000 RPM for 20 seconds. The backside SOG was then cured on a digital hotplate at temperatures ranging from $100-200{ }^{\circ} \mathrm{C}$ for $\sim 165$ minutes.

Finally, the wafer was diced and then etched in TMAH to suspend the microbridges/ cantilevers. The substrate was diced into quad and duo die, as per the Suggested Wafer Dicing Diagram in Appendix VIII. TMAH solution was chosen to etch the (100) substrate instead of $\mathrm{KOH}$, as it was expected to not degrade the SOG (or $\mathrm{SiO}_{2}$ ) layers, unlike $\mathrm{KOH}$ which noticeably attacks $\mathrm{SiO}_{2}$. A special basket (shown in Figure 88) was fashioned to hold the die in the etchant solution. Small holes were drilled throughout a three-inch-diameter Fluoroware wafer container lid, which was then inverted and secured inside the bottom of a Teflon ${ }^{\mathrm{TM}}$ wafer basket with 


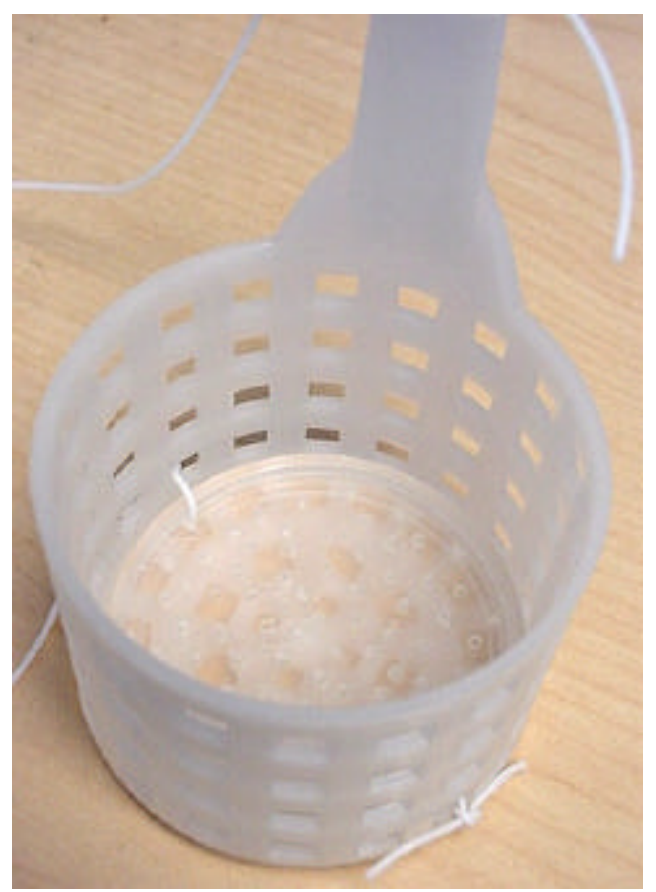

FIGURE 88 - $\quad$ Photo of the customized Teflon ${ }^{\mathrm{TM}}$ die basket used for $\mathrm{TMAH} / \mathrm{KOH}$ etching. This was used for anisotropic etching of individual (100) silicon substrate die to suspend microbridges/cantilevers.

0.47-inch PTFE cord. The holes were drilled in the lid to facilitate slow immersion of the basket into the etchant solution without significantly perturbing or flipping-over the dice. As shown in Figure 89 , a silicon substrate anisotropic etching configuration was set up by pouring fresh $25 \%$ wt. TMAH solution into a quartz beaker on a closed-loop-control, digitally-programmable hotplate. A Teflon ${ }^{\mathrm{TM}}$-coated magnetic stir bar was added to the solution, and the hotplate was programmed to stir at $200 \mathrm{RPM}$. The solution temperature was set at $80^{\circ} \mathrm{C}$. Prior to TMAH etching, each flow sensor quad or duo die was immersed in HF dip (40:1) for $10-30$ seconds, and then rinsed in DI water to remove any residual or native $\mathrm{SiO}_{2}$ layers on exposed silicon areas (since TMAH does not attack $\mathrm{SiO}_{2}$ ). Then the dice were placed face-up in the basket, and it was carefully lowered into the heated TMAH solution. A reflux condenser (with flowing cold tap water) was placed on top of the beaker. To avoid significant concentration changes in the TMAH solution over time, electrical tape was affixed to the interface between the reflux condenser and the quartz beaker in an attempt to create a viable vapor seal. After four hours of elevated-temperature etching, depths of $80-90 \mu \mathrm{m}$ were etched into the silicon substrate. Some die flipped over during etching (due 


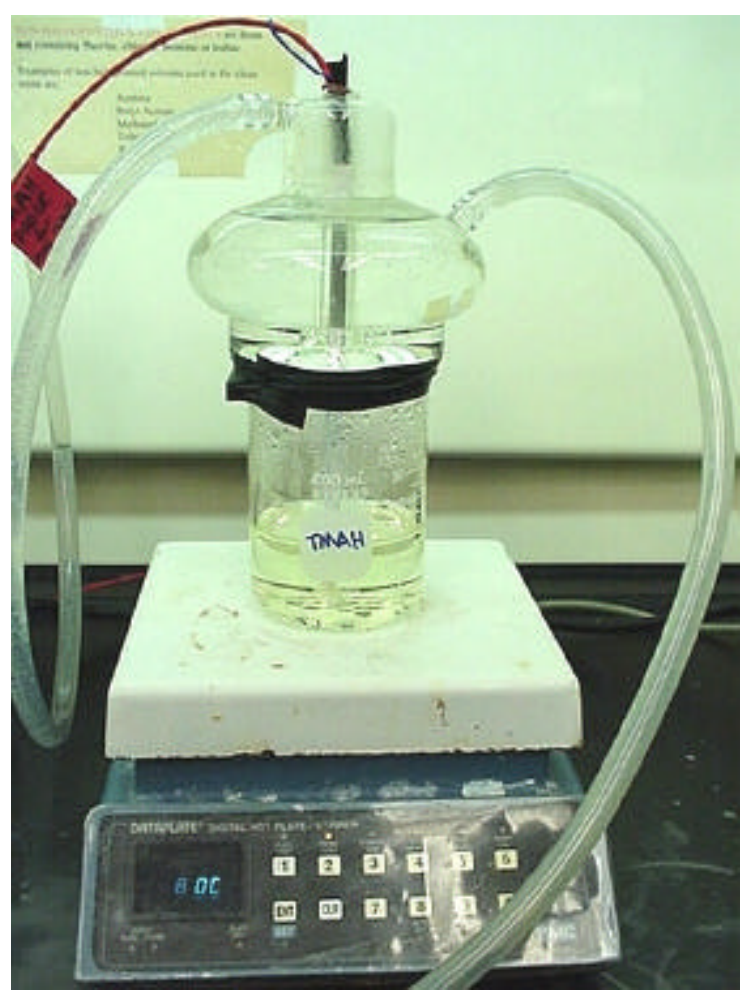

FIGURE 89 - Photo of the TMAH anisotropic (100) silicon etching system. The system had a quartz beaker, reflux condenser, and digital hotplate, used for etching SOG die. Electrical tape was used to seal the reflux condenser to the beaker to minimize changes in TMAH concentration due to escaping water vapor during etching.

to bubbles that formed on them in the solution) which may have reduced their etch rates. After TMAH etching, the top $\mathrm{Cr} / \mathrm{Au}$ etch-window masking layer was removed from each die by careful immersion in $\mathrm{KI}$ and then chrome etch solution.

Only a few suspended microbridges survived this processing methodology intact, despite meticulously careful die handling procedures (e.g. die were not placed under flowing DI water streams after TMAH etching). Those which did survive were curled or buckled along the lateral axis of each suspended structure, as shown in the SEM montage of Figure 90.

The lateral curling of the microbridges might have been caused by the dissimilar curing processes used for the bottom and top layer SOG. The bottom SOG layer was more thoroughly cured at $900^{\circ} \mathrm{C}$ than the top layer, at $400{ }^{\circ} \mathrm{C}$. Since most microbridges did not survive intact, and since those which were intact were also laterally buckled, this SOG sandwich methodology was abandoned for further exploration. 


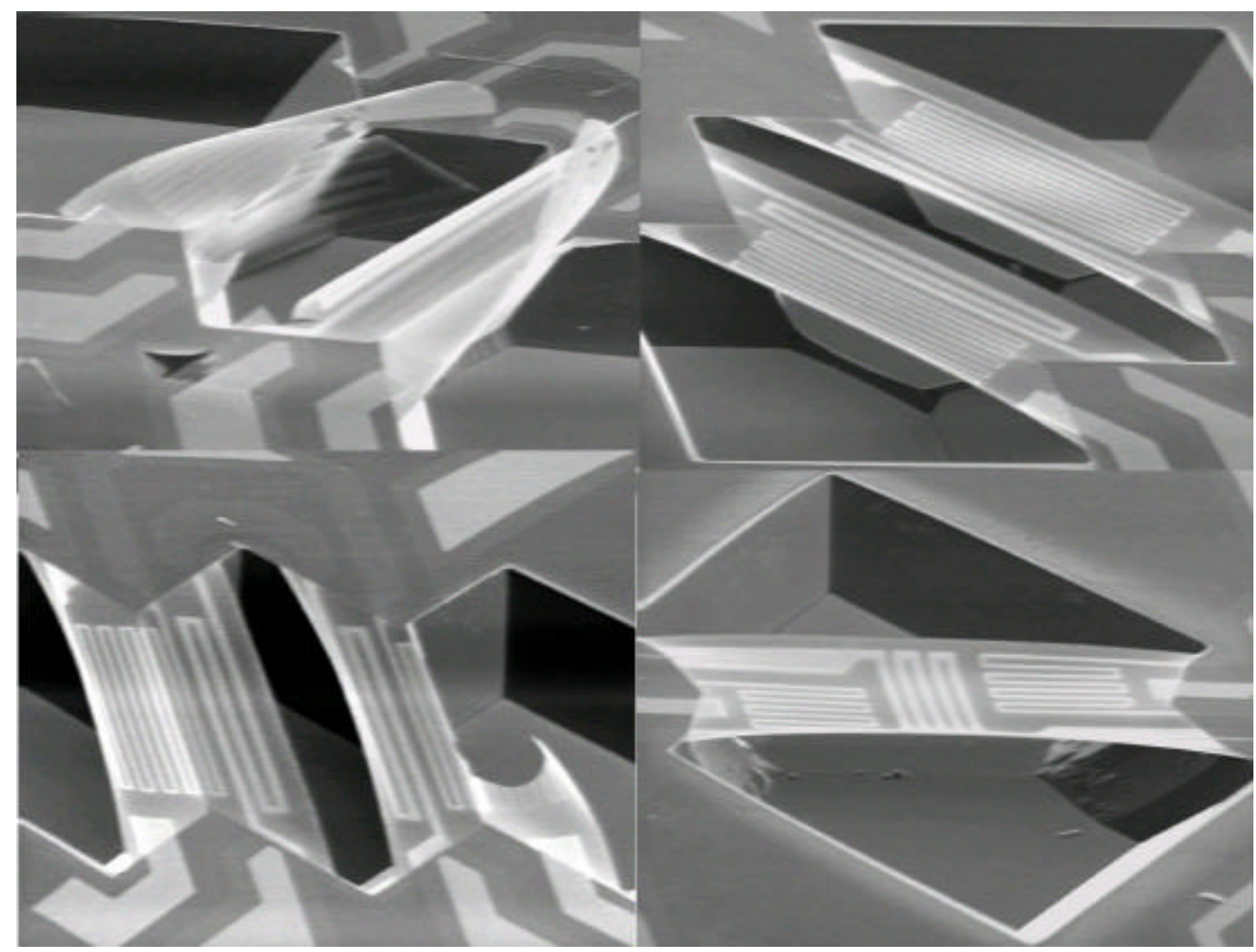

FIGURE 90 - An SEM photo montage of laterally buckled SOG microbridges.

\section{The Low-Stress LPCVD Si $3 \underline{N}_{4}$ Thin Film Method}

An illustrated fabrication summary is shown in Appendix XIII (but a slightly different one is reprinted in Table XVII for convenience), and a detailed fabrication process description is given in Appendix XIV for a method to fabricate flow sensors that proved to be extremely successful. This process used outsourced wafers with low-stress, tensile $0.5 \mu m$-thick LPCVD silicon nitride as the flow sensor microbridge/cantilever structural layer, and thin-film, delineated platinum for the resistor metallization. A gold metallization layer was sputter-deposited (to cover bonding-pad and routing areas), although it was found to be optional, because platinum was discovered to be inert in the anisotropic silicon etchant, $\mathrm{KOH}$. A thin $\mathrm{Si}_{x} \mathrm{~N}_{\mathrm{y}}$ layer was sputter-deposited to provide passivation between the gold metallization layer and the top chromium/gold RIE/KOH etchwindow-masking layer. This top chromium/gold layer was sputter-deposited and subsequently 
TABLE XVII

SUCCESSFUL LPCVD $\mathrm{SI}_{3} \mathrm{~N}_{4}$ FLOW SENSOR FABRICATION SUMMARY

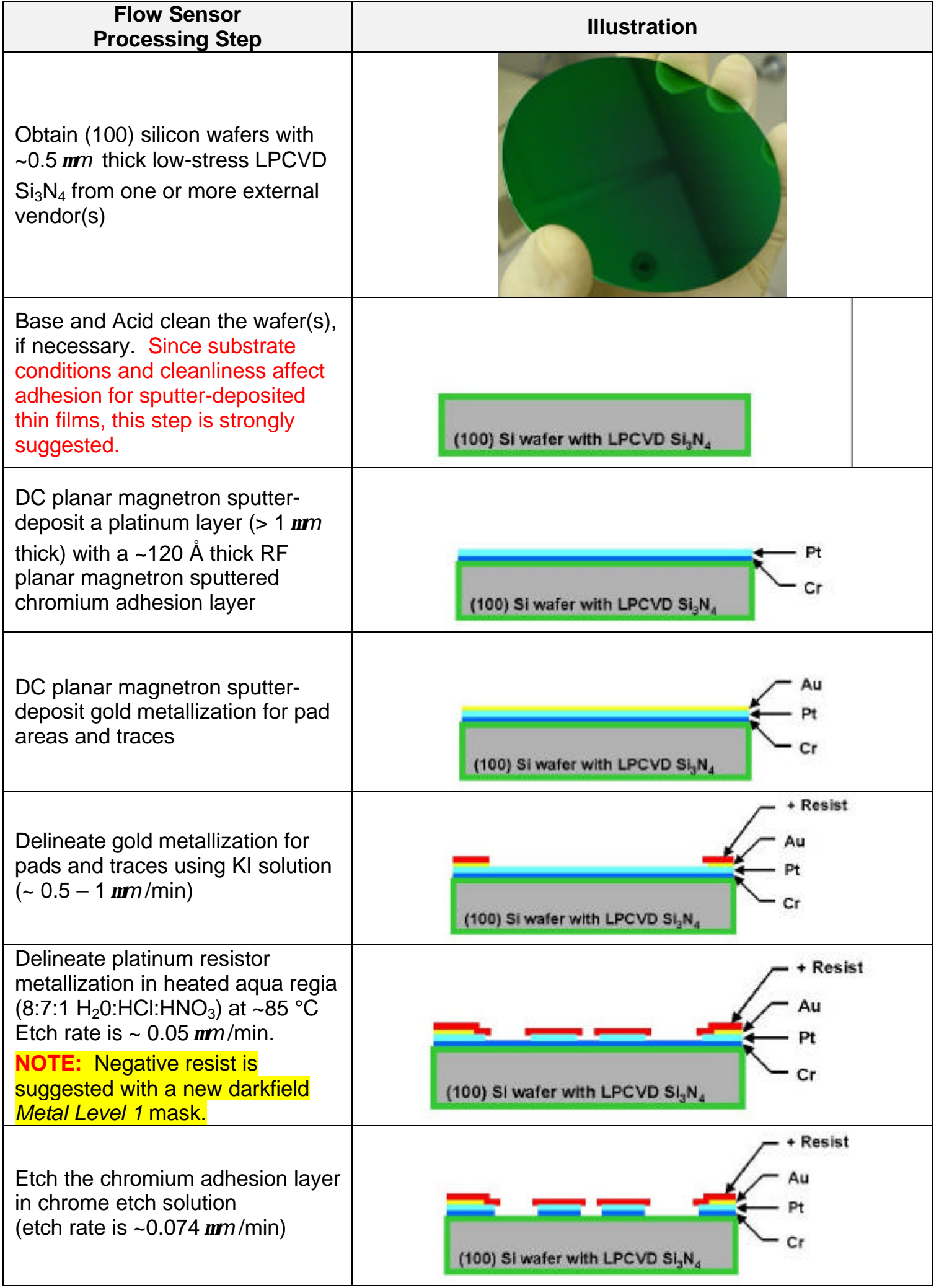




\begin{tabular}{|c|c|c|}
\hline $\begin{array}{l}\text { RF planar magnetron sputter- } \\
\text { deposit a thin silicon nitride } \\
\text { passivation layer }\end{array}$ & (100) Si wafer with LPCVD Sis & $\overbrace{}^{-\mathrm{Sil}_{\mathrm{X}} \mathrm{N}_{\mathrm{r}}}$ \\
\hline $\begin{array}{l}\text { Sputter-deposit the top RIE/KOH- } \\
\text { masking-layer metals: RF planar } \\
\text { magnetron sputter-deposit a } \\
\text { chromium adhesion layer and DC } \\
\text { planar magnetron sputter-deposit } \\
\text { a gold top layer }\end{array}$ & (100) Si wafer with LPCVD $\mathrm{Si}_{3} \mathrm{~N}_{4}$ & $\begin{aligned}-\mathrm{Si}_{\mathrm{x}} \mathrm{N}_{\mathrm{Y}} \\
-\mathrm{Au} \\
-\mathrm{Pt}\end{aligned}$ \\
\hline $\begin{array}{l}\text { Delineate top gold and then } \\
\text { chromium adhesion layers using } \\
\text { the Etch Window Level } 3 \text { darkfield } \\
\text { photomask }\end{array}$ & (100) Si waler with LPCVD Si, $\mathrm{N}_{4}$ & $\mathrm{At}$ \\
\hline $\begin{array}{l}\text { OPTIONAL: Apply layer of } \\
\text { positive resist to backside of the } \\
\text { wafer for silicon nitride RIE } \\
\text { protection (slight etching will } \\
\text { occur on the backside in the RIE) }\end{array}$ & (100) SI wafer with LPCVD $\mathrm{SI}_{3} \mathrm{~N}_{4}$ & $\begin{array}{l}\mathrm{Au} \\
\mathrm{Pt} \\
\mathrm{Cr}\end{array}$ \\
\hline $\begin{array}{l}\text { Dice apart the die into } \\
\text { "quad" and "duo" blocks as per } \\
\text { the Suggested Wafer Dicing } \\
\text { Diagram in Appendix VIII }\end{array}$ & & \\
\hline $\begin{array}{l}\text { Dice apart quad-(die)-sized } \\
\text { microscope glass spacers for } \\
\text { subsequent sensor DIP-24 } \\
\text { packaging }\end{array}$ & & \\
\hline $\begin{array}{l}\text { Reactive lon Etch through all } \\
\text { exposed silicon nitride layers on } \\
\text { individual die, or on the entire } \\
\text { wafer at once. Until an RIE } \\
\text { process is well characterized, } \\
\text { etch a few dice in each run. RIE } \\
\text { etch rate data are in Appendix } X\end{array}$ & (100) Si wafer with LPCVD Sis & $\begin{array}{l}\mathrm{Pt} \\
\mathrm{Cr}\end{array}$ \\
\hline
\end{tabular}




\begin{tabular}{|c|c|c|}
\hline $\begin{array}{l}\text { Bulk-micromachine individual } \\
\text { quad/dual die in } \mathrm{KOH} \text { to release/ } \\
\text { suspend sensor microbridge and } \\
\text { cantilever structures. Empirical } \\
\mathrm{KOH} \text { etch-rate data are included } \\
\text { in Appendix VI. }\end{array}$ & (100) Si wafer with LPCVD SII $\mathrm{N}_{4}$ & \\
\hline $\begin{array}{l}\text { Remove gold and chromium } \\
\text { masking layers from individual } \\
\text { quad/dual die by immersion in KI } \\
\text { and then chrome etch solutions. }\end{array}$ & (100) Si wafer with LPCVD Sis $N_{4}$ & \\
\hline $\begin{array}{l}\text { Using the Wentworth Labs probe } \\
\text { station, test individual flow sensor } \\
\text { resistor electrical continuity and } \\
\text { viability before attempting to } \\
\text { package and wire-bond. } \\
\text { Locate devices with } \\
\mathrm{R}_{U} \approx \mathrm{R}_{\mathrm{D}} \text { (resistances within } \sim 5 \% \\
\text { of each other). }\end{array}$ & & \\
\hline $\begin{array}{l}\text { Package individual quad or duo- } \\
\text { die flow sensors into DIP- } 24 \text { shell. } \\
\text { Use Epo-Tek® } 353 \text { ND epoxy. } \\
\text { Bond a } 45^{\circ} \text { rotated glass-die } \\
\text { spacer to bottom of DIP- } 24 \text { device } \\
\text { cavity and a quad flow sensor die } \\
\text { onto the top of the spacer. } \\
\text { Cure epoxy in an oven at } \sim 140^{\circ} \mathrm{C} \\
\text { for } \sim 4-5 \text { minutes. }\end{array}$ & 具 & \\
\hline $\begin{array}{l}\text { Wire-bond devices which passed } \\
\text { electrical continuity et al. tests, } \\
\text { and devices with } R_{U} R \text { D. } \\
\text { IMPORTANT: For testing in the } \\
\text { ColeJet } 2000^{T M} \text { machine, do NOT } \\
\text { wirebond to DIP pins } 2,6,9,10 \text {, } \\
\text { 11, and } 24\end{array}$ & (100) Si wafer with LPCVD Si & \\
\hline $\begin{array}{l}\text { Test fabricated mass airflow } \\
\text { sensors in the ColeJet } 2000^{T M} \\
\text { machine for flow rates up to } 14 \\
\text { SLPM. Follow operating } \\
\text { instructions in Appendix XXI to } \\
\text { use the machine. For measuring } \\
\text { direct sense-resistor Wheatstone } \\
\text { bridge voltage output, connect an } \\
\text { external DMM with iV resolution, } \\
\text { as described in Appendix XXI. }\end{array}$ & 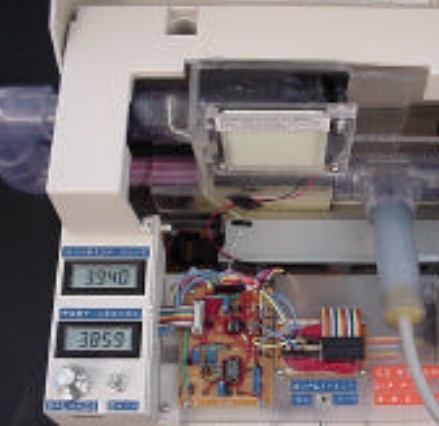 & 10 \\
\hline
\end{tabular}


patterned using the Etch Window Level 3 photomask to provide an inert etch-window metallization mask for both etching through the silicon nitride layer in the March RIE system, and anisotropic etching of the silicon substrate in $\mathrm{KOH}$ to suspend the microbridges/cantilevers. The dice were diced apart prior to reactive ion etching and anisotropic silicon etching to avoid potentially damaging suspended structures with the programmable dicing saw. A speciallymodified Teflon ${ }^{\mathrm{TM}}$ basket (like the one shown in Figure 88) was used to hold the separated die in elevated-temperature, magnetically-stirred $45 \%$ wt. $\mathrm{KOH}$ solution during anisotropic silicon substrate bulk micromachining. Individual die were mounted and epoxied in a standard DIP-24 package, in which up to three flow sensors (out of four maximum) in each quad-die could be directly wire-bonded to the package. Dynamic device testing was performed using the customdesigned ColeJet 2000 machine, which was capable of providing laminar airflow at a volumetric flow rate of up to 14 SLPM across flow sensor die in a $33 \mathrm{~mm}$.-diameter channel.

For this process, four low-stress LPCVD $\mathrm{Si}_{3} \mathrm{~N}_{4}$ wafers were obtained from Chronos Integrated Microsystems (3021 Cornwallis Rd.; Research Triangle Park, NC 27709; 919-2481455), at a price of US $\$ 120$ each, with the following specifications:

- $\quad<100>$ double-side-polished $10 \mathrm{~cm}$. silicon substrate, $400 \mu \mathrm{m}$ thick;

- Low-stress LPCVD silicon nitride on both sides, $0.5 \mu \mathrm{m}$ typical thickness $(0.46-0.54$ $\mu m$ );

- Residual stress in silicon nitride layer, specified to be typically +60 MPa and tensile (ranging from +20 MPa to +140 MPa);

- Index of refraction of silicon nitride, 2.15-2.30.

Each wafer was then base and acid cleaned (as previously described in the Anodically Bonded Borosilicate Glass Method, and in Appendix XIV) to prepare for platinum resistor and gold metallization sputter deposition. Platinum-based devices were successfully fabricated on only one-half of one of the four wafers (hereafter designated PT1), as other unsuccessful metallization efforts (including annealing) were attempted on the remaining wafers.

A platinum resistor metallization layer and top gold metallization layer were DC planar magnetron sputter-deposited in the Technics sputtering system onto wafer PT1. Since platinum adhesion to these $\mathrm{Si}_{3} \mathrm{~N}_{4}$ substrates was found to be very poor (platinum layers easily peeled off with an electrical-tape-test), a thin adhesion layer of chromium was used (for convenience), 
because it is well known for its excellent adhesive properties with respect to nearly all other materials. It should also be noted that titanium and tantalum would be suitable pure-elemental adhesion layers for platinum $[81,82]$. The chromium adhesion layer was RF planar magnetron sputter-deposited (after reaching a base pressure of $\sim 7 \times 10^{-6}$ Torr) at 250 Watts and $10 \mathrm{mTorr}$ in argon for 30 seconds (with the chiller at $20^{\circ} \mathrm{C}$ ) to achieve an approximate thickness of $120 \AA$ (with a $~ 24-29.2 \mathrm{~nm} / \mathrm{min}$ deposition rate). Without breaking vacuum in the system, platinum was DC sputter-deposited at 200 Watts and 10 mTorr in argon for 10 minutes, achieving a deposition rate of $\sim 6.8-8.7 \mathrm{~nm} / \mathrm{min}$. The thickness of both the platinum and chromium adhesion layers was measured to be $\sim 70-87 \mathrm{~nm}$ with the Tencor profilometer. Next, optional gold metallization was sputter-deposited on top of the platinum layer. Since platinum does not oxidize easily, wafer PT1 was temporarily exposed to the air without adversely affecting gold adhesion. Vacuum in the sputtering system was broken while the platinum-foil target was replaced with a gold target, and within ten minutes the chamber was again being evacuated with wafer PT1 inside. A gold layer was then DC planar magnetron sputter-deposited at 350 Watts and 10 mTorr in argon for eight minutes (with an initial base pressure below $\sim 7 \times 10^{-6}$ Torr) to achieve a Tencorprofilometer-measured film thickness of $0.285-0.375 \mu \mathrm{m}$. An electrical tape test on the edge of the wafer showed that $\mathrm{Cr} / \mathrm{Pt} / \mathrm{Au}$ adhesion to the substrate was excellent, and gold did not peel off of the underlying platinum layer.

The top gold bonding-pad-area-and-routing layer was then delineated using a standard photolithographic process, the details for which are given in Appendix XIV, where applied photoresist was exposed through the Gold Metal Level 2 photomask in the Kasper aligner. Shipley 1813 photoresist applied to the wafer was either soft-baked for $20-30$ minutes at $95{ }^{\circ} \mathrm{C}$ with forced air convection in a Lindberg/Blue $\mathrm{M}$ oven or for approximately five minutes at $115^{\circ} \mathrm{C}$ on a hotplate, in atmosphere. After hard-baking the photoresist for approximately 20 minutes at $95^{\circ} \mathrm{C}$ in a Lindberg/Blue $\mathrm{M}$ oven, the gold layer was patterned by immersing wafer $\mathrm{PT} 1$ in $\mathrm{KI}$ solution (with agitation) for less than one minute.

Next, the platinum resistor metallization layer was photolithographically patterned. Shipley 1827 positive photoresist was used to delineate this layer in heated, diluted aqua regia, 
because it was hoped that a thicker photoresist layer might survive better. However, because both 1827 and 1813 photoresist peeled away from the platinum layer in the aqua regia solution, potential device yield was reduced by as much as $50 \%$. After applying and patterning the photoresist (with standard techniques) using the Resistor Metal Level 1 photomask in the Kasper aligner, wafer PT1 was hard-baked for 70 minutes. A diluted aqua regia solution was set up to etch the platinum layer [77], as shown in Figure 91, where $400 \mathrm{~mL}$ of $\mathrm{DI} \mathrm{H}_{2} \mathrm{O}$ (8 parts) was mixed with $350 \mathrm{~mL}$ or $\mathrm{HCl}$ (7 parts) and $50 \mathrm{~mL}$ of $\mathrm{HNO}_{3}$ (1 part) in a Pyrex ${ }^{\mathrm{TM}}$ beaker and heated to $~ 85$ ${ }^{\circ} \mathrm{C}$ on a hotplate, with occasional manual stirring. The solution bubbled with its color changing from transparent, to yellow, as it was heated. Another Pyrex ${ }^{\mathrm{TM}}$ beaker with $\sim 1000 \mathrm{~mL}$ of DI water was heated to $\sim 80^{\circ} \mathrm{C}$ on another hotplate to stop platinum etching without thermally shocking the delicate photoresist in room temperature DI water (so etching could be continued, if needed), The photoresist tended to lose adhesion to the underlying platinum layer and become brittle in aqua regia. Once immersed in the diluted aqua regia solution, the $\sim 680-820 \AA$ thick platinum layer on PT1 was expected to etch very quickly. Photoresist on the wafer immediately turned a deep red color upon immersion. The silvery platinum layer etched away starting from the outside periphery of the wafer, "creeping" inwards (as sputtering typically results in maximum film thickness at the center of a substrate, decreasing in thickness radially outwards), slowly changing the color of the wafer from silvery to dull green (the color of the green LPCVD $\mathrm{Si}_{3} \mathrm{~N}_{4}$ layer with a thin, translucent chromium adhesion layer on top). Within 70 seconds, the discoloration change

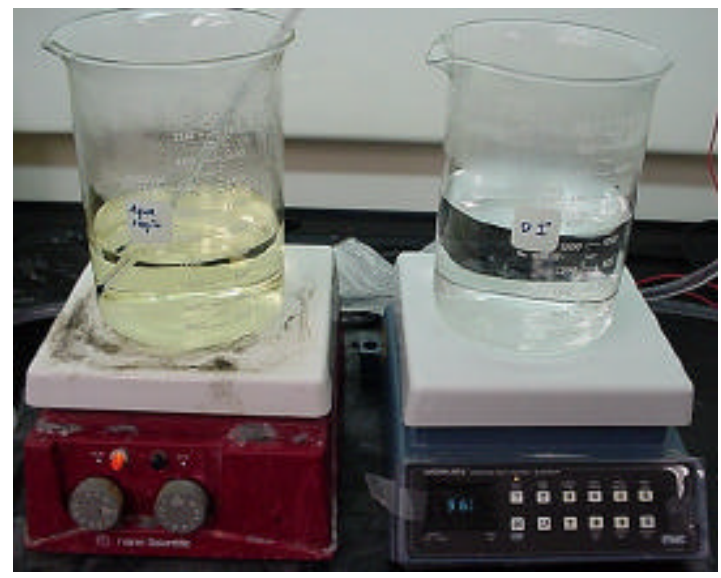

FIGURE 91 - A photo of diluted, heated aqua regia set-up for delineating platinum. Aqua regia is on the left and heated DI rinse water is shown on the right. 
across the wafer had stopped, and it was assumed platinum etching was finished. The wafer was immediately removed from the aqua regia solution, and immersed in the warm, static DI water bath for approximately two minutes to stop platinum etching. The wafer was then allowed to cool in air before immersion in a static room-temperature DI water bath for approximately five minutes. Wafer PT1 was then gently blow-dried with high-purity nitrogen. After high-magnification inspection under a microscope, a hazy "snow" effect was seen around $5 \mu \mathrm{m}$-line-width resistors that were not sharply delineated. Thus, PT1 was re-immersed in the aqua regia solution for 20 seconds to more sharply delineate the platinum layer and then rinsed in tap DI water for about five minutes. Finally, the wafer was immersed for about eight seconds in chrome etch solution to remove the exposed chromium adhesion layer, rinsed in DI water, and dried with nitrogen. It was very obvious when the chrome etching process was finished, as the wafer surface changed from a dull to bright-green color. It was important to not unnecessarily prolong this chromium adhesion-layer etching step, as it was found that over-etching could undercut many of the platinum $5 \mu \mathrm{m}$-line-width resistors (removing the Pt adhesion layer), causing resistors to lift-off the wafer. Wafer PT1 is shown in Figure 92 after platinum and chromium-adhesion-layer etching, and before residual photoresist was removed from it. After a total of $\sim 90$ seconds of immersion in the platinum-etch solution, about $50 \%$ of the $5 \mu \mathrm{m}$-line-width resistors on the wafer survived.

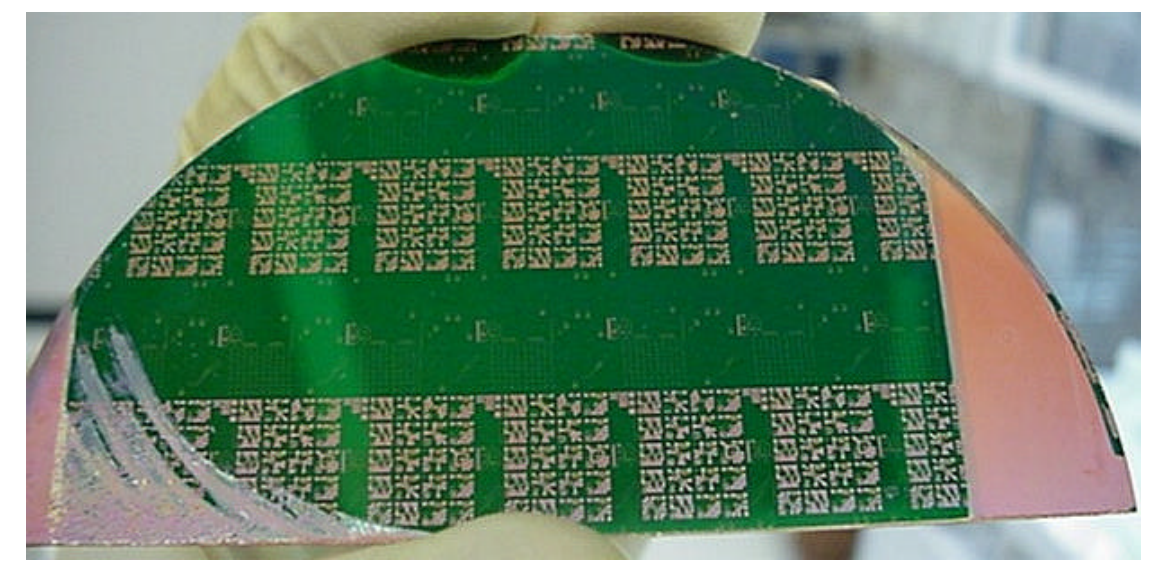

FIGURE 92 - Photo of wafer PT1 after delineating the platinum metal layer. Platinum resistor metallization was patterned in a diluted, heated aqua regia mix. The photoresist appeared charred and changed to a red color (seen in this photo) after platinum etching. Most all of the $5 \mu \mathrm{m}$-line-width resistors in blocks of devices along the bottom straight edge of the wafer did not survive platinum etching intact. 
After platinum etching, inspection under a microscope showed significant damage to, or destruction of, delineated platinum resistor areas due to photoresist charring, cracking, or peeling away from the platinum layer in the aqua regia solution, resulting in a yield of about $50 \%$ for this wafer. This solution also degraded or destroyed any gold metallization areas that were exposed by photoresist lift-off (which would theoretically not damage flow sensor viability). Figure 93 shows examples of photoresist lift-off problems experienced, and Figure 94 shows examples of $5 \mu m$-line-width resistors which were over-etched due to undercutting of the photoresist by the

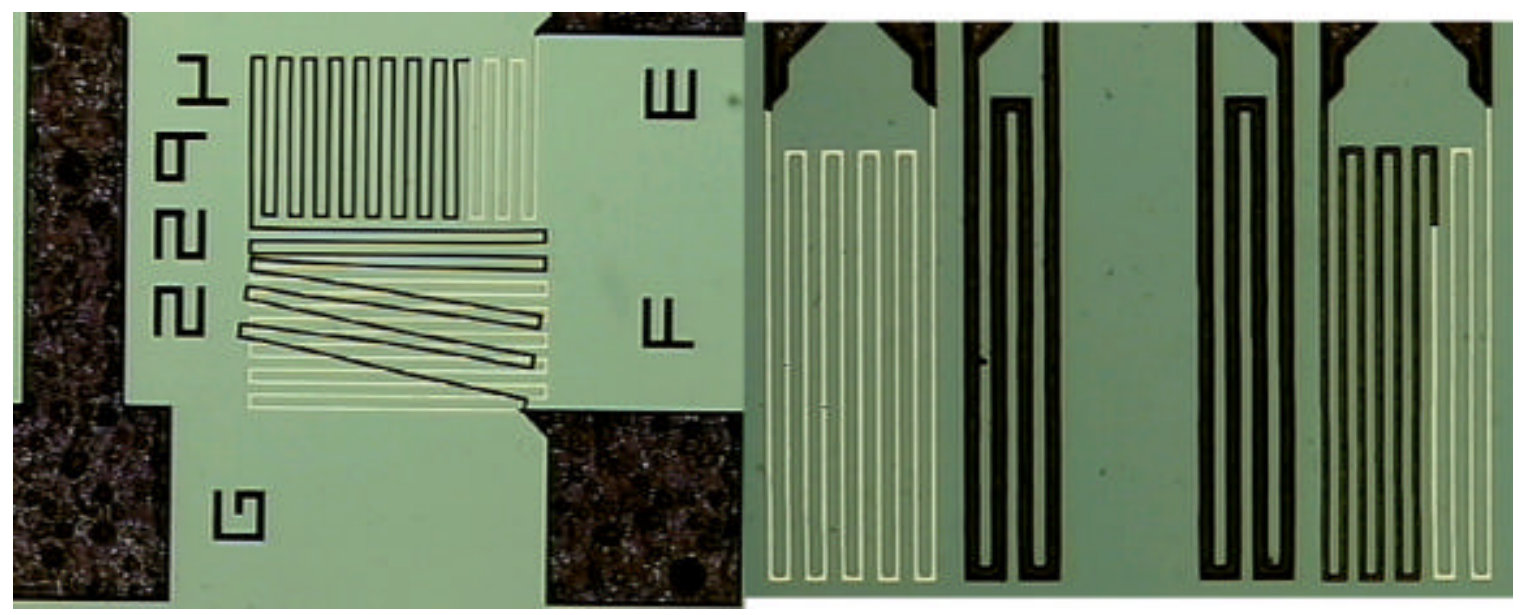

FIGURE 93 - Examples of poor positive photoresist adhesion to Pt. after delineation. Lift-off on $5 \mu \mathrm{m}$-line-width resistors occurred during platinum delineation in aqua regia. In some cases, brittle photoresist may have peeled off during nitrogen blowdrying of the wafer.

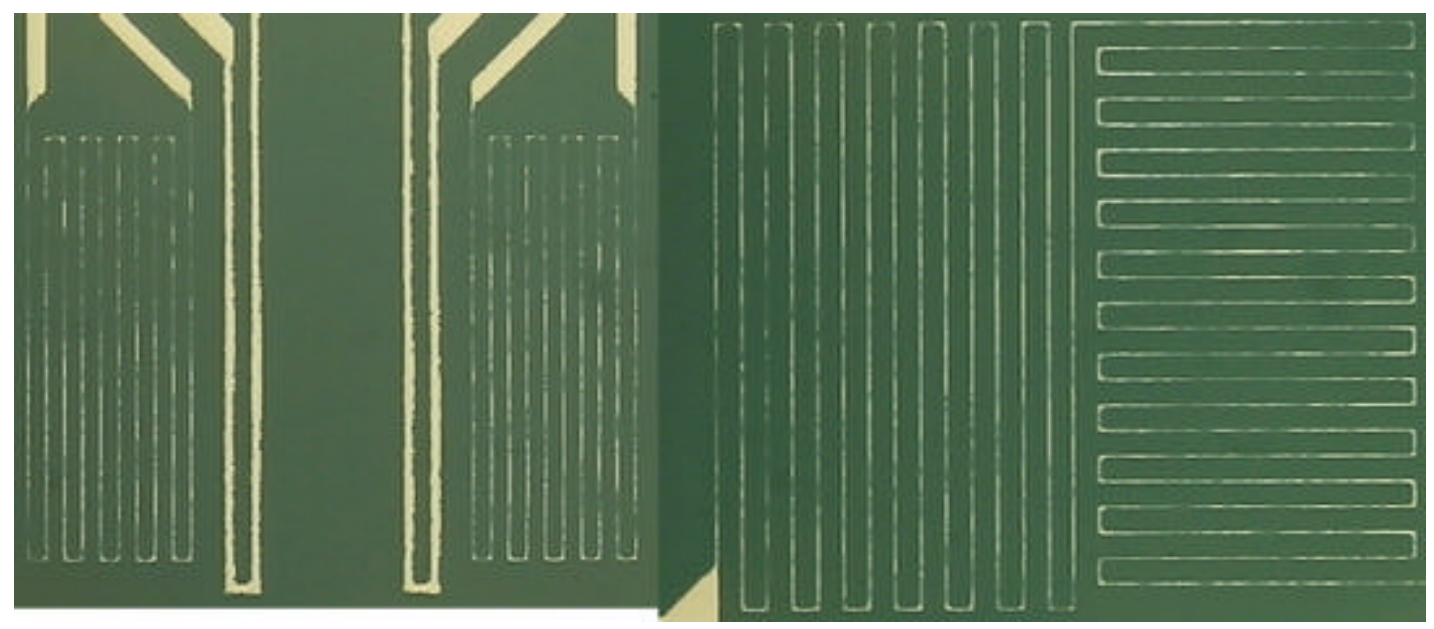

FIGURE 94 - Photos of over-etched $5 \mu \mathrm{m}$-line-width platinum resistors. Excessive undercutting of the photoresist occurred in the aqua regia solution. 
aqua regia solution. It is important to note that this platinum layer was very thin $(\sim 68-82 \mathrm{~nm})$, and the etch duration quite short, yet device yield was very poor. If a sputter-deposited platinum thin film were desired with maximum TCR characteristics, it should be thicker than $350 \mathrm{~nm}$ [18], which would take at least four to five times longer to delineate in this aqua regia mix. It is, therefore, not likely that these $5 \mu \mathrm{m}$-line-width resistors would be delineated, intact, after etching in the solution for this duration. This is perhaps why platinum thin films are typically dry-etched with RIE chemistries, rather than wet-etched.

The next step was to sputter-deposit a $\mathrm{Si}_{\mathrm{x}} \mathrm{N}_{\mathrm{y}}$ thin-film passivation layer on top of the platinum and gold resistor metallization layers on the wafer, prior to depositing and patterning the top gold RIE/KOH etch-window-masking layer. This passivation layer was needed to separate the resistor and gold metallization layers (a $\mathrm{Cr} / \mathrm{Pt} / \mathrm{Au}$ sandwich) from the top $\mathrm{RIE} / \mathrm{KOH}$ etchwindow-masking layer (an additional $\mathrm{Cr} / \mathrm{Au}$ sandwich). Without this separation, the process of removing the chromium adhesion layer for the top RIE/KOH etch-window-gold-masking layer (after microbridges/cantilevers were separated from the substrate in $\mathrm{KOH}$ ) could also degrade the chromium adhesion layer under platinum resistors, cause platinum lift-off, cause gold lift-off, or all three. In other words, with this $\mathrm{Cr} / \mathrm{Pt} / \mathrm{Au} / \mathrm{Cr} / \mathrm{Au}$ sandwich, it was not clear whether removing the chromium layer between the gold layers would also cause lift-off of the underlying gold layer. After residual photoresist was removed from $P T 1$, an $\mathrm{Si}_{x} \mathrm{~N}_{\mathrm{y}}$ thin film was $\mathrm{RF}$ planar magnetron sputter-deposited (with the chiller at $20^{\circ} \mathrm{C}$, after achieving a base pressure of $1.5 \times 10^{-5} \mathrm{Torr}$ ) at 450 Watts, in nitrogen, at 15 mTorr, for seven minutes to achieve a desired target thickness of $\sim 50 \mathrm{~nm}$.

Next, a top inert gold RIE/KOH etch-window masking layer was sputter-deposited onto the wafer, with a chromium adhesion layer underneath, and then both layers were patterned. It should be noted that this etch-window-masking layer could instead be aluminum, since no masking layer was needed in $\mathrm{KOH}$ (as platinum, gold, and the LPCVD $\mathrm{Si}_{3} \mathrm{~N}_{4}$ layer were all found to be inert in $\mathrm{KOH}$ ), but a masking layer was needed to anisotropically etch through the $\mathrm{Si}_{3} \mathrm{~N}_{4}$ thin film in the RIE. A disadvantage of using gold was that the process of removing it after anisotropic etching of the substrate in $\mathrm{KOH}$, would also degrade exposed gold at the bonding-pad areas (but 
this would not result in untestable devices, unless wire-bonds with good ohmic contact could not be made to platinum bonding pads). This was similar to the problem experienced in the SputterDeposited Silicon Nitride Thin Film process for fabricating flow sensors, which was somewhat resolved by depositing a bonding-pad gold metallization layer that was at least twice as thick as the top gold etch-window-masking layer. The chromium adhesion layer was RF planar magnetron sputter-deposited (after achieving a base pressure of $7.4 \times 10^{-6}$ Torr) at 250 Watts, in argon, at $10 \mathrm{mTorr}$ for 30 seconds to achieve a nominal $\sim 12 \mathrm{~nm}$ thickness. Without breaking vacuum, a gold layer was DC planar magnetron sputter-deposited at 350 Watts, and 10 mTorr in argon for four minutes, to achieve a target thickness of $\sim 165 \mathrm{~nm}$. The top gold and chromium layers were then patterned by standard photolithographic techniques, exposing 1813 positive photoresist through the Etch Window Level 3 photomask, etching the gold layer in $\mathrm{KI}$, and etching the thin chromium adhesion layer in chrome etch solution.

Before RIE and $\mathrm{KOH}$ processing, wafer PT1 was diced into quad and duo-die arrangements (as shown in Appendix VIII) with the Micro-Automation Programmable Dicing Saw. Before dicing, a protective layer of positive photoresist was spun onto the backside of the wafer to inhibit reactive ion etching of the backside $\mathrm{Si}_{3} \mathrm{~N}_{4}$ thin film in the March RIE system. The photoresist was then cured in a Lindberg/Blue $\mathrm{M}$ oven at $100^{\circ} \mathrm{C}$ for 15 minutes. The dicing saw was programmed as per the Micro-Automation Dicing Saw SOP, in Appendix IX, with the following parameters, in metric units, prior to cutting:

1. Mode: 60 (for progressive-mode cuts);

2. $\mathbf{1}^{\text {st }}$ Index: $2.3 \mathrm{~mm}$. (the street width between individual flow sensors);

3. $2^{\text {nd }}$ Index: $2.3 \mathrm{~mm}$.;

4. Height (amount of substrate to leave undiced): 0.150 or $0.200 \mathrm{~mm}$;

5. Angle: $90^{\circ}$;

6. Speed: $1.27 \mathrm{~mm} / \mathrm{s}$ (the slowest possible speed for cutting).

The wafer was treated as a "round" specimen, with its maximum dimension (in mm.) entered as the Diameter parameter. The versatile blue adhesive film was applied to the backside of $P T 1$ (while eliminating and popping air pockets), and then cut out around (and in the shape of) the wafer. This film was used to hold the die together during the dicing process. The total micrometer-measured thickness of the substrate and blue film was then entered (in $\mathrm{mm}$.) as the Thickness parameter. Typically, the blue film had a measured thickness of $70-75 \mu \mathrm{m}$. A 
progressive-depth-cut-distance parameter was entered (with the zero key on the saw) to allow for three passes per cut (this was approximately the Thickness of the sample divided by three). The saw was operated in single-cut, manual mode for each pass after the diamond blade was aligned to cut through each street.

Glass microscope slides were then diced into "quad-die" sizes using the dicing saw. These were needed to provide simple spacers in the DIP-24 package to elevate each quad (or duo-) flow sensor die out of the package cavity and into the flow stream (flowing longitudinally across the main axis of the package, as shown in Figure 74). The dicing saw was programmed as per the Micro-Automation Dicing Saw SOP, in Appendix IX, with the following parameters, in metric units, prior to cutting:

1. Mode: 60 (for progressive mode cuts);

2. $\mathbf{1}^{\text {st }}$ Index: $4.6 \mathrm{~mm}$. (the street width spacing between two contiguous sensors);

3. $2^{\text {nd }}$ Index: $4.6 \mathrm{~mm}$.;

4. Height (amount of glass to leave uncut): $0.400 \mathrm{~mm}$;

5. Angle: $90^{\circ}$;

6. Speed: $1.27 \mathrm{~mm} / \mathrm{s}$ (the slowest possible speed for cutting).

The total micrometer-measured thickness of the glass slide was then entered (in $\mathrm{mm}$.) as the Thickness parameter. The adhesive blue film was not used (due to the larger Height of glass left after each cut). A progressive-depth-cut-distance parameter was entered (with the zero key on the saw) to allow for two passes per cut (this was approximately the Thickness of the sample divided by two). The saw was operated in single-cut, manual mode for each pass. No alignment was required between passes, other than to manually index the saw to the next street for cutting.

Next, each die (or, up to approximately six quad or duo-die) was individually reactive ion etched in the March RIE system. Although, in practice, all dice in PT1 could have been etched simultaneously (after removing the adhesive blue-plastic-film backing), measured theoretical etch rates for a given RIE chemistry typically decreased with increased loading of the chamber, and an increased etch duration might also etch through (i.e. oxidize by RF cathodic pulverization) the entire top gold RIE/KOH masking layer. The patterned top gold layer on each die was first wiped off with an acetone-soaked cotton swab to remove contaminants left by the dicing operation, and any residual photoresist on top of the die (because residual photoresist on top of exposed $\mathrm{Si}_{3} \mathrm{~N}_{4}$ would inhibit etching). It should also be noted that photoresist would have likely provided a 
suitable masking layer, but a gold metallization top-masking layer was utilized simply to reduce chances of failure due to excessive degradation of a photoresist masking layer in the RIE. Previous March RIE system plasma etch chemistry experiments with $\mathrm{Si}_{3} \mathrm{~N}_{4}$ dice from other Chronos LPCVD low-stress nitride wafers in this batch provided etch rate data, which are shown graphically in Appendix X. From these data, a moderately-aggressive nitride etching chemistry was selected: with a $\mathrm{CF}_{4} \mathrm{MFC}$ flow rate of $15.375 \mathrm{sccm}$, an etching pressure of 200 mTorr (with Independent or Automatic Pressure Control enabled), an RF power setting of 300 Watts, and a chiller temperature of $\sim 20^{\circ} \mathrm{C}$. Individual quad or duo-die were separated from the adhesive blueplastic-film backing and placed in the RIE chamber. Once loaded with one to six quad/duo-die, the RIE chamber was pumped down to a base pressure of $\sim 57 \mathrm{mTorr}$ prior to etching. Previous experiments showed the nitride etch rate was $\sim 251.5 \mathrm{~nm} / \mathrm{min}$ with this chemistry. After 270 seconds of etching, the nitride layer was etched through with a total Tencor-profilometermeasured etched depth of $0.96-1.25 \mu \mathrm{m}$ into the substrate (about $\sim 0.5 \mu \mathrm{m}$ of which was the nitride layer). For these dice, the gold/chrome RIE/KOH etch window masking layer survived etching with residual gold on top, and had a measured thickness of $\sim 0.17-0.19 \mu \mathrm{m}$. Four minutes of etching with this chemistry resulted in measured etch depths of $0.66-0.69 \mu \mathrm{m}$ into the die substrates. It is important to note that measured $\mathrm{Si}_{3} \mathrm{~N}_{4}$ etch rates were originally established from etching experiments where the nitride layer was not etched completely through (as these $4-4.5$ minute etch runs have combined silicon and silicon nitride etch rates). After etching in the RIE system, each die was immersed in acetone to remove all residual photoresist (especially from the backside). In between etching runs, the RIE system chamber was cleaned of organic residues with an oxygen plasma, at maximum MFC high-purity oxygen flow, at 400 Watts $\mathrm{RF}$, for at least five minutes. If the chamber appeared excessively coated with Teflon ${ }^{\mathrm{TM}}$ residue, it was mechanically/abrasively cleaned with a Scotch-Brite ${ }^{\circledR}$ pad and then vacuumed.

To prepare for anisotropic silicon etching of the die substrates in heated, magneticallystirred $45 \%$ wt. $\mathrm{KOH}$ solution, a basket was made to hold the die. This was the same type of basket previously described and shown in Figure 88. Small holes were drilled throughout a threeinch-diameter Fluoroware wafer container lid, which was then secured (with the top, face-down) 
inside the bottom of a Teflon ${ }^{\mathrm{TM}}$ wafer basket with 0.47 -inch diameter PTFE cord. The $\mathrm{KOH}$ solution was setup in a quartz beaker with reflux condenser on a closed-loop-control programmable Dataplate ${ }^{\mathrm{TM}}$ hotplate, as shown in Figure 61 . Three separate $\mathrm{KOH}$ etches were setup at different solution temperatures, depending upon the desired etch duration (see Appendix VI for $\mathrm{KOH}$ etch-rate data). One etch lasted for 13.67 hours in $57^{\circ} \mathrm{C}$ solution with $150 \mathrm{RPM}$ stirring (with a Teflon ${ }^{\mathrm{TM}}$-coated magnetic stir-bar), achieving a depth of between 100 and $110 \mu \mathrm{m}$ into the silicon substrate. Another etch was performed for several dice in $64^{\circ} \mathrm{C}$ solution with $150 \mathrm{RPM}$ stirring for 13.78 hours, resulting in an etched depth of $\sim 170 \mu \mathrm{m}$ into the dice substrates. A third etch was performed in $70{ }^{\circ} \mathrm{C}$ solution with 150 RPM stirring for 7.17 hours, with a resulting etched depth of between 140 and $150 \mu \mathrm{m}$ into the substrates. To release all 20 flow sensor microbridges/cantilevers, a total etched depth of about $180 \mu \mathrm{m}$ into the substrate was needed (to release the largest microbridge in Flow Sensor \#10). After $\mathrm{KOH}$ etching, the die basket was carefully immersed in several static DI water baths for rinsing. None of the dice were rinsed in running tap DI water, and none of the dice were blow dried with high-purity nitrogen. Tweezers were used to carefully remove each quad/duo die from the Teflon ${ }^{\mathrm{TM}}$ basket. Water was evaporated off of the dice in air, to avoid damaging the delicate microstructures with blow-drying.

After anisotropic silicon substrate etching of each die in $\mathrm{KOH}$, the top gold/chromium $\mathrm{RIE} / \mathrm{KOH}$ masking layer was removed by brief immersion in $\mathrm{KI}$ (for no more than 30 seconds) and then chrome etch solution (for about ten seconds). This was a tedious process, as etching was performed on each die, separately. Static rinsing baths were used in which to immerse the die after gold/chromium layer etching (to avoid damaging the delicate microstructures).

After removing the RIE/KOH metallization masking layers it was observed that the $\mathrm{Si}_{x} \mathrm{~N}_{\mathrm{y}}$ thin-film sputtered layer peeled partially or completely off most dice. Microbridges and cantilevers on dice where this layer completely disappeared in $\mathrm{KOH}$ were straight and unbuckled (implying the presence of residual tensile stress in the LPCVD nitride thin film). SEM photos of flow sensor die shown in Appendix XV are of devices where this top passivation layer was missing, where the superb structural quality of these suspended structures can be seen. These devices were glorious to behold. Furthermore, it was observed that regions of platinum resistor metallization 
that were exposed to the $\mathrm{KOH}$ etchant were undamaged. Even at high optical microscope magnification, exposed platinum surfaces did not appear pitted, discolored, or otherwise damaged. Further etching tests showed that this sputter-deposited platinum layer was completely resistant to degradation in $\mathrm{KOH}$ (for all practical purposes).

Each die was packaged after testing for electrical viability, by testing the continuity of flow sensor resistors with the Wentworth Labs probe station. Dice were singled out which had upstream and downstream sense resistors in individual flow sensor microbridges/cantilevers that had matching resistances to within about $5 \%$. A small quantity of Epo-Tek® $353 N D$ epoxy resin was mixed (10 parts of "A" solution with 1 part "B" hardener) to glue a $45^{\circ}$ rotated glass-slide quad-die spacer into a DIP-24 package cavity, and to glue a quad or duo flow sensor die on top of the spacer, as shown in Figure 74 (where a quad die is shown epoxied on top of a spacer, epoxied inside the DIP-24 package cavity, rotated at $\left.45^{\circ}\right)$. The DIP-24 package with the epoxied die and glass-spacer was then placed in an oven at $\sim 140^{\circ} \mathrm{C}$ for about four minutes to cure the epoxy.

After packaging, each die in a DIP-24 package was wire-bonded with 0.001-inch aluminum silicon alloy wire to the package using the K\&S Model 4123 ultrasonic wedge wirebonder. Note that, due to the design of the ColeJet ${ }^{T M} 2000$ flow sensor test machine, flow sensor resistors were not wire-bonded to DIP-24 package pins 2, 6, 9, 10,11, or 24. Depending upon the wire-bonding configuration, typically three out of four flow sensors in a quad die could be wirebonded to the package (sharing some pins between flow sensors, since only one flow sensor can be tested at a time in the ColeJet $2000^{T M}$ machine). Finally, the wire-bonded flow sensors in each DIP-24 package were tested in the ColeJet $2000^{T M}$ machine (see the testing and ColeJet $2000^{\text {TM }}$ configuration instructions included in Appendix XXI). 


\section{Metallization Methods Attempted}

\section{Bulk vs. Thin Film Electrical Properties [83]}

At the time the 1997 photomask set was designed, along with a conceptual theoretical framework for how to potentially fabricate the flow sensors, it was incorrectly assumed that there would be little or no difference between the bulk and sputter-deposited, thin-film properties of a selected resistor metallization layer. This assumption was grossly in error. Thin film growth on a substrate, by sputtering for example, involves the formation of independently nucleated particles or islands, which grow together to form a continuous film as the deposition process continues. The physical and electrical properties of a deposited thin film are, thus, affected by the nature of the substrate, rate of film deposition, and the thickness, structure, and stoichiometric composition of the film itself [84]. Thus, when a metal is deposited as a thin film, its electrical properties differ from those of the bulk form: e.g., resistivity is generally higher, and thermal conductivity is lower. The stability of the sheet resistance of a film (which refers to its volume resistivity normalized to the film thickness) to temperature changes is characterized by its TCR. It should be emphasized that thin films typically have very low TCR's compared to bulk metals (four to ten times less), due to their deposited microstructure/morphology, thinness, impurities, intrinsic stress/strain, and high resistivity. A thin-film's TCR is a function of its nominal thickness (related to the mean free path of electrons in the material), in part because a sputtered film's composition and microstructure may vary.

Deviations from bulk properties can also be caused by high impurity concentrations in thin films. These could be sputtered chamber-structure materials, or as in the case of sputtered films, the "background" gases such as argon or residual oxygen and nitrogen may be partially incorporated into the film's microstructure, influencing its electrical and mechanical properties. High background pressures can lead to porous coatings, such as with argon absorption to grain surfaces [85]. Crystallites in thin films are often smaller than the film thickness because of high impurity concentrations. 
Thin films are likely to have high residual intrinsic strains due to such factors as distortions of the film lattice by the substrate lattice (a lattice mismatch) and a mismatch in the thermal coefficient of expansion between the film and substrate. One disadvantage of sputterdepositing a thin film is that there may be considerable heating of the substrate, thereby depositing the film at elevated temperatures. The sputtering chamber background gas pressure also affects whether the deposited thin metal film will have residual tensile stress (sometimes nearing or exceeding the yield strength, causing cracking, as shown in Figure 95), or compressive stress. Large stress may give rise to void formation upon subsequent thermal

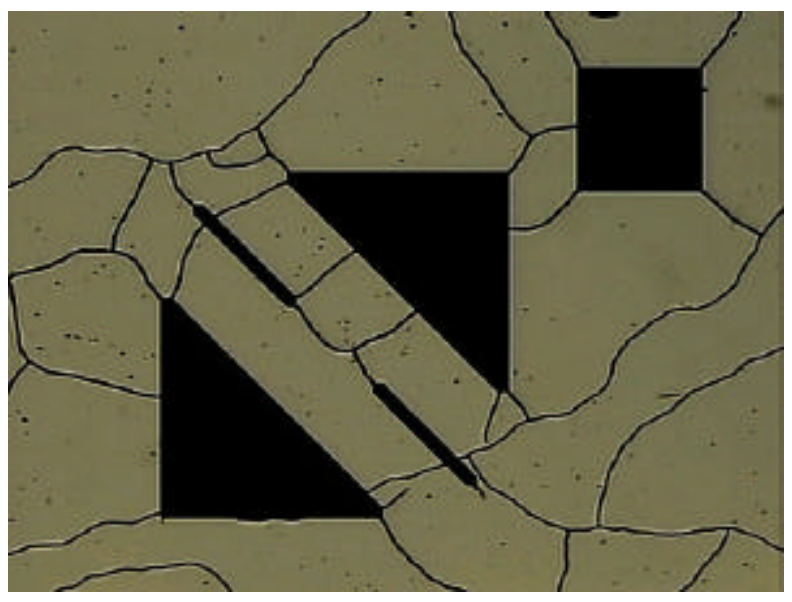

FIGURE 95 - A severely cracked chromium layer on a 4-inch borosilicate glass wafer. The residual tensile stress in the sputtered film likely exceeded its ultimate yield strength, which is $\sim 300-400 \mathrm{MPa}$. The film was RF planar magnetron sputterdeposited at 250 Watts, and 10 mTorr in argon for 34 minutes, with a profilometer-measured thickness of $0.62-0.86 \mu \mathrm{m}$.

cycling where the strain state provides a driving force for grain boundary diffusion. As shown illustratively in Figure 96, at lower working pressures the deposited film's stress transitions from tensile to compressive, with the pressure at which this transition occurs increasing with atomic mass. For these reasons, thin film resistivities are often several times greater than the bulk metal value. Film resistivity is a sensitive function of the base pressure and gas composition in the sputtering chamber [44], and it will depend, in part, upon its residual intrinsic strain. Since thin metal film resistors are strain-sensitive, their TCR's may be a function of the mismatch between the thermal expansion coefficients of the thin film and substrate (e.g. the choice of substrate 


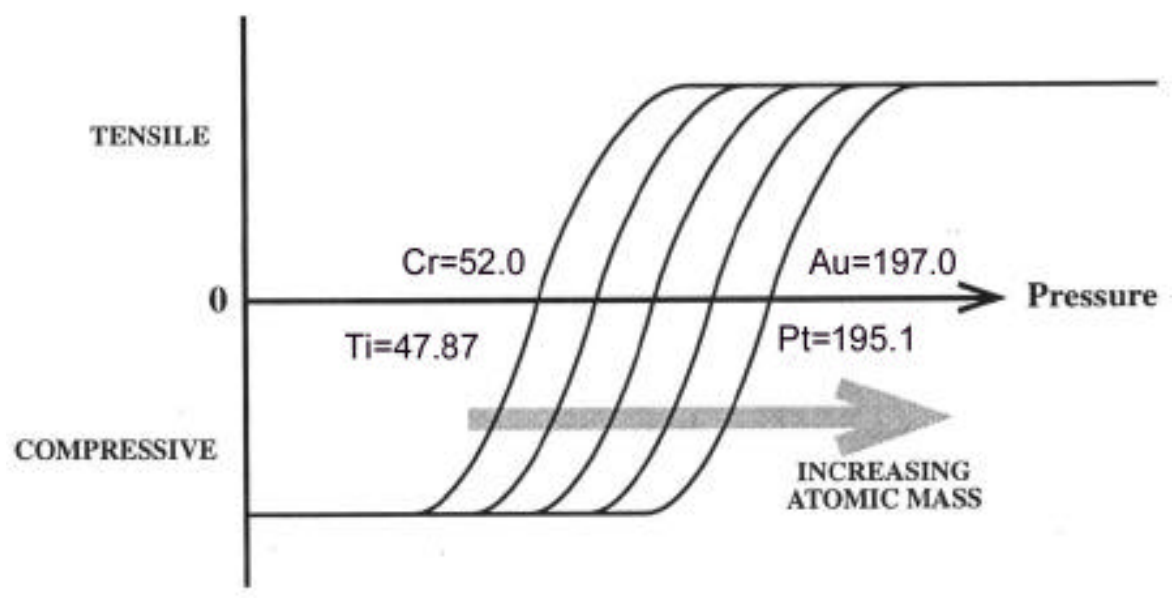

FIGURE 96 - A graph of sputtering parameter relationships to metal film stress. The relationship among background gas (e.g. argon), sputtering pressure, atomic mass, and residual stress is related to stress in a sputtered, metal thin film. This has been proven for most metals, especially $\mathrm{Cr}, \mathrm{Ti}, \mathrm{Ni}, \mathrm{Mo}, \mathrm{Ta}$, etc. Atomic masses are shown with thin metal films evaluated for the experimental flow sensors: $\mathrm{Cr}, \mathrm{Ti}, \mathrm{Au}$, and Pt. High tensile stress in the deposited film can cause cracking, if the yield strength of the metal is exceeded. Adapted from ref. [86].

alone may affect the TCR of a thin film deposited in the same way, with the same thickness on multiple substrates). Furthermore, a film's resistivity can be a function of crystallographic orientation in a noncubic metal, and there may be a preferred orientation of crystallites in a film, causing its resistivity to depend on the growth habit of the film.

Thin film properties, such as intrinsic stress, roughness, and resistivity can be changed by control of the film microstructure during sputter-deposited growth. Microstructure is determined primarily by the surface and near-surface environment during film growth, and it is strongly influenced by the thin-film deposition parameters and technique. Annealing or rapid thermal annealing techniques can be used to both stabilize the microstructure of thin films and improve their electrical characteristics to more closely match bulk values. The task is to select controlled deposition and post deposition thermal annealing conditions to preferentially form the desired thin-film compound or phase.

\section{Permalloy $\left(\mathrm{Ni}_{81} \underline{\mathrm{Fe}}_{19}\right)$ Resistors}

This nickel-iron alloy was the original choice for flow sensor resistor metallization when the 1997 photomask set and fabrication process were designed, because it had already been 
proven to be viable in the Honeywell flow sensor design. Permalloy was preferred in the Honeywell design for several reasons [18]. It has a resistance vs. temperature characteristic similar to platinum's $\left(\sim 4 \times 10^{-3} \Omega / \Omega /{ }^{\circ} \mathrm{C}\right)$. Permalloy is cheaper than platinum, reducing the cost of fabricated sensors. It has a resistivity that is approximately twice that of platinum's. Permalloy achieves a maximum TCR at less than half the thickness of platinum required (160 $\mathrm{nm}$ for maximum TCR, but $80 \mathrm{~nm}$ can be used with only a slightly smaller TCR). Platinum elements require a thickness of more than $350 \mathrm{~nm}$ to achieve maximum TCR. Consequently, for a given resistance platinum or permalloy resistor element, permalloy requires as little as one-eighth the surface area that would be required for a platinum element. This increases the thermal efficiency of the anemometer heating and sensing elements, reducing the required surface area, and lowering unit costs.

In the 1997 flow sensor design methodology, the permalloy metallization layer was to be RF planar magnetron sputter-deposited and patterned by a lift-off technique. Although not investigated, it was also possible to use ion milling for delineation [18]. A 99.95\% pure $6.35 \mathrm{~mm}$ thick, four-inch diameter sputtering target with $\mathrm{Ni}_{81} \mathrm{Fe}_{19}$ composition (similar to the $\mathrm{Ni}_{80} \mathrm{Fe}_{20}$ thin film composition used in the Honeywell flow sensor) was ordered from Angstrom Sciences (1200 Lebanon Rd.; West Mifflin, PA 15122; 412-462-2777) in preparation for RF planar magnetron sputter-deposition in the Technics system.

Permalloy has a resistance versus temperature characteristic similar to that of bulk platinum, $4 \times 10^{-3} 1 /{ }^{\circ} \mathrm{C}$ at $0{ }^{\circ} \mathrm{C}$, where, as a thin film, it achieves maximum TCR in a deposition thickness range of 80 to $160 \mathrm{~nm}$ [18]. Anisotropic magneto-resistive (AMR) permalloy thin films have TCR's near $2.8 \times 10^{-3} 1 /{ }^{\circ} \mathrm{C}$ [87]. Its resistivity, which may differ from published bulk material values, is reasonable at $2.4 \times 10^{-7} \Omega \cdot \mathrm{m}$ (measured for a permalloy layer that was $80 \mathrm{~nm}$ thick) [25]. Thus, for flow sensor upstream or downstream sense resistors in the 1997 design that were $80 \mathrm{~nm}$ thick with 194 to 521 designed "squares" (as shown in Table VIII), the total resistance would have been approximately 580 to 1560 ohms. As shown in Equation (51), the sheet resistance of the resistor thin-film is inversely proportional to its measured thickness. So, in this example, if the permalloy metallization layer thickness were reduced to $40 \mathrm{~nm}$, the estimated 
range of sense resistor resistances would be 1.16 to 3.12 kilohms.

Permalloy also has desirable reported electromigration characteristics. Electromigration is a temperature-dependent failure mechanism that occurs when current densities exceed a critical limit within a metal film, where the movement of atoms due to momentum transfer from electrons during current flow causes voids in some regions and hillocks in others. Voids can eventually result in open circuits in interconnect or resistor routing, and hillocks can cause shortcircuits between closely spaced conductors. The critical current density limit for permalloy is approximately $10 \times 10^{6} \mathrm{~A} / \mathrm{cm}^{2}$ at $25^{\circ} \mathrm{C}$. In the Honeywell design, for a flow sensor heater resistance between 600 and 1000 ohms, a heater current of $2-3 \mathrm{~mA}$, a line width not less than 5 $\mu m$, and a nominal thickness of $0.08 \mu m$, the current density was about $0.6 \times 10^{6} \mathrm{~A} / \mathrm{cm}^{2}$, which was significantly less than the reported critical value $[18,88]$.

While permalloy's electrical properties were initially attractive from a design perspective, there were significant problems with sputter-depositing and potentially delineating it as a thin-film metallization layer. Since permalloy contains nickel, which is a magnetic material, a sufficiently large magnetic field $\left(\sim 50 \times 10^{-4} \mathrm{~T}\right)$ above the target surface was required during magnetron sputter deposition to confine electrons [89]. A special magnetron cathode configuration, with a field strength about three times higher than normal, could also be potentially used [90]. Many problems were experienced in attempting to RF planar magnetron sputter-deposit permalloy films, as it was very difficult to ignite the RF plasma and/or keep the plasma lit for the duration of a sputtering run. It was concluded that the paramagnetic properties of the permalloy sputtering target combined with the potentially insufficient magnetic field produced in the sputtering chamber, made reproducible thin-film deposition with this target impractical. However, it is possible a sputtering recipe and technique at a very high RF power could be found to successfully deposit permalloy films. Other problems in 1997 with the Technics sputtering system made high RF-power depositions difficult and potentially damaging to its RF power supply, as it was later discovered that the internal cabling which supplied RF power to the sputtering target was damaged (an intermittent discontinuity). Thus, another thin film resistor metallization layer with similar electrical properties, which could be more easily sputter-deposited 
at reasonable RF powers (200 - 400 Watts), namely chromium, was explored.

\section{Chromium Resistors}

Some selected properties of chromium metal films are shown in Table XVIII. Chromium was among the first metals investigated as a thin film resistor material, in part because of its

TABLE XVIII

PROPERTIES OF CHROMIUM METAL FILMS $\left(\right.$ AT $\left.27^{\circ} \mathrm{C}\right)[91]$

\begin{tabular}{|c|c|c|c|c|c|}
\hline $\begin{array}{c}\text { Resistivity } \\
\rho_{r} \\
\left(10^{-6} \Omega \cdot \mathrm{cm}\right)\end{array}$ & $\begin{array}{c}\text { TCR, bulk } \\
\left(10^{-3} \Omega / \Omega /{ }^{\circ} \mathrm{C}\right)\end{array}$ & $\begin{array}{l}\text { TCR, thin film } \\
\left(10^{-3} \Omega / \Omega /{ }^{\circ} \mathrm{C}\right)\end{array}$ & $\begin{array}{c}\text { Density } \\
\rho \\
\left(\mathrm{g} / \mathrm{cm}^{3}\right)\end{array}$ & $\begin{array}{c}\text { Thermal } \\
\text { Conductivity } \\
\left(0-100^{\circ} \mathrm{C}\right) \\
(W / m \cdot K)\end{array}$ & $\begin{array}{c}\text { Thermal } \\
\text { Exp. } \\
\text { Coeff.* } \\
\left(10^{-6} 1 / K\right)\end{array}$ \\
\hline \multirow{2}{*}{$13.2-13.5$} & \multirow{2}{*}{$2.14-2.3$} & \multirow{2}{*}{$0.1-0.6$} & \multirow{2}{*}{7.16} & \multirow{2}{*}{$90-9$} & 5.1 \\
\hline & & & & & 6.5 (bulk) \\
\hline
\end{tabular}

* Or, the coefficient of linear thermal expansion.

high resistivity and excellent chemical stability. It is also frequently used as an underlayer to provide adherence for other films [92]. Chromium was the second resistor metallization layer explored because it had desirable bulk electrical properties (resistivity and TCR) for resistor delineation, was easily and very controllably wet-etchable, and could provide a convenient adhesion layer for a top gold metallization layer (added in the 1998/1999 photomask design). As shown in Table XVIII, chromium's thin-film TCR is significantly less than its bulk value.

Unfortunately, in 1997 when it was decided to explore the use of chromium as a potential resistor metallization layer, its bulk TCR was erroneously assumed to be the same as its thin film TCR.

The Resistor Metallization photomask in the 1997 set was designed for using a lift-off technique with positive photoresist to delineate a blanket-sputter-deposited, thin metallization film (originally permalloy). In this technique photoresist side walls underneath the deposited metal film are left uncoated to allow the solvent (acetone) to penetrate under the sputtered layer. As shown graphically in Figure 97, the vertical incidence of the deposit is easily achieved with evaporative sputtering, but special techniques are required for magnetron sputtering to achieve similar results (such as using a source capable of operating below 3 mTorr to reduce gas 


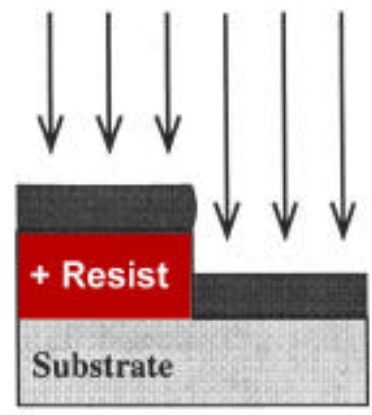

EVAPORATION

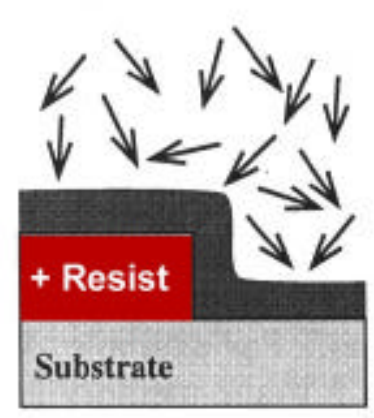

PLANAR SPUTTERING

FIGURE 97 - Step coverage comparison for magnetron sputtering vs. evaporative sputtering. As shown on the left, the vertical side-wall of delineated photoresist is exposed in an evaporative-sputtered thin film, where subsequent immersion in acetone would easily result in achieving lift-off of the thin metal film on top of the resist layer. However, with planar magnetron thin-film sputtering, the vertical side-wall of the photoresist is often not exposed, making successful lift-off difficult or impossible. Adapted from ref. [86].

scattering effects) [89]. Many problems were experienced in 1997 with using lift-off as a means to delineate chromium resistor metallization (after the use of permalloy was abandoned). In many instances, lift-off was only achieved after immersing wafers in an ultrasonically-agitated acetone bath, which also resulting in greater tearing of the thin film metal layer. After performing lift-off, the tearing of the thin-film, deposited chromium metal sheet layer (e.g. around 5- $\mu m$ line-width delineated sense resistors on a microbridge) would leave protruding vertical edges that presented problems for sputter-depositing a top passivation layer (over a jagged, non-planar surface) that would provide adequate protection for the chromium layer in the anisotropic silicon etchant, $\mathrm{KOH}$. It was found that $\mathrm{KOH}$ would substantially degrade or destroy chromium around the protruding edges, rendering many delineated serpentine resistors unusable. Consequently, the original 1997 photomask design was redesigned to ameliorate delineation of the thin-film resistor metallization layer by replacing the lift-off strategy with isotropic wet-chemical etching, and to add a top gold metallization layer that would be inert in $\mathrm{KOH}$, protecting previously-exposed bondingpad resistor metallization areas from degradation.

The measured resistivity of chromium thin-films was much less than the theoretical value. Sheet resistance was measured using four probes at the Wentworth Labs probe station and chromium-only delineated Van der Pauw structures. A Keithley 224 programmable current 
source and Keithley 196 digital multimeter were connected to the structures as shown in Figure 34, and Equation (51) was used to calculate sheet resistance. The average measured resistivity for a chromium thin film that was RF planar magnetron sputter-deposited in argon at 250 Watts and 10 mTorr for 6 minutes with a measured Van der Pauw structure thickness of $0.16 \mu \mathrm{m}$, was $2.046 \times 10^{-6} \Omega \cdot \mathrm{cm}$. This low resistivity value may be due, in part, to the presence of a native oxide layer on the metal film.

An RF planar magnetron sputter-deposited chromium thin film can be annealed to both stabilize its microstructure and potentially improve its electrical characteristics (i.e. TCR). A metastable, impurity-locked phase of chromium, called $\delta$-A15, may be present in thin, sputterdeposited films, depending upon sputtering working pressure, deposition temperature, time, and the presence of impurities in the as-deposited thin-film crystal structure (especially oxygen and carbon). The $\delta-\mathrm{A} 15 \mathrm{Cr}$ is a high-temperature allotropic chromium phase that forms under nonequilibrium conditions (quenched-in during film deposition), becoming a metastable phase at room temperature. The complete phase transformation from $\delta-\mathrm{A} 15 \mathrm{Cr}$ into an equilibrium $\delta$ BCC $\mathrm{Cr}$ phase is an irreversible exothermic transition that will occur at vacuum thermal annealing temperatures between $350^{\circ} \mathrm{C}$ and $550^{\circ} \mathrm{C}$ (especially near $\left.427^{\circ} \mathrm{C}\right)$. The higher the impurity concentration in the film, the higher the required temperature needed to completely transform an A15 structure into a BCC one. Impurities such as oxygen and carbon may be incorporated into the chromium film from residual gases in the sputtering deposition chamber atmosphere or from the sputtering target, or both. As the $\delta-\mathrm{A} 15 \mathrm{Cr}$ phase will transform into an equilibrium $\delta-\mathrm{BCC}$ Cr phase upon heating, it becomes unfavorable to form under processing conditions that increase the film's surface-temperature (e.g. "in-situ thermal annealing"), such as lengthy deposition times or elevated deposition temperatures. Post deposition annealing can be performed by annealing in a vacuum chamber: heating a sample with the chromium thin film to the desired temperature, followed by an immediate quench to preserve the high-temperature characteristics of the film structure $[93,94,95]$.

Annealing was experimentally attempted in atmosphere (i.e. with flowing nitrogen) in a furnace and in argon on a high-temperature hotplate to stabilize the TCR characteristics of thin, 
sputter-deposited chromium films. For the annealing tests, chromium and gold layers were delineated on a microscope glass slide with the Resistor Metal Level 1 and Gold Metal Level 2 photomasks, and the glass dice were then diced apart. Gold bonding-pad metallization was used to provide good ohmic contact to chromium resistors after annealing, because a high-impedance, thick oxide layer formed on the thin-film chromium resistors, making obtaining resistance values by probe-tip-contact to the chrome layer after high-temperature annealing $\left(>500^{\circ} \mathrm{C}\right)$ impossible. TCR measurements were obtained by using the Wentworth Labs probe station and integrated ThermoChuck ${ }^{\mathrm{TM}}$, made by Temptronic Corp. with digitally-programmable temperature controls. A temperature differential of $100^{\circ} \mathrm{C}$ above the ambient was used to measure the change in resistance values, which were measured by a Fluke $8500 \mathrm{~A}$ rack-mount, lab-bench digital multimeter. In general, before annealing, chromium resistors exhibited hysteresis, with drifting resistance values at elevated temperatures, and a negatively-correlated TCR. After annealing at temperatures no greater than $500^{\circ} \mathrm{C}$, the TCR was stable, and positively-correlated with temperature. Post deposition annealing above $500{ }^{\circ} \mathrm{C}$ caused the resistance of thin film resistors to increase to unmeasurable values, most likely due to oxidation of the chromium thin-film layer. The thermal annealing results are summarized in Table XIX.

Ideally, thermal annealing should be performed in a vacuum, to reduce the chances of oxidizing the chromium metallization layer, but no vacuum furnace was available. Thus, annealing was performed with nitrogen flow in a Lindberg Moldatherm hinged tube furnace or in a Labconco Protector Glove Box environmental chamber, pressurized with argon. Thermal annealing in "positive-pressure argon" was performed in the Labconco chamber, with the Thermolyne Type 1900 high-temperature hotplate, controller, and sample(s) to be annealed inside. After evacuating the chamber (by repeatedly pumping air out, injecting argon, pumping it out, re-injecting argon etc.), high-purity argon was injected until a pressure of $\sim+2$ " $\mathrm{H}_{2} \mathrm{O}$ to +3 " $\mathrm{H}_{2} \mathrm{O}$ was achieved, or enough to create a slight positive pressure differential that would prevent outside air from leaking into the chamber. Positive pressure was used, as opposed to pulling a vacuum in the chamber, as it was discovered that the chamber seals leaked air with a vacuum inside. The gold layer on most samples was discolored after thermal annealing. 
TABLE XIX

CHROMIUM THIN FILM* ANNEALING EXPERIMENTAL RESULTS

\begin{tabular}{|l|c|c|}
\hline \multicolumn{1}{|c|}{ Thermal Annealing Parameters } & $\begin{array}{c}\text { Avg. TCR } \\
\text { Before Anneal } \\
\left(\sim 20-120^{\circ} \mathrm{C}\right) \\
10^{-3} \Omega / \Omega /{ }^{\circ} \mathrm{C}\end{array}$ & $\begin{array}{c}\text { Avg. TCR } \\
\text { After Anneal } \\
\left(\sim 20-120^{\circ} \mathrm{C}\right) \\
10^{-3} \Omega / \Omega /{ }^{\circ} \mathrm{C}\end{array}$ \\
\hline $\begin{array}{l}300^{\circ} \mathrm{C} \text { for } 190 \mathrm{~min} \text {. in furnace with } \mathrm{N}_{2} \text { flow; } \\
\text { ramp up/down at } 10^{\circ} \mathrm{C} / \text { min. }\end{array}$ & -0.1093 & +0.174 \\
\hline $\begin{array}{l}450^{\circ} \mathrm{C} \text { for } 1 \mathrm{hr} \text { in furnace with high } \mathrm{N}_{2} \text { flow; } \\
\text { ramp up at } 10^{\circ} \mathrm{C} / \text { min.; rapid quench to ambient }\end{array}$ & -0.1054 & +0.2083 \\
\hline $\begin{array}{l}450^{\circ} \mathrm{C} \text { for } 1 \mathrm{hr} \text { on hotplate in chamber with positive- } \\
\text { pressure argon; } 1 \mathrm{hr} \text { ramp up; } \\
\text { rapid quench to ambient }\end{array}$ & -0.0989 & +0.2507 \\
\hline $\begin{array}{l}450^{\circ} \mathrm{C} \text { for } 2 \mathrm{hrs} \text { on hotplate in chamber with positive- } \\
\text { pressure argon; } 70 \text { min. ramp up; } \\
\text { rapid quench to ambient }\end{array}$ & -0.1345 & +0.2452 \\
\hline $\begin{array}{l}450^{\circ} \mathrm{C} \text { for } 5 \text { min. on hotplate in chamber with positive- } \\
\text { pressure argon; } ~ 55 \text { min. ramp up; } \\
\text { rapid quench to ambient }\end{array}$ & -0.1447 & +0.2552 \\
\hline $\begin{array}{l}500^{\circ} \mathrm{C} \text { in furnace with } \mathrm{N}_{2} \text { flow; } \\
\text { ramp up/down at } 3{ }^{\circ} \mathrm{C} / \text { min. }\end{array}$ & -0.0936 & +0.480 \\
\hline
\end{tabular}

${ }^{*}$ RF planar magnetron sputter-deposited at 250 Watts and 10 mTorr in argon for 6 minutes, achieving a film thickness of $144-175 \mathrm{~nm}$.

Figure 98 shows the difference in appearance of the chromium and gold metallized glass slide before and after annealing, where oxidation of the chromium layer is quite apparent. In conclusion, while thermal annealing of RF planar magnetron sputter-deposited chromium thin film resistor metallization stabilized its microstructure and TCR properties, it did not appreciably
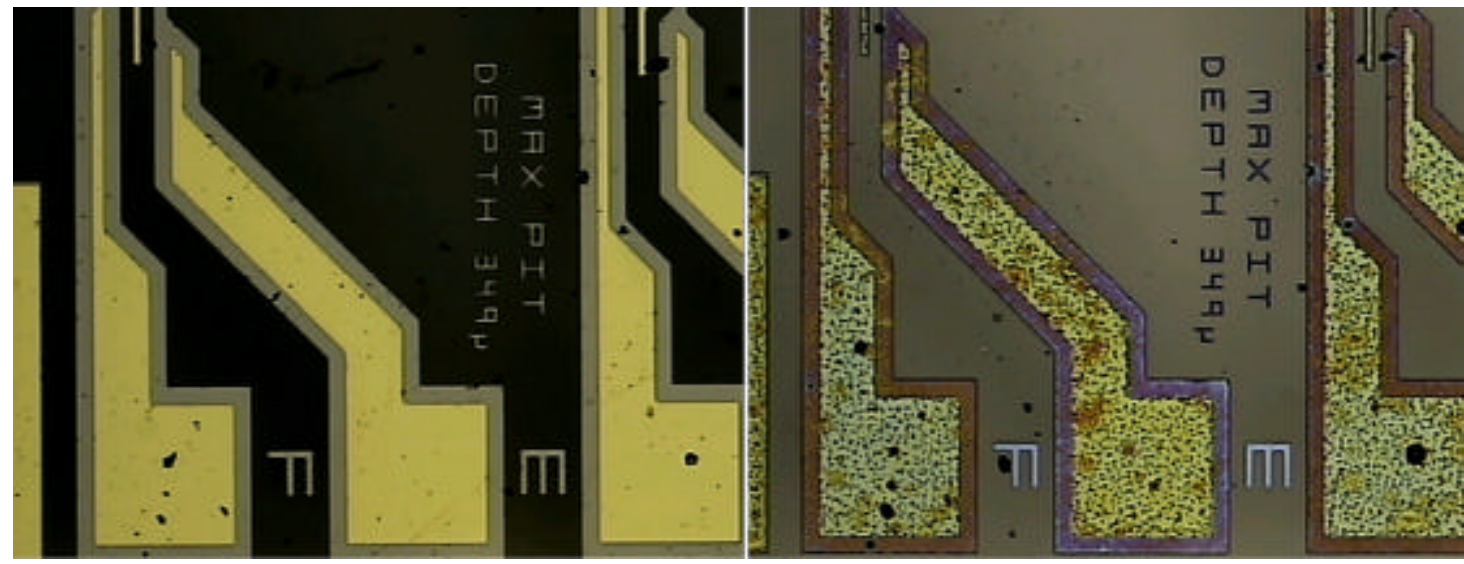

FIGURE 98 - Photos comparing a chromium layer's appearance before/after annealing. Oxidation of the chromium is obvious on a glass substrate after thermal annealing at $500{ }^{\circ} \mathrm{C}$ for one hour. The photo on the right shows the appearance after annealing (note also the discoloration of the gold layer). 
improve the TCR beyond $0.25 \times 10^{-3} \Omega / \Omega /{ }^{\circ} \mathrm{C}$. Furthermore, thermal annealing generally caused a native oxide film to grow on the chromium metallization surface (because of residual air and moisture present in the furnace and environmental chamber during annealing), making good ohmic contact difficult after annealing runs at temperatures in excess of $500{ }^{\circ} \mathrm{C}$. Since a TCR of at least $2 \times 10^{-3} \Omega / \Omega /{ }^{\circ} \mathrm{C}$ was desired, chromium was abandoned for potential use as flow sensor resistor metallization.

\section{Titanium Resistors}

\section{TABLE XX}

PROPERTIES OF BULK TITANIUM [50]

\begin{tabular}{|c|c|c|c|c|}
\hline $\begin{array}{c}\text { Resistivity } \\
\text { at } 20^{\circ} \mathrm{C} \\
\rho_{r} \\
\left(10^{-6} \Omega \cdot \mathrm{cm}\right)\end{array}$ & $\begin{array}{c}\text { TCR, bulk } \\
\left(10^{-3} \Omega / \Omega /{ }^{\circ} \mathrm{C}\right)\end{array}$ & $\begin{array}{c}\text { Density } \\
\rho \\
\left(\mathrm{g} / \mathrm{cm}^{3}\right)\end{array}$ & $\begin{array}{c}\text { Thermal } \\
\text { Conductivity } \\
\left(0-100^{\circ} \mathrm{C}\right) \\
(W / m \cdot K)\end{array}$ & $\begin{array}{c}\text { Coefficient of } \\
\text { Linear Thermal } \\
\text { Expansion } \\
\left(0-100^{\circ} \mathrm{C}\right) \\
(1 / K)\end{array}$ \\
\hline $54-55$ & $3.3-3.8$ & 4.507 & $21.6-21.9$ & $8.9 \times 10^{-6}$ \\
\hline
\end{tabular}

Titanium metallization was evaluated as a potential flow sensor resistor metallization layer because it had excellent theoretical bulk electrical properties, and would provide a superb and convenient adhesion layer for the top gold bonding-pad metallization layer, which was added in the 1998/1999 photomask design. Bulk properties for titanium are shown in Table XX. Since the resistivity of titanium is slightly more than four times that of chromium, for the same thin-film resistor metallization thickness and for a given flow sensor resistor, a titanium one would have four times the resistance of a chromium one [see Equation (51)]. Thus, for a given resistor, the same resistance in titanium could be achieved for a thin-film thickness that is one-fourth that of a chromium one. Generally, higher resistance values for flow sensor resistors were not expected to be problematic.

Delineating titanium resistor metallization layers with various dilutions of "titanium etch" solution (20:1:1 $\left.\mathrm{H}_{2} \mathrm{O}: \mathrm{H}_{2} \mathrm{O}_{2}: \mathrm{HF}\right)$ proved to be difficult, with typically low yields (50 - 70\%). The published etch rate was $0.88 \mu \mathrm{m} / \mathrm{min}$ for this solution [96]. Allowing etching to proceed just a few seconds beyond resistor delineation would often result in complete isotropic undercutting of 
photoresist on many 5- $\mu \mathrm{m}$ line-width resistors on a given substrate. Furthermore, to compound this problem, since RF planar magnetron sputter-deposited titanium thin films were typically much thicker in the center of a substrate than along the outer periphery, halfway through the etching process resistors in the center of the wafer would still need more time to be fully etched/patterned while photoresist on many 5- $\mu m$ line-width delineated resistors at the periphery would be completely undercut by the etchant. Since undercutting of photoresist occurred isotropically, best results were achieved with the thinnest possible titanium metallization films (which also achieved the shortest etch durations) and with maximum dilution of the titanium etch solution, where the most-controllable results were obtained with a mix of $80: 1: 1 \mathrm{H}_{2} \mathrm{O}: \mathrm{H}_{2} \mathrm{O}_{2}: \mathrm{HF}$.

The measured resistivity of titanium thin-films was much less than the theoretical value. Sheet resistance was measured using the Wentworth Labs probe station, programmable current source, digital multimeter, and titanium-only delineated Van der Pauw structures. The average measured resistivity for a titanium layer RF planar magnetron sputter-deposited in argon at 250 Watts and 10 mTorr for 4 minutes with a measured Van der Pauw structure thickness of $81.5 \mathrm{~nm}$, was $5.726 \times 10^{-6} \Omega \cdot \mathrm{cm}$. Stable voltage measurements were difficult, with significant drift (hysteresis), possibly due in part to the presence of a native titanium oxide layer. This low resistivity value may be due, in part, to the presence of an oxide layer on the metal film. Other researchers have reported argon sputter-deposited titanium thin film (33 nm thick) resistivities of $72 \times 10^{-6} \Omega \cdot \mathrm{cm}$ with a TCR of $2.8 \times 10^{-3} \Omega / \Omega /{ }^{\circ} \mathrm{C}[97]$.

Initial TCR measurements with sputter-deposited thin-film titanium resistors were very disappointing, as experimentally measured values differed significantly from bulk values. Thermal annealing was again attempted to potentially improve the film's microstructure and stabilize its TCR characteristics. The results of the titanium thermal annealing experiments are summarized in Table XXI. Because no data for annealing thin-film titanium resistors were found, various temperature and technique thermal anneals were attempted (as with the chromium metallization annealing trials). A microscope glass slide was prepared by delineating titanium and gold layers with the Resistor Metal Level 1 and Gold Metal Level 2 photomasks, and dicing apart individual quad glass die. Gold bonding-pad metallization was used in an effort to 
TABLE XXI

TITANIUM THIN FILM* ANNEALING EXPERIMENTAL RESULTS

\begin{tabular}{|l|c|c|}
\hline \multicolumn{1}{|c|}{ Thermal Annealing Parameters } & $\begin{array}{c}\text { Avg. TCR } \\
\text { Before Anneal } \\
\left(\sim 20-120^{\circ} \mathrm{C}\right) \\
10^{-3} \Omega / \Omega /{ }^{\circ} \mathrm{C}\end{array}$ & $\begin{array}{c}\text { Avg. TCR } \\
\text { After Anneal } \\
\left(\sim 20-120^{\circ} \mathrm{C}\right) \\
10^{-3} \Omega / \Omega /{ }^{\circ} \mathrm{C}\end{array}$ \\
\hline $\begin{array}{l}300^{\circ} \mathrm{C} \text { for } 190 \mathrm{~min} \text {. in furnace with } \mathrm{N}_{2} \text { flow; } \\
\text { ramp up/down at } 10^{\circ} \mathrm{C} / \text { min. }\end{array}$ & $\begin{array}{c}-0.0103 \\
+0.0203\end{array}$ & -0.1225 \\
\hline $\begin{array}{l}400^{\circ} \mathrm{C} \text { for } 280 \mathrm{~min} \text {. in furnace in air; } \\
\text { ramp up/down at } 10^{\circ} \mathrm{C} / \text { min. }\end{array}$ & -0.0863 & -1.837 \\
\hline $\begin{array}{l}450^{\circ} \mathrm{C} \text { for } 1 \mathrm{hr} \text { in furnace with high } \mathrm{N}_{2} \text { flow; } \\
\text { ramp up at } 10^{\circ} \mathrm{C} / \text { min.; rapid quench to ambient }\end{array}$ & $\begin{array}{l}-0.0327 \\
+0.0632\end{array}$ & -2.1805 \\
\hline $\begin{array}{l}450^{\circ} \mathrm{C} \text { for } 1 \mathrm{hr} \text {. on hotplate in chamber with positive- } \\
\text { pressure argon; } ~ 1 \mathrm{hr} \text { ramp up; } \\
\text { rapid quench to ambient }\end{array}$ & +0.1016 & -5.399 \\
\hline $\begin{array}{l}450^{\circ} \mathrm{C} \text { for } 5 \mathrm{~min} \text { on hotplate in chamber with positive- } \\
\text { pressure argon; } 55 \text { min. ramp up; } \\
\text { rapid quench to ambient }\end{array}$ & +0.3609 & -0.5753 \\
\hline
\end{tabular}

* RF planar magnetron sputter-deposited at 250 Watts and 10 mTorr in argon for 4 minutes, achieving a film thickness of $\sim 800 \AA$.

potentially provide good ohmic contact to titanium resistors after annealing. A high-impedance, thick oxide layer formed on the thin-film resistors, making measuring resistance values by probetip-contact to the titanium layer after high-temperature annealing $\left(>450^{\circ} \mathrm{C}\right)$ quite difficult or impossible. Above $370^{\circ} \mathrm{C}$ and below $450^{\circ} \mathrm{C}$, titanium forms colored surface oxide films which slowly thicken with time. Above $\sim 650^{\circ} \mathrm{C}$, titanium suffers from a lack of long-term oxidation resistance and will become brittle due to the increased diffusion of oxygen into the metal. Additionally, above $800{ }^{\circ} \mathrm{C}$ excessive diffusion of nitrogen may cause metal embrittlement [98]. As with chromium annealing experiments, titanium TCR measurements were obtained using the heated Thermochuck ${ }^{\mathrm{TM}}$ with temperatures up to $100^{\circ} \mathrm{C}$ above the ambient. Annealing was attempted in atmosphere (flowing nitrogen) in the Lindberg Moldatherm hinged tube furnace and in argon on the Thermolyne Type 1900 high-temperature hotplate in the Labconco environmental chamber.

An example of the appearance of the titanium and gold layers on a glass substrate before and after annealing is shown in Figure 99. After the thermal annealing trials, the titanium metallization layer was more discolored, with a higher impedance native oxide and was, thus, 

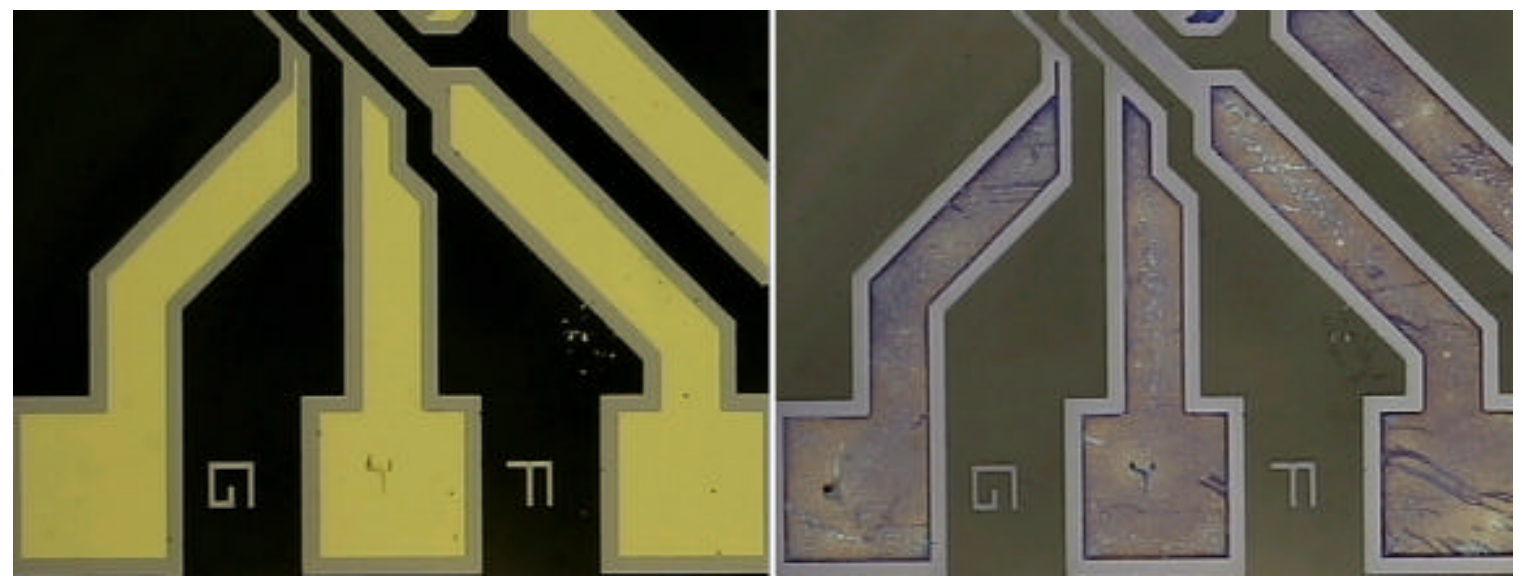

FIGURE 99 - Photos comparing a titanium layer's appearance before/after annealing. The titanium and gold metallization layers on a glass slide before (left) and after (right) a $500{ }^{\circ} \mathrm{C}$ thermal anneal for one hour are shown. The titanium layer was so severely oxidized that measured resistance values exceeded the range of the digital ohmmeter. The gold layer was also discolored after annealing.

significantly more oxidized. Achieving good probe-tip ohmic contact with the residual gold layer was sometimes difficult, and typically impossible on the oxidized titanium metal. Oxidation occurred because both the furnace and "positive-pressure" argon atmosphere environmental chamber could have easily had residual oxygen or water vapor in them. Here again, if a vacuum furnace set-up had been available for thermal annealing trials, the titanium layer might not have so significantly oxidized. Initial titanium thin-film TCRs were both negatively and positively correlated with temperature. Post-annealing TCR's were all negatively correlated. Since bulk titanium's TCR is positively correlated, these post-anneal TCR values are likely due to the severe oxidation of the titanium layer. Due to the low TCR values obtained, the heavy oxidation of the titanium layer, and the lack of significant improvement in TCR results after annealing, titanium was abandoned as a potential resistor metallization layer.

\section{Gold Top Layer Metallization and RIE/KOH Masking}

Gold has excellent resistance to corrosion, and good conductivity with a low sheet resistance (down to less than $0.1 \mathrm{ohm}$ per square), making it a superb low-impedance interconnect metal. Various relevant properties of gold metal films are shown in Table XXII. Gold can be readily isotropically wet-etched in $\mathrm{KI}$ solution (at $\sim 0.5-1 \mu \mathrm{m} / \mathrm{min}$ ). Gold was the obvious 
TABLE XXII

PROPERTIES OF GOLD METAL FILMS (AT $\left.27^{\circ} \mathrm{C}\right)[91,99]$

\begin{tabular}{|c|c|c|c|c|c|}
\hline $\begin{array}{c}\text { Resistivity } \\
\rho_{r} \\
\left(10^{-6} \Omega \cdot \mathrm{cm}\right)\end{array}$ & $\begin{array}{c}\text { TCR } \\
\left(10^{-3} \Omega / \Omega /{ }^{\circ} \mathrm{C}\right)\end{array}$ & $\begin{array}{l}\text { Limiting } \\
\text { Current } \\
\text { Density } \\
\left(\mathrm{MA} / \mathrm{cm}^{2}\right)\end{array}$ & $\begin{array}{c}\text { Density } \\
\rho \\
\left(\mathrm{g} / \mathrm{cm}^{3}\right)\end{array}$ & $\begin{array}{c}\text { Thermal } \\
\text { Conductivity } \\
(W / m \cdot K)\end{array}$ & $\begin{array}{c}\text { Thermal } \\
\text { Exp. } \\
\text { Coeff.* } \\
(1 / K)\end{array}$ \\
\hline \multirow{2}{*}{$2.2-2.35$} & $\begin{array}{l}1.6-2.8 \\
\text { (thin film) }\end{array}$ & \multirow{2}{*}{70} & \multirow{2}{*}{19.3} & \multirow{2}{*}{315.5 - 318} & \multirow{2}{*}{$14.2 \times 10^{-6}$} \\
\hline & $\begin{array}{c}3.67-4.0 \\
\text { (bulk) }\end{array}$ & & & & \\
\hline
\end{tabular}

* Or, the coefficient of linear thermal expansion.

choice for of thin-film, second-layer metallization in the 1998/1999 flow sensor design which could provide a corrosion-resistant top layer at the bonding pad areas, between the underlying resistor metallization (and adhesion) layer and exposure to the anisotropic silicon etchant, $\mathrm{KOH}$, after reactive ion etching through the top dielectric $\left(\mathrm{Si}_{x} \mathrm{~N}_{\mathrm{y}}\right)$ passivation layer. One reason the 1997 mask set was redesigned was to add this additional metallization layer. Gold was also designed to be delineated as a low-impedance interconnect routing metal between bonding pads and flow sensor resistors.

Gold was successfully used as an inert RIE/KOH masking layer (delineated with the Etch Window Level 3 photomask in the 1998/1999 mask set) with sputtered $\mathrm{Si}_{x} \mathrm{~N}_{\mathrm{x}}$ and low stress LPCVD $\mathrm{Si}_{3} \mathrm{~N}_{4}$ device fabrication attempts. This masking layer was needed to improve device yield, especially where sputtered $\mathrm{Si}_{x} \mathrm{~N}_{\mathrm{x}}$ was used as the microbridge/cantilever structural layer, because the sputtered film was of poor quality (porous, cracked, etc). Micropores and cracks in an unmasked $\mathrm{Si}_{\mathrm{x}} \mathrm{N}_{\mathrm{x}}$ top passivation layer would allow the anisotropic silicon etchant to not only etch into the substrate in undesirable locations, but also degrade the $\mathrm{Si}_{x} \mathrm{~N}_{\mathrm{x}}$ layer itself.

To provide adherence to silicon, glass, polymer, and other dielectric substrates, gold requires a mutually soluble interface, or bonding layer, of chromium or titanium to achieve acceptable adhesion and electrical contact. The adhesion layer is typically 10 to $20 \mathrm{~nm}$ thick. Chromium and titanium were evaluated as potential resistor metallization layers because each not only had desirable bulk electrical properties, but also could provide excellent adhesion layers for gold. 
A significant, albeit simple, discovery resulted from worsening sputter-deposited gold adhesion problems which very nearly brought this research to a halt. Gold adhesion to a chromium or titanium underlayer on a silicon substrate may be significantly adversely affected by the cleanliness of the vacuum chamber in the (Technics) sputtering system. Any residual build up of grease, dust, or old coatings can trap moisture and other contaminants, both impeding the ability to achieve a high vacuum and increasing the risk of depositing a gold film with poor adhesion. Surface roughness in deposited films and trace chemical impurities can be sometimes linked to dirty sputtering chambers or targets [100]. Trapped moisture in old coatings in the chamber which is released during chromium adhesion layer sputtering (for example) will release oxygen, which may become incorporated into the chromium underlayer. Gold will not adhere well to any oxidized (chromium) adhesion layer. Thus, both the sputtering chamber and sputtering targets should be typically, periodically cleaned. The sputtering target should be wiped with acetone, methanol, or similar solvent before each use. The sputtering chamber should also be wiped down before each use, and a thorough periodic bead-blasting should be done to remove contaminants and layered films. Gold adhesion in the Technics system deteriorated rapidly after the system had been in use for approximately three years. Adhesion problems were not immediately obvious when substrates were removed from the system. A simple tape test would confirm immediately whether or not gold adhesion was acceptable. Otherwise, if gold layers lifted off in sheets in $\mathrm{KI}$ etch solution during gold patterning (as opposed to a shifting brown discoloration moving across the top gold surface as it was isotropically etched), it could generally be assumed that adhesion was unacceptably poor. Initial attempts to improve adhesion resulted in using a "pre-sputtering" strategy for adhesion layer metals. Improved gold adhesion can be obtained by "pre-sputtering" the adhesion-layer sputtering targets (titanium or chromium) to clean them prior to deposition. To pre-sputter, the plasma was ignited before opening the shutter so that any thin native oxide layer (or other particulates) on top of the target would be deposited onto the backside of the shutter instead of onto the substrate. Pre-sputtering of reactive species, such as chromium, also getters the residual reactive gases such as $\mathrm{O}_{2}, \mathrm{H}_{2} \mathrm{O}$, and $\mathrm{N}_{2}$ from the chamber, both by gas phase reactions and by coating the back surface of the shutter with a highly reactive 
film. All adhesion layer chromium or titanium RF planar magnetron sputter depositions (prior to gold depositions) were done after sputtering the target onto the shutter for at least five minutes and after purging the argon (or nitrogen) gas lines at maximum flow for at least ten minutes to remove oxygen and water vapor from the system. Yet, these techniques did not ameliorate adhesion. It was not until the chamber was bead-blasted and chemically cleaned that adhesion properties of sputter-deposited gold thin-films were once again acceptable.

Other factors may also influence gold adhesion in the Technics system. Since initial substrate conditions affect adhesion, substrate preparation and cleaning was essential. Substrate microfractures could adversely affect adhesion. If the substrate is a spin on glass (SOG), it should be fully cured. The high-purity argon and nitrogen (process) gas lines should be periodically tested for leaks. The turbo pump bearings should be periodically lubricated. The use of adhesive tapes in the sputtering chamber could cause outgassing. And, of course, the sputtering pressure during gold deposition partially determines whether the film will have residual tensile or compressive stress, consequently, affecting its adhesion properties.

During attempts to fabricate low-stress LPCVD $\mathrm{Si}_{3} \mathrm{~N}_{4}$ flow sensors without a top $\mathrm{Si}_{x} \mathrm{~N}_{\mathrm{y}}$ passivation layer, problems were experienced with using a top sputter-deposited gold (and underlying chromium adhesion) RIE/KOH masking layer on top of resistor (and gold) metallization layers. Layering schemes such as $\mathrm{Cr} / \mathrm{Au} / \mathrm{Cr} / \mathrm{Au}$ for chromium resistor metallization and $\mathrm{Ti} / \mathrm{Au} / \mathrm{Cr} / \mathrm{Au}$ for titanium resistor metallization, resulted in gold (shown in green) peeling or lifting off of the resistor metallization when the top gold and chromium RIE/KOH masking layer was removed by wet etching. This occurred after etching away the chromium adhesion layer (shown in blue) for the gold RIE/KOH masking layer. The only way to avoid these layering problems was to use a top sputter-deposited $\mathrm{Si}_{x} \mathrm{~N}_{\mathrm{y}}$ thin passivation layer between the resistor and gold metallization layers and top chromium/gold RIE/KOH masking layer. 


\section{Platinum Resistor Metallization}

Some selected properties of platinum films are shown in Table XXIII. Platinum was selected for evaluation of its potential use as a resistor metallization layer upon learning it is frequently used in RTDs and flow sensors because it has a very stable and near-linear resistance

TABLE XXIII

PROPERTIES OF PLATINUM METAL FILMS [26,50]

\begin{tabular}{|c|c|c|c|c|c|}
\hline $\begin{array}{l}\text { Resistivity } \\
\rho_{r} \\
\left(10^{-6} \Omega \cdot \mathrm{cm}\right)\end{array}$ & $\begin{array}{c}\text { TCR, bulk } \\
\left(10^{-3} \Omega / \Omega /{ }^{\circ} \mathrm{C}\right)\end{array}$ & $\begin{array}{l}\text { TCR, thin film } \\
\left(10^{-3} \Omega / \Omega /{ }^{\circ} \mathrm{C}\right)\end{array}$ & $\begin{array}{c}\text { Density } \\
\rho \\
\left(\mathrm{g} / \mathrm{cm}^{3}\right)\end{array}$ & $\begin{array}{c}\text { Thermal } \\
\text { Conductivity } \\
\left(0-100^{\circ} \mathrm{C}\right) \\
(W / m \cdot K)\end{array}$ & $\begin{array}{c}\text { Thermal } \\
\text { Exp. } \\
\text { Coeff.* } \\
(1 / K)\end{array}$ \\
\hline $\begin{array}{c}9.8-10.58 \\
\text { (bulk) }\end{array}$ & \multirow{2}{*}{3.9289} & \multirow{2}{*}{$3.75-3.85$} & \multirow{2}{*}{21.45} & \multirow{2}{*}{71.5} & \multirow{2}{*}{$9 \times 10^{-6}$} \\
\hline $\begin{array}{c}9.83-10.4 \\
\text { (film) }\end{array}$ & & & & & \\
\hline
\end{tabular}

${ }^{*}$ Or, the coefficient of linear thermal expansion.

versus temperature function [modeled by Equations (15) and (16)], and excellent reproducibility of electrical characteristics $[80,101,102]$. Platinum has poor adhesion to silicon substrates, requiring an adhesion layer or typically chromium, tantalum, or titanium. It was also found that platinum would not adhere to low-stress $\mathrm{Si}_{3} \mathrm{~N}_{4}$, without using an adhesion layer. Chromium was used because of its excellent adhesive properties with respect to platinum [103].

The DC planar magnetron sputter-deposited platinum thin film used in the successfully fabricated low-stress LPCVD $\mathrm{Si}_{3} \mathrm{~N}_{4}$ flow sensors had a chromium adhesion layer which was approximately $12 \mathrm{~nm}$ thick (estimated, based upon the sputtering deposition parameters). The profilometer-measured thickness of both the platinum and chromium layers was measured to be 70 - $87 \mathrm{~nm}$ across the wafer (this wafer is shown in Figure 92 just after platinum delineation in aqua regia). The platinum thin-film layer was deposited in the Technics sputtering system with an argon chamber pressure of 10 mTorr at 200 Watts, DC, for 10 minutes, after depositing a chromium adhesion layer, sputtered in argon at a pressure of 10 mTorr, 250 Watts, for 30 seconds. 
The TCR characteristic for these platinum thin film resistors was measured on both the Wentworth Labs probe station heated Thermochuck ${ }^{\mathrm{TM}}$ (at $20-120^{\circ} \mathrm{C}$ ) and with an oven test. The average TCRs were found to be $2.008 \times 10^{-3} \Omega / \Omega /{ }^{\circ} \mathrm{C}$ and $2.169 \times 10^{-3} \Omega / \Omega /{ }^{\circ} \mathrm{C}$, respectively. These results were lower than the expected thin-film TCR, which should have been larger than $3.0 \times 10^{-3} \Omega / \Omega /{ }^{\circ} \mathrm{C}$. However, as previously shown in Figures 15 and 16 , platinum's TCR is highly dependent upon film thickness, especially below $1 \mu \mathrm{m}$. To potentially improve the microstructure of the as-deposited platinum thin film, one unsuccessful annealing trial in air was attempted to increase its TCR. Several "quad" platinum flow sensor die (where the top gold layer had been removed) were placed on an alumina substrate in the Lindberg tube furnace, which was ramped up at $10^{\circ} \mathrm{C} / \mathrm{min}$ to $1150^{\circ} \mathrm{C}$ and held for one hour, before ramping down to the ambient at the same rate (allowing the furnace to cool to ambient overnight). After annealing, ohmic contact to the platinum layer was impossible, as it was both potentially oxidized and a residual $\mathrm{Pt} / \mathrm{Au}$ eutectic layer was visible (the gold layer was etched off of the platinum layer in KI prior to annealing).

One U.S. patent $(\# 4,739,657)$ describes the use of serpentine flow sensor resistors that combine a small length of permalloy with platinum to achieve a more linear TCR characteristic, with an experimentally measured sheet resistance value of 4.2 ohms-per-square for a $50 \mathrm{~nm}$ thick platinum thin-film. This corresponds to a measured thin-film resistivity of $2.1 \times 10^{-7} \Omega \cdot \mathrm{m}$ [see Equation (17)]; whereas, the bulk value for platinum is $1.058 \times 10^{-7} \Omega \cdot \mathrm{m}[50,101]$. This thin-film value of resistivity closely matched that of the platinum thin film that was DC planar magnetron sputter-deposited to fabricate the viable low-stress LPCVD $\mathrm{Si}_{3} \mathrm{~N}_{4}$ flow sensors. The average measured resistivity of these devices was calculated to be $2.025 \times 10^{-7} \Omega \cdot \mathrm{m}$, using Equation (51) and six measurements from Van der Pauw structures comprised of $\sim 67 \mathrm{~nm}$ thick platinum. This corresponded to a sheet resistance of between $2.469 \Omega$ /square and $2.978 \Omega$ /square for the unannealed platinum deposition, which had a Tencor-profilometer-measured thickness variation across the wafer of $\sim 68-82 \mathrm{~nm}$. 


\section{DEVICE TESTING: DESIGN OF THE COLEJET 2000TM MACHINE}

\section{A. Design Overview}

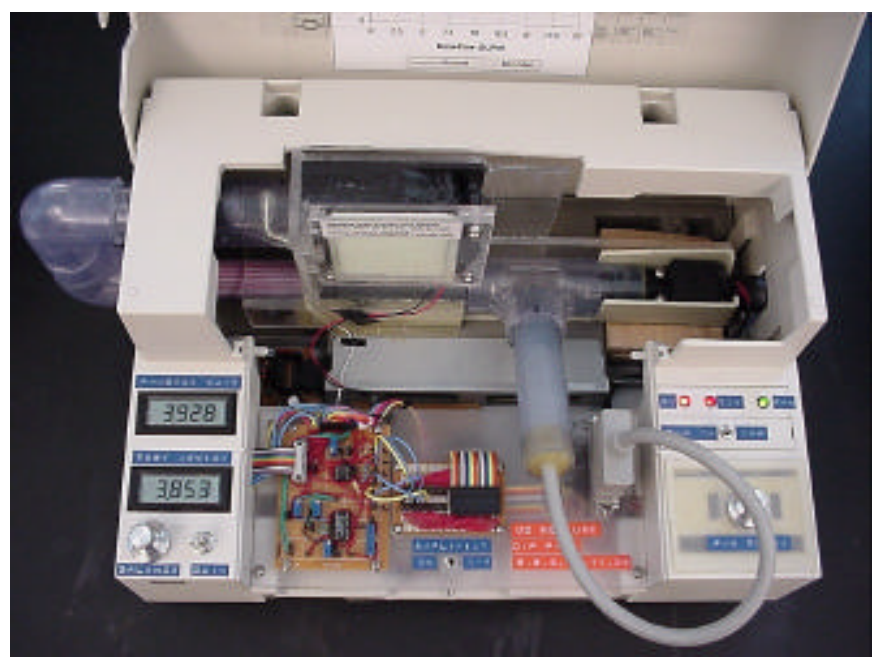

FIGURE 100 - A top view of the ColeJet 2000'M flow sensor testing machine. This machine was built for controlled laminar flow testing of fabricated mass airflow sensors.

A configurable machine, branded the ColeJet $2000^{T M}$, which could produce theoretical laminar airflow across fabricated and packaged mass airflow sensor devices was designed and built. ColeJet $2000^{T M}$, shown in Figure 100, was developed with the previously elaborated device testing and evaluation goals in mind, including: allowing for reconfigurable DIP-24 device wirebonding, interchangeable device testing, adjustable/controllable laminar airflow rates, and a measurable flow rate obtained from a calibrated Honeywell flow sensor installed in series within the flow channel. An aging, warehoused/scrapped HP DeskJet ${ }^{\mathrm{TM}}$ printer was selected for the outer shell, chassis, and basic structure of the flow sensor test machine, because its shape and size closely matched what was needed. The DeskJet ${ }^{\mathrm{TM}}$ printer was gutted, except for the power supply (and external transformer) which could be used to power circuitry in ColeJet 2000TM. The front of the printer was altered by cutting flush, the output paper tray and adding a top acrylic panel to accommodate interface circuitry, Device Under Test wiring, a reconfigurable wirebonding area, switches, digital voltmeter displays, and user adjustments for operating the flow 
sensor test machine.

The original HP DeskJet ${ }^{\mathrm{TM}}$ power supply board was left in place inside the printer shell. Three voltage taps (+5 VDC, +19 VDC, and +30 VDC) out of the six available were utilized to power components inside ColeJet $2000^{\text {TM }}$. Since a +10 VDC and a variable DC supply were also needed (for the calibrated Honeywell flow sensor and the flow stream blower, respectively), an additional power supply board, shown in Figure 101, was added to the machine.

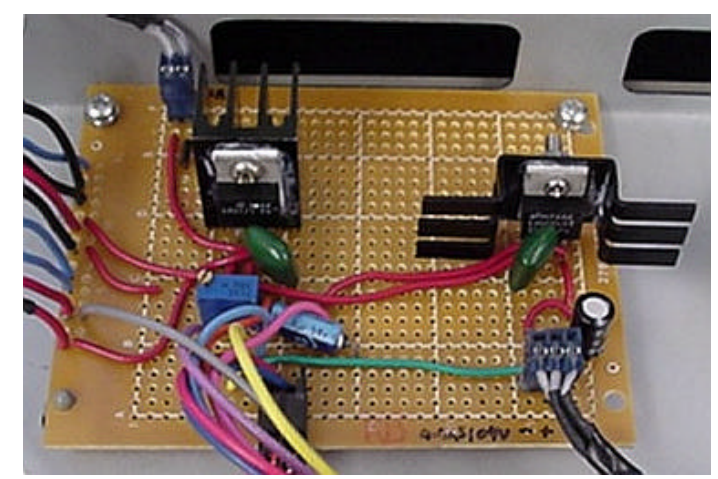

FIGURE 101 - A photo of the supplemental power supply in ColeJet 2000'M. This supply had an LM317 adjustable DC regulator and a LM2940 +10 VDC regulator.

Appendices XVI through $\mathrm{XXI}$ contain material related to the design and operation of this machine. Appendices $\mathrm{XVI}$ and $\mathrm{XVII}$ show tabulated values of relevant air and nitrogen gas properties and various design parameter relationships (respectively) used during the conceptual development of this machine. Appendix XVIII contains selected excerpts from datasheets of major components used in the design of ColeJet $2000^{\text {TM }}$. Detailed machine schematic diagrams are shown in Appendix XIX. Photographs and descriptions of significant machine components and subassemblies are displayed in Appendix XX. A condensed set of operating instructions (which also explain how to attach an ohmmeter to the heater driver, interface board to measure potentiometer values) is included in Appendix XXI.

\section{B. Straightforward Wire Bonding and Packaging Scheme}

Early in the initial design stages for ColeJet $2000^{\mathrm{TM}}$ it was decided that a standard packaging methodology should be utilized to mount and wire-bond the flow sensors in order to 
provide students with hands-on experience in using common Dual Inline Packages (DIPs) for IC devices. The next decision was then which size package(s) to utilize and how to direct flow across each package. The flow direction was designed to be at a $45^{\circ}$ angle with respect to the longitudinal axes of individual flow sensor microbridges/cantilevers. Thus, if mounted within the device cavity of a DIP, the flow sensor die would have to be rotated $45^{\circ}$ for flow directed either along the longitudinal (or main) axis of the package (as shown in Figure 74), or orthogonally across the package. Otherwise, if the flow sensor die were not rotated $45^{\circ}$ within the DIP, a channel would have to be constructed to direct flow across the top of the DIP at a $45^{\circ}$ angle with respect to the longitudinal axis of the package (or the DIP inserted into a flow channel with its main axis rotated $45^{\circ}$ with respect to the flow direction within the channel). For simplicity, it was decided to develop a design in which flow would be directed across the longitudinal axis of the Dual Inline Package.

Considerable thought was given as to how many flow sensor devices should be mounted within each DIP. Since for a given volumetric flow rate, higher velocity flows could be achieved in smaller diameter flow channels (providing greater flow sensor dynamic range testing), ideally, each device die could be diced apart, mounted, wire-bonded, and tested within the smallest possible DIP. However, not only was the handling of individual die proven to be quite difficult (especially etching individual die in $\mathrm{KOH}$ or $\mathrm{TMAH}$ ), but also the dicing of processed wafers into individual die was tedious and very time consuming. Furthermore, since dicing apart die sometimes damaged periphery pad areas, devices which were more fully-intact could be processed by dicing the sensors into blocks of two or four die each. These "duo" or "quad" flow sensor blocks proved to be both much easier to handle, especially during etching, and easier to test multiple device geometries within one package (i.e. more flow sensors could be tested in fewer DIPs), by wire-bonding to all devices in the DIP and using the reconfigurable wire-bonding design of the test machine. On the other hand, larger blocks of two or four flow sensor dice required a DIP with a sufficiently large device cavity to accommodate a $45^{\circ}$ rotated block. Consequently, a DIP-24 (24 pin) package style was selected that met this large device-cavity requirement. 
Since the overall depth of the device cavity in the DIP-24 package significantly exceeded the thickness of flow sensor substrates $(\sim 500 \mu \mathrm{m})$, it was found that a glass spacer was needed to elevate the substrates to the plane of the top surface of the DIP-24. A standard microscope slide was diced into "quad" die sizes and used as spacers for device packaging and testing.

\section{Easily Interchangeable Device Testing}

Although Honeywell commercial flow sensors used customized packaging and a very small high-impedance flow channel (with a $0.05 \times 0.25 \mathrm{~cm}^{2}$ cross section) above each sensor, early in the ColeJet $2000^{T M}$ design process the idea of students (in the microfabrication laboratory) building and integrating their own customized flow channel packaging solution (as well as fabricating the devices themselves) and gluing their custom-designed flow channels to each

wirebonded DIP-24 package was ruled out as both inconvenient and not expedient for consistent, reproducible testing of multiple packaged devices. Customized flow channel packaging for each fabricated flow sensor might also introduce more errors into the testing process itself. A positive consequence of selecting a standard DIP-24 packaging strategy for students to mount and wirebond fabricated flow sensors was that the DIP could be inserted into a pre-configured flow channel, rather than machining and mounting (i.e. gluing) a customized flow channel to each package. Thus, a design was developed in which the DIP (with a mounted flow sensor die), somehow mounted within a "plug", could be inserted into a machined, fixed-position flow channel for device testing. For easy DIP insertion and removal, a low-profile Zero Insertion Force (ZIF) socket was implemented to hold each DIP-24 package inside the plug.

For the DIP-24 ZIF-socket packaging scheme, both 0.75 and 1-inch inside-diameter rigid, clear PVC tubing, and T-shaped fittings (into which a Device Under Test plug could be inserted into the flow channel) obtained from McMaster-Carr supply company were evaluated for possible use as flow channels. Since a clear PVC 1-inch, T-shaped fitting could be machined to accommodate the insertion of a low-profile, 24-pin ZIF socket (holding a DIP-24 package) mounted inside the end of a short length of 1-inch PVC tubing, 1-inch size tubing was selected for the flow channel dimension. 


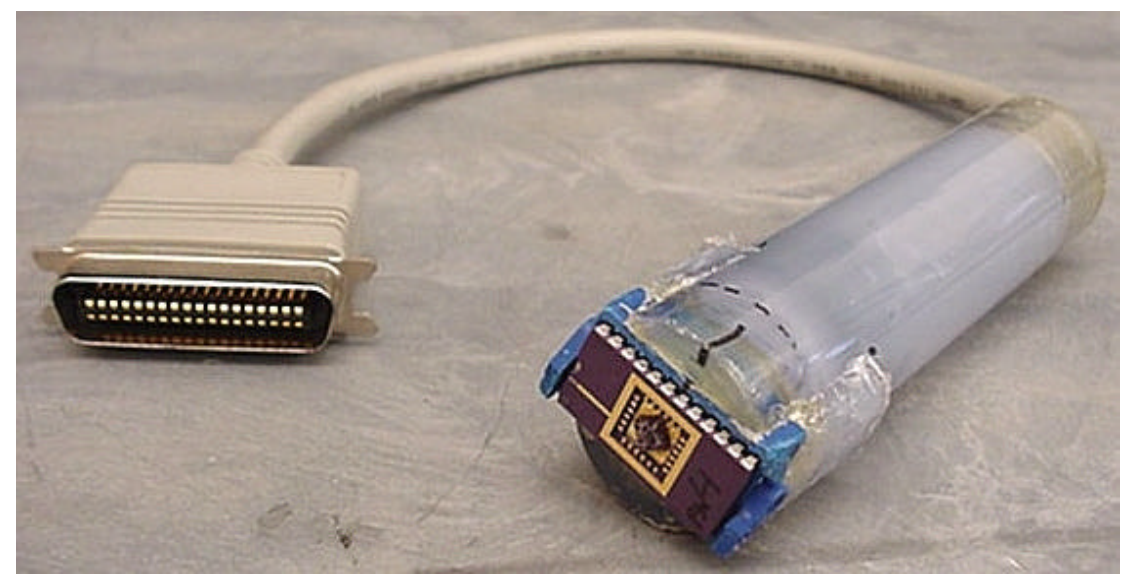

FIGURE 102 - Photo of the Device Under Test insertion tube assembly with 24-pin ZIF.

As shown in Figure 102, a Device Under Test (DUT) plug, which could be inserted into a modified, 1-inch "T" section, was created using a short section of 1-inch PVC tubing, a low-profile 24-pin ZIF socket, and an end of a parallel printer cable with a 36-pin Centronics-style connector. Epoxy resin and silicone sealant were used to permanently affix the ZIF socket inside the PVC plug. The PVC "T" fitting (shown in Figure 103) was machined with a dremel tool to accommodate (the $90^{\circ}$ edges of) a slightly modified, low-profile, 24-pin ZIF socket, which was glued into the small section of 1-inch tubing, through which wires from the socket were routed to the Centronics connector at the end of the cable. The "T" fitting was also machined to allow for insertion of the DUT plug at any depth into the flow channel (to measure the velocity profile of the flow with test flow sensors). Silicone sealant was used to fill gaps around the DUT plug to minimize air leakage out of the "T" section during flow sensor testing with the plug in place. This

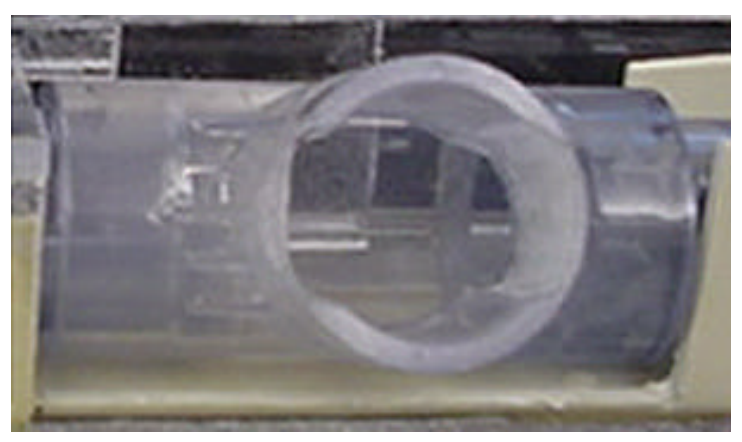

FIGURE 103 - Photo of the machined 1-inch PVC "T" section where the DUT is inserted. This is where devices are inserted into the flow stream during testing. 
sealant can be seen underneath the ZIF socket, on the outside of the clear PVC tube in Figure 102. The Centronics connector cable was wired to the ZIF socket and used to allow for complete removal of the DUT tube assembly from the machine, for storage, cleaning, modification, etc.

\section{Reconfigurable Wire-Bonding Scheme}

In conjunction with the objective for easy device testing interchangeability, ColeJet $2000^{T M}$ was developed to permit different wire-bonding schemes for individual DIP-24 packages. The ZIF socket in the Device Under Test tube was wired, through the Centronics connectors, to a patch board with 24 rows of two test-points (or jumper posts) each. The patch board was wired to the (DIP-24) ZIF socket such that the arrangement of pins on the board had a one-to-one geometrical correspondence with the location of pins in the DIP, as shown in Figure 104. Each pair of jumper posts was wired to a single (DIP-24) ZIF socket pin. A set of jumper wires was created to allow for connecting jumper-post nodes on the patch board to the flow sensor
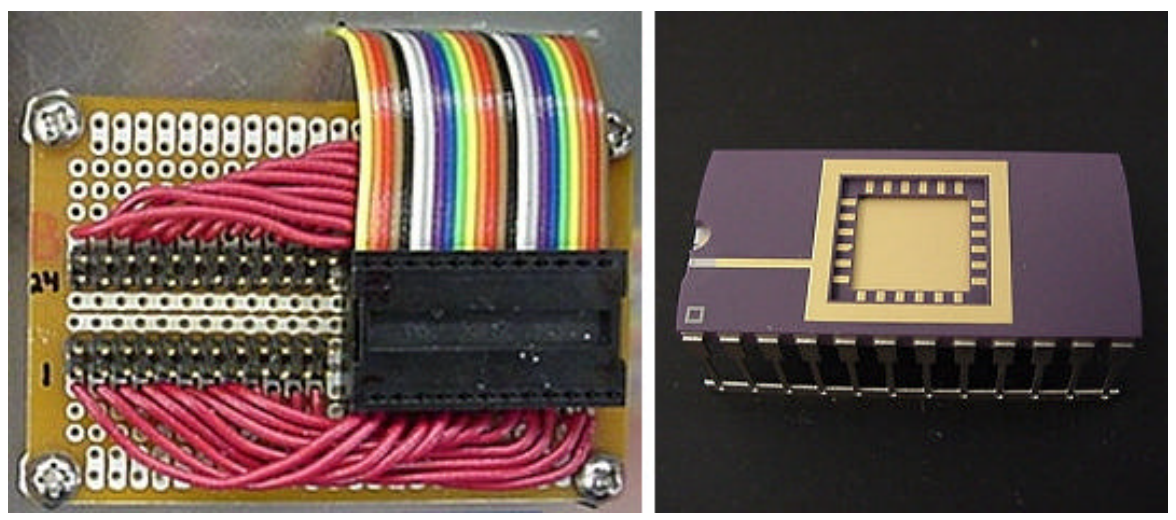

FIGURE 104 - Side-by-side photos showing patch wiring board pin correspondence. The patch board jumper wire posts had a one-to-one correspondence to DIP-24 package pins. On the left, the DUT patch board where jumper wires from the flow sensor interface/driver board can be plugged onto posts corresponding to DIP-24 pins. Each row of two posts corresponds to one DIP-24 package pin (except for pins $2,6,9,10,11$, and 24 , which are unconnected). On the right, a DIP-24 package arranged with pin alignments corresponding to the jumper post array on the left, with pin \#1 at the bottom left and pin \#24 on the top left.

interface/driver board. The re-configurable patch board and the flow sensor interface/driver board are shown together, with attached jumper wires, in Figure 105. Unfortunately, due to the internal wiring of the sealed Centronics connector, it was not possible to connect DIP pins \#2, 6, 9, 10, 


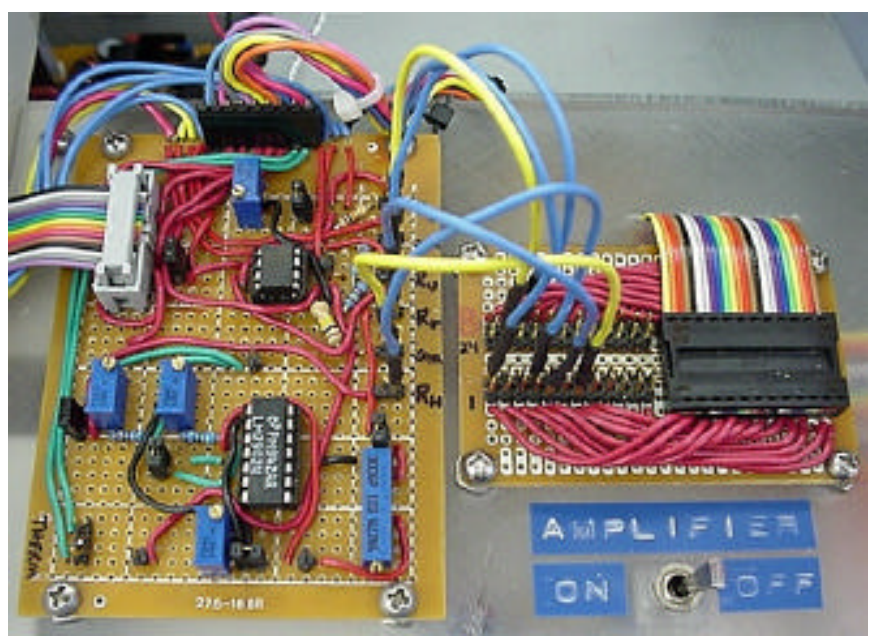

FIGURE 105 - Photo of the flow sensor interface/heater-driver board and patch board. The flow sensor interface/driver board is shown, on the left, with attached jumper wires to the reconfigurable patch board, on the right. The "Amplifier On/Off" switch controls whether the Device Under Test digital voltmeter display shows the $\mathrm{mV}$ output from the flow sensor sense-resistor Wheatstone bridge or the output from a differential instrumentation amplifier included in the design, which amplifies the output from the sense resistor Wheatstone bridge.

11 , and 24 to the patch board. Therefore, these DIP pins were not available to use for wirebonding and testing of flow sensors. An explanation for how to wire/configure these boards is shown in the condensed operating instructions for ColeJet 2000'M in Appendix XXI.

\section{E. Laminar Gas Flow Design}

One of the most important design objectives for consideration was how to develop a machine with laminar flow capabilities in a flow channel sufficiently dimensioned for interchangeable testing of fabricated flow sensors. Based on the requirements for easy device interchangeability, 1-inch inside-diameter PVC tubing was to be utilized as the flow channel. The next issues to resolve were how to produce the flow in a controllable fashion, whether to use a compressed gas or air for the flow, what should be the maximum volumetric flow rate for laminar flow in the 1-inch tubing, how in incorporate a calibrated Honeywell flow sensor in the design, and how to achieve laminar flow in a reasonably compact ColeJet $2000^{T M}$ machine design. Initially, the choice for the flow medium was between compressed high-purity nitrogen gas, or air. As is discussed later, compressed nitrogen gas was abandoned in favor of using air to keep the assumption of gas "incompressibility" valid for the system. For an incompressible flow stream 
through a straight, circular pipe, laminar flow characteristics will dominate for Reynolds numbers below 2000 [see Equation (28)]. Using Equation (26) with the "Properties of Air" Table XXXI in Appendix XVI ( $\rho=1.2006 \mathrm{~kg} / \mathrm{m}^{3}$ and $\left.\mu=1.8253 \times 10^{-5} \mathrm{~kg} / \mathrm{m} \cdot \mathrm{s}\right)$, for air at $21^{\circ} \mathrm{C}$ in a 1 -inch insidediameter pipe, maintaining laminar flow corresponds to a maximum volumetric flow rate of 36.4 SLPM (or $36.28 \mathrm{~L} / \mathrm{min}$ for pure nitrogen). Thus, the upper limit for volumetric flow within the 1inch diameter PVC tubing was 36.4 SLPM for air at room temperature to insure laminar characteristics. Assuming this flow rate could be achieved within this straight tubing (at a Reynolds number of 2000), from Equation (29), the entrance length required for $99 \%$ fully developed laminar flow would be 2.95 meters, which would obviously make the machine much larger than optimally desired.

To keep the size of ColeJet $2000^{T M}$ reasonably small and portable, to produce fullydeveloped (theoretical) laminar flow at a maximum flow rate of 36.4 SLPM within a shorter length (than 2.95 meters) of 1 -inch diameter tubing, a clever technique was employed to split the flow into multiple smaller-diameter channels to achieve laminar flow in a shorter distance. To accomplish this, a "laminar flow section" of $68,3-\mathrm{mm}$-diameter cocktail straws was added to the 1-inch diameter clear PVC flow channel tubing, as shown in Figure 106, to completely fill a section of tubing with the straws. Thus, the total volumetric flow within the PVC tube would be divided equally among the 68 straws, assuming the diameter of each straw was the same, none of the straws were compressed (thereby reducing their cross-sectional area), and there were no gaps between straws. Obviously, there were some gaps around the straws, and some of them were compressed, as is shown in Figure 106. Nonetheless, if the total volumetric flow in the 1inch PVC tube were divided equally among the 68 straws (replacing $Q_{v}$ with $36.4 / 68$ in Equation (26), for the flow in each straw), for air at $21^{\circ} \mathrm{C}$ at a maximum flow rate of 36.4 SLPM in the PVC tube, a maximum Reynolds number of 249 is obtained for each 3-mm diameter straw. Then, to achieve 99\% fully developed laminar flow in each straw, as per Equation (29), each straw length must be at least $4.33 \mathrm{~cm}$. Since each straw was slightly more than $13 \mathrm{~cm}$. long, fully developed laminar flow should exit each straw (at 36.4 SLPM in the PVC tube), and once summated in the 1-inch tubing, the flow across a test flow sensor located farther downstream should be laminar. 


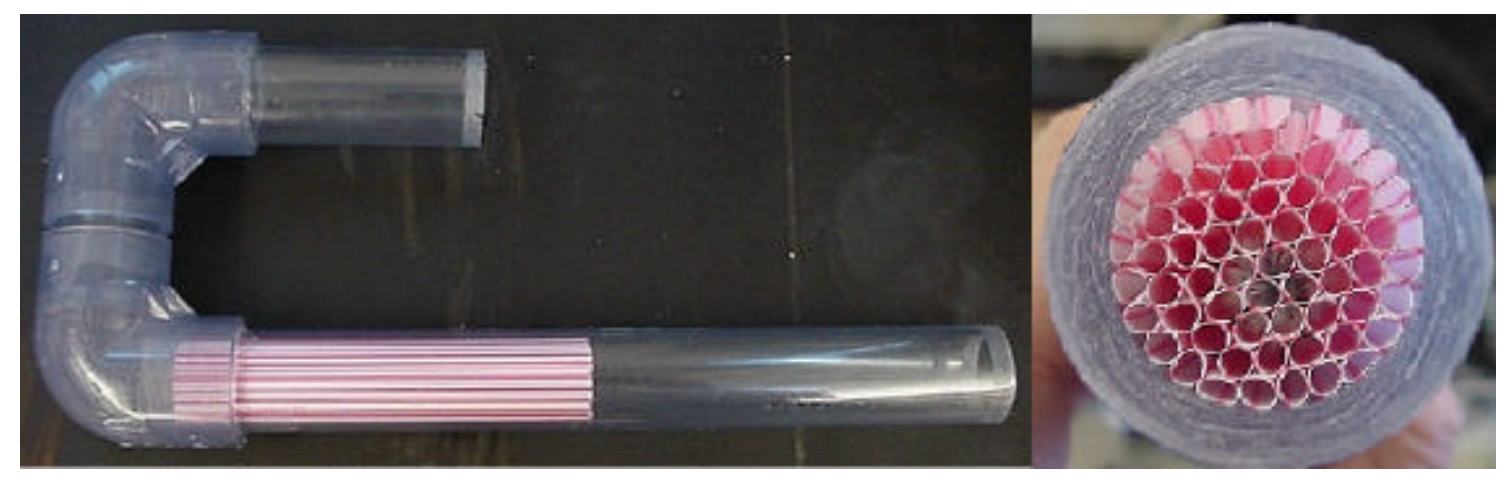

FIGURE 106 - Photos of the laminar-flow cocktail straw section of clear PVC tubing. The cocktail-straw section of 1" PVC tubing was designed for achieving fullydeveloped laminar flow in $2.5 \mathrm{~cm}$. at 20 SLPM. There are 68 straws in the tube with a maximum diameter of $3 \mathrm{~mm}$. each.

Thus, with this cocktail-straw "laminar flow section" within the 1-inch PVC tubing, the length of an upstream, straight section of the PVC tubing (attached to the "T" where flow sensors were to be inserted and tested) could be reduced from more than three meters to somewhat longer than the cocktail straws themselves, to achieve fully developed laminar air flow in the tube at 36.4 SLPM.

The PVC tube section, shown in Figure 106 (one end of which was to be inserted into the "T" where the DUT tube would be inserted with a test DIP-24 flow sensor), was designed to also be removable from ColeJet $2000^{\text {TM }}$. This would allow students and other users to visually examine the device insertion region within the tube, shown in Figure 107, to see where a flow

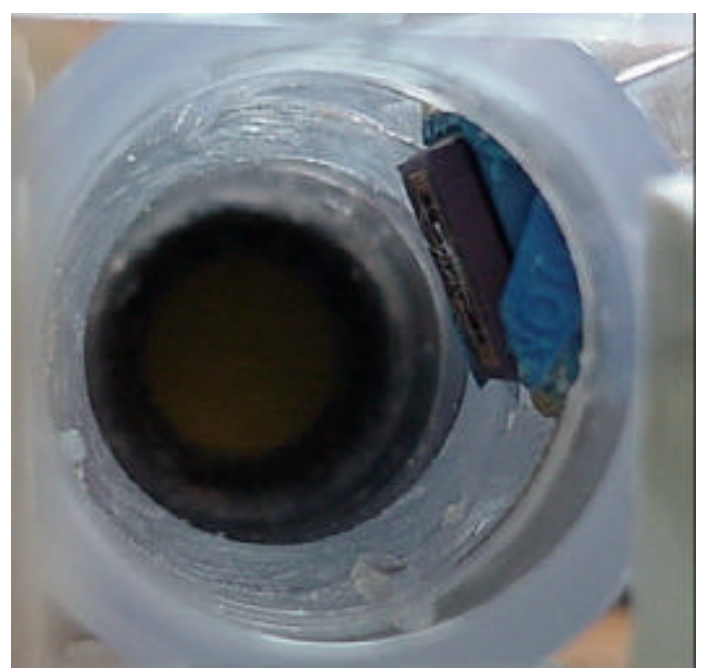

FIGURE 107 - A view of the Device Under Test device insertion region inside the PVC "T" section. The cocktail straw "laminar flow section" J-shaped tube, shown in Figure 106, has been removed to look down into the flow channel at this area. 
sensor being tested was placed within the flow channel. Theoretically, with fully-developed laminar flow, the flow sensor would measure maximum flow in the center of the tube, as shown in the velocity profiles for fully-developed, laminar flow in a pipe shown in Figures 20 and 21. Additionally, to improve "smooth" flow characteristics within the flow channel, all ends of PVC tubing were tapered with the dremel tool to reduce "turbulence" effects at these interfaces.

\section{F. Assumption of "Incompressible" Gas Flow}

In order to use the Bernoulli equation [in Equations (31) - (35)] to calculate the pressure differential between pipe ports within the system and to generally simplify flow calculations, ColeJet $2000^{T M}$ was designed such that an assumption of gas "incompressibility" could be applied. In this case, incompressibility would imply that density variations within the flow stream could be neglected. Gas flows may be considered incompressible if their flow velocities are small relative to the speed of sound in the medium. With a Mach number less than 0.3 (corresponding to an air flow less than $103 \mathrm{~m} / \mathrm{s}$ at $20^{\circ} \mathrm{C}$ ), changes in density are less than $2 \%$ of the mean value. In this system, a maximum volumetric flow rate of 36.4 SLPM for laminar flow conditions corresponds to an average flow velocity of $1.20 \mathrm{~m} / \mathrm{s}$ in the 1-inch PVC tube. Thus, just based upon the flow rate, this maximum flow velocity was sufficiently small to apply the assumption of air incompressibility.

Although the gas velocity was sufficiently small to apply the assumption of incompressibility to the flow stream, the choice of flow media was also important to keep this assumption valid. The original choice for media to test fabricated flow sensors was either to use ambient air or to use compressed, high-purity nitrogen gas. The choice of using compressed nitrogen gas would significantly complicate testing, because both its temperature and density would change throughout the system after exiting the compressed gas storage canister. Initially, the nitrogen would cool substantially upon exiting its compressed state in the tank and would then slowly warm to the ambient throughout the system. Typically a gas "holding tank" would be used in such a system to stabilize the nitrogen temperature before routing it across any test flow sensors. It was quickly concluded that using compressed nitrogen would require the design of a 
much more complicated flow sensor testing system, including a holding tank and temperature sensors to monitor any aggregate changes in gas temperature across the flow sensor being tested. Thus, instead of using compressed nitrogen, filtered, ambient air was to be forced through the system with a DC fan. For theoretical flow rate calculations, a Thermometrics MA100 thermistor was also included in the machine design (inserted into the flow stream just after the DC fan) to measure the stream temperature. Its resistance can be measured by an externallyconnected ohmmeter attached to jumper posts on the flow sensor interface/driver board on the top of the machine. Appendix XVIII contains a data sheet for this thermistor, showing its resistance vs. temperature characteristic, which is shown graphically in Figure 108.

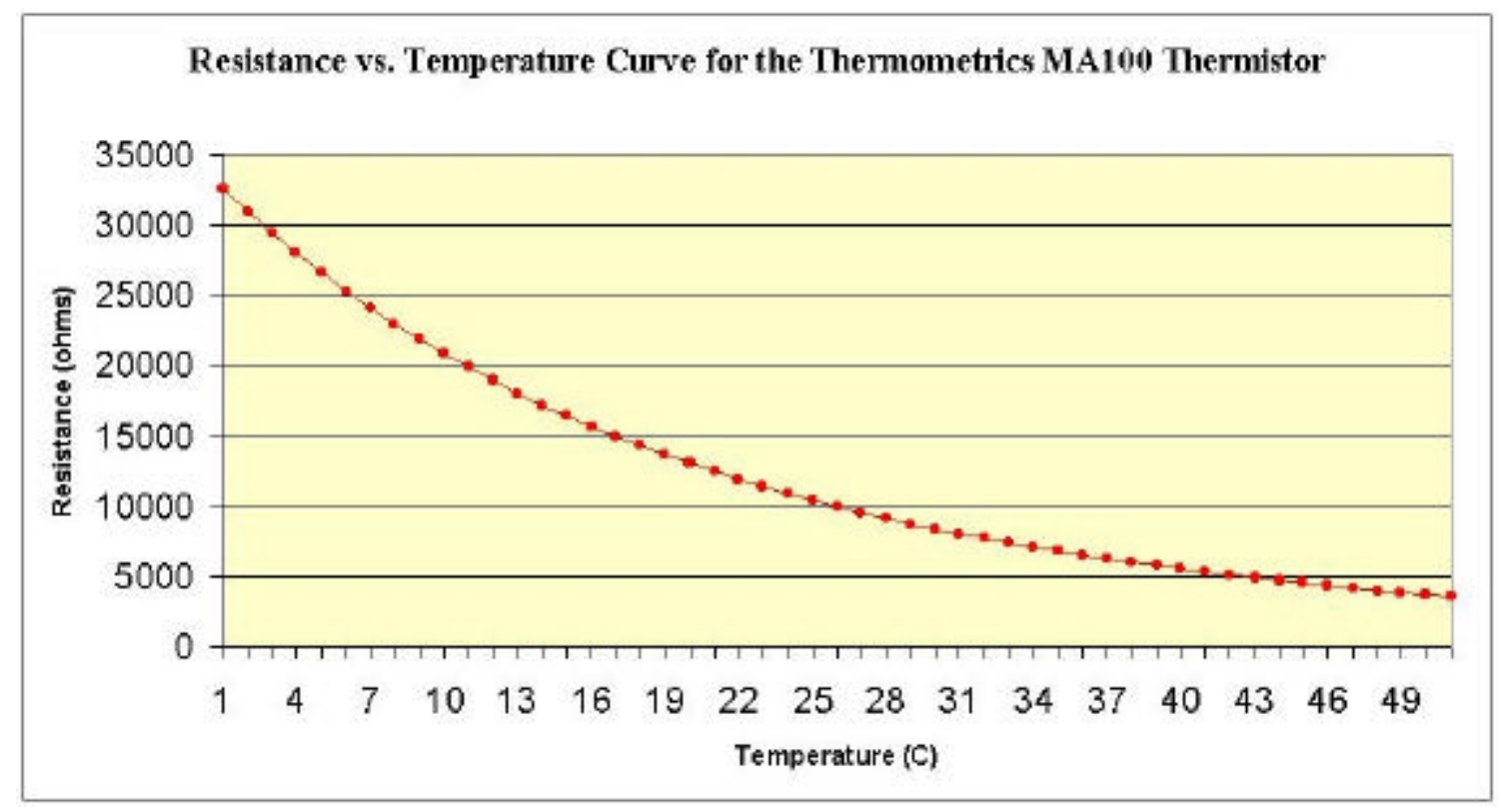

FIGURE 108 - A plot of the resistance vs. temperature characteristic for the MA103 thermistor. This thermistor was installed within the flow channel air stream.

\section{G. Measuring Actual Flow with a Calibrated Honeywell Flow Sensor}

To measure actual flow within the 1-inch diameter PVC tube, a commercial Honeywell flow sensor (from its Micro Switch sensing and control division) was to be included within the ColeJet $2000^{T M}$ design. The choice of which commercial sensor to utilize was based upon whether it would be incorporated in a bypass or in-line (series) configuration within the flow channel design. 
Although they are not specific to the AWM5000 series flow sensor, recom-mendations and design considerations for bypass flow-sensing configurations are included among the data sheets provided in Appendix I. In an in-line configuration, the commercial flow sensor would be installed such that all flow within the channel would be routed through the sensor. A bypass configuration, in which the Honeywell sensor would be installed in parallel with the main 1-inch flow channel, would allow for measuring flow rates that exceed the specifications for the commercial sensor, because only a portion of the total flow would pass into the bypass channel and through the sensor. The majority of the flow would pass through the main PVC channel. An added complexity of the bypass configuration was that the total calculated pressure drop across the sensor in the bypass channel must be less than or equal to the typical full scale pressure drop of the commercial flow sensor. In other words, the estimated maximum total pressure drop "seen" by the flow sensor in the bypass channel, relative to flow in the main PVC channel, must be within its dynamic measurement range. As shown in Figure 109, the full scale pressure drop range for all commercially available Honeywell flow sensors was 49.8 - $1992 \mathrm{~Pa}$.

Tables included in Appendix XVII show various bypass-channel design possibilities considered in developing the flow channel arrangement for the ColeJet $2000^{\text {TM }}$ machine.

\begin{tabular}{|c|c|c|c|c|}
\hline Product & Rated Flow & Rated Pressure & Output Type & $\begin{array}{l}\text { Select by Typical Full } \\
\text { Scale Pressure Drop }\end{array}$ \\
\hline AWM2100V & $\pm 200 \mathrm{sccm}$ & N.A. & $\pm 44.5 \mathrm{mv}$ & $20 " \mathrm{H}_{2} \mathrm{O}$ or $49.8 \mathrm{~N} / \mathrm{m}^{2}$ \\
\hline AWM2150V & $\pm 30 \mathrm{sccm}$ & N.A. & $\pm 14 \mathrm{mv}$ & $.20 " \mathrm{H}_{2} \mathrm{O}$ or $49.8 \mathrm{~N} / \mathrm{m}^{2}$ \\
\hline AWM2200V & N.A. & $0-2^{\prime \prime} \mathrm{H}_{2} \mathrm{O}$ & $\pm 31.75 \mathrm{mv}$ & $2.0^{\prime \prime} \mathrm{H}_{2} \mathrm{O}$ or $498 \mathrm{~N} / \mathrm{m}^{2}$ \\
\hline AWM2300V & $0-1000 \mathrm{sccm}$ & N.A. & $\pm 55.5 \mathrm{mv}$ & $1.3^{\prime \prime} \mathrm{H}_{2} \mathrm{O}$ or $324 \mathrm{~N} / \mathrm{m}^{2}$ \\
\hline AWM3100V & $0-200$ sccm & N.A. & $1.5 \mathrm{Vdc}$ & $20 " \mathrm{H}_{2} \mathrm{O}$ or $49.8 \mathrm{~N} / \mathrm{m}^{2}$ \\
\hline AWM3150V & $0-30 \mathrm{sccm}$ & N.A. & $1.5 \mathrm{Vdc}$ & $.20 " \mathrm{H}_{2} \mathrm{O}$ or $49.8 \mathrm{~N} / \mathrm{m}^{2}$ \\
\hline AWM3200CR & N.A. & $0-2^{\prime \prime} \mathrm{H}_{2} \mathrm{O}$ & 4-20 mA & $2.0^{\prime \prime} \mathrm{H}_{2} \mathrm{O}$ or $498 \mathrm{~N} / \mathrm{m}^{2}$ \\
\hline AWM3201CR & N.A. & $0-0.5^{\prime \prime} \mathrm{H}_{2} \mathrm{O}$ & $4-20 \mathrm{~mA}$ & $.50 " \mathrm{H}_{2} \mathrm{O}$ or $125 \mathrm{~N} / \mathrm{m}^{2}$ \\
\hline AWM3200V & N.A. & $0-2^{\prime \prime} \mathrm{H}_{2} \mathrm{O}$ & $1.5 \mathrm{Vdc}$ & $2.0 " \mathrm{H}_{2} \mathrm{O}$ or $498 \mathrm{~N} / \mathrm{m}^{2}$ \\
\hline AWM3300V & $0-1000 \mathrm{sccm}$ & N.A. & $1.5 \mathrm{Vdc}$ & $1.3^{\prime \prime} \mathrm{H}_{2} \mathrm{O}$ or $324 \mathrm{~N} / \mathrm{m}^{2}$ \\
\hline AWM42150VH & $\pm 25 \mathrm{sccm}$ & N.A. & $\pm 8.5 \mathrm{mv}$ & $.20 " \mathrm{H}_{2} \mathrm{O}$ or $49.8 \mathrm{~N} / \mathrm{m}^{2}$ \\
\hline AWM42300V & $0-1000 \mathrm{sccm}$ & N.A. & $\pm 55.5 \mathrm{mv}$ & $.90^{\prime \prime} \mathrm{H}_{2} \mathrm{O}$ or $224 \mathrm{~N} / \mathrm{m}^{2}$ \\
\hline AWM43300V & $0-1000 \mathrm{sccm}$ & N.A. & $1.5 \mathrm{Vdc}$ & $.90 " \mathrm{H}_{2} \mathrm{O}$ or $224 \mathrm{~N} / \mathrm{m}^{3}$ \\
\hline AWM43600V & $0-6 \mathrm{sim}$ & N.A. & $1.5 \mathrm{Vdc}$ & $8 " \mathrm{H}_{2} \mathrm{O}$ or $1992 \mathrm{~N} / \mathrm{m}^{4}$ \\
\hline
\end{tabular}

FIGURE 109 - Table showing full-scale pressure drop values for Honeywell flow sensors. This was derived from a Bypass Design Considerations data sheet showing the full scale pressure drop (FSPD) values for different sensors. Although not shown, the AWM5000 series, 20 SLPM sensor has a FSPD of $\sim 560 \mathrm{~N} / \mathrm{m}^{2}$. 
Calculations for numerical data in Appendix XVII were based upon equations provided in Honeywell's Bypass Design Considerations data sheet, contained in Appendix I. Equations (24), (25), (26), (34), and (35) can also be used to calculate parameters shown in Appendix XVII.

Table XXIV, shows a set of bypass design parameters for the maximum volumetric flow rate through the 1-inch diameter flow channel to have theoretical laminar flow. As shown, even with a bypass length of one meter (which was impractical), the total pressure drop across the bypass flow sensor $\left(\Delta P_{\text {total }}\right)$ would be less than $2.4 \mathrm{~Pa}$. Since this is $1 / 21^{\text {st }}$ the full-scale pressure drop for Honeywell flow sensors with the smallest full scale pressure drop range (49.8 Pa), even for an AWM3100V sensor with a $200 \mathrm{sccm}$ maximum rated flow, this might represent only $10 \mathrm{sccm}$ of flow through the device at maximum 36.4 SLPM flow. It was concluded that the bypass configuration was potentially too problematic and impractical to achieve good dynamic range with the system. It was believed that it would be better use more of the calibrated flow sensor's dynamic range: i.e. to measure a full-scale flow rate range of $0-20$ SLPM in a series configuration, than to measure a $0-10 \mathrm{sccm}$ flow range in a one-meter bypass channel, $0-36.4$ SLPM configuration.

\section{TABLE XXIV}

\section{DESIGN PARAMETERS WITH AN AWM5000 SERIES BYPASS SENSOR FOR AIR AT $21^{\circ} \mathrm{C}$}

\begin{tabular}{|c|c|c|c|c|c|c|c|}
\hline $\begin{array}{c}\text { Main } \\
\text { Flow } \\
\text { Channel } \\
\text { ID (Area) } \\
\mathrm{mm}\left(\mathrm{mm}^{2}\right)\end{array}$ & $\begin{array}{c}\text { Bypass } \\
\text { Channel } \\
\text { Length } \\
(\mathrm{m})\end{array}$ & $\begin{array}{c}\text { Max. } \\
\text { Design } \\
\begin{array}{c}\text { Flow } \\
\text { Rate } \\
(\mathrm{L} / \mathrm{min})\end{array}\end{array}$ & $\begin{array}{c}\text { Velocity } \\
\text { of Flow, } \\
\bar{v} \\
(\mathrm{~m} / \mathrm{s})\end{array}$ & $\begin{array}{c}\text { Reynolds } \\
\text { Number, } \\
\mathrm{R}_{\mathrm{e}}\end{array}$ & $\begin{array}{c}\text { Entrance } \\
\text { Length, } \\
\mathrm{L}_{\mathrm{e}} \\
(\mathrm{m})\end{array}$ & $\Delta \mathrm{P}_{\text {flow }}$ & $\Delta \mathrm{P}_{\text {total }}$ \\
\hline $25.4(507)$ & 0.356 & 36.4 & 1.2 & 2000 & 2.95 & 0.385 & 1.676 \\
\hline $25.4(507)$ & 1.0 & 36.4 & 1.2 & 2000 & 2.95 & 1.084 & 2.374 \\
\hline
\end{tabular}

Since an in-line, or series, calibrated flow sensor configuration was to be used in the 1inch flow channel, a sensor with the maximum possible measurable flow range was needed. An AWM5104VN flow sensor (shown in Figure 110) with a maximum calibrated measurable flow rate of 20 SLPM, and $1 / 4$ " ports was purchased (for US\$155 from Newark Electronics). A flow rate of 20 SLPM in the 1-inch diameter PVC flow channel corresponded to a Reynolds number of 1099, which was theoretically laminar. Data sheets for the AWM5104VN are included in Appendix I. 


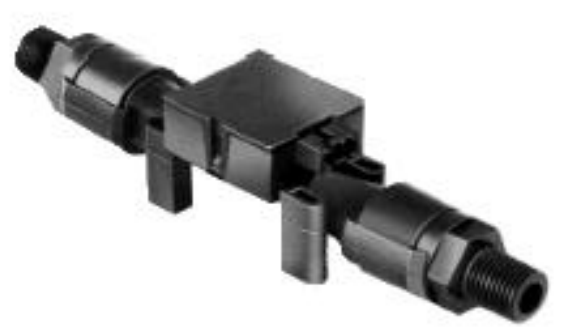

FIGURE 110 - A photo of the commercial Honeywell AWM5104VN flow sensor. It has a measurable flow range of $0-20$ SLPM. This sensor was calibrated for nitrogen.

The sensor was positioned within the flow channel as close to the flow sensor(s) being tested as possible, located after the "T" section where the DUT would be inserted into the flow stream. A threaded $1 / 4$ " adapter on the inlet port end of the sensor was removed, and a short section of clear PVC pipe was epoxied to the sensor housing. Once a flow channel mounting assembly was constructed within the HP DeskJet ${ }^{\mathrm{TM}}$ shell (with acrylic sheets, a wood block, a plastic trough, and epoxy), the AWM5104VN was held in place with four screws through its mounting holes. Air exiting the Honeywell sensor also exited the machine through the sensor's outlet port, which protruded beyond the plastic top-cover shell of the machine.

Since the sensor's calibration was only guaranteed for a precise supply voltage of 10 VDC ( \pm 0.01), an LM2940T 10V regulator was installed on the supplemental power supply board to power the AWM5104VN. A data sheet for this power supply is included in Appendix XVIII.

\section{H. Precisely and Finely Controllable Gas Flow Rate}

After compressed nitrogen gas was abandoned as a potential flow medium, a means was needed to controllably push ambient air through the flow channel. Direct Current fans were evaluated as they were believed to offer superb controllability for the flow rate. A comparison of various fans evaluated is included in Table XXV. Initially, axial-style fans with wide operating voltage ranges of up to $30 \mathrm{VDC}$ were investigated. It became quickly apparent, however, that blower-style ("squirrel cage") fans could develop greater static air pressure in the stream and push more air through the channel. The Panasonic blower highlighted in red in the table was actually implemented in ColeJet $2000^{T M}$. Note that even though the Panasonic axial fan highlighted in blue in the table has a nearly identical, rated static air pressure capability, the 
TABLE XXV

COMPARISION BETWEEN VARIOUS AXIAL AND BLOWER FANS EVALUATED

\begin{tabular}{|c|c|c|c|c|c|c|c|}
\hline $\begin{array}{c}\text { Manufacturer } \\
\text { and } \\
\text { Model } \\
\text { Number }\end{array}$ & $\begin{array}{l}\text { Fan } \\
\text { Type }\end{array}$ & $\begin{array}{l}\text { Oper. } \\
\text { Voltage } \\
\text { Range } \\
\text { (V) }\end{array}$ & $\begin{array}{c}\begin{array}{c}\text { Rated } \\
\text { Current } \\
(\mathrm{mA})\end{array}\end{array}$ & $\begin{array}{l}\text { Nominal } \\
\text { Speed } \\
\text { (RPM) }\end{array}$ & $\begin{array}{l}\text { Max. } \\
\text { Air } \\
\text { Flow } \\
(\text { LPM) }\end{array}$ & $\begin{array}{c}\text { Max. } \\
\text { Static } \\
\text { Air } \\
\text { Pressure } \\
(\mathrm{Pa}) \\
\end{array}$ & $\begin{array}{c}\text { Max. } \\
\text { Measured } \\
\text { Flow* }^{*} \\
\text { (SLPM) }\end{array}$ \\
\hline $\begin{array}{l}\text { Panasonic } \\
\text { FBK04F12L }\end{array}$ & Axial & $7-13.8$ & 75 & 4700 & 110.44 & 24.5 & $\sim 2.125$ \\
\hline $\begin{array}{l}\text { Comair-Rotron } \\
\text { CR0412HB-50 }\end{array}$ & Axial & $6-14$ & 130 & 8500 & 170.0 & 51.76 & $\sim 4.625$ \\
\hline $\begin{array}{l}\text { Panasonic } \\
\text { FBK04F24U }\end{array}$ & Axial & $14-27.6$ & 100 & 9500 & 260 & 117.7 & $\sim 6.15$ \\
\hline $\begin{array}{l}\text { Panasonic, } \\
\text { FAL6F12LH }\end{array}$ & Blower & $7-13.8$ & 500 & 2550 & 650 & 110.3 & $\sim 16.5$ \\
\hline $\begin{array}{l}\text { Comair- } \\
\text { Rotron, } \\
\text { VK24B3 }\end{array}$ & Blower & $12-28$ & 800 & 4000 & 934.4 & 423.03 & $?$ \\
\hline
\end{tabular}

blower forced nearly $168 \%$ more air (volumetrically) through the channel. The last blower listed in the table was not evaluated as no retailer could be found which had it in stock and deliverable on short notice. The Panasonic blower (FAL6F12LH) was installed in ColeJet $2000^{\text {TM }}$ by mounting it to the acrylic assembly that housed and supported the flow channel. A 1-inch PVC coupling (which was one receptacle for the removable "J" shaped laminar flow tube section) was glued to the blower housing, and the blower outlet was sealed to the PVC tube. A Eureka vacuum cleaner micron filter with a custom acrylic filter holder assembly was added to the air intake of the blower to reduce particulate contamination in the flow stream during fabricated flow sensor testing. The typical maximum achievable flow rates with this blower were 15-16.5 SLPM in the channel, corresponding to a maximum flow velocity of $\sim 0.543 \mathrm{~m} / \mathrm{s}(1.78 \mathrm{ft} / \mathrm{s})$.

An LM-317 adjustable DC power regulator circuit was installed on the supplemental power supply board for the blower. A data sheet for this regulator is included in Appendix XVIII. As part of this circuit, for precise user adjustment of the output voltage to the blower (and hence the flow rate in the channel), a 20-turn precision potentiometer was installed on the top, right, panel of the machine (and labeled FAN SPEED). 


\section{Flow Sensor Interface Circuitry Design}

The theory of operation for how flow sensors were to be tested with ColeJet $2000^{\mathrm{TM}}$ was essentially the same as how the original Honeywell flow sensor was driven. The interface circuitry for testing fabricated flow sensors was developed based upon designs and specifications suggested by Honeywell in data sheets for using its current unamplified mass airflow sensor product line. All circuitry developed for ColeJet $2000^{T M}$ is shown in schematic diagrams included in Appendix XIX. Where possible, wiring to installed major components or circuits had connectors to permit easier replacement, testing, etc. The flow sensor Device Under Test interface circuit was mounted on top of the machine, and included six jumper posts for connections to the patch board. A constant DC voltage Wheatstone half-bridge configuration (with $10 \mathrm{~V}$ supply from the LM2940T regulator) was implemented for the upstream and downstream sense resistors. A 20-turn precision potentiometer was included to provide user adjustment of the Wheatstone bridge BALANCE. The thermal anemometer heater-driver circuit was designed to operate at a constant temperature differential, approximately $150-220^{\circ} \mathrm{C}$, above the ambient.

An optional Burr-Brown INA128 differential instrumentation amplifier was added to the interface circuit to provide an amplified sense-resistor Wheatstone bridge output, if needed. A 20-turn precision GAIN potentiometer was included on the top left panel for user adjustment of the INA128 amplifier circuit gain. Another smaller 10-turn potentiometer on the interface circuit board may be adjusted to set the reference voltage of the amplifier at no flow (see Appendix XXI).

The heater driver circuit was similar to the design shown in Figure 10, in which the microbridge/cantilever heated resistor was positioned in one bridge arm and a temperaturecompensating, heat-sunk, substrate, reference resistor was positioned in the other bridge arm. The rest of the bridge was comprised of three 10-turn potentiometers (located on the circuit board) which could be adjusted to compensate for nonlinearities in the metallization TCR characteristic of the flow sensors. An error integrator using one of the LM2902 operational amplifiers provided the supply voltage to the bridge. A total of four 10-turn circuit board potentiometers (including the three previously mentioned) were included in this circuit to allow for 
custom configuration (and comparative circuit modeling) of the heater driver. Additionally, each potentiometer can be disconnected from the circuit by removing a jumper, and an ohmmeter connected to jumper posts on the board to measure the potentiometer's resistance. The condensed operating instructions included in Appendix XXI (the Technical Guide) include a description of how to measure and adjust each potentiometer's resistance.

Two C-TON DK743 digital LCD voltmeter panel meter displays $(0-19.999$ VDC) were added to the top left panel of ColeJet $2000^{\mathrm{TM}}$. The top display was wired to the output voltage of the Honeywell AWM5104VN sensor to continuously indicate the measured flow rate in the PVC flow channel. Depending upon the position of the AMP ON/OFF switch, the bottom display was connected to either the $\mathrm{mV}$ output of the sense resistor Wheatstone bridge or the output of the optional, differential instrumentation amplifier. The AMP ON/OFF switch only controlled which voltage nodes the bottom LCD meter displays, as it did not actually disconnect power to the amplifier. The amplifier was always connected to the output of the sense resistor Wheatstone bridge, unless jumper JP5 was removed (see Appendices XIX and XXI). 


\section{EXPERIMENTAL RESULTS}

Successfully fabricated flow sensors were tested using the ColeJet $2000^{T M}$ machine. A more detailed description of how to use the machine is included in the condensed operating instructions in Appendix XXI. Before packaging and wire-bonding each die, the Wentworth Labs probe station was utilized to test electrical continuity of resistors on each flow sensor. Devices were singled out which had measured upstream and downstream resistor resistances that were symmetrical, or within approximately $5 \%$ of each other. Each "quad" or "duo" die which had viable sensors to be tested was then mounted in a DIP-24 package, on top of a "quad-size" glass-slide spacer, using oven-cured Epo-Tek® 353ND epoxy. Each die was then wire-bonded to its DIP-24 package, skipping wire-bonding to DIP pins \#2, 6, 9, 10, 11, and 24, because those pins were not electrically connected to the patch board via the Centronics connector. Note that creative wire-bonding schemes could be utilized to share DIP pin nodes with multiple flow sensors, such as using a single DIP pin for two or more Wheatstone bridge common connections (e.g. either the common node used for the heater resistor, $R_{h}$, and substrate resistor, $R_{r}$, or the common node used for the upstream resistor, $R_{u}$, and downstream resistor, $\left.R_{d}\right)$. Each DIP-24 was inserted into the DUT plug ZIF socket, which was marked with a "1" to indicate the correct orientation of the package and location of pin \#1 in the socket. The plug was then inserted into the flow channel. After insuring the Centronics connector was snugly plugged into its receptacle on the top panel of the machine, patch cables were then connected between flow sensor nodes (jumper posts) on the patch board (corresponding to the wire-bonded device to be tested) and the six flow sensor driver nodes on the interface/driver board. Once configured, the machine was turned on with the FAN switch in the OFF position. The BALANCE knob (on the top left panel) was then carefully adjusted to null the output of the sense resistor Wheatstone bridge to nearly 0 VDC at no flow. Next, the FAN switch was turned $O N$ to power the blower, and the desired flow rate was adjusted with the FAN SPEED potentiometer dial. The Honeywell AWM5104VN sensor measured flow rate in the channel was displayed in the top left LCD panel meter in volts, which 
was converted to SLPM using the following formula:

$$
Q_{v}=5(V-1)
$$

where $Q_{v}$ was the measured volumetric air flow rate in SLPM and $V$ was the displayed output voltage of the sensor in volts (which was valid from 1 VDC at no flow to 5 VDC at 20 SLPM). Depending upon the position of the AMPLIFIER ON/OFF switch, the bottom LCD panel meter either directly displayed the output voltage across the sense-resistor Wheatstone bridge, or the amplified bridge output from the differential instrumentation amplifier. If the output of the amplifier was selected to be displayed, its gain could be adjusted with the GAIN knob on the left, top panel of the machine. The gain setting could be determined (as described in Appendix XXI) by removing jumper JP6 and connecting an external ohmmeter to Test Point 11 (TP11) and a Jumper Post at JP6 to measure $R_{G}$. The gain could then be calculated from:

$$
G=1+\frac{50}{R_{G}}
$$

where $R_{G}$ is the measured setting in $\mathrm{K} \Omega$ of the $5 \mathrm{~K}, 10$-turn, Bourns $G A I N$ potentiometer (labeled $R_{7}$ in the schematics provided in Appendix XIX). Additionally, an external high-input-impedance digital multimeter (DMM) could be connected to the sense-resistor Wheatstone bridge output by wiring it to appropriate posts on the patch board (which could also be already connected to the interface/driver board with jumper wires for testing). The use of an external DMM, in $20 \mathrm{mV}$ or $200 \mathrm{mV}$ full-scale measurement range, was especially useful for measuring the small $\mathrm{mV}$ sense resistor Wheatstone bridge outputs (without amplification) during testing, since the built-in LCD panel meter had a 19.999 VDC full scale range with minimum increments of $1 \mathrm{mV}$.

The configuration and measurement of error integrator heater driver circuit resistances $\left(R_{1}, R_{a}, R_{b}\right.$, and $\left.R_{c}\right)$ is described in Appendix XXI. For test results described herein, these resistances were set to typical values suggested by Honeywell for interface circuitry to use with 
its unamplified flow sensor products (some of which also use platinum resistor metallization):

$\mathrm{R}_{1}=5 \mathrm{~K} \Omega, \mathrm{R}_{\mathrm{a}}=501 \Omega, \mathrm{R}_{\mathrm{b}}=3 \mathrm{~K} \Omega$, and $\mathrm{R}_{\mathrm{c}}=1.5 \mathrm{~K} \Omega$.

Out of all the processes explored and documented in this research (over three years), the low-stress LPCVD $\mathrm{Si}_{3} \mathrm{~N}_{4}$ (structure) flow sensors with DC planar magnetron sputter-deposited platinum thin-film metallization (and chromium adhesion layer) were the only viable devices that were fabricated. Chromium and titanium metallization-based devices (some of which were fabricated on LPCVD $\mathrm{Si}_{3} \mathrm{~N}_{4}$ wafers) were deemed electrically nonviable because of their extremely low and hysteretic TCR characteristics. The half-wafer (PT1) shown in Figure 92, which was the only platinum metallization wafer processed, contained $30-50 \%$ device yields, because positive photoresist lifted-off underlying resistor metallization during delineation in heated aqua regia, allowing the solution to etch through $5-\mu m$ line-width resistors in some regions.

Figures 111, 112, and 113 show response characteristics for two identical-geometry flow sensor devices (Flow Sensor \#1 in the 1998/1999 photomask design) from wafer PT1 on two

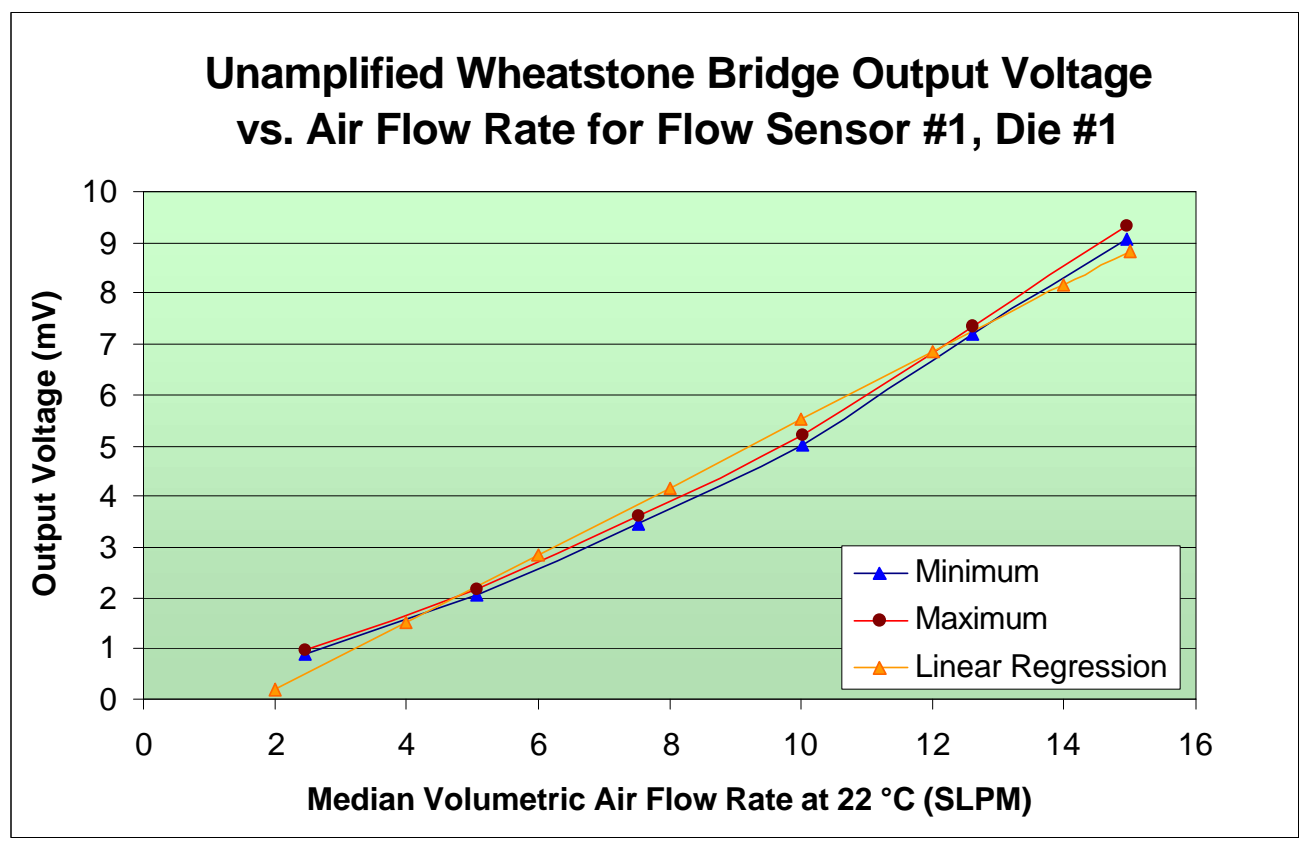

FIGURE 111 - Graph of bridge output voltage vs. flow rate for Flow Sensor \#1, die \#1. A graph of the experimentally-measured sense resistor Wheatstone bridge output response of Flow Sensor \#1 in die \#1, vs. volumetric air flow rate in the ColeJet $2000^{T M}$ machine. Both the output voltage and measured air flow rates fluctuated during measurement. By linear regression, the sensitivity was calculated to be $0.67 \mathrm{mV} / \mathrm{SLPM}$. 


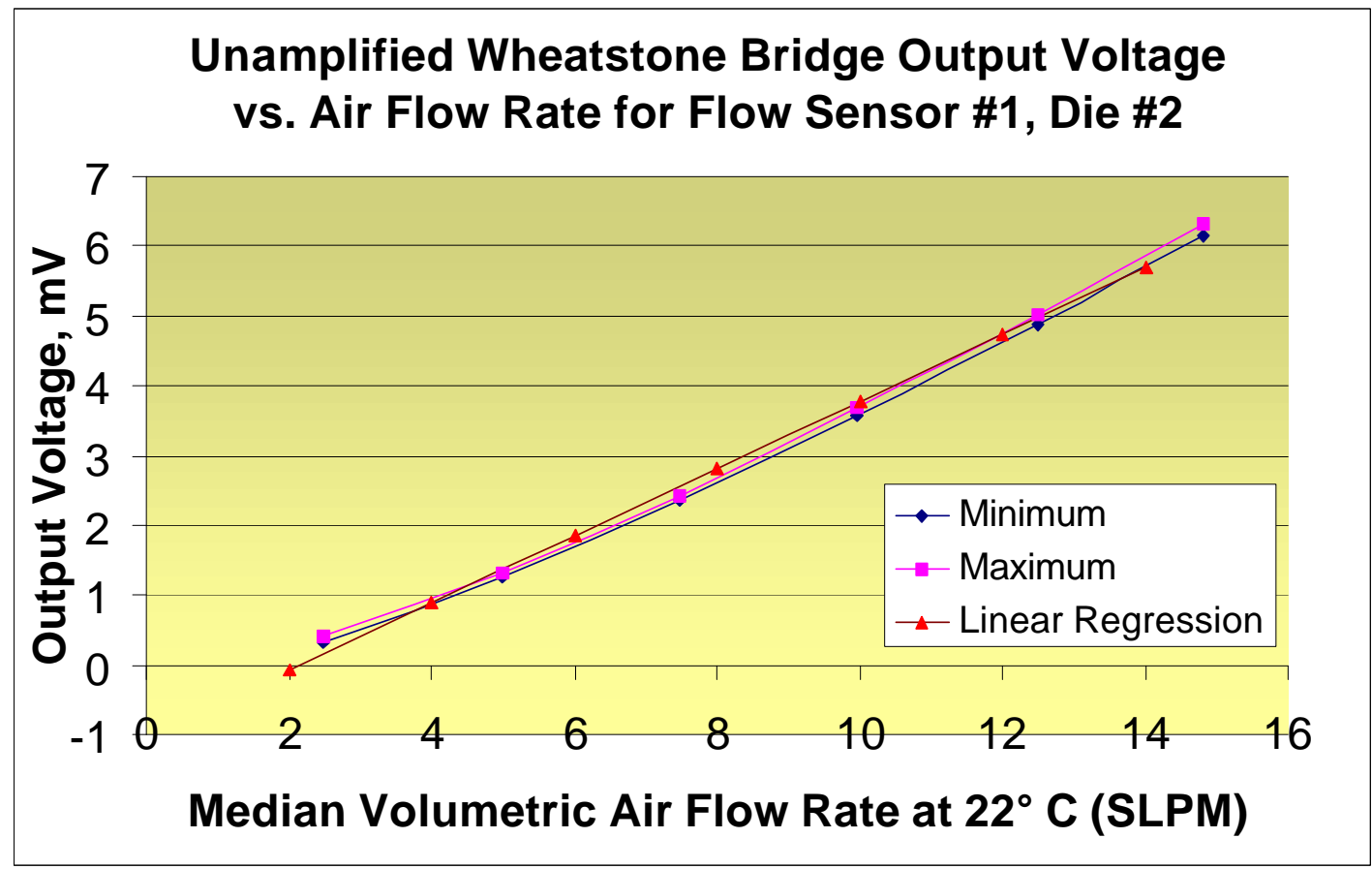

FIGURE 112 - Graph of bridge output voltage vs. flow rate for Flow Sensor \#1, die \#2. A graph of the experimentally-measured sense resistor Wheatstone bridge output response of Flow Sensor \#1 in die \#2, vs. volumetric air flow rate in the ColeJet $2000^{T M}$ machine. Both the output voltage and measured air flow rates fluctuated during measurement. From linear regression, the sensitivity was calculated to be $0.48 \mathrm{mV} / \mathrm{SLPM}$.

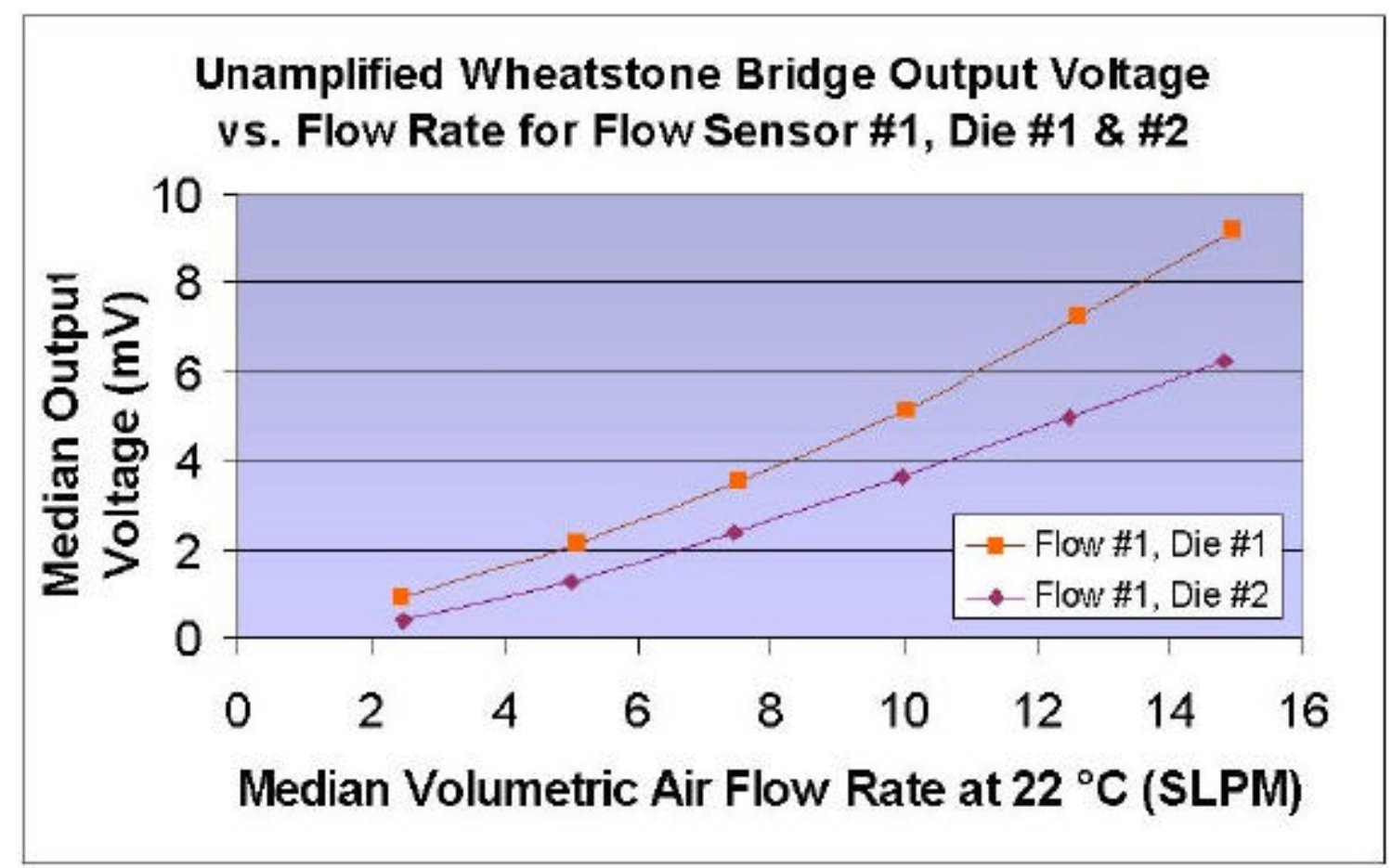

FIGURE 113 - Graph of Wheatstone bridge output voltage vs. flow rate, combined results. 
separate quad dice. Each graph also shows a linear regression curve through the data for calculating each sensor's sensitivity. Sense resistor Wheatstone bridge unamplified $(\mathrm{mV})$ output voltages were measured with an externally-connected Fluke $8500 \mathrm{~A}$ lab multimeter. It is important to note that exact reproducibility of response characteristics for individual flow sensors (e.g. for the same flow rate tests on the same sensor performed on different days) was difficult, and sometimes impossible, because:

1. The response characteristics were strongly influenced by the exact placement of the DIP-24 Device Under Test plug within the flow stream, due to the geometry and flow characteristics of the "T" pipe fitting into which the DUT plug was inserted;

2. Flow rates randomly fluctuated, with variation spreads of as much as $300 \mathrm{sccm}$ for a given adjusted speed of the blower fan (set with the FAN SPEED dial), due to flow characteristics and speed variations of the Panasonic blower motor design itself for a given supply voltage;

3. The response characteristic was slightly different for each set of unique, adjusted resistor values $\left(R_{1}, R_{a}, R_{b}\right.$, and $\left.R_{c}\right)$ set in the heater driver circuit; and

4. The response changed slightly depending upon the initial, finely-adjusted balance setting (or nulling) of the sense resistor Wheatstone bridge at no flow conditions, which was determined by the setting of the BALANCE potentiometer.

For comparison purposes, Figure 114, compares the unamplified sense resistor Wheatstone bridge output voltage characteristics to median flow velocity between the two tested flow sensors and two commercially-marketed Honeywell flow sensors. The AWM42150VH sensor is rated to measure flows of up to $30 \mathrm{sccm}$, and the AWM2100V is rated for flows of up to $200 \mathrm{sccm}$.

Linear regression was performed on data generated from testing the two fabricated flow sensors and on data provided in Honeywell's AWM42150VH and AWM2100V data sheets for comparison. Regression of response data from Flow Sensor \#1 on die \#1 resulted in an approximate sensitivity of $0.67 \mathrm{mV} / \mathrm{SLPM}$, and similar calculations for Flow Sensor \#1 on die \#2 produced a sensitivity of $0.48 \mathrm{mV} / \mathrm{SLPM}$. Linear regression of the AWM42150VH sensor's response characteristics resulted in a calculated sensitivity of $222.5 \mathrm{mV} / \mathrm{SLPM}$. From Figure 114 


\section{Comparision of Unamplified Wheatstone Bridge Output vs. Flow Velocity}

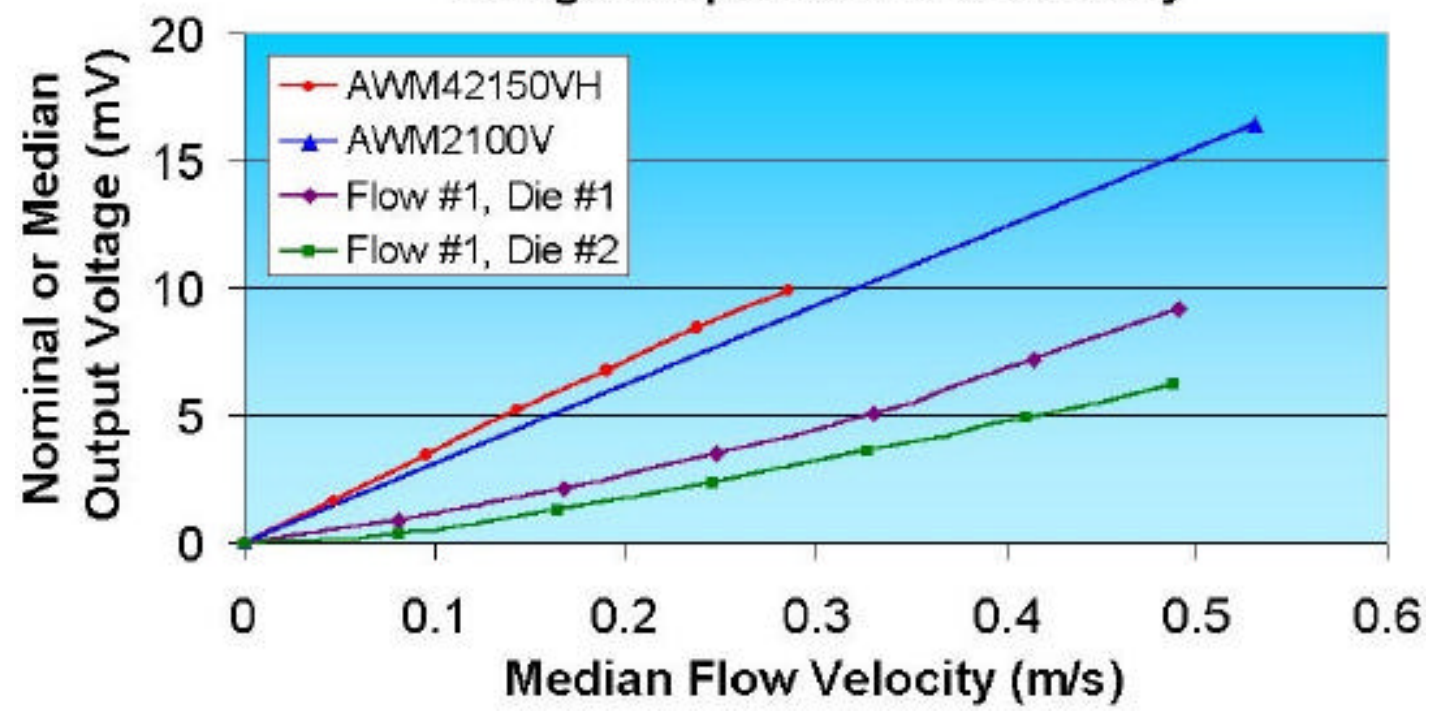

FIGURE 114 - A graph comparing fabricated sensor output responses to Honeywell's. This compares the unamplified Wheatstone bridge output voltage to the nominal/ median flow rate velocity between the fabricated flow sensors and two commercially marketed Honeywell flow sensors. Data for the Honeywell flow sensors were extracted from data sheets. The cross-sectional area above the AWM42150VH microbridge chip was specified to be $1.75 \mathrm{~mm}^{2}$, and the area above the AWM2100V microchip was $1.57 \mathrm{~mm}^{2}$. The cross-sectional area above the fabricated flow sensors was $\sim 506.7 \mathrm{~mm}^{2}$.

and these calculations, the Honeywell sensors are clearly more responsive (i.e. producing a larger output voltage swing than the fabricated sensors at a given flow rate). This may be due to different heater resistor operating points between the Honeywell devices and the fabricated sensors, but the most likely explanation is that the Honeywell sensors have a resistor metallization layer with a much higher TCR than the fabricated sensors, which could be as high as $4.0 \times 10^{-3} \Omega / \Omega /{ }^{\circ} \mathrm{C}$ if it is permalloy. A larger sense-resistor TCR would imply a larger change in resistance in the sense resistor for a given temperature gradient, and a greater unbalancing of the sense resistor Wheatstone bridge, producing a larger output voltage. Also, the Honeywell devices have the advantage of integrated, precision, laser-trimmed resistors to balance the sense resistor and heater/substrate resistor Wheatstone bridges.

The average measured resistivity of the unannealed platinum thin film was calculated to be $2.025 \times 10^{-7} \Omega \cdot \mathrm{m}$, using Equation (51) and six measurements from Van der Pauw structures 
comprised of $\sim 67 \mathrm{~nm}$ thick platinum. This corresponded to a sheet resistance of between 2.469 $\Omega$ /sq. and $2.978 \Omega$ /sq. for the thin film, which had a measured thickness variation across the wafer of $\sim 68-82 \mathrm{~nm}$. Thus, for Flow Sensor \#1, upstream or downstream sense resistors in the 1998/1999 photomask design that had 521 design squares (from Table XI), their measured resistances were expected to be between $\sim 1,286$ and 1,552 $\Omega$ (just based solely on platinum thickness variations across the wafer). Actual measured resistances for these resistors in Flow Sensor \#1 on wafer PT1 were 2,818-5,578 $\Omega$. For the measured platinum resistivity on PT1, the measured resistance of the large serpentine 5- $\mu \mathrm{m}$ designed-line-width substrate resistor with 1966 designed "squares" (named $R_{\text {alt }}$ in Table XI), present in Flow Sensor designs \#1, \#2, \#3, and \#4, was expected to be between $\sim 4,855$ and $5,855 \Omega$. However, the actual measured resistance of this substrate resistor in Flow Sensor \#1 (on the two die tested) was $\sim 22 \mathrm{~K} \Omega$.

These substantial differences between expected and measured resistances were largely explained by effects of the platinum delineation process itself, in which $5 \mu \mathrm{m}$ designed resistor line widths were reduced to approximately 2.5 to $3 \mu \mathrm{m}$ by undercutting of the positive photoresist on many devices. A reduction from 5 to $3 \mu \mathrm{m}$ average line widths corresponded to a $67 \%$ increase in the number of "squares" for a given serpentine resistor (with the same overall design length). A reduction in 5 to $2.5 \mu \mathrm{m}$ average resistor line widths corresponded to a $100 \%$ increase in the number of "squares" for a given resistor. Thus, if the sense resistors in Flow Sensor \#1 (which were designed to have $5 \mu \mathrm{m}$ line widths), with 521 designed "squares" had actual average line widths of $3 \mu \mathrm{m}$ after platinum delineation in aqua regia, the actual number of squares in each resistor would be $868\left(521^{\star} 5 / 3\right)$. This corresponded to an expected measured resistance range of $2,144-2,586 \Omega$. If these sense resistors had actual average line widths of $2.5 \mu \mathrm{m}$ after delineation, the actual number of squares would be $1,042\left(521^{*} 5 / 2.5\right)$, corresponding to an expected measured resistance range of $2,573-3,103 \Omega$. Similarly, if the large serpentine sense resistor, $R_{a l t}$, with 1966 designed squares had an average line width of $3 \mu \mathrm{m}$ after delineation, its number of squares would increase to 3,277 , corresponding to an expected measured resistance range of $8,090-9,758 \Omega$. If its actual average line width were $2.5 \mu \mathrm{m}$ after delineation, its 
number of squares would increase to 3,932 , resulting in an expected resistance range of $9,708-$ $11,709 \Omega$. Of course, in reality the line widths of these resistors, especially long serpentine ones (such as the substrate temperature-sensing resistor, $R_{a l t}$ ), were not the same over the entire length of the resistor. Narrower sections of these resistors would, therefore, have larger numbers of actual squares than designed, resulting in even higher measured resistances than those indicated above for "average" reduced resistor line widths of 2.5 or $3 \mu \mathrm{m}$ after delineation. The use of a more chemically inert or impervious photoresist, such as negative resist, to delineate the platinum resistor metallization layer would produce resistors with more uniform line widths, but of course, isotropic undercutting of the resist would still occur.

Platinum TCR characteristics for this batch were measured using two techniques. The first was to place each flow sensor die on a heated Thermochuck ${ }^{\mathrm{TM}}$ in the Wentworth Labs probe station, and measure the change in resistance of a given substrate resistor while heating the Thermochuck ${ }^{\mathrm{TM}}$ to temperatures up to $100^{\circ} \mathrm{C}$ above the ambient, using a Fluke Model $8500 \mathrm{~A}$ digital lab multimeter. The second method used an "oven test" in which a wire-wrap 24-pin socket was placed in an oven with a wire-bonded flow-sensor DIP-24 package in the socket and wires wrapped around appropriate socket pins. The wires were routed outside the oven to a Fluke $8500 \mathrm{~A}$ multimeter to measure a substrate resistor's change in resistance with increasing temperature. The average measured TCR for unannealed platinum flow sensor substrate resistors in the low-stress LPCVD $\mathrm{Si}_{3} \mathrm{~N}_{4}$ batch on the heated Thermochuck ${ }^{\mathrm{TM}}\left(20-120^{\circ} \mathrm{C}\right)$ was 2.008 $\times 10^{-3} \Omega / \Omega /{ }^{\circ} \mathrm{C}$; and, the average measured TCR with the oven test was $2.169 \times 10^{-3} \Omega / \Omega /{ }^{\circ} \mathrm{C}$. These TCRs were much lower than that which was expected (see Table XXIII). This was due, in part, to the characteristics of the deposition process itself, the fact that the resulting microstructure was not stabilized by thermal annealing, and to the very small thickness of the platinum layer, which was much less than $350 \mathrm{~nm}$ (near where platinum's thin film maximum TCR is achieved). As previously shown in Figures 15 and 16, the TCR for platinum decreases with decreasing thickness, especially below $1 \mu \mathrm{m}$.

Tables XXVI and XXVII show heat transfer measurements that were made (or estimated) with the two flow sensors that were tested. Heat transfer measurements at no flow and maximum 
TABLE XXVI

HEAT TRANSFER MEASUREMENTS FOR FLOW SENSOR \#1, DIE \#1

\begin{tabular}{|l|c|}
\hline Temperature Coefficient of Resistance $\left(\times 10^{-3} \Omega / \Omega /{ }^{\circ} \mathrm{C}\right)$ & 2.17 \\
\hline Measured resistance of heater resistor, $\mathrm{R}_{\mathrm{h}}$ & $758.5 \Omega$ \\
\hline Measured resistance of substrate resistor, $\mathrm{R}_{\mathrm{r}}$ & $21,998 \Omega$ \\
\hline Measured resistance of upstream resistor, $\mathrm{R}_{\mathrm{u}}$ & $5,487 \Omega$ \\
\hline Measured resistance of downstream resistor, $\mathrm{R}_{\mathrm{d}}$ & $5,578 \Omega$ \\
\hline Temp. change in upstream resistor, $\Delta \mathrm{T}_{\mathrm{Ru}}$, heater off, then on at no flow & $186.2^{\circ} \mathrm{C}$ \\
\hline Temp. change in downstream resistor, $\Delta \mathrm{T}_{\mathrm{Rd}}$, heater off then on, no flow & $192.9^{\circ} \mathrm{C}$ \\
\hline Temp. change in upstream resistor, $\Delta \mathrm{T}_{\mathrm{Ru}}$, heater on at no flow; max. flow & $-2.45^{\circ} \mathrm{C}$ \\
\hline Temp. change in downstr. resistor, $\Delta \mathrm{T}_{\mathrm{Rd}}$, heater on at no flow; max. flow & $0.87^{\circ} \mathrm{C}$ \\
\hline $\begin{array}{l}\text { Estimated temp. change of heater resistor, } \Delta \mathrm{T}_{\mathrm{Rh}}, \text { at no flow, assuming } \\
\Delta \mathrm{T}_{\mathrm{Ru}} 0.7 \cdot \Delta \mathrm{T}_{\mathrm{Rh}}, \text { where } \Delta \mathrm{T}_{\mathrm{Rh}} \text { is the temp. difference in the heater } \\
\text { between when it is on and off at no flow }\end{array}$ & $266{ }^{\circ} \mathrm{C}$ \\
\hline $\begin{array}{l}\text { Estimated change in resistance of the heater resistor, } \Delta \mathrm{R}_{\mathrm{h}}, \text { when power } \\
\text { is applied at no flow, where } \Delta \mathrm{R}_{\mathrm{h}}=\left(\Delta \mathrm{T}_{\mathrm{Rh}}\right)\left(\mathrm{R}_{\mathrm{h}}\right)(\mathrm{TCR})\end{array}$ & $+438 \Omega$ \\
\hline Estimated heater resistance when power is applied at no flow & $1,196 \Omega$ \\
\hline Measured heater current at no flow & $4.727 \mathrm{~mA}$ \\
\hline Measured heater current at max. flow & $4.731 \mathrm{~mA}$ \\
\hline Estimated power dissipated in the heater at no flow & $26.73 \mathrm{~mW}$ \\
\hline Estimated power dissipated in the heater at max. flow & $26.78 \mathrm{~mW}$ \\
\hline
\end{tabular}

TABLE XXVII

HEAT TRANSFER MEASUREMENTS FOR FLOW SENSOR \#1, DIE \#2

\begin{tabular}{|l|c|}
\hline Temperature Coefficient of Resistance $\left(\times 10^{-3} \Omega / \Omega /{ }^{\circ} \mathrm{C}\right)$ & 2.17 \\
\hline Measured resistance of heater resistor, $\mathrm{R}_{\mathrm{h}}$ & $1,092 \Omega$ \\
\hline Measured resistance of upstream resistor, $\mathrm{R}_{\mathrm{u}}$ & $3,014 \Omega$ \\
\hline Measured resistance of downstream resistor, $\mathrm{R}_{\mathrm{d}}$ & $2,818 \Omega$ \\
\hline Temp. change in upstream resistor, $\Delta \mathrm{T}_{\mathrm{Ru}}$, heater off, then on at no flow & $156.7^{\circ} \mathrm{C}$ \\
\hline Temp. change in downstream resistor, $\Delta \mathrm{T}_{\mathrm{Rd}}$, heater off then on, no flow & $150.1^{\circ} \mathrm{C}$ \\
\hline Temp. change in upstream resistor, $\Delta \mathrm{T}_{\mathrm{Ru}}$, heater on at no flow; max. flow & $-1.71^{\circ} \mathrm{C}$ \\
\hline Temp. change in downstr. resistor, $\Delta \mathrm{T}_{\mathrm{Rd}}$, heater on at no flow; max. flow & $0.86{ }^{\circ} \mathrm{C}$ \\
\hline $\begin{array}{l}\text { Estimated temp. change of the heater resistor, } \Delta \mathrm{T}_{\mathrm{Rh}}, \text { at no flow, } \\
\text { assuming } \Delta \mathrm{T}_{\mathrm{Ru}} 0.7 \cdot \Delta \mathrm{T}_{\mathrm{Rh}}, \text { where } \Delta \mathrm{T}_{\mathrm{Rh}} \text { is the temp. difference in the } \\
\text { heater between when it is on and off at no flow }\end{array}$ & $224{ }^{\circ} \mathrm{C}$ \\
\hline $\begin{array}{l}\text { Estimated change in resistance of the heater resistor, } \Delta \mathrm{R}_{\mathrm{h}}, \text { when power } \\
\text { is applied at no flow, where } \Delta \mathrm{R}_{\mathrm{h}}=\left(\Delta \mathrm{T}_{\mathrm{Rh}}\right)\left(\mathrm{R}_{\mathrm{h}}\right)(\mathrm{TCR})\end{array}$ & $+530 \Omega$ \\
\hline Estimated heater resistance when power is applied at no flow & $1,622 \Omega$ \\
\hline Measured heater current at no flow & $4.220 \mathrm{~mA}$ \\
\hline Measured heater current at max. flow & $4.223 \mathrm{~mA}$ \\
\hline Estimated power dissipated in the heater at no flow & $28.89 \mathrm{~mW}$ \\
\hline Estimated power dissipated in the heater at max. flow & $28.94 \mathrm{~mW}$ \\
\hline
\end{tabular}


flow conditions were made by applying power to the microbridge flow sensor heater and measuring the corresponding resistance change in the upstream and downstream resistors on the same bridge. Additionally, the power dissipated in the heater resistor was estimated. In the tables temperature change refers to a temperature differential from the ambient air temperature during testing. Based on data in the original Honeywell U.S. Patent \#4,501,144 for a similar $\mathrm{Si}_{3} \mathrm{~N}_{4}$, thermally-isolated, dual-microbridge (permalloy sandwich) flow sensor design, approximately $70 \%$ of the temperature differential in the heater was estimated to be transferred to the sense resistors at no flow. However, the Honeywell design was slightly different from the devices tested in that it used permalloy metallization sandwiched between silicon nitride layers, having a total thickness of $\sim 0.8 \mu \mathrm{m}$. These flow sensors had platinum metallization on top of a single $\sim 0.5$ $\mu m$-thick, low-stress LPCVD $\mathrm{Si}_{3} \mathrm{~N}_{4}$ layer. Thermodynamic modeling of this design would be required to determine more precisely how much heat is actually transferred at no flow conditions. As Tables XXVI and XXVII show, very small changes in sense resistor temperatures were measured at maximum flow (at approx. 15 SLPM, or $\sim 97.1 \mathrm{ft} / \mathrm{min}$ ). This is not unusual for this type of sensor. As shown in Figure 115, the approx. differential in sense resistor temperature (i.e. $\left.\left|\Delta T_{\mathrm{Ru}}\right|+\left|\Delta \mathrm{T}_{\mathrm{Rd}}\right|\right)$ in the original Honeywell design, for the same $\sim 97 \mathrm{ft} / \mathrm{min}$ (max.) flow rate, would have been $\sim 5^{\circ} \mathrm{C}$. This sense resistor temperature differential (from Tables XXVI and XXVII) was measured to be $3.3^{\circ} \mathrm{C}$ and $2.6^{\circ} \mathrm{C}$ for Flow Sensor \#1 on die \#1 and die \#2, respectively.

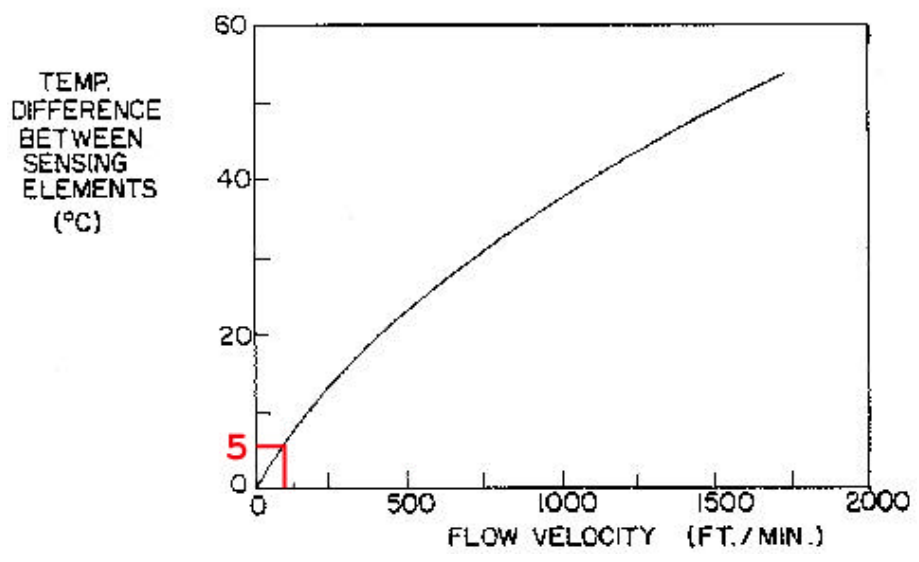

FIGURE 115 - Flow velocity vs. measured temp. difference between sense resistors in the Honeywell flow sensor. The red lines indicate what the temp. difference would have been for this type of flow sensor at a flow rate of $\sim 97 \mathrm{ft} / \mathrm{min}$., which is the max. achievable in the ColeJet $2000^{T M}$ machine. Adapted from [18]. 


\section{CONCLUSIONS AND RECOMMENDATIONS}

Originally a hot-wire anemometer, thermal-type of flow sensor was chosen as the method to measure gas flow because it was one of the oldest technologies available for developing a microfabricated mass airflow sensor. Initially there were high expectations that this design method would have the greatest probability of success, since the technology for creating and miniaturizing a hot-wire anemometer type of sensor was mature. Thus, this development effort was not intended to create a unique, "cutting-edge" flow sensor design, but was instead focused upon designing, fabricating, and testing a device which could be built by students in a microfabrication laboratory, giving them hands-on learning experience with a MEMS device.

Despite the maturity of the hot-wire anemometer design, this extensive effort was characterized by two fundamental problem solving dimensions, which were not easily resolved and which were typically mutually exclusive: how to fabricate, with a controllable thickness, a high-quality suspended microbridge/cantilever (tensile) thin-film structure for the flow sensors; and what metal to use for the resistor metallization layer, which would have acceptable thin-film electrical properties. Six unique processes were explored to create the thin-film (microbridge/ cantilever) structure of the devices, and only the low-stress LPCVD $\mathrm{Si}_{3} \mathrm{~N}_{4}$ process proved to be successful (and reproducible). Permalloy $\left(\mathrm{Ni}_{81} \mathrm{Fe}_{19}\right)$, chromium, titanium, and platinum were investigated for potential use as resistor metallization, with only platinum proving to have desirable, reproducible (typically non-hysteretic) electrical characteristics, with a particularly good TCR. Finding a usable metal for the thin-film metallization layer was significantly complicated by the pronounced difference discovered between a metal's bulk and measured, thin-film electrical properties. Below $1 \mu \mathrm{m}$ in thickness, a platinum metal layer's TCR decreased substantially with decreasing thickness. Methods such as thermal annealing (with or without rapid quenching) were explored to improve the TCR properties of the thin metal film layers. High-temperature furnace annealing in air and nitrogen, and lower-temperature hot-plate annealing in argon were investigated. While annealing generally stabilized a given metal's microstructure and improved 
its TCR characteristics, depending upon the annealing temperature and duration, most metals were oxidized afterwards, sometimes changing the material properties of the metal itself (e.g. titanium high-temperature annealing). Although thermal annealing did not improve the TCR properties of platinum for the successfully fabricated flow sensors, it still needs to be further explored for improving TCR properties of platinum layers with thicknesses greater than $1 \mu \mathrm{m}$. In particular, high-temperature, vacuum furnace annealing/tempering in the range of $1000-1200$ ${ }^{\circ} \mathrm{C}$ should be investigated.

The half-wafer (PT1) shown in Figure 92, which was the only platinum metallization wafer processed, contained $30-50 \%$ device yields, because positive photoresist lifted-off underlying resistor metallization during delineation of platinum in heated aqua regia, allowing the solution to etch through 5- $\mu \mathrm{m}$ line-width resistors in some regions. An improved approach that would result in significantly better device yields would be to delineate platinum resistor metallization in heated aqua regia with negative photoresist. However, the current resistor delineation photomask (the Resistor Metal Level 1 mask) is a lightfield mask that works properly with positive photoresist. Switching to the use of negative photoresist to delineate platinum metallization would require obtaining a new darkfield photomask for the resistor metallization L-Edit ${ }^{\mathrm{TM}}$ design file layer.

The recommended process for fabricating flow sensors is similar to the fabrication summary and detailed process outline provided in Appendices XIII and XIV, but with a few notable exceptions. Although many sensor geometries were available in the 1998/1999 mask set, suggested flow sensors to fabricate include those which are dual microbridge designs with combined sense resistor nodes (Flow Sensor \#1, \#4, or \#17) as these sensors were easiest to fabricate and test, and have the largest resistance sense resistors. Flow Sensor \#17 has the smallest dual microbridges, which are undercut and suspended in the anisotropic silicon etchant the fastest (compared to other dual microbridge sensors in the design). A low-stress LPCVD (or possibly annealed PECVD) $\mathrm{Si}_{3} \mathrm{~N}_{4}$ on (100) silicon substrate is suggested for the structure of the flow sensors. Even with some residual compressive stress in the nitride layer, microbridge-based devices can still survive processing and provide testable sensors. Although test structures were included in the 1998/1999 mask set design and may be used, no tests were performed to 
measure residual stress/strain in thin films with these structures, and thus the assessment of their usefulness is left for future research. A good quality $\mathrm{Si}_{3} \mathrm{~N}_{4}$ layer (as was obtained with the outsourced low-stress nitride wafers used to fabricate viable flow sensors) is also important, so that it can withstand exposure to the anisotropic silicon etchant with minimal, or no, degradation. Thin-film platinum metallization is recommended, with a thickness near $1 \mu \mathrm{m}$, delineated in either heated aqua regia solution by photolithographic patterning techniques using negative photoresist and a darkfield Resistor Metal Level 1 photomask, or by ion milling (RIE). Once the platinum layer is delineated, thermal annealing at $\sim 1000-1200{ }^{\circ} \mathrm{C}$ in a vacuum furnace is also suggested to potentially improve the TCR characteristic. $\mathrm{An} \mathrm{Si}_{3} \mathrm{~N}_{4}$ sandwich approach which was used in the Honeywell design, with the metallization layer in the center, is not necessary. However, the process described in Appendices XIII and XIV suggests sputter-depositing a thin $\mathrm{Si}_{\mathrm{x}} \mathrm{N}_{\mathrm{y}}$ layer (on top of delineated platinum) and top $\mathrm{Cr} / \mathrm{Au}$ RIE masking layer to pattern the $\mathrm{Si}_{3} \mathrm{~N}_{4}$ layer prior to anisotropic silicon etching. The gold metallization second-layer is optional, unless wire-bonding problems (to platinum) are experienced with platinum-only devices. Since platinum is, itself, inert in the anisotropic silicon etchant, $\mathrm{KOH}$, it may be exposed to the etchant without degradation.

To improve the electrical properties of platinum films used for resistors in these flow sensors, further work is needed. The TCR characteristics of platinum thin-films that are planarmagnetron-deposited in the Technics system should be evaluated as a function of nominal film thickness and any applicable post-deposition annealing that is experimentally utilized to stabilize the film's microstructure. For example, a vacuum-annealed platinum layer that is at least $1.0 \mu \mathrm{m}$ thick would theoretically achieve optimal TCR properties (of up to $3.5-3.85 \times 10^{-3} \Omega / \Omega /{ }^{\circ} \mathrm{C}$ ). Furthermore, ion milling (or reactive ion etching) should be investigated as a potential means to anisotropically delineate the platinum resistors.

In hindsight, while the objective to design a flow sensor test apparatus with easy device interchangeability was a reasonable goal, it was implemented at the cost of using a much larger flow channel with correspondingly low velocity flows (unless a much larger blower were utilized). While ColeJet $2000^{\mathrm{TM}}$ is useful for testing the flow sensors, it does so over a very small flow velocity range. A test apparatus capable of a broader flow velocity gamut is needed to better 
assess the dynamic range of fabricated devices. ColeJet $2000^{T M}$ is capable of velocity flow rates of up to $0.54 \mathrm{~m} / \mathrm{s}$ (at $16.5 \mathrm{SLPM}$ ) in a 1-inch diameter flow channel. The patented Honeywell flow sensor had a reported maximum, unamplified Wheatstone bridge output voltage of $100 \mathrm{mV}$ at a flow velocity of $7.62 \mathrm{~m} / \mathrm{s}$ [18]. If this response were linear, this would correspond to an output voltage of $7.1 \mathrm{mV}$ at $0.54 \mathrm{~m} / \mathrm{s}$, which is close to the output achieved with the fabricated flow sensors at maximum flow (6.24 - 9.2 mV max.).

Furthermore ColeJet $2000^{T M}$ should have been designed with a device insertion scheme that had a fixed "stop" in the flow channel to provide reproducible device insertion depths into the flow channel. This might lead to improved reproducibility in flow sensor test results.

As with the Honeywell flow sensor implementation, measured maximum resistance and temperature changes in the upstream resistor were at least twice that of the downstream resistor. This is shown graphically in Figure 9 for the patented Honeywell sensor. Due to the very low maximum flow velocity achievable in the ColeJet $2000^{\text {TM }}$ machine, the maximum measured temperature change of the upstream and downstream resistors in the successfully fabricated LPCVD $\mathrm{Si}_{3} \mathrm{~N}_{4}$ thin-film platinum devices was $2-4$ ohms.

Poor cleanliness of the Technics sputtering system chamber (combined with poor lab techniques) severely, adversely affected gold adhesion, due to (presumed) outgassing of oxygen (and moisture) from residual films in the chamber, which was gettered into adhesion-layer films (such as chromium) during sputtering. To prevent adhesion problems and consistently produce higher-quality (minimally-contaminated) thin films, the Technics system should be more thoroughly, periodically cleaned, and potential upgrades should be considered to improve control of sputtering processes. A periodic bead-blasting cleaning of the system should be performed at least annually, along with wiping the chamber and sputtering target(s) with solvents (such as acetone and methanol) prior to each use. It is strongly suggested that the system be upgraded with mass flow controllers to more precisely control the flow rates of sputtering gases used in the system. Better control would potentially result in deposited thin films with more consistent properties between runs that have the same sputtering parameters. For example, film thicknesses vary not only across substrates because of the substrate's geometrical placement 
above the target (and choice of static vs. dynamic/rotate mode), but also because of the inability to precisely control the sputtering gas flow rate and sputtering pressure on different runs with the same parameters. The addition of mass flow controllers to the system, affording better control of the chamber pressure, might also allow for a more thorough characterization of sputtering parameters needed to transition from residual compressive stress to tensile stress in a deposited thin film. In general, properties of thin films sputter-deposited in the Technics system need to be vigorously investigated, along with experimental methods to deposit better quality films. For example, how do the sputtering parameters (choice of gas, gas mix if applicable, gas pressure, DC or RF power, choice of static vs. dynamic mode, and chiller temperature) affect a thin film's microstructure, residual stress/strain properties and gradients, stoichiometry, resistivity, and TCR? How can film's be deposited with better thickness uniformity across substrates? Is in-situ thin film annealing possible with this system? Is it possible to deposit multiple layers of the same material using different sputtering parameters to produce a film with better physical and electrical properties than just a single layer?

In hindsight, certain difficulties in this project could have been easily avoided. For example, a thorough analysis of the electrical properties (e.g. resistivity, sheet resistance, and TCR) of sputter-deposited, thin-film metallization layers performed before any fabrication attempts to build flow sensors would have allowed for the choice of an optimal resistor layer with acceptable electrical (and isotropic, wet-etch) properties. Stagnation in the development process could have been avoided by more vigorous efforts to seek help and experience synergism through the power of collaboration to generate new and more insightful fabrication ideas. $A$ "design of experiments" approach could have been utilized in the design of different flow sensor geometries, especially for the $1998 / 1999$ photomask set. To provide better experimental accuracy in the estimation of silicon etch rates, the incremental resolution of the square etchdepth measurement structures could be improved to provide $5 \mu \mathrm{m}$ resolution, instead of $10 \mu \mathrm{m}$. Rather than requiring the external connection of a DMM to measure the sense resistor Wheatstone bridge unamplified $\mathrm{mV}$ output, an LCD panel meter with a smaller range than 19.999 VDC (e.g. $200 \mathrm{mV}$ ) should have been installed in ColeJet 2000тM. 
For the flow sensor structural layer, it is also possible that thermally-grown oxide could be used providing certain conditions are met. If a thin $0.5 \mu \mathrm{m}$ oxide layer could be thermally annealed at high temperatures to reduce/relieve residual intrinsic compressive stresses, it could be potentially viable, provided that it is the only structural layer (with no top layer passivation), and that it is annealed prior to metallization deposition and delineation. The addition of a top passivation layer could add a residual stress nonuniformity through the total thickness of the thin film (due to dissimilar intrinsic material properties between the two layers), causing buckling of structures once they are released from the substrate.

Many opportunities exist for future research with this flow sensor design. Of course, the response characteristics of all twenty flow sensors should eventually be evaluated (except for Flow Sensor \#15, which is untestable, due to a design flaw, as explained in Appendix XXIII), possibly in a different flow stream setup which could achieve higher gas velocities of up to $8 \mathrm{~m} / \mathrm{s}$ across the sensors, for example. An analytical or iterative computational model could be developed to mathematically relate a given flow sensor's thermodynamic and flow characteristics to its electrical properties, such as the heater current or power delivered to the heater resistor for a given air flow velocity $[104,105]$. A model could be developed to calculate the temperature of the heater resistor at its operating point, based on the thermodynamic, material, and electrical properties of the thin film (platinum) metallization and $\mathrm{Si}_{3} \mathrm{~N}_{4}$ thermally-isolated structural layers. Finally, a "smoke test" could be performed with the ColeJet $2000^{T M}$ machine, by routing an opaque smoke into the intake of the blower fan (with the prefilter assembly removed), to analyze and better understand characteristics of the flow stream within the designed PVC flow channel. 


\section{APPENDICES TABLE OF CONTENTS}

\begin{tabular}{|c|c|c|}
\hline APPENDIX & TITLE & PAGE \\
\hline Appendix I & Honeywell AWM5000 Series Flow Sensor Data Sheets & 230 \\
\hline Appendix II & Honeywell AWM2150V Flow Sensor Die Photos & 250 \\
\hline Appendix III & Chemical List and Compositions & 253 \\
\hline Appendix IV & Laboratory Apparatus Equipment Photographs & 256 \\
\hline Appendix V & Summary of Flow Sensor Methodologies Attempted & 265 \\
\hline Appendix VI & KOH Etch Rate Data Graphs & 268 \\
\hline Appendix VII & Borosilicate Glass RIE Data Graphs & 271 \\
\hline Appendix VIII & Suggested Wafer Dicing Diagram & 274 \\
\hline Appendix IX & Micro-Automation Dicing Saw Revised SOP & 277 \\
\hline Appendix X & Silicon Nitride Sputtering Deposition and RIE Data Graphs & 296 \\
\hline Appendix XI & SEM Photos of Sputtered Silicon Nitride Flow Sensors & 299 \\
\hline Appendix XII & Filmtronics 700 Series Spin-On Glass Data Sheet & 302 \\
\hline Appendix XIII & Successful LPCVD $\mathrm{Si}_{3} \mathrm{~N}_{4}$ Flow Sensor Fabrication Summary & 305 \\
\hline Appendix XIV & Detailed Outline of LPCVD $\mathrm{Si}_{3} \mathrm{~N}_{4}$ Flow Sensor Fabrication & 311 \\
\hline Appendix XV & SEM Photos of Low-Stress LPCVD $\mathrm{Si}_{3} \mathrm{~N}_{4}$ Pt Flow Sensors & 319 \\
\hline Appendix XVI & Air and Nitrogen Properties & 322 \\
\hline Appendix XVII & ColeJet $2000^{\text {TM }}$ Parts' Design Considerations & 325 \\
\hline Appendix XVIII & ColeJet $2000^{\text {TM }}$ Parts' Data Sheets & 328 \\
\hline Appendix XIX & ColeJet $2000^{\text {TM Machine Schematics }}$ & 361 \\
\hline Appendix XX & ColeJet $2000^{T M}$ Photographs and Descriptions & 367 \\
\hline Appendix XXI & ColeJet $2000^{T M}$ Condensed Operating Instructions & 375 \\
\hline Appendix XXII & 1997 Two-Layer L-Edit'M Design File Images/Descriptions & 386 \\
\hline Appendix XXIII & 1998/99 3-Layer L-EditTM Design File Images/Descriptions & 396 \\
\hline
\end{tabular}




\section{APPENDIX I.}

\section{HONEYWELL AWM5000 SERIES FLOW SENSOR DATA SHEETS*}

\footnotetext{
${ }^{*}$ Available on the World Wide Web at http://content.honeywell.com/sensing/prodinfo/massairflow/
} 
Airflow Sensors

Microbridge Mass Airflow

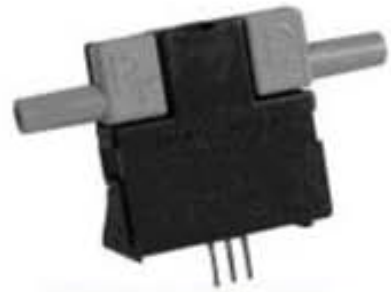

AWM 1000/2000/3000 Series

FEATURES

- State-cl-the-art silicon

micromachining

- Sensitive to low flows - 0.1 sccm to 20 SLPM

- Adaptable for use with higher flows (See Application Note 2 page 128.)

- Fast response time

- Analog output

- Low power consumption

\section{OPERATION}

The microbridge mass airflow sensor operates on the theory of heat transfer. Mass airflow is directed across the surface of the sensing elements. Output voltage varies in proportion to the mass air or other gas flow through the inlet and outlet ports of the package. The specially designed housing precisely directs and controls the airficw across the microstructure sense element. Mechanical design of the package allows it to be easily mounted to printed circuit boards.

The microbridge mass airflow sensor has a unique silicon chip based on advanced microstructure technology. It consists of a thin-film, thermally isolated bridge structure containing heater and temperature sensing elements. The bridge struc. ture provides a sensitive and fast response to the flow of air or other gas over the chip. Dual sensing elements positioned on both sides of a central heating element indicate flow direction as well as flow rate. Laser trimmed thick film and thin film resistors provide consistent interchangeability from one device to the next.

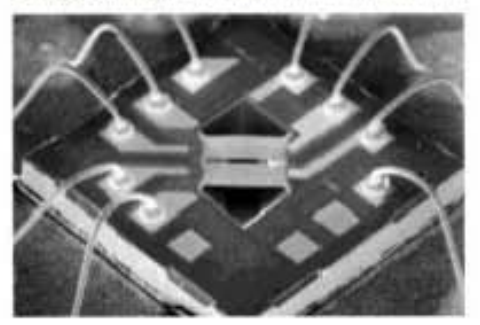

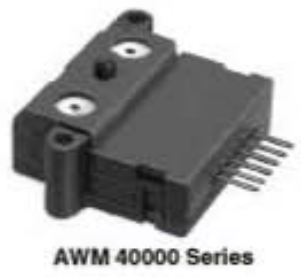

- Repeatable response

- Laser-trimmed interchangeability

- Accurate, cost effective flow sensing

- In-line printed circuit board terminals

- Standard $0.100^{\prime \prime}(2,54 \mathrm{~mm})$ mounting centers

- Accurate sensing of low pressure $0.001^{\prime \prime}$ to $4.0^{\gamma} \mathrm{H}_{2} \mathrm{O}$ (.003 to $10 \mathrm{mBar}$ )

The microbridge mass airflow sensor us es temperature-sensitive resistors de posited within a thin film of silicon nitride. They are suspended in the form of two bridges over an etched cavity in the silicon, shown below. The chip is located in a precisely dimensioned airflow channel to provide a repeatable flow response. Highly effective thermal isolation for the heater and sensing resistors is attained by etching the cavity space beneath the flow sensor bridges. The small size and thermal isolation of the microbridge mass airflow sensor are responsible for the extremely fast response and high sensitivity to flows.

Dual Wheatstone bridges control airflow measurement - one provides closed loop heater control, the other contains the dual sensing elements. The heater circuit minimizes shift due to ambient temperature changes by providing an output proportional to mass flow. The circuit keeps the heater temperature at a constant differential $\left(160^{\circ} \mathrm{C}\right)$ above ambient air temperature which is sensed by a heat-sunk resistor on the chip. The ratiomotric voltege output of the device corresponds to the differential voltage across the Wheatstone bridge circuit.

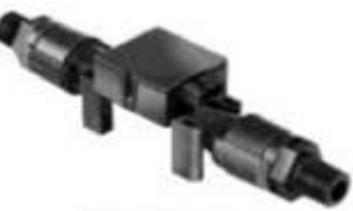

AWM 5000 Series

APPLICATIONS

- Damper control for heating, ventilation and air conditioning systems

- Gas analyzers

- Low vacuum control

- Process control

- Medical respirators and ventilators

- Oxygen concentrators

- Leak detection equipment

- Vent hoods

- Anesthesia control

- Gas metering

- Gas chromatography

\section{NOTICE}

Dust contamination may be possible in some applications, the effects of which can be minimized. By design, dust particles that may be present in the air stream will flow past the chip parallel to the chip surface. In addition, the microstructure chip produces a thermophoretic effect, which repels micrometer-sized dust particles away from the bridge structure

Dust adherence to chip edges and channel surfaces can be prevented us. ing a simple filter. A disposable fivemicron filter used in series on the upstream side of the airflow device will provide adequate filtering in most applications. For a list of possible filter sourc. es, see Filter Manufacturers, page 126.

\section{CAUTION}

PAODUCT DAMAGE

AWM Series Microbridge Mass Airflow Sensors are NOT designed to sense liquid flow and will be damaged by liquid flow through the sensor. 


\section{REFERENCE AND APPUICATION DATA \\ Microbridge Airflow Sensors}

\section{Measuring Low Differential Pressures Altitude and Gas Density Correction - Note \#1}

Microbridge mass airflow sensors measure actual mass flow of a gas media (assuming thermal conductivity of the gas is held constant). The actual flow of the gas media is driven by a pressure gradient flowing from a higher pressure region to a lower pressure region. The pressure differential required to drive flow through the microbridge mass flow sensor is considered very low, typically less than two inches water column (less than five $\mathrm{mBar}$ ) full scale.

As a result, microbridge mass flow sensors are commonly used to measure differential pressures as low as $0.001^{\prime \prime} \mathrm{H}_{2} \mathrm{O}$ ( $0.002 \mathrm{mBar}$ ). In these applications, the microbridge sensors actually sense mass gas flow. However, the product is specified and calibrated against differentia pressure. To measure differential pres sure, the application must be able to provide gas flow through the sensor (gas density remaining constant).
This can be referenced to the Ideal Gas Law which states: $P V=n R T$. This implies that while measuring differential pressures, the sensor gain will be directly proportional to the absolute pressure (absolute density) of the gas. Microbridge mass flow sensors are calibrated at 850 feet (260 meters) above sea level with the absolute pressure at approximately 740 torr.

At sea level with absolute pressure at 760 torr, the sensor voltage output will be higher by an approximate factor of:

$$
\frac{1+(760-740)}{740} \text { or } 1.027
$$

Additionally, if located in Salt Lake City Utah, where the altitude is 4,200 feet $(1,270$ meters) above sea level, the standard absolute pressure is 650 torr.
This will result in the sensor voltage output being reduced by an approximate factor of:

$\frac{1+(650-740)}{740}$ or 0.8784

See Table 1 below for additional approximations for various altitude corrections.

When using microbridge mass flow sensors to measure low differential pressures, the temperature of the gas can also affect the relative gas density. Density changes due to temperature can cause a shift in the sensor output gain. The shift will be proportional to the change in the absolute gas density (referenced to $\left.23^{\circ} \mathrm{C}\right)$.

NOTE: When measuring actual mass flow, the microbridge sensor is insensitive to altitude and gas density changes.

Table 2- Approximate Altitude Correction Factors

\begin{tabular}{llllll}
\hline Altitude & $\begin{array}{l}\text { Absolute Pressure } \\
\text { Torr }\end{array}$ & $\begin{array}{l}\text { mBar } \\
\text { kPa }\end{array}$ & $\begin{array}{l}\text { Approximate } \\
\text { Correction Factors }\end{array}$ & Representative Cities \\
\hline $0 \mathrm{~m}(0 \mathrm{ft})$. & 760 & 1000 & 100 & $1+(760-740) / 740=1.027$ & London (U.K.), New York, NY (US), Tokyo (Japan) \\
\hline $250 \mathrm{~m}(820 \mathrm{ft})$. & 740 & 984 & 98 & $1+(740-740) / 740=1.000$ & Minneapolis, MN (US), New Delhi (India), Turin (Italy) \\
\hline $500 \mathrm{~m}(1,650 \mathrm{ft})$. & 720 & 958 & 96 & $1+(720-740) / 740=0.973$ & Munich (Germany), Santiago (Chile), Spokane, WA (US) \\
\hline $750 \mathrm{~m}(2,500 \mathrm{ft})$. & 694 & 925 & 92 & $1+(694-740) / 740=0.938$ & Ankara (Turkey), Tucson, AZ (US) \\
\hline $1,500 \mathrm{~m}(5,000 \mathrm{ft}$ ) & 632 & 842 & 84 & $1+(632-740) / 740=0.854$ & Denver, CO (US), Johannesburg (S. Africa) \\
\hline $2,250 \mathrm{~m}(7,500 \mathrm{ft})$. & 575 & 766 & 77 & $1+(575-740) / 740=0.777$ & Addis Ababa (N.E. Africa), Mexico City (Mexico) \\
\hline $3,000 \mathrm{~m}(10,000 \mathrm{ft})$. & 523 & 697 & 70 & $1+(523-740) / 740=0.707$ & La Paz (Bolivia), Leadville, CO (US) \\
\hline
\end{tabular}




\section{Microbridge Airflow Sensors}

\section{High Flow Capability Bypass Design Considerations - Note \#2}

Many users would like to take advantage of the cost-effective, high-performance characteristics of the microbridge mass airflow sensor. Some of these applications require the ability to measure flow ranges higher than the capability of existing microbridge sensors. Others may simply wish to take advantage of the flow range capability of the high-flow AWM5000 series, but require the small size and fast response times of the AWM2000, AWM3000 series.

One way to achieve higher flow range capability is through the use of a bypass configuration. This provides a higher main flow channel than the lower bypass (sensor) flow channel. In this configuration, only a sample of the total flow actually gets directed through the bypass channel and the sensor. The amount of flow directed through the microbridge device is determined by the "bypass ratio." The smaller the ratio, the more predictable and stable the sensor output throughout the measured flow range.

Simple bypass configurations can be easily incorporated into most applications. The bypass ratio can be calculated by determining the cross sectional area of the flow channel above the microbridge chip compared to the cross sectional area of the main flow channel at its point of greatest restriction. The cross sectional area above the microbridge chip is determined according to the internal design of the sensor flow tube (See Table 1).

Figure 1 Typical bypass design

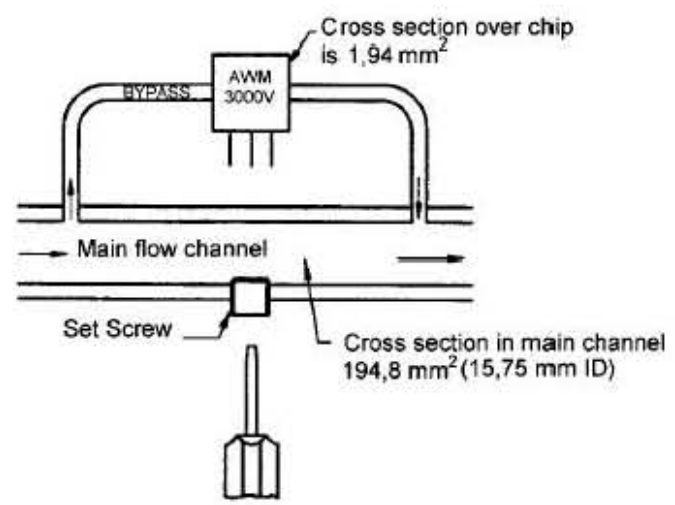

Figure 1 is a typical bypass configuration. In this design, the main flow channel has an ID of $15,75 \mathrm{~mm}$ which results in a cross sectional area of $194,83 \mathrm{~mm}^{2}$. The cross sectional area above the microbridge chip is $1,95 \mathrm{~mm}^{2}$. This yields an approximate bypass ratio of 100 to 1 . This design incorporates a variable flow restriction (set screw) that can be used to calibrate a specific bypass ratio.
Applications that incorporate bypass designs with ratios of 100 to 1 or greater may experience noticeable errors near zero flow. Large bypass ratios require a larger pressure drop to adequately direct flow to the sensor. Under very low flow conditions, there may not be sufficient pressure drop to drive flow through the bypass (sensor) flow channel. There may also be considerable variation in performance due to variety of bypass channel designs and geometries. In addition, device-to-device variation may be amplified when used in conjunction with high bypass ratios.

For example, an application that needs 5 SLPM flow measurement capability could potentially use an AWM3300V (1,000 $\mathrm{sccm}$ flow range device). This would simply require the use of two T-connections and a bypass flow channel using $1 / 8$ inch ID tubing. This is the same tubing size recommended for connecting the sensor. With this configuration, the bypass ratio is 4 to 1 . This will allow 4 liters of flow through the bypass channel with 1 liter of flow through the sensor channel, permitting a total of 5 liters of flow through the system.

The same size tubing, $1 / 8$ inch ID, to bypass an AWM5000 series sensor would provide a 1 to 1 bypass ratio, thus doubling the flow capability of this sensor. Further, using a $3 / 8$ inch ID tubing to bypass the AWM5000 series would provide a 3 to 1 bypass ratio, thus quadrupling the flow range capability for the AWM5000 sensor. This 3 to 1 bypass configuration used with an AWM5104VN (20 SLPM flow range) would provide flow measurement capability up to 80 SLPM.

Table 1 Cross-sectional area above microbridge chip

\begin{tabular}{|c|c|}
\hline Catalog Listing & Area* \\
\hline AWM2100V & $1.57 \mathrm{~mm}^{2}$ \\
AWM2300V & $1.94 \mathrm{~mm}^{2}$ \\
AWM3100V & $1.57 \mathrm{~mm}^{2}$ \\
AWM3300V & $1.94 \mathrm{~mm}^{2}$ \\
AWM42150VH & $1.75 \mathrm{~mm}^{2}$ \\
AWM42300V & $1.75 \mathrm{~mm}^{2}$ \\
AWM43300V & $1.75 \mathrm{~mm}^{2}$ \\
AWM43600V & $12.07 \mathrm{~mm}^{2}$ \\
AWM5000 Series & $45.60 \mathrm{~mm}^{2}$ \\
\hline
\end{tabular}

*All values are approximate. 


\section{REFERENCE AND APPLICATION DATA}

\section{Microbridge Airflow Sensors}

High Flow Capability

Bypass Design Considerations - Note \#2

In applications where the desired flow rate exceeds the flow specifications for standard MICRO SWITCH products, a bypass flow channel (see Figure 2.) can be configured. A bypass (appropriately sized) consists of a Microbridge sensor on parallel with the main flow channel. In this configuration, only a portion of the total flow rate will pass through the bypass channel and sensor, while the majority of the total flow passes through the main flow channel. Below is an example of calculations needed to properly size the bypass configuration and help select an appropriate Microbridge Sensor.

In this example, a multi-channel bypass with a Microbridge Sensor is configured for $100 \mathrm{liter} / \mathrm{min}$ flow rate.

Step 1: Convert desired flow rate from liters $/ \mathrm{min}$ to $\mathrm{m}^{3} / \mathrm{sec}$ by the following formula:

$$
\begin{aligned}
& \text { Enter Desired Flow Rate }=\quad 100 \quad \text { liters } / \mathrm{min} \\
& \text { liter } / \mathrm{min} \text { X.00001666667 }=0.001666667 \mathrm{~m} / \mathrm{sec}
\end{aligned}
$$

Enter the above calculated value into INPUT area below as the Total Volumetric Flow (Qtotal).

Step 2: Calculate Reynold's number for desired volumetric flow. It is important that desired volumetric flow is in the laminar flow range. This means that the Reynold's number must be 2000 or less. Enter desired volumetric flow in INPUT area below. Make adjustments in INPUT area to diameter $(\mathrm{D})$ and quantity $(\mathrm{N})$ of flow channels for a Reynold's number (Re) of 2000 or less (Laminar Flow):

$$
\operatorname{Re}=\frac{4{ }^{*} \rho{ }^{*} \mathrm{Q} / \mathrm{N}}{\pi^{*} \mathrm{D}{ }^{*} \mu}=\frac{0.000347826}{1.80539 \mathrm{E}-07}=1926.59302
$$

Step 3: Calculate approximate pressure drop across Laminar Flow Area (due to flow). Use the following equation:

$$
\Delta \text { Pflow }=\frac{128^{*} \mu^{*}(\mathrm{Q} / \mathrm{N}) * L}{\pi^{\star} \mathrm{D}^{4}}=\frac{8.52851 \mathrm{E}-09}{3.19246 \mathrm{E}-10}=\frac{26.71 \mathrm{~N} / \mathrm{m}^{3}}{0.107} \mathrm{H}+\mathrm{O}
$$

Step 4: Calculate approximate pressure loss at inlet and outlet. Use the following formula:

$$
\text { first, calculate velocity: } V=\frac{4^{*} Q}{\pi^{*} N^{*} D^{4}}=9.2 \mathrm{~m} / \mathrm{sec}
$$

\begin{tabular}{|c|c|c|c|c|}
\hline Definitions & & $\begin{array}{l}\text { Input } \\
\text { Range }\end{array}$ & $\begin{array}{c}\text { Constant } \\
@ 20^{\circ} \mathrm{C}\end{array}$ & $\begin{array}{c}\text { Metric } \\
\text { Units }\end{array}$ \\
\hline$\Delta \mathrm{P}=$ Delta Pressure & $=$ & - & & $\mathrm{N} / \mathrm{m}^{2}$ \\
\hline$\mu=$ Viscosity Air & $=$ & 1.81E-05 & - & $\left(\mathrm{N}^{*} \mathrm{~s}\right) / \mathrm{m}^{2}$ \\
\hline$p=$ Density Air & $=$ & 1.20 & - & $\mathrm{kg} / \mathrm{m}^{3}$ \\
\hline$\pi=\mathrm{PI}$ & $=$ & - & 3.141592654 & - \\
\hline$D=$ Diameter of Single Flow Channel & $=$ & 0.003175 & - & $m$ \\
\hline $\mathrm{N}=$ Number of channels & $=$ & 23 & - & - \\
\hline$L=$ Length of By-Pass & $=$ & 0.0508 & - & $\mathrm{m}$ \\
\hline $\mathrm{Q}_{\text {mas }}=$ Total Volumetric Flow Rate & $=$ & 0.001666667 & - & $\mathrm{m}^{1 / 3 e c}$ \\
\hline $\mathrm{K}_{\operatorname{mat}}=$ Inlet Loss Coefficients & $=$ & 0.5 & - & - \\
\hline $\mathrm{K}_{\text {outa }}=$ Outlet Loss Coefficients & $=$ & 1.0 & - & - \\
\hline $\mathrm{g}=$ Acceleration of gravity constant & $=$ & - & 9.806 & $\mathrm{~m} / \mathrm{sec}^{2}$ \\
\hline
\end{tabular}

next, use calculated velocity $(\mathrm{V})$ in equation below and calculate Inlet and Outlet pressure loss:

$$
\Delta P_{\text {inlet/outlet loss }}=\frac{\left(K_{\text {inlet }}+K_{\text {outlet }}\right) * V *{ }^{*} \rho}{2}=\frac{75.39 \mathrm{~N} / \mathrm{m}^{2}}{0.303} " \mathrm{H}+\mathrm{O}
$$

Table 1 


\section{REFERENCE AND APPLICATION DATA}

\section{Microbridge Airflow Sensors}

\section{High Flow Capability \\ Bypass Design Considerations - Note \#2}

Step 5: Calculate approximate Total Pressure drop across Laminar Flow Area. Use the following formula:

$$
\Delta P_{\text {total }}=\Delta P_{\text {inlet } / \text { outlet loss }}+\Delta P_{\text {flow }}=\frac{102.11}{0.41} \mathrm{~N} / \mathrm{m}^{2}
$$

Note: $\Delta P_{\text {total }}$ is an approximate value. Testing or further analysis is needed for each specific design.
Step 6: Select appropriate Microbridge sensor that has a full scale pressure drop that is equal to or greater than calculated Total Pressure Drop ( $\left.\Delta \mathrm{P}_{\text {total }}\right)$ using Table 2 below:

Table 2

\begin{tabular}{|c|c|c|c|c|}
\hline Product & Rated Flow & Rated Pressure & Output Type & $\begin{array}{l}\text { Select by Typical Full } \\
\text { Scale Pressure Drop }\end{array}$ \\
\hline AWM2100V & $\pm 200 \mathrm{sccm}$ & N.A. & $\pm 44.5 \mathrm{mv}$ & $20 " \mathrm{H}_{2} \mathrm{O}$ or $49.8 \mathrm{~N} / \mathrm{m}^{2}$ \\
\hline AWM2150V & $\pm 30 \mathrm{sccm}$ & N.A. & $\pm 14 \mathrm{mv}$ & $20^{\prime \prime} \mathrm{H}=\mathrm{O}$ or $49.8 \mathrm{~N} / \mathrm{m}^{2}$ \\
\hline AWM2200V & N.A. & $0-2 " \mathrm{H}_{2} \mathrm{O}$ & $\pm 31.75 \mathrm{mv}$ & $2.01 \mathrm{H} \mathrm{H}_{2} \mathrm{O}$ or $498 \mathrm{~N} / \mathrm{m}^{2}$ \\
\hline AWM2300V & $0-1000 \mathrm{sccm}$ & N.A. & $\pm 55.5 \mathrm{mv}$ & $1.3^{\prime \prime} \mathrm{H}_{2} \mathrm{O}$ or $324 \mathrm{~N} / \mathrm{m}^{2}$ \\
\hline AWM3100V & $0-200 \mathrm{sccm}$ & N.A. & $1-5 \mathrm{Vdc}$ & $20 " \mathrm{H}_{2} \mathrm{O}$ or $49.8 \mathrm{~N} / \mathrm{m}^{2}$ \\
\hline AWM3150V & $0-30 \mathrm{sccm}$ & N.A. & $1.5 \mathrm{Vdc}$ & $.20 " \mathrm{H}_{2} \mathrm{O}$ or $49.8 \mathrm{~N} / \mathrm{m}^{2}$ \\
\hline AWM3200CR & N.A. & $0-2{ }^{\prime \prime} \mathrm{H}_{2} \mathrm{O}$ & $4-20 \mathrm{~mA}$ & $2.0^{\prime \prime} \mathrm{H}_{2} \mathrm{O}$ or $498 \mathrm{~N} / \mathrm{m}^{2}$ \\
\hline AWM3201CR & N.A. & $0-0.5 " \mathrm{H}_{2} \mathrm{O}$ & $4.20 \mathrm{~mA}$ & $.50 " \mathrm{H}, \mathrm{O}$ or $125 \mathrm{~N} / \mathrm{m}^{2}$ \\
\hline AWM3200V & N.A. & $0-2 " \mathrm{H}_{2} \mathrm{O}$ & $1-5 \mathrm{Vdc}$ & $2.0 " \mathrm{H}_{2} \mathrm{O}$ or $498 \mathrm{~N} / \mathrm{m}^{2}$ \\
\hline AWM3300V & $0-1000 \mathrm{sccm}$ & N.A. & $1-5 \mathrm{Vdc}$ & $1.3^{\prime \prime} \mathrm{H}_{2} \mathrm{O}$ or $324 \mathrm{~N} / \mathrm{m}^{2}$ \\
\hline AWM42150VH & $\pm 25 \mathrm{sccm}$ & N.A. & $\pm 8.5 \mathrm{mv}$ & $20 " \mathrm{H}_{2} \mathrm{O}$ or $49.8 \mathrm{~N} / \mathrm{m}^{2}$ \\
\hline AWM42300 V & $0-1000 \mathrm{sccm}$ & N.A. & $\pm 55.5 \mathrm{mv}$ & $.90 " \mathrm{H}_{2} \mathrm{O}$ or $224 \mathrm{~N} / \mathrm{m}^{2}$ \\
\hline AWM $43300 \mathrm{~V}$ & $0-1000 \mathrm{sccm}$ & N.A. & $1-5 \mathrm{Vdc}$ & $.90 " \mathrm{H}_{2} \mathrm{O}$ or $224 \mathrm{~N} / \mathrm{m}^{3}$ \\
\hline AWM43600V & $0-6 \mathrm{sim}$ & N.A. & $1-5 \mathrm{Vdc}$ & $8^{\prime \prime} \mathrm{H}_{2} \mathrm{O}$ or $1992 \mathrm{~N} / \mathrm{m}^{*}$ \\
\hline
\end{tabular}

In this case, using the By-Pass Configurations for flows up to $100 \mathrm{LPM}$ with a $\triangle \mathrm{P}=$ 0.37 " $\mathrm{H}_{2} \mathrm{O}$, the following listings would work: AWM2200V, AWM2300V, AWM3200V, AWM3300V, AWM $3200 \mathrm{CR}$, AWM42300V, and AWM43600V depending on desired outputs and flow ranges.

Figure 2

BY-PASS CONFIGURATION DRAWING

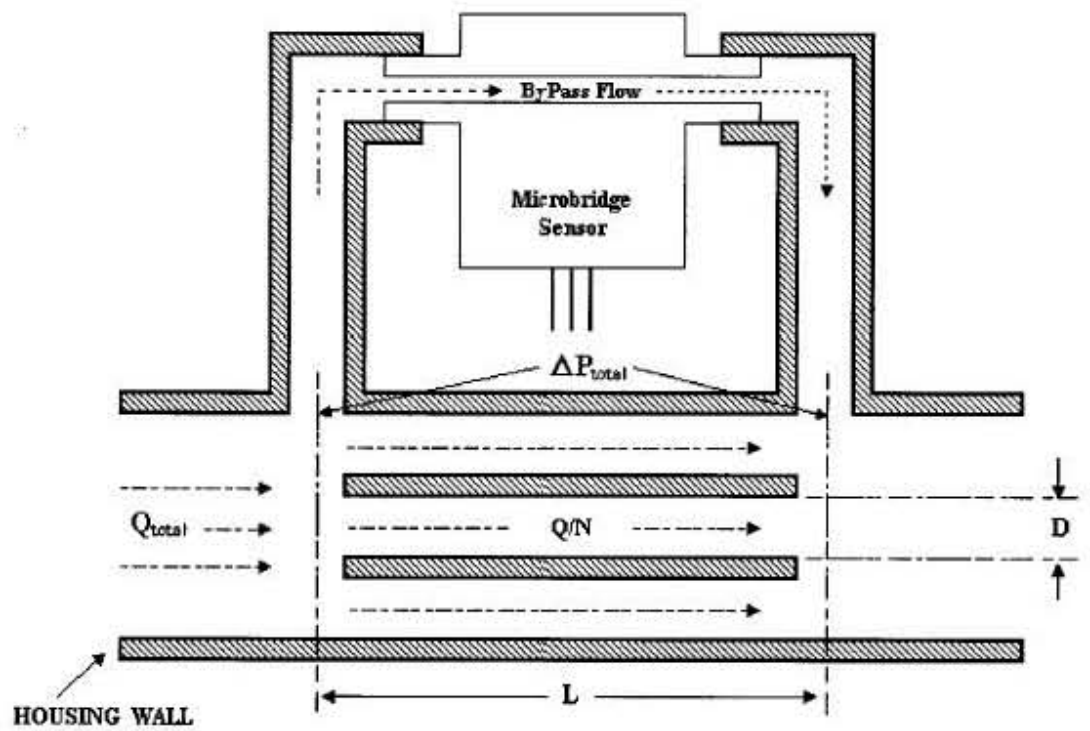




\section{REFERENCE AND APPLICATION DATA \\ Microbridge Airflow Sensors \\ Gas Correction Factors - Note \#3}

Microbridge mass airflow sensors operate by measuring the rate of relative heat transfer from a heater resistor to a temperature sensing resistor located on either side of the heater. The heat transfer is proportional to the mass flow. Offsets in the sensor sensitivity (thermal efficiency) will occur if the thermal properties of the medium change. The dominate factor is the thermal conductivity of the gas being measured. Additionally, heat capacity and gas viscosity cause lesser effects.

Heat capacity and gas viscosity are constants for a given gas. However, if the gas composition changes, these properties may also change. Fortunately, air, nitrogen, and oxygen have nearly identical properties.

The $0.5 \%$ Argon ( $\mathrm{Ar}$ ) in air is not significant, nor is the relative humidity from 0 to $99 \%$ at temperatures less than $40^{\circ} \mathrm{C}\left(104^{\circ} \mathrm{F}\right)$. Humidity levels of $100 \%$, with temperatures greater than $40^{\circ} \mathrm{C}$ $\left(104^{\circ} \mathrm{F}\right)$, indicate that more than $1 \%$ of the atmosphere is water. This may cause a measurable increase in sensor output. The actual offsets from Argon and $100 \%$ humidity are in opposite directions and may partially cancel each other at temperatures less than $40^{\circ} \mathrm{C}\left(104^{\circ} \mathrm{F}\right)$.

\section{Approximate Gas Correction Factors}

\begin{tabular}{|lc|}
\hline Gas Type & $\begin{array}{c}\text { Approximate } \\
\text { Correction Factor }\end{array}$ \\
\hline Helium $(\mathrm{He})$ & $0.5^{\star}$ \\
Hydrogen $\left(\mathrm{H}_{2}\right)$ & $0.7^{\star}$ \\
Argon $(\mathrm{Ar})$ & 0.95 \\
Nitrogen $\left(\mathrm{N}_{2}\right)$ & 1.0 \\
Oxygen $\left(\mathrm{O}_{2}\right)$ & 1.0 \\
Air & 1.0 \\
Nitric oxide $(\mathrm{NO})$ & 1.0 \\
Carbon monoxide $(\mathrm{CO})$ & 1.0 \\
Methane $\left(\mathrm{CH}_{2}\right)$ & 1.1 \\
Ammonia $\left(\mathrm{NH}_{2}\right)$ & 1.1 \\
Nitrous oxide $\left(\mathrm{N}_{2} \mathrm{O}\right)$ & 1.35 \\
Nitrogen dioxide $\left(\mathrm{NO}_{2}\right)$ & 1.35 \\
Carbon dioxide $\left(\mathrm{CO}_{2}\right)$ & 1.35 \\
\hline
\end{tabular}

Note: Gas correction factors are referenced to nitrogen $\left(\mathrm{N}_{2}\right)$ as calibration gas type. Approximate gas correction factors are provided as guidelines only. Individual gas types may perform differently at temperature extremes and varying flow rates.

*When sensing Hydrogen $\left(\mathrm{H}_{2}\right)$ or Helium $(\mathrm{He})$ it may be necessary to power the mass flow sensors using increased supply voltage: Hydrogen, 12 VDC typical, and Helium, 15 VDC typical.
Carbon monoxide $(\mathrm{CO})$ and nitric oxide (NO) have properties similar to air. Carbon dioxide $\left(\mathrm{CO}_{2}\right)$ will have increased sensitivity compared to that of air (roughly $135 \%$, this may vary with flow rate). Gases similar to $\mathrm{CO}_{2}$ are nitrous oxide $\left(\mathrm{N}_{2} \mathrm{O}\right)$ and nitrogen dioxide $\left(\mathrm{NO}_{2}\right)$.

Helium has such a high thermal conductivity that it will saturate the heater control circuit on the sensor unless supply voltage is increased to 15 VDC*. Helium sensitivity is then reduced to the point where two liters of Helium will produce an output eqivalent to one liter $(1,000 \mathrm{sccm})$ mass flow of air or nitrogen. The correction factor will be dependent upon temperature and actual flow rate.

Hydrogen flow measurement requires the use of a special sensor. These devices provide normal operation when sensing hydrogen flow and are designated with an " $\mathrm{H}$ " at the end of the catalog listing. Established hydrogen stable listings include AWM2100VH, AWM2300VH and AWM42150VH. 


\section{REFERENCE AND APPLICATION DATA \\ Microbridge Airflow Sensors}

\section{Gas Media Compatibility - Note \#4}

The microbridge mass airflow sensor incorporates a limited number of wetted materials in the construction of the device. The wetted materials in the sensor are fairly non-reactive and are compatible with a wide variety of gaseous media. Table 1 and Table 2 list wetted sensor materials and associated media compatibility.

\section{NOTICE}

Filtering is highly recommended for use in applications that may contain dust particle contamination that can degrade sensor performance over time. See Particle Contamination and Filter Manufacturers, page 126, for more information.

\section{CAUTION}

PRODUCT DAMAGE

AWM Series microbridge mass airflow sensors are NOT designed to sense liquid flow and will be damaged by liquid flow through the sensor.

MICROBRIDGE-WETTED SENSOR MATERIALS

\begin{tabular}{|c|c|c|c|c|c|}
\hline Sensor material & AWM1000 & AWM2000 & AWM 3000 & AWM40000 & AWM5000 \\
\hline Silicon & $\mathrm{x}$ & $\mathrm{x}$ & $\mathrm{x}$ & $x$ & $\mathrm{x}$ \\
\hline Silicon nitride & $\mathrm{x}$ & $\mathrm{x}$ & $\mathrm{x}$ & $\mathrm{x}$ & $\mathrm{x}$ \\
\hline Gold & $\mathrm{x}$ & $x$ & $\mathrm{x}$ & $x$ & $x$ \\
\hline Aluminum oxide & $\mathrm{x}$ & $\mathrm{x}$ & $\mathrm{x}$ & $x$ & $x$ \\
\hline Epoxy sealant & $\mathrm{x}$ & $\mathrm{x}$ & $\mathrm{x}$ & $\mathrm{x}$ & $\mathrm{x}$ \\
\hline Fluorocarbon & & & & $\mathrm{x}$ & $x$ \\
\hline Polyester & & & & & $\mathrm{x}$ \\
\hline Polyetherimide & $\mathrm{x}$ & $\mathrm{x}$ & $\mathrm{x}$ & 6 SLPM Only & \\
\hline 316 Stainless steel & AWM1200V & AWM2200V & AWM3200V & & \\
\hline
\end{tabular}

\section{MICROBRIDGE-GAS MEDIA COMPATIBILITY}

\begin{tabular}{|c|c|c|c|c|c|}
\hline Gas Media & AWM1000 & AWM2000 & AWM3000 & AWM40000 & AWM5000 \\
\hline Air & $\mathrm{x}$ & $\mathrm{x}$ & $\mathrm{x}$ & $\mathrm{x}$ & $\mathrm{x}$ \\
\hline Nitrogen & $\mathrm{x}$ & $\mathrm{x}$ & $\mathrm{x}$ & $\mathrm{x}$ & $\mathrm{x}$ \\
\hline Oxygen & $\mathbf{x}$ & $\mathrm{x}$ & $\mathrm{x}$ & $\mathrm{x}$ & $\mathrm{x}$ \\
\hline Argon & $x$ & $x$ & $\mathrm{x}$ & $x$ & $\mathrm{x}$ \\
\hline Helium & 15 VDC Supply & 15 VDC Supply & 15 VDC Supply & 15 VDC Supply & 15 VDC Supply \\
\hline Hydrogen* & Special listing & Special listing & Special listing & Special listing & Special listing \\
\hline Natural Gas & $\mathrm{x}$ & $\mathrm{x}$ & $\mathrm{x}$ & $\mathrm{x}$ & $x$ \\
\hline Nitrous oxide & $\mathrm{x}$ & $x$ & $\mathrm{x}$ & $\mathrm{x}$ & $\mathrm{x}$ \\
\hline Anesthetic Gasses & $\mathrm{N} / \mathrm{A}$ & N/A & $\mathrm{N} / \mathrm{A}$ & N/A (Except 6 SLPM) & $\mathrm{x}$ \\
\hline Carbon dioxide & $\mathrm{x}$ & $\mathrm{x}$ & $\mathrm{x}$ & $\mathrm{x}$ & $\mathrm{x}$ \\
\hline Nitric oxide & Dry Gas Only & Dry Gas Only & Dry Gas Only & Dry Gas Only & Dry Gas Only \\
\hline Sulfur oxide & Dry Gas Only & Dry Gas Only & Dry Gas Only & Dry Gas Only & Dry Gas Only \\
\hline Water vapor & Non-Condensing & Non-Condensing & Non-Condensing & Non-Condensing & Non-Condensing \\
\hline Ammonia gas & Dry Gas Only, $<1 \%$ & Dry Gas Only, $<1 \%$ & Dry Gas Only, $<1 \%$ & Dry Gas Only, $<1 \%$ & Dry Gas Only, $<1 \%$ \\
\hline Chlorine gas & $<0.1 \%$ & $<0.1 \%$ & $<0.1 \%$ & $<0.1 \%$ & $<0.1 \%$ \\
\hline Hydrogen sulfide & $<0.1 \%$ & $<0.1 \%$ & $<0.1 \%$ & $<0.1 \%$ & $<0.1 \%$ \\
\hline
\end{tabular}

*Note: Applications involving hydrogen gas sensing require the use of a sensor identified by the letter " $\mathrm{H}$ " in the catalog order guide. Current hydrogen stable catalog listings are: AWM2100VH, AWM2300VH, and AWM42150VH. Other listings may be established as hydrogen stable devices. Please contact the Application Center for assistance at 1-800-537-6945. 


\section{NOTICE}

Dust particle contamination may be present in some applications. Appropriate measures should be taken to minimize the effect of particulate contamination.

The sensor design directs dust particles in the air stream flow past the sense element parallel to its surface. In addition, the microstructure IC produces a thermophoretic effect, which repels micrometer-sized dust particles away from the microbridge structure.

Dust adherence to chip edges and channel surfaces can be prevented using a simple filter. A disposable fivemicron filter used in series on the upstream side of the airflow devide will provide adequate filtering in most applications.

\section{CAUTION}

PRODUCT DAMAGE

AWM Microbridge Mass Airflow Sensors are NOT designed to sense liquid flow and will be damaged by liquid flow through the sensor.

\section{U.S. Suppliers}

Pall Corporation

2200 Northern Blvd.

East Hills, NY 11548-1289

Tel: (516) 484-5400

1-800-645-6532 (USA Only)

Fax: (516) 484-6164

Internet: www.pall.com

\section{Pall - DFFH200}

These filters exhibit little lot-to-lot variation. Pressure drop at $1000 \mathrm{sccm}$ mass flow is less than $0.010^{\prime \prime} \mathrm{H}_{2} \mathrm{O}$. They are relatively expensive and larger in size.

\section{Pall Gelman Sciences}

600 South Wagner Road

Ann Arbor, Ml 48103-9019

Tel: (734) 665-0651

1-800-521-1520 (USA Only)

Fax: (734) 913-66114

Internet: www.pal.com/gelman

\section{Gelman Acrodisc - 4199}

These filters exhibit roughly $25 \%$ lot-to-lot variation. Differential pressure drop is approximately $0.130^{\prime \prime}$ to $0.160^{\prime \prime} \mathrm{H}_{2} \mathrm{O}$ at 100 sccm and $0.600^{\prime \prime}$ to $0.900^{\prime \prime} \mathrm{H}_{2} \mathrm{O}$ at 500 sccm mass flow. These filters are considered medically sterile and are relatively small in size.

\section{Gelman Acro - 504258}

These filters are highly efficient and exhibit little lot-to-lot variation. Typical pressure drop across the filter is $0.030^{\prime \prime} \mathrm{H}_{2} \mathrm{O}$ at 100 sccm mass flow. They are larger in size, medium priced and considered medically sterile.

Parker Hannifin Corp. - Filtration Group Finite Filter Company

500 Glaspie Street

Oxford, MI 48371

Tel: (810) 628-6400

Fax: $(810) 628-1850$

Internet: www.parker.com

\section{Finite Filter - IDN-14G}

Finite filters exhibit minor lot-to-lot variation. Differential pressure drop is less than $0.020^{r} \mathrm{H}_{2} \mathrm{O}$ at $100 \mathrm{sccm}$ mass flow and less than $0.060^{\prime \prime} \mathrm{H}_{2} \mathrm{O}$ at $500 \mathrm{sccm}$ mass flow. These filters are smaller in size and made of transparent plastic for ease of inspection.

\section{International Suppliers}

AUSTRALIA

Pall Gelman Sciences

P.O. Box 4100

Lane Cove DC, Sydney

NSW 2066

Tel: $(61-29)$ 428-2333

Fax: $(61-29)$ 428-5610

FRANCE

Pall Gelman Sciences

Cite Descartes - 10 allee

Lorentz

77420 Champs sur Marne

Tel: (33-1) 6461-5252

Fax: (33-1) 6461-5262

\section{GERMANY}

Pall Gelman Sciences

Arheilger Weg 6

D-64380 Roßdorf

Tel: (49-6) 154-60220

Fax: (49-6) 154-602260

JAPAN

Pall Gelman Sciences

1-9-12 Kita-Ueno

Taito-ku, Tokyo 110

Tel: (81-3) 3844-5411

Fax: (81-3) $3844-5433$

\section{UNITED KINGDOM}

Gelman Sciences, Ltd.

Brackmills Business Park

Caswell Road

Northampton NN4 7EZ

Tel: (441-604) 70-4704

Fax: (441-604) 70-4724

BRAZIL

Parker Hannifin Industria

e Comercio Ltda.

Irlemp Filter Division

Via Anhanguera, KM, 25,5 - Trevo Perus

05276-000 Sao Paulo, SP, Brazil

Tel: (55) (11) 847-1222

Fax: (55) (11) $847-1610$

\section{FINLAND}

Parker Hannifin Corporation

Finn Filter Division

Fin-31700

Urjala AS., Finland

Tel: (358) 37-54100

Fax: (358) 37-5410 100

\section{UNITED KINGDOM}

Parker Hannifin Corporation

Filter Division Morley

Peel Street

Morley, Leeds

LS27 8EL England

Tel: (44) 113 253-7921

Fax: (44) 113 252-7815 


\section{AWM5104VN}

Airflow Sensor, Signal Conditioning: Amplified; Flow/Pressure Range: 0 SLPM to 20 SLPM; Port Style: Threaded, $1 / 4$ NPT

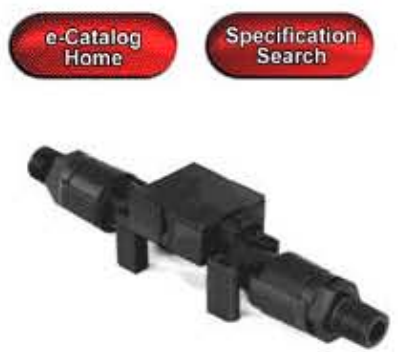

Representative photograph, actual product appearance may vary.

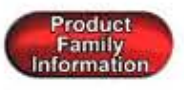

\section{FEATURES}

- Linear voltage output

- Venturi design

- Remote mounting capability

- Active laser trimming improves interchangeability

- Separate gas calibration types: $-\operatorname{Ar}(\operatorname{argon})$ $-\mathrm{N}_{2}$ (nitrogen) or $-\mathrm{CO}_{2}$ (carbon dioxide)

In-Line Flow Measurement

AWM5000 Series Microbridge Mass Airflow Sensors feature a venturi type flow housing. They measure flow as high as 20 standard liters per minute (SLPM) while inducing a maximum pressure drop of $2.25^{\prime \prime} \mathrm{H}_{2} \mathrm{O}$. The microbridge chip is in direct contact with the flow stream, greatly reducing error possibilities due to orifice or bypass channel clogging.

\section{Rugged, Versatile Package}

The rugged plastic package has been designed to withstand common mode pressures up to $50 \mathrm{psi}$, and the small sensing element allows $100 \mathrm{gs}$ of shock without compromising performance. The included "AMP" compatible connector provides reliable connection in demanding applications.

On-board Signal Conditioning

Each AWM5000 sensor contains circuitry which performs amplification, linearization, temperature compensation, and gas calibration. A 1 to $5 \mathrm{Vdc}$ linear output is possible for all listings regardless of flow range $(5,10,15$, or 20 SLPM) or calibration gas (nitrogen, carbon dioxide, nitrous oxide, or argon). All calibration is performed by active laser trimming.

\begin{tabular}{|c|c|}
\hline \multicolumn{2}{|l|}{ Searchable Features } \\
\hline Signal Conditioning & Amplified \\
\hline Flow/Pressure Range & 0 SL.PM to 20 SL.PM \\
\hline Output Voltage @ Trim Point & 5 Vdc@ Full Scale Flow \\
\hline Port Style & Threaded, $1 / 4$ NPT \\
\hline \multicolumn{2}{|l|}{ Informational Features } \\
\hline Null Shift over Temperature & \pm 0.050 Vdc typ., \& $@ 1770.20 \mathrm{Vdc} \max$. \\
\hline Output Shift over Temperature & $\pm 7.0 \%$ Reading \\
\hline Maximum change in flow rate & $5 \mathrm{SLPM} / \mathrm{s}$ \\
\hline \multicolumn{2}{|c|}{ Max. Repeatability \& Hysteresis Error $\pm 0.50 \%$ Reading } \\
\hline Null Offset & 0.95 Vdc min., 1 Vdc typ., 1.05 Vdc max. \\
\hline Response Time & $60 \mathrm{~ms}$ max. \\
\hline Supply Voltage & 8 Vde min., 10 Vdc typ., 15 Vdc max. \\
\hline Maximum Common Mode Pressure & $50 \mathrm{psi}$ \\
\hline Power Consumption & $100 \mathrm{~mW}$ max. \\
\hline Operating Temperature Range & $-20^{\circ} \mathrm{C}$ to $70^{\circ} \mathrm{C}\left[-4^{\circ} \mathrm{F}\right.$ to $\left.158^{\circ} \mathrm{F}\right]$ \\
\hline Storage Temperature Range & $-20^{\circ} \mathrm{C}$ to $70^{\circ} \mathrm{C}\left[-4^{\circ} \mathrm{F}\right.$ to $\left.158^{\circ} \mathrm{F}\right]$ \\
\hline Media Compatibility & Dry gas only \\
\hline Weight & $60 \mathrm{~g}$ \\
\hline Shock & $100 \mathrm{~g}$ peak $6 \mathrm{~ms}$ half-sine ( 3 drops, each direction of 3 axes) \\
\hline Availability & Global \\
\hline Comment & Nitrogen calibration gas. This calibration is identical to using oxygen or air as calibration gas. \\
\hline
\end{tabular}


Honeywell

Installation Instructions for the

ISSUE 2

\section{MICRO SWITCH AWM5000 Series Microbridge PK 88762 Mass Airflow Sensor}

\section{GENERAL INFORMATION}

AWM5000 Series Microbridge Mass Airflow Sensors operate on the theory that airflow directed across the surface of a sensing element causes heat transfer. Output voltage varies in proportion to the mass of air or other gas flowing through a given sensor's inlet and outlet ports.

Current sink/source. Maximum current ratings are $10 \mathrm{~mA}$ sinking and $20 \mathrm{~mA}$ sourcing, governed by an LM224 operational amplifier in the final stage of the instrumentation amplifier.

\section{MEDIA CONTAMINATION}

Media flowing through the sensor should be free of condensing moisture and particulate contaminants. An inexpensive 5 micron filter upstream of the sensing element substantially reduces the risk of damage due to contaminants.

\section{MOUNTING INSTRUCTIONS}

Mount AWM5000 Series sensors with 6-32 screws. Use of washers below screw head is recommended. Mounting torque is $1.1 \mathrm{Nm}$ (9.75 in/lb) max. for steel screws, or $0.75 \mathrm{Nm}(6.75 \mathrm{in} / \mathrm{lb})$ max. for brass screws.

\section{NOTICE}

When making flow connections to mounted sensor, the AWM5000 must be supported at the flow adapter.

If end adapters are twisted with respect to the flow tube during installation, the seal between $\mathrm{O}$-ring and flowtube will be broken, causing a small temporary leak. The leak can be as high as 1 psi, or may remain within specification. It will self-heal as the 0 ring conforms. About $85 \%$ of the leak will be gone in 24 hours, with complete recovery within 48 hours.

Do not expose ports to forces greater than $1 \mathrm{~kg} \mathrm{(2}$ pounds) in a direction perpendicular to the port centerline.

Torque on ports should not exceed $4,52 \mathrm{Nm}$ (40 in/lb).

\section{ELECTRICAL CONNECTION}

The AWM5000 Series accepts a latch detente connector, such as:

1. Amp part number $103956-3$.

2. MICRO SWITCH part number SS-12143.

Information and literature on latch detente connectors is available from Amp Product Information Center, 1-800-522-6752 or the Customer Hotline, 1-800-722-1111.

\section{RECOMMENDED AMP LITERATURE}

\begin{tabular}{ll}
\hline 82160 & $\begin{array}{l}\text { MTE Interconnection System } \\
\text { (AMPMODU) Catalog }\end{array}$ \\
\hline $108-25034$ & $\begin{array}{l}\text { Product Specification (technical } \\
\text { performance information) }\end{array}$ \\
\hline $114-25026$ & $\begin{array}{l}\text { Application Specification (describes } \\
\text { product, proper assembly, full tooling } \\
\text { information) }\end{array}$ \\
\hline IS 6919 & Instruction Sheet for assembly procedure \\
\hline
\end{tabular}

TO MAKE ELECTRICAL CONNECTIONS

1. Remove (unlatch) the connector from the AWM5000.

2. Hand-crimp the interface wire to the appropriate pin on connector. Suggested tool: AMP HandCrimp Tool, part number IS9407.

3. Insert the terminal contacts into the connector housing after carrier strip (lead-frame) is removed.

4. Reconnect (latch) connector to AWM5000 device.

\section{CLEANING}

\section{NOTICE}

Do not use ultrasonics when cleaning. This may damage the microstructure.

Cover the ends of the tube during cleaning, since certain solvents may attack the epoxy which seals the chip tube to the ceramic substrate. Do not use: III Tri-chloroethane, methylene chloride, methyl pyrrolidone, or any oxidizing type acid such as formic acid.

MICRO SWITCH Sensing and Control 


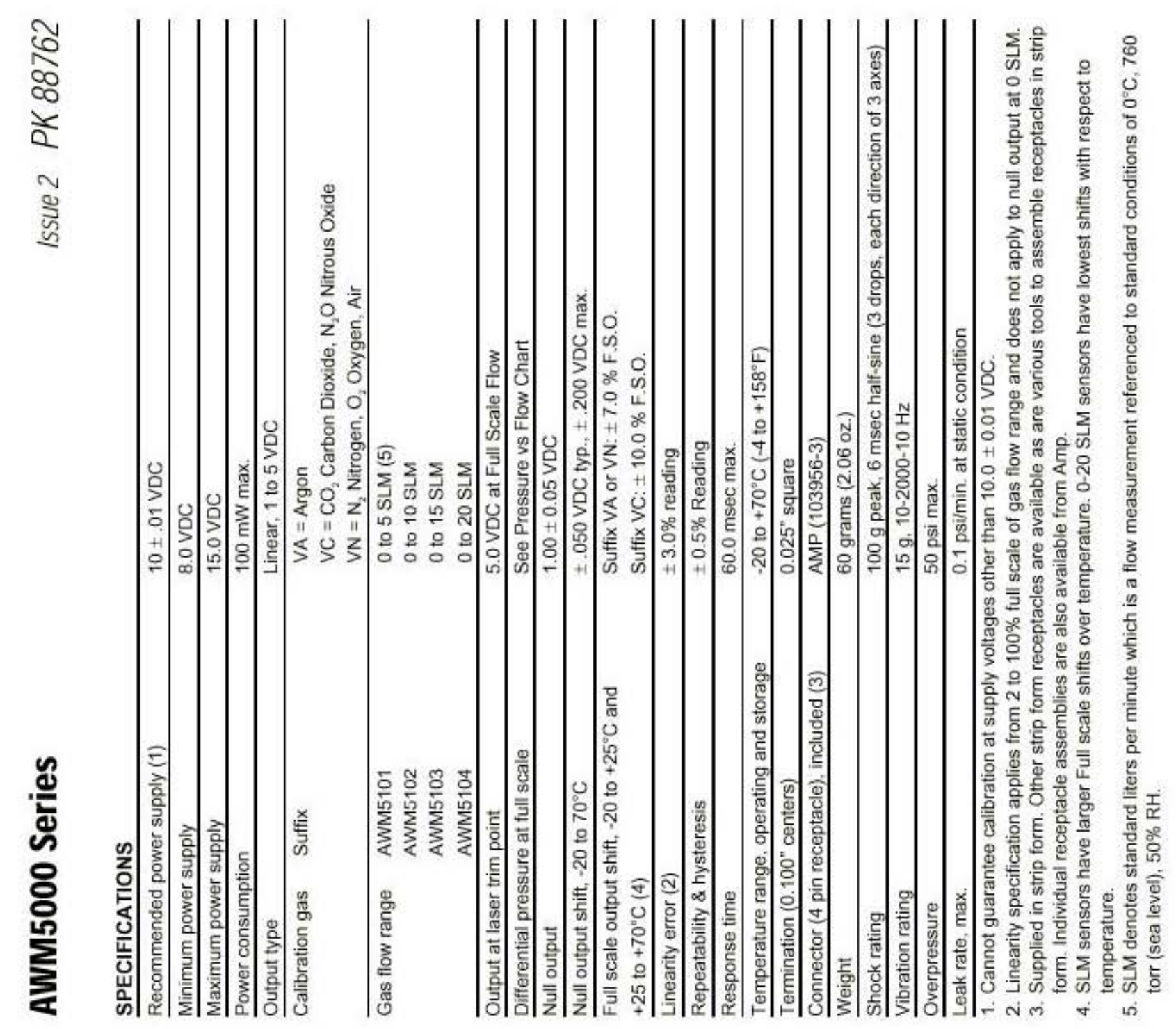




\section{CHARACTERISTICS}

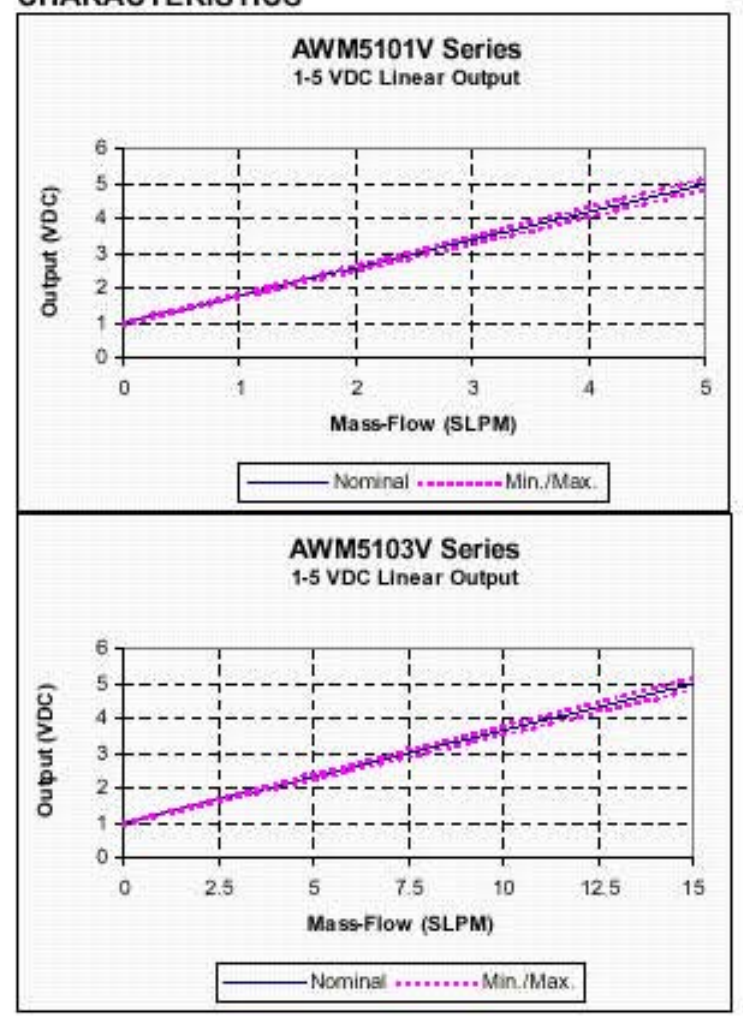

PRESSURE vS. AIRFLOW

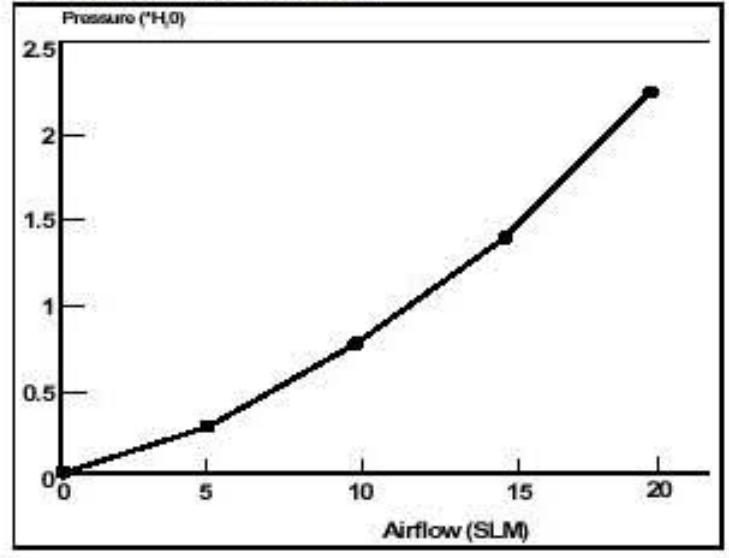

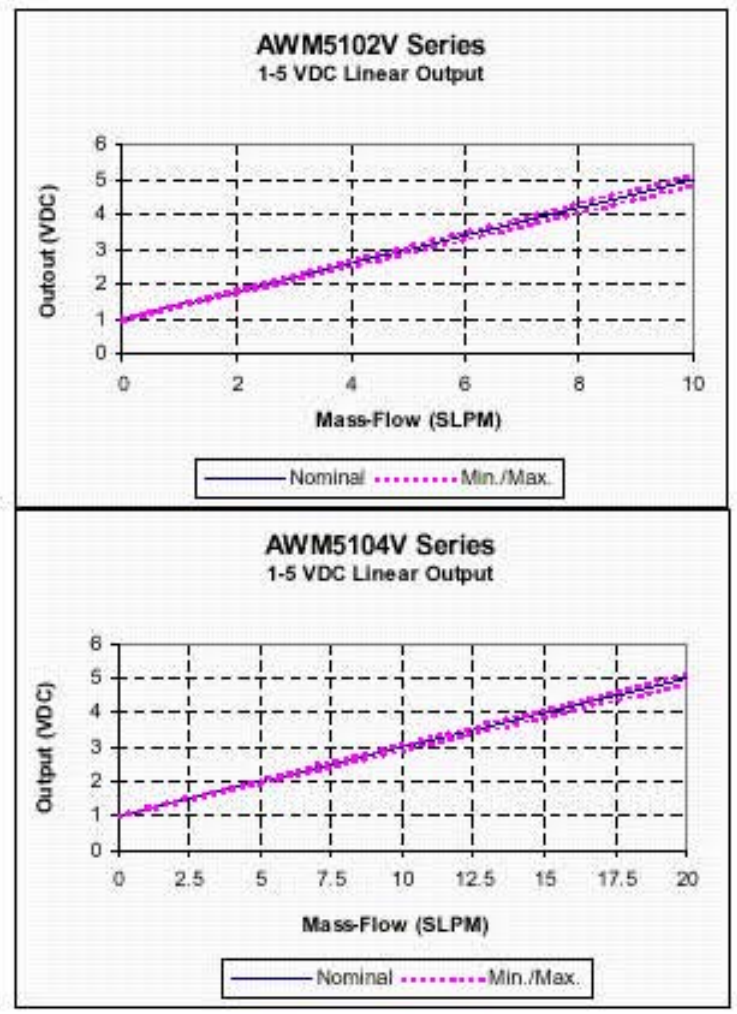

PRODUCT DESCRIPTION

\begin{tabular}{ll}
\hline Catalog Listing & Flow Range \\
\hline AWM5101VA & 5 SLPM, Argon calibration \\
\hline AWM5101VC & 5 SLPM, CO, calibration \\
\hline AWM5101VN & 5 SLPM, $N_{2}$ calibration \\
\hline AWM5102VA & 10 SLPM, Argon calibration \\
\hline AWM5102VC & 10 SLPM, CO, calibration \\
\hline AWM5102VN & 10 SLPM, $N_{2}$ calibration \\
\hline AWM5103VA & 15 SLPM, Argon calibration \\
\hline AWM5103VC & 15 SLPM, CO, calibration \\
\hline AWM5103VN & 15 SLPM, $N_{2}$ calibration \\
\hline AWM5104VA & 20 SLPM, Argon calibration \\
\hline AWM5104VC & 20 SLPM, CO, calibration \\
\hline AWM5104VN & 20 SLPM, $N_{2}$ calibration \\
\hline
\end{tabular}

All listings have 1 - 5 VDC linear output with 10 VDC supply over given flow range for a given gas. 
MOUNTING DIMENSIONS (for reference only)

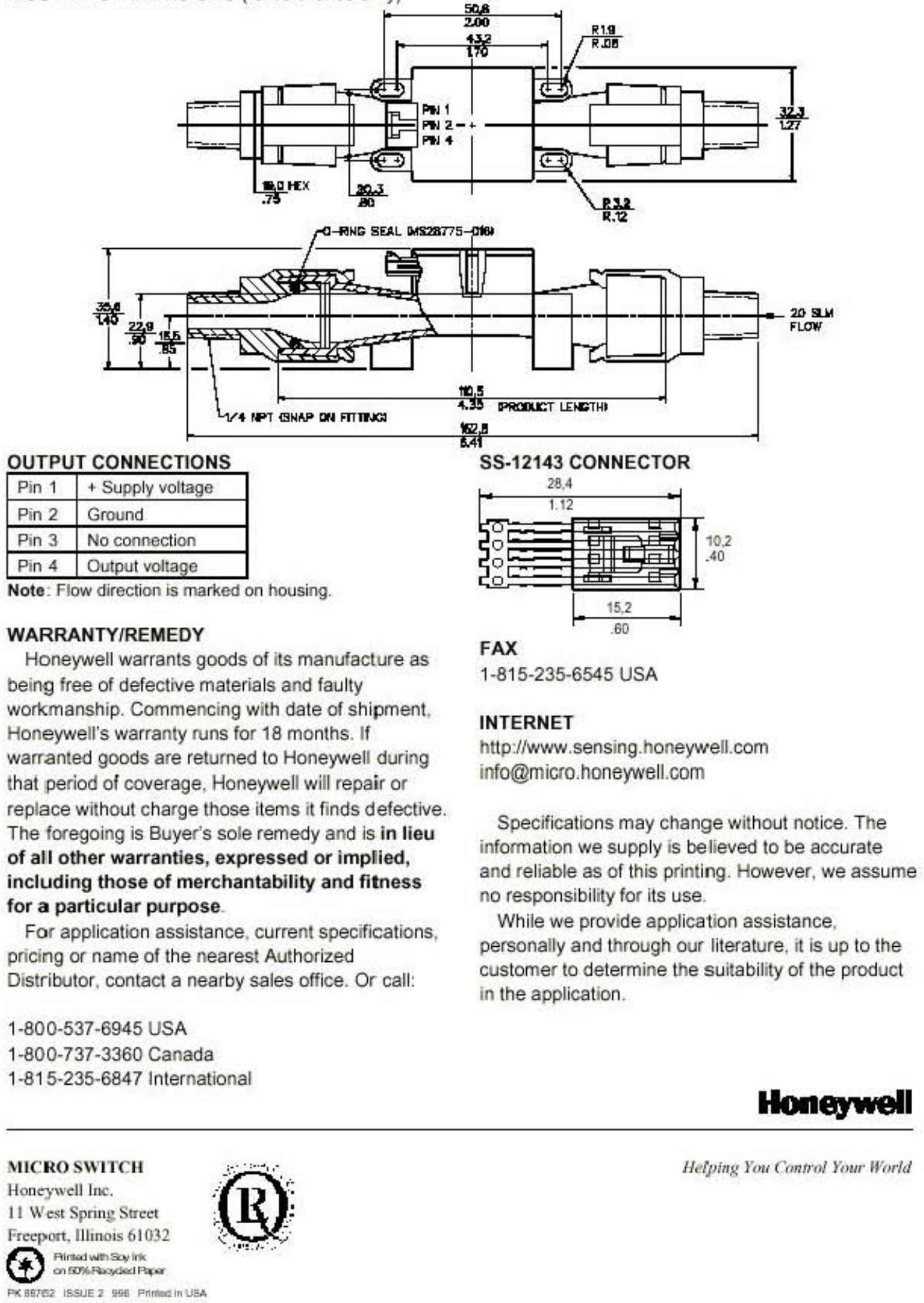


Airflow Sensors

AWM5000 Series High Flow Mass Airflow/Amplified

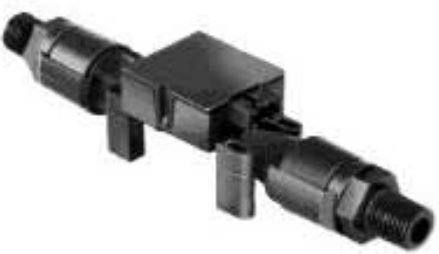

In-Line Flow Measurement AWM5000 Series Microbridge Mass Airflow Sensors feature a venturi type flow housing. They measure flow as high as 20 standard liters per minute (SLPM) while inducing a maximum pressure drop of $2.25^{\circ} \mathrm{H}_{3} \mathrm{O}$. The microbridge chip is in direct contact with the flow stream, greatly redicing error possibilities due to orifice or bypass channel clogging.

Rugged, Versatile Package

The rugged plastic package has been designed to withstand common mode pressures up to $50 \mathrm{psi}$, and the small sensing element allows 100 gs of shock withsut compromising performance. The included "AMP" compatible connector provides reliable connection in demanding applications.

\section{On-board Signal Conditioning}

Each AWM5000 sensor contains circuitry which performs amplification, linearization, temperature compensation, and gas calibration. Figure 1 (Heater Control Circuit) and Figure 2 (Sensor Bridge Circuit and Amplification Linearization Circuit) itlustrate the on-board electrical circuitry for the AWM5000 Series. A 1 to 5 VDC linear output is possible for all listings regardless of flow range $(5,10,15$, or 20 SLPV) or calibration gas (nitrogen, carbon dioxide, nitrous oxide, or argon). All calibration is performed by active laser trimming.
FEATURES

- Linear voltage output

- Venturi design

- Remote mounting capability

- Active laser trimming improves interchange ability

- Separate gas calibration types:

- Ar (argon)

- $\mathrm{N}_{2}$ (nitrogen) or

- $\mathrm{CO}_{2}$ (carbon dioxide)

\section{Figure 1}

Heater Control Circuit

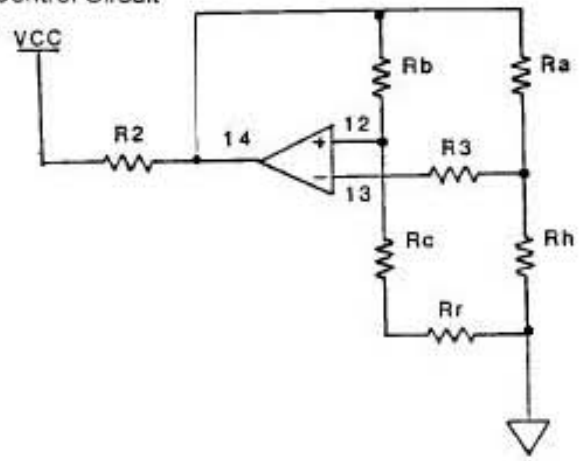

Figure 2

Sensor Bridge Circuit and hmplification Linearization Circuit

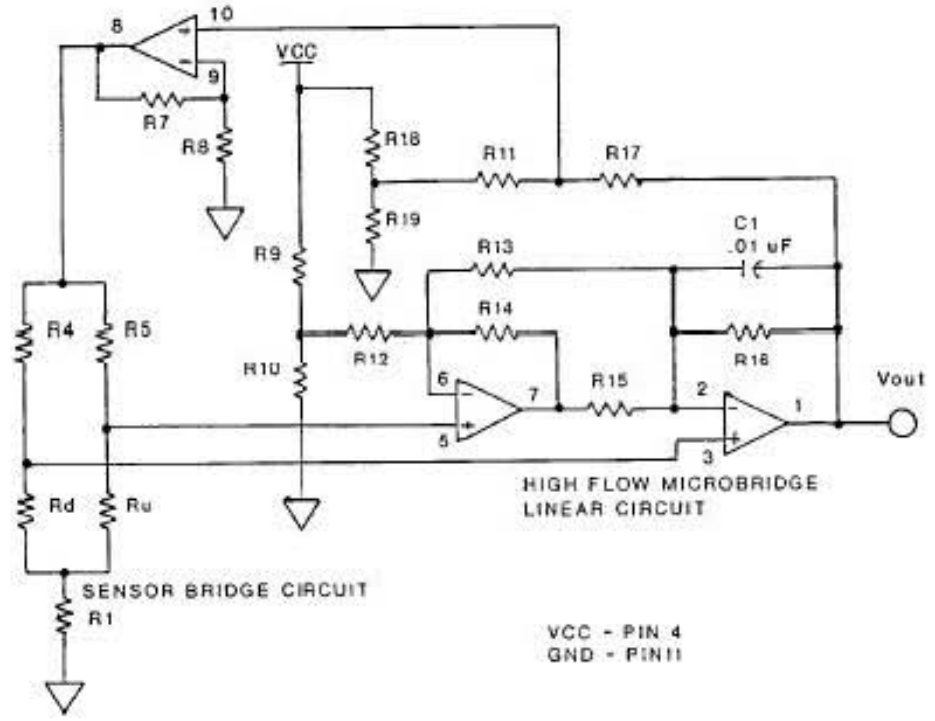


Airflow Sensors

AWM5000 Series

Highflow Mass Airflow/Amplified

SPECIFICATIONS (Performance Characteristics@ $10.0 \pm 0.01$ VDC, $25^{\circ} \mathrm{C}$ )

\begin{tabular}{|c|c|c|c|c|}
\hline & AWM5101 & AWM5102 & AWM5103 & AWM5104 \\
\hline Flow Range (Note 3) & $0-5$ SLPM & 0-10 SLPM & $0-15$ SLPM & 0-20 SLPM \\
\hline \multirow[t]{2}{*}{ Suffix - Calibration gas } & \multicolumn{2}{|c|}{ VA - Argon (Ar) } & VC - Carbon dioxide $\left(\mathrm{CO}_{2}\right)$ & \multirow[t]{2}{*}{ VN - Nitrogen $\left(\mathrm{N}_{2}\right)$} \\
\hline & Min. & & & \\
\hline Excitation VDC & 8 & \multicolumn{2}{|c|}{$10 \pm 0.01$} & 15 \\
\hline Power consumption (mW) & - & \multicolumn{2}{|c|}{ - } & 100 \\
\hline Response time (msec) & - & \multicolumn{2}{|c|}{-} & 60 \\
\hline Null output VDC & 0.95 & \multicolumn{2}{|c|}{1} & 1.05 \\
\hline $\begin{array}{l}\text { Null output shift } \\
-20^{\circ} \text { to } 70^{\circ} \mathrm{C}\end{array}$ & - & \multicolumn{2}{|c|}{ $\pm 0.050 \mathrm{VDC}$} & $\pm 200 \mathrm{VDC}$ \\
\hline $\begin{array}{l}\text { Common Mode } \\
\text { Pressure (psi) }\end{array}$ & - & \multicolumn{2}{|c|}{ - } & 50 \\
\hline Temperature range & \multicolumn{4}{|c|}{$-20^{\circ}$ to $+70^{\circ} \mathrm{C},\left(-4^{\circ}\right.$ to $\left.158^{\circ} \mathrm{F}\right\}$} \\
\hline Weight & \multicolumn{4}{|c|}{60 grams $(2.12 \mathrm{oz})}$. \\
\hline Shock ratings & \multicolumn{4}{|c|}{$100 \mathrm{~g}$ peak, 6 msec half-sine (3 drops, each direction of 3 axes) } \\
\hline Output @ laser trim point & \multicolumn{4}{|c|}{5 VDC@ Full Scale Flow } \\
\hline $\begin{array}{l}\text { Output voltage shift } \\
+20^{\circ} \text { to }-25^{\circ} \mathrm{C},+20^{\circ} \text { to } 70^{\circ} \mathrm{C}\end{array}$ & \multicolumn{4}{|c|}{ Suffix VA or VN $\pm 7.0 \%$ Reading, Suffix VC $\pm 10.0 \%$ Reading } \\
\hline Linearity error (2) & \multicolumn{4}{|c|}{ $\pm 3.0 \%$ Feading $(\max )$} \\
\hline Repeatability \& Hysteresis & \multicolumn{4}{|c|}{ $\pm 0.5 \%$ Reading (max.) } \\
\hline $\begin{array}{l}\text { Connector (Included) } \\
\text { - Four pin receptacle }\end{array}$ & \multicolumn{4}{|c|}{ MICRO SWITCH (SS12143)/AMP (103956-3) } \\
\hline Leak rate, $\max$ & \multicolumn{4}{|c|}{$0.1 \mathrm{ps} / \mathrm{min}$. at static candition, (Note 2) } \\
\hline
\end{tabular}

Notes:

1. Linearity specification applies from 2 to $100 \%$ full scale of gas flow range, and does not apply to null output at O SLPM.

2. The AWM5000 series product has a leakage spec of less than 0.1 psi per minute at 50 psi common mode pressure. If during installation, the end adapters are twisted with respect to the flowtube, this may compromise the seal between the o-ring and the flowtube and may cause a temporary leak. This leak might be as high as 1 psi or might remain in specification. It will self-reseal as the o-ring takes a new set.

Approximately $85 \%$ of the leakage will dissipate in 24 hours. Within 48 hours, complete recovery will take place.

3. SLPM denotes standard liters per minute, which is a flow measurement referenced to standard conditions of $0^{\circ} \mathrm{C} / 1$ bar (sea level), $50 \%$ RH.

\section{NOTICE}

\section{AWM5000-Chimney Effect}

AWM microbridge mass airflow sensors detect mass airflow caused by heat transfer. The thermally isolated

microbridge structure consists of a heater resistor positioned between two temperature sensing resistors.

The heater resistor maintains a constant temperature, $160^{\circ} \mathrm{C}$ above ambient, during sensor operation. Airflow moving past the chip transfers heat from the heater resistor. This airflow warms the downstream resistor and cools the upstream resistor. The temperature change and the resulting change in resistance of the

temperature resistors is proportional to the mass airflow across the sensing element.

When the sensor is mounted in a vertical position, under zero flow conditions, the sensor may produce an output that is the result of thermally induced convection current. This occurrence is measurable in the

AWM5000 Series, particularly in the 5 SLPM versions. When designing the sensor into applications where nul

stability is critical, avoid mounting the sensor in a vertical position. 
Airflow Sensors

Microbridge Mass Airflow - High Flow/Amplified AWM5000

AWM5000 Series

OUTPUT CURVES Performance Characteristics @ 10.0 Vdc +/- $0.01 \mathrm{Vdc}, 25^{\circ} \mathrm{C}$

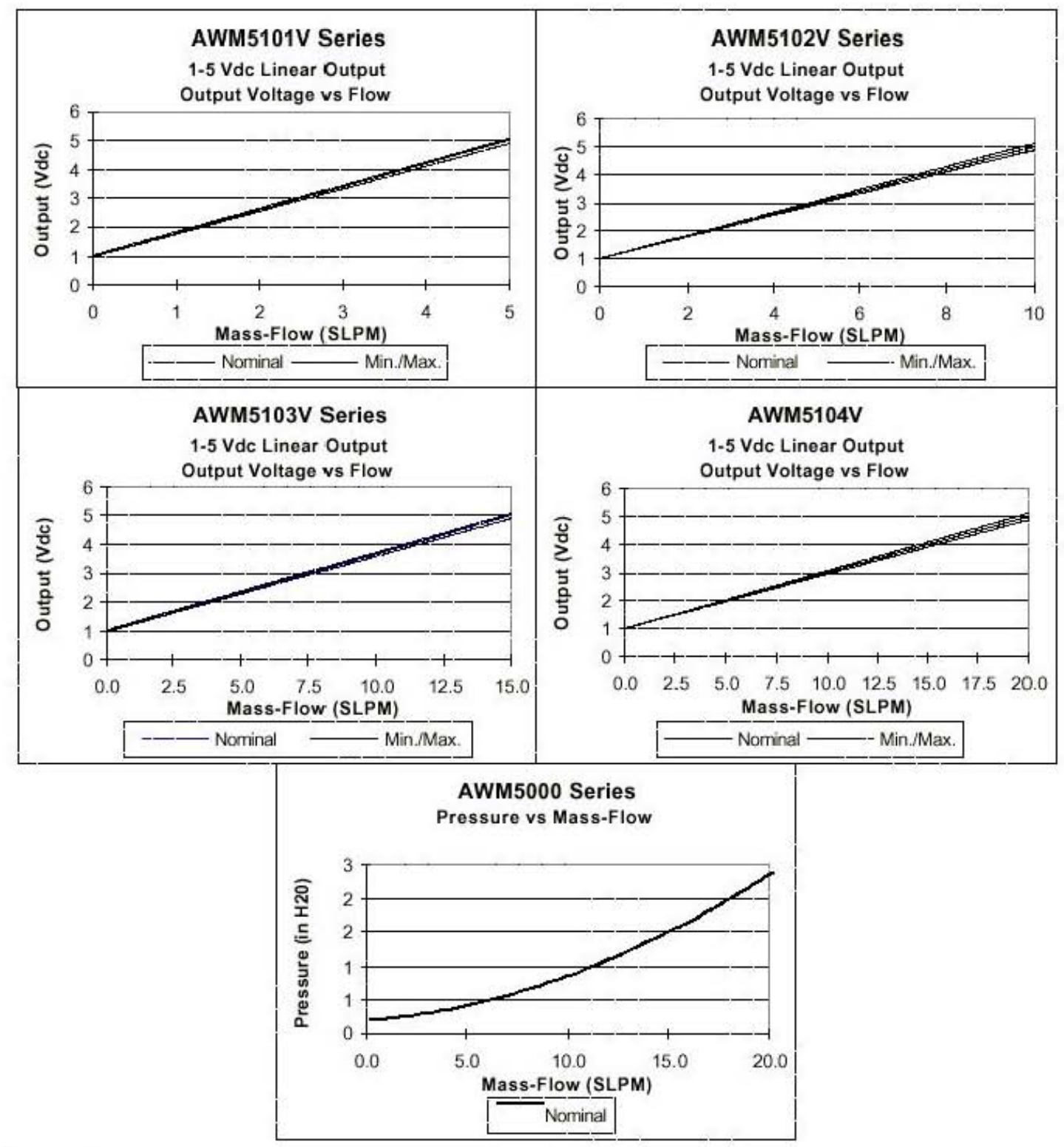

NOTICE

AWM5000-Ehimney Effect

AWM microbridge mass airflow sensors detect mass airflow caused by heat transfer. The thermally isolated microbridge structure consists of a heater resistor positioned between two temperature sensing resistors.

The heater resistor maintains a constant temperature, $160^{\circ} \mathrm{C}$ above ambient, during sensor operation. Airflow moving past the chip transfers heat from the heater resistor. This aifflow warms the downstream resistor and cools the upstream resistor. The temperature change and the resulting change in resistance of the temperature resistors is proportional to the mass airflow across the sensing element.

When the sensor is mounted in a vertical position, under zero flow conditions, the sensor may produce an output that is the result of thermally induced convection current. This occurrence is measurable in the AWM5000 Series, particularly in the 5 SLPM versions. When designing the sensor into applications where null stability is critical, avoid mounting the sensor in a vertical position. 
Highflow Mass Airflow/Amplified

AWM5000 ORDER GUIDE
\begin{tabular}{|c|l|} 
Catalog Listing & Flow Range \\
\hline AWM5101VA & 5 SLPM, Argon calibration \\
\hline AWM5101VC & 5 SLPM, CO, calibration (2) \\
\hline AWM5101VN & 5 SLPM, N, calibration (1) \\
\hline AWM5102VA & 10 SLPM, Argon calibration \\
\hline AWM5102VC & 10 SLPM, CO, calibration (2) \\
\hline AWM5102VN & 10 SLPM, Na calibration (1) \\
\hline AWM5103VA & 15 SLPM, Argon calibration \\
\hline AWM5103VC & 15 SLPM, CO, calibration (2) \\
\hline AWM5103VN & 15 SLPM, Na calibration (1) \\
\hline AWM5104VA & 20 SLPM, Argon calibration \\
\hline AWM5104VC & 20 SLPM, CO, calibration (2) \\
\hline AWM5104VN & 20 SLPM, Nan calibration (1) \\
\hline
\end{tabular}

\section{CONNECTOR ORDER GUIDE}

\section{Catalog Listing}

SS12143
Description

Four pin Electrical connector

Connectors use Amp 103956-3

Note: All listings have 1 - 5 VDC linear output with 10 VDC supply over given flow range for a specific calibration gas.

1. $\mathrm{N}_{n}$ calibration is identical to $\mathrm{O}_{\mathrm{n}}$ and air calibration.

2. $\mathrm{CO}$, calibration is identical to $\mathrm{N}_{\text {O }}$ calibration

3. For additional gas correction factors, see Application Note 3.

\section{OUTPUT CONNECTIONS}

Pin 1 + Supply voltage

Pin 2 Ground

Pin 3 No connection

Pin 4 Output voltage

Arrow on bottom of housing indicates direction of flow.

MOUNTING DIMENSIONS (for reference only)
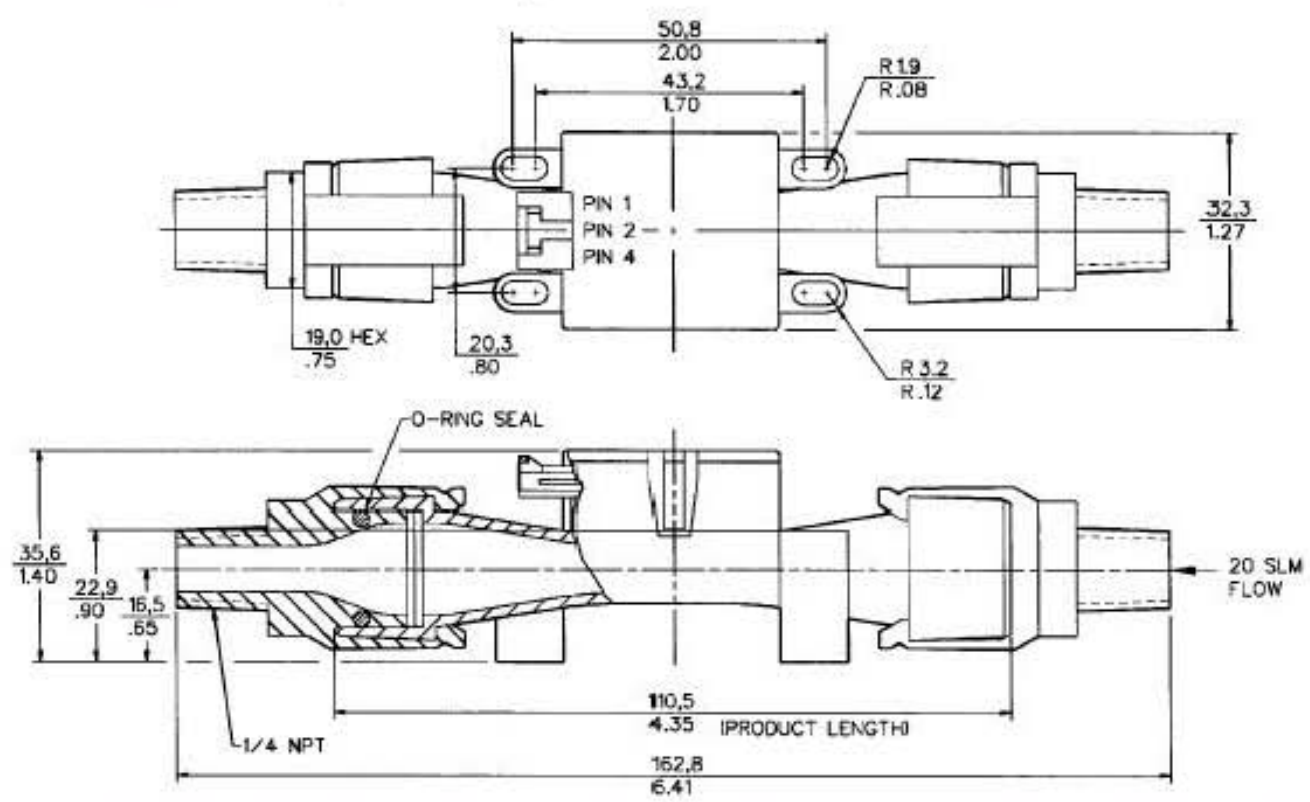


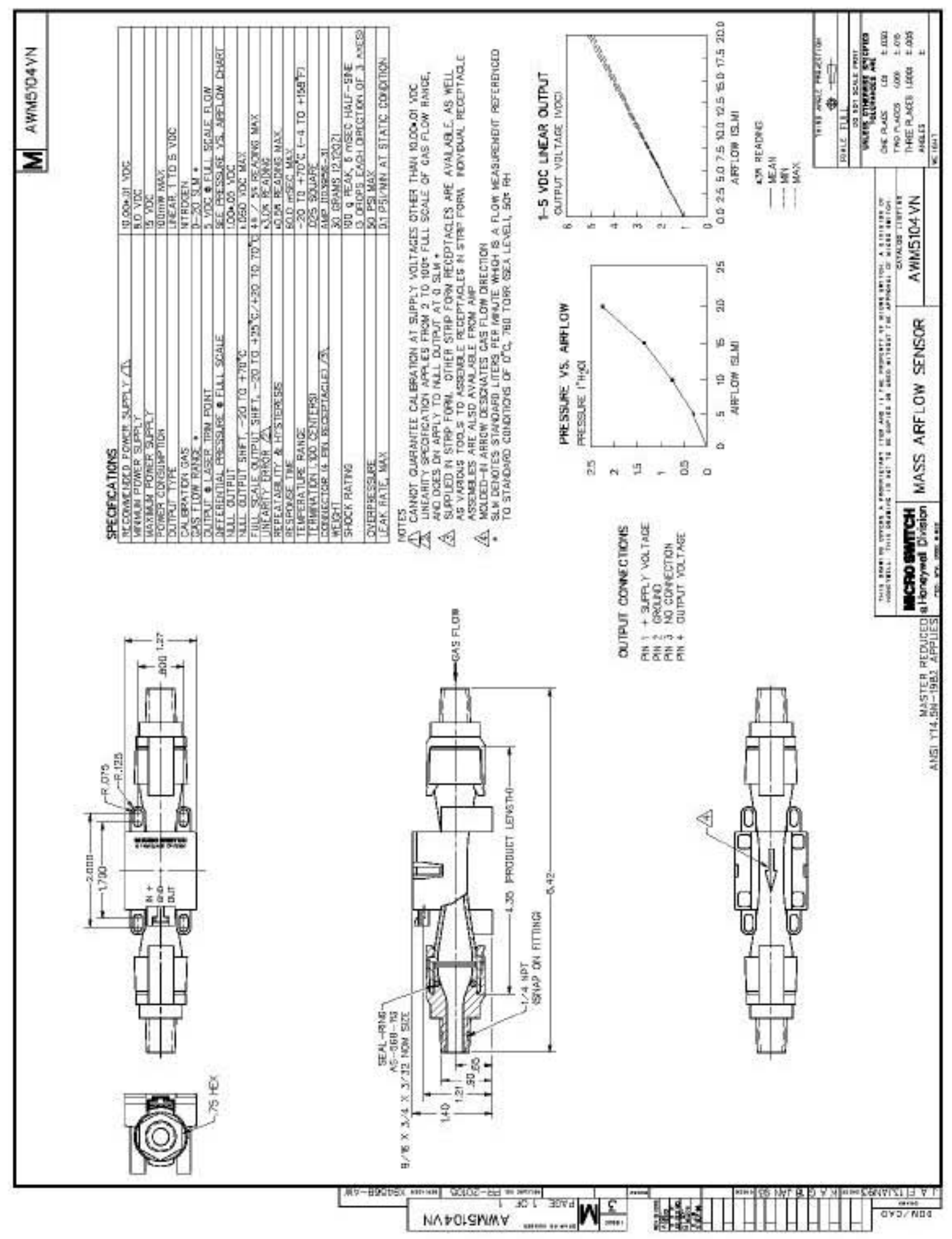




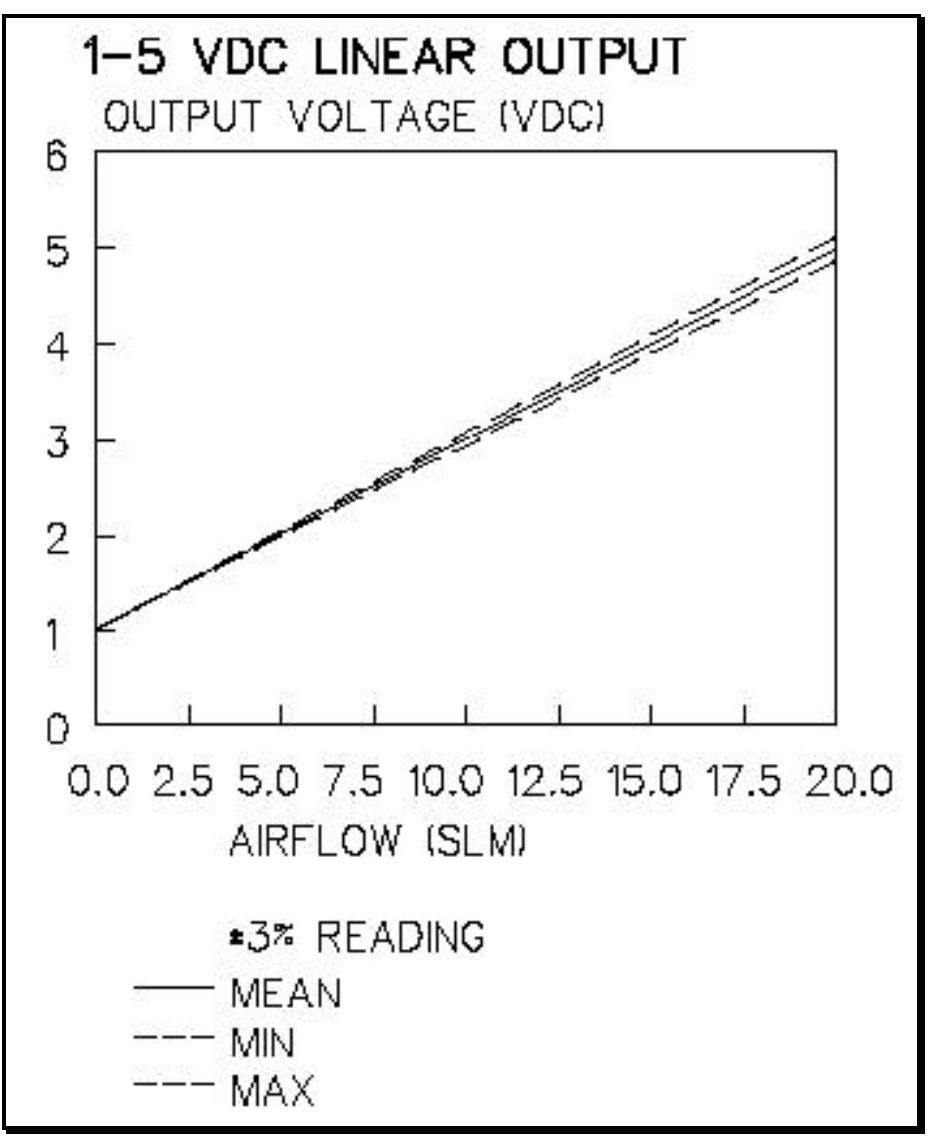

FIGURE 116 - AWM5104VN flow vs. output voltage characteristic

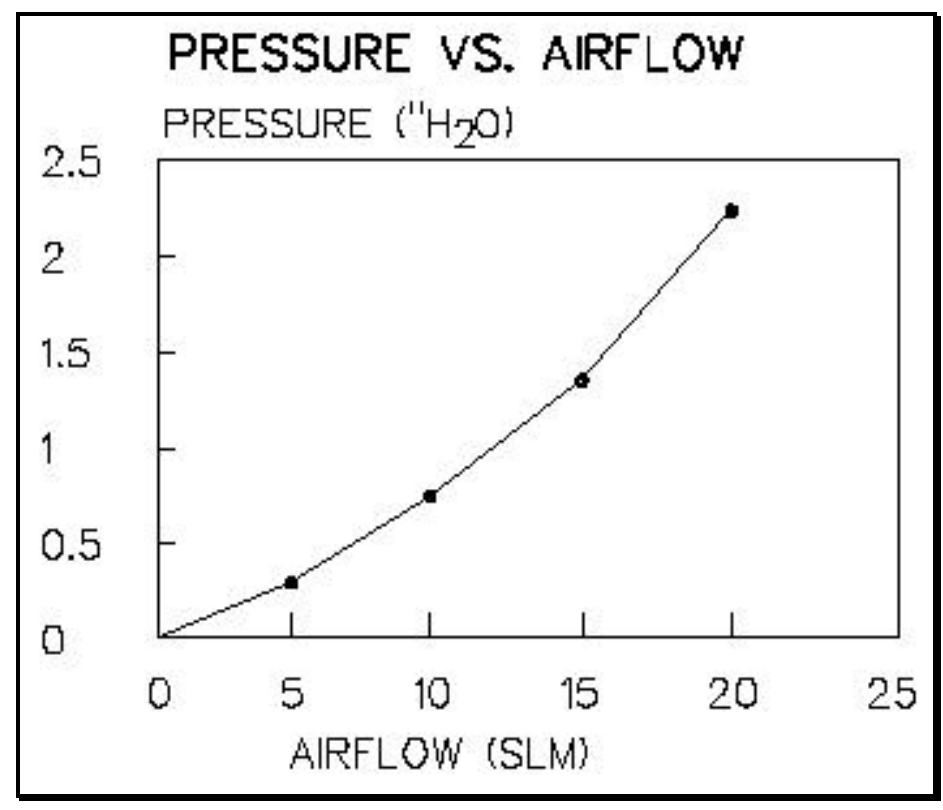

FIGURE 117 - AWM5104VN flow vs. differential pressure characteristic 


\section{APPENDIX II.}

HONEYWELL AWM2150V FLOW SENSOR DIE PHOTOS 


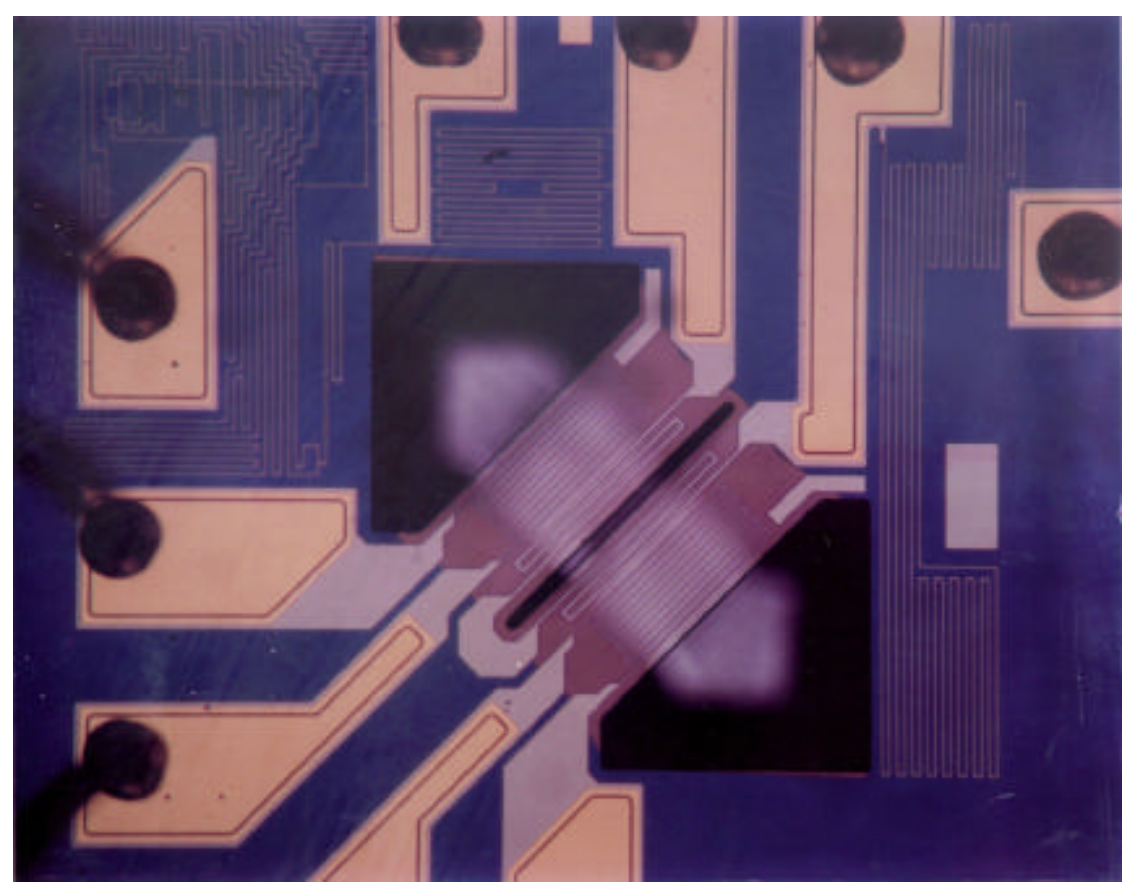

FIGURE 118 - Photograph of dual $\mathrm{Si}_{3} \mathrm{~N}_{4}$ suspended microbridge structure in the AWM2150V mass airflow sensor

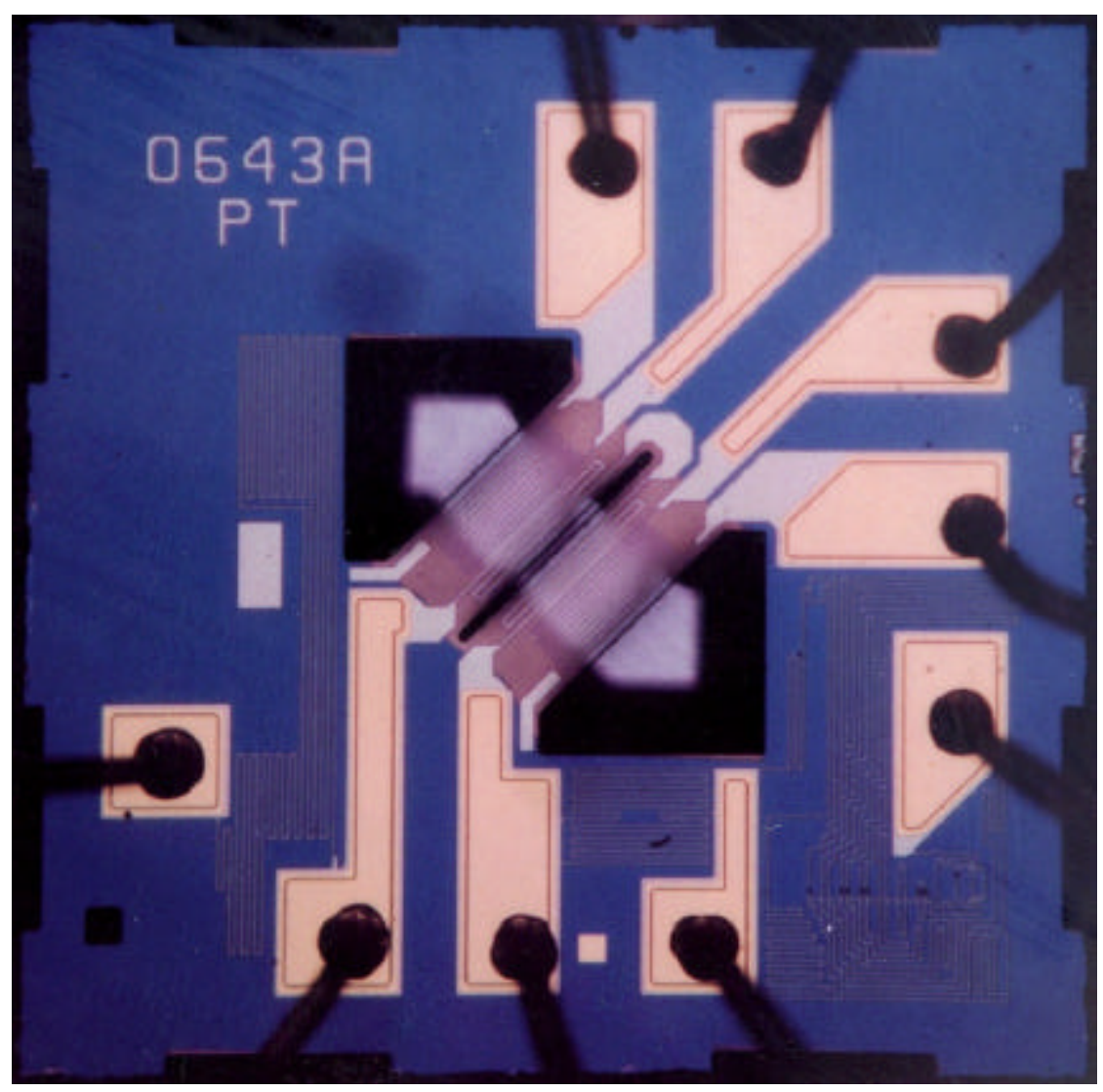

FIGURE 119 - Photograph of AWM2150V mass airflow sensor die 


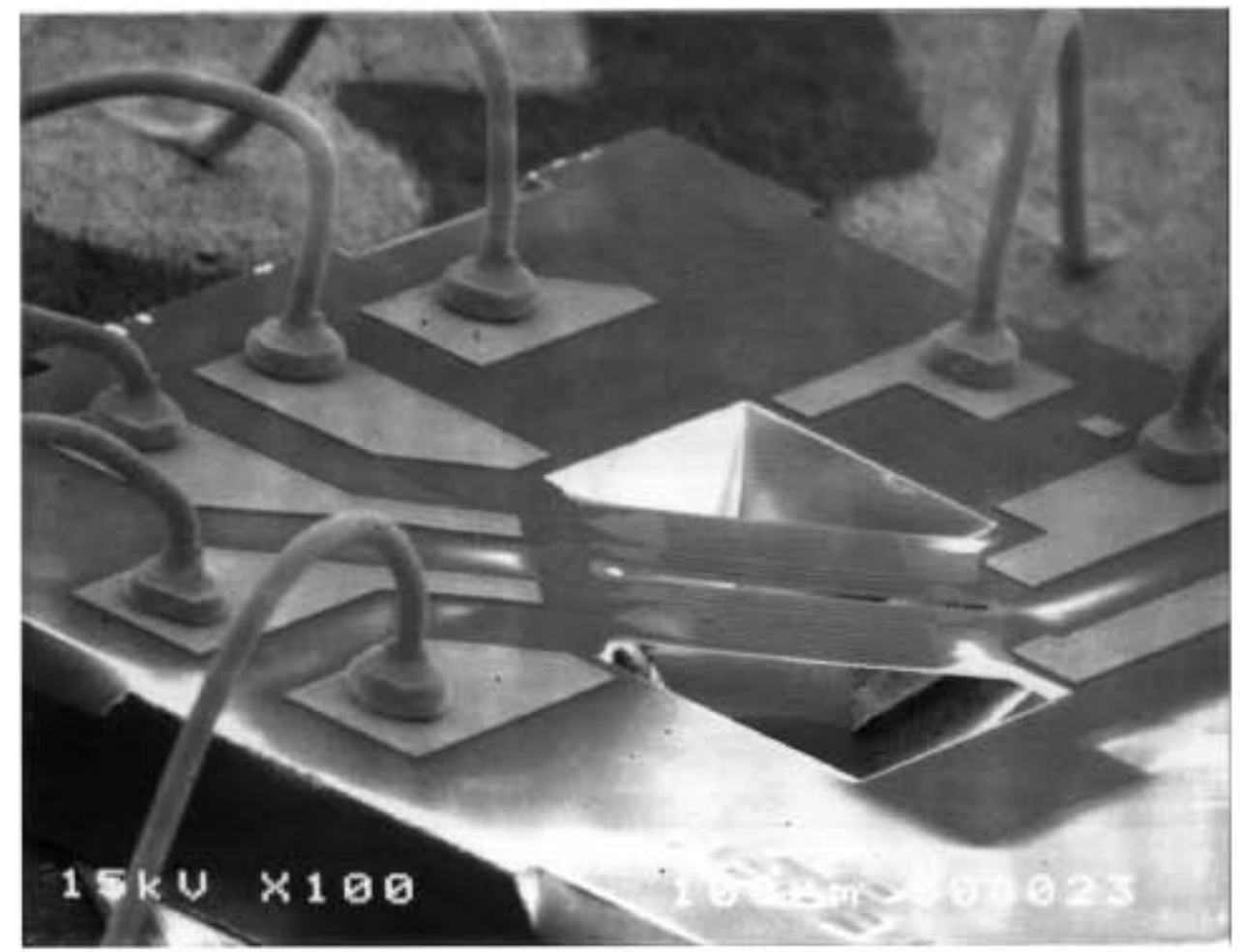

FIGURE 120 - SEM photograph at 100X magnification of AWM2150V die

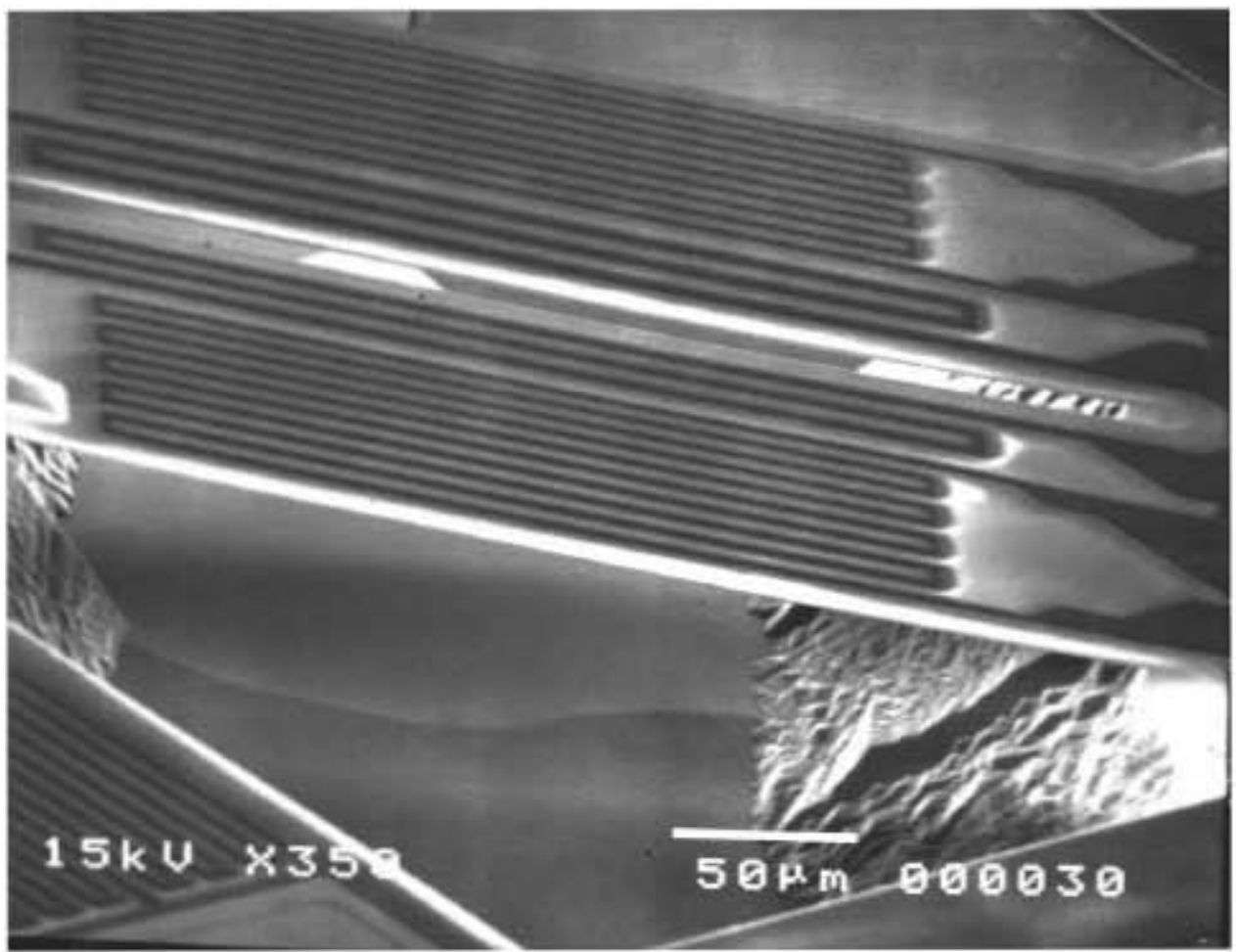

FIGURE 121 - SEM photograph at 350X of microbridges in AWM2150V flow sensor 
APPENDIX III.

CHEMICAL LIST AND COMPOSITIONS 
TABLE XXVIII

LABORATORY CHEMICAL DESCRIPTIONS

\begin{tabular}{|c|c|c|c|}
\hline Name & Mix Ratio & Vendor & Constituent Description \\
\hline Acetone & & $\begin{array}{c}\text { J.T. Baker } \\
\text { Phillipsburg, NJ }\end{array}$ & - $\quad\left(\mathrm{CH}_{3}\right)_{2} \mathrm{CO}, 99.5 \%$ assay \\
\hline Acid Clean & $\begin{array}{c}4: 1: 1 \\
\mathrm{H}_{2} \mathrm{O}: \mathrm{HCl}: \mathrm{H}_{2} \mathrm{O}_{2}\end{array}$ & Mixed & $\begin{array}{ll}- & \mathrm{DI} \mathrm{H}_{2} \mathrm{O}, 18 \mathrm{M} \Omega \\
\text { - } & \text { Hydrochloric Acid (HCl), } 37 \% \\
& \text { assay, CMOS Electronic Grade } \\
& \text { (J.T. Baker) } \\
\text { - } & \text { Hydrogen Peroxide }\left(\mathrm{H}_{2} \mathrm{O}_{2}\right) 30 \% \\
& \text { assay CMOS Electronic Grade } \\
& \text { (J.T. Baker) }\end{array}$ \\
\hline $\begin{array}{l}\text { Aluminum } \\
\text { Etch }\end{array}$ & & J.T. Baker & 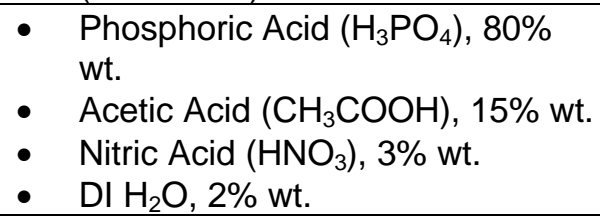 \\
\hline Base Clean & $\begin{array}{l}5: 1: 1 \\
\mathrm{H}_{2} \mathrm{O}: \mathrm{NH}_{4} \mathrm{OH}: \mathrm{H}_{2} \mathrm{O}_{2}\end{array}$ & Mixed & $\begin{array}{ll}- & \mathrm{DI} \mathrm{H} \mathrm{H}_{2} \mathrm{O}, 18 \mathrm{M} \Omega \\
- & \text { Ammonium Hydroxide }\left(\mathrm{NH}_{4} \mathrm{OH}\right) \\
& 30 \% \text { assay CMOS Electronic } \\
& \text { Grade (J.T. Baker) } \\
\text { - } & \text { Hydrogen Peroxide }\left(\mathrm{H}_{2} \mathrm{O}_{2}\right) 30 \% \\
& \text { assay, CMOS Electronic Grade } \\
& \text { (J.T. Baker) }\end{array}$ \\
\hline $\begin{array}{c}\text { BOE, } \\
\text { Buffered } \\
\text { Oxide Etch }\end{array}$ & $\begin{array}{c}\text { 6:1:1 } \\
\mathrm{H}_{2} \mathrm{O}: \mathrm{HF}: \mathrm{NH}_{4} \mathrm{~F}\end{array}$ & $\begin{array}{c}\text { J.T. Baker } \\
\text { Phillipsburg, NJ }\end{array}$ & $\begin{array}{ll}- & \text { DI water, } 18 \mathrm{M} \Omega \\
\text { - } & \text { Hydrofluoric Acid (HF), 49\% Low } \\
& \text { Sodium CMOS Electronic Grade } \\
\text { - } & \text { Ammonium Fluoride }\left(\mathrm{NH}_{4} \mathrm{~F}\right) 30 \% \\
& \text { Low Sodium CMOS Electronic } \\
& \text { Grade }\end{array}$ \\
\hline $\begin{array}{l}\text { Chloro- } \\
\text { benzene }\end{array}$ & & $\begin{array}{c}\text { Fisher } \\
\text { Scientific } \\
\text { Fair Lawn, NJ }\end{array}$ & - $\quad \mathrm{C}_{6} \mathrm{H}_{5} \mathrm{Cl}, 99 \%$ assay \\
\hline $\begin{array}{l}\text { Chrome } \\
\text { Etch }\end{array}$ & & $\begin{array}{c}\text { Micro-Chrome } \\
\text { Technology, Inc. } \\
\text { San Jose, CA } \\
(408) 259-4948\end{array}$ & - $\quad$ CEP-200 Micro-Chrome Etchant \\
\hline $\begin{array}{l}\text { Gold Etch } \\
\text { (KI) }\end{array}$ & & Mixed & $\begin{array}{ll}\text { - } & 4 \mathrm{~g} . \mathrm{KI} \\
\text { - } & 1 \mathrm{g.} \mathrm{I} \\
\text { - } & 40 \mathrm{~mL} \mathrm{DI} \mathrm{H} \mathrm{O}, 18 \mathrm{M} \Omega\end{array}$ \\
\hline $\mathrm{HF}$ & & $\begin{array}{l}\text { J.T. Baker } \\
\text { Phillipsburg, NJ }\end{array}$ & $\begin{array}{l}\text { - Hydrofluoric Acid (HF), 49\% } \\
\text { assay Low Sodium CMOS } \\
\text { Electronic Grade }\end{array}$ \\
\hline HF Dip & $\begin{array}{c}40: 1 \\
\mathrm{H}_{2} \mathrm{O}: \mathrm{HF} \text { solution }\end{array}$ & $\begin{array}{c}\text { J.T. Baker } \\
\text { Phillipsburg, NJ }\end{array}$ & $\begin{array}{ll}- & \mathrm{DI} \mathrm{H}_{2} \mathrm{O} \\
\text { - } & \text { Hydrofluoric Acid (HF), 49\% Low } \\
& \text { Sodium CMOS Electronic Grade }\end{array}$ \\
\hline $\begin{array}{l}\text { HMDS } \\
\text { Primer }\end{array}$ & $\begin{array}{c}\text { MS7300 } \\
\text { UPG/HMDS Ultra } \\
\text { Pure Grade }\end{array}$ & $\begin{array}{l}\text { MicroSi, Inc. } \\
\text { Phoenix, AZ }\end{array}$ & - Hexamethyldisilazane, $100 \%$ \\
\hline
\end{tabular}




\begin{tabular}{|c|c|c|c|}
\hline $\begin{array}{l}\text { Isopropyl } \\
\text { Alcohol } \\
\text { (2 Propanol) }\end{array}$ & & $\begin{array}{c}\text { J.T. Baker } \\
\text { Phillipsburg, NJ }\end{array}$ & - $\mathrm{CH}_{3} \mathrm{CHOHCH}_{3}, 99.5 \%$ assay \\
\hline $\mathrm{KOH}$ & & $\begin{array}{c}\text { J.T. Baker } \\
\text { Phillipsburg, NJ }\end{array}$ & $\begin{array}{l}\text { - Potassium Hydroxide }(\mathrm{KOH}) \text {, } \\
45.4 \% \text { wt. solution }\end{array}$ \\
\hline Methanol & & $\begin{array}{c}\text { J.T. Baker } \\
\text { Phillipsburg, NJ }\end{array}$ & - $\mathrm{CH}_{3} \mathrm{OH}, 99.9 \%$ assay \\
\hline $\begin{array}{l}\text { MF®-319 } \\
\text { Microposit@ } \\
\text { Developer }\end{array}$ & & $\begin{array}{c}\text { Shipley } \\
\text { Marlborough, MA }\end{array}$ & $\begin{array}{ll}\text { - } & \text { TMAH, } 2 \% \text { wt. } \\
\text { - } & \text { DI } \mathrm{H}_{2} \mathrm{O},<98 \% \text { wt. }\end{array}$ \\
\hline $\begin{array}{l}\text { HMDS } \\
\text { Primer } \\
\end{array}$ & & $\begin{array}{c}\text { Shipley } \\
\text { Marlborough, MA }\end{array}$ & $\bullet$ \\
\hline $\begin{array}{l}\text { Micropositß } \\
\text { S1813 } \\
\text { Photoresist }\end{array}$ & & $\begin{array}{c}\text { Shipley } \\
\text { Marlborough, MA }\end{array}$ & $\begin{array}{ll}- & \text { Propylene glycol monomethyl } \\
\text { ether acetate, } 72 \% \\
\text { - Novolak resins and photoactive } \\
\text { compounds, } 28 \%\end{array}$ \\
\hline $\begin{array}{l}\text { Microposit@ } \\
\text { S1827 } \\
\text { Photoresist }\end{array}$ & & $\begin{array}{c}\text { Shipley } \\
\text { Marlborough, MA }\end{array}$ & $\begin{array}{l}\text { - Propylene glycol monomethyl } \\
\text { ether acetate, } 67 \% \\
\text { - Novolak resins and photoactive } \\
\text { compounds, } 33 \%\end{array}$ \\
\hline $\begin{array}{l}\text { Nano- } \\
\text { Strip® }\end{array}$ & & $\begin{array}{l}\text { Cyantek Corp. } \\
\text { Fremont, CA } \\
\text { (510) 651-3341 }\end{array}$ & $\begin{array}{l}\text { - } \begin{array}{l}\text { Sulfuric Acid }\left(\mathrm{H}_{2} \mathrm{SO}_{4}\right), 90 \% \text { wt. } \\
\text { - }\end{array} \text { Hydrogen Peroxide }\left(\mathrm{H}_{2} \mathrm{O}_{2}\right),<1 \% \\
\text { wt. } \\
\text { - } \\
\text { Peroxymonosulfuric Acid, } 5 \% \text { wt. }\end{array}$ \\
\hline $\begin{array}{l}\text { Platinum } \\
\text { Etch }\end{array}$ & $\begin{array}{c}\text { 8:7:1 } \\
\mathrm{H}_{2} \mathrm{O}: \mathrm{HCl}: \mathrm{HNO}_{3}\end{array}$ & Mixed & $\begin{array}{ll}\cdot & \mathrm{DI} \mathrm{H}_{2} \mathrm{O}, 18 \mathrm{M} \Omega \\
- & \text { Hydrochloric Acid (HCl), } 37 \% \\
& \text { CMOS Electronic Grade (J.T. } \\
& \text { Baker) } \\
\text { Nitric Acid }\left(\mathrm{HNO}_{3}\right), 70 \% \text { assay, } \\
\text { (J.T. Baker) }\end{array}$ \\
\hline $\begin{array}{l}\text { Titanium } \\
\text { Etch }\end{array}$ & $\begin{array}{c}20: 1: 1 \\
\mathrm{H}_{2} \mathrm{O}: \mathrm{H}_{2} \mathrm{O}_{2}: \mathrm{HF} \\
40: 1: 1 \\
\mathrm{H}_{2} \mathrm{O}: \mathrm{H}_{2} \mathrm{O}_{2}: \mathrm{HF} \\
80: 1: 1 \\
\mathrm{H}_{2} \mathrm{O}: \mathrm{H}_{2} \mathrm{O}_{2}: \mathrm{HF}\end{array}$ & Mixed & $\begin{array}{ll}\text { - } & \mathrm{DI} \mathrm{H}_{2} \mathrm{O}, 18 \mathrm{M} \Omega \text { dilution slows } \\
\text { down etching and improves } \\
\text { uniformity } \\
\text { - } \\
\text { Hydrogen Peroxide }\left(\mathrm{H}_{2} \mathrm{O}_{2}\right) 30 \% \\
\text { assay CMOS Electronic Grade } \\
\text { (J.T. Baker) } \\
\text { Hydrofluoric Acid (HF), 49\% } \\
\text { assay Low Sodium CMOS } \\
\text { Electronic Grade }\end{array}$ \\
\hline TMAH & & $\begin{array}{c}\text { Alfa Aesar } \\
\text { (stock \#20932) } \\
\text { Ward Hill, MA } \\
\text { 1-800-343-0660 }\end{array}$ & $\begin{array}{l}\text { - Tetramethylammonium Hydroxide } \\
\left(\mathrm{CH}_{3}\right)_{4} \mathrm{NOH}, 25 \% \text { wt. aqueous } \\
\text { solution, electronic grade } \\
\text { - } \quad \mathrm{DI} \mathrm{H}_{2} \mathrm{O}, 75 \% \text { wt. } \\
\end{array}$ \\
\hline $\begin{array}{l}\text { TMAH } \\
\text { Silicate }\end{array}$ & & $\begin{array}{c}\text { Alfa Aesar } \\
\text { (stock \#41719) } \\
\text { Ward Hill, MA } \\
\text { 1-800-343-0660 } \\
\end{array}$ & $\begin{array}{l}\text { Tetramethylammonium silicate, } \\
\left(\mathrm{CH}_{3}\right)_{4} \mathrm{NOH} \cdot 2 \mathrm{SiO}_{2}, 16 \% \text { aqueous } \\
\text { solution }\end{array}$ \\
\hline
\end{tabular}




\section{APPENDIX IV.}

LABORATORY APPARATUS

EQUIPMENT PHOTOGRAPHS 


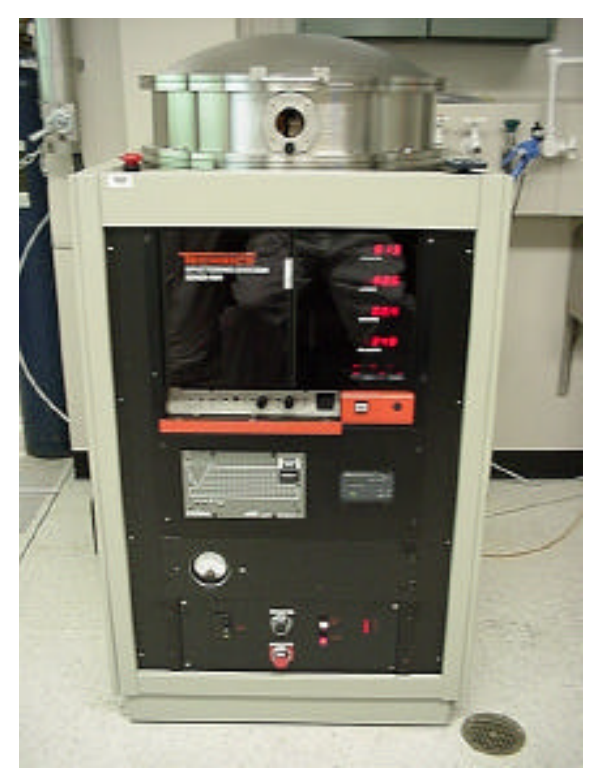

FIGURE 122 - Technics Model 4604 Manual Control Sputtering System (Technics, Inc.; 7060300 Koll Center Pkwy.; Pleasanton, CA 94566).

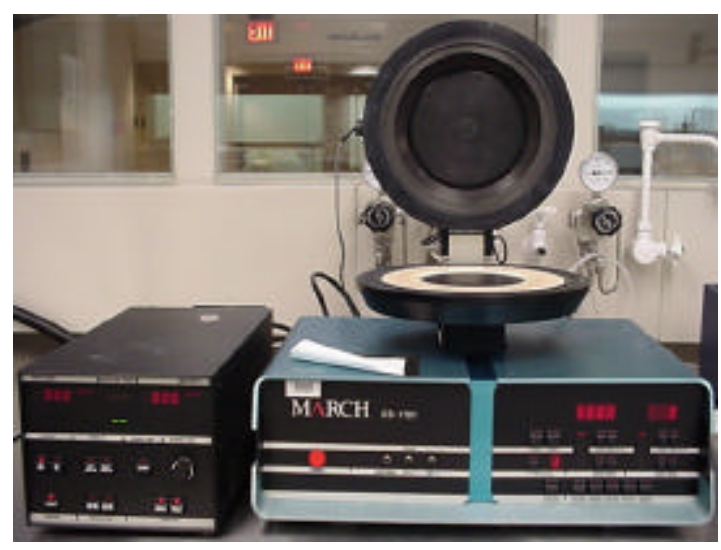

FIGURE 123 - March CS-1701 Reactive Ion Etcher system (March Instruments, Inc.; 125 Mason Circle, Suite J; Concord, CA 94520).

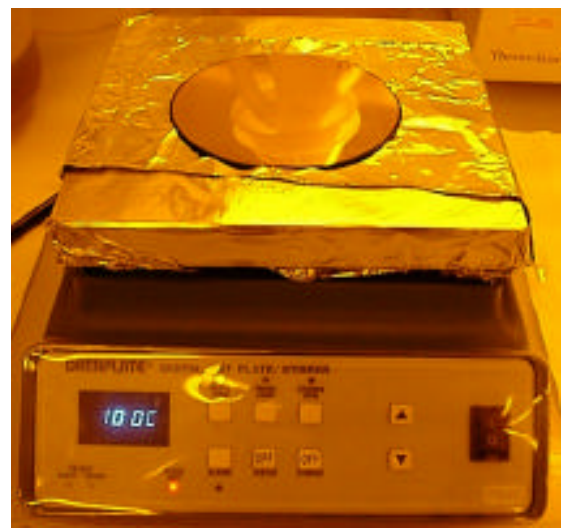

FIGURE 124 - Dataplate® Series 720 programmable digital hotplate with stirrer (PMC Industries, Inc.; 8880 Rehco Rd.; San Diego, CA 92121). 


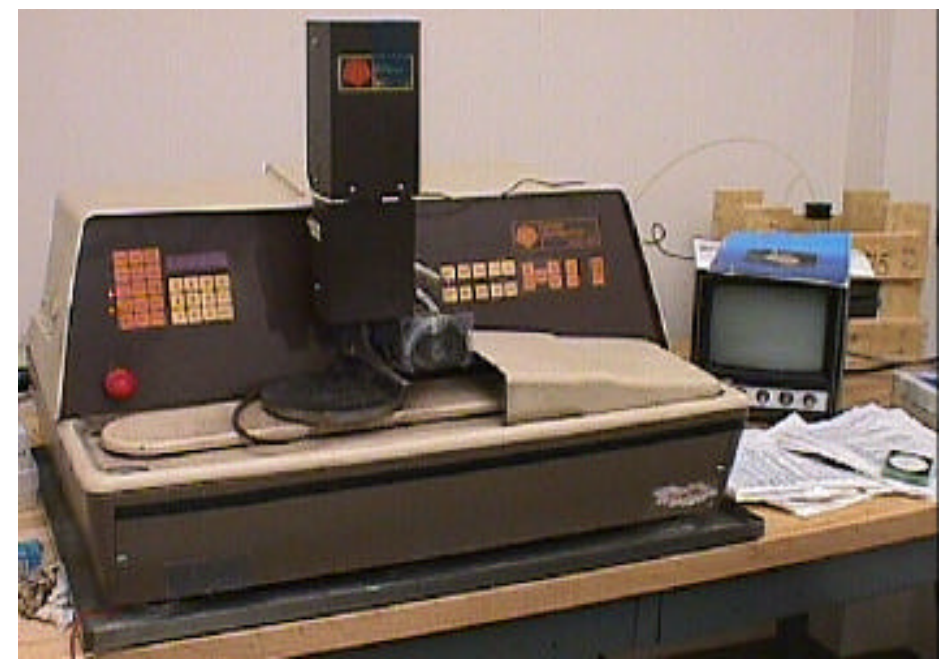

FIGURE 125 - Micro Automation Model 1006 programmable dicing saw.

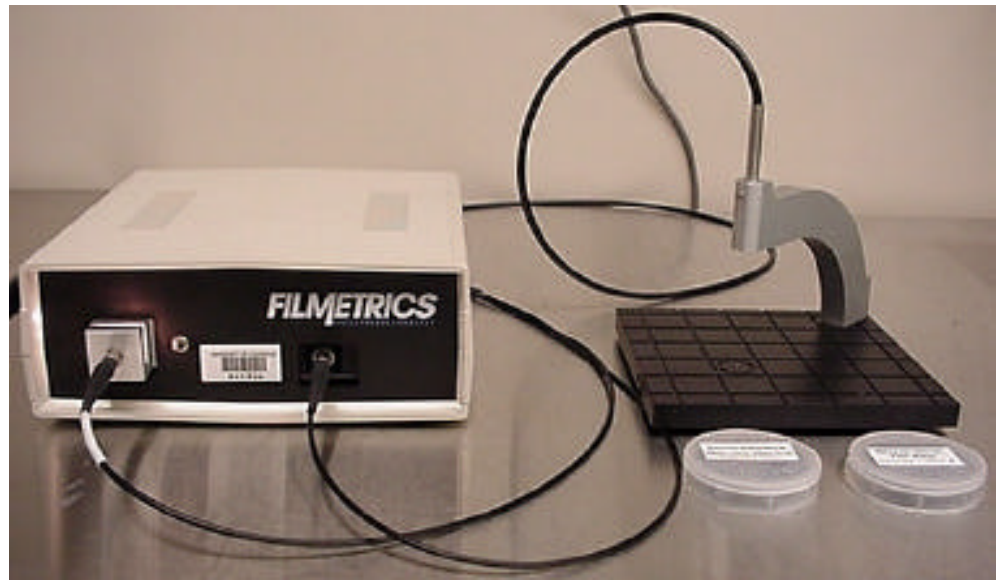

FIGURE 126 - Filmetrics F20 Thin-Film Measurement System (Filmetrics, Inc.; 10655 Roselle St., Suite G; San Diego, CA 92121).

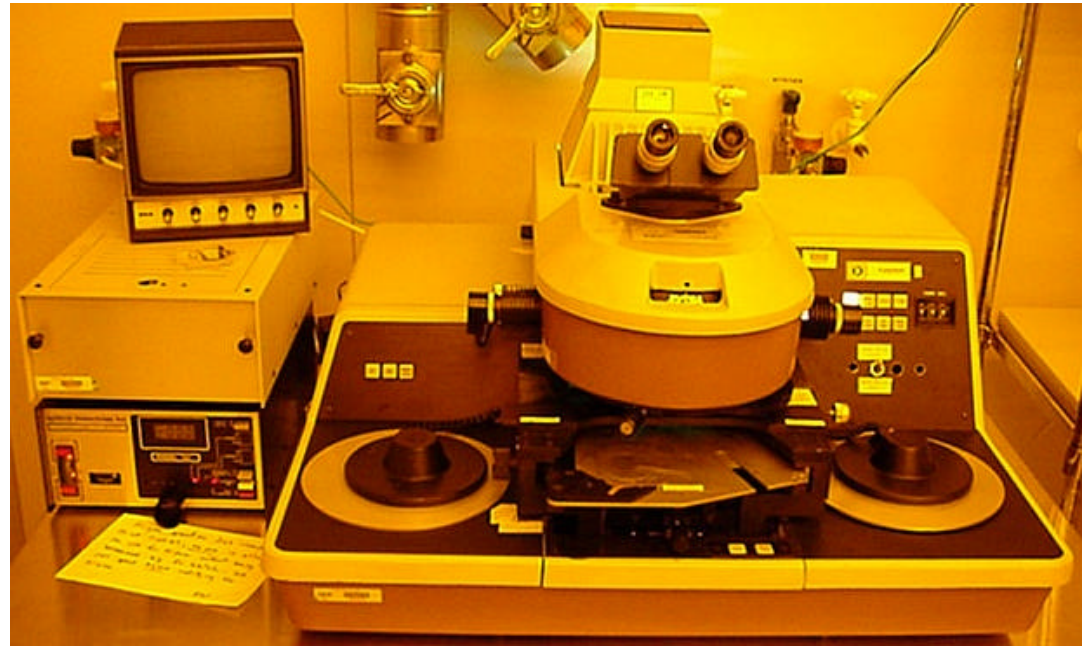

FIGURE 127 - Kasper 2001 contact mask aligner Wafer Alignment System. 


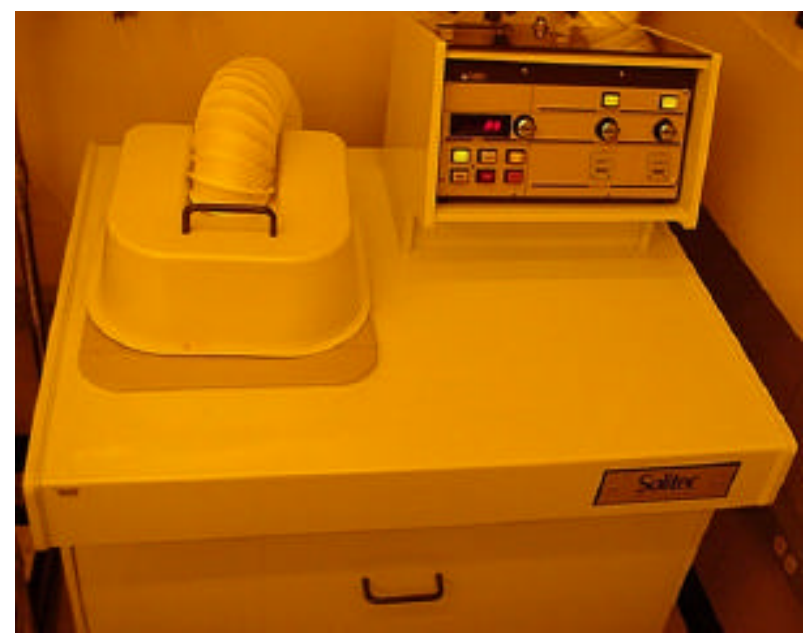

FIGURE 128 - Solitec Model 5100 Manual Single-Head Wafer/Substrate Spinner (Solitec Wafer Processing, Inc.; 685 River Oaks Pkwy.; San Jose, CA 95134).

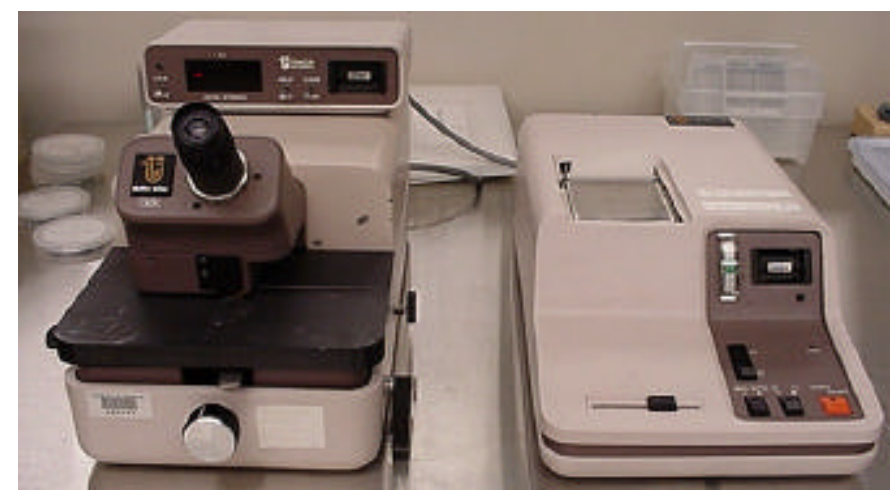

FIGURE 129 - Tencor Alpha-Step 100 Profilometer (Tencor Instruments; 2400 Charleston Road; Mountain View, CA 94043).

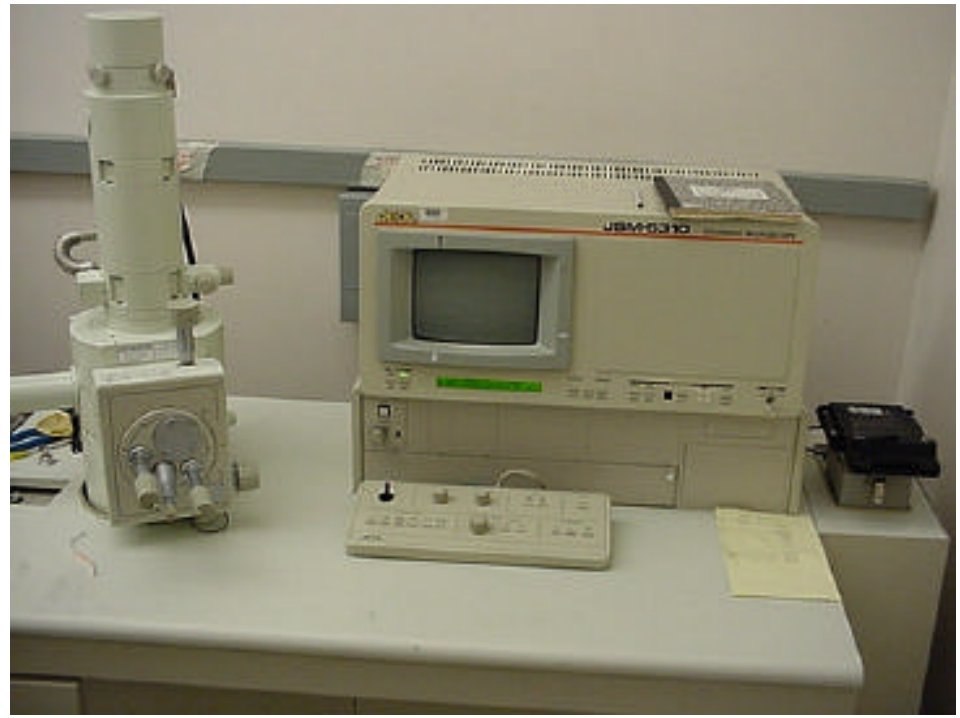

FIGURE 130 - Jeol JSM-5310 Scanning Electron Microscope (Jeol USA, Inc.; 1015 East Meadow Circle; Palo Alto, CA 94303). 


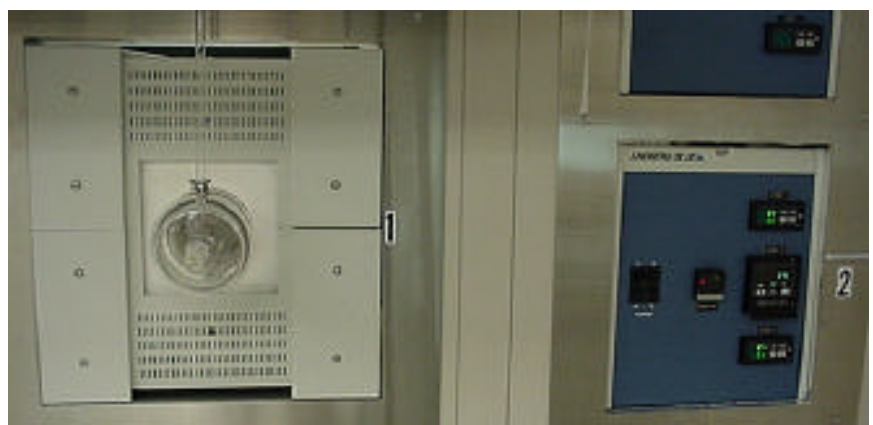

FIGURE 131 - Lindberg Model 55122 Moldatherm Hinged Tube Furnace with Three-Zone 1200 ${ }^{\circ} \mathrm{C}$ Blue M Controller (Lindberg, A Unit of General Signal; 304 Hart Street; Watertown, WI 53094).

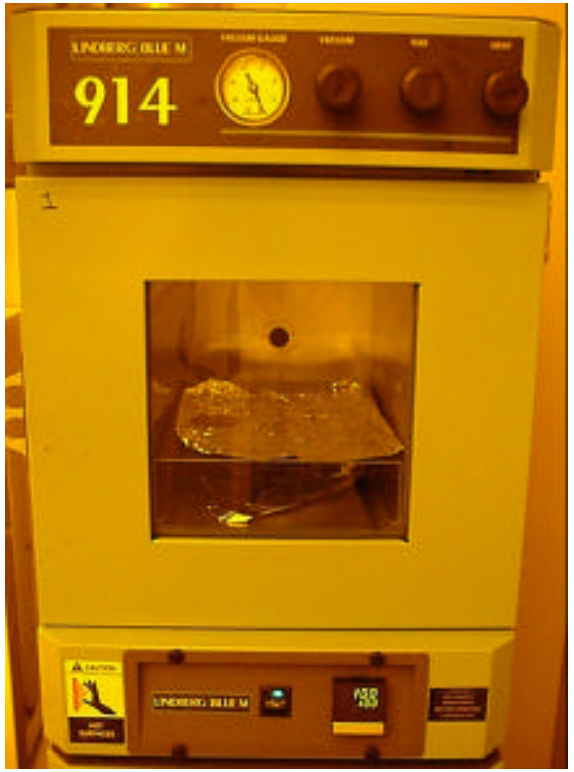

FIGURE 132 - Lindberg/Blue M Model 914 and 914A Laboratory Vacuum Ovens (Blue M Electric; 2218 W. $138^{\text {th }}$ St.; Blue Island, IL 60406).

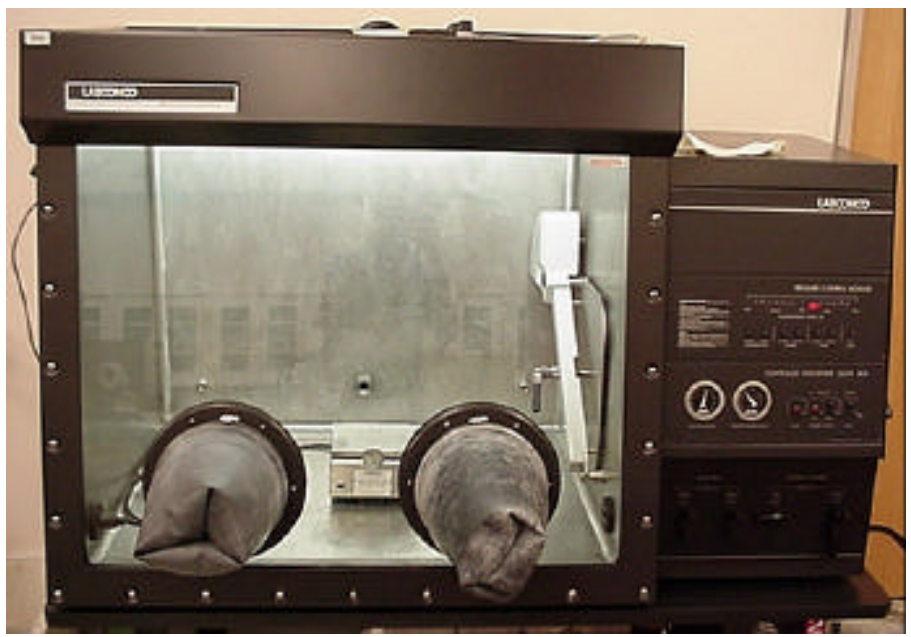

FIGURE 133 - Labconco Protector Glove Box environmental chamber. 


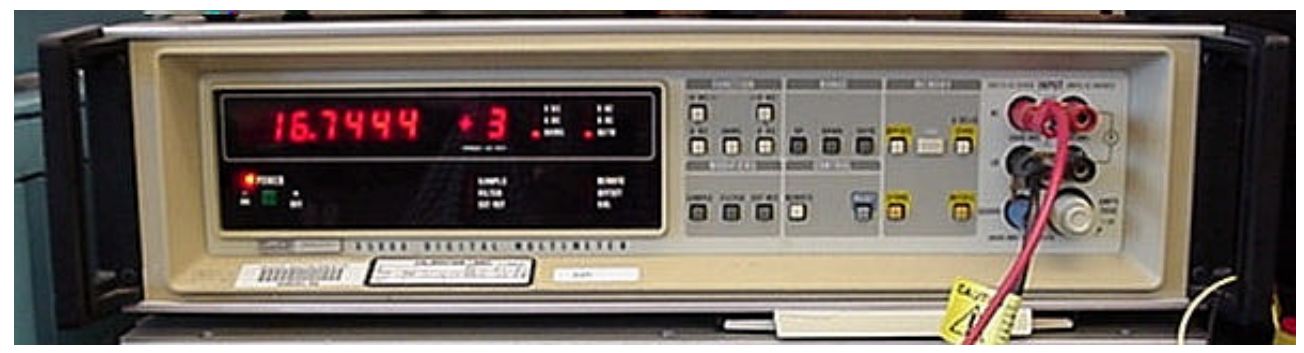

FIGURE 134 - Fluke Model 8500A digital multimeter.

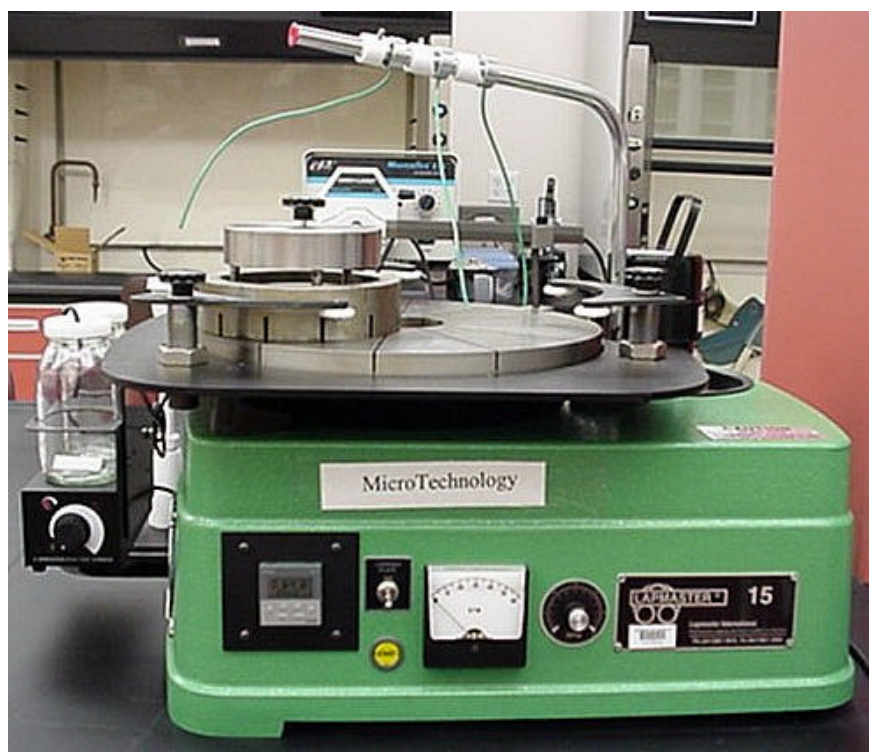

FIGURE 135 - Lapmaster Model 15 Lapping/Polishing Machine (Lapmaster International; 6400 W. Oakton St.; Morton Grove, IL 60053).

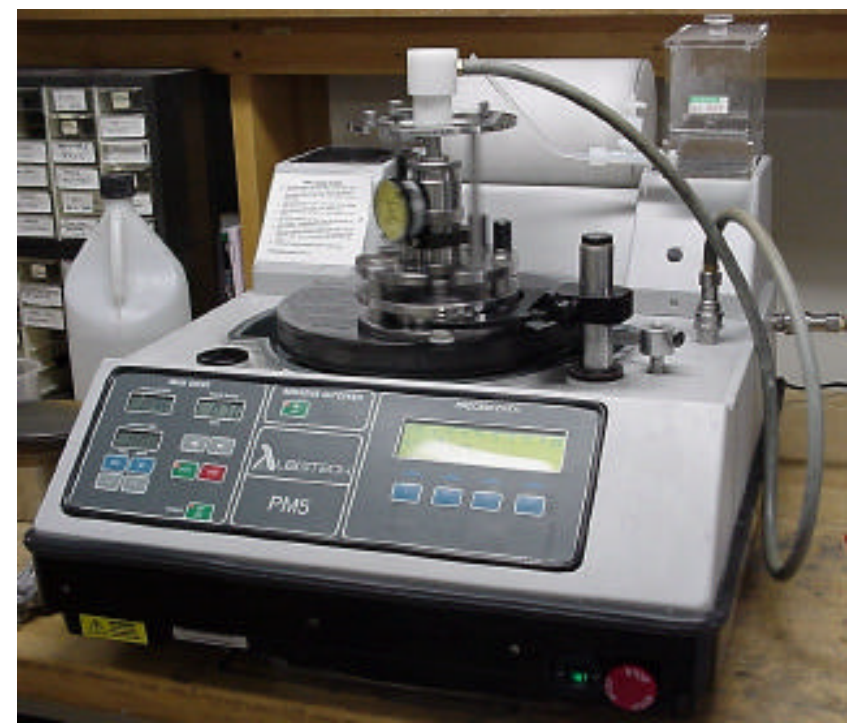

FIGURE 136 - Logitech Model PM5 Lapping and Polishing Machine. 


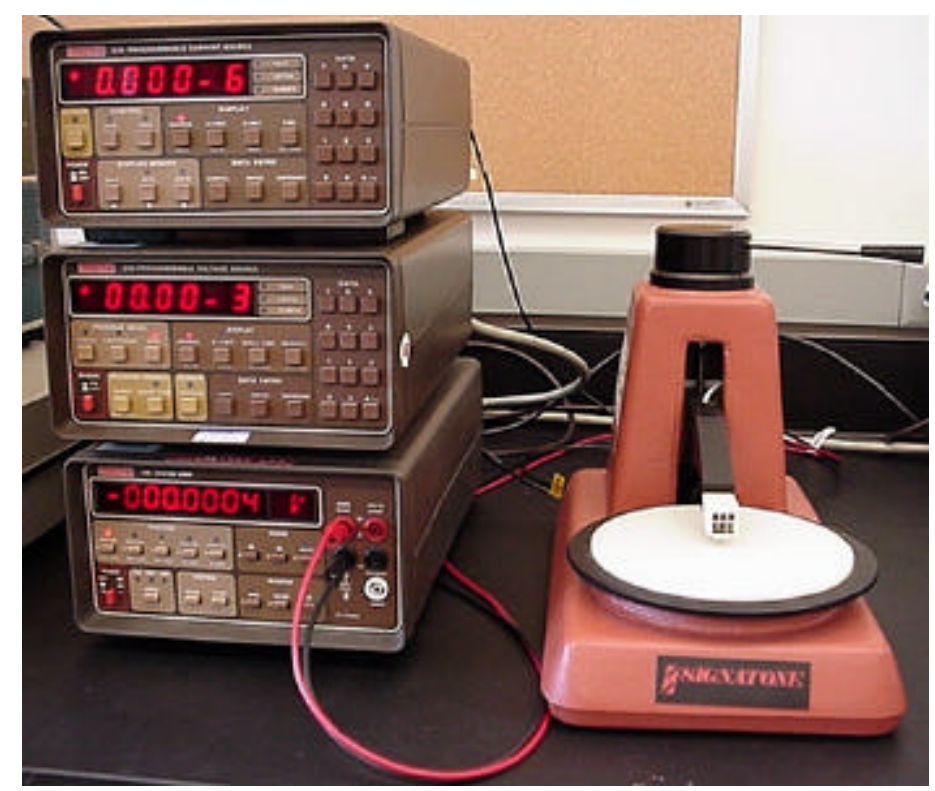

FIGURE 137 - Lucas/Signatone SYS-301 Four-point Resistivity Probe Station with Keithley 224 Programmable Current Source, Keithley 230 Programmable Voltage Source, and Keithley 196 System Digital Multimeter (Lucas/Signatone; 393-J Tomkins Ct.; Gilroy, CA 95020).

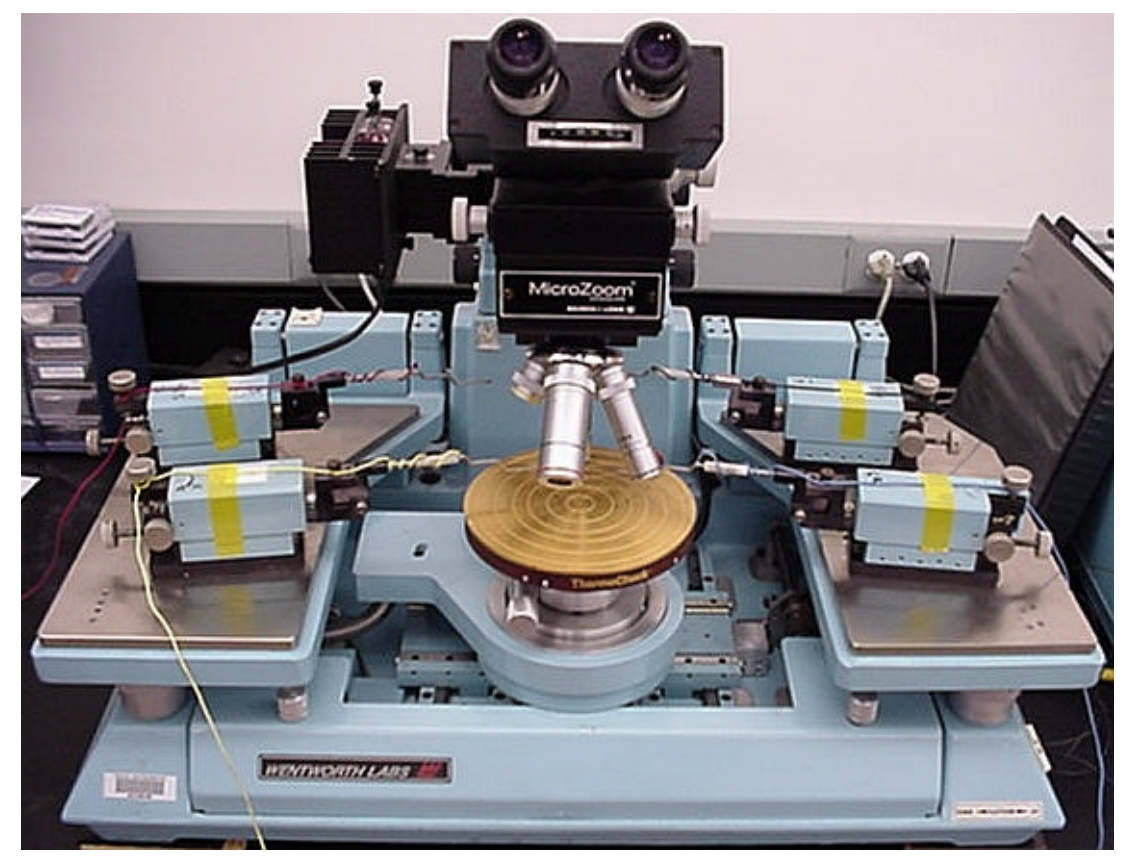

FIGURE 138 - Wentworth Labs probe station with MP-1100 Series Controller (Wentworth Labs; 2659 Guadalupe Rd., Suite D205; Mesa, AZ 85202). 


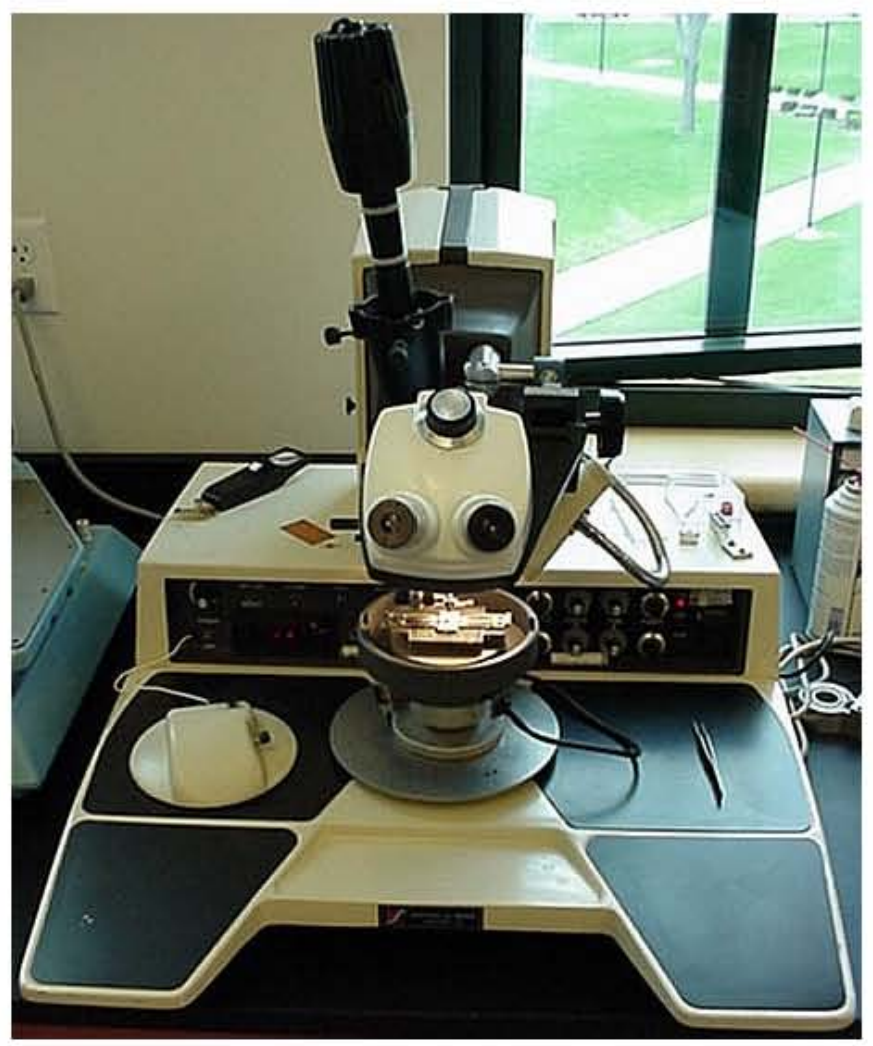

FIGURE 139 - Kulicke and Soffa Industries, Inc. Ultrasonic Wedge Bonder Model 4123.

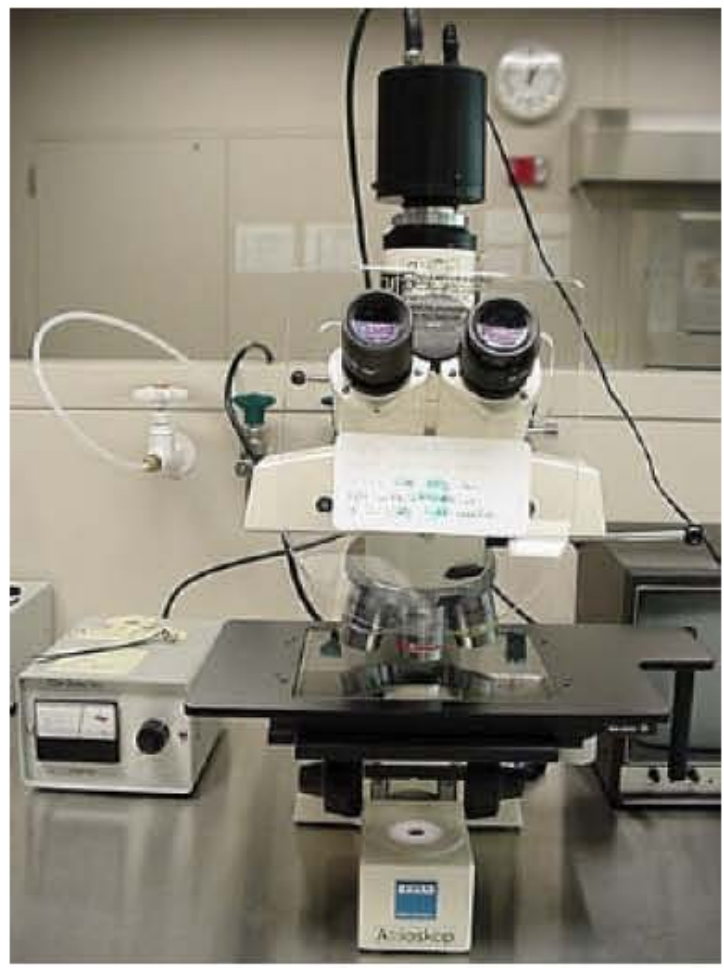

FIGURE 140 - Zeiss Axioskop inspection microscope (Carl Zeiss, Inc.; Microscope Division; One Zeiss Drive; Thronwood, NY 10594). 


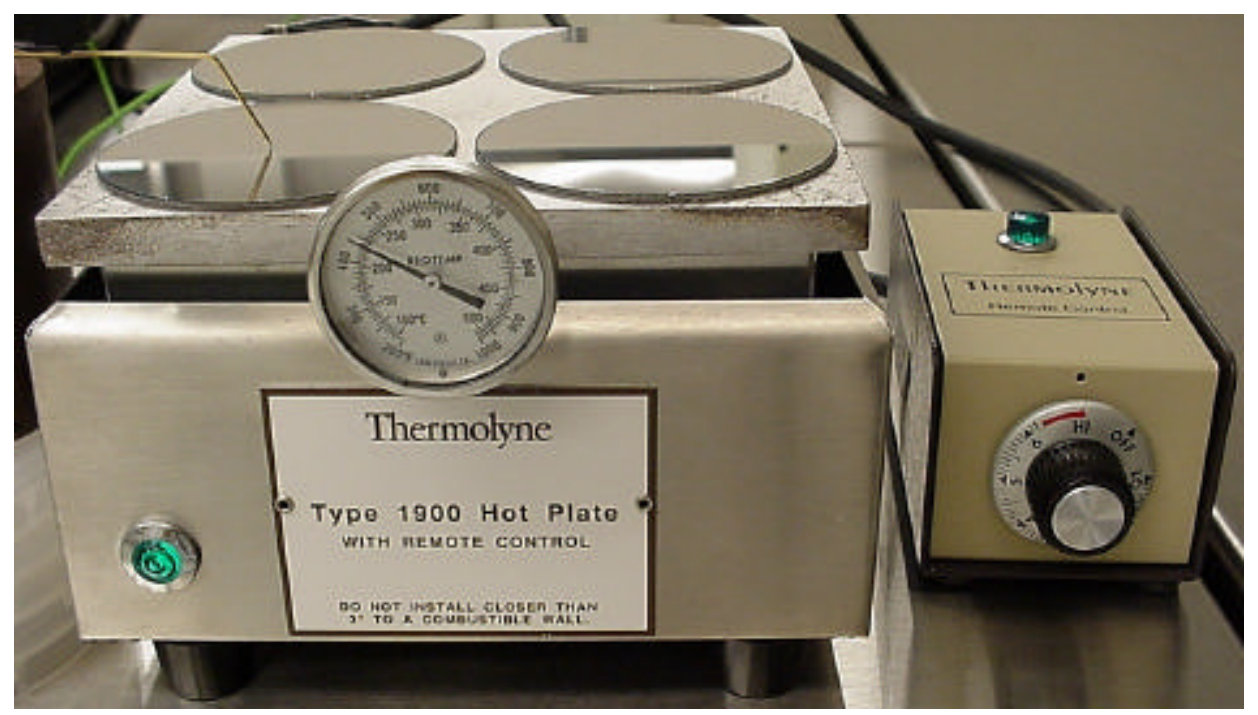

FIGURE 141 - The Thermolyne Type 1900 high temperature hot plate, with remote control (used for anodic bonding at $450^{\circ} \mathrm{C}$ ).

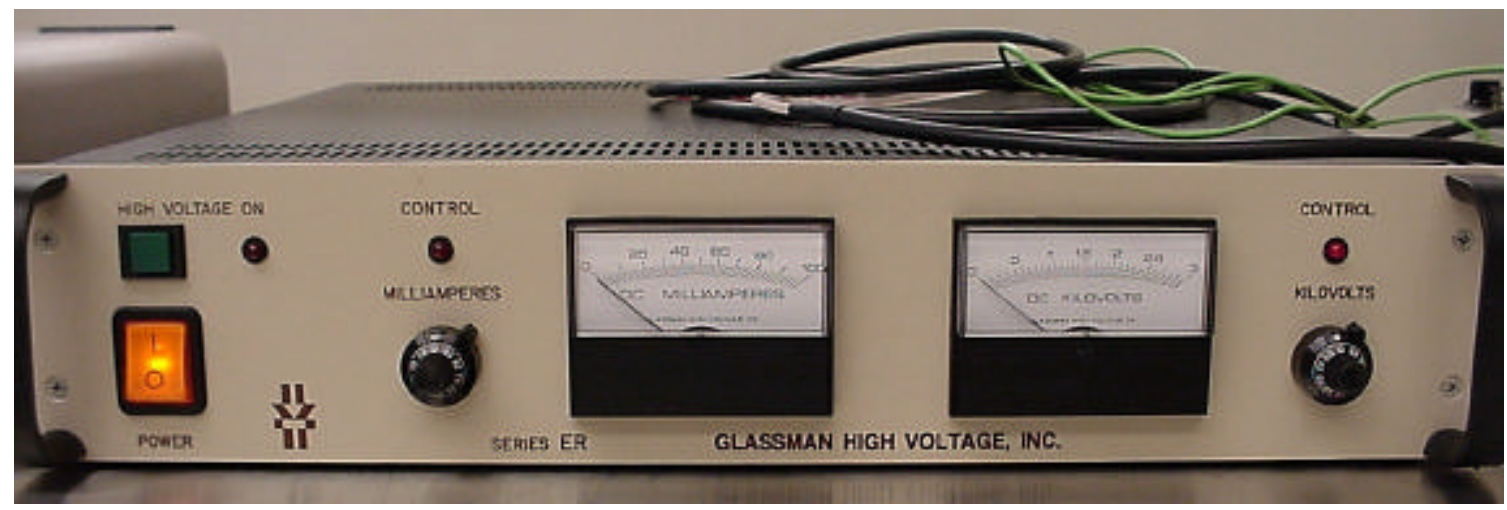

FIGURE 142 - The Glassman High Voltage, Inc., Model ER3R100 reversible polarity 0-3 kV, 0-100 mA panel-mount power supply used for anodic bonding (Glassman High Voltage, Inc.; Salem Industrial Park; P.O. Box 551; Whitehouse Station, NJ 08889; 908-534-9007).

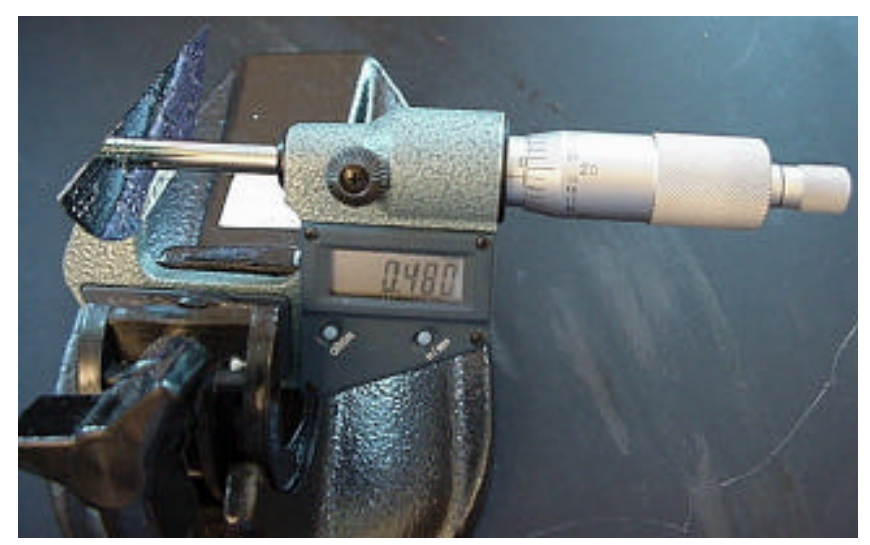

FIGURE 143 - One of two Mitutoyo precision digital micrometers used to measure substrate or glass thicknesses (Model number 293-761-30, shown). 
APPENDIX V.

SUMMARY OF FLOW SENSOR FABRICATION METHODOLOGIES ATTEMPTED 
TABLE XXIX

COMPREHENSIVE FABRICATION METHODOLOGIES EXPLORED

\begin{tabular}{|c|c|c|c|}
\hline $\begin{array}{c}\text { Thin-Film } \\
\text { Microbridge } \\
\text { Material }\end{array}$ & $\begin{array}{c}\text { Pre-Etch }(100) \mathrm{Si} \\
\text { Substrate with KOH }\end{array}$ & $\begin{array}{l}\text { Post-Etch (100) Si } \\
\text { Substrate with KOH }\end{array}$ & $\begin{array}{l}\text { Post-Etch (100) Si } \\
\text { Wafer with TMAH }\end{array}$ \\
\hline $\begin{array}{c}\text { RF Planar } \\
\text { Magnetron Sputter- } \\
\text { Deposited Silicon } \\
\text { Nitride }\left(\mathrm{Si}_{\times} \mathrm{N}_{\mathrm{y}}\right)\end{array}$ & N/A; Not Practical & $\begin{array}{l}\text { Resulted in a porous } \\
\text { thin-film with significant } \\
\text { residual compressive } \\
\text { stress (buckled/warped } \\
\text { bridges) }\end{array}$ & $\begin{array}{c}\text { Possible, but not } \\
\text { evaluated. }\end{array}$ \\
\hline $\begin{array}{c}\text { Thermally-grown } \\
\text { Oxide, } \mathrm{SiO}_{2} \text {, Glass } \\
\text { for Bottom Layer, \& } \\
\text { Sputter-Deposited } \\
\text { Si }_{x} \mathrm{~N}_{\mathrm{y}} \text { for Top } \\
\text { Passivation Layer }\end{array}$ & N/A; Not Practical & $\begin{array}{l}\text { Evaluated in } 1997 \text { with } \\
\text { poor results as } \mathrm{KOH} \\
\text { degraded chromium } \\
\text { metallization, and the } \\
\text { thin-film was under } \\
\text { compression. Thermally } \\
\text { grown } \mathrm{SiO}_{2} \text { will typically } \\
\text { have residual } \\
\text { compressive stress. } \\
\text { Might be successful } \\
\text { with ex-situ annealing } \\
\text { and top } \mathrm{Cr}+\text { Au RIE/ } \\
\mathrm{KOH} \text { masking layer. }\end{array}$ & Not Evaluated. \\
\hline $\begin{array}{l}\text { Low-Stress LPCVD } \\
\text { Silicon Nitride }\end{array}$ & N/A; Not Practical & $\begin{array}{l}\text { Successful! Good } \\
\text { quality structures under } \\
\text { tension with } \mathrm{Cr}+\mathrm{Au} \\
\mathrm{KOH} \text { masking layer. No } \\
\mathrm{KOH} \text { masking layer } \\
\text { needed with } \mathrm{Pt}(+\mathrm{Au}) \\
\text { metallization. }\end{array}$ & Not Evaluated. \\
\hline $\begin{array}{c}\text { Anodically-Bonded } \\
\sim 1.1 \mathrm{~mm} \text { Thick } \\
\text { Borosilicate Glass }\end{array}$ & $\begin{array}{l}\text { Glass was wet- } \\
\text { etched and machined } \\
\text { to } \sim 12-30 \mu m \text { thick. } \\
\text { Glass was too thick } \\
\text { with too much RIE } \\
\text { isotropy to etch } \\
\text { through without } \\
\text { damaging bridge } \\
\text { resistors. Some } \\
\text { cavities under pad } \\
\text { areas ruptured during } \\
\text { wirebonding } \\
\text { attempts. } \\
\text { Diaphragm devices } \\
\text { (without an RIE step) } \\
\text { might function well as } \\
\text { flow sensors. }\end{array}$ & $\begin{array}{l}\text { Possible, but not } \\
\text { evaluated as this would } \\
\text { be more practical with } \\
\text { thermally-grown oxide } \\
\left(\mathrm{SiO}_{2}\right) \text { or spin-on-glass } \\
\text { thin film microbridges/ } \\
\text { cantilevers. }\end{array}$ & $\begin{array}{l}\text { Possible, but not } \\
\text { evaluated as this } \\
\text { would be more } \\
\text { practical with } \\
\text { thermally-grown } \\
\text { oxide }\left(\mathrm{SiO}_{2}\right) \text { or spin- } \\
\text { on-glass thin film } \\
\text { microbridges/ } \\
\text { cantilevers. }\end{array}$ \\
\hline
\end{tabular}

Note that if an etch-window photomask had been created and used that allowed for patterning etch window areas at the microbridges only (and not also at pad areas) these pre-etched $\mathrm{KOH}$ silicon wafers might have worked with sacrificial layer resist and spin on glass or anodically-bonded-and-machined glass. It is assumed that cavities under the pads generally cause wire-bonding at pad regions to fail during subsequent processing. 


\begin{tabular}{|c|c|c|c|}
\hline $\begin{array}{l}\text { Thin-Film } \\
\text { Microbridge } \\
\text { Material }\end{array}$ & $\begin{array}{l}\text { Pre-Etch (100) Si } \\
\text { Substrate with KOH }\end{array}$ & $\begin{array}{l}\text { Post-Etch (100) Si } \\
\text { Substrate with KOH }\end{array}$ & $\begin{array}{l}\text { Post-Etch (100) Si } \\
\text { Wafer with TMAH }\end{array}$ \\
\hline $\begin{array}{c}\text { Thermally-grown } \\
\text { Oxide, } \mathrm{SiO}_{2} \text {, for } \\
\text { Bottom Layer with } \\
\text { Spin On Glass as } \\
\text { Top Layer }\end{array}$ & N/A; Not Practical & $\begin{array}{l}\text { Possible, but not } \\
\text { evaluated, as } \mathrm{KOH} \\
\text { attacks glass and any } \\
\text { aluminum masking } \\
\text { layers. Thin-film } \\
\text { microbridges/ } \\
\text { cantilevers comprised } \\
\text { of thermally-grown } \\
\text { oxide will typically have } \\
\text { residual compressive } \\
\text { stress causing buckling, } \\
\text { which may be relieved } \\
\text { by ex-situ annealing. }\end{array}$ & $\begin{array}{l}\text { Might be successful } \\
\text { with Au TMAH } \\
\text { masking layer (or by } \\
\text { using an Al masking } \\
\text { layer and TMAH } \\
\text { silicate), but with } \\
\text { some bridge } \\
\text { buckling due to } \\
\text { intrinsic } \\
\text { compressive stress } \\
\text { in thermally-grown } \\
\mathrm{SiO}_{2} \text {. }\end{array}$ \\
\hline $\begin{array}{c}\text { Cured Spin-On } \\
\text { Glass (SOG) for } \\
\text { Bottom and Top } \\
\text { Layers }\end{array}$ & $\begin{array}{l}\text { Sacrificial layer } \\
\text { wafers prepared with } \\
\text { positive photoresist } \\
\text { or Shipley Surface } \\
\text { Coating in cavities } \\
\text { and machined } \\
\text { smooth, but deemed } \\
\text { ineffective due to } \\
\text { explosive pressure } \\
\text { differential developed } \\
\text { across thin-film } \\
\text { diaphragms during } \\
\text { metallization sputter } \\
\text { deposition. } \\
\text { Sputtering might be } \\
\text { feasible if Si } \\
\text { substrates are } \\
\text { sufficiently thin to } \\
\text { have cavities etched } \\
\text { completely through } \\
\text { the wafer (for trapped } \\
\text { gas evacuation from } \\
\text { the sacrificial layer } \\
\text { through the back-side } \\
\text { openings in cavities). }\end{array}$ & $\begin{array}{l}\text { Possible, but deemed } \\
\text { impractical and not } \\
\text { evaluated, as } \mathrm{KOH} \\
\text { attacks } \mathrm{SiO}_{2} \text { (unless at } \\
\text { very low etch } \\
\text { temperatures with } \\
\text { sufficiently thick } \mathrm{SiO}_{2} \text { ) } \\
\text { and any aluminum } \\
\text { masking layers. }\end{array}$ & $\begin{array}{l}\text { Attempted, but failed } \\
\text { due to difficulties in } \\
\text { properly curing SOG } \\
\text { without lateral } \\
\text { curling of brittle } \\
\text { microbridges/ } \\
\text { cantilevers. Most } \\
\text { structures fractured } \\
\text { and broke off. } \\
\text { Needs further } \\
\text { exploration with } \\
\text { same curing process } \\
\text { for bottom and top- } \\
\text { layer SOG. }\end{array}$ \\
\hline
\end{tabular}


APPENDIX VI.

KOH ETCH RATE DATA GRAPHS 


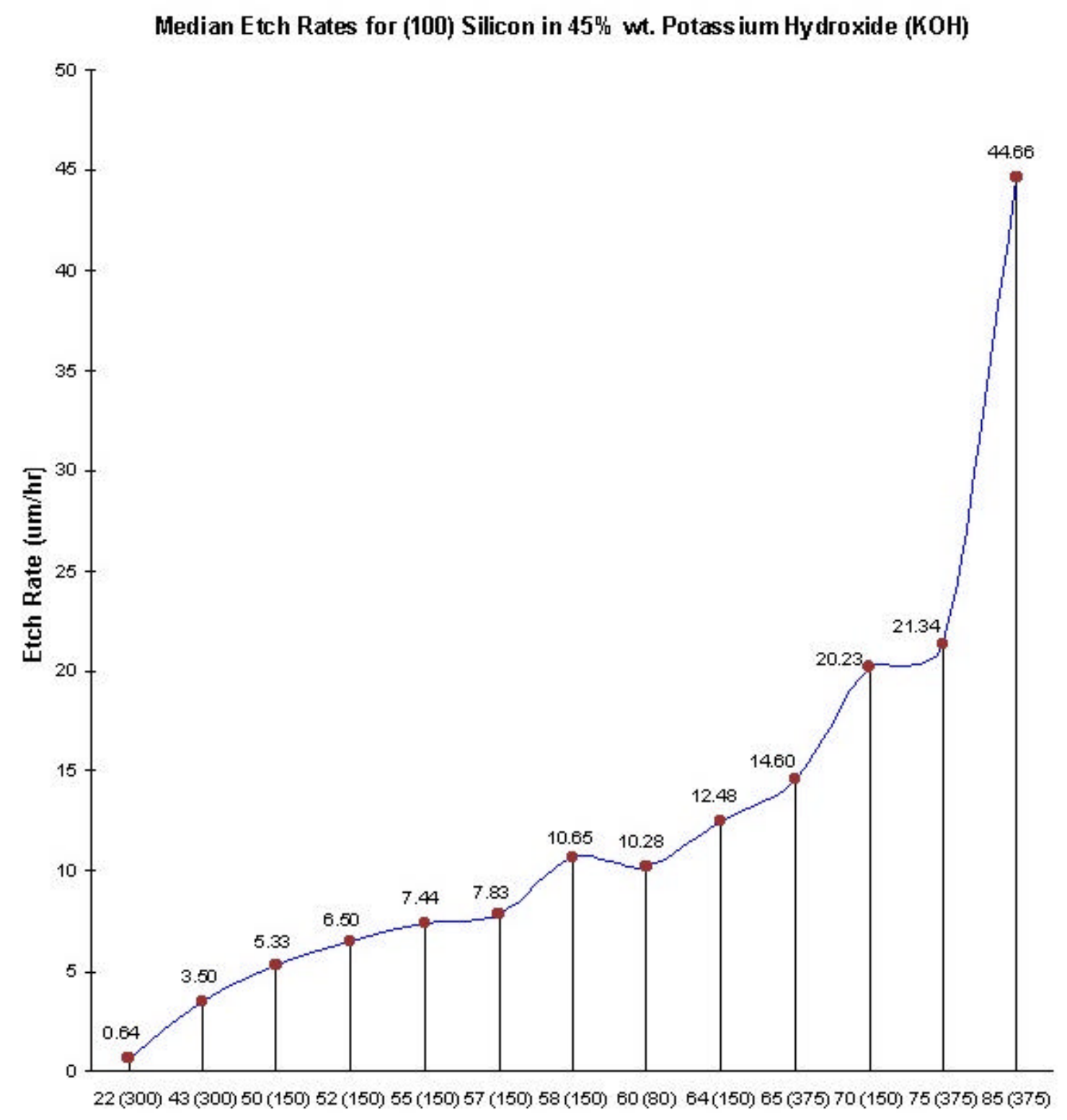

Temperature, deg. C (Stirring RPhi)

FIGURE 144 - Aggregate experimentally-measured median etch rate data for (100)-oriented silicon in $45 \%$ wt. $\mathrm{KOH}$ as a function of temperature (with stir-rate RPM in parentheses) using the Dataplate digital programmable hotplate and reflux condenser. Note that etch rates are affected by the stirring RPM, solution $\mathrm{pH}$, concentration, temperature variations (which are typically $\pm 2{ }^{\circ} \mathrm{C}$ max.), age, and impurities/contaminants. 
Silicon Dioxide Etch Rates in $45 \%$ wt. Potassium Hydroxide ( $\mathrm{KOH})$

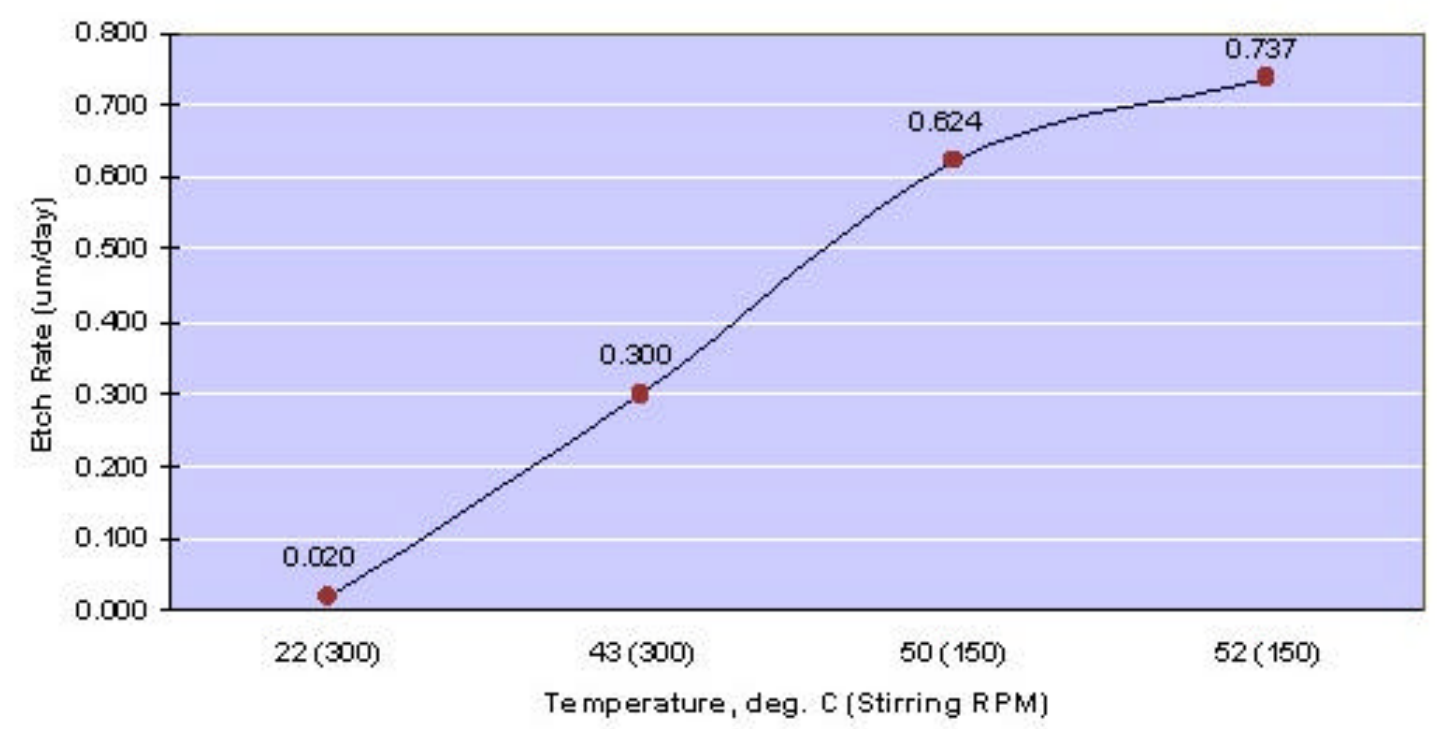

FIGURE 145 - Experimentally-measured thermally-grown $\mathrm{SiO}_{2}$ etch rates in $45 \%$ wt. $\mathrm{KOH}$ as a function of temperature (with RPM stir rate in parentheses).

\section{Relative Etch R ate of (100) Silicon to Silicon Dioxide in $45 \%$ wt. Potassium Hydroxide (KOH)}

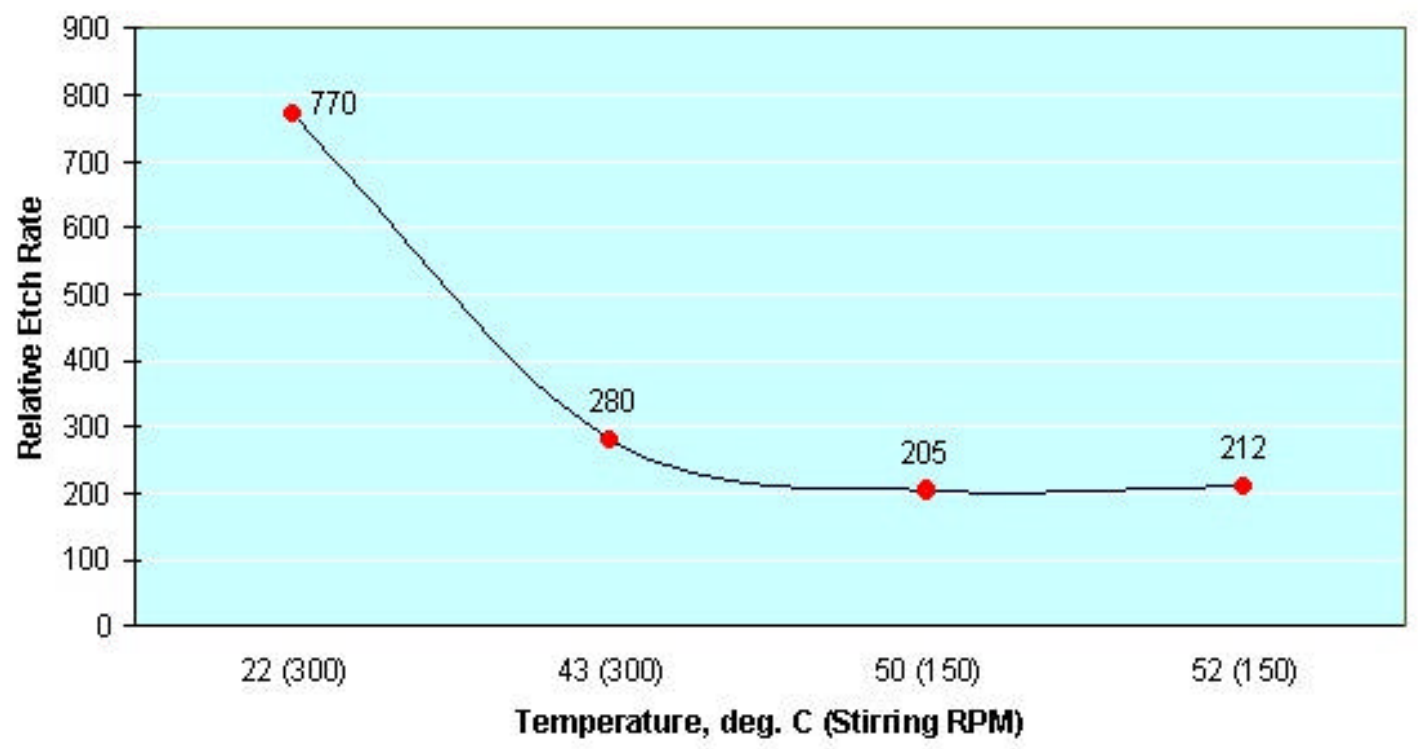

FIGURE 146 - Experimentally-measured relative etch rates of (100) silicon to thermally-grown $\mathrm{SiO}_{2}$ in $45 \%$ wt. $\mathrm{KOH}$ solution as a function of temperature (with stir rate RPM in parentheses). 
APPENDIX VII.

BOROSILICATE GLASS RIE DATA GRAPHS 


\section{Median Etch Rates for Borosilicate Glass with Positive Resist Masking Layer}

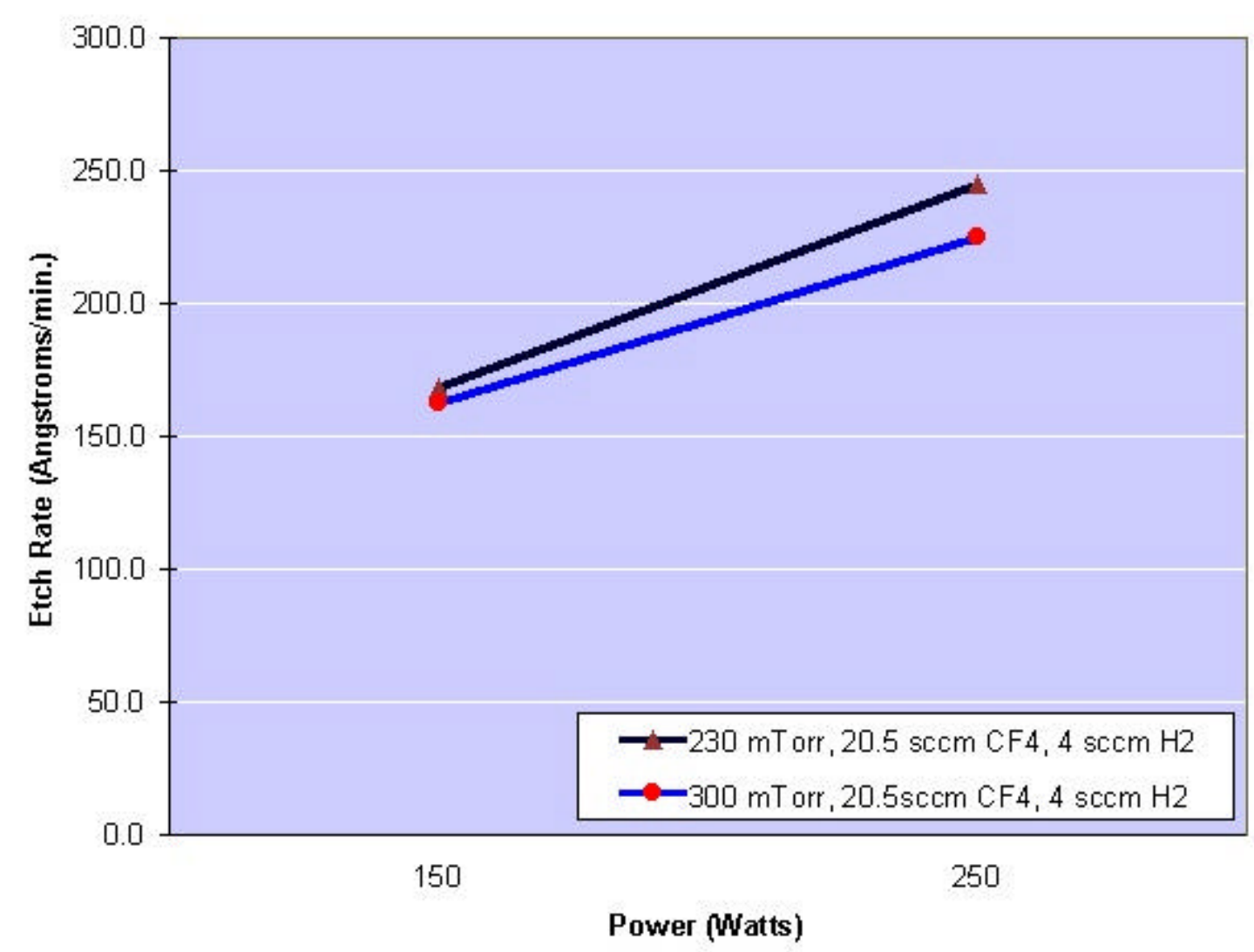

FIGURE 147 - Experimentally-measured median etch rates for Schott borosilicate glass with a Shipley 1813 positive photoresist masking layer in the March CS-1701 RIE System with automatic pressure control enabled, $57 \mathrm{mTorr}$ base pressure, and chiller at $25^{\circ} \mathrm{C}$. 


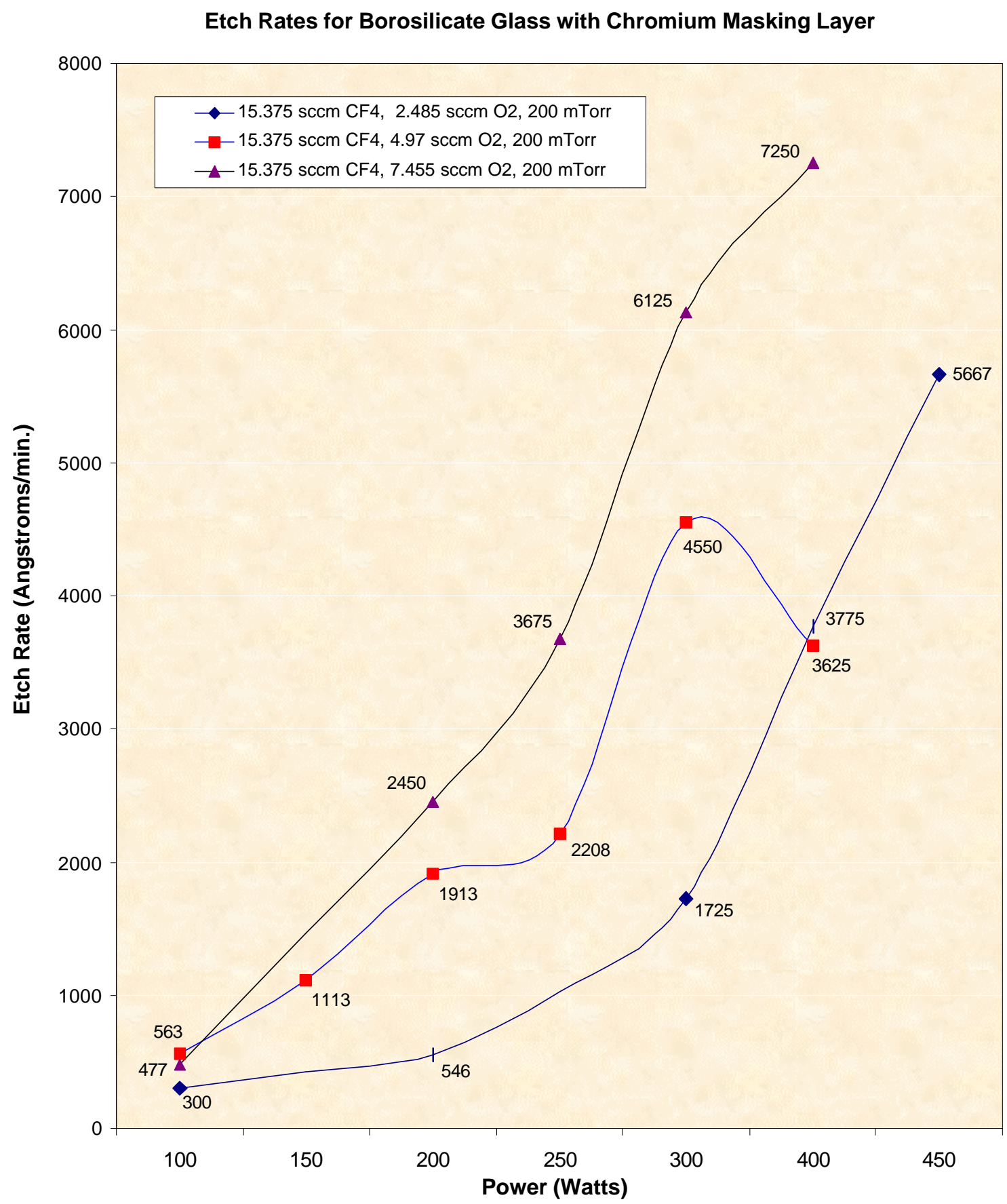

FIGURE 148 - Experimentally-measured median etch rates as a function of RF power and oxygen partial pressure for Schott borosilicate glass with a chromium masking layer in the March CS-1701 RIE system with automatic pressure control enabled, $57 \mathrm{mTorr}$ base pressure, and chiller at $25^{\circ} \mathrm{C}$. 


\section{APPENDIX VIII.}

SUGGESTED WAFER DICING DIAGRAM 


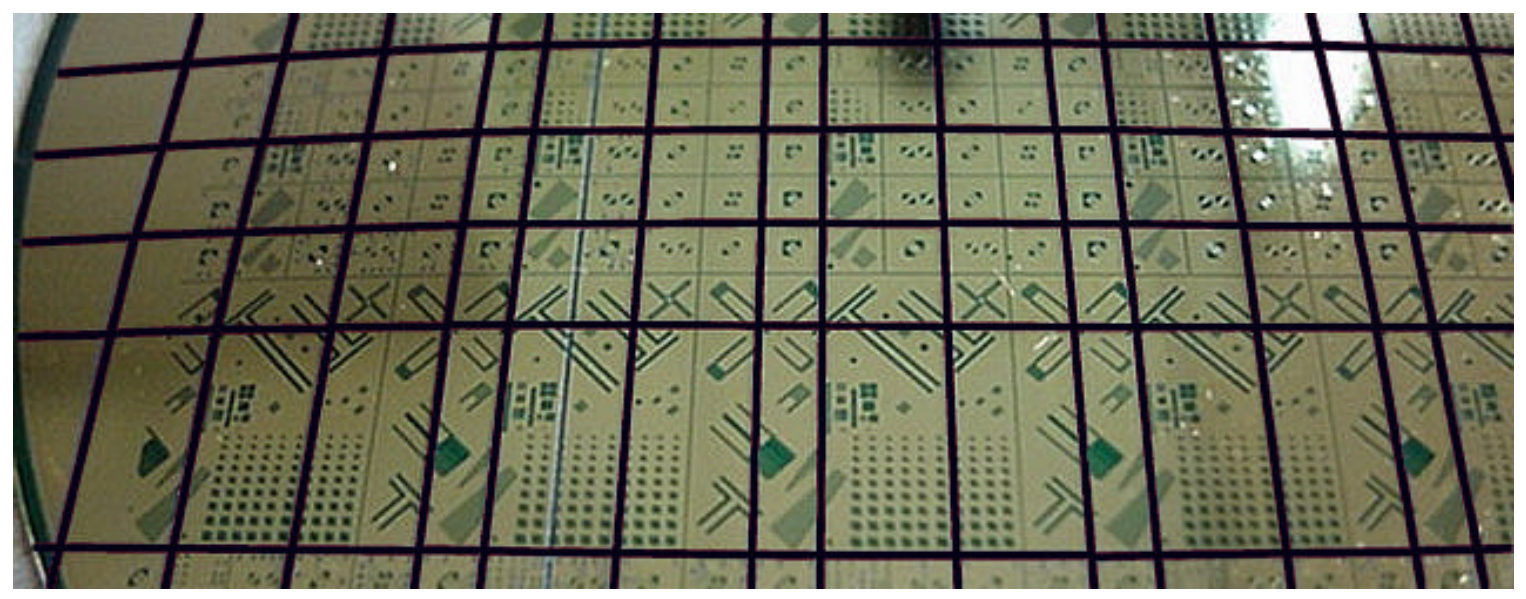

FIGURE 149 - Photo of suggested wafer dicing pattern. 


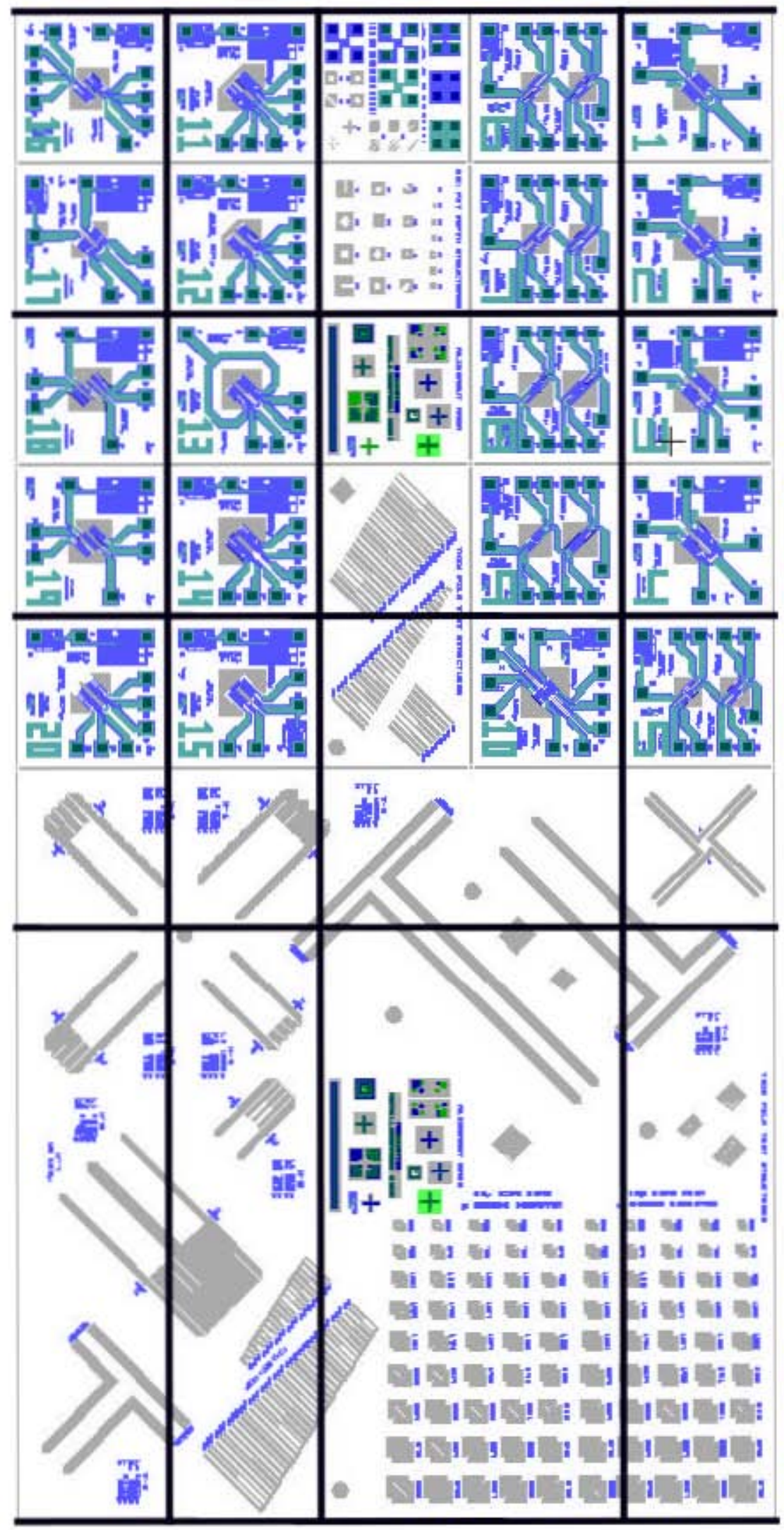

FIGURE 150 - Suggested dicing pattern shown for single die (replicated throughout the wafer). 


\section{APPENDIX IX.}

MICRO-AUTOMATION PROGRAMMABLE DICING SAW REVISED SOP 
Enhanced Sop \#53

Revised: March/April 1999

Last Revised: February 2000

\section{Title: Micro Automation Programmable Dicing Saw, Model 1006}

\section{Purpose}

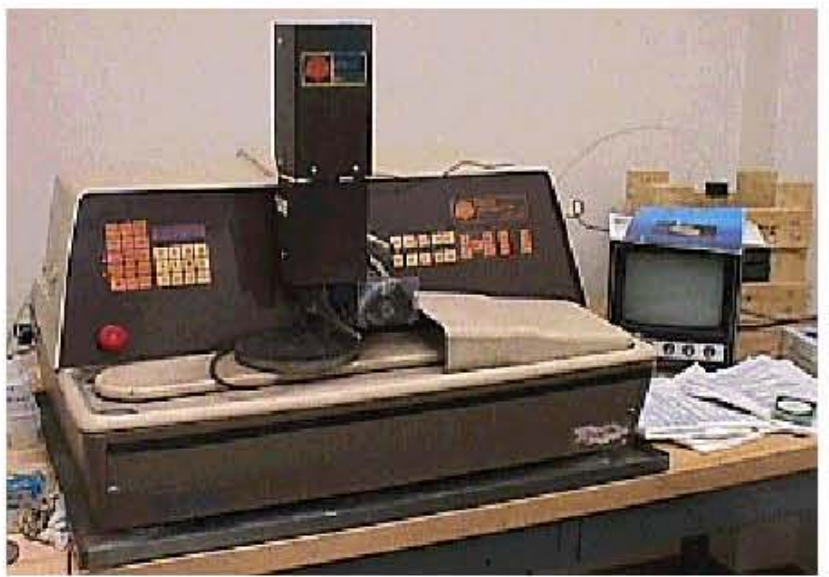

Operational and procedural instructions used to separate individual die on a silicon wafer or glass substrate.

Precautions easily cut through the skin during regular handling.

(2) There is high risk of damage to the delicate blades during handling. Handle the blades with extreme care, as they break very easily. Never touch the cutting edge of a blade, as even the slightest touch can bend, deform, and/or break a blade.

Do not flex or bend these thin blades!

(3) The spindle should never be turned on without the clear plastic guard tightly in place.

(4) You are strongly encouraged to wear hearing protection, as the spindle is agonizingly loud when it is running.

(5) Due to the age and unpredictability of this machine, do not leave it running unattended. For example, it is not uncommon for the spindle to slow down, jam, or halt suddenly, causing one or more internal fuses to blow inside the machine, disabling the saw. If the spindle is damaged or destroyed, it may cost at least $\$ 20,000$ to replace! If you are nearby watching or listening for these problems while operating the dicing saw, you should immediately push the STANDBY key to stop the spindle, if it seems to be slowing down, jamming, or operating unstably (which you can easily detect by a dramatic change in pitch of the loud noise it emits when running). 
(6) The red pushbutton switch, labelled STOP , is used to turn the saw's main power on and off. To turn the saw on, turn the button to the right. To turn it off, push the button in.

Although this button may be used to immediately stop the saw in a crisis situation, do not turn off the main power when the spindle is running

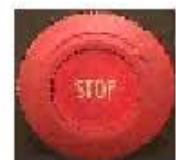
(instead, press the $S T A N D B Y$ key to stop the spindle).

(7) The operating controls are clustered into two groups of pressure/touch-sensitive switches on either side of the spindle. Operate them by pressing on the switch area with your finger. You will hear a beep when you press these buttons. Due to the age of this machine, you may experience problems in " double-pressing" these switches when you touch them. If this occurs, simply touch the button again, so that it is pressed only once (and then press the enter key, if applicable). You can damage these switches by pushing them with metal tools.

(8) IMPORTANT NOTE: If you are dicing silicon wafers or other samples with delicate or suspended microstructures, you are strongly encouraged to spin on photoresist to protect your sample from saw blade and vibration damage before you begin cutting your sample.

Procedure: Please read this entire document before operating the saw.

\section{PRELIMINARIES}

1. Obtain a copy of and fill out the "SAW PROGRAM " sheet (see section II., below)

2. Check the water level in the Vacuum Water Trap. This trap is located on top of the saw table to the right of the saw.

a.If the water level is above the tape on the side of the flask, empty the flask. To empty it, carefully remove the stopper (leave the two tubes in the stopper), leave the flask in the wooden base, and remove both. Hold the neck of the flask

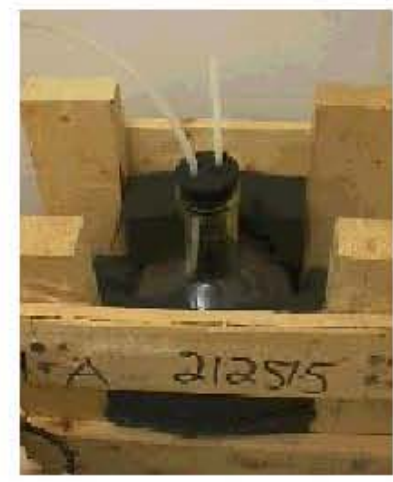
and tilt the base into a sink. Slowly pour water out into the sink. 
3. Check the water level in the Air Line Filter. This filter is located at the right, rear of the saw, at the table level. This Filter is also a regulator. This device may be labeled Saw Air Utility Filter/Regulator.

Remember the location of this filter, as it will be mentioned later.

a.If the water level is above the tape, empty the filter.

To empty it, turn the bottom plastic screw clockwise.

4. Check the four drain holes at the corners on top of the machine (in the water basin area) for visible obstructions. Ideally, these holes should all be covered with a wire mesh to keep large pieces of debris from clogging the drains. Once operating the dicing saw, if you find that water is spilling over the top water basin area, you may need to either unclog one or more of the drain tubes or reduce the flow rate (flow rates around 50 $55 \mathrm{GPH}$ for the vacuum-chuck-and-diamond-cutting-blade cooling water will not cause water to spill over the top basin, if all four drain holes are unclogged!).

5. Check to insure that all of the water drain tubes are properly situated/mounted to drain into a nearby sink or water drain. There should be two large (one-half-inch diameter) tubes for draining chuck and cutting blade cooling water out of the top water basin, and two smaller tubes (one to drain spindle cooling water and another to drain the bottom steal pan in which the dicing saw is sitting).

6. Decide how you will mount the sample to be cut or diced (e.g. with or without tape or blue film under the sample). Discuss mounting your sample with Dr. Walsh. Your sample to be diced or cut with any mount underneath (if applicable) will be hereafter referred to as the SPECIMEN .

7. Check to see if there is a blade sheet present on top of or near the saw. This document describes the blade which is currently mounted on the saw (grit, cutting depth, and thickness).

a. Visually inspect the blade to make sure it is not damaged or broken.

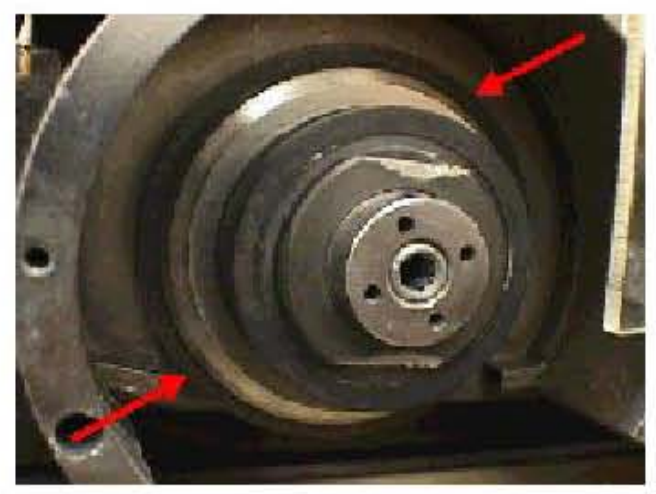


b.If you need to change the blade, refer to and follow instructions provided in the Saw Blade Changing SOP ( SOP \#55)

8. If the dicing saw itself is not already plugged in, plug it in. If you do not see any LED's on the front panels lit, rotate the red STOP button to turn on the saw. If the saw is still not on, check to make sure the top-cover interlock switch in the back of the unit is pushed down and latched (it can be latched down, even if the top cover is not on the saw).

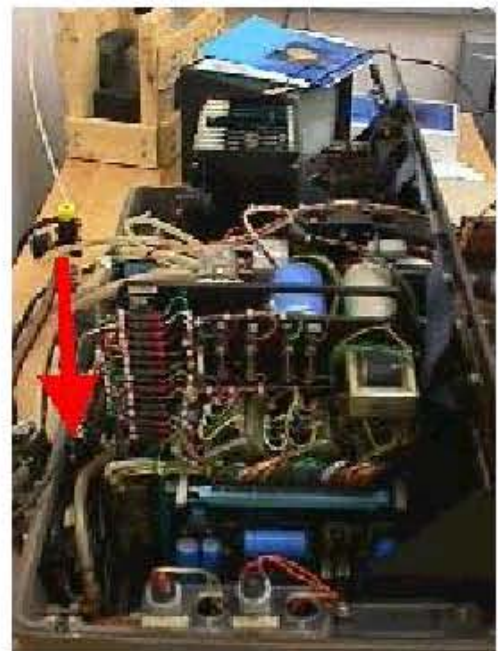

Location of the top-cover interlock switch.

$</$ tr $>$

II. PROGRAM

1. These are the left-panel program and data-entry keys you will use.

a.

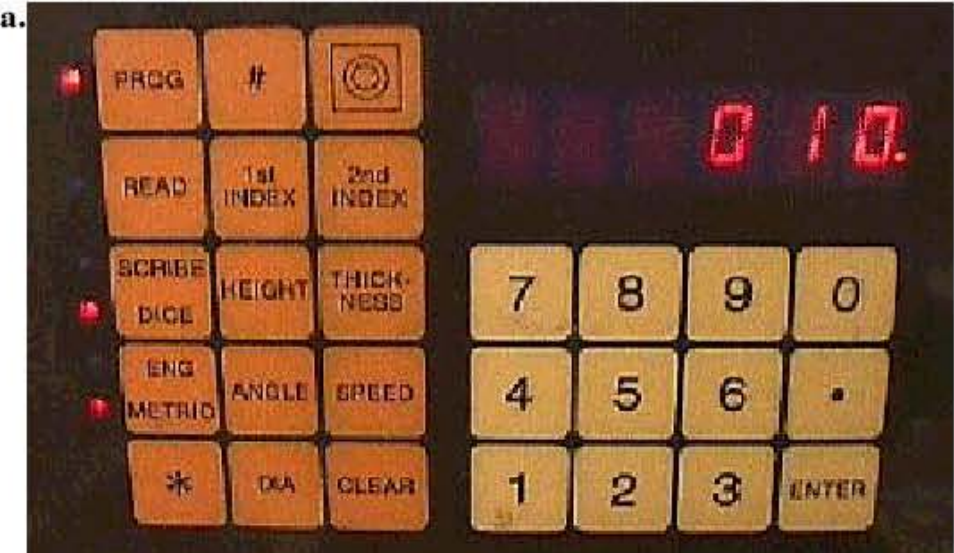

b. These are the right-panel program and chuck movement keys. 


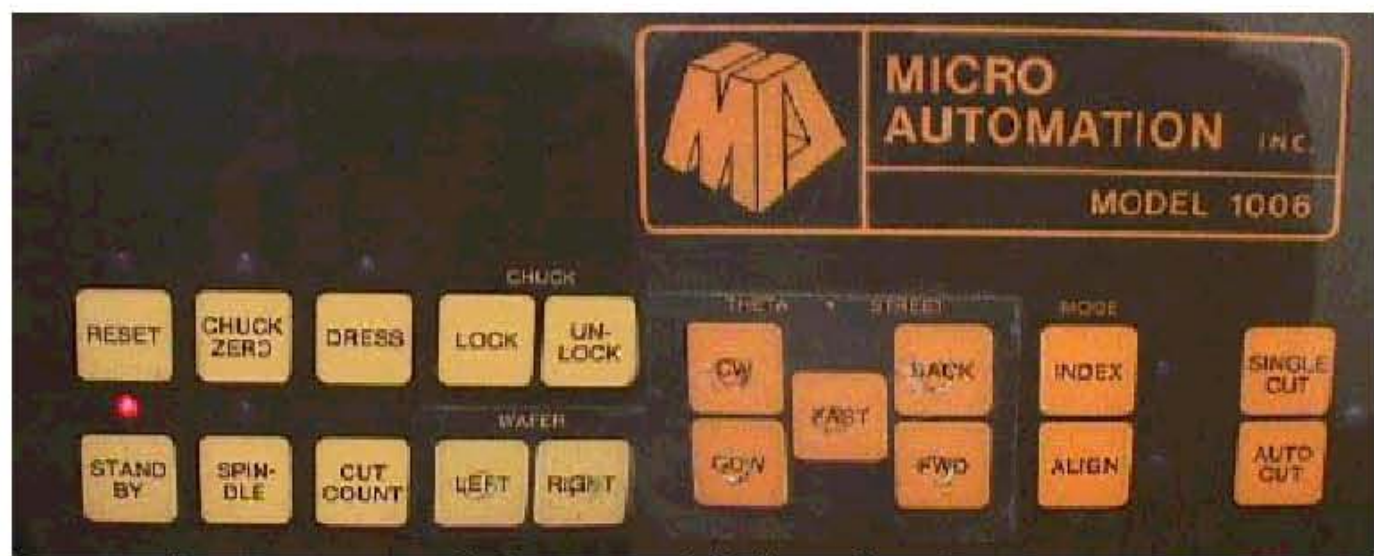

2. a.You must first store a program before you can initialize and use the saw.

First, press the PROGRAM key:

\section{b. General Program Rules and Notes}

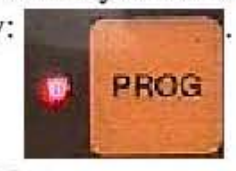

i. Once you have begun to program the saw, you must finish (a value must be entered for each cutting parameter).

ii. A non-zero number must be entered for each parameter (except CUT COUNT).

iii. When the red LED next to the PROGRAM key is lit, the saw is in program mode. The saw cannot be operated unless the PROGRAM LED is not lit (i.e. all sufficient program parameters have been entered).

iv. If the saw is started up from the standby condition, the program stored and used in the last operation will be used.

v. If during programming, you hear a beep and receive a flashing error code, refer to the error code list in Section V (below). Typically these can be cleared by pressing CLEAR, re-entering the number or parameter, then pressing $\boldsymbol{E N T E R}$. Because the program-entry keys are very sensitive to touch, a key may register multiple touches or presses, generating an error. Carefully re-entering the number or parameter usually corrects this problem.

vi. The numbers entered and displayed (on the red 7-segment LED display) are either in mils $(0.001 \mathrm{in}$.) or $\mathrm{mm}$., depending on the state of the ENG/METRIC switch.

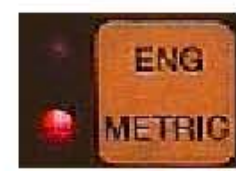

vii. If you accidently enter a wrong number, press $C \boldsymbol{L E A R}$,

change the number, then press $\boldsymbol{E N T E R}$.

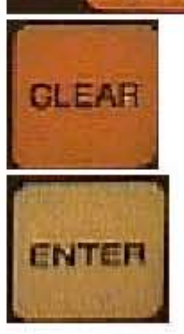


3. a.Press the MODE key:

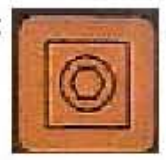

enter a mode number (see below) on the numeric keypad,

and press ENTER.

VALUE (MODE):

b. Mode

Number

Description

10 For round specimens, with alignment required for each pass.

11 For round specimens with no alignment required for the second pass. (Blank wafers)

20 For round specimens, hexagonal die, where alignment is required for each pass (Triple alignment option).

21 For round specimens, hexagonal die, with no alignment required for the last two passes. (Blank wafers)

22 For round specimens, hexagonal die, with no alignment required for the second pass. The third pass alignment is made to an intersection of cuts made during the first and second passes. (Blank wafers)

30 For rectangular specimens, with alignment required for each pass.

31 For rectangular specimens, where no alignment is required for the second pass. (Blank wafers)

60 Progressive depth cuts for round specimens, with alignment required for each pass.

61 Progressive depth cuts for round specimens, with no alignment required for the second pass. (Blank wafers)

70 Progressive depth cuts for rectangular specimens, with alignment required for each pass.

71 Progressive depth cuts for rectangular specimens, where no alignment is required for the second pass. (Blank wafers)

4. Select which base units system you will use when entering cut parameters.

Press the $\boldsymbol{E N G / M E T R I C}$ key to toggle between mils ( 0.001 inch) and millimeters $(\mathrm{mm})$. The

LED indicator to the left of this key will indicate which base units the saw is currently configured to use.

5. Press the SCR/DICE,

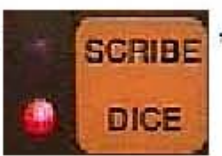

, button (if needed) to toggle the red LED to the DICE

mode.

6. Press the $1 S T$ INDEX key, enter the street-to-street distance for the first cut pass, then press $\boldsymbol{E N T E R}$.

The range of possible street widths is 0.125 mils $-4,000$ mils, or 0.0032 mm. $-101.6 \mathrm{~mm}$.

VALUE (1ST INDEX): 
7. Press the THICKNESS key, enter your specimen's total (maximum) thickness, then press $\boldsymbol{E N T E R}$.

Thickness is programmable from 0.25 mils - 511 mils, or $0.0064 \mathrm{~mm} .-13$ mm.

WARNING: Make sure you know, accurately, your specimen's maximum thickness (measure it with a micrometer) to avoid cutting into the vacuum chuck.

VALUE (THICKNESS):

8. Press the HEIGHT key, enter the amount of the specimen you want left after the cut, then press ENTER. The depth of the cut equals the THICKNESS of the specimen minus this programmed HEIGHT parameter.

The height range is 0.25 mils - 511 mils, or $0.0064 \mathrm{~mm}$. $-13 \mathrm{~mm}$.

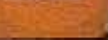

VALUE (HEIGHT):

9. Press the $A N G L E$ key, enter the theta-axis angle the vacuum chuck will rotate between cut passes, then press ENTER.

The normal range is $1^{\circ}$ to $121^{\circ}$ (except in 'Hex" cutting mode where the largest angle is $60.1^{\circ}$ ).

VALUE (ANGLE):

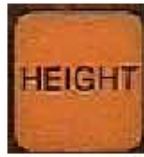

10. Next, you will enter parameters to specify whether your specimen is round or $\mathbf{r}$ ectangular.

a.For round specimens, press the $\boldsymbol{D I A}$ key, enter the specimen diameter, then press $\boldsymbol{E N T E R}$.

The range of possible values is 0.25 mils - 6000 mils (6 inches), or 0.0064 mm. $-152 \mathrm{~mm}$.

VALUE (ROUND):

b.For rectangular specimens, press the DIA key, enter the specimen's width dimensi on, then press $\boldsymbol{E N T E R}$.

VALUE (RECT):

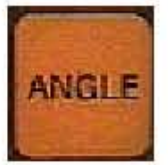

Then press the * key, enter the specimen's length dimension, then press

ENTER.

VALUE (RECT):

The range is 0.25 mils - 6000 mils (6 inches), or $0.0064 \mathrm{~mm} .-152 \mathrm{~mm}$.

11. Press the $2 N D$ INDEX key, enter the street-to-street dimension for the second cut pass (which is the pass that occurs after your programmed theta-axis $\boldsymbol{A N G L E}$ rotation of the vacuum chuck), then press $\boldsymbol{E N T E R}$. The range of possible street width values is 0.125 mils $-4,000$ mils, or $0.0032 \mathrm{~mm}$. $-101.6 \mathrm{~mm}$.

VALUE (2ND INDEX):

12. Press the $\boldsymbol{S P E E D}$ key, enter the speed of $\mathrm{X}$-axis travel (i.e. the wafer cutting speed), then press $E N T E R$. The best cutting SPEED is largely determined by the specimen material's hardness.

Speed is programmable from $50 \mathrm{mils} / \mathrm{sec}$ - $19,999 \mathrm{mils} / \mathrm{sec}$, or 1.27 $\mathrm{mm} . / \mathrm{sec}$. $-508 \mathrm{~mm} . / \mathrm{sec}$.

NOTE: A recommended starting speed through silicon samples is 50 $\mathrm{mils} / \mathrm{sec}$.

VALUE (SPEED): 
13. If you want to use progressive-depth cuts, press the $\boldsymbol{\theta}$ (zero) key, enter the desired incremental depth cut distance, then press ENTER.

This is only used if you have already programmed $M O D E$ number 60, 61, 70 , or 71 .

The incremental-depth cut range is $1 \mathrm{mil}-15 \mathrm{mil}$, or $0.025 \mathrm{~mm} .-0.381$ $\mathrm{mm}$.

\section{VALUE (PROGRESSIVE DEPTH CUT DISTANCE):}

a.Progressive depth cuts are used the thickness of your specimen is too thick to be effectively, cleanly cut all at once. In this cutting mode, the saw will make a series of progressively deeper cuts on the same street before moving to the next street.

b.An example of using progressive depth cuts, is to cut through a thick glass specimen to reduce blade wear.

14. Press the PROGRAM key to indicate that you have entered all cut parameters for your specimen.

15. Press the RESET key,

\section{RESET}

III. OPERATION

1. Turn ON the TV monitor, that is located beside the saw, by rotating the right-most knob (below the monitor screen) clockwise.

2. Turn on the lab air (compressed air) valve by rotating the knob COUNTER-CLOCKWISE.
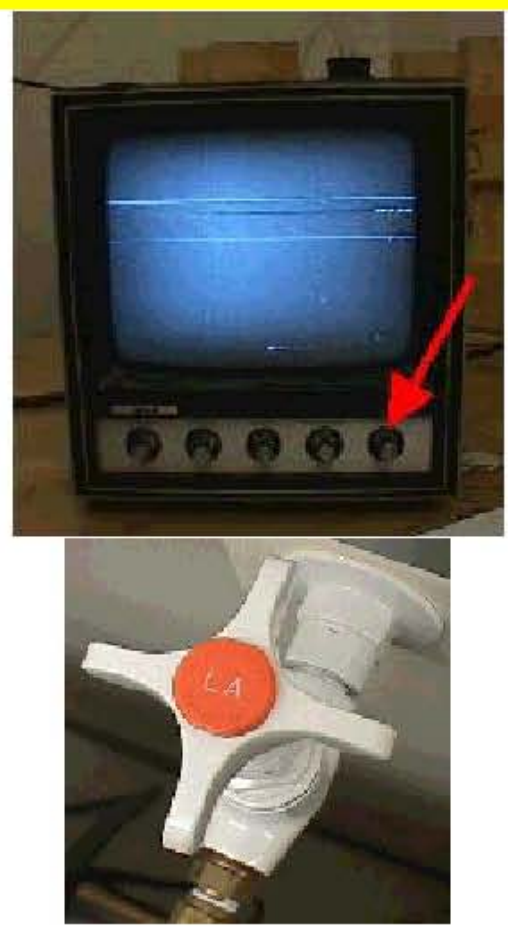

http://mitghmr.spd.louisville.edu/lutz/resources/sops/sop53.html (8 of 18) [5/10/2000 9:36:14 PM] 
3. If needed, turn on the Saw Air Utility Filter/Regulator. Typically this regulator valve has already been opened to the correct PSI setting.

Turn the top yellow knob CLOCKWISE until the regulator gauge reads 90 PSI (as of March 1999). The nominal PSI range over which the saw has been operated is $65-75$ PSI. (The previous operating pressure setting was 70 PSI.)

4. Turn on the vacuum pump (which is typically on the floor to the right of the saw table), by plugging it into an electrical outlet.

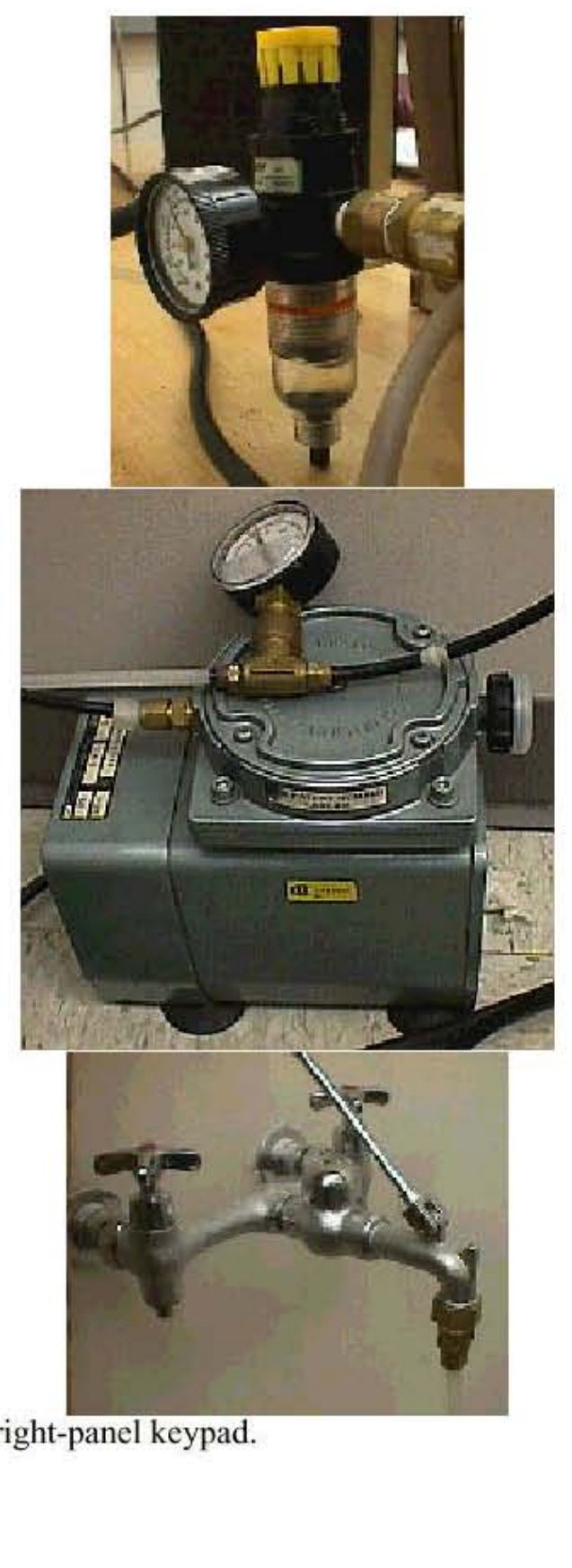

http://mitghmr.spd.louisville.edu/lutz/resources/sops/sop53.html (9 of 18) [5/10/2000 9:36:14 PM] 
a. After pressing the SPINDLE key, carefully watch the blade assembly through the clear plastic cover and verify that the spindle begins to rotate.

WARNING: If the spindle fails to start rotating within a few seconds, immediately press the $S T A N D B Y$ key to abort start-up. Failure to do this may damage the expensive spindle motor assembly.

NOTE: If the lab air or cooling water is not already turned on, the spindle may not start-up.

b. Cooling water should begin flowing to the spindle once it is started. Verify that the right GPH flow meter (for spindle cooling water) is reading approx. $26 \mathrm{GPH}$. The flow meter has a black mark on it to indicate where the flow rate should be. If needed, adjust the flow rate by turning the black knobs at the bottom of each meter as needed.
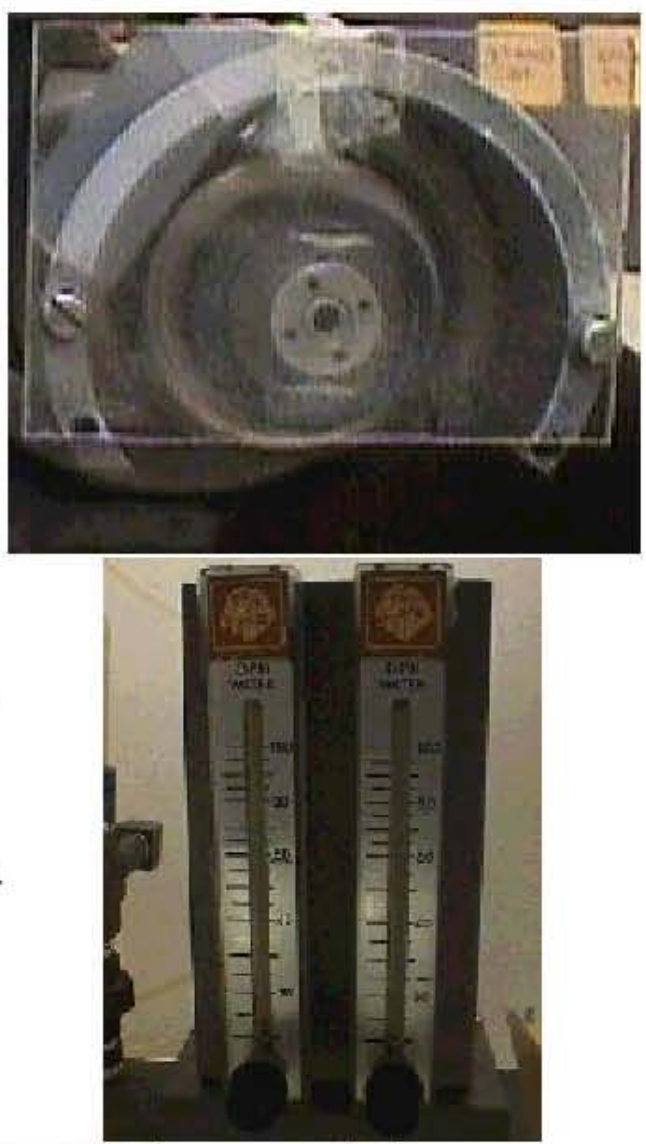

7. Next you need to "zero" the saw blade with respect to the vacuum chuck.

a. First, locate the round metal gauge disk, which should be stored near the saw on top of the saw table, or on top of the nearby black filing cabinet. This metal disk is about 4 inches in diameter and about $1 / 8$ th of an inch thick.

b. Make sure that the gauge disk and vacuum chuck assembly are dry.

NOTE: Make sure the gauge disk and vacuum chuck assembly are dry. Also make sure the water spray tube (which protrudes out over the vacuum chuck assembly) is not dripping water onto the chuck. During chuck zero calibration (step 7.g., below), the vacuum chuck and gauge disk are slowly elevated until the gauge disk is brought into brief contact with the saw blade, closing an electrical circuit. Thus, if the gauge disk is

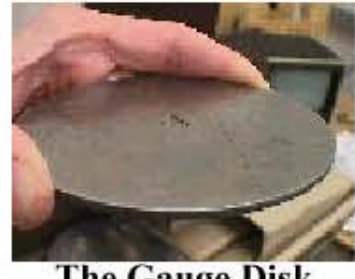

The Gauge Disk

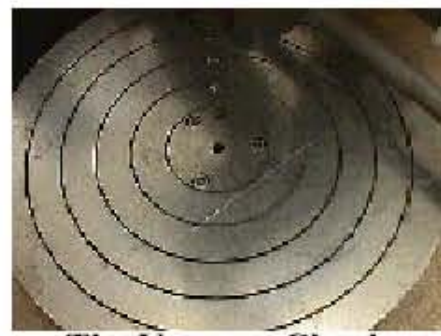

The Vacuum Chuck 
wet, erroneous chuck zero calibration will

result, because the water will conduct

current causing the saw to calibrate for a

chuck that is thicker than it really is.

c. Center the gauge disk and gently drop it onto the chuck

d. If you need to move the gauge disk around on the chuck, since the vacuum pump should already be running, press the $U N L O C K$ button.

e. Due to the age of the saw, the lock/unlock solenoid usually does not function properly. If you cannot move the gauge disk around after pressing the $\boldsymbol{U} \boldsymbol{N L O C K} \mathrm{key}$, unplug the vacuum hose from where it attaches to the vacuum chuck to remove vacuum from the chuck. Then move the gauge disk into place and reconnect the hose to re-establish vacuum adhesion.

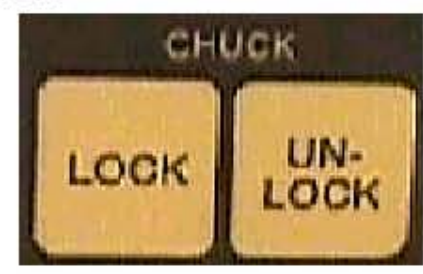

f. Press $\mathbf{L O C K}$ to activate the chuck vacuum (even if the lock/unlock solenoid is not working properly, you cannot proceed to the next step until this is done).

g. Press the CHUCK ZERO key to "zero" the vacuum chuck assembly. The chuck should slowly raise the gauge disk up under the rotating saw blade until it actually comes into brief contact with the blade, and then the chuck should lower and reset.

NOTE: If you receive error codes when trying to "zero" the chuck, refer to Section $\mathbf{V}$,below to identify and clear them. A common error often occurs when the saw

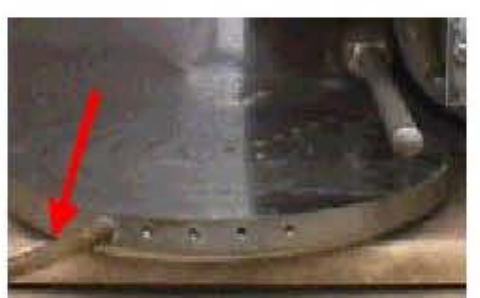

The vacuum hose attachment point does not register that the gauge disk has been locked onto the vacuum chuck assembly. Try repeatedly pressing the LOCK, UNLOCK, LOCK, then CHUCK ZERO keys.

h. After the vacuum chuck assembly has finished "zeroizing," press the UNLOCK key, remove the gauge disk, and re-store it next to the saw. Also, it may be necessary to unplug the vacuum hose from the vacuum chuck to release the vacuum (leave the hose disconnected until you have placed your specimen onto the chuck). 
8. a. There are a series of "set" screws in the vacuum grooves of the top surface of the vacuum chuck which can be removed as desired to enlarge the diameter of vacuum suction for the specimen. Depending upon the size of your specimen, you may need to remove additional set screws.

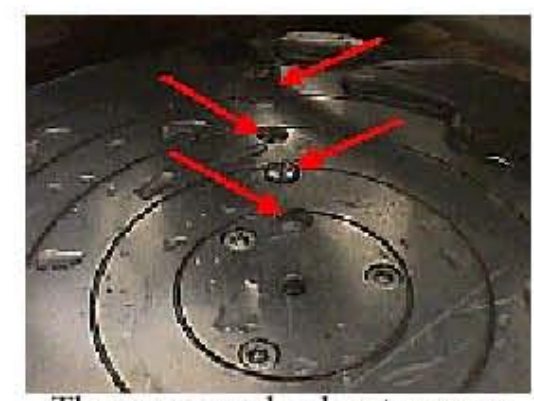

The vacuum chuck set screws

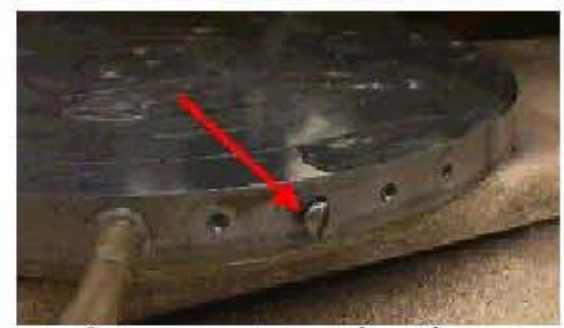

Set screw storage locations

c. Now, center and gently place your specimen onto the vacuum chuck.

9. Re-connect the chuck vacuum hose (if you had to disconnect it to release the vacuum) and press the $\mathbf{L O C K}$ key.

10. Next you need to optically align the saw blade with respect to the specimen on the vacuum chuck.

a. Press the $A \boldsymbol{A L I} \boldsymbol{I}$ key to allow you to align the saw's first cut on your specimen.

b. The TV monitor shows a split-field image of your specimen. The image on the right side of the screen (and right side of your specimen) may be obscured by the cooling-water outlet tube. Thus, you may have to gently push or bend the cooling-water outlet tube towards the center of the vacuum chuck (i.e. to the left) in order to see your specimen clearly on the TV monitor during this alignment sequence (especially as you scroll to the left and right across your specimen to check the alignment).

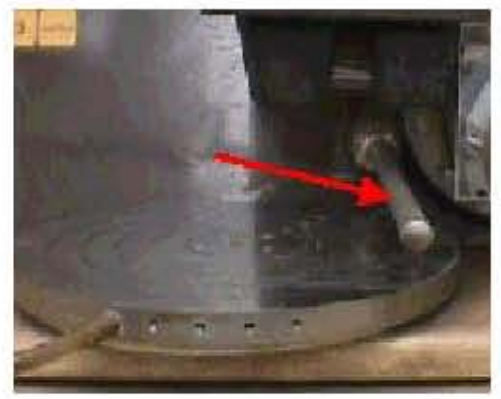


c. You should see two white, horizontal "reticle" lines on the black- and-white TV monitor. Use the red buttons on the right side of the camera assembly (positioned above the vacuum chuck area) to position a reticle line just above, and another reticle line just below where your specimen's streets (or other horizontal features to use for alignment) are visible on the monitor. Use the top two red buttons to move BOTH reticle lines up or down on the screen, and use the bottom two red buttons to move the reticle lines closer together or farther apart on the monitor.

NOTE: These reticle lines are actually used to evaluate where the saw blade will cut into your specimen. To precisely determine where the blade will cut your specimen, you may wish to first make a single cut into a "test wafer" or specimen. After making a single-pass cut, scroll across the specimen to see exactly where the blade cut into it. Then, use the red reticle-position and spacing keys to move the horizontal reticle lines to the outer edges of the cut trench. After you remove the test specimen, replace it with the actual specimen, and follow this alignment routine (10 a.-e.), you can then use the position of the reticle lines to see where cuts will actually be made.

d.Align the streets to the plane of the cutting

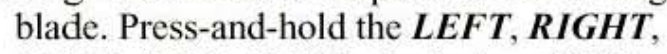
$B A C K, F W D, C W$ (clockwise), or $C C W$ (counter-clockwise) keys on the right panel keypad to align your specimen. The $\boldsymbol{L E F T}$ and $\boldsymbol{R I G H T}$ keys move the specimen rapidly along the horizontal $\mathrm{X}$-axis, while the $\boldsymbol{B} \boldsymbol{A C K}$ and $\boldsymbol{F} \boldsymbol{W D}$ keys move the specimen along the vertical Y-axis. The $\boldsymbol{C W}$ (clockwise) and

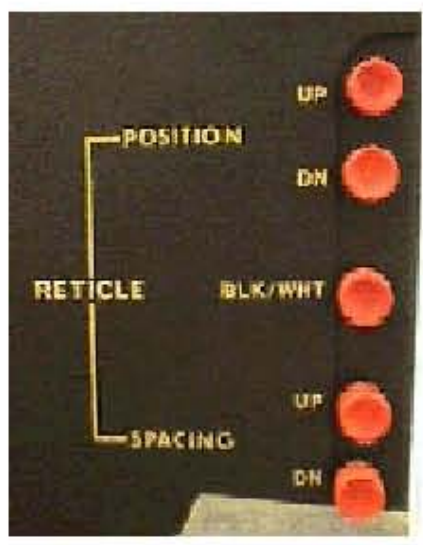
$\boldsymbol{C C W}$ (counter- clockwise) keys rotate the specimen. By default, pressing-and-holding the $B A C K, F W D, C W$, or $C C W$ key moves the specimen very slowly for fine alignments. For faster, coarse alignment, first press the $\boldsymbol{F A S T}$ key, followed by pressing-and-holding the $\boldsymbol{B A C K}, \boldsymbol{F} \boldsymbol{W D}, \boldsymbol{C W}$, or $\boldsymbol{C C W}$ key (the $\boldsymbol{F A S T}$ key only works for

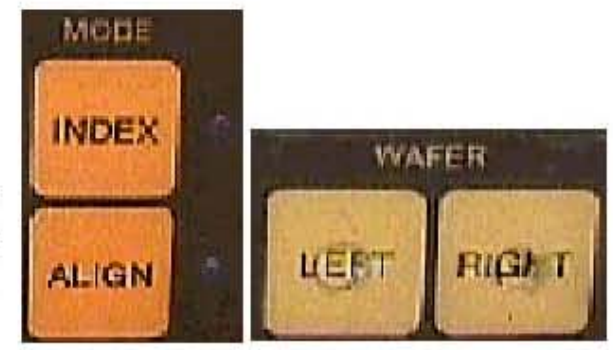

http://mitghmr.spd.louisville.edu/lutz/resources/sops/sop53.html (13 of 18) [5/10/2000 9:36:14 PM] 
the next key pressed). You should attempt to align horizontal features (such as streets) on your specimen to the horizontal reticle lines. This is done by horizontally moving across your specimen to see if your horizontal features or streets are parallel to the reticle lines. If not, rotate the wafer (by pressing-and-holding the $\boldsymbol{C} \boldsymbol{W}$ or $\boldsymbol{C C W}$ key)

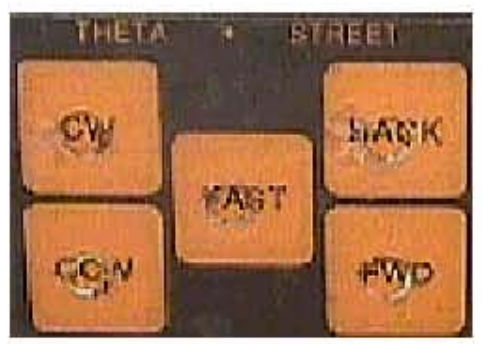
and continue moving across the wafer until $\mathrm{X}$-axis features or streets are parallel to the reticle lines.

e. You can move to every street on your specimen by first pressing the INDEX key followed by the $\boldsymbol{F} \boldsymbol{W D}$ or $\boldsymbol{B} \boldsymbol{A C K}$ key. You should see your specimen step to the next (or previous) cutting street (through a distance specified by the IST INDEX program parameter).

By first pressing the $I N D E X$ key followed by the $\boldsymbol{C W}$ or $\boldsymbol{C C W}$ key, the saw will rotate

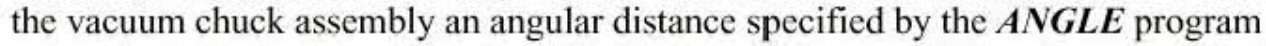
parameter to prepare your specimen for the 2 nd cutting pass. Thereafter, pressing the $\boldsymbol{I N D E X}$ key followed by the $\boldsymbol{F} \boldsymbol{W D}$ or $\boldsymbol{B} \boldsymbol{A C K}$ key, should move your specimen to the next (or previous) cutting street, through a distance specified by the $2 N D I N D E X$ program parameter.

NOTE: If you press the FAST key before pressing the INDEX key, the Y-axis will continue to step the programmed vertical distance as long as the INDEX button is held down, or until the end of the wafer is reached.

11. Next, decide how you will dice your specimen.

a. To execute only a single pass and cut through only the current street visible on the monitor (in either INDEX or $\boldsymbol{A L I G N}$ modes), press the SINGLE CUT key. Water should immediately begin to flow onto the vacuum chuck assembly. Make sure the blade-and-chuck cooling water is draining properly into the sink drain basin. Position the large, clear Lexan ${ }^{\circledR}$ plastic shield (stored on the floor near the saw

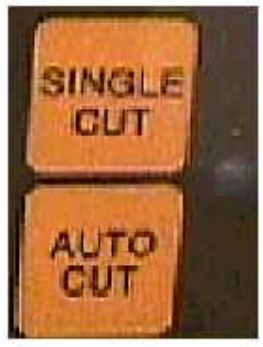
table) in front of the saw to avoid splashing water onto the floor. 
b. To cut through all streets in the X-axis (horizontal) dimension (and possibly the Y-axis streets on the second pass, depending on the programmed $M O D E$ number) of your specimen, press the AUTO CUT key. If the saw is in ALIGN mode when AUTO CUT is pressed, it will begin cutting in the first street. If the saw is in INDEX mode when AUTO CUT is pressed, it will begin cutting in the next street.

Water should immediately begin to flow onto the vacuum chuck assembly once $A U T O$ $\boldsymbol{C U T}$ is pressed. Make sure the blade-and-chuck cooling water is draining properly into the sink drain basin. Position the large, clear Lexan ${ }^{\circledR}$ plastic shield (stored on the floor near the saw table) in front of the saw to avoid splashing water onto the floor.

i. If the saw has been programmed in MODE $10,30,60$, or 70 , you have aligned the streets to the blade along only the X-axis (for the first pass), and you have pressed AUTO CUT, the saw will make all cuts for the first pass, rotate the specimen through the programmed $\boldsymbol{A N G L E}$, return the specimen to the align position, and beep to tell you that you can re-align your specimen prior to the 2 nd cutting pass. You can then align for the 2nd pass, then press AUTO CUT again to cut all of the specimen's streets in the 2nd pass.

ii. If the saw has been programmed in $M O D E 10,30,60$, or 70 , you have aligned your specimen for the first pass, then rotated the specimen, aligned the streets for the 2 nd cutting pass, and you have pressed $A \boldsymbol{U} \boldsymbol{T} \boldsymbol{O} \boldsymbol{C U T}$, the saw will make all cuts for the first pass, rotate the specimen through the programmed $A N G L E$, and make all cuts for the second pass without operator intervention. A beep will sound when the saw has finished all dicing.

iii. If the saw has been programmed in $M O D E 11,31,61$, or 71 , the specimen and chuck will rotate through the programmed $A N G L E$ after cutting through the first pass and then make the second pass without pausing for operator alignment. There is no provision for alignment on the second pass (the center of the chuck is used for an alignment reference)

c. After pressing SINGLE CUT or AUTO

CUT, verify that the left GPH flow meter (for vacuum chuck and diamond cutting blade cooling water) is reading approximately 58-60 GPH and the right GPH flow meter (for spindle cooling water) is reading $26 \mathrm{GPH}$. The meters each have a black mark on them to indicate where the flow rate should be. Adjust the flow rates by turning the black knobs at the bottom of each meter as needed. NOTE: If you find that water is spilling over the top basin when the dicing saw is running, you may need to unclog one or more of the four drain tube holes at each corner of the basin, or you may solve the problem by reducing the vacuum chuck and diamond cutting blade cooling water flow rate to 50 - 55 GPH).

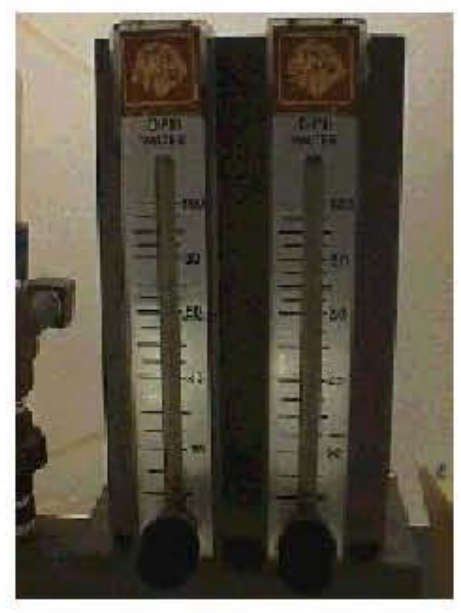

http://ritghmr.spd.louisville.edu/lutz/resources/sops/sop53.html (15 of 18) [5/10/2000 9:36:14 PM] 
12. To dry off your specimen and verify proper alignment after cutting, an air-dry button is accessible under the saw. Reach your hand under the bottom left side corner of the saw, feel for, and press a pushbutton switch to release the air.

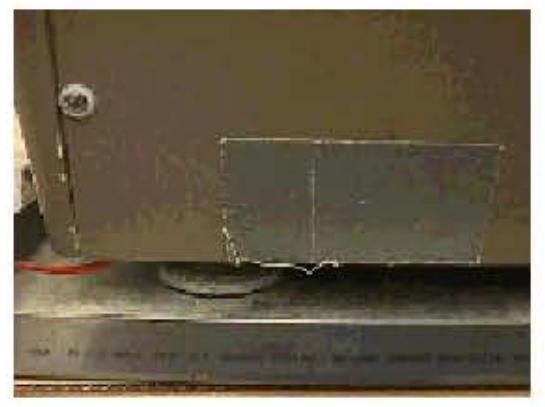

IV. SHUTDOWN

1. After your specimen has been diced, press the $S T A N D B Y$ key to stop the rotating spindle and saw blade and to return the chuck to its original position. The saw will go through a homing sequence to return to standby status. The chuck zero will be lost, but your working file program will remain stored.

NOTE: Other methods of stopping the saw or for stopping the saw to make alignments during a cut are described in the table below.

a. Alternate Precedures for Stopping the Dicing Saw During Cuts

i. PANIC STOP Press the red EMERGENCY OFF pushbutton switch located below the left-side programming panel to immediately shut down everything.

ii. RESET

Press the RESET key to enter a mode similar to $S T A N D B Y$, except that the spindle will continue to run, the blade will remain spinning, and chuck zero will not be lost.

iii. INDEX

Press the $\boldsymbol{I N D E X}$ key, and the saw will finish the current cut it is making, t hen return to the Index position. When AUTO CUT is pressed, cutting will start at the next street. Chuck zero is retained.

iv. ALIGN

Press the $\boldsymbol{A L I G N}$ key, and the saw will finish the current cut and then stop in the Align position. However, when AUTO CUT is pressed, sawing will begin at the front of the wafer. Chuck zero is retained.

v. CLEAR

In SINGLE CUT mode, when the saw is within the second pass ("PASS 2" is displayed on the LED display), pressing $\boldsymbol{C L E A R}$ at any time will reverse the vacuum chuck and specimen by the programmed $\boldsymbol{A N G L E}$ to return to the first pass orientation and halt.

2. Turn the water faucet OFF [rotate the knob(s) CLOCKWISE].

3. Turn the lab compressed air OFF by rotating the knob CLOCKWISE.

a. Optional: Turn off the A

4. Wipe off the vacuum chuck area with a clean, dry cloth. 
SOP \#53: Micro Automation Programmable Dicing Saw (with diamond blades)

5. If the saw will be unattended and unused for a prolonged period, unplug it.

\section{LIST OF COMMON ERROR CODES 1}

Error

Code

E001 Saw is currently in $S T A N D B Y$ mode.

\section{Cause or Corrective Action}

The RESET button must be pressed before attempting to turn the spindle on or otherwise operate the saw.

E002 The operator tried to operate the machine See if the PROG or $\boldsymbol{R E A D}$ LED's are lit. while in PROGRAM or READ modes.

Press the appropriate switch to extinguish the LED's.

E003 A number parameter entered is out of the valid range.

An attempt was made to enter a numerical parameter which is outside the design capability of the saw.

Press $\boldsymbol{C L E A R}$ and program an acceptable number.

E004 The dimension entered for height is greater than the dimension entered for wafer/specimen thickness. This results in no dicing of the wafer/specimen.

E005 The dimension entered for INDEX is Press $\boldsymbol{C L E A R}$ and enter the correct dimensions. greater than the dimension entered for the dimensions. wafer/specimen size. This implies that the dies are larger than the wafer/specimen.

E006 The saw has detected the lower chuck limit. This is likely caused by an excessive THICKNESS number.

E101 More than one switch was pressed simultaneously.

Press $\boldsymbol{C L E A R}$ and enter the correct THICKNESS.

Press $\boldsymbol{C L E A R}$. The panel program buttons are very sensitive to touch. If this error code is displayed there may be a shorted switch or connector.

E102 An attempt has been made to operate the saw without the chuck $L O C K$ ed.

Press chuck $\boldsymbol{L O C K}$ (and, then perhaps chuck $U N L O C K$, and $L O C K$ again).

E103 An attempt has been made to operate the saw with one or more program parameters missing.

Make sure all parameters have been programmed into the saw (check this by pressing a program parameter key, such as $\boldsymbol{H E I G H T}$, to display the stored parameter, then press $\boldsymbol{E N T E R}$, and so on). Check the asterisk (*) entry key if in rectangular MODE 30 or 31 . Check the decimal (.) entry key if in progressive depth cut MODE 60, 61, 70, or 71 . 
E104 Chuck zero is required.

E105 Chuck zero was attempted before the spindle was up to operating speed.

E106 A switch was pressed while the spindle was ramping up to its operating speed.

E108 Program storage is full.

E109 An attempt was made to retrieve a program that does not exist from storage.

E110 There is already a program stored in the memory location you requested, and if you continue, an existing stored program will be altered.

E111 An attempt was made to turn on the spindle while the machine was in a service mode.
Place the gauge disk on the vacuum chuck and perform a chuck zeroization (III., 7.g., above). Chuck zeroization is required when exiting $S T A N D B Y$ or RESET modes.

Wait until the spindle reaches its operating speed (you should hear a beep when this occurs).

Wait until the spindle reaches its operating speed (you should hear a beep when this occurs).

This error code indicates that all memory spaces are full. If you still want to store a program, you must erase or write over an existing program to make room for a new one.

Check the program ID number requested.

This error code is a warning that an existing program is about to be altered.

The spindle will not run while in a service mode.

Select the proper mode.

E113 The chuck was not unlocked after a chuck Press the UNLOCK key to remove this zero operation. block.

${ }^{I}$ Note: Details on how to store and retrieve programs and a complete list of error and fault codes are provided in the "1006 Dicing Saw Operation Manual" stored near the dicing saw table. 


\section{APPENDIX X.}

\section{SILICON NITRIDE SPUTTERING DEPOSITION}

AND RIE ETCH RATE GRAPHS 


\section{Etch Rate of Low-Stress LPCVD Silicon N itride}

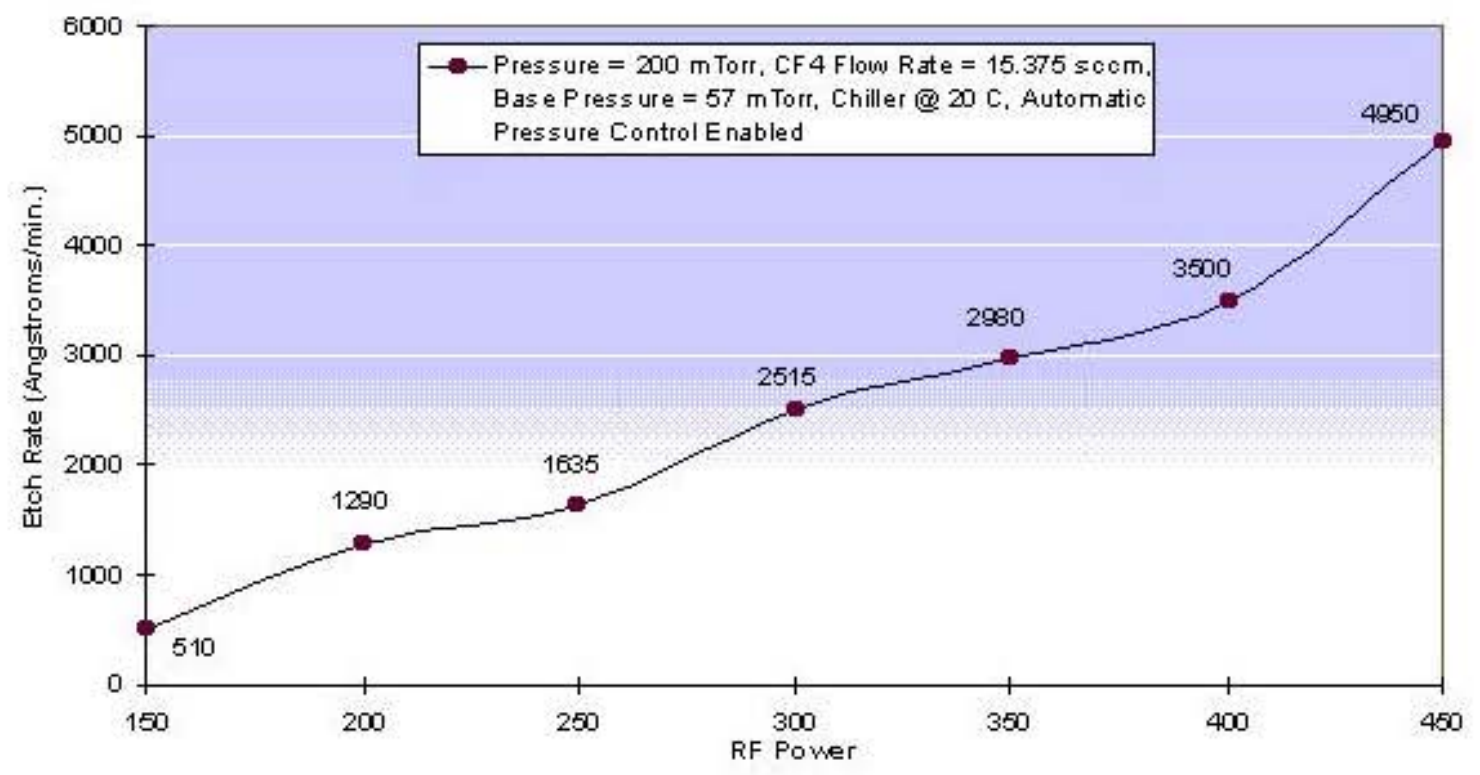

FIGURE 151 - Etch rate as a function of RF power setting for Chronos low-stress LPCVD silicon nitride wafers using a March CS-1701 RIE System.

\section{Etch Rates for Silcon Nitride RF-Sputtered in Nitrogen Gas at $450 \mathrm{~W}$ and $15 \mathrm{mTorr}$}

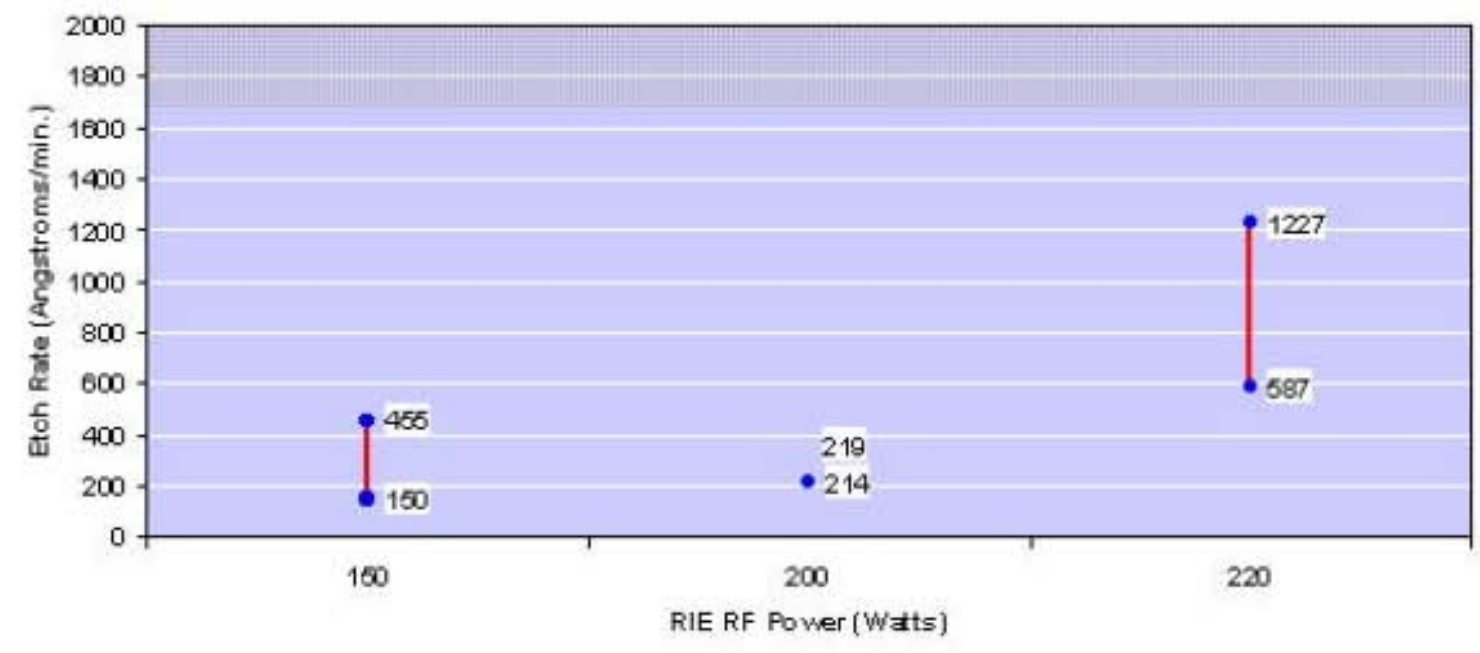

FIGURE 152 - Measured etch rates for $\mathrm{N}_{2}$-RF-planar-magnetron-sputter-deposited silicon nitride (deposited at $450 \mathrm{~W}$ and $15 \mathrm{mTorr}$ ) as a function of RF power setting in the March CS-1701 RIE System at 200 mTorr (automatic pressure control enabled), with $\mathrm{CF}_{4}$ flow rate at $15 \mathrm{sccm}$, base pressure of $40 \mathrm{mTorr}$, and chiller temperature at $25^{\circ} \mathrm{C}$. 


\section{Nitrogen RF-Sputtered Silicon Nitride Deposition Rates}

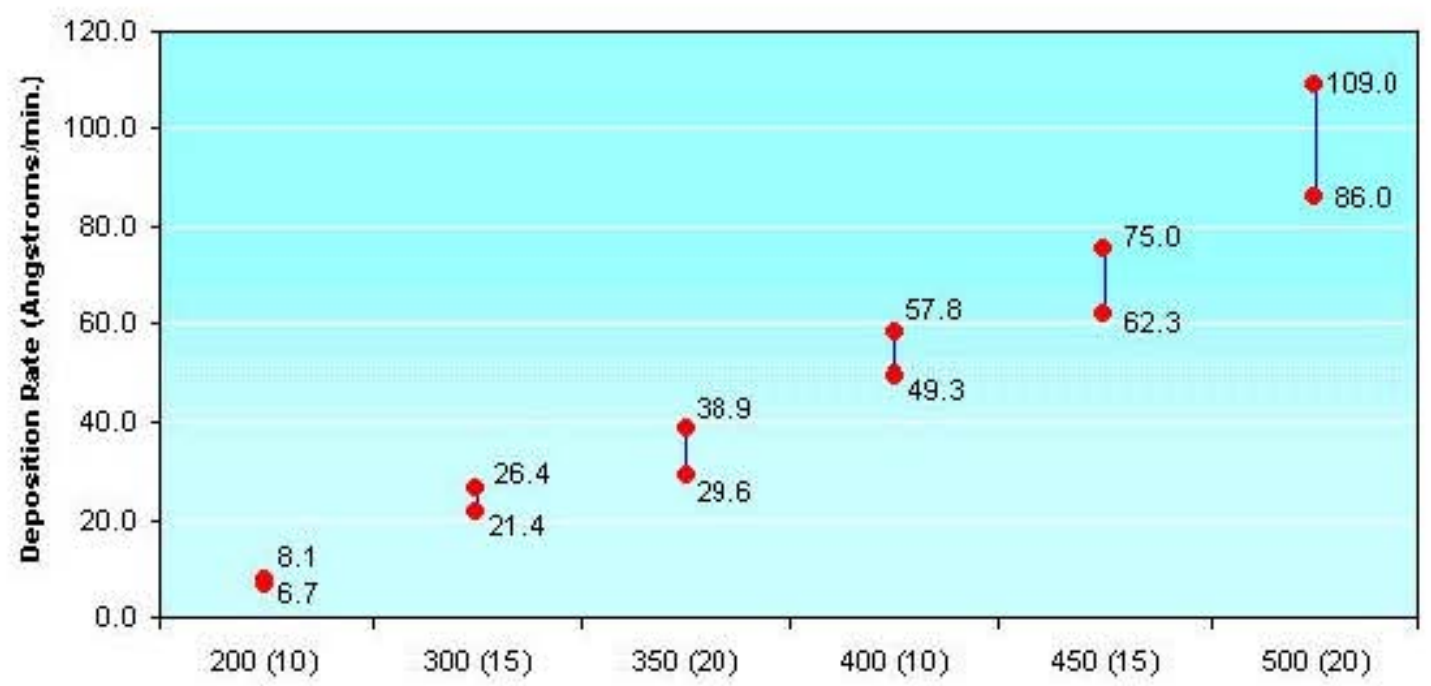

RF Power in Watts (Sputtering Pressure in mTorr)

FIGURE 153 - Sputtering deposition rate ranges for silicon nitride using nitrogen gas at various pressures and RF power settings in the Technics Model 4604 sputtering system. Depths of deposited silicon nitride were measured with both the Tencor profilometer and the Filmetrics F-20 Thin-Film Measurement system.

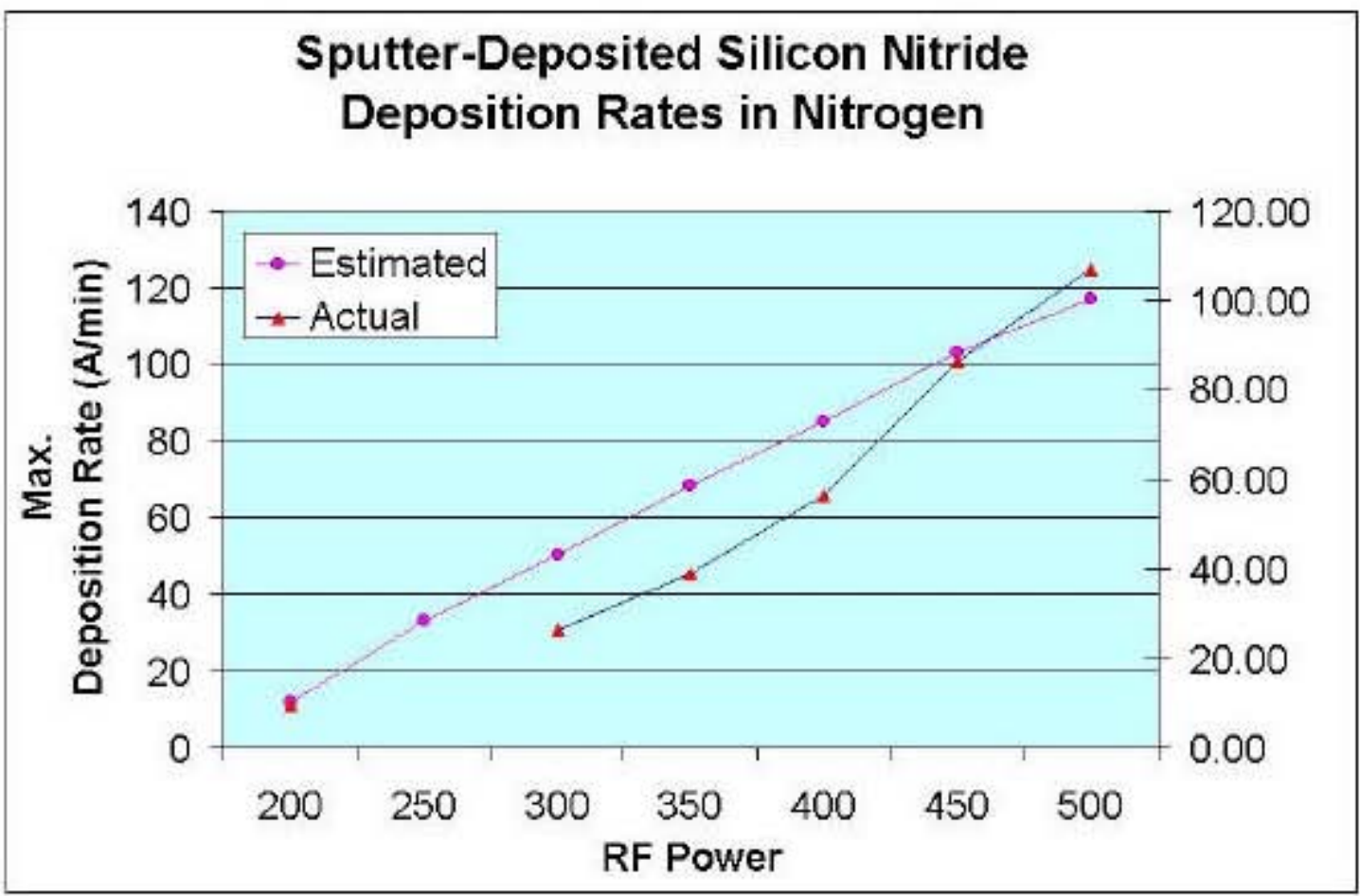

FIGURE 154 - Filmetrics-measured RF planar magnetron sputtering deposition rates for $\mathrm{Si}_{x} \mathrm{~N}_{\mathrm{y}}$ thin films sputtered in nitrogen. 


\section{APPENDIX XI.}

\section{SEM PHOTOS OF SPUTTERED \\ SILICON NITRIDE FLOW SENSORS}



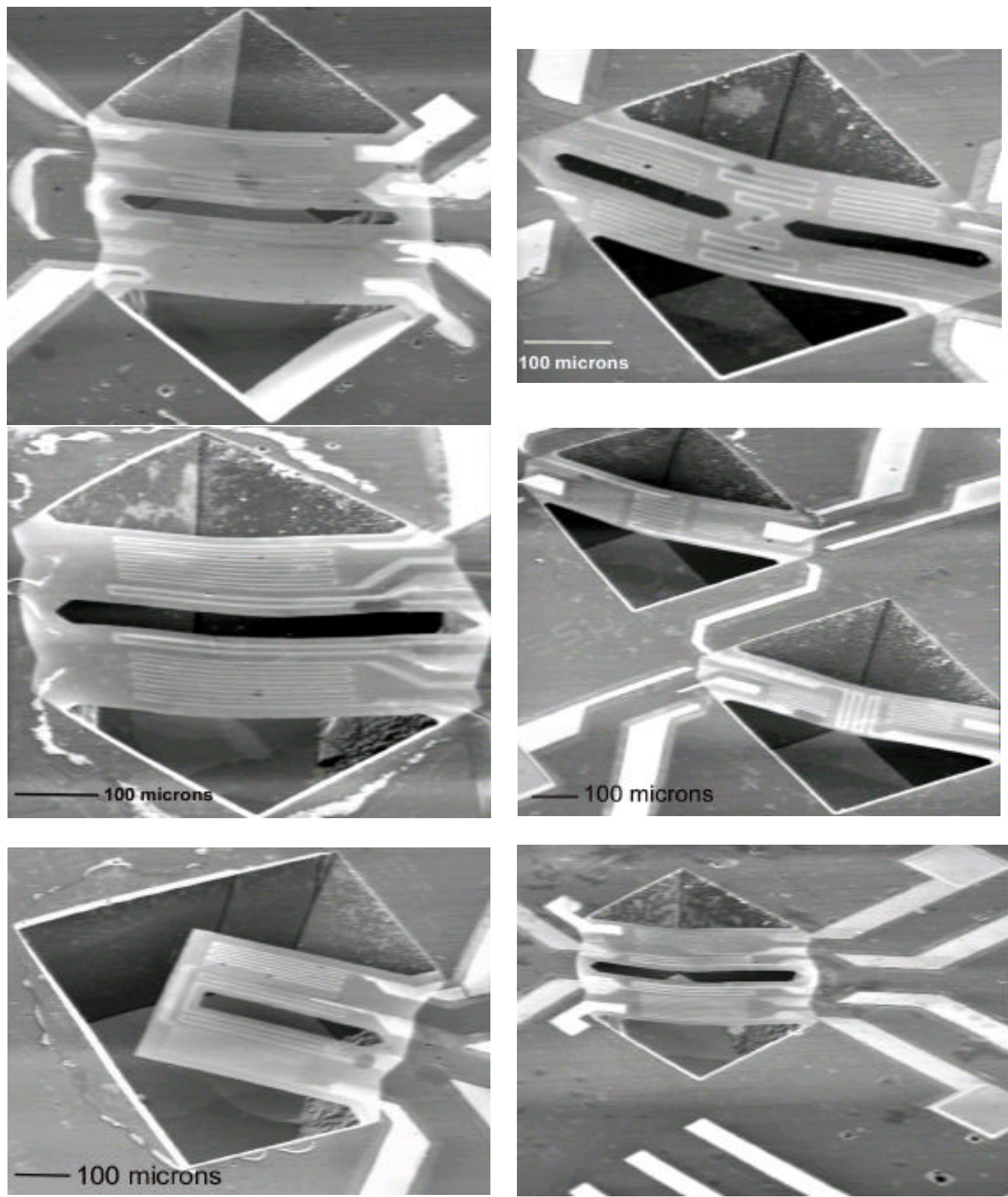

FIGURE 155 - A collage of SEM photos showing flow sensor devices and test structures made with nitrogen-sputtered $\mathrm{Si}_{x} \mathrm{~N}_{\mathrm{y}}$ thin films. Note the buckling of doubly-supported microbridges and warping of simply-supported cantilever structures. 

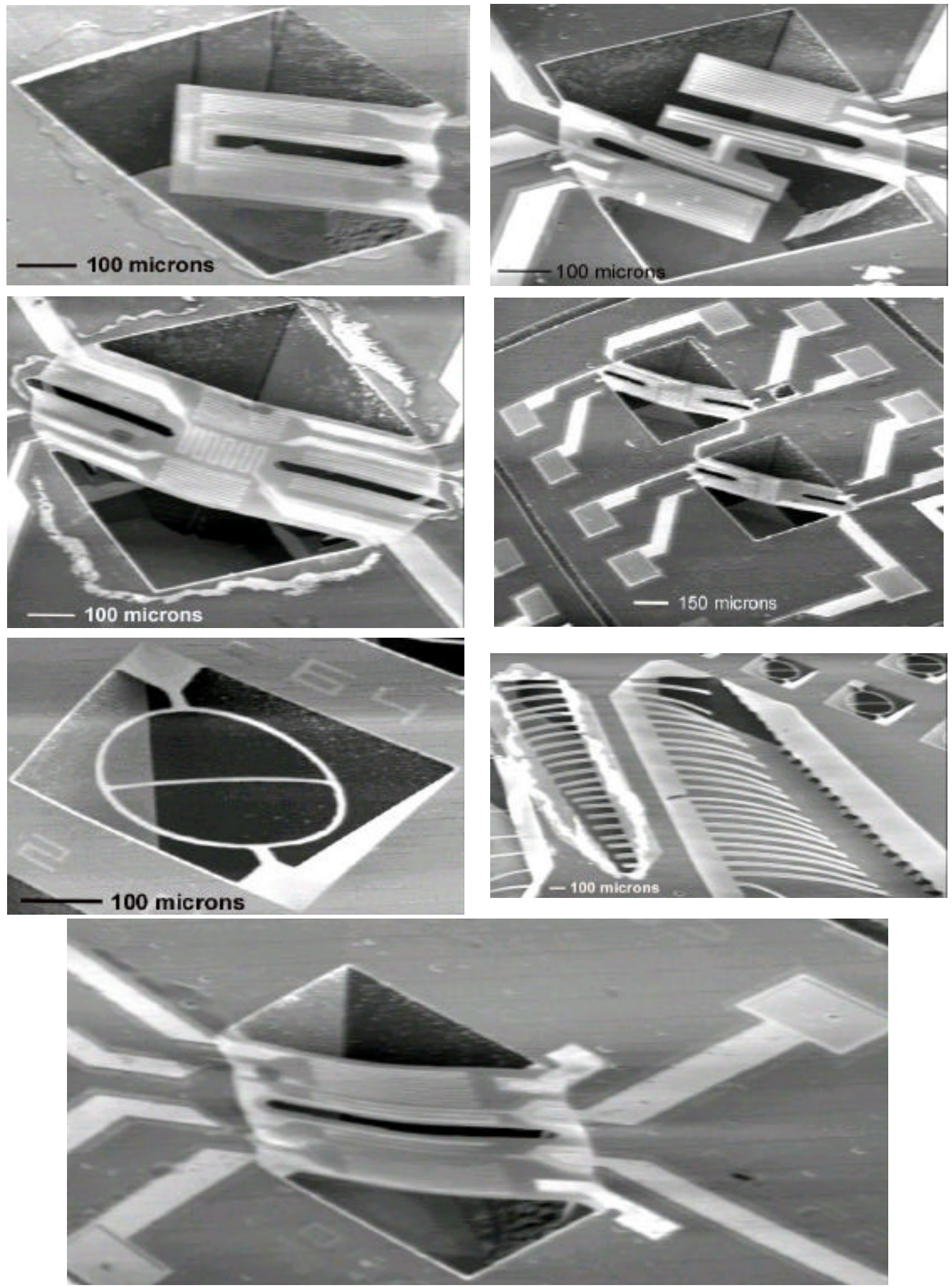

FIGURE 156 - A collage of SEM photos showing flow sensor devices and test structures made with nitrogen-sputtered $\mathrm{Si}_{x} \mathrm{~N}_{\mathrm{y}}$ thin films. Note the buckling of doubly-supported microbridges and warping of simply-supported cantilever structures. 
APPENDIX XII.

FILMTRONICS 700 SERIES SPIN-ON GLASS DATA SHEET 


\section{$\begin{array}{llllllllllllllllll}P & R & O & D & U & C & T & D & E & S & C & R & \text { I } & P & T & \text { I } & O & \mathrm{~N}\end{array}$ SILICATE $\mathrm{SiO}_{2} 700$ SERIES SPIN-ON GLASSES}

Filmtronics' $\mathrm{SiO}_{2}$ film is a solution of glass forming compound. When applied to a surface such as a silicon wafer, it dries to yield a pure film of $\mathrm{SiO}_{2}$. The glass solutions may be applied to a clean, dry substrate and spun at $1500-6000 \mathrm{rpm}$ to form a thin glass layer which is cured at $200^{\circ} \mathrm{C}-900^{\circ} \mathrm{C}$ to densify the resultant silicon dioxide film. The cured films are highly amorphous, crack-free and have a very low density of pinholes and particulates.

$\mathrm{SiO}_{2}$ solution is an alcohol based solution filtered through 0.1 micron filters. Thickness may be varied with spin speed or dilution with isopropanol or ethanol. A shelf life of more than 3 months is possible at $4{ }^{\circ} \mathrm{C}\left(40^{\circ} \mathrm{F}\right)$. Metallic impurities are well below $0.05 \mathrm{ppm}$. After film is spun-on and cured at $400^{\circ} \mathrm{C}$ for one hour, it has a density of $2.1 \mathrm{~g} / \mathrm{cc}$, a refactive index of 1.43 and thickness variation of $1 \%$ with no striations or pinholes.

This undoped oxide film may be used for a diffusion mask in semiconductor processing which is capable of being patterned by standard photolithographic techniques. The solution is also useful in solar cell production and planarization of integrated circuits as an interlevel dielectric.

$\mathrm{SiO}_{2} 700$ is suitable for those applications in microelectronics processing where the film thickness is between $1000 \mathrm{~A}$ to $3000 \mathrm{~A}$. If a thickness greater than $3000 \mathrm{~A}$ is needed, several applications of $\mathrm{SiO}_{2} 700$ may be utilized with a cure of 10 minutes at $200^{\circ} \mathrm{C}$ or 5 minutes at $400^{\circ} \mathrm{C}$ between sucessive spin-ons. The maximum thickness of these films should be limited to $7000 \mathrm{~A}$ to avoid film cracking.

$\mathrm{SiO}_{2} 700$ may also be used as a mask for liquid phase crystal growth on Gallium Arsenide Substrates, or for a capping layer in group III-V semiconductor processing or for a back sealing layer prior to epitaxial silicon deposition in MOS applications.

Filmtronics' $\mathrm{SiO}_{2}$ film may also be used to getter metallic impurities from silicon. The submicroporous structure of the silicon dioxide film has the ability to getter metallic impurities from silicon surfaces at temperatures of $1050^{\circ} \mathrm{C}$ to $1150^{\circ} \mathrm{C}$. Then the silicon slices are cooled at a rate of $1{ }^{\circ} \mathrm{C}$ to $3^{\circ} \mathrm{C}$ per minute.

$\mathrm{SiO}_{2} 700$ has excellent bonding to thermal $\mathrm{SiO}_{2}$, silicon and itself. Adhesion to $\mathrm{CVD}$ films containing phosphorous deteriorates with increasing phosphorous content of CVD film. Adhesion to aluminum depends on aluminum surface and cleaniness. There is a possibility of $\mathrm{SiO}, 700$ crazing or cracking on aluminum features greater than few square microns upon curing at $400^{\circ} \mathrm{C}$ due to poor adhesion or differences in coefficient of expansions. If this occurs, one of our spin-on glasses may be employed. See our SOG Catalogue.

For smoothing uneven wafer topologies or for dielectric layer applications, $\mathrm{SiO}_{2} 700$ may be applied either before or after the deposition of a CVD glass layer as shown at top of next page.
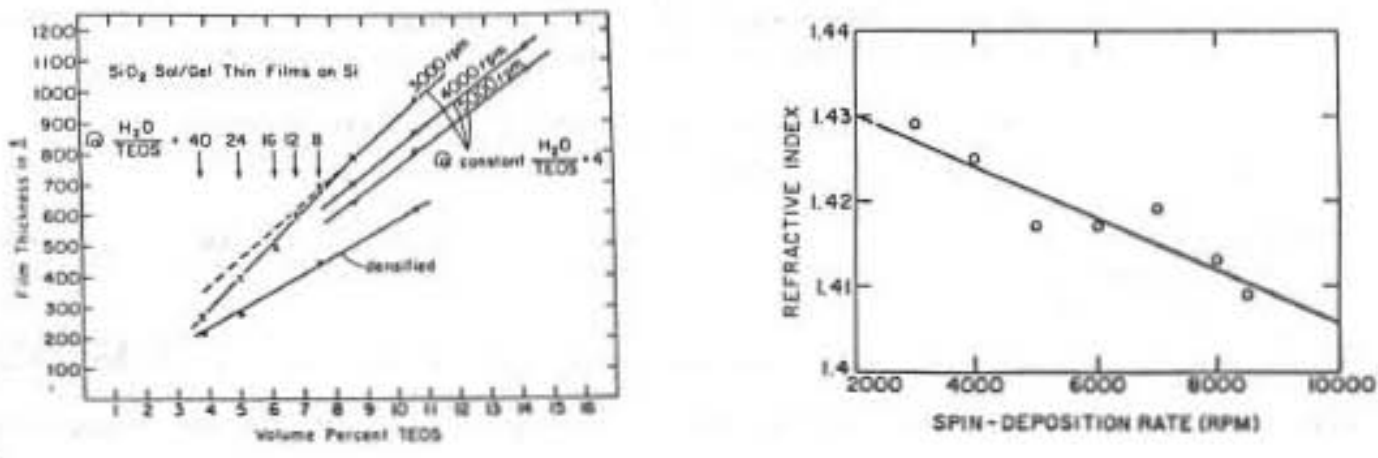

$\cdot 32$. 


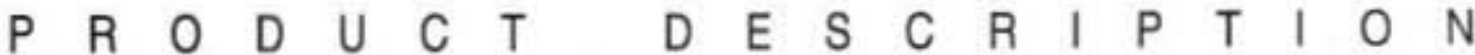 SPIN-ON GLASS 700 A \& B}

\section{Before CVD}

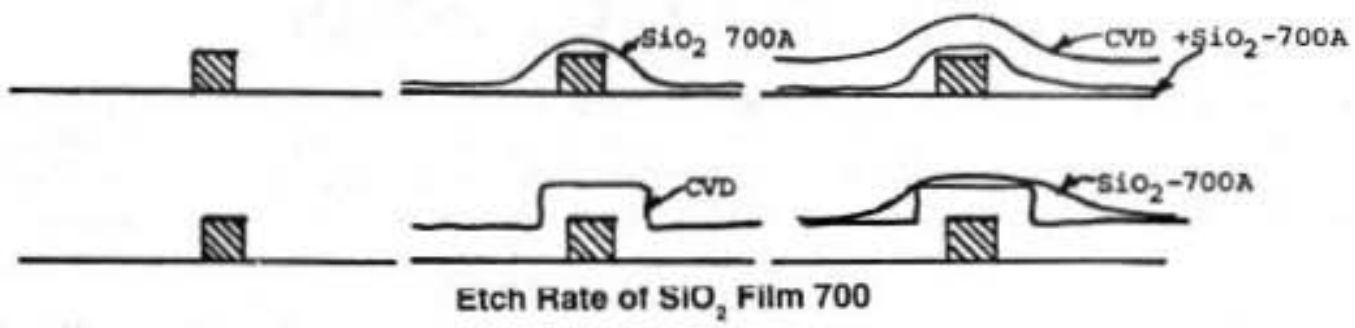

Etch Rate, A/minute

Type of Etch

100:1 BOE, $20^{\circ} \mathrm{C}$

$50: 1 \mathrm{BOE}, 20^{\circ} \mathrm{C}$

$\mathrm{CF}_{1}+\mathrm{O}_{2}$ plasma $\mathrm{CHF}$, plasma

\begin{tabular}{c}
\hline $\mathrm{SiO}_{2} 700\left(400^{\circ} \mathrm{C}\right)$ \\
275 \\
660 \\
1730 \\
600
\end{tabular}

$\mathrm{SIO} \mathrm{O}_{2} 700\left(900^{\circ} \mathrm{C}\right)$

$\begin{array}{rr}50 & 50 \\ 130 & 130 \\ 1140 & 1100 \\ 440 & 440\end{array}$

BOE stands for buffered oxide etch and is made by mixing 100 or 50 parts by volume of $40 \% \mathrm{NH}_{4} \mathrm{~F}$ solution in water with 1 part of $49 \%$ HF.

\begin{tabular}{|c|c|c|c|c|}
\hline & CURE & 700 FILM & & \\
\hline Aefractive index & $\begin{array}{l}425^{\circ} \mathrm{C} \text {, Air } \\
425^{\circ} \mathrm{C} \text {, Air }\end{array}$ & $\begin{array}{l}1.45,0.002 \\
6.7\end{array}$ & Thickness variation (across waler) & $<2 \%$ \\
\hline $\begin{array}{l}\text { Dielectric conatant } \\
\text { (thermal } \mathrm{SiO}_{2}=4.1 \text { ) }\end{array}$ & $\begin{array}{l}425^{\circ} \mathrm{C} \text {, Alr } \\
600^{\circ} \mathrm{C} \text {, Oxygen } \\
800^{\circ} \mathrm{C}, \text { Oxyoen }\end{array}$ & $\begin{array}{l}6.7 \\
5 \cdot 6 \\
4.1\end{array}$ & Padiel striations: & $<1 \%$ (ot Thickness) \\
\hline $\begin{array}{l}\text { Fim Shrinkage, } \% \\
\text { (from } 150^{\circ} \mathrm{C} \text { to } 425^{\circ} \mathrm{C} \text { ) }\end{array}$ & $425^{\circ} \mathrm{C}$, Air & $15+2$ & Intrinsic film stress: & $2 \times 10^{4}$ dynes $\mathrm{cm}^{2}$ \\
\hline $\begin{array}{l}\text { Exch rate in } 50.1 \text { B.O.E. } \\
\text { (rolative to thermal codide) }\end{array}$ & $\begin{array}{l}425^{\circ} \mathrm{C}, \text { Alr } \\
600^{\circ} \mathrm{C} \text {, Oxygen } \\
800^{\circ} \mathrm{C} \text {, Orygen } \\
\text { B00 C, Steam }\end{array}$ & $\begin{array}{l}4.0 \\
3.0 \\
2.2 \\
1.2\end{array}$ & $\begin{array}{l}\text { Coefficient of stress: } \\
\text { Moblle ion contamination: }\end{array}$ & $\begin{array}{l}1 \times 10^{4} \mathrm{~K}^{-1} \text { (essimated) } \\
5 \times 10^{4} \mathrm{~cm}^{2} \text { (typical) }\end{array}$ \\
\hline
\end{tabular}

$\mathrm{SiO}_{2}$ films should be used in a well ventilated area to avoid inhalation of vapors as well as skin contact.

Any standard dopant (or photoresist) spinner that is well ventilated can be used. No special ambient conditions are required.

1. The solution should be brought to room temperature 24 hours prior to spin-on application.

2. Dispense enough material on to the wafer to cover one-third to half of the wafer.

3. Spin the wafer for 20 seconds at $2000-6000 \mathrm{rpm}$ to obtain the desired film thickness.

4. Probake at $90^{\circ} \mathrm{C}-100^{\circ} \mathrm{C}$ for 10 minutes in air (this step is not necessary if the temperature is ramped in the cure step).

5. Cure the film at $400^{\circ} \mathrm{C}-450^{\circ} \mathrm{C}$ (in air) for 1 hour in an oven or on a hot plate. Higher cure temperature will improve film properties. It is suggested that the spun glass films be cured for 15 minutes at $100^{\circ} \mathrm{C}$ followed by 60 minutes at $400^{\circ} \mathrm{C}$ depending on application of film.

Film Thickness As A Function Of Spin-Speed

(One hour cure at $400^{\circ} \mathrm{C}$ )

Spin Speed, rpm

$\mathrm{SiO}_{2}-700 \mathrm{~A}$ Thickness, A

$\mathrm{SiO}_{2}-700 \mathrm{~B}$ Thickness, $\mathrm{A}$

$\begin{array}{lll}2000 & 3000 & 4000 \\ 2000 & 1620 & 1395 \\ 2970 & 2240 & 2100\end{array}$

5000

1210

1820 


\section{APPENDIX XIII.}

SUCCESSFUL LPCVD $\mathrm{SI}_{3} \mathrm{~N}_{4}$ FLOW SENSOR FABRICATION SUMMARY 
TABLE XXX

SUCCESSFUL LPCVD $\mathrm{SI}_{3} \mathrm{~N}_{4}$ FLOW SENSOR FABRICATION SUMMARY MATCHED WITH EXISTING MICROFAB COURSE LABORATORY EXPERIMENTS

\begin{tabular}{|c|c|c|}
\hline $\begin{array}{c}\text { Flow Sensor } \\
\text { Processing Step }\end{array}$ & Illustration & $\begin{array}{c}\text { Laboratory } \\
\text { Experiment(s) }\end{array}$ \\
\hline $\begin{array}{l}\text { Cleanroom protocol } \\
\text { and other } \\
\text { preliminaries }\end{array}$ & & $\begin{array}{l}\text { Laboratory } \\
\text { Introduction: } \\
\text { Cleanroom Safety, } \\
\text { Protocols, and } \\
\text { Operational Procedures } \\
\text { Experiment \#2: } \\
\text { Introduction To Basic } \\
\text { Cleanroom Equipment } \\
\end{array}$ \\
\hline \multicolumn{3}{|l|}{$\begin{array}{l}\text { Obtain (100) silicon } \\
\text { wafers with low-stress } \\
\text { LPCVD } \mathrm{Si}_{3} \mathrm{~N}_{4} \text { from } \\
\text { one or more external } \\
\text { vendor(s) }\end{array}$} \\
\hline Initial Wafer Clean & (100) Si wafer with LPCVD Sl, $\mathrm{N}_{4}$ & $\begin{array}{l}\text { Experiment \#3: Wafer } \\
\text { Preparation and Initial } \\
\text { Cleaning (Base and Acid } \\
\text { Clean) }\end{array}$ \\
\hline $\begin{array}{l}\text { Flow sensor resistor } \\
\text { metallization } \\
\text { deposition: DC planar } \\
\text { magnetron sputtering } \\
\text { of platinum with RF } \\
\text { planar magnetron } \\
\text { sputtered chromium } \\
\text { adhesion layer }\end{array}$ & (100) Si wafer with LPCVD Sil $\mathrm{N}_{4}$ & $\begin{array}{l}\text { Experiment \#9: Film } \\
\text { Deposition Using } \\
\text { Sputtering }\end{array}$ \\
\hline $\begin{array}{l}\text { Flow sensor gold } \\
\text { metallization } \\
\text { deposition for pad } \\
\text { areas and traces }\end{array}$ & (100) Si wafer with LPCVD Sij $N_{4}$ & $\begin{array}{l}\text { Experiment \#9: Film } \\
\text { Deposition Using } \\
\text { Sputtering }\end{array}$ \\
\hline $\begin{array}{l}\text { Application of positive } \\
\text { photoresist and } \\
\text { subsequent } \\
\text { photolithographic } \\
\text { imaging/patterning }\end{array}$ & (100) Si wafer with LPCVD SI, $N_{4}$ & $\begin{array}{l}\text { Experiment \#7a: } \\
\text { Photolithography Using } \\
\text { Etching Techniques. } \\
\text { Use the Gold Metal } \\
\text { Level } 2 \text { lightfield } \\
\text { photomask. }\end{array}$ \\
\hline
\end{tabular}




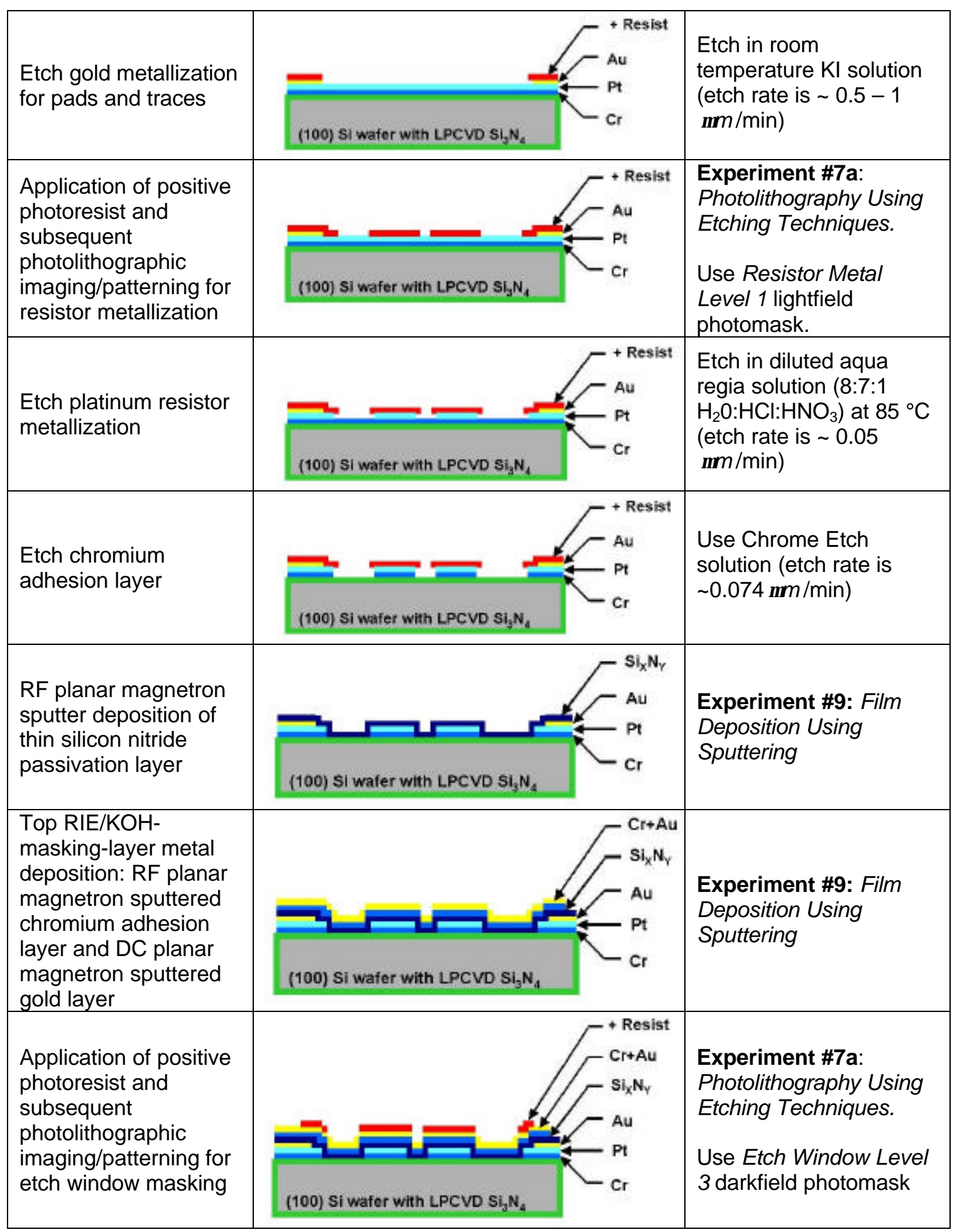




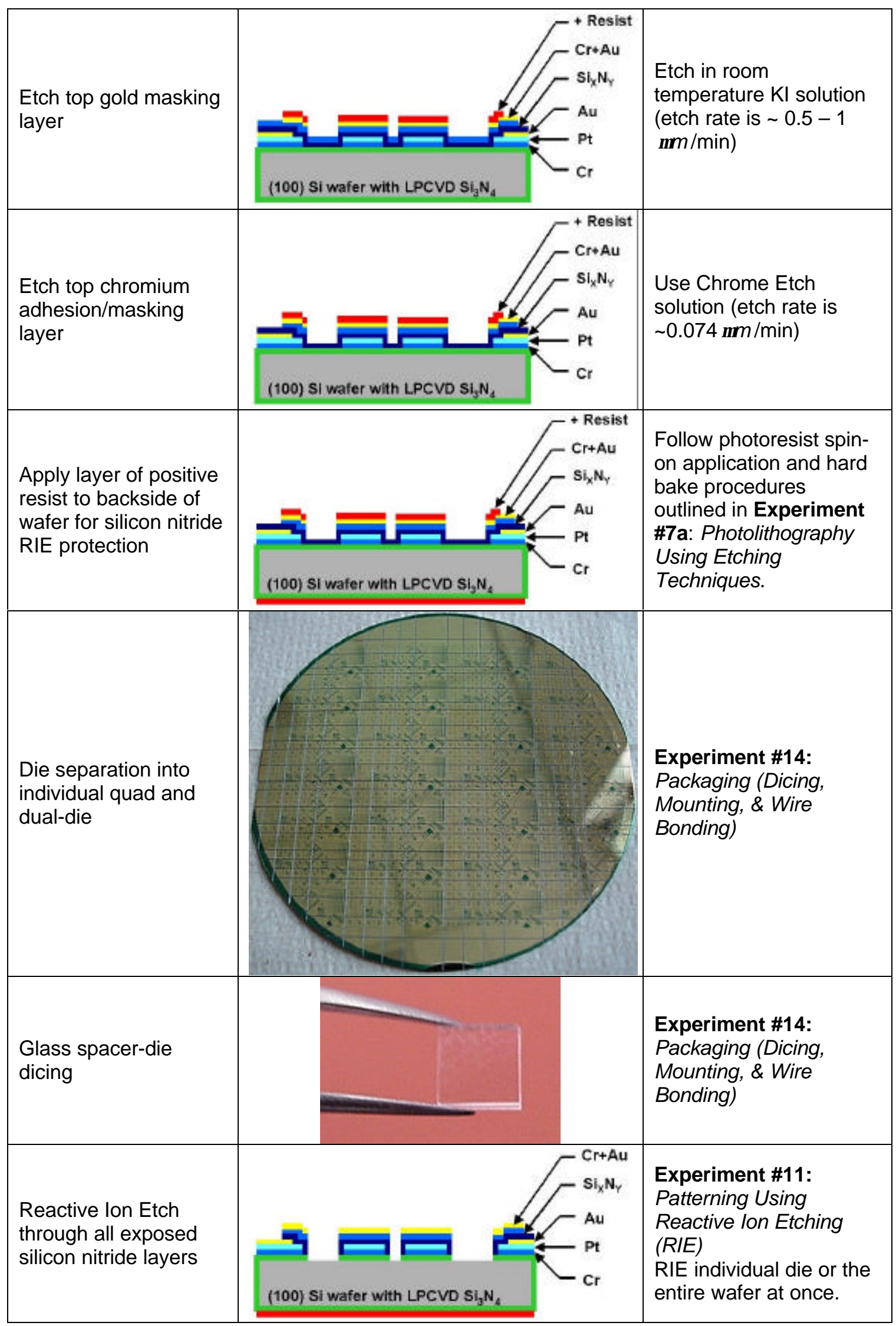




\begin{tabular}{|c|c|c|}
\hline $\begin{array}{l}\text { Individual quad/duo } \\
\text { die } \mathrm{KOH} \text { anisotropic } \\
\text { bulk-micromachining } \\
\text { etch to suspend } \\
\text { sensor microbridge } \\
\text { and cantilever } \\
\text { structures }\end{array}$ & (100) Si wafer with LPCVD Sis & $\begin{array}{l}\text { Experiment \#1: Bulk } \\
\text { Micromachining (with } \\
\mathrm{KOH} \text { ) }\end{array}$ \\
\hline $\begin{array}{l}\text { Remove gold and } \\
\text { chromium masking } \\
\text { layers from individual } \\
\text { quad/dual die }\end{array}$ & (100) Si wafer with LPCVD Si, $N_{4}$ & $\begin{array}{l}\text { Etch in room } \\
\text { temperature } \mathrm{KI} \text { solution } \\
(\sim 0.5-1 \mu \mathrm{m} / \mathrm{min}) \\
\text { followed by etching in } \\
\text { Chrome Etch solution } \\
(\sim 0.074 \mu \mathrm{m} / \mathrm{min}) .\end{array}$ \\
\hline $\begin{array}{l}\text { Flow sensor electrical } \\
\text { continuity and viability } \\
\text { testing }\end{array}$ & and & $\begin{array}{l}\text { Use the Wentworth Labs } \\
\text { probe station to } \\
\text { investigate individual } \\
\text { flow sensor electrical } \\
\text { continuity and viability } \\
\text { before packaging is } \\
\text { attempted. } \\
\text { Locate devices with } \\
\mathrm{R}_{\cup} \approx \mathrm{R}_{\mathrm{D}} \text { (resistances } \\
\text { within } \sim 5 \% \text { of each } \\
\text { other). }\end{array}$ \\
\hline $\begin{array}{l}\text { Flow sensor DIP-24 } \\
\text { device packaging }\end{array}$ & 算 & $\begin{array}{l}\text { Using Epo-Tek® 353ND } \\
\text { epoxy, thoroughly mix } \\
\text { together } 10 \text { parts of "A" } \\
\text { solution with } 1 \text { part "B" } \\
\text { (hardener) using the end } \\
\text { of a paper clip. } \\
\text { Bond a } 45^{\circ} \text { rotated } \\
\text { glass-die spacer to } \\
\text { bottom of DIP-24 device } \\
\text { cavity and a quad flow } \\
\text { sensor die onto the top } \\
\text { of the spacer. } \\
\text { Cure epoxy in an oven } \\
\text { at } \sim 140^{\circ} \mathrm{C} \text { for } \sim 4-5 \\
\text { minutes. }\end{array}$ \\
\hline Wire-bonding & (100) Si wafer with LPCVD Sij, & $\begin{array}{l}\text { Wire-bond good devices } \\
\text { with } \mathrm{R}_{\mathrm{U}} \approx \mathrm{R}_{\mathrm{D}} \text { following } \\
\text { guidelines in } \\
\text { Experiment \#14: } \\
\text { Packaging (Dicing, } \\
\text { Mounting, \& Wire } \\
\text { Bonding) } \\
\text { IMPORTANT: Do NOT } \\
\text { wirebond to DIP pins } 2 \text {, } \\
6,9,10,11 \text {, and } 24 .\end{array}$ \\
\hline
\end{tabular}




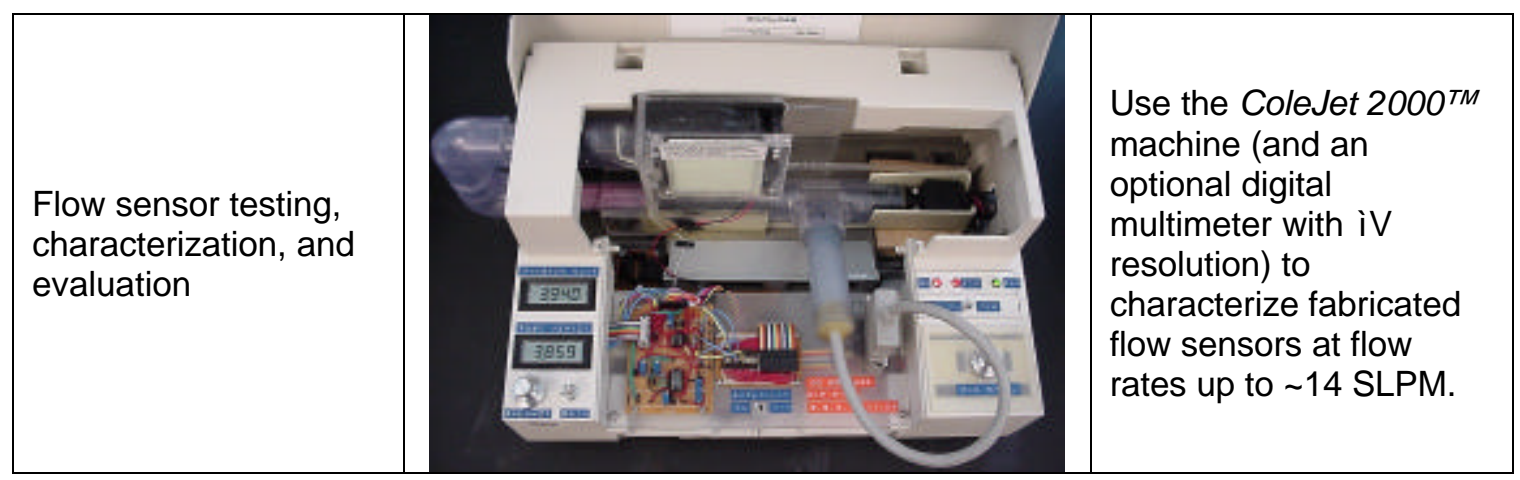

* This refers to on-line laboratory experiments at $h t t p: / / m i t g h m r . s p d . l o u i s v i l l e . e d u /$ which provide general procedural guidance to students using the University of Louisville's clean room equipment and processes for the microfabrication course laboratories. 


\section{APPENDIX XIV.}

DETAILED OUTLINE OF LPCVD $\mathrm{SI}_{3} \mathrm{~N}_{4}$ FLOW SENSOR FABRICATION 


\begin{tabular}{|c|c|}
\hline Process Step & "Description \\
\hline Outsourcing & $\begin{array}{l}\text { Obtain (100)-oriented silicon wafers with } \sim 0.5 \mu \mathrm{m} \text { of low- } \\
\text { stress LPCVD } \mathrm{Si}_{3} \mathrm{~N}_{4} \text { from external vendor(s). }\end{array}$ \\
\hline \multirow[t]{2}{*}{ Wafer Cleaning: Base Clean } & $\begin{array}{l}\text { Base clean wafer for } 15 \text { min. in } 5: 1: 1 \mathrm{H}_{2} \mathrm{O}: \mathrm{NH}_{4} \mathrm{OH}: \mathrm{H}_{2} \mathrm{O}_{2} \\
\text { mix. Heat } \mathrm{DI} \mathrm{H}_{2} \mathrm{O} \text { and } \mathrm{NH}_{4} \mathrm{OH} \text { solutions together to } \\
70( \pm 5){ }^{\circ} \mathrm{C} \text { in a glass beaker on a hotplate, then add } \mathrm{H}_{2} \mathrm{O}_{2} \text {. } \\
\text { Immerse wafer once solution bubbles vigorously. }\end{array}$ \\
\hline & Rinse thoroughly under running DI water (3-5 minutes). \\
\hline \multirow[t]{3}{*}{ Wafer Cleaning: Acid Clean } & $\begin{array}{l}\text { Clean wafer for } 15 \text { min. in fresh } 4: 1: 1 \mathrm{H}_{2} \mathrm{O}: \mathrm{HCl}: \mathrm{H}_{2} \mathrm{O}_{2} \text { mix. } \\
\text { Heat } \mathrm{DI} \mathrm{H}_{2} \mathrm{O} \text { and } \mathrm{HCl} \text { solutions together to } 70( \pm 5)^{\circ} \mathrm{C} \text { in a } \\
\text { glass beaker on a hotplate, then add } \mathrm{H}_{2} \mathrm{O}_{2} \text {. } \\
\text { Immerse wafer once solution bubbles vigorously. }\end{array}$ \\
\hline & Rinse thoroughly under running DI water (3-5 minutes). \\
\hline & Blow dry wafer with $\mathrm{N}_{2}$ gun. \\
\hline \multirow[t]{2}{*}{$\begin{array}{l}\text { Sputter Deposition: } \\
\text { Chromium Adhesion Layer }\end{array}$} & $\begin{array}{l}\text { Chiller for sputtering system should be at } \sim 20^{\circ} \mathrm{C} \text {. } \\
\text { Purge argon line with needle valve fully open for at least } 10 \\
\text { min. prior to actual sputtering. } \\
\text { Sputter chromium plasma onto shutter for at least } 5 \text { min. } \\
\text { prior to actual deposition (for oxygen gettering and to } \\
\text { remove oxide layer from sputtering target). }\end{array}$ \\
\hline & $\begin{array}{l}\text { RF planar magnetron sputter } 99.99 \% \text { pure chromium } \\
\text { (maximum target size for all sputtering: } 3.99 \text { in. dia. by } 0.25 \\
\text { in. thick) at } 250 \text { Watts in argon at } 10 \text { mTorr for } 30 \text { seconds } \\
\text { for desired thickness of } \sim 120 \AA \text { ( } 240-292 \AA / \text { min } \\
\text { deposition rate) with initial base press. }<1.0 \times 10^{-5} \text { Torr. }\end{array}$ \\
\hline Sputter Deposition: Platinum & $\begin{array}{l}\text { Without breaking vacuum from chromium deposition, } \\
\text { DC planar magnetron sputter-deposit } 99.99 \% \text { pure } \\
\text { platinum at } 200 \text { Watts in argon at } 10 \mathrm{mTorr} \text { for } 10 \mathrm{~min} \text {. for } \\
\text { target thickness of } \sim 700-870 \AA \text { ( } ~ \\
\text { rate) with initial base pressure below } 1.0 \times 10^{-5} \text { Torr. }\end{array}$ \\
\hline \multirow[t]{3}{*}{ Sputter Deposition: Gold } & $\begin{array}{l}\text { Although platinum does not easily oxidize, it is strongly } \\
\text { suggested that gold deposition immediately follow platinum } \\
\text { deposition and breaking vacuum on the sputtering system. }\end{array}$ \\
\hline & $\begin{array}{l}\text { Chiller for sputtering system should be at } \sim 20^{\circ} \mathrm{C} \text {. } \\
\text { Purge argon line with needle valve fully open for at least } 10 \\
\text { min. prior to deposition. }\end{array}$ \\
\hline & $\begin{array}{l}\text { DC planar magnetron sputter-deposit } 99.9969 \% \text { pure gold } \\
\text { at } 350 \text { Watts in argon at } 10 \mathrm{mTorr} \text { for } 8 \mathrm{~min} \text {. for target } \\
\text { thickness of } 0.260-0.375 \mu \mathrm{m}(\sim 325-517 \AA \text { A } / \mathrm{min} \text {. } \\
\text { deposition rate) with initial base press. }<1.0 \times 10^{-5} \text { Torr. }\end{array}$ \\
\hline OPTIONAL Adhesion Test & $\begin{array}{l}\text { Using electrical tape, or similar, check adhesion of } \mathrm{Cr} / \mathrm{Pt} / \mathrm{Au} \\
\text { metallization to } \mathrm{Si}_{3} \mathrm{~N}_{4} \text { substrate in a "non-device" area. } \\
\text { Note that electrical tape leaves a residue that will affect } \\
\text { subsequent photolithographic patterning. This residue may } \\
\text { be removed with mineral spirits. }\end{array}$ \\
\hline \multirow[t]{2}{*}{ Gold Pad/Routing Patterning } & $\begin{array}{l}\text { Spin on Shipley } 1813 \text { positive photoresist on top side of } \\
\text { wafer with SPREAD at } 500 \text { RPM for } 2 \text { sec. and SPIN at } \\
4500 \text { RPM for } 12 \text { sec. }\end{array}$ \\
\hline & $\begin{array}{l}\text { Soft bake for either } 20-30 \text { min. at } 95^{\circ} \mathrm{C} \text { in a vented oven } \\
\text { or for } 5 \text { min. at } 115^{\circ} \mathrm{C} \text { on a hotplate. }\end{array}$ \\
\hline
\end{tabular}




\begin{tabular}{|c|c|}
\hline & $\begin{array}{l}\text { Under a microscope, inspect Pt resistor metallization areas. } \\
\text { Look for damaged or peeling-off photoresist. A hazy } \\
\text { "snow" effect around (not sharply defined) resistor edges } \\
\text { under high magnification means more Pt etching is needed. } \\
\text { If this is the case, try } 10-20 \text { sec. more etching, with } \\
\text { heated and normal DI rinse described above. }\end{array}$ \\
\hline \multirow[t]{2}{*}{$\begin{array}{l}\text { Chromium Adhesion Layer } \\
\text { Patterning/Delineation }\end{array}$} & $\begin{array}{l}\text { Immerse wafer in Chrome Etch solution for } \sim 5-8 \text { sec. } \\
\text { WARNING: Do not unnecessarily prolong this step. Too } \\
\text { much chromium etching will adversely affect Pt adhesion, } \\
\text { and may cause liftoff of most or all of the Pt layer. }\end{array}$ \\
\hline & Rinse in DI water for approx. 3 min. \\
\hline \multirow[t]{2}{*}{ Remove Residual Photoresist } & $\begin{array}{l}\text { Remove charred photoresist with acetone and a } \\
\text { subsequent } 5 \text { min. Nano-Strip® soak, with agitation. }\end{array}$ \\
\hline & Rinse wafer in running DI water and dry with $\mathrm{N}_{2}$ gun. \\
\hline OPTIONAL: Dehydrate Bake & $\begin{array}{l}\text { Place wafer on } 150^{\circ} \mathrm{C} \text { hotplate to evaporate all moisture } \\
\text { for faster sputtering pump-down in next step. }\end{array}$ \\
\hline \multirow[t]{3}{*}{$\begin{array}{l}\text { Si }_{X} \mathrm{~N}_{Y} \text { Passivation Layer } \\
\text { Sputter Deposition }\end{array}$} & $\begin{array}{l}\text { Chiller for sputtering system should be at } \sim 20^{\circ} \mathrm{C} \text {. } \\
\text { Turn-on } \mathrm{N}_{2} \text { tank and connect } \mathrm{N}_{2} \text { line to GAS \#2 port on } \\
\text { sputterer (with } \mathrm{N}_{2} \text { flowing as connection is made to keep air } \\
\text { out of the line), or temporarily disconnect nitrogen purge } \\
\text { line and connect to GAS \#2 port. } \\
\text { Purge nitrogen line with needle flow valve fully open for at } \\
\text { least } 10 \text { min. prior to deposition. }\end{array}$ \\
\hline & $\begin{array}{l}\mathrm{RF} \text { planar magnetron sputter-deposit } 99.9 \% \text { pure } \mathrm{Si}_{3} \mathrm{~N}_{4} \text { at } \\
450 \text { Watts in nitrogen at } 15 \mathrm{mTorr} \text { for } \sim 7 \text { min. for target } \\
\text { thickness of } \sim 0.05 \mu \mathrm{m} \text { with initial base pressure below } \\
2.0 \times 10^{-5} \text { Torr. }\end{array}$ \\
\hline & $\begin{array}{l}\text { After deposition, disconnect } \mathrm{N}_{2} \text { line from GAS \#2 port and } \\
\text { reconnect to nitrogen purge line before bringing sputtering } \\
\text { chamber up to atmospheric pressure. }\end{array}$ \\
\hline \multirow[t]{2}{*}{$\begin{array}{l}\text { Sputter Deposition: Chromium } \\
\text { Adhesion Layer for RIE/KOH } \\
\text { Mask }\end{array}$} & $\begin{array}{l}\text { Chiller for sputtering system should be at } \sim 20^{\circ} \mathrm{C} \text {. } \\
\text { Purge argon line with needle valve fully open for at least } 10 \\
\text { min. prior to actual sputtering. } \\
\text { Sputter chromium plasma onto shutter for at least } 5 \text { min. } \\
\text { prior to actual deposition. }\end{array}$ \\
\hline & $\begin{array}{l}\text { RF planar magnetron sputter } 99.99 \% \text { pure chromium at } \\
250 \text { Watts in argon at } 10 \text { mTorr for } 30 \text { seconds for desired } \\
\text { thickness of } \sim 120 \AA \text { ( } \sim 240-292 \AA / \text { min deposition rate }) \\
\text { with initial base pressure below } 1.0 \times 10^{-5} \text { Torr. }\end{array}$ \\
\hline $\begin{array}{l}\text { Sputter Deposition: Top Gold } \\
\text { RIE/KOH Mask }\end{array}$ & $\begin{array}{l}\text { Without breaking vacuum from chromium deposition, } \\
\text { DC sputter-deposit } 99.9969 \% \text { pure gold at } 350 \text { Watts in } \\
\text { argon at } 10 \text { mTorr for } 4 \text { min. for target thickness of } \sim 0.165 \\
\mu m(\sim 325-517 \AA \text { Amin. deposition rate }) \text { with initial base } \\
\text { pressure below } 1.0 \times 10^{-5} \text { Torr. }\end{array}$ \\
\hline \multirow[t]{3}{*}{$\begin{array}{l}\text { Top Layer RIE/KOH Gold Mask } \\
\text { Patterning }\end{array}$} & $\begin{array}{l}\text { Spin on Shipley } 1813 \text { positive photoresist on top side of } \\
\text { wafer with SPREAD at } 500 \text { RPM for } 2 \text { sec. and SPIN at } \\
4500 \text { RPM for } 10 \text { sec. }\end{array}$ \\
\hline & $\begin{array}{l}\text { Soft bake for either } 20-30 \text { min. at } 95^{\circ} \mathrm{C} \text { in a vented oven } \\
\text { or for } 5 \text { min. at } 115^{\circ} \mathrm{C} \text { on a hotplate. }\end{array}$ \\
\hline & $\begin{array}{l}\text { Use mask alignment/registration features/areas on wafer to } \\
\text { align to Etch Window Level } 3 \text { darkfield photomask and } \\
\text { expose in contact mask aligner for } 15 \mathrm{sec} \text { to } 436 \mathrm{~nm} \\
\text { wavelength UV light at } 6.4 \mathrm{~mW} / \mathrm{cm}^{2} \text { intensity. }\end{array}$ \\
\hline
\end{tabular}




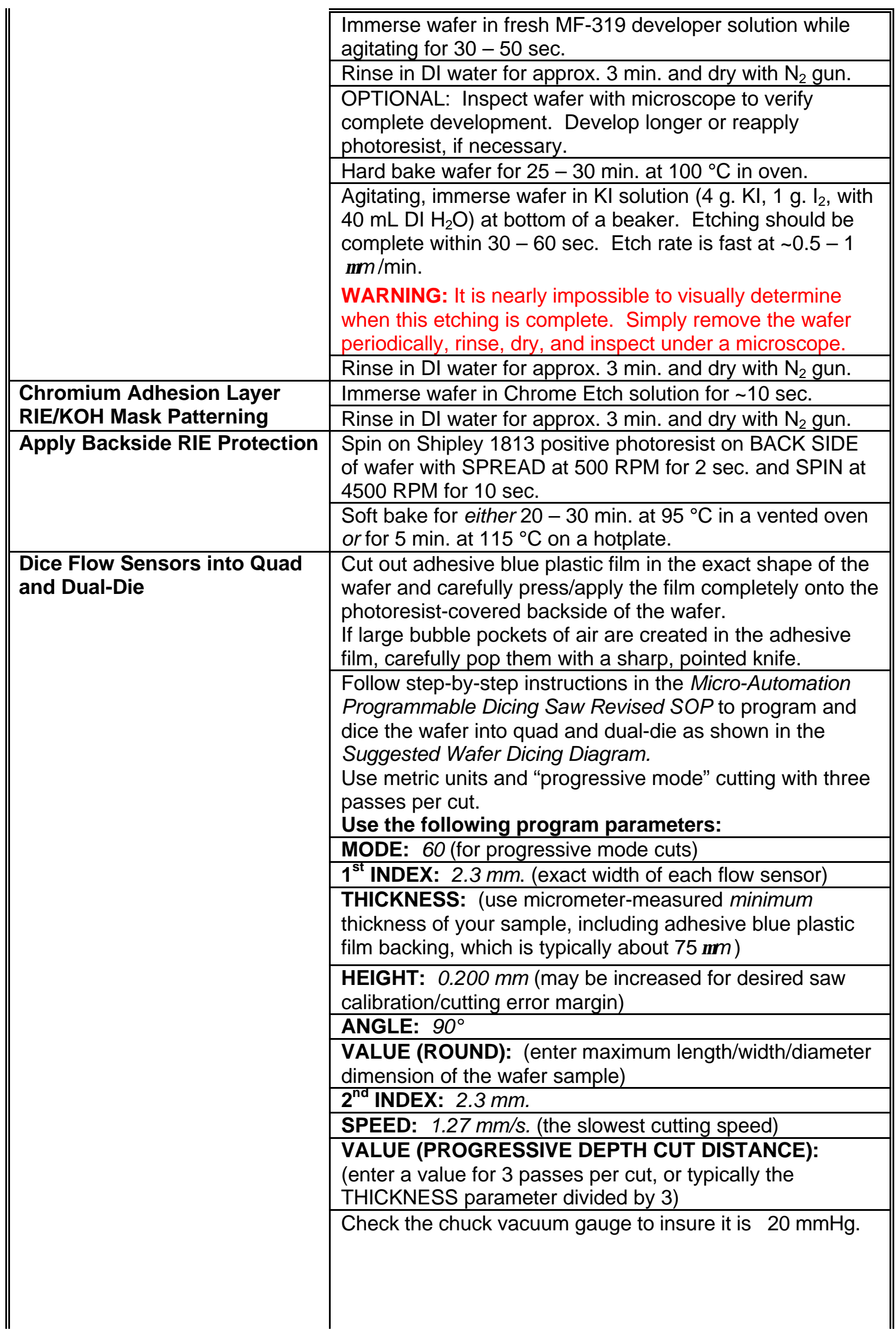




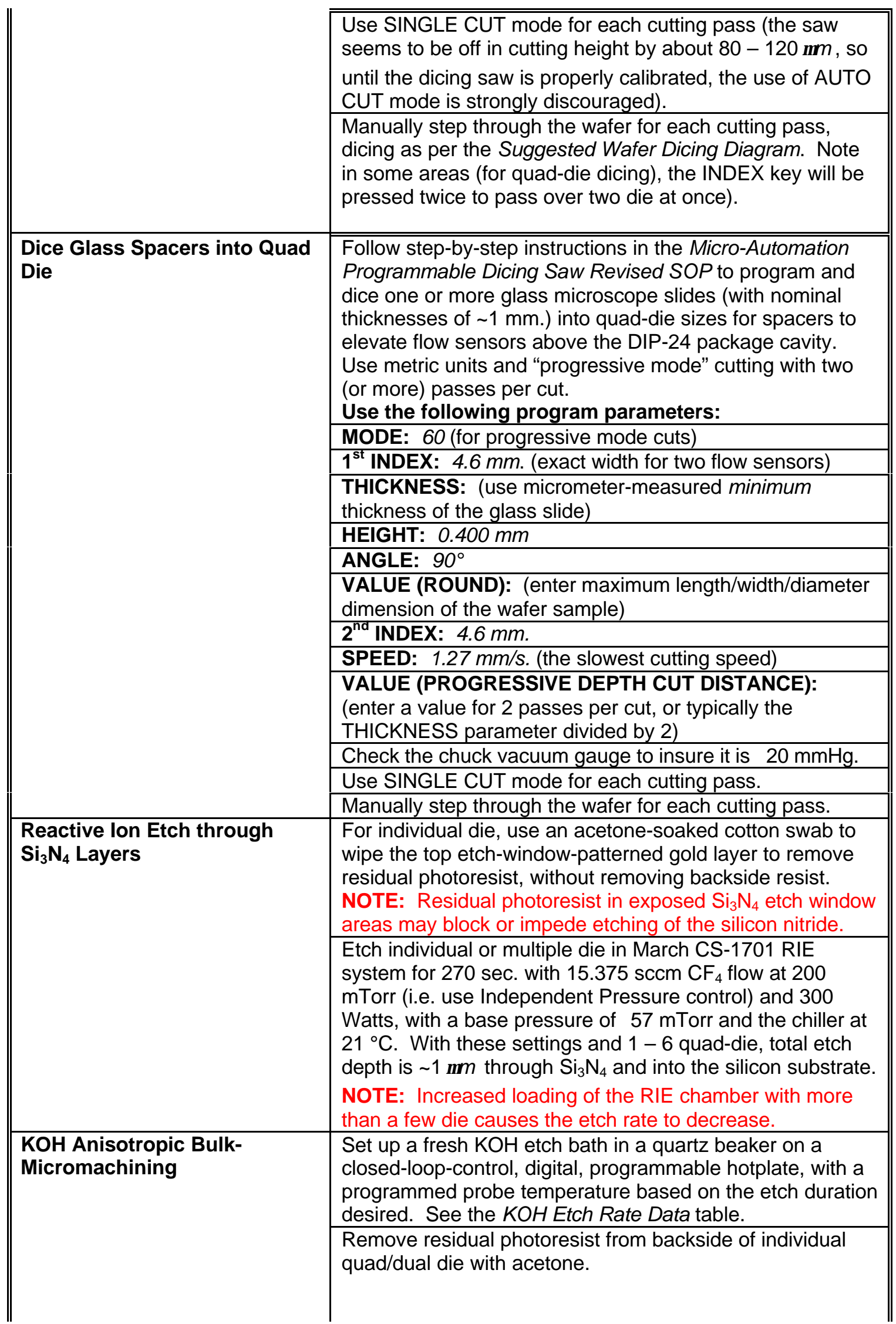




\begin{tabular}{|c|c|}
\hline & $\begin{array}{l}\text { Using the specially-designed die basket, place quad/dual } \\
\text { die into it and immerse in KOH solution (already at desired } \\
\text { temperature). Use } \sim 150 \mathrm{RPM} \text { stirring. }\end{array}$ \\
\hline & $\begin{array}{l}\text { Remove die basket from } \mathrm{KOH} \text { etch bath after } \\
\text { predetermined etch time has elapsed and immerse basket } \\
\text { in static } \mathrm{DI} \text { water bath in a beaker for } \sim 2 \text { min. }\end{array}$ \\
\hline & $\begin{array}{l}\text { Remove die basket, dump and refill beaker with fresh DI, } \\
\text { and re-immerse die basket for an additional } \sim 2 \mathrm{~min} \text {. of } \\
\text { static rinsing. }\end{array}$ \\
\hline & $\begin{array}{l}\text { Remove die basket from DI water rinse bath, carefully } \\
\text { remove die from basket, and allow it/them to dry in air. } \\
\text { Quad/dual die will air-dry faster if turned on edge on a dry, } \\
\text { lint-free cloth to force water to soak into the cloth. } \\
\text { Do NOT blow dry die with the } \mathrm{N}_{2} \text { gun, or microbridges may } \\
\text { be damaged or destroyed. }\end{array}$ \\
\hline & $\begin{array}{l}\text { Inspect die under a microscope to insure desired etch } \\
\text { depth has been achieved. Re-immerse die in } \mathrm{KOH} \text { if } \\
\text { necessary, or continue processing. } \\
\text { NOTE: The thin sputtered top } \mathrm{Si}_{3} \mathrm{~N}_{4} \text { passivation layer may } \\
\text { be damaged, destroyed, or missing after } \mathrm{KOH} \text { etching. }\end{array}$ \\
\hline \multirow[t]{2}{*}{$\begin{array}{l}\text { Top Layer RIE/KOH Gold Mask } \\
\text { Removal }\end{array}$} & $\begin{array}{l}\text { Immerse individual quad/dual die in } \mathrm{KI} \text { solution }(4 \mathrm{~g} . \mathrm{KI}, 1 \mathrm{~g} \text {. } \\
\mathrm{I}_{2} \text {, with } 40 \mathrm{~mL} \mathrm{DI} \mathrm{H}_{2} \mathrm{O} \text { ) for approx. } 30 \mathrm{sec} \text {. to remove top } \\
\text { gold masking layer. } \\
\text { WARNING: Since the exposed gold pads (which are about } \\
\text { twice as thick as the top gold masking layer) are also being } \\
\text { etched, if die are left in } \mathrm{KI} \text { solution for too long, gold will } \\
\text { etch completely off of the pads. }\end{array}$ \\
\hline & Rinse gently in static DI water bath. Do NOT dry with $\mathrm{N}_{2}$. \\
\hline \multirow[t]{2}{*}{$\begin{array}{l}\text { Top Chromium Adhesion } \\
\text { Layer RIE/KOH Mask Removal }\end{array}$} & $\begin{array}{l}\text { Immerse individual quad/dual die in Chrome Etch solution } \\
\text { for } \sim 10 \mathrm{sec} \text {. }\end{array}$ \\
\hline & Rinse in static DI water bath. Do NOT dry with $\mathrm{N}_{2}$ gun. \\
\hline \multirow[t]{3}{*}{$\begin{array}{l}\text { Electrical Continuity and } \\
\text { Device Viability Testing }\end{array}$} & $\begin{array}{l}\text { Use the Wentworth Labs probe station and lab multimeters } \\
\text { to test continuity and resistances of individual flow sensor } \\
\text { sense, heater, and substrate resistors on each die. }\end{array}$ \\
\hline & Identify which flow sensors are viable and nonviable. \\
\hline & $\begin{array}{l}\text { Locate/single-out die with devices that have } R_{U} \approx R_{D} \text {, or } \\
\text { upstream and downstream resistors with resistances within } \\
\sim 5 \% \text { of each other). }\end{array}$ \\
\hline \multirow[t]{2}{*}{ Device Mounting/Packaging } & $\begin{array}{l}\text { Using Epo-Tek® 353ND high-temperature epoxy, mix } 10 \\
\text { parts of "A" solution with } 1 \text { part "B" (hardener). Use the end } \\
\text { of a paper clip to dab } 10 \text { (or } 20 \text { ) drops of part "A" solution } \\
\text { onto a piece of paper, and mix with } 1 \text { (or } 2 \text { ) drop(s) of part } \\
\text { "B" solution. Mix together thoroughly. } \\
\text { NOTE: Since the pot life of this mixed epoxy is } \sim 4 \text { hrs., } \\
\text { there is no hurry in bonding the die to the package. }\end{array}$ \\
\hline & $\begin{array}{l}\text { Apply a very thin layer of epoxy mix to the bottom of the } \\
\text { device cavity in the DIP- } 24 \text { package. Place a quad-glass- } \\
\text { die spacer into the cavity, rotated at } 45^{\circ} \text {. }\end{array}$ \\
\hline
\end{tabular}




\begin{tabular}{|c|c|}
\hline & $\begin{array}{l}\text { Carefully apply a thin layer of epoxy mix to the top of the } \\
\text { rotated glass-die spacer. } \\
\text { Make sure the flow sensor microbridges (longitudinal axes) } \\
\text { are aligned transverse or perpendicular to the top-to- } \\
\text { bottom axis of the DIP-24 package. } \\
\text { Gently drop a rotated quad-flow-sensor die onto the top of } \\
\text { the spacer. Using the fine tip of die tweezers, press the } \\
\text { quad die onto the glass spacer at the center of the die } \\
\text { (where there is no resistor metallization or microbridges). }\end{array}$ \\
\hline & $\begin{array}{l}\text { Cure the epoxy in an oven at } 140^{\circ} \mathrm{C} \text { for } 4-5 \text { minutes. } \\
\text { When curing is complete, the epoxy will turn from a } \\
\text { translucent into a reddish color. }\end{array}$ \\
\hline \multirow[t]{3}{*}{ Flow Sensor Wire-Bonding } & $\begin{array}{l}\text { Use the K\&S Model } 4123 \text { Wedge Bonder to wire-bond the } \\
\text { flow sensor die to the DIP-24 package cavity bonding pads. }\end{array}$ \\
\hline & $\begin{array}{l}\text { Use ultrasonic mode with } 0.001 \text { in. diameter aluminum } \\
\text { silicon alloy wire. }\end{array}$ \\
\hline & $\begin{array}{l}\text { WARNING: Do not wire bond to DIP-24 package pins 2, } 6 \text {, } \\
9,10,11 \text {, and } 24 \text {, as there is no interface for these } \\
\text { connections in the Flow Test } 2000 \text { machine. }\end{array}$ \\
\hline \multirow[t]{3}{*}{$\begin{array}{l}\text { Flow Sensor Testing and } \\
\text { Characterization }\end{array}$} & $\begin{array}{l}\text { Use the ColeJet } 2000^{T M} \text { machine to characterize fabricated } \\
\text { flow sensors at air flow rates up to } \sim 14 \text { SLPM in a } 33 \mathrm{~mm} \\
\text { dia. flow channel. }\end{array}$ \\
\hline & $\begin{array}{l}\text { For more accurate measurements of sense resistor } \\
\text { Wheatstone bridge voltage outputs, connect an external } \\
\text { digital multimeter with iV resolution to the ColeJet } 2000^{T M} \\
\text { machine (according to instructions accompanying the } \\
\text { machine). }\end{array}$ \\
\hline & $\begin{array}{l}\text { To measure the air stream temperature (for theoretical } \\
\text { flow-rate calculations using the density of air), connect an } \\
\text { external multimeter to the Thermometrics MA100 } \\
\text { thermistor test points (according to instructions provided } \\
\text { with the ColeJet } 2000^{T M} \text { machine in Appendix XXI) to } \\
\text { measure its resistance and use the chart provided with the } \\
\text { ColeJet } 2000^{T M} \text { machine to correlate the resistance with } \\
\text { temperature. }\end{array}$ \\
\hline
\end{tabular}




\section{APPENDIX XV.}

SEM PHOTOS OF LOW-STRESS LPCVD SILICON NITRIDE PLATINUM/GOLD FLOW SENSORS 

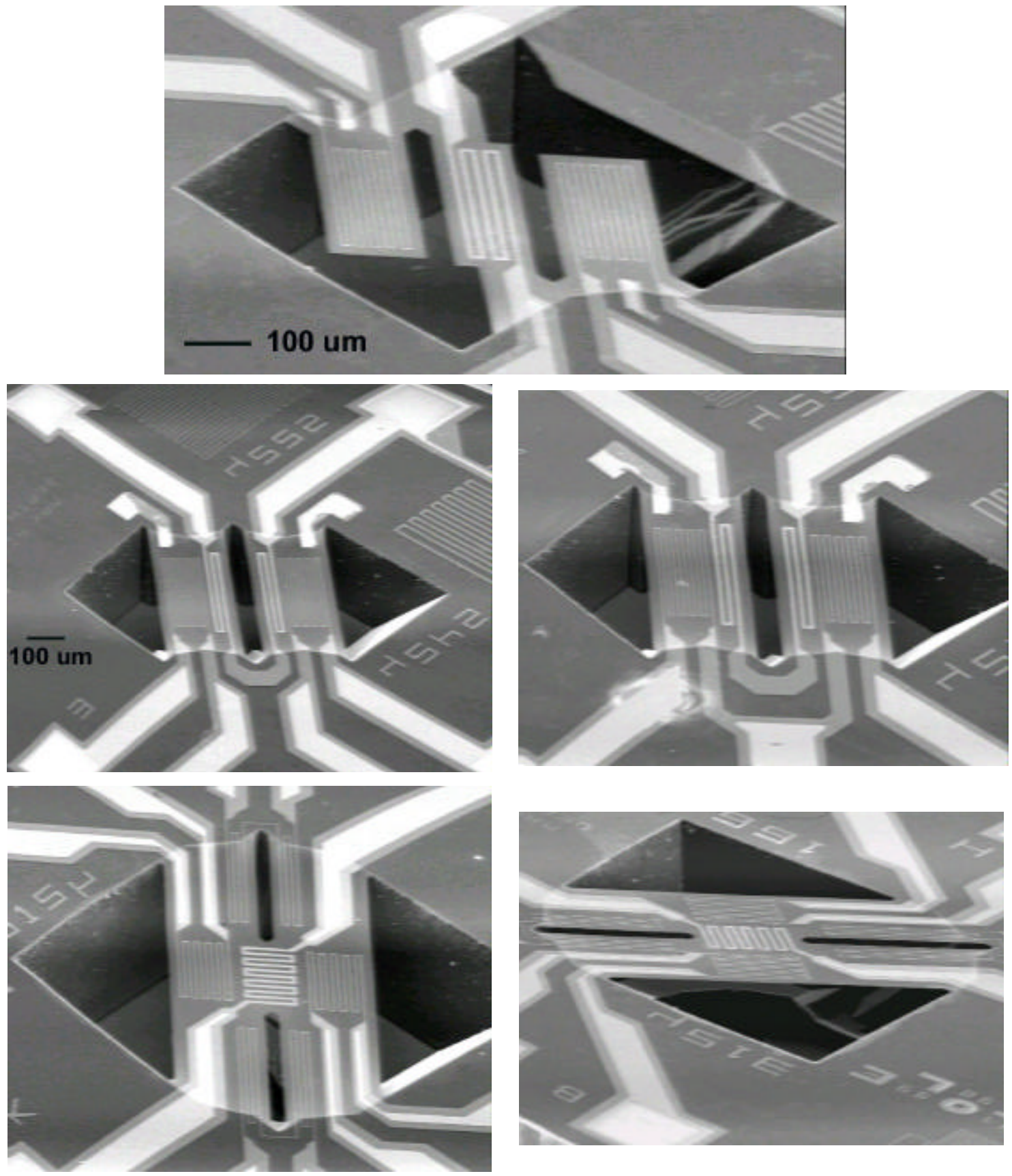

FIGURE 157 - A collage of SEM photos showing low-stress $0.5 \mu m$-thick LPCVD $\mathrm{Si}_{3} \mathrm{~N}_{4}$ flow sensors with thin film platinum and gold metallization. Note the cantilever and microbridge structures are neither buckled nor warped. Etch depths were at least $140 \mu \mathrm{m}$ and not more than $150 \mu \mathrm{m}$ into the (100) silicon substrate. 

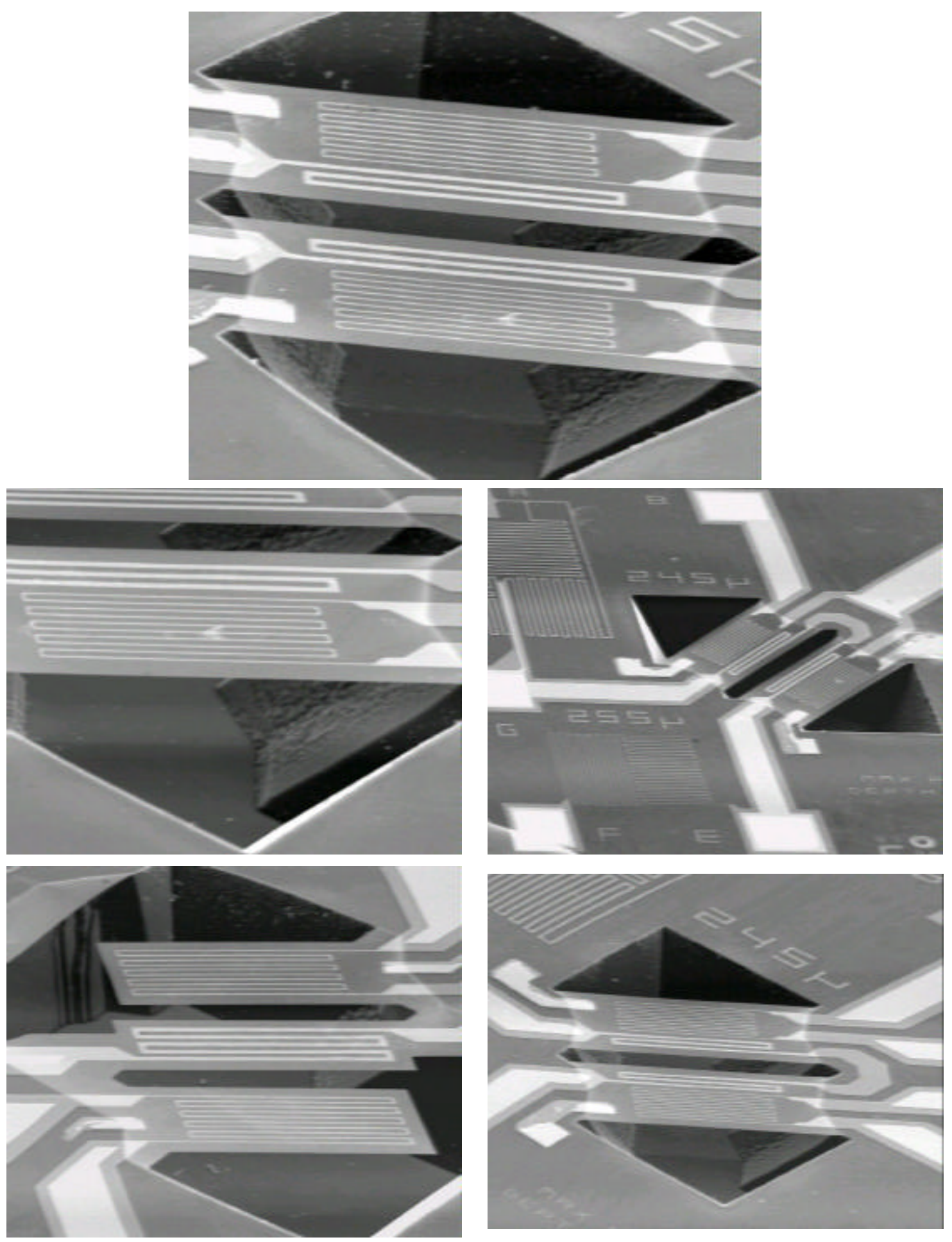

FIGURE 158 - A collage of SEM photos showing low-stress $0.5 \mu m$-thick LPCVD $\mathrm{Si}_{3} \mathrm{~N}_{4}$ flow sensors with thin film platinum and gold metallization. Note the cantilever and microbridge structures are neither buckled nor warped. Etch depths were at least $140 \mu \mathrm{m}$ and not more than $150 \mu \mathrm{m}$ into the (100) silicon substrate. 


\section{APPENDIX XVI.}

AIR AND NITROGEN GAS PROPERTIES 
TABLE XXXI

PROPERTIES OF AIR*

\begin{tabular}{|c|c|c|c|c|c|c|c|}
\hline C & $\begin{array}{c}\text { Density } \\
\rho \\
\left(\mathrm{kg} / \mathrm{m}^{3}\right)\end{array}$ & $\begin{array}{c}\text { Dynamic } \\
\text { Viscosity } \\
\mu \\
\left(\mathrm{kg} / \mathrm{m}^{*} \mathrm{~s}\right)\end{array}$ & $\begin{array}{c}\text { Kinematic } \\
\text { Viscosity } \\
v \\
\left(\mathrm{~m}^{2} / \mathrm{s}\right)\end{array}$ & $\begin{array}{c}\text { Specific } \\
\text { Heat } \\
\mathrm{C}_{\mathrm{p}} \\
\left(\mathrm{J} / \mathrm{kg}^{\star} \mathrm{K}\right)\end{array}$ & $\begin{array}{c}\text { Conductivity } \\
k \\
\left(\mathrm{~W} / \mathrm{m}^{\star} \mathrm{K}\right)\end{array}$ & $\begin{array}{c}\text { Thermal } \\
\text { Diffusivity } \\
\left(\mathrm{m}^{2} / \mathrm{s}\right)\end{array}$ & $\begin{array}{c}\text { Thermal } \\
\text { Expansion } \\
\text { Coefficient } \\
\alpha \\
(1 / \mathrm{K}) \\
\end{array}$ \\
\hline 15 & 1.2257 & 1.7965E-5 & 1.4657E-5 & 1005.9 & 0.025219 & $2.0454 \mathrm{E}-5$ & $3.4704 E-3$ \\
\hline 16 & 1.2215 & 1.8013E-5 & 1.4747E-5 & 1006.0 & 0.025295 & $2.0586 \mathrm{E}-5$ & 3.4584E-3 \\
\hline 17 & 1.2172 & 1.8061E-5 & 1.4838E-5 & 1006.0 & 0.025370 & $2.0718 \mathrm{E}-5$ & 3.4465E-3 \\
\hline 18 & 1.2130 & 1.8109E-5 & 1.4929E-5 & 1006.0 & 0.025445 & $2.0851 \mathrm{E}-5$ & 3.4347E-3 \\
\hline 19 & 1.2089 & 1.8157E-5 & $1.5020 \mathrm{E}-5$ & 1006.1 & 0.025520 & $2.0984 \mathrm{E}-5$ & 3.4229E-3 \\
\hline 20 & 1.2047 & 1.8205E-5 & $1.5111 \mathrm{E}-5$ & 1006.1 & 0.025596 & $2.1117 \mathrm{E}-5$ & $3.4112 \mathrm{E}-3$ \\
\hline 21 & 1.2006 & 1.8253E-5 & 1.5203E-5 & 1006.1 & 0.025671 & $2.1251 \mathrm{E}-5$ & 3.3996E-3 \\
\hline 22 & 1.1965 & $1.8301 \mathrm{E}-5$ & 1.5295E-5 & 1006.2 & 0.025745 & $2.1385 \mathrm{E}-5$ & 3.3881E-3 \\
\hline 23 & 1.1925 & $1.8348 \mathrm{E}-5$ & $1.5387 \mathrm{E}-5$ & 1006.2 & 0.025820 & $2.1519 \mathrm{E}-5$ & 3.3767E-3 \\
\hline 24 & 1.1885 & 1.8396E-5 & 1.5479E-5 & 1006.2 & 0.025895 & $2.1654 \mathrm{E}-5$ & 3.3653E-3 \\
\hline 25 & 1.1845 & $1.8444 \mathrm{E}-5$ & $1.5571 \mathrm{E}-5$ & 1006.3 & 0.025969 & $2.1789 \mathrm{E}-5$ & $3.3540 \mathrm{E}-3$ \\
\hline 26 & 1.1805 & 1.8491E-5 & 1.5664E-5 & 1006.3 & 0.026044 & $2.1924 \mathrm{E}-5$ & $3.3428 \mathrm{E}-3$ \\
\hline 27 & 1.1765 & $1.8538 \mathrm{E}-5$ & 1.5757E-5 & 1006.3 & 0.026118 & $2.2059 \mathrm{E}-5$ & 3.3317E-3 \\
\hline 28 & 1.1726 & $1.8586 \mathrm{E}-5$ & $1.5850 \mathrm{E}-5$ & 1006.4 & 0.026193 & $2.2195 \mathrm{E}-5$ & 3.3206E-3 \\
\hline 29 & 1.1687 & 1.8633E-5 & 1.5943E-5 & 1006.4 & 0.026267 & $2.2331 \mathrm{E}-5$ & 3.3096E-3 \\
\hline 30 & 1.1649 & $1.8680 \mathrm{E}-5$ & 1.6036E-5 & 1006.5 & 0.026341 & $2.2468 \mathrm{E}-5$ & $3.2987 \mathrm{E}-3$ \\
\hline
\end{tabular}

* Source: $h$ ttp://www.mhtl.uwaterloo.ca/old/onlinetools/airprop/airprop.htm/ on 2/22/2000. 
TABLE XXXII

PROPERTIES OF NITROGEN GAS*

\begin{tabular}{|c|c|c|c|c|c|c|c|}
\hline C & $\begin{array}{c}\text { Density } \\
\rho \\
\left(\mathrm{kg} / \mathrm{m}^{3}\right)\end{array}$ & $\begin{array}{c}\text { Dynamic } \\
\text { Viscosity } \\
\mu \\
\left(\mathrm{kg} / \mathrm{m}^{*} \mathrm{~s}\right)\end{array}$ & $\begin{array}{c}\text { Kinematic } \\
\text { Viscosity } \\
v \\
\left(\mathrm{~m}^{2} / \mathrm{s}\right)\end{array}$ & $\begin{array}{c}\text { Specific } \\
\text { Heat } \\
\mathrm{C}_{\mathrm{p}} \\
\left(\mathrm{J} / \mathrm{kg}^{\star} \mathrm{K}\right)\end{array}$ & $\begin{array}{c}\text { Conductivity } \\
k \\
\left(\mathrm{~W} / \mathrm{m}^{*} \mathrm{~K}\right)\end{array}$ & $\begin{array}{c}\text { Thermal } \\
\text { Diffusivity } \\
\left(\mathrm{m}^{2} / \mathrm{s}\right)\end{array}$ & $\begin{array}{c}\text { Thermal } \\
\text { Expansion } \\
\text { Coefficient } \\
\alpha \\
(1 / \mathrm{K}) \\
\end{array}$ \\
\hline 15 & 1.1854 & 1.7317E-5 & 1.4609E-5 & 1041.1 & 0.025106 & $2.0347 \mathrm{E}-5$ & $3.4704 \mathrm{E}-3$ \\
\hline 16 & 1.1812 & $1.7364 \mathrm{E}-5$ & $1.4700 \mathrm{E}-5$ & 1041.1 & 0.025179 & $2.0477 \mathrm{E}-5$ & 3.4584E-3 \\
\hline 17 & 1.1772 & $1.7411 \mathrm{E}-5$ & 1.4790E-5 & 1041.1 & 0.025252 & $2.0608 \mathrm{E}-5$ & $3.4465 \mathrm{E}-3$ \\
\hline 18 & 1.1731 & 1.7457E-5 & $1.4881 \mathrm{E}-5$ & 1041.1 & 0.025324 & $2.0739 \mathrm{E}-5$ & 3.4347E-3 \\
\hline 19 & 1.1691 & $1.7504 \mathrm{E}-5$ & 1.4972E-5 & 1041.1 & 0.025396 & $2.0870 \mathrm{E}-5$ & $3.4229 \mathrm{E}-3$ \\
\hline 20 & 1.1651 & 1.7550E-5 & 1.5064E-5 & 1041.1 & 0.025468 & $2.1002 \mathrm{E}-5$ & 3.4112E-3 \\
\hline 21 & 1.1611 & $1.7597 \mathrm{E}-5$ & $1.5155 \mathrm{E}-5$ & 1041.1 & 0.025541 & $2.1134 \mathrm{E}-5$ & 3.3996E-3 \\
\hline 22 & 1.1572 & 1.7643E-5 & 1.5247E-5 & 1041.1 & 0.025613 & $2.1266 \mathrm{E}-5$ & 3.3881E-3 \\
\hline 23 & 1.1532 & 1.7689E-5 & 1.5339E-5 & 1041.1 & 0.025684 & $2.1398 \mathrm{E}-5$ & 3.3767E-3 \\
\hline 24 & 1.1493 & 1.7736E-5 & $1.5431 \mathrm{E}-5$ & 1041.1 & 0.025756 & $2.1531 \mathrm{E}-5$ & 3.3653E-3 \\
\hline 25 & 1.1455 & 1.7782E-5 & 1.5523E-5 & 1041.1 & 0.025828 & $2.1664 \mathrm{E}-5$ & 3.3540E-3 \\
\hline 26 & 1.1416 & $1.7828 \mathrm{E}-5$ & 1.5616E-5 & 1041.1 & 0.025899 & $2.1797 \mathrm{E}-5$ & $3.3428 \mathrm{E}-3$ \\
\hline 27 & 1.1378 & 1.7874E-5 & 1.5709E-5 & 1041.1 & 0.025971 & $2.1931 \mathrm{E}-5$ & 3.3317E-3 \\
\hline 28 & 1.1340 & 1.7919E-5 & 1.5802E-5 & 1041.1 & 0.026042 & $2.2065 \mathrm{E}-5$ & 3.3206E-3 \\
\hline 29 & 1.1303 & 1.7965E-5 & 1.5895E-5 & 1041.1 & 0.026113 & 2.2199E-5 & 3.3096E-3 \\
\hline 30 & 1.1265 & $1.8011 \mathrm{E}-5$ & 1.5988E-5 & 1041.1 & 0.026184 & $2.2334 \mathrm{E}-5$ & 3.2987E-3 \\
\hline
\end{tabular}

* Source: http://www.mhtl.uwaterloo.ca/old/onlinetools/airprop/airprop.htm/ on 2/22/2000. 


\section{APPENDIX XVII.}

COLEJET 2000TM DESIGN CONSIDERATIONS 
TABLE XXXIII

FLOW TUBE CHANNEL DESIGN PARAMETER RELATIONSHIPS

FOR A GIVEN VOLUMETRIC FLOW RATE

\begin{tabular}{|c|c|c|c|c|c|}
\hline $\begin{array}{c}\text { Pipe } \\
\text { Diameter, } \\
D, \text { and } \\
\text { Cross- } \\
\begin{array}{c}\text { Sectional } \\
\text { Area, } A\end{array}\end{array}$ & $\begin{array}{c}\text { Bypass } \\
\text { Ratio } \\
\text { Change }\end{array}$ & $\begin{array}{c}\text { Change in } \\
\text { Fluid Mean } \\
\text { Velocity, } \\
\bar{v}\end{array}$ & $\begin{array}{c}\text { Change in } \\
\text { Reynolds } \\
\text { Number, } \\
R e\end{array}$ & $\begin{array}{c}\text { Change in } \\
\text { Pressure Drop } \\
\text { Across } \\
\text { Laminar } \\
\text { Flow Area }\end{array}$ & $\begin{array}{c}\text { Change in } \\
\text { Inlet and } \\
\text { Outlet } \\
\text { Pressure Loss } \\
\text { for Laminar } \\
\text { Flow Area }\end{array}$ \\
\hline Increase & Increase & Decrease & Decrease & Decrease & Decrease \\
\hline Decrease & Decrease & Increase & Increase & Increase & Increase \\
\hline
\end{tabular}


TABLE XXXIV

DESIGN PARAMETERS FOR COLEJET ${ }^{T M} 2000$ WITH AN AWM5000 SERIES BYPASS

FLOWSENSOR USING AIR AT $21^{\circ} \mathrm{C}$

\begin{tabular}{|c|c|c|c|c|c|c|c|c|}
\hline $\begin{array}{c}\text { Main } \\
\text { Flow } \\
\text { Channel } \\
\text { ID (Area) } \\
\text { mm } \\
\left(\mathrm{mm}^{2}\right)\end{array}$ & $\begin{array}{l}\text { Bypass } \\
\text { Channel } \\
\text { Length } \\
\text { (m) }\end{array}$ & $\begin{array}{c}\text { Bypass } \\
\text { Ratio }\end{array}$ & $\begin{array}{l}\text { Max. } \\
\text { Design } \\
\text { Flow } \\
\text { Rate } \\
\text { (L/min) }\end{array}$ & $\begin{array}{c}\text { Velocity } \\
\text { of Flow, } \\
\bar{v} \\
(\mathrm{~m} / \mathrm{s})\end{array}$ & $\begin{array}{c}\text { Reynolds } \\
\text { Number, } \\
R_{e}\end{array}$ & $\begin{array}{c}\text { Entrance } \\
\text { Length, } \\
L_{e} \\
(\mathrm{~m})\end{array}$ & $\begin{array}{c}\Delta \mathrm{P}_{\text {flow }} \\
(\mathrm{Pa})\end{array}$ & $\begin{array}{c}\Delta \mathrm{P}_{\text {total }} \\
(\mathrm{Pa})\end{array}$ \\
\hline $\begin{array}{l}19.05 \\
(285)\end{array}$ & 0.254 & $6.25: 1$ & 40 & 2.33899 & 2931 & 1.675 & 0.95622 & 4.92626 \\
\hline $\begin{array}{l}19.05 \\
(285)\end{array}$ & 0.254 & $6.25: 1$ & 30 & 1.75424 & 2198 & 1.256 & 0.71716 & 2.77102 \\
\hline $\begin{array}{l}19.05 \\
(285)\end{array}$ & 0.900 & $6.25: 1$ & 30 & 1.75424 & 2198 & 1.256 & 3.38817 & 2.77102 \\
\hline $\begin{array}{l}19.05 \\
(285)\end{array}$ & 0.3556 & $6.25: 1$ & 20 & 1.16950 & 1465 & 0.837 & 0.66935 & 1.23157 \\
\hline $\begin{array}{l}19.05 \\
(285)\end{array}$ & 0.508 & $6.25: 1$ & 20 & 1.16950 & 1465 & 0.837 & 0.95622 & 1.23157 \\
\hline $\begin{array}{l}25.4 \\
(507)\end{array}$ & 0.254 & $11: 1$ & 40 & 1.31568 & 2198 & 1.675 & 0.30255 & 1.55870 \\
\hline $\begin{array}{r}25.4 \\
(507)\end{array}$ & 0.508 & $11: 1$ & 40 & 1.31568 & 2198 & 1.675 & 0.60511 & 1.55870 \\
\hline $\begin{array}{l}25.4 \\
(507)\end{array}$ & 0.254 & $11: 1$ & 30 & 0.98676 & 1649 & 1.257 & 0.22691 & 0.87677 \\
\hline $\begin{array}{r}25.4 \\
(507) \\
\end{array}$ & 0.508 & $11: 1$ & 30 & 0.98676 & 1649 & 1.257 & 0.45383 & 0.87677 \\
\hline $\begin{array}{l}25.4 \\
(507)\end{array}$ & 0.900 & $11: 1$ & 30 & 0.98676 & 1649 & 1.257 & 1.07204 & 0.87677 \\
\hline $\begin{array}{r}25.4 \\
(507) \\
\end{array}$ & 0.3556 & $11: 1$ & 20 & 0.65784 & 1099 & 0.837 & 0.21179 & 0.38967 \\
\hline $\begin{array}{l}25.4 \\
(507)\end{array}$ & 0.508 & $11: 1$ & 20 & 0.65784 & 1099 & 0.837 & 0.30255 & 0.38967 \\
\hline $\begin{array}{l}25.4 \\
(507)\end{array}$ & 0.508 & $11: 1$ & 10 & 0.32892 & 550 & 0.41874 & 0.15128 & 0.24870 \\
\hline $\begin{array}{r}25.4 \\
(507)\end{array}$ & 0.3556 & $11: 1$ & 10 & 0.32892 & 550 & 0.41874 & 0.10589 & 0.20331 \\
\hline $\begin{array}{c}25.4 \\
(507)\end{array}$ & 0.254 & $11: 1$ & 10 & 0.32892 & 550 & 0.41874 & 0.07564 & 0.17306 \\
\hline $\begin{array}{c}25.4 \\
(507)\end{array}$ & 0.3556 & $11: 1$ & 5 & 0.16446 & 275 & 0.20937 & 0.05295 & 0.07730 \\
\hline $\begin{array}{r}25.4 \\
(507) \\
\end{array}$ & 0.254 & $11: 1$ & 5 & 0.16446 & 275 & 0.20937 & 0.03782 & 0.06217 \\
\hline
\end{tabular}


APPENDIX XVIII.

COLEJET $2000^{T M}$ SELECTED PARTS' DATA SHEETS 

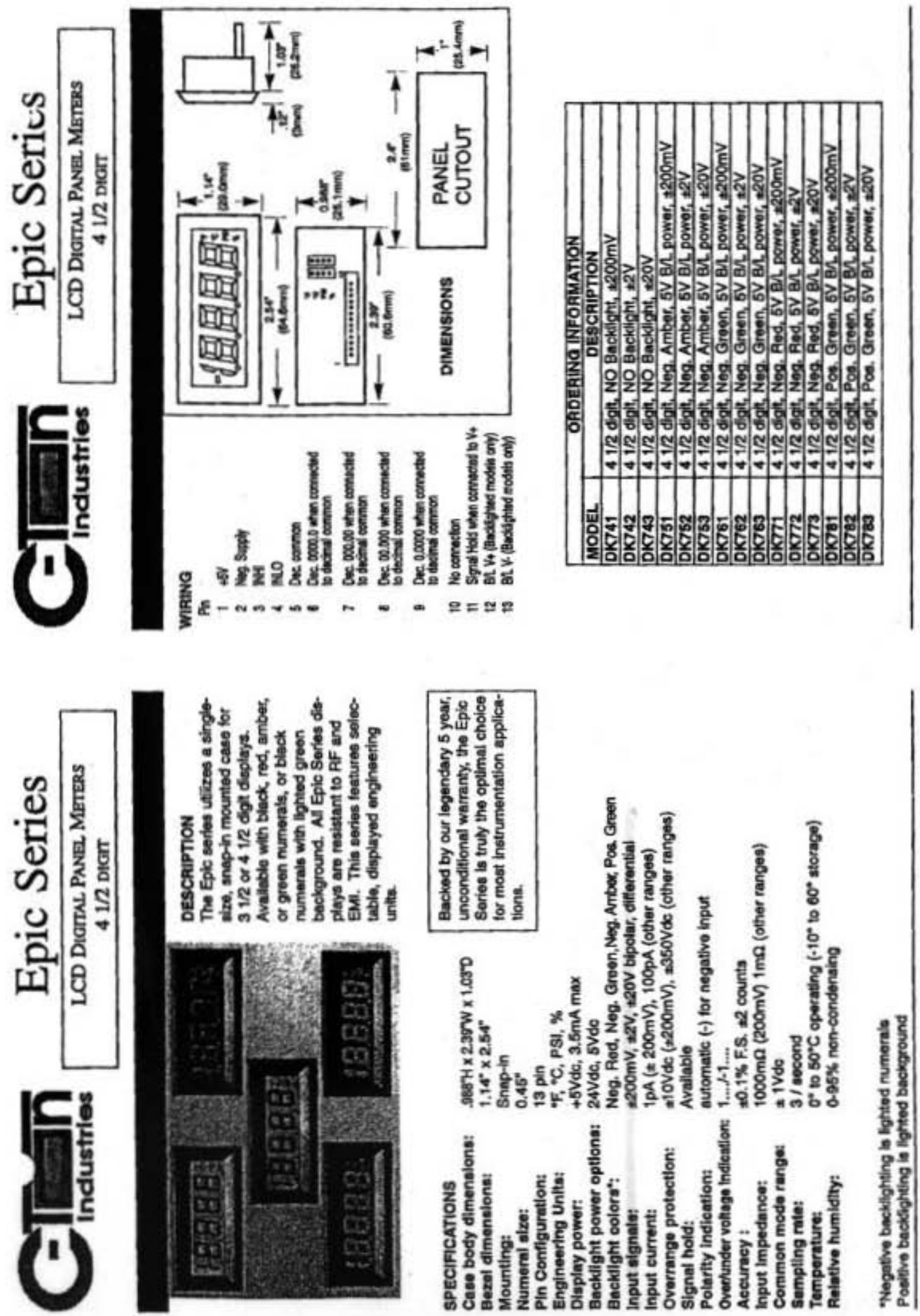

FIGURE 159 - Data sheet for the C-Ton Industries DK-743 LCD voltmeter. 


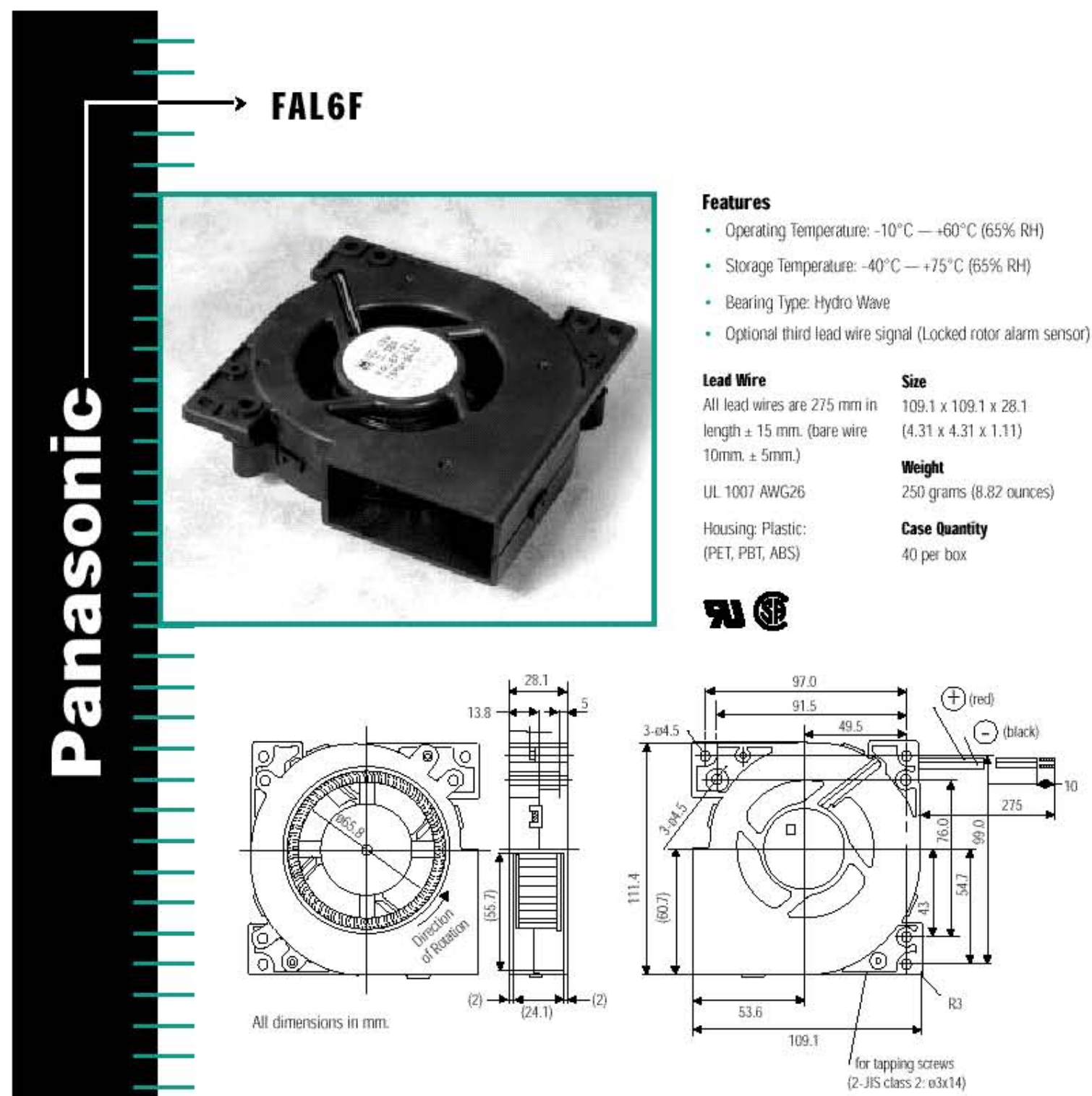

Conversion Factors

$1 \mathrm{~mm} \mathrm{H}_{2} \mathrm{O}=9.8 \mathrm{PA}$

Basic Data (nominal at room temperature)

$1 \mathrm{~m} 3 / \mathrm{min}=35.313 \mathrm{CFM}$

\begin{tabular}{|c|c|c|c|c|c|c|c|c|c|c|}
\hline & \multirow{2}{*}{$\begin{array}{l}\text { Rated } \\
\text { Voliage } \\
\text { (I) }\end{array}$} & \multirow{2}{*}{$\begin{array}{c}\text { Operating } \\
\text { Vollage } \\
\text { M }\end{array}$} & \multirow{2}{*}{$\begin{array}{c}\text { Rated } \\
\text { Current } \\
(\mathrm{mA})\end{array}$} & \multirow{2}{*}{$\begin{array}{l}\text { Rated } \\
\text { Input } \\
\text { (W) }\end{array}$} & \multirow{2}{*}{$\begin{array}{l}\text { Noninal } \\
\text { Speed } \\
\text { (rhinin) }\end{array}$} & \multicolumn{2}{|c|}{$\begin{array}{l}\text { Maxinum } \\
\text { Air Flow }\end{array}$} & \multicolumn{2}{|c|}{$\begin{array}{l}\text { Maximum } \\
\text { Air Pressure }\end{array}$} & \multirow{2}{*}{$\begin{array}{l}\text { Noise } \\
(\mathrm{dB}-\mathrm{A})\end{array}$} \\
\hline & & & & & & (m3/ain) & (Crm) & $(\mathrm{mm} H 20)$ & (Pa) & \\
\hline FAL6F12L & 12 & $7-13.8$ & 280 & 3.36 & 2100 & 0.53 & 18.7 & 11.25 & 176.5 & 40.0 \\
\hline FAL6F12LH & 12 & $7-13.8$ & 500 & 6.00 & 2550 & 0.65 & 23.0 & 18.00 & 110.3 & 45.0 \\
\hline FAL6F24L & 24 & $14-27.0$ & 155 & 3.72 & 2100 & 0.53 & 18.7 & 11.50 & 193.2 & 40.0 \\
\hline FAL6F24LH & 24 & $14-27.0$ & 295 & 7.08 & 2550 & 0.67 & 23.7 & 19.70 & 112.8 & 45.0 \\
\hline
\end{tabular}

28

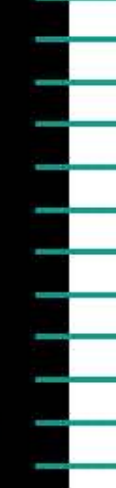

FIGURE 160 - Data sheet for the Panasonic Scirocco FAL6F12LH blower. 


\section{FAL6F}

\section{Characteristics}
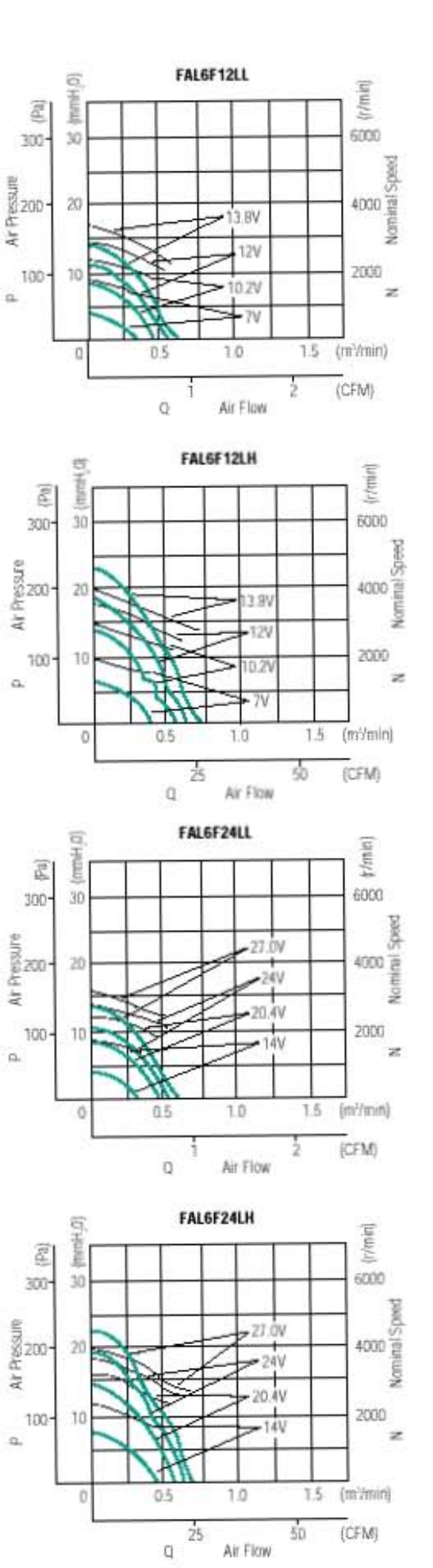

FIGURE 161 - Data sheet (pg. 2) for the Panasonic Scirocco FAL6F12LH blower. 


\begin{tabular}{|c|c|c|c|c|c|c|c|c|c|c|c|c|c|}
\hline \multirow[t]{2}{*}{$\begin{array}{l}\text { Iypo } \\
\text { and Size }\end{array}$} & \multirow[t]{2}{*}{ Hodel } & \multirow{2}{*}{$\begin{array}{l}\text { Ratod } \\
\text { Voltage } \\
\text { (M) }\end{array}$} & \multirow{2}{*}{$\begin{array}{l}\text { Operating } \\
\text { Uoltage } \\
\text { (V) }\end{array}$} & \multirow{2}{*}{$\begin{array}{l}\text { Rated } \\
\text { Current } \\
\text { (mi) }\end{array}$} & \multirow{2}{*}{$\begin{array}{l}\text { Rated } \\
\text { Inpet } \\
\text { (M) }\end{array}$} & \multirow{2}{*}{$\begin{array}{l}\text { Neninal } \\
\text { Speed } \\
\text { (rimin) }\end{array}$} & \multicolumn{2}{|c|}{$\begin{array}{l}\text { Maxinimin } \\
\text { Air flow }\end{array}$} & \multicolumn{2}{|c|}{$\begin{array}{l}\text { Maximim } \\
\text { Air Pressure }\end{array}$} & \multirow[t]{2}{*}{$\begin{array}{l}\text { Hoise } \\
\text { (cB) }\end{array}$} & \multirow{2}{*}{$\begin{array}{l}\text { Saloty } \\
\text { Standard } \\
\text { UL CSA }\end{array}$} & \multirow{2}{*}{$\begin{array}{l}\text { Locke: } \\
\text { Roter } \\
\text { Alarm } \\
\text { Senso }\end{array}$} \\
\hline & & & & & & & $\overline{(\text { m3/min })}$ & (CFM) & (minh20) & $\overline{(P a)}$ & & & \\
\hline FBKO4F & FBK04F05L & 5 & $4.5-6.0$ & 120 & 0.60 & 4700 & 0.11 & 3.9 & 250 & 24.5 & 24.0 & . & - \\
\hline $40 \mathrm{~mm}$ & FBK04F05H & 5 & $4.5-6.0$ & 2000 & 1.40 & 7500 & 0.19 & 6.1 & 6.60 & 64.7 & 33.0 & - & - \\
\hline \multirow[t]{5}{*}{ DC axial fans } & FBK04F12L & 12 & $7.0-13.8$ & 75 & 0.90 & 4700 & 0.11 & 3.9 & 2.50 & 24.5 & 23.0 & - & $\cdot$ \\
\hline & FBK04F $12 \mathrm{H}$ & 12 & $7.0-13.8$ & 150 & 1.80 & 7500 & 0.19 & 6.7 & 6.60 & 64.7 & 320 & . & - \\
\hline & FBKO4F $12 \mathrm{U}$ & 12 & $7.0-13.8$ & 170 & 2.04 & 9500 & 0.26 & 9.2 & 12.00 & 117.7 & 42.0 & - & $\cdot$ \\
\hline & FBKO $04 \mathrm{~F} 24 \mathrm{H}$ & 24 & $14.0-27.6$ & 92 & 2.21 & 7500 & 0.19 & 6.7 & 6.60 & 64.7 & 33.0 & - & $\cdot$ \\
\hline & FEK04F24U & 24 & $14.0-27.6$ & 100 & 2.40 & 9500 & 0.26 & 9.2 & 12.00 & 117.7 & 42.0 & . & $\cdot$ \\
\hline FBAOGAA & FBA0GA1ZLIA & 12 & $7.0-13.8$ & 110 & 1.32 & 3200 & 0.40 & 14.1 & 3.10 & 30.4 & 24.0 & . & $\cdot$ \\
\hline $60 \mathrm{~mm}$ & FBA06A12M1A & 12 & $7.0-13.8$ & 130 & 1.56 & 3700 & 0.47 & 16.6 & 3.95 & 38.7 & 28.0 & .. & $\cdot$ \\
\hline \multirow[t]{5}{*}{ DC axial fans } & FBA06A1ZHIA & 12 & $7.0-13.8$ & 130 & 2.04 & 4200 & 0.54 & 19.1 & 5.20 & 51.0 & 32.0 & .. & - \\
\hline & FBAD6A12111A & 12 & $7.0-13.8$ & 210 & 2.52 & 4550 & 0.58 & 20.5 & 6.0 & 58.0 & 37.0 & . & $\cdot$ \\
\hline & FBAOGA24L1A & 24 & $14.0-27.6$ & 70 & 1.68 & 3200 & 0.40 & 14.1 & 3.10 & 30.4 & 24.0 & .. & 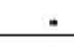 \\
\hline & FBA06A24M1A & 24 & $14.0-27.6$ & 90 & 2.16 & 3700 & 0.47 & 16.6 & 3.95 & 38.7 & 28.0 & - $\cdot$ & - \\
\hline & FBAD6A24H1A & 24 & $14.0-27.6$ & 105 & 2.52 & 4200 & 0.54 & 19.1 & 5.20 & 51.0 & 320 & - & $\cdot$ \\
\hline FBAOGT & FBA06T12L & 12 & $7.0-13.8$ & 88 & 1.06 & 3200 & 0.34 & 12.0 & 280 & 27.5 & 24.0 & - $\cdot$ & $\cdot$ \\
\hline $60 \mathrm{~mm}$ & FBA06T12H & 12 & $7.0-13.8$ & 145 & 1.74 & 4200 & 0.47 & 16.6 & 4.75 & 46.6 & 32.0 & . & - \\
\hline \multirow[t]{2}{*}{ Thin } & FBA06T24L & 24 & $14-27.6$ & 56 & 1.34 & 32000 & 0.34 & 12.0 & 280 & 27.5 & 24.0 & . & - \\
\hline & FBA06I24H & 24 & $14-27.6$ & 83 & 1.99 & 4200 & 0.47 & 16.6 & 4.75 & 46.6 & 320 & - $\quad$ & - \\
\hline FBACBA & FBADBA12L1A & 12 & $7.0-13.8$ & 68 & 0.82 & 1900 & 0.68 & 24.0 & 1.57 & 15.4 & 21.0 & - $\cdot$ & $\cdot$ \\
\hline gomm & FBA0BA12M1A & 12 & $7.0-13,8$ & 124 & 1.49 & 2450 & 0.91 & 32.1 & 2.51 & 24.6 & 28.0 & -. & - \\
\hline \multirow[t]{5}{*}{ DC axial fans } & FBA0BA12H1A & 12 & $7.0-13,8$ & 173 & 2.000 & 2950 & 1.12 & 39.6 & 3.78 & 37.1 & 32.0 & . & $\cdot$ \\
\hline & FBA0BA12U1A & 12 & $7.0-13.8$ & 270 & 3.24 & 3450 & 1.33 & 46.9 & 4.80 & 47.0 & 38.2 & . & $\cdot$ \\
\hline & FBA0BA24L1A & 24 & $14-27.6$ & 54 & 1.30 & 1900 & 0.68 & 24.0 & 1.57 & 15.4 & 210 & . & 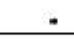 \\
\hline & FBA0BA24M1A & 24 & $14-27.6$ & 71 & 1.70 & 2450 & 0.91 & 32.1 & 2.51 & 24.6 & 28.0 & . & - \\
\hline & FBA0BA24H1A & 24 & $14-27.6$ & 105 & 2.52 & 2950 & 1.12 & 39.6 & 3.78 & 37.1 & 320 & . & $\cdot$ \\
\hline FBAOBT & FBA0BT12L & 12 & $7.0-13.8$ & 79 & 0.95 & 2000 & 0.62 & 21.9 & 1.55 & 15.2 & 23.0 & . & $\cdot$ \\
\hline $80 \mathrm{~mm}$ & FBA08T12H & 12 & $7.0-13.8$ & 200 & 2.40 & 3100 & 0.95 & 33.5 & 3.20 & 31.4 & 34.0 & - . & - \\
\hline \multirow[t]{2}{*}{ Thin } & FBAOBI24L & 24 & $14-27.6$ & 47 & 1.13 & 2000 & 0.62 & 21.9 & 1.55 & 152 & 23.0 & . & - \\
\hline & FBA0BT24H & 24 & $14-27.6$ & 110 & 2.64 & 3100 & 0.95 & 33.5 & 3.20 & 31.4 & 34.0 & .. & $\cdot$ \\
\hline FBAD9A & FBA99A12L1A & 12 & $7.0-13.8$ & 110 & 1.32 & 2100 & 1.21 & 42.7 & 2.30 & 22.6 & 27.0 & .. & $*$ \\
\hline $90 \mathrm{~mm}$ & FBA09A12M1A & 12 & $7.0-13.8$ & 150 & 1.80 & 2450 & 136 & 48,0 & 3.00 & 29.4 & 30.0 & - & - \\
\hline \multirow[t]{5}{*}{ DC axial fans } & FBA09A12H1A & 12 & $7.0-13.8$ & 225 & 2.70 & 2850 & 1.61 & 56.8 & 3.98 & 39.0 & 35.0 & -. & $\cdot$ \\
\hline & FBA09A12U1A & 12 & $7.0-13.8$ & 430 & 5.16 & 3450 & 1.95 & 68.8 & 5.0 & 49.0 & 43.0 & . & - \\
\hline & FBA09A24L1A & 24 & $14-27.6$ & 80 & 1.92 & 2100 & 1.21 & 42.7 & 230 & 22.6 & 27.0 & - & - \\
\hline & FBA09A24M1A & 24 & $14-27.6$ & 110 & 2.64 & 2450 & 1.36 & 48.0 & 3.00 & 29.4 & 30.0 & . & $\cdot$ \\
\hline & FBA09A24H१А & 24 & $14-27.6$ & 140 & 3.36 & 2850 & 1.61 & 56.8 & 3.98 & 39.0 & 35.0 & . & $\cdot$ \\
\hline FBA12G & FBA12G12L1A & 12 & $7.0-13.8$ & 180 & 2.16 & 1700 & 1.95 & 68.9 & 3.30 & 32.4 & 30.0 & . & $\cdot$ \\
\hline $120 \mathrm{~mm}$ & FBA12G12M1A & 12 & $7.0-13.8$ & 340 & 4.06 & 2100 & 2.45 & 86.5 & 4.89 & 47.1 & 35.5 &. &. \\
\hline \multirow[t]{5}{*}{ DC axial fans } & FBA12G12H1A & 12 & $7.0-13.8$ & 460 & 5.52 & 2500 & 2.34 & 103.8 & 6.80 & 66.7 & 41.5 & . & $\cdot$ \\
\hline & FBA12G12U1A & 12 & $7.0-13.8$ & 510 & 6.12 & 2750 & 325 & 114.7 & 8.0 & 39.0 & 45.5 & . & - \\
\hline & FBA12G24L1A & 24 & $14-27.6$ & 110 & 2.64 & 1700 & 1.95 & 68.9 & 3.30 & 32.4 & 30.0 & . & $\cdot$ \\
\hline & FBA12G24M1A & 24 & $14-27.6$ & 160 & 3.84 & 2100 & 2.45 & 86.5 & 4.80 & 47.1 & 35.5 & . & - \\
\hline & FBA12G24H1A & 24 & $14-27.6$ & 230 & 5.5 .2 & 2500 & 2.94 & 103.8 & 6.89 & 66.7 & 41.5 & - & $\cdot$ \\
\hline
\end{tabular}

FIGURE 162 - Product specifications comparison table for the Panasonic axial fans and Scirocco blowers. 


\begin{tabular}{|l|c|c|}
\hline \multicolumn{1}{|c|}{ Series } & Still Air & Still Water* \\
\hline $\begin{array}{l}\text { MA100 } \\
\text { Catheter Assembly }\end{array}$ & $15 \mathrm{sec}$. & $2.0 \mathrm{sec}$. \\
\hline $\begin{array}{l}\text { MA200 } \\
\text { Oral-Roctal Assembly }\end{array}$ & $35 \mathrm{sec}$. & $0.6 \mathrm{sec}$. \\
\hline $\begin{array}{l}\text { MA300 } \\
\text { Sinin Surface Assembly }\end{array}$ & $45 \mathrm{sec}$. & $2.0 \mathrm{sec}$. \\
\hline
\end{tabular}

* Response time provided is for assembly plunged from $25^{\circ} \mathrm{C}$ air to $5^{\circ} \mathrm{C}$ still water

TOLERANCE CODE AND TEMPERATURE RANGE

\begin{tabular}{|c|c|c|c|}
\hline \multirow[b]{2}{*}{$\begin{array}{c}\text { Temperature } \\
\text { Range } \\
\text { "C }\end{array}$} & \multicolumn{3}{|c|}{ Tolerance Code } \\
\hline & $\begin{array}{c}\text { A } \\
\pm \mathrm{C}\end{array}$ & $\begin{array}{c}\text { B } \\
\pm^{-c} \mathrm{C}\end{array}$ & $\begin{array}{l}\mathrm{C} \\
\pm \mathrm{C}\end{array}$ \\
\hline 0.20 & .15 & .2 & .25 \\
\hline $20-35$ & .1 & .15 & .2 \\
\hline $35 \cdot 39$ & .05 & .1 & .15 \\
\hline $39 \cdot 42$ & .075 & .15 & .2 \\
\hline $42-45$ & .1 & .15 & .2 \\
\hline $45-50$ & .15 & .2 & .25 \\
\hline
\end{tabular}

\section{DATA:}

Our biomedical series thermistor chips and sub assemblies are designed to be interchangeable over a $0^{\circ} \mathrm{C}$ to $50^{\circ} \mathrm{C}$ range. Best overall stability is maintained when exposure and storage temperatures remain below $70^{\circ} \mathrm{C}$.

\section{CODING: \\ MA $\quad X X X X X$ - $X X X$ - $X$}

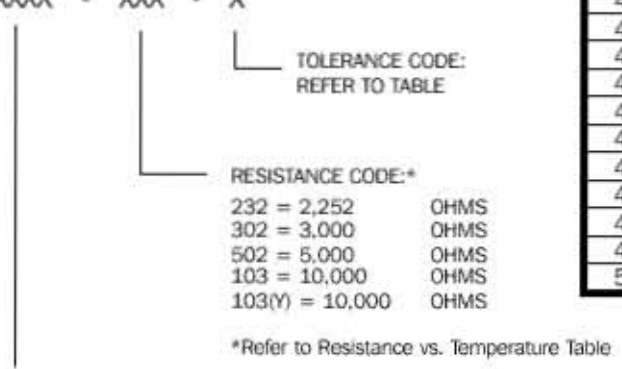

\begin{tabular}{|c|c|c|c|c|c|}
\hline$\underset{{ }^{\circ} \mathrm{C}}{\text { TEMP }}$ & $\begin{array}{l}2252 \\
\text { OHMS }\end{array}$ & $\begin{array}{c}3 \mathrm{k} \\
\mathrm{OHMS}\end{array}$ & $\begin{array}{c}5 \mathrm{k} \\
\text { OHMS }\end{array}$ & $\begin{array}{c}10 \mathrm{k} \\
\text { OHMS }\end{array}$ & $\begin{array}{l}103(Y) \\
\text { OHMS }\end{array}$ \\
\hline 0 & 7352.90 & 9795.16 & 16325.3 & 32650.5 & 29491.24 \\
\hline 1 & 6988.42 & 9309.62 & 15516.0 & 31032.1 & 28157.49 \\
\hline 2 & 6643.38 & 8849.98 & 14750.0 & 29499.9 & 26891.19 \\
\hline 3 & 6317.41 & 8415.73 & 14026.2 & 28052.4 & 25688.61 \\
\hline 4 & 6009.39 & 8005.39 & 13342.3 & 26684.6 & 24546.22 \\
\hline 5 & 5718.10 & 7617.37 & 12695.6 & 25391.2 & 23460.72 \\
\hline 6 & 5442.68 & 7250.46 & 12084.1 & 24168.2 & 22428.99 \\
\hline 7 & 5182.12 & 6903.35 & 11505.6 & 23011.2 & 21448.12 \\
\hline 8 & 4935.54 & 6574.88 & 10958.1 & 21916.3 & 20515.34 \\
\hline 9 & 4702.12 & 6263.93 & 10439.9 & 20879.8 & 19628.07 \\
\hline 10 & 4481.09 & 5969.48 & 9949.14 & 19898.3 & 18783.87 \\
\hline 11 & 4271.72 & 5690.57 & 9484.28 & 18968.6 & 17980.43 \\
\hline 12 & 4073.33 & 5426.28 & 904380 & 180876 & 17215.58 \\
\hline 13 & 3885.28 & 5175.78 & 8626.30 & 17252.6 & 16487.30 \\
\hline 14 & 3706.99 & 4938.27 & 8230.45 & 16460.9 & 15793.65 \\
\hline 15 & 3537.90 & 4713.01 & 7855.01 & 15710.0 & 15132.82 \\
\hline 16 & 3377.47 & 4499.30 & 7498.83 & 14997.7 & 14503.11 \\
\hline 17 & 3225.23 & 4296,48 & 7160.80 & 14321.6 & 2.89 \\
\hline 18 & 3080.70 & 4103.95 & 6839.92 & 13679.8 & 13330.64 \\
\hline 19 & 2943.46 & 3921.13 & 6535.22 & 13070.4 & 4.92 \\
\hline 20 & 2813.11 & 3747.48 & 6245.80 & 12491.6 & 12264.39 \\
\hline 21 & 2689.26 & 3582.49 & 5970.82 & 11941.6 & 11767.75 \\
\hline 22 & 2571.54 & 3425.68 & 5709.47 & 11418.9 & 11293.80 \\
\hline 23 & 2459.64 & 6276.61 & 5461.01 & 10922.0 & 10841.39 \\
\hline 24 & 2353.22 & 3134.84 & 5224.74 & 10449.5 & 10409.44 \\
\hline 25 & 2252.00 & 3000.00 & 5000.00 & 10000.0 & 10000.00 \\
\hline 26 & 2155.69 & 2871.70 & 4786.16 & 9572.32 & 9602.89 \\
\hline 27 & 2064.02 & 2749.59 & 4582.64 & 9165.29 & 9226.41 \\
\hline 28 & 1976.76 & 2633.34 & 4388.89 & 8777.79 & 8866.62 \\
\hline 29 & 1893.67 & 2522.10 & 4204.34 & 8408.68 & 8522.70 \\
\hline 30 & 1814.51 & 2417.19 & 4028.66 & 8057.31 & 8193.89 \\
\hline 31 & 1739.09 & 2316.73 & 3861.22 & 7722.43 & 7879.43 \\
\hline 32 & 1667.22 & 2220.99 & 3701.65 & 7403.29 & 7578.65 \\
\hline 33 & 1598.51 & 2129.52 & 3549.20 & 7098.42 & 7290.88 \\
\hline 34 & 1533.20 & 2042.50 & 3404.18 & 6808.36 & 7015.50 \\
\hline 35 & 1470.89 & 1959.39 & 3265.65 & 6531.31 & 6751.92 \\
\hline 36 & 1411.58 & 1880.47 & 3134.12 & 6265.75 & 6499.57 \\
\hline 37 & 1354.91 & 1804.94 & 3008.23 & 6016.47 & 6257.93 \\
\hline 38 & 1300.77 & 1732.82 & 2888.03 & 5776.05 & 6026.49 \\
\hline 39 & 1249.08 & 1663.96 & 2773.26 & 5546.53 & 5804.78 \\
\hline 40 & 1199.72 & 1598.20 & 2663.67 & 5327.34 & 5592.33 \\
\hline 41 & 1152.57 & 1535.39 & 2558,99 & 5117.97 & 5388.73 \\
\hline 42 & 1107.52 & 1475.38 & 2458.97 & 4917.94 & 5193.56 \\
\hline 43 & 1064.47 & 1418.03 & 2363.39 & 4726.77 & 5006.43 \\
\hline 44 & 1023.30 & 1363.17 & 2271.95 & 4543.91 & 4826.98 \\
\hline 45 & 983.97 & 1310.80 & 2184.66 & 4369.33 & 4654.86 \\
\hline 46 & 946.02 & 1260.25 & 2100.92 & 4200.84 & 4489.73 \\
\hline 47 & 909.99 & 1212.24 & 2020.40 & 4040.81 & 5331.28 \\
\hline 48 & 875.92 & 1166.85 & 1944.76 & 3889.51 & 4179.20 \\
\hline 49 & 842.96 & 1122.95 & 1871.59 & 3743.17 & 4033.22 \\
\hline 50 & 811.42 & 1080.93 & 1801.55 & 3603.10 & 3893.05 \\
\hline
\end{tabular}

STANDARD ASSEMBLIES

100FA - Series 100, .070" diameter molded plastic tip, 30 gauge PVC insulated ribbon cable.

100FD - Series 100, .070" diameter molded plastic tip, 32 gauge bifilar heavy isomid insulation.

100DD - Series 100, .050" diameter kapton sleeve with 32 gauge bifilar heavy isomid insulation.

100BF - Series $100, .030^{\circ}$ diameter kapton sleeve with 38 gauge bifilar heavy isomid insulation.
200LC - Series 200, .156" diameter aluminum tip. 30 gauge teflon leads.

300TA - Series 300, .375" diameter stainless steel cup, 30 gauge PVC insulated ribbon cable.

300тв - Series 300, .375" diameter stainless steel cup, 30 gauge teflon insulated ribbon cable.

100GG - Series, 100, .080" diameter molded plastic tip, 28 gauge kynar insulated twisted pair.

FIGURE 163 - Data sheet (pg. 3) for the Thermometrics MA100 10K $\Omega$ thermistor. 


\section{LM117/LM317A/LM317 3-Terminal Adjustable Regulator}

\section{General Description}

The LM117 series of adjustable 3-terminal positive voltage regulators is capable of supplying in excess of $1.5 \mathrm{~A}$ over a $1.2 \mathrm{~V}$ to $37 \mathrm{~V}$ output range. They are exceptionally easy to use and require only two external resistors to set the output voltage. Further, both line and load regulation are better than standard fixed regulators. Also, the LM117 is packaged in standard transistor packages which are easily mounted and handled.

In addition to higher performance than fixed regulators, the L.M117 series offers full overload protection available only in IC's. Included on the chip are current limit, thermal overioad protection and safe area protection. All overload protection circuitry remains fully functional even if the adjustment terminal is disconnected.

Normally, no capacitors are needed unless the device is situated more than 6 inches from the input filter capacitors in which case an input bypass is needed. An optional output capacitor can be added to improve transient response. The adjustment terminal can be bypassed to achieve very high ripple rejection ratios which are difficult to achieve with standard 3-terminal regulators.

Besides replacing fixed regulators, the LM117 is useful in a wide variety of other applications. Since the regulator is "floating" and sees onlyy the input-to-output differential volt-

\section{Typical Applications}

1.2V-25V Adjustable Regulator

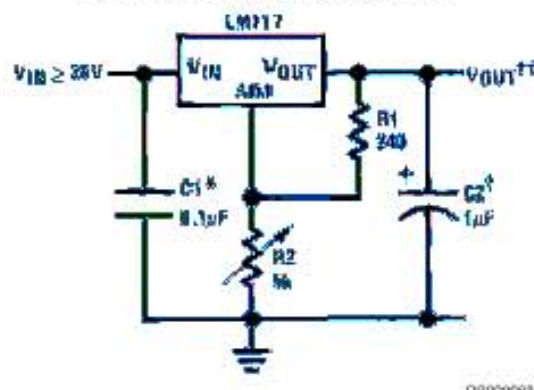

0900008s-1

Full output current not available at high inpud-output voltages

"Needed if device is more then 6 inches from filter capacitors.

tOpticnal - irreroves transient response. Oulput capacitors in the range of 1 ff to $1000 \mu \mathrm{F}$ of alizninum or tantalum electrobytic are commonty used to provide improved outpuat impedance and rejection of transients.

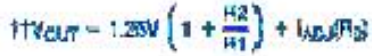

age, supplies of several hundred volts can be regulated as long as the maximum input to output differential is not exceeded, L.e., avoid short-circuiting the output.

Aiso, it makes an especially simple adjustable switching regulator, a programmable output regulator, or by connecting a fixed resistor between the adjustment pin and output, the LM117 can be used as a precision current regulator. Supplies with electronic shutdown can be achieved by clamping the adjustment terminal to ground which programs the output to $1.2 \mathrm{~V}$ where most loads draw little current.

For applications requiring greater output current, see LM150 series (3A) and LM138 series (5A) data sheets. For the negative complement, see LM137 series data sheet.

\section{Features}

- Guaranteed $1 \%$ output voltage tolerance (LM317A)

- Guaranteed max. $0.01 \% N$ line regulation (LM317A)

- Guaranteed max. 0.3\% load regulation (LM117)

- Guaranteed 1.5A output current

- Adjustable output down to $1.2 \mathrm{~V}$

- Current limit constant with temperature

- P+ Product Enhancement tested

- $80 \mathrm{~dB}$ ripple rejection

- Output is short-circuit protected

\section{LM117 Series Packages}

\begin{tabular}{|c|c|c|}
\hline $\begin{array}{c}\text { Part Number } \\
\text { Suffix }\end{array}$ & Package & $\begin{array}{c}\text { Design } \\
\text { Load } \\
\text { Current }\end{array}$ \\
\hline K & TO-3 & $1.5 \mathrm{~A}$ \\
\hline H & TO-39 & $0.5 \mathrm{~A}$ \\
\hline T & TO-220 & $1.5 \mathrm{~A}$ \\
\hline E & LCC & $0.5 \mathrm{~A}$ \\
\hline S & TO-263 & $1.5 \mathrm{~A}$ \\
\hline EMP & SOT-223 & $1 \mathrm{~A}$ \\
\hline MDT & TO-252 & $0.5 \mathrm{~A}$ \\
\hline
\end{tabular}

\section{SOT-223 vs D-Pak (TO-252) Packages}

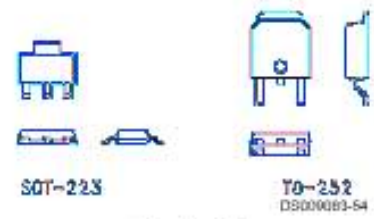

Scale 1:1

FIGURE 164 - Data sheet (pg. 1) for the LM317T (blower) voltage regulator. 


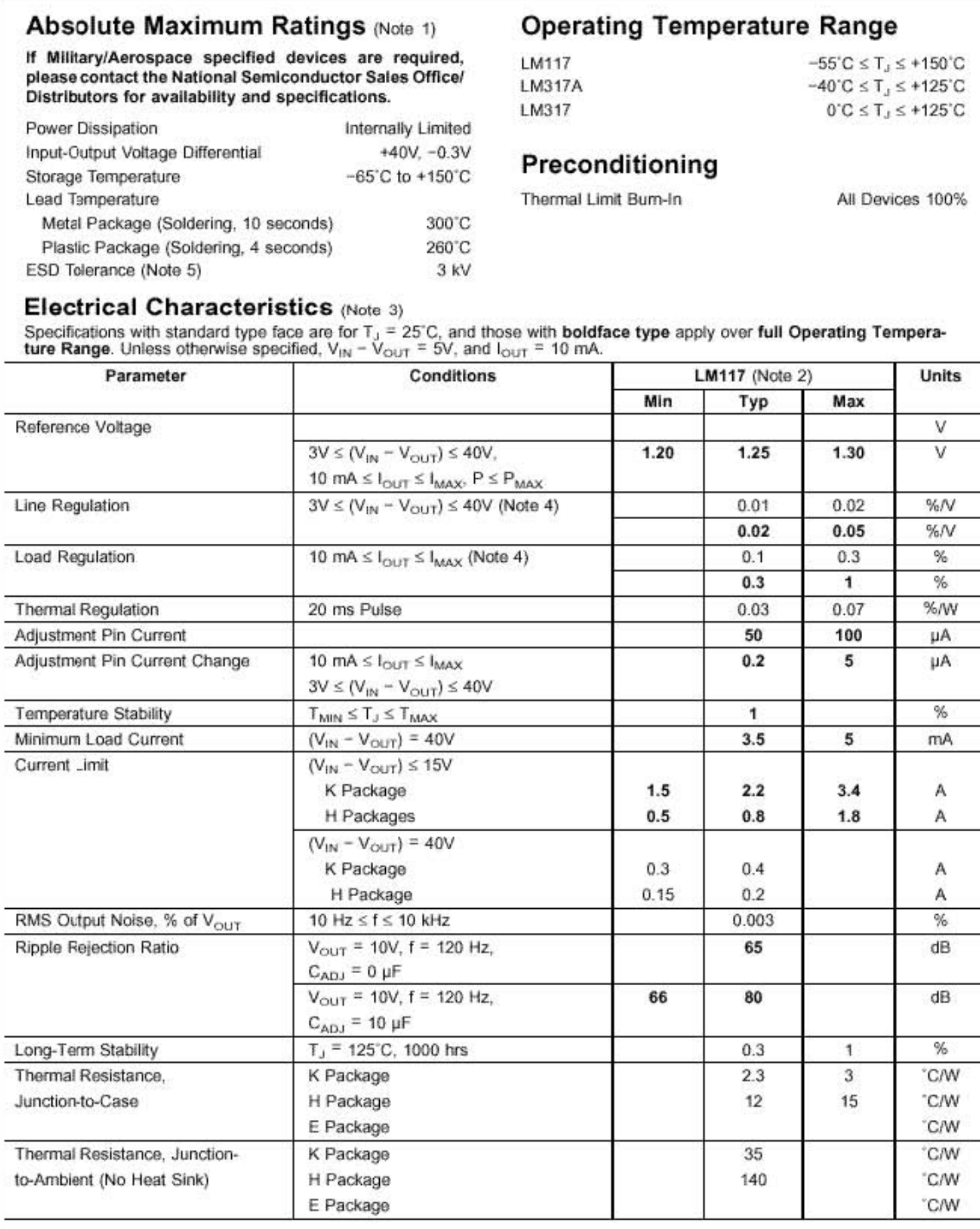

FIGURE 165 - Data sheet (pg. 2) for the LM317T (blower) voltage regulator. 


\section{Application Hints}

In operation, the LM117 develops a nominal $1.25 \mathrm{~V}$ reference voltage, $V_{R E F}$, between the output and adjustment teminal. The reference voltage is impressed across program resistor $\mathrm{R} 1$ and, since the voltage is constant, a constant current $I_{\text {, }}$ then flows through the output set resistor R2, giving an output voltage of

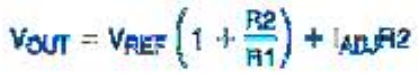

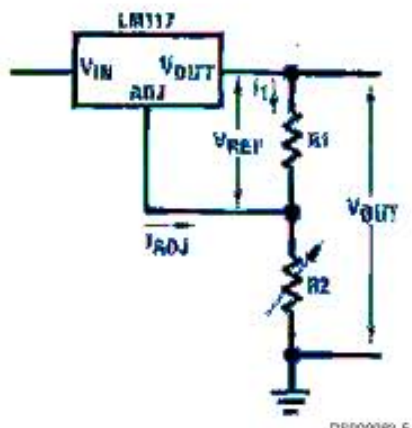

FIGURE 1.

Since the $100 \mu \mathrm{A}$ current from the adjustment terminal represents an error term, the LM117 was designed to minimize $I_{A D}$ and make it very constant with line and load changes. To do this, all quiescent operating current is returned to the output establishing a minimum load current requirement. If there is insuficient load on the output, the output will rise.

\section{External Capacitors}

An input bypass capacitor is recommended. A $0.1 \mu \mathrm{F}$ disc or $1 \mu \mathrm{F}$ solid tantalum on the input is suitable input bypassing for almost all applications. The device is more sensitive to the absence of input bypassing when adjustment or output capacitors are used but the above values will eliminate the possibility of problems.

The adjustment terminal can be bypassed to ground on the LM117 to improve ripple rejection. This bypass capacitor prevents ripple from being amplified as the output voltage is increased. With a $10 \mu \mathrm{F}$ bypass capacitor $80 \mathrm{~dB}$ ripple rejection is obtainable at any output level. Increases over $10 \mu \mathrm{F}$ do not appreciably improve the ripple rejection at frequencies above $120 \mathrm{~Hz}$. If the bypass capacitor is used, it is sometimes necessary to include protection diodes to prevent the capacitor from discharging through internal low current paths and damaging the device.

In general, the best type of capacitors to use is solid tantalum. Solid tantalum capacitors have low impedance even at high frequencies. Depending upon capacitor construction, if takes about $25 \mu \mathrm{F}$ in aluminum electrolytic to equal $1 \mu \mathrm{F}$ solid tantalum at high frequencies. Ceramic capacitors ane also good at high frequencies; but some types have a large decrease in capacitance at frequencies around $0.5 \mathrm{MHz}$. For this reason, $0.01 \mu \mathrm{F}$ disc may seem to work better than a 0.1 $\mu \mathrm{F}$ disc as a bypass.

Aithough the LM117 is stable with no output capacitors, like any feedback circuit, certain values of external capacitance can cause excessive ringing. This occurs with values between $500 \mathrm{pF}$ and $5000 \mathrm{pF}$. A $1 \mu \mathrm{F}$ solid tantalum (or $25 \mu \mathrm{F}$ aluminum electrolytic) on the output swamps this effect and insures stability. Any increase of the load capacitance larger than $10 \mu \mathrm{F}$ will merely improve the loop stability and output impedance.

\section{Load Regulation}

The LM117 is capable of providing extremely good load regulation but a few precautions are needed to obtain maximum performance. The current set resistor connected between the adjustment terminal and the output terminal (usually $240 \Omega 2$ ) should be tied directly to the output (case) of the regulator rather than near the load. This eliminates line drops from appearing effectively in series with the reference and degrading regulation. For example, a $15 \mathrm{~V}$ regulator with $0.05 \Omega$ resistance between the regulator and load will have a load regulation due to line resistance of $0.05 \Omega \times \mathrm{I}_{\mathrm{L}}$. If the set resistor is connected near the load the effective line resistance will be $0.05 \Omega(1+R 2 / R 1)$ or in this case, 11.5 times worse.

Figure 2 shows the effect of resistance between the regulator and $240 \Omega$ set resistor.

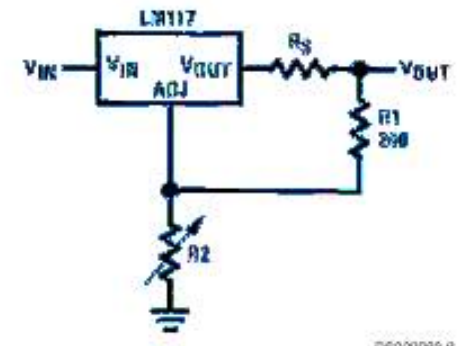

FIGURE 2. Regulator with LIne Resistance in Output Lead

With the TO-3 package, it is easy to minimize the resistance from the case to the set resistor, by using two separate leads to the case. However, with the TO-39 package, care should be taken to minimize the wire length of the output lead. The ground of R2 can be retumed near the ground of the load to provide remote ground sensing and improve load regulation.

\section{Protection Diodes}

When external capacitors are used with any IC regulator it is sometimes necessary to add protection diodes to prevent the capacitors from discharging through low current points into the regulator. Most $10 \mu \mathrm{F}$ capacitors have low enough internal series resistance to deliver 20A spikes when shorted. Although the surge is short, there is enough energy to damage parts of the IC.

When an output capacitor is connected to a regulator and the input is shorted, the output capacitor will discharge into the output of the regulator. The discharge current depends on the value of the capacitor, the output voltage of the regulator, and the rate of decrease of $\mathrm{V}_{\mathrm{IN}}$. In the LM117, this discharge path is through a large junction that is able to sustain $15 \mathrm{~A}$ surge with no problem. This is not true of other types of positive regulators. For output capacitors of $25 \mu \mathrm{F}$ or less, there is no need to use diodes.

The bypass capacitor on the adjustment terminal can discharge through a low current junction. Discharge occurs when either the input or output is shorted. Internal to the LM117 is a $50 \Omega$ resistor which limits the peak discharge current. No protection is needed for output voltages of $25 \mathrm{~V}$ or

FIGURE 167 - Data sheet (pg. 5) for the LM317T (blower) voltage regulator. 


\section{Application Hints (Continued)}

less and $10 \mu \mathrm{F}$ capacitance. Figure 3 shows an LM117 with protection diodes included for use with outputs greater than $25 \mathrm{~V}$ and high values of output capacitance.

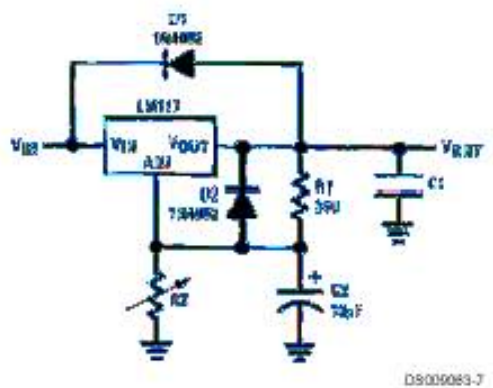

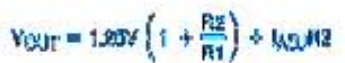

D1 protects aganst C1

D2 protects aganst C2

\section{FIGURE 3. Regulator with Protection Diodes}

When a value for $\theta_{\langle i-A)}$ is found using the equation shown. a heatsink must be selected that has a value that is less than or equal to this number.

$\theta_{\langle\mathrm{H}-\mathrm{A}\rangle}$ is specified numerically by the heatsink manufacturer in the catalog, or shown in a curve that plots temperature rise vs power dissipation for the heatsink.

\section{HEATSINKING TO-263, SOT-223 AND TO-252 PACKAGE} PARTS

The TO-263 ("S"), SOT-223 ("MP") and TO-252 ("DT") packages use a copper plane on the PCB and the PCB itself as a heatsink. To optimize the heat sinking ability of the plane and PCB. solder the tab of the package to the plane.

Figure 4 shows for the TO-263 the measured values of $\theta_{(j-A)}$ for different copper area sizes using a typical PCB with 1 ounce copper and no solder mask over the copper area used for heatsinking

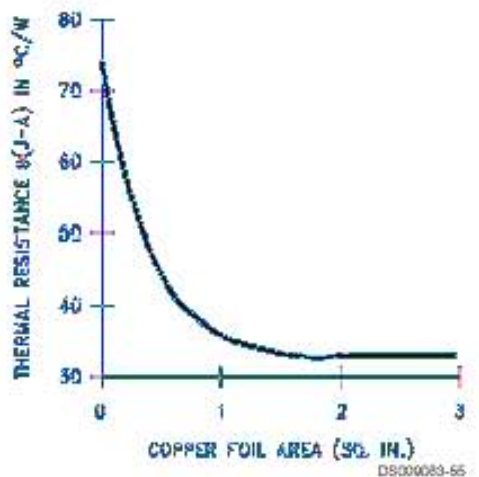

FIGURE 4. $\theta_{(J-A)}$ Vs Copper (1 ounce) Area for the TO-263 Package

As shown in the figure, increasing the copper area beyond 1 square inch produces very little improvement. It should also be observed that the minimum value of $\theta_{\{, 3-A)}$ for the TO-263 package mounted to a $\mathrm{PCB}$ is $32^{\circ} \mathrm{C}$.
As a design aid, Figure 5 shows the maximum allowable power dissipation compared to ambient temperature for the TO-263 device (assuming $\theta_{(j-\mathrm{A})}$ is $35^{\circ} \mathrm{C} / \mathrm{W}$ and the maximum junction temperature is $125^{\circ} \mathrm{C}$ ).

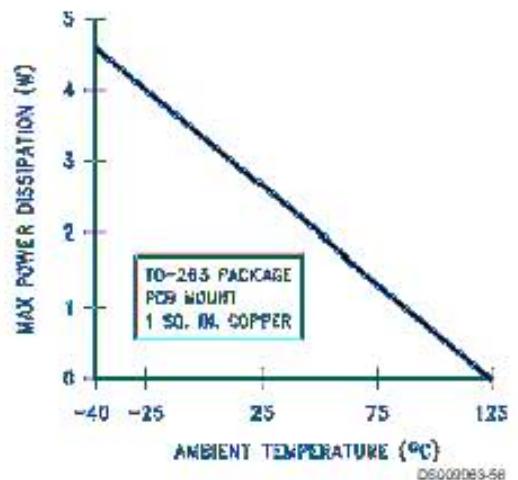

FIGURE 5. Maximum Power Dissipation vs $T_{A M B}$ for the TO-263 Package

Figure 6 and Figure 7 show the information for the SOT-223 package. Figure 7 assumes a $\theta_{(J-A)}$ of $74^{\circ} \mathrm{C} / W$ for 1 ounce copper and $51^{\circ} \mathrm{C} / \mathrm{W}$ for 2 ounce copper and a maximum junction temperature of $125^{\circ} \mathrm{C}$.

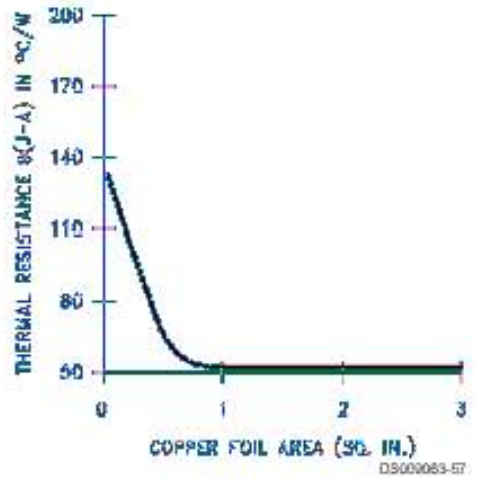

FIGURE 6. $\theta_{(J-A)}$ vs Copper ( 2 ounce) Area for the SOT-223 Package

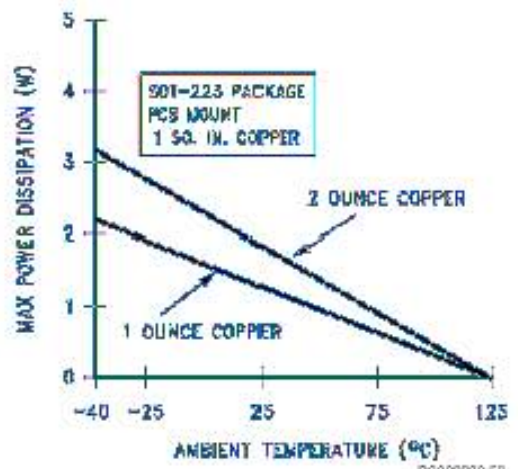

FIGURE 7. Maximum Power Dissipation vs $T_{A M B}$ for the SOT-223 Package

FIGURE 168 - Data sheet (pg. 6) for the LM317T (blower) voltage regulator. 


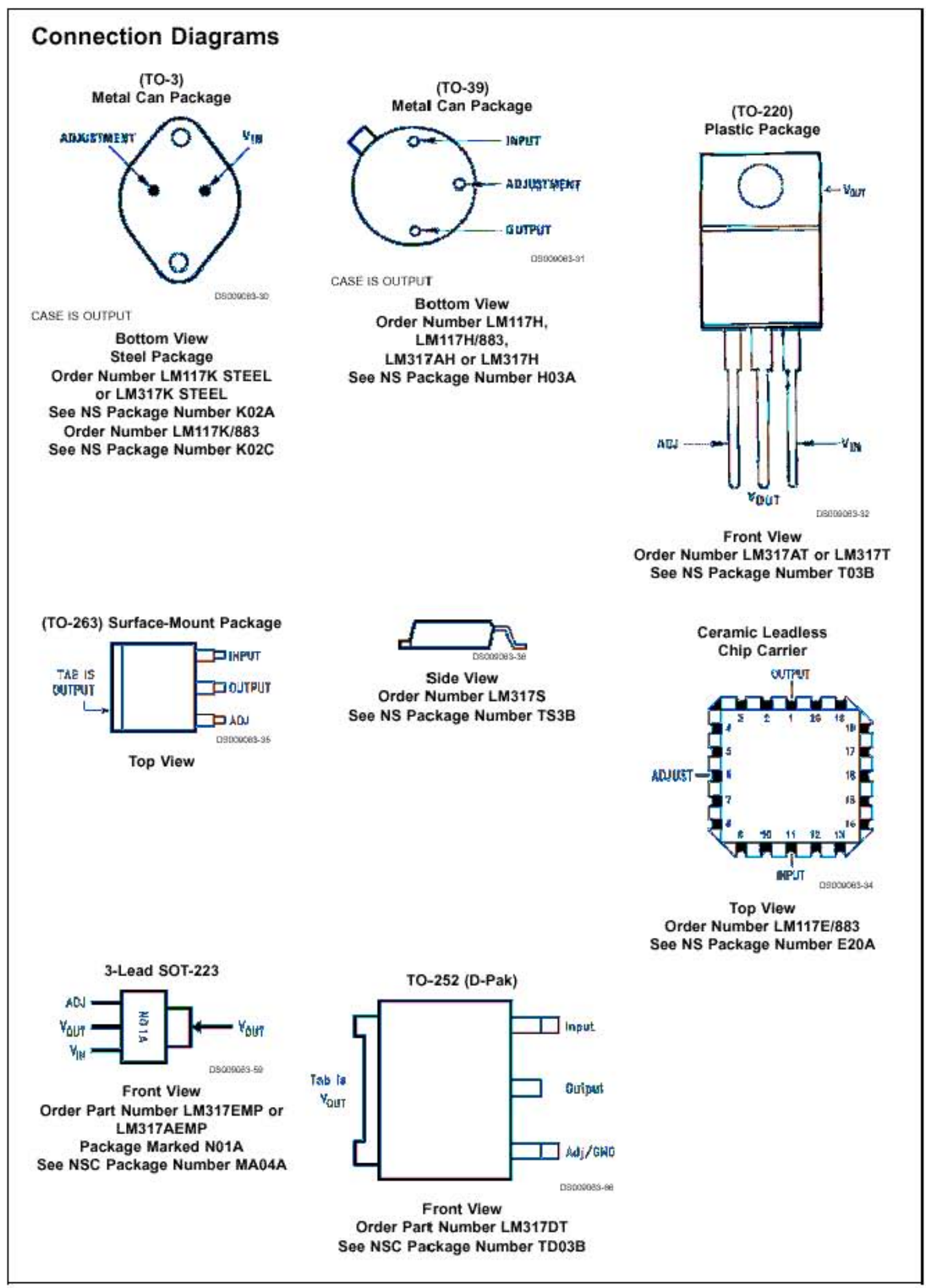

19

miwinational.com

FIGURE 169 - Data sheet (pg. 19) for the LM317T (blower) voltage regulator. 


\section{LM2940/LM2940C}

\section{A Low Dropout Regulator}

\section{General Description}

The LM2940/LM2940C positive voltage regulator features the ability to source $1 \mathrm{~A}$ of output current with a dropout voltage of typically $0.5 \mathrm{~V}$ and a maximum of $1 \mathrm{~V}$ over the entire temperature range. Furthermore, a quiescent current reduction circuit has been included which reduces the ground current when the differential between the input voltage and the output voltage exceeds approximately $3 \mathrm{~V}$. The quiescent current with $1 \mathrm{~A}$ of output current and an input-output differential of $5 \mathrm{~V}$ is therefore only $30 \mathrm{~mA}$. Higher quiescent currents only exist when the regulator is in the dropout mode $\left(\mathrm{V}_{\mathbb{I N}}-\mathrm{V}_{\text {OUT }} \leq 3 \mathrm{~V}\right)$.

Designed also for vehicular applications, the LM2940/ LM2940C and all regulated circuitry are protected from reverse battery installations or 2-battery jumps. During line transients, such as load dump when the input voltage can momentarily exceed the specified maximum operating voltage, the regulator will automatically shut down to protect both the internal circuits and the load. The LM2940/ LM2940C cannot be harmed by temporary mirror-image insertion. Familiar regulator features such as short circuit and thermal overload protection are also provided.

\section{Features}

- Dropout voltage typically $0.5 \mathrm{~V} \oplus \mathrm{I}_{\mathrm{O}}=1 \mathrm{~A}$

- Output current in excess of $1 \mathrm{~A}$

- Output voltage trimmed before assembly

- Reverse battery protection

- Internal short circuit current limit

- Mirror image insertion protection

- $\mathrm{P}^{+}$Product Enhancement tested

\section{Typical Application}

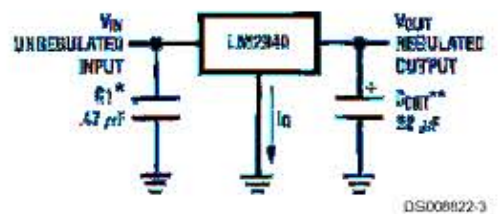

"Required if regulator is located far from power supply fitter.

*Cout must be at least $22 \mu \mathrm{F}$ to maintain stability. May be increased without bound to maintain regulation during transients. Locate as close as possible to the regulator. This capacitor must be rated over the same operating temperature range as the regulator and the ESR is critical; see curve.

\section{Ordering Information}

\begin{tabular}{|c|c|c|c|c|c|c|c|c|}
\hline \multirow{2}{*}{$\begin{array}{c}\text { Temperature } \\
\text { Range }\end{array}$} & \multicolumn{7}{|c|}{ Output Voltage } & \multirow{2}{*}{ Package } \\
\hline & 5.0 & \multicolumn{2}{|c|}{8.0} & 9.0 & 10 & 12 & 15 & \\
\hline \multirow[t]{2}{*}{$0^{\prime} \mathrm{C} \leq \mathrm{T}_{\mathrm{J}} \leq 125^{*} \mathrm{C}$} & LM2940CT-5.0 & & & LM2940CT-9.0 & & \multirow{2}{*}{$\begin{array}{l}\text { LM2940CT-12 } \\
\text { LM2940CS-12 }\end{array}$} & LM2940CT-15 & TO-220 \\
\hline & LM2940CS. 5.0 & & & LM2940CS-9.0 & & & LM2940CS-15 & TO-263 \\
\hline \multirow[t]{2}{*}{$-40^{\circ} \mathrm{C} \leq \mathrm{T}_{\mathrm{J}} \leq 125^{\prime} \mathrm{C}$} & LM2940T-5.0 & \multirow{2}{*}{\multicolumn{2}{|c|}{$\begin{array}{l}\text { LM2940T }-8.0 \\
\text { LM2940S-8.0 }\end{array}$}} & LM2940T-9.0 & LM2940T-10 & \multirow{2}{*}{$\begin{array}{l}\text { LM2940T-12 } \\
\text { LM2940S-12 } \\
\end{array}$} & & TO-220 \\
\hline & LM2940S-5.0 & & & LM2940S-9.0 & LM2940S-10 & & & TO-263 \\
\hline \multirow[t]{2}{*}{$-40^{\prime} \mathrm{C} \leq \mathrm{T}_{\mathrm{J}} \leq 85^{\circ} \mathrm{C}$} & LM2940IMP 5.0 & \multicolumn{2}{|c|}{ LM2940IMP=8.0 } & LM29401MP $=9.0$ & LM2940IMP $=10$ & LM29401MP=12 & LM2.940IMP=15 & SOT $=223$ \\
\hline & LM2940IMPX-5. & \multicolumn{2}{|c|}{ LM2940IMPX-8. } & LM2940IMPX-9.9 & LM2940IMPX-10 & LM2940IMPX-12 & LM2940IMPX-10 & $\begin{array}{l}\text { SOT-223 } \\
\text { in Tape } \\
\text { and Reel }\end{array}$ \\
\hline $\begin{array}{l}\text { SOT-223 Package } \\
\text { Marking }\end{array}$ & L.53B & \multicolumn{2}{|c|}{ L54B } & LOEB & L55B & L56B & L70B & \\
\hline \multicolumn{9}{|c|}{ The physical size of the SOT-223 is too small to contain the full device part number. The package markings indicated are what will appear on the actual devict } \\
\hline \multirow{2}{*}{$\begin{array}{c}\text { Temperature } \\
\text { Range }\end{array}$} & \multicolumn{7}{|c|}{ Output Voltage } & \multirow[t]{2}{*}{ Package } \\
\hline & 5.0 & & & 8.0 & 12 & & 15 & \\
\hline \multirow[t]{2}{*}{$-55^{\circ} \mathrm{C} \leq \mathrm{T}_{J} \leq 125^{\circ} \mathrm{C}$} & \multicolumn{2}{|c|}{$\begin{array}{l}\text { LM2940J-5.0/883 } \\
5962-8958701 \mathrm{EA}\end{array}$} & \multicolumn{2}{|c|}{$\begin{array}{l}\text { LM2940J-8.0/883 } \\
5962-9088301 \text { QEA }\end{array}$} & $\begin{array}{l}\text { LM2940J-12/88: } \\
5962-9088401 \mathrm{QE}\end{array}$ & \multicolumn{2}{|c|}{$\begin{array}{c}\text { LM2940J-15/883 } \\
5962-9088501 \text { QEA }\end{array}$} & J16A \\
\hline & \multicolumn{2}{|c|}{$\begin{array}{l}\text { LM2940WG5.0/883 } \\
5962-8958701 X A\end{array}$} & & & & & & WG16A \\
\hline \multicolumn{9}{|c|}{ For information on military temperature range products, please go to the MivAero Web Site at http:-i/www.national.com/appinfo/milaerorindex. himl. } \\
\hline
\end{tabular}

FIGURE 170 - Data sheet (pg. 1) for the LM2940T 10-volt regulator. 


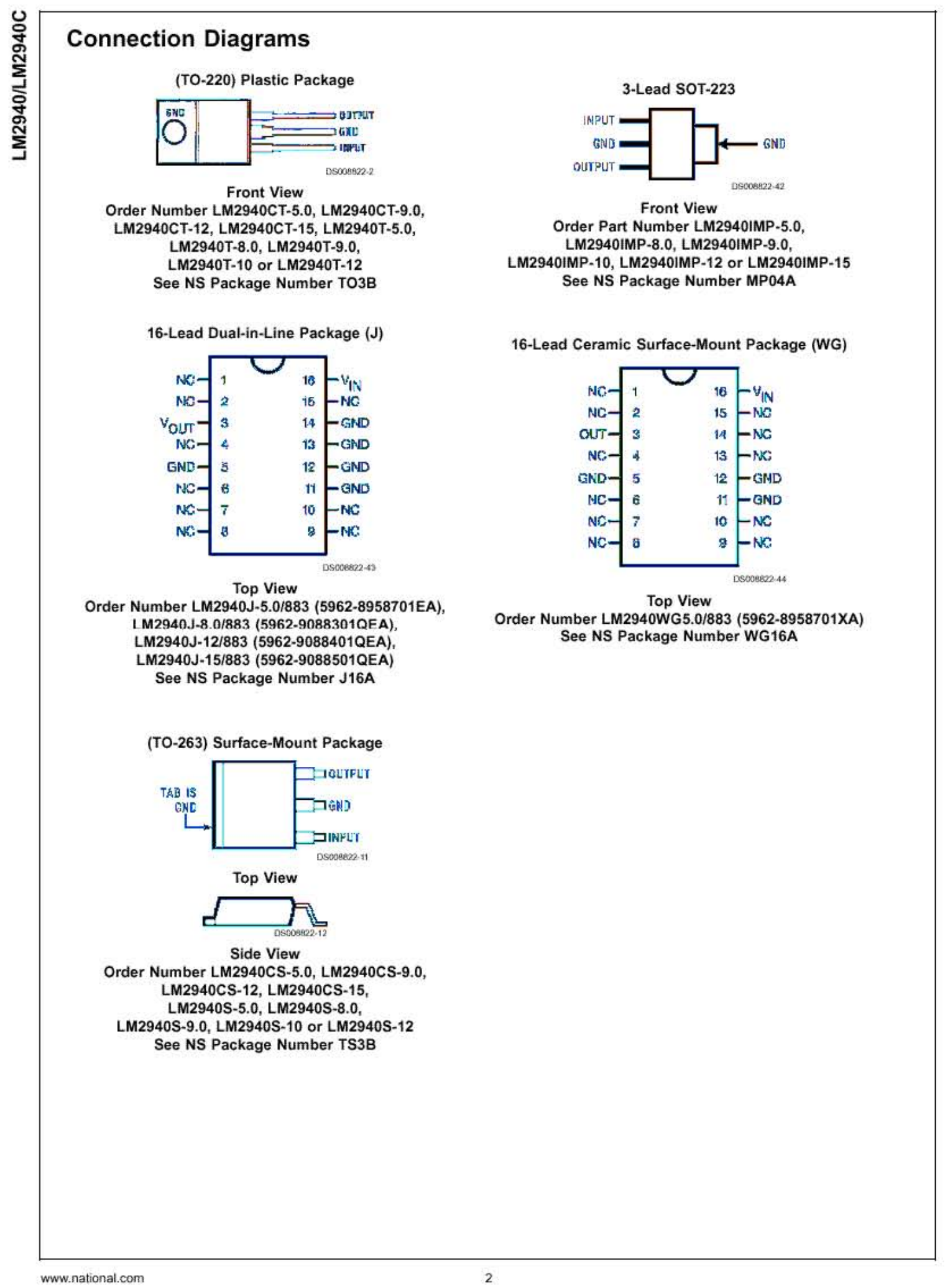

FIGURE 171 - Data sheet (pg. 2) for the LM2940T 10-volt regulator. 


\begin{tabular}{|c|c|c|c|c|c|c|c|c|}
\hline \multicolumn{9}{|c|}{$\begin{array}{l}\text { Electrical Characteristics (Continued) } \\
V_{1 N}=V_{O}+5 V, I_{O}=1 A, C_{O}=22 \mu F \text {, unless otherwise specified. Boldface limits apply over the entire operating tempera- } \\
\text { ture range of the indicated device. All other specifications apply for } T_{A}=T_{J}=25^{\circ} \mathrm{C} \text {. }\end{array}$} \\
\hline \multicolumn{2}{|c|}{ Output Voltage $\left(\mathrm{V}_{\mathrm{o}}\right)$} & \multicolumn{3}{|c|}{$5 \mathrm{~V}$} & \multicolumn{3}{|c|}{$8 \mathrm{~V}$} & \multirow[b]{2}{*}{ Units } \\
\hline Parameter & Conditions & Typ & $\begin{array}{l}\text { LM2940 } \\
\text { Limit } \\
\text { (Note 4) }\end{array}$ & $\begin{array}{c}\text { LM2940/883 } \\
\text { Limit } \\
\text { (Note 5) }\end{array}$ & Typ & $\begin{array}{l}\text { LM2940 } \\
\text { Limit } \\
\text { (Note 4) }\end{array}$ & $\begin{array}{c}\text { LM2940/883 } \\
\text { Limit } \\
\text { (Note 5) }\end{array}$ & \\
\hline $\begin{array}{l}\text { Maximum Line } \\
\text { Transient }\end{array}$ & $\begin{array}{l}R_{\mathrm{O}}=100 \Omega \\
\mathrm{LM} 2940, \mathrm{~T} \leq 100 \mathrm{~ms} \\
\mathrm{LM} 2940 / 883, T \leq 20 \mathrm{~ms} \\
\mathrm{LM} 2940 \mathrm{C}, \mathrm{T} \leq 1 \mathrm{~ms}\end{array}$ & $\begin{array}{l}75 \\
55\end{array}$ & $\begin{array}{c}60 / 60 \\
45\end{array}$ & $40 / 40$ & $\begin{array}{l}75 \\
55\end{array}$ & $\begin{array}{c}60 / 60 \\
45\end{array}$ & $40 / 40$ & $V_{\text {MIN }}$ \\
\hline $\begin{array}{l}\text { Reverse Polarity } \\
\text { DC Input Voltage }\end{array}$ & $\begin{array}{l}R_{\mathrm{O}}=100 \Omega \\
\mathrm{LM} 2940, \mathrm{LM} 2940 / 883 \\
\mathrm{LM} 2940 \mathrm{C}\end{array}$ & $\begin{array}{l}-30 \\
-30 \\
\end{array}$ & $\begin{array}{c}-15 /-15 \\
-15 \\
\end{array}$ & $-15 /-15$ & $\begin{array}{l}-30 \\
-30 \\
\end{array}$ & $\begin{array}{c}-15 /-15 \\
-15\end{array}$ & $-15 /-15$ & $V_{\text {MIN }}$ \\
\hline $\begin{array}{l}\text { Reverse Polarity } \\
\text { Transient Input } \\
\text { Voltage }\end{array}$ & $\begin{array}{l}R_{\mathrm{O}}=100 \Omega \\
\mathrm{LM} 2940, T \leq 100 \mathrm{~ms} \\
\mathrm{LM} 2940 / 883, T \leq 20 \mathrm{~ms} \\
\mathrm{LM} 2940 \mathrm{C}, T \leq 1 \mathrm{~ms}\end{array}$ & $\begin{array}{l}-75 \\
-55\end{array}$ & $\begin{array}{l}-50 /-50 \\
-45 /-45\end{array}$ & $-45 /-45$ & -75 & $-50 /-50$ & $-45 /-45$ & $V_{\text {MIN }}$ \\
\hline
\end{tabular}

\section{Electrical Characteristics}

$V_{I N}=V_{O}+5 V, L_{O}=1 A, C_{O}=22 \mu F$, unless otherwise specified. Boldface limits apply over the entire operating temperature range of the indicated device. All other specifications apply for $T_{A}=T_{J}=25^{\circ} \mathrm{C}$.

\begin{tabular}{|c|c|c|c|c|c|c|}
\hline \multicolumn{2}{|c|}{ Output Voltage $\left(\mathrm{V}_{\mathrm{o}}\right)$} & \multicolumn{2}{|c|}{$9 \mathrm{~V}$} & \multicolumn{2}{|c|}{$10 \mathrm{~V}$} & \multirow[b]{2}{*}{ Units } \\
\hline Parameter & Conditions & Typ & $\begin{array}{l}\text { LM2940 } \\
\text { Limit } \\
\text { (Note 4) }\end{array}$ & Typ & $\begin{array}{l}\text { LM2940 } \\
\text { Limit } \\
\text { (Note 4) }\end{array}$ & \\
\hline \multirow[b]{2}{*}{ Output Voltage } & \multirow[b]{2}{*}{$5 \mathrm{~mA} \leq \mathrm{l}_{\mathrm{O}} \leq 1 \mathrm{~A}$} & \multicolumn{2}{|c|}{$10.5 \mathrm{~V} \leq \mathrm{V}_{\mathrm{IN}} \leq 26 \mathrm{~V}$} & \multicolumn{2}{|c|}{$11.5 \mathrm{~V} \leq \mathrm{V}_{\mathrm{IN}} \leq 26 \mathrm{~V}$} & \multirow[b]{2}{*}{$\begin{array}{l}V_{\text {MIN }} \\
V_{\text {MAX }}\end{array}$} \\
\hline & & 9.00 & $\begin{array}{l}8.73 / 8.55 \\
9.27 / 9.45\end{array}$ & 10.00 & $\begin{array}{c}9.70 / 9.50 \\
10.30 / 10.50\end{array}$ & \\
\hline Line Regulation & $\begin{array}{l}V_{O}+2 V \leq V_{1 N} \leq 26 V \\
I_{0}=5 \mathrm{~mA}\end{array}$ & 20 & 90 & 20 & 100 & $\mathrm{mV}_{\text {MAX }}$ \\
\hline Load Regulation & $\begin{array}{l}50 \mathrm{~mA} \leq \mathrm{I}_{\circ} \leq 1 \mathrm{~A} \\
\mathrm{LM} 2940 \\
\mathrm{LM} 2940 \mathrm{C}\end{array}$ & $\begin{array}{l}60 \\
60\end{array}$ & $\begin{array}{c}90 / 150 \\
90\end{array}$ & 65 & $100 / 165$ & $\operatorname{mV} V_{\text {MAX }}$ \\
\hline Output Impedance & $\begin{array}{l}100 \mathrm{mADC} \text { and } \\
20 \mathrm{mArms}, \\
\mathrm{f}_{\mathrm{o}}=120 \mathrm{~Hz}\end{array}$ & 60 & & 65 & & $\mathrm{~m} \Omega$ \\
\hline \multirow[t]{2}{*}{$\begin{array}{l}\text { Quiescent } \\
\text { Current }\end{array}$} & $\begin{array}{l}\mathrm{V}_{\mathrm{O}}+2 \mathrm{~V} \leq \mathrm{V}_{\mathrm{IN}}<26 \mathrm{~V}, \\
\mathrm{I}_{\mathrm{O}}=5 \mathrm{~mA} \\
\mathrm{LM} 2940 \\
\mathrm{LM} 2940 \mathrm{C}\end{array}$ & $\begin{array}{l}10 \\
10\end{array}$ & $\begin{array}{c}15 / 20 \\
15\end{array}$ & 10 & $15 / 20$ & $\mathrm{~mA}_{\text {MAX }}$ \\
\hline & $V_{I N}=V_{O}+5 V, I_{O}=1 A$ & 30 & $45 / 60$ & 30 & $45 / 60$ & $\mathrm{~mA}_{\text {MAX }}$ \\
\hline $\begin{array}{l}\text { Output Noise } \\
\text { Voltage }\end{array}$ & $\begin{array}{l}10 \mathrm{~Hz}-100 \mathrm{kHz}, \\
\mathrm{I}_{0}=5 \mathrm{~mA}\end{array}$ & 270 & & 300 & & $\mu \mathrm{V}_{\mathrm{rms}}$ \\
\hline Ripple Rejection & $\begin{array}{l}\mathrm{f}_{\mathrm{O}}=120 \mathrm{~Hz}, 1 \mathrm{~V}_{\mathrm{rms}} \\
\mathrm{I}_{\mathrm{O}}=100 \mathrm{~mA} \\
L M 2940 \\
L M 2940 \mathrm{C}\end{array}$ & $\begin{array}{l}64 \\
64\end{array}$ & $\begin{array}{c}52 / 46 \\
52\end{array}$ & 63 & $51 / 45$ & $\mathrm{~dB}_{\mathrm{MIN}}$ \\
\hline $\begin{array}{l}\text { Long Term } \\
\text { Stability }\end{array}$ & & 34 & & 36 & & $\begin{array}{c}\mathrm{mV} / \\
1000 \mathrm{Hr}\end{array}$ \\
\hline \multirow[t]{2}{*}{ Dropout Voltage } & $I_{0}=1 \mathrm{~A}$ & 0.5 & $0.8 / 1.0$ & 0.5 & $0.8 / 1.0$ & $V_{\text {MAX }}$ \\
\hline & $I_{0}=100 \mathrm{~mA}$ & 110 & $150 / 200$ & 110 & $150 / 200$ & $\operatorname{mV} V_{\text {MAX }}$ \\
\hline
\end{tabular}

www.national.com

FIGURE 172 - Data sheet (pg. 4) for the LM2940T 10-volt regulator. 


\begin{tabular}{|c|c|c|c|c|c|c|c|c|}
\hline \multicolumn{9}{|c|}{$\begin{array}{l}V_{1 N}=V_{O}+5 \mathrm{~V}, I_{O}=1 \mathrm{~A}, \mathrm{C}_{0}=22 \mu \mathrm{F} \text {, unless otherwise specified. Boldface limits apply over the entire operating tempera- } \\
\text { ture range of the indicated device. All other specifications apply for } T_{A}=T_{J}=25 \mathrm{C} \text {. }\end{array}$} \\
\hline \multicolumn{3}{|c|}{ Output Voltage $\left(\mathrm{V}_{\mathrm{o}}\right)$} & \multicolumn{2}{|c|}{$9 \mathrm{~V}$} & \multicolumn{3}{|c|}{$10 \mathrm{~V}$} & \multirow[b]{2}{*}{ Units } \\
\hline Parameter & \multicolumn{2}{|l|}{ Conditions } & Typ & $\begin{array}{l}\text { LM2940 } \\
\text { Limit } \\
\text { (Note 4) }\end{array}$ & Typ & \multicolumn{2}{|c|}{$\begin{array}{c}\text { LM2940 } \\
\text { Limit } \\
\text { (Note 4) }\end{array}$} & \\
\hline $\begin{array}{l}\text { Short Circuit } \\
\text { Current }\end{array}$ & \multicolumn{2}{|l|}{ (Note 6) } & 1.9 & 1.6 & 1.9 & \multicolumn{2}{|c|}{1.6} & $A_{\text {MIN }}$ \\
\hline $\begin{array}{l}\text { Maximum Line } \\
\text { Transient }\end{array}$ & $\begin{array}{l}\mathrm{R}_{\mathrm{O}}=100 \Omega \\
\mathrm{T} \leq 100 \mathrm{~ms} \\
\mathrm{LM} 2940 \\
\mathrm{LM} 2940 \mathrm{C}\end{array}$ & & $\begin{array}{l}75 \\
55\end{array}$ & $\begin{array}{c}60 / 60 \\
45\end{array}$ & 75 & \multicolumn{2}{|c|}{$60 / 60$} & $V_{\text {MIN }}$ \\
\hline $\begin{array}{l}\text { Reverse Polarity } \\
\text { DC Input Voltage }\end{array}$ & $\begin{array}{l}R_{\mathrm{O}}=100 \Omega \\
\mathrm{LM} 2940 \\
\mathrm{LM} 2940 \mathrm{C}\end{array}$ & & $\begin{array}{l}-30 \\
-30\end{array}$ & $\begin{array}{c}-15 /-15 \\
-15\end{array}$ & -30 & \multicolumn{2}{|c|}{$-15 /-15$} & $\mathrm{~V}_{\mathrm{MIN}}$ \\
\hline $\begin{array}{l}\text { Reverse Polarity } \\
\text { Transient Input } \\
\text { Voltage }\end{array}$ & $\begin{array}{l}R_{\mathrm{O}}=100 \Omega \\
\mathrm{T} \leq 100 \mathrm{~ms} \\
\mathrm{LM} 2940 \\
\mathrm{LM} 2940 \mathrm{C}\end{array}$ & & $\begin{array}{l}-75 \\
-55\end{array}$ & $\begin{array}{l}-50 /-50 \\
-45 /-45\end{array}$ & -75 & \multicolumn{2}{|c|}{$-50 /-50$} & $V_{\text {MIN }}$ \\
\hline \multicolumn{9}{|c|}{$\begin{array}{l}\text { Electrical Characteristics } \\
V_{I N}=V_{O}+5 \mathrm{~V}, I_{0}=1 \mathrm{~A}, C_{O}=22 \mu \mathrm{F} \text {, unless otherwise specified. Boldface limits apply over the entire operating tempera- } \\
\text { ture range of the indicated device. All other specifications apply for } T_{A}=T_{J}=25 \mathrm{C} \text {. }\end{array}$} \\
\hline \multicolumn{2}{|c|}{ Output Voltage $\left(\mathrm{V}_{\mathrm{o}}\right)$} & \multicolumn{3}{|c|}{$12 \mathrm{~V}$} & \multicolumn{3}{|c|}{$15 \mathrm{~V}$} & \multirow[b]{2}{*}{ Units } \\
\hline Parameter & Conditions & Typ & $\begin{array}{l}\text { LM2940 } \\
\text { Limit } \\
\text { (Note 4) }\end{array}$ & \begin{tabular}{|c|c|c|c|} 
LM2940/833 \\
Limit \\
(Note 5)
\end{tabular} & Typ & $\begin{array}{l}\text { LM2940 } \\
\text { Limit } \\
\text { (Note 4) }\end{array}$ & \begin{tabular}{|c|c|c|c|} 
LM2940/833 \\
Limit \\
(Note 5)
\end{tabular} & \\
\hline \multirow[b]{2}{*}{ Output Voltage } & \multirow[b]{2}{*}{$5 \mathrm{~mA} \leq \mathrm{l}_{\mathrm{O}} \leq 1 \mathrm{~A}$} & \multicolumn{3}{|c|}{$13.6 \mathrm{~V} \leq \mathrm{V}_{\mathrm{IN}} \leq 26 \mathrm{~V}$} & \multicolumn{3}{|c|}{$16.75 \mathrm{~V} \leq \mathrm{V}_{\mathrm{iN}} \leq 26 \mathrm{~V}$} & \multirow[b]{2}{*}{$\begin{array}{l}V_{\text {MIN }} \\
V_{\text {MAX }}\end{array}$} \\
\hline & & 12.00 & $\begin{array}{l}11.64 / 11.40 \\
12.36 / 12.60\end{array}$ & $\begin{array}{l}11.64 / 11.40 \\
12.36 / 12.60\end{array}$ & 15.00 & $\begin{array}{l}14.55 / 14.25 \\
15.45 / 15.75\end{array}$ & $\begin{array}{l}14.55 / 14.25 \\
15.45 / 15.75 \\
\end{array}$ & \\
\hline Line Regulation & $\begin{array}{l}\mathrm{V}_{\mathrm{O}}+2 \mathrm{~V} \leq \mathrm{V}_{\mathrm{IN}} \leq 26 \mathrm{~V}, \\
\mathrm{I}_{\mathrm{O}}=5 \mathrm{~mA}\end{array}$ & 20 & 120 & $75 / 120$ & 20 & 150 & $95 / 150$ & $m V_{\text {MAX }}$ \\
\hline Load Regulation & $\begin{array}{l}50 \mathrm{~mA} \leq \mathrm{I}_{0} \leq 1 \mathrm{~A} \\
\mathrm{LM} 2940, \mathrm{LM} 2940 / 883 \\
\text { LM2940C }\end{array}$ & $\begin{array}{l}55 \\
55\end{array}$ & $\begin{array}{c}120 / 200 \\
120\end{array}$ & $120 / 190$ & 70 & 150 & $150 / 240$ & $\mathrm{mV}_{\text {MAX }}$ \\
\hline $\begin{array}{l}\text { Output } \\
\text { Impedance }\end{array}$ & $\begin{array}{l}100 \mathrm{mADC} \text { and } \\
20 \mathrm{mArms} \\
\mathrm{f}_{\mathrm{O}}=120 \mathrm{~Hz}\end{array}$ & 80 & & $1000 / 1000$ & 100 & & $1000 / 1000$ & $\mathrm{~m} \Omega$ \\
\hline $\begin{array}{l}\text { Quiescent } \\
\text { Current }\end{array}$ & $\begin{array}{l}V_{O}+2 V \leq V_{I N} \leq 26 V, \\
I_{O}=5 \mathrm{~mA} \\
L M 2940, L M 2940 / 883 \\
L M 2940 \mathrm{C}\end{array}$ & $\begin{array}{l}10 \\
10\end{array}$ & $\begin{array}{c}15 / 20 \\
15\end{array}$ & $15 / 20$ & 10 & 15 & $15 / 20$ & $\mathrm{~mA}_{\operatorname{MAX}}$ \\
\hline & $V_{I N}=V_{O}+5 V, I_{O}=1 A$ & 30 & $45 / 60$ & $50 / 60$ & 30 & $45 / 60$ & $50 / 60$ & $\mathrm{~mA}_{\text {MAX }}$ \\
\hline $\begin{array}{l}\text { Output Noise } \\
\text { Voltage }\end{array}$ & $\begin{array}{l}10 \mathrm{~Hz}-100 \mathrm{kHz}, \\
\mathrm{I}_{\mathrm{O}}=5 \mathrm{~mA}\end{array}$ & 360 & & $1000 / 1000$ & 450 & & $1000 / 1000$ & $\mu \mathrm{V}_{\mathrm{ms}}$ \\
\hline Ripple Rejection & $\begin{array}{l}f_{O}=120 \mathrm{~Hz}, 1 \mathrm{~V}_{\mathrm{rms}} \\
\mathrm{I}_{\mathrm{O}}=100 \mathrm{~mA} \\
\mathrm{LM} 2940 \\
\text { LM2940C }\end{array}$ & $\begin{array}{l}66 \\
66\end{array}$ & $\begin{array}{c}54 / 48 \\
54\end{array}$ & & 64 & 52 & & $\mathrm{~dB}_{\text {MIN }}$ \\
\hline & $\begin{array}{l}\mathrm{f}_{\mathrm{O}}=1 \mathrm{kHz}, 1 \mathrm{~V}_{\mathrm{ms}} \\
\mathrm{I}_{\mathrm{O}}=5 \mathrm{~mA}\end{array}$ & & & $52 / 46$ & & & $48 / 42$ & $\mathrm{~dB}_{\text {MIN }}$ \\
\hline
\end{tabular}

FIGURE 173 - Data sheet (pg. 5) for the LM2940T 10-volt regulator. 


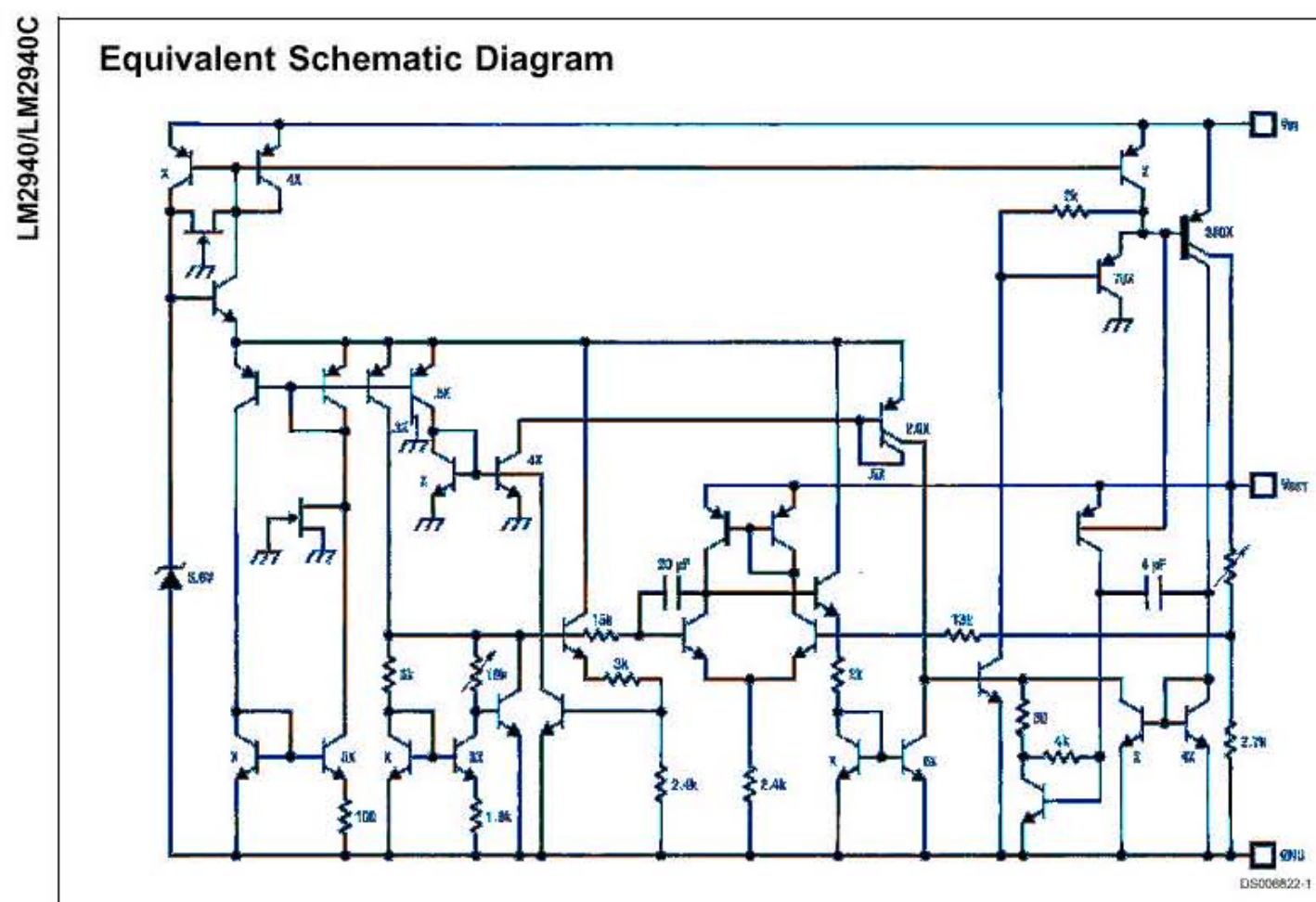

\section{Application Hints}

\section{EXTERNAL CAPACITORS}

The output capacitor is critical to maintaining regulator stability, and must meet the required conditions for both ESR (Equivalent Series Resistance) and minimum amount of capacitance.

MINIMUM CAPACITANCE:

The minimum output capacitance required to maintain stability is $22 \mu \mathrm{F}$ (this value may be increased without limit) Larger values of output capacitance will give improved transient response.

ESR LIMITS

The ESR of the output capacitor will cause loop instability if it is too high or too low. The acceptable range of ESR plotted versus load current is shown in the graph below. It is essential that the output capacitor meet these requirements, or oscillations can result.

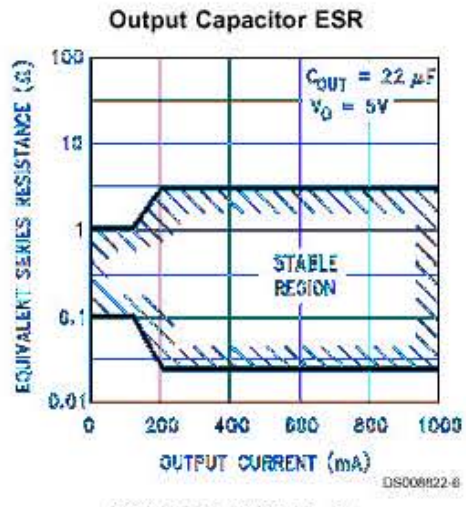

FIGURE 1. ESR Limits

It is important to note that for most capacitors, ESR is specified only at room temperature. However, the designer must ensure that the ESR will stay inside the limits shown over the entire operating temperature range for the design.

For aluminum electrolytic capacitors, ESR will increase by about $30 \mathrm{X}$ as the temperature is reduced from $25^{\circ} \mathrm{C}$ to $-40^{\circ} \mathrm{C}$. This type of capacitor is not well-suited for low temperature operation.

Solid tantalum capacitors have a more stable ESR over temperature, but are more expensive than aluminum electrolytics. A cost-effective approach sometimes used is to parallel

FIGURE 174 - Data sheet (pg. 10) for the LM2940T 10-volt regulator. 


\section{Application Hints (Continued)}

an aluminum electrolytic with a solid Tantalum, with the total capacitance split about $75 / 25 \%$ with the Aluminum being the larger value.

If two capacitors are paralleled, the effective ESR is the parallel of the two individual values. The "flatter" ESR of the Tantalum will keep the effective ESR from rising as quickly at low temperatures.

\section{HEATSINKING}

A heatsink may be required depending on the maximum power dissipation and maximum ambient temperature of the application. Under all possible operating conditions, the junction temperature must be within the range specified under Absolute Maximum Ratings.

To determine if a heatsink is required, the power dissipated by the regulator, $P_{D}$, must be calculated.

The figure below shows the voltages and currents which are present in the circuit, as well as the formula for calculating the power dissipated in the regulator:

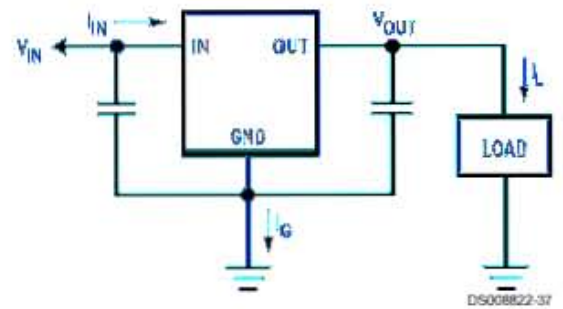

$h_{N}=I_{L}+I_{G}$

$P_{D}=\left(V_{B A}-V_{\text {OUT }}\right) h_{L}+\left(V_{D N}\right) i_{G}$

FIGURE 2. Power Dissipation Diagram

The next parameter which must be calculated is the maximum allowable temperature rise, $T_{R}(\max )$. This is calculated by using the formula:

$$
T_{R}(\max )=T_{J}(\max )-T_{A}(\max )
$$

where: $T_{j}$ (max) is the maximum allowable junction temperature, which is $125^{\circ} \mathrm{C}$ for commercial grade parts

$T_{A}$ (max) is the maximum ambient temperature which will be encountered in the application.

Using the calculated values for $T_{R}(\max )$ and $P_{D}$, the maximum allowable value for the junction-to-ambient thermal resistance, $\theta_{(\mathrm{J}-\mathrm{A})}$, can now be found:

$$
\theta_{(J-A)}=T_{R}(\max ) / P_{D}
$$

IMPORTANT: If the maximum allowable value for $\theta_{(\mathrm{J}-\mathrm{A})}$ is found to be $\geq 53^{\circ} \mathrm{C} / \mathrm{W}$ for the TO-220 package, $\geq 80^{\circ} \mathrm{C} / \mathrm{W}$ for the TO-263 package, or $\geq 174^{\circ} \mathrm{C} M$ for the SOT-223 package, no heatsink is needed since the package alone will dissipate enough heat to satisfy these requirements.

If the calculated value for $\theta_{(J-A)}$ falls below these limits, a heatsink is required.

\section{HEATSINKING TO-220 PACKAGE PARTS}

The TO-220 can be attached to a typical heatsink, or secured to a copper plane on a PC board. If a copper plane is to be used, the values of $\theta_{(\mathrm{J}-\mathrm{A})}$ will be the same as shown in the next section for the TO-263.
If a manufactured heatsink is to be selected, the value of heatsink-to-ambient thermal resistance, $\theta_{(H-A)}$, must first be calculated:

$$
\theta_{(\mathrm{H}-\mathrm{A})}=\theta_{(\mathrm{J}-\mathrm{N})}-\theta_{\left(\mathrm{C}-H_{0}\right)}-\theta_{(\mathrm{J}-\mathrm{C})}
$$

Where: $\theta_{(\mathrm{J}-\mathrm{C})}$ is defined as the thermal resistance from the junction to the surface of the case. A value of $3^{\circ} \mathrm{C} / \mathrm{W}$ can be assumed for $\theta_{(\mathrm{J}-\mathrm{C})}$ for this calculation.

$\theta_{(\mathrm{C}-\mathrm{H})}$ is defined as the thermal resistance between the case and the surface of the heatsink. The value of $\theta_{(\mathrm{C}-\mathrm{H})}$ will vary from about $1.5^{\prime} \mathrm{C} / \mathrm{W}$ to about $2.5^{\circ} \mathrm{C} / \mathrm{W}$ (depending on method of attachment, insulator, etc.). If the exact value is unknown, $2 \mathrm{C} / \mathrm{W}$ should be assumed for $\theta_{(\mathrm{C}-H)}$.

When a value for $\theta_{(H-A)}$ is found using the equation shown, a heatsink must be selected that has a value that is less than or equal to this number.

$\theta_{(H-A)}$ is specified numerically by the heatsink manufacturer in the catalog, or shown in a curve that plots temperature rise vs power dissipation for the heatsink.

\section{HEATSINKING TO-263 AND SOT-223 PACKAGE PARTS}

Both the TO-263 ("S") and SOT-223 ("MP") packages use a copper plane on the PCB and the PCB itself as a heatsink. To optimize the heat sinking ability of the plane and PCB solder the tab of the package to the plane.

Figure 3 shows for the TO-263 the measured values of $\theta_{(\mathrm{J}-\mathrm{A})}$ for different copper area sizes using a typical PCB with 1 ounce copper and no solder mask over the copper area used for heatsinking.

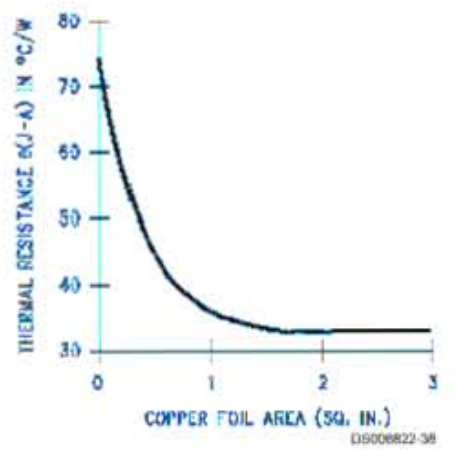

FIGURE 3. $\theta_{(J-A)}$ vs Copper (1 ounce) Area for the TO-263 Package

As shown in the figure, increasing the copper area beyond 1 square inch produces very little improvement. It should also be observed that the minimum value of $\theta_{(\mathrm{J}-\mathrm{A})}$ for the TO-263 package mounted to a $\mathrm{PCB}$ is $32^{\prime} \mathrm{C} / \mathrm{W}$.

As a design aid, Figure 4 shows the maximum allowable power dissipation compared to ambient temperature for the TO-263 device (assuming $\theta_{(\mathrm{J}-\mathrm{A})}$ is $35^{\circ} \mathrm{C} / \mathrm{W}$ and the maximum junction temperature is $125^{\circ} \mathrm{C}$ ).

FIGURE 175 - Data sheet (pg. 11) for the LM2940T 10-volt regulator. 


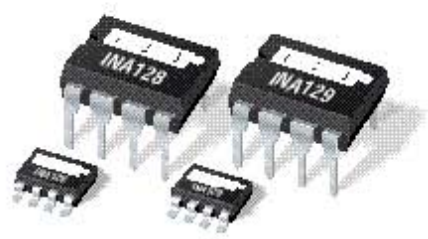

\section{Precision, Low Power INSTRUMENTATION AMPLIFIERS}

\section{FEATURES}

- LOW OFFSET VOLTAGE: $50 \mu \mathrm{V}$ max

- LOW DRIFT: $0.5 \mu \mathrm{V} /{ }^{\circ} \mathrm{C}$ max

- LOW INPUT BIAS CURRENT: 5nA max

- HIGH CMR: $120 \mathrm{~dB}$ min

- INPUTS PROTECTED TO $\pm 40 \mathrm{~V}$

- WIDE SUPPLY RANGE: \pm 2.25 to $\pm 18 \mathrm{~V}$

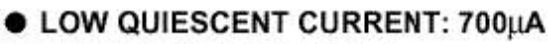

- 8-PIN PLASTIC DIP, SO-8

\section{APPLICATIONS}

\section{- BRIDGE AMPLIFIER \\ - THERMOCOUPLE AMPLIFIER \\ - RTD SENSOR AMPLIFIER \\ - MEDICAL INSTRUMENTATION \\ - DATA ACQUISITION}

\section{DESCRIPTION}

The INA128 and INA129 are low power, general purpose instrumentation amplifiers offering excellent accuracy. Their versatile 3-op amp design and small size make them ideal for a wide range of applications. Current-feedback input circuitry provides wide bandwidth even at high gain $(200 \mathrm{kHz}$ at $\mathrm{G}=100)$.

A single external resistor sets any gain from 1 to 10,000 . INA128 provides an industry standard gain equation; INA129's gain equation is compatible with the AD620.

The INA128/INA129 is laser trimmed for very low offset voltage $(50 \mu \mathrm{V})$, drift $\left(0.5 \mu \mathrm{V} /{ }^{\circ} \mathrm{C}\right)$ and high common-mode rejection ( $120 \mathrm{~dB}$ at $\mathrm{G} \geq 100$ ). It operates with power supplies as low as $\pm 2.25 \mathrm{~V}$, and quiescent current is only $700 \mu \mathrm{A}$-ideal for battery operated systems. Internal input protection can withstand up to $140 \mathrm{~V}$ without damage.

The INA128/INA129 is available in 8-pin plastic DIP, and SO-8 surface-mount packages, specified for the $-40^{\circ} \mathrm{C}$ to $+85^{\circ} \mathrm{C}$ temperature range. The INA1 28 is also available in dual configuration, the INA2128.

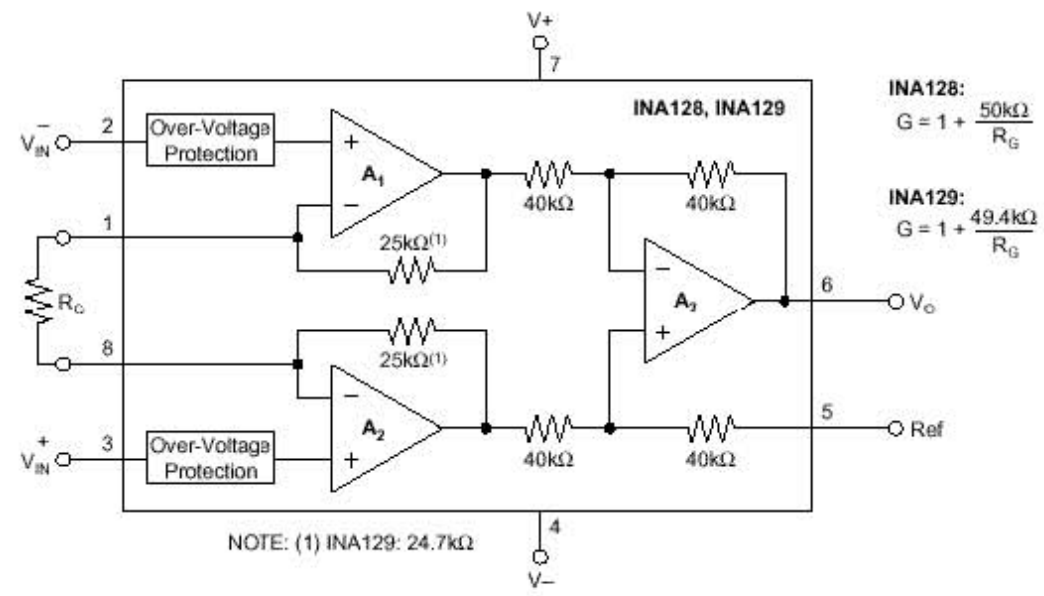

International Airport industrial Park - Maling Address: PO 30x 11400, Tucson, AZ 95734 - Street Address: 6730 S. Tucson Blvd., Tucson, AZ 85706 - Tel: (520) 746-1111 - Twx: 910-952-1111 Internet hitp:iliwww burr-brown.com/ - FAXLine: (800) 5:8-6133 (USiCanada Only) - Cable: BBRCORP - Telex: 066-6491 - FAX (520) 889-1510 - Immediate Product info: (8000) 548-6132

Q1995 Burr-Brown Corponation

PDS- 12960

Printed in U.S.A. October, 1996

FIGURE 176 - Data sheet (p. 1) for the Burr-Brown INA128 instrumentation amp. 


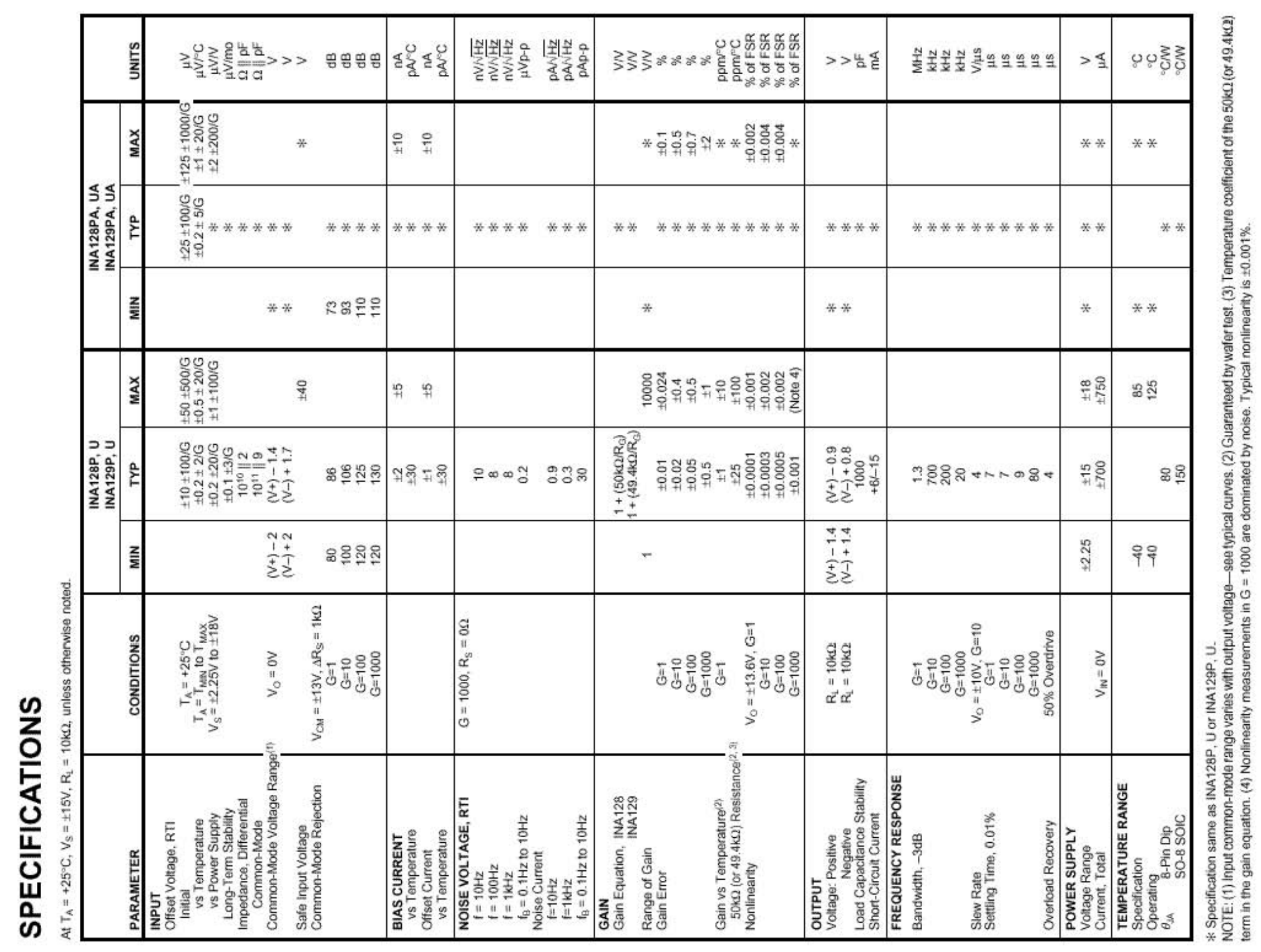

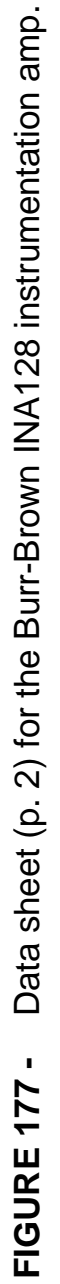




\section{PIN CONFIGURATION}

8-Pin DIP and SO-8

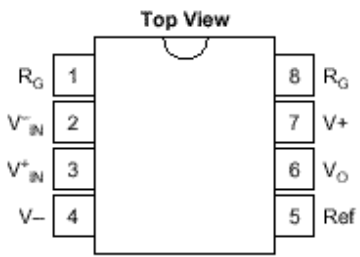

\section{ABSOLUTE MAXIMUM RATINGS}

\begin{tabular}{|c|}
\hline $\begin{array}{l}\text { Supply Voltage } \\
\text { Analog Input Voltage Range }\end{array}$ \\
\hline Output Short-Circuit (to ground) ............ \\
\hline Operating Temperature ............... \\
\hline 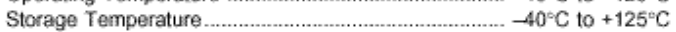 \\
\hline Junction Temperature \\
\hline Lead Temperature (soldering, 10s) ......... \\
\hline
\end{tabular}

ELECTROSTATIC 16 DISCHARGE SENSITIVITY

This integrated circuit can be damaged by ESD. Burr-Brown recommends that all integrated circuits be handled with appropriate precautions. Failure to observe proper handling and installation procedures can cause damage.

ESD damage can range from subtle performance degradation to complete device failure. Precision integrated circuits may be more susceptible to damage because very small parametric changes could cause the device not to meet its published specifications.

\section{ORDERING INFORMATION}

\begin{tabular}{|l|c|c|c|}
\hline PRODuCT & PACKAGE & $\begin{array}{c}\text { PACKAGE } \\
\text { DRAWING } \\
\text { NUMBER }\end{array}$ & $\begin{array}{c}\text { (1) } \\
\text { TEMPERATURE } \\
\text { RANGE }\end{array}$ \\
\hline INA128PA & 8-Pin Plastic DIP & 006 & $-40^{\circ} \mathrm{C}$ to $+85^{\circ} \mathrm{C}$ \\
INA128P & 8-Pin Plastic DIP & 006 & $-40^{\circ} \mathrm{C}$ to $+85^{\circ} \mathrm{C}$ \\
INA128UA & SO-8 Surface-Mount & 182 & $-40^{\circ} \mathrm{C}$ to $+85^{\circ} \mathrm{C}$ \\
INA128U & SO-8 Surface-Mount & 182 & $-40^{\circ} \mathrm{C}$ to $+85^{\circ} \mathrm{C}$ \\
\hline INA129PA & 8-Pin Plastic DIP & 006 & $-40^{\circ} \mathrm{C}$ to $+85^{\circ} \mathrm{C}$ \\
INA129P & 8-Pin Plastic DIP & 006 & $-40^{\circ} \mathrm{C}$ to $+85^{\circ} \mathrm{C}$ \\
INA129UA & SO-8 Surface-Mount & 182 & $-40^{\circ} \mathrm{C}$ to $+85^{\circ} \mathrm{C}$ \\
INA129U & SO-8 Surface-Mount & 182 & $-40^{\circ} \mathrm{C}$ to $+85^{\circ} \mathrm{C}$ \\
\hline
\end{tabular}

NOTE: (1) For detailed drawing and dimension table, please see end of data sheet, or Appendix C of Burr-Brown IC Data Book.

FIGURE 178 - Data sheet (p. 3) for the Burr-Brown INA128 instrumentation amp. 


\section{TYPICAL PERFORMANCE CURVES}

At $T_{A}=+25^{\circ} \mathrm{C}, V_{S}= \pm 15 \mathrm{~V}$, unless othenwise noted
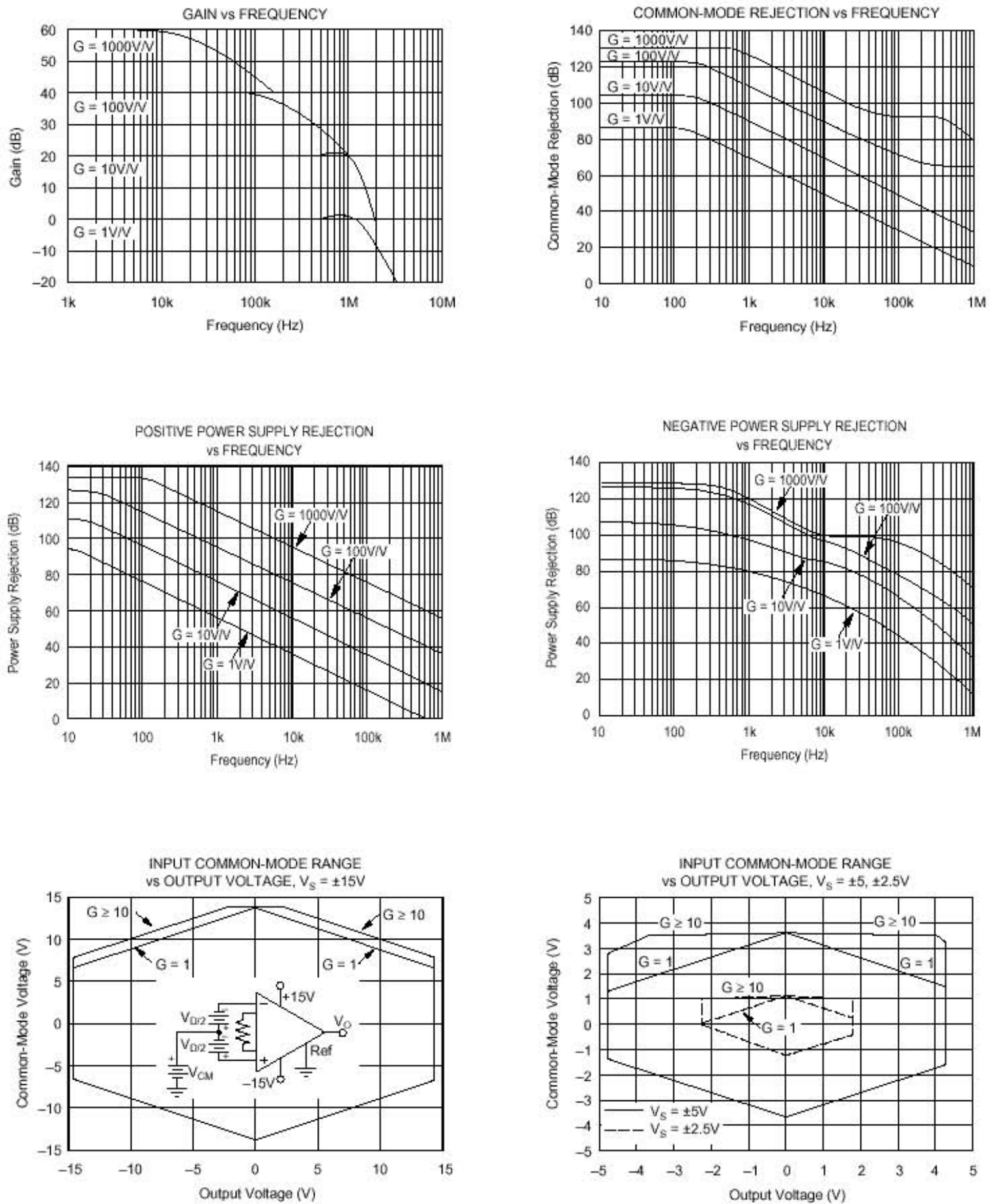

FIGURE 179 - Data sheet (p. 4) for the Burr-Brown INA128 instrumentation amp. 


\section{TYPICAL PERFORMANCE CURVES (CONT)}

A: $T_{A}=+25^{\circ} \mathrm{C}, V_{S}= \pm 15 \mathrm{~V}$, unless othermse noted.
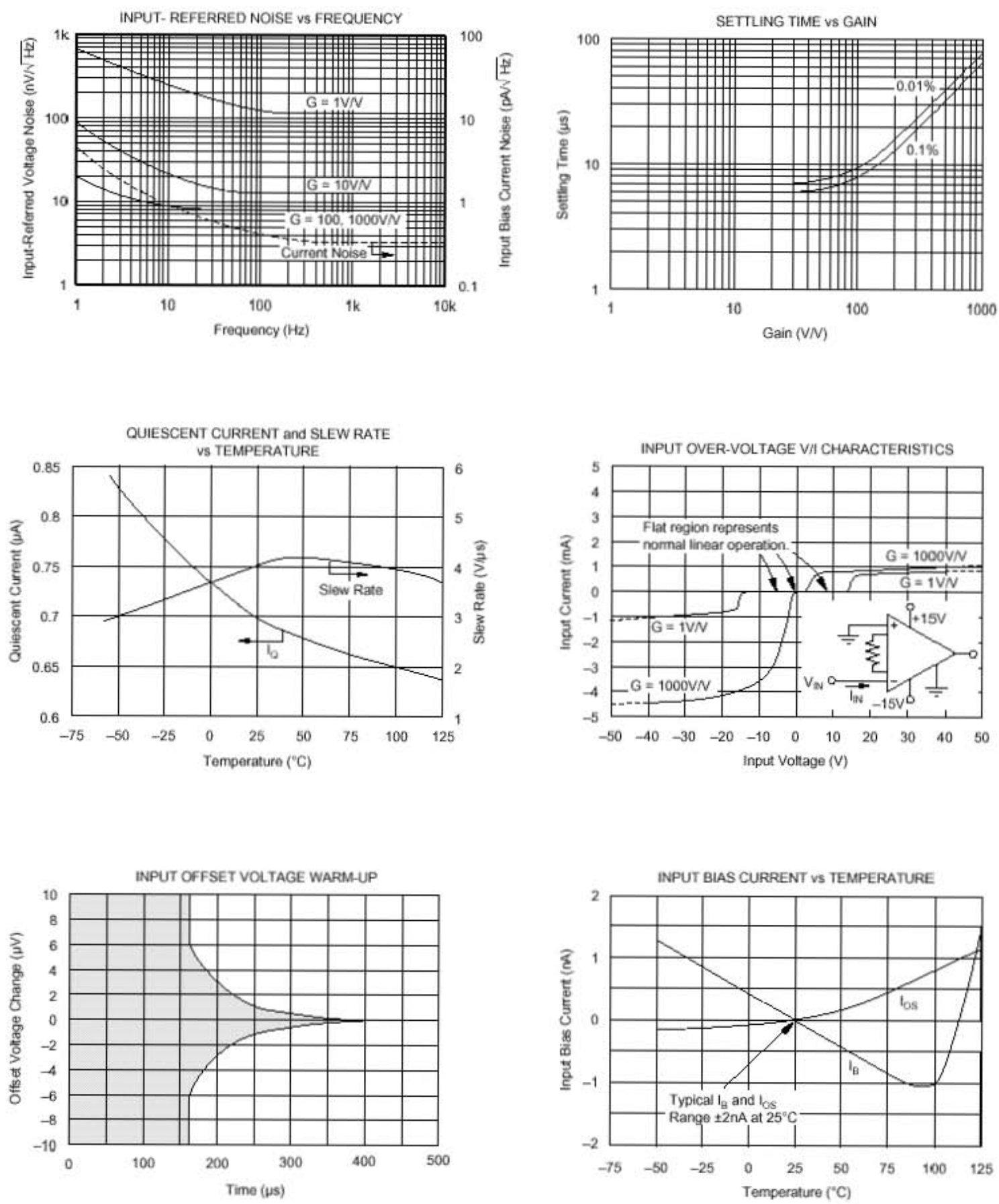

FIGURE 180 - Data sheet (p. 5) for the Burr-Brown INA128 instrumentation amp. 
TYPICAL PERFORMANCE CURVES (CONT)

At $T_{A}=+25^{\circ} \mathrm{C}, V_{S}= \pm 15 \mathrm{~V}$, uniess otherwise noted.
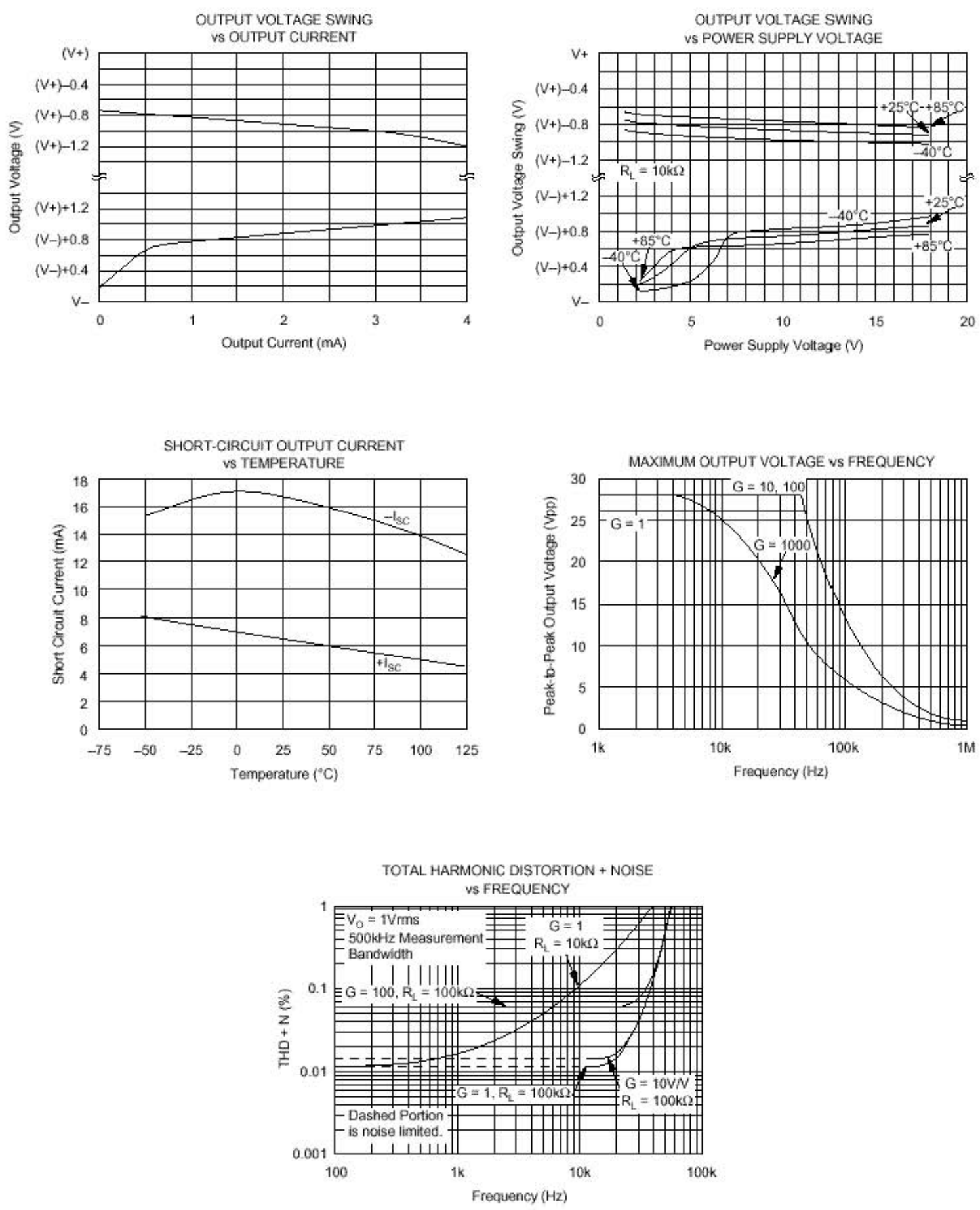

FIGURE 181 - Data sheet (p. 6) for the Burr-Brown INA128 instrumentation amp. 


\section{APPLICATION INFORMATION}

Figure 1 shows the basic connections required for operation of the INA128/INA129. Applications with noisy or high impedance power supplies may require decoupling capacitors close to the device pins as shown.

The output is referred to the output reference (Ref) terminal which is normally grounded. This must be a low-impedance connection to assure good common-mode rejection. A resistance of $8 \Omega$ in series with the Ref pin will cause a typical device to degrade to approximately $80 \mathrm{~dB} C M R(G=1)$.

\section{SETTING THE GAIN}

Gain is set by connecting a single external resistor, $\mathrm{R}_{\mathrm{G}}$, connected between pins 1 and 8 :

$$
\begin{aligned}
& \text { INA128: } \quad \mathrm{G}=1+\frac{50 \mathrm{k} \Omega}{\mathrm{R}_{\mathrm{G}}} \\
& \text { INA129: } \quad \mathrm{G}=1+\frac{49.4 \mathrm{k} \Omega}{\mathrm{R}_{\mathrm{G}}}
\end{aligned}
$$

Commonly used gains and resistor values are shown in Figure 1.

The $50 \mathrm{k} \Omega$ term in Equation $1(49.4 \mathrm{k} \Omega$ in Equation 2$)$ comes from the sum of the two internal feedback resistors of $A_{1}$ and $A_{2}$. These on-chip metal film resistors are laser trimmed to accurate absolute values. The accuracy and temperature coefficient of these internal resistors are included in the gain accuracy and drift specifications of the INA128/INAI29.

The stability and temperature drift of the external gain setting resistor, $R_{G}$, also affects gain. $R_{G}$ 's contribution to gain accuracy and drift can be directly inferred from the gain equation (1). Low resistor values required for high gain can make wiring resistance important. Sockets add to the wiring resistance which will contribute additional gain error (possibly an unstable gain error) in gains of approximately 100 or greater.

\section{DYNAMIC PERFORMANCE}

The typical performance curve "Gain vs Frequency" shows that, despite its low quiescent current, the INA128/INA129 achieves wide bandwidth, even at high gain. This is due to the current-feedback topology of the input stage circuitry. Settling time also remains excellent at high gain.

\section{NOISE PERFORMANCE}

The INA128/INA129 provides very low noise in most applications. Low frequency noise is approximately $0.2 \mu \mathrm{Vp}-\mathrm{p}$ measured from 0.1 to $10 \mathrm{~Hz}$ ( $\mathrm{G} \geq 100$ ). This provides dramatically improved noise when compared to state-of-theart chopper-stabilized amplifiers.

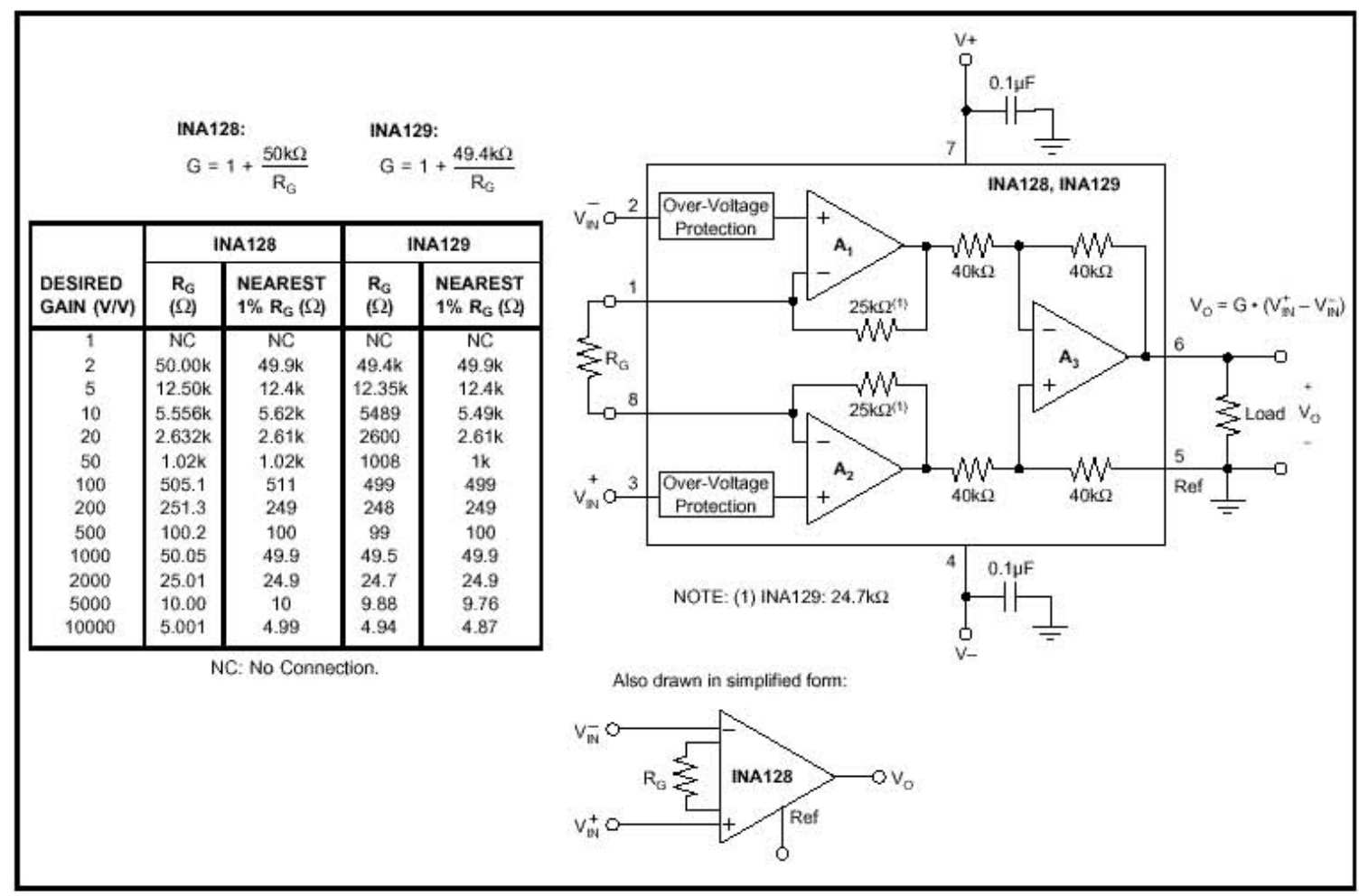

FIGURE 1. Basic Connections.

FIGURE 182 - Data sheet (p. 8) for the Burr-Brown INA128 instrumentation amp. 


\section{OFFSET TRIMMING}

The INA128/INA129 is laser trimmed for low offset voltage and offset voltage drift. Most applications require no external offset adjustment. Figure 2 shows an optional circuit for trimming the output offset voltage. The voltage applied to Ref terminal is summed with the output. The op amp buffer provides low impedance at the Ref terminal to preserve good common-mode rejection.

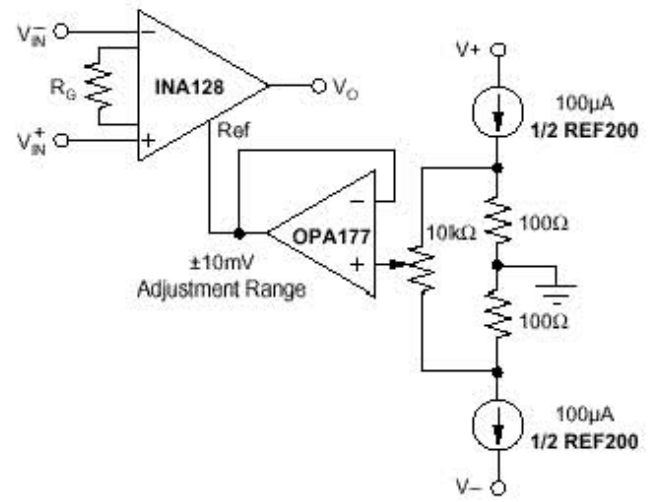

FIGURE 2. Optional Trimming of Output Offset Voltage.

\section{INPUT BIAS CURRENT RETURN PATH}

The input impedance of the INA128/INA129 is extremely high - approximately $10^{10} \Omega$. However, a path must be provided for the input bias current of both inputs. This input bias current is approximately $\pm 2 \mathrm{nA}$. High input impedance means that this input bias current changes very little with varying input voltage.

Input circuitry must provide a path for this input bias current for proper operation. Figure 3 shows various provisions for an input bias current path. Without a bias current path, the inputs will float to a potential which exceeds the commonmode range, and the input amplifiers will saturate.

If the differential source resistance is low, the bias current return path can be connected to one input (see the thermocouple example in Figure 3). With higher source impedance, using two equal resistors provides a balanced input with possible advantages of lower input offset voltage due to bias current and better high-frequency common-mode rejection.

\section{INPUT COMMON-MODE RANGE}

The linear input voltage range of the input circuitry of the INA128/INA129 is from approximately $1.4 \mathrm{~V}$ below the positive supply voltage to $1.7 \mathrm{~V}$ above the negative supply. As a differential input voltage causes the output voltage increase, however, the linear input range will be limited by the output voltage swing of amplifiers $A_{1}$ and $A_{2}$. So the

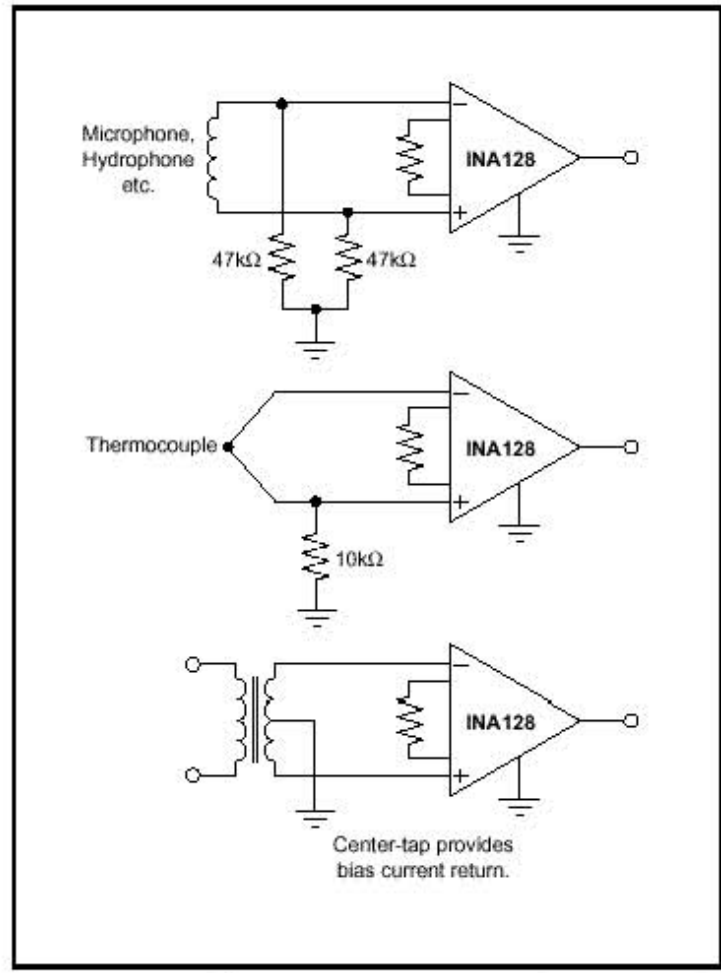

FIGURE 3. Providing an Input Common-Mode Current Path.

linear common-mode input range is related to the output voltage of the complete amplifier. This behavior also depends on supply voltage - see performance curves "Input Common-Mode Range vs Output Voltage".

Input-overload can produce an output voltage that appears normal. For example, if an input overload condition drives both input amplifiers to their positive output swing limit, the difference voltage measured by the output amplifier will be near zero. The output of $\mathrm{A}_{3}$ will be near $0 \mathrm{~V}$ even though both inputs are overloaded.

\section{LOW VOLTAGE OPERATION}

The INA128/INA129 can be operated on power supplies as low as $\pm 2.25 \mathrm{~V}$. Performance remains excellent with power supplies ranging from $\pm 2.25 \mathrm{~V}$ to $\pm 18 \mathrm{~V}$. Most parameters vary only slightly throughout this supply voltage range-see typical performance curves. Operation at very low supply voltage requires careful attention to assure that the input voltages remain within their linear range. Voltage swing requirements of internal nodes limit the input commonmode range with low power supply voltage. Typical performance curves, "Input Common-Mode Range vs Output Voltage" show the range of linear operation for $\pm 15 \mathrm{~V}, \pm 5 \mathrm{~V}$, and $\pm 2.5 \mathrm{~V}$ supplies.

FIGURE 183 - Data sheet (p. 9) for the Burr-Brown INA128 instrumentation amp. 


\section{LM124/LM224/LM324/LM2902 Low Power Quad Operational Amplifiers}

\section{General Description}

The LM124 series consists of four independent, high gain, intemally frequency compensated operational amplifiers which were designed specifically to operate from a single power supply over a wide range of voltages. Operation from split power supplies is also possible and the low power supply current drain is independent of the magnitude of the power supply voltage.

Application areas include transducer amplifiers, DC gain blocks and all the conventional op amp circuits which now can be more easily implemented in single power supply systerns. For example, the LM124 series can be directly operated off of the standard $+5 \mathrm{~V}$ power supply voltage which is used in digital systems and will easily provide the required interface electronics without requiring the additional $\pm 15 \mathrm{~V}$ power supplies.

\section{Unique Characteristics}

- In the linear mode the input common-mode voltage range includes ground and the output voltage can also swing to ground, even though operated from only a single power supply voltage

- The unity gain cross frequency is temperature compensated

- The input bias current is also temperature compensated

\section{Advantages}

- Eliminates need for dual supplies

- Four internally compensated op amps in a single package

- Allows directly sensing near GND and $V_{\text {out }}$ also goes to GND

- Compatible with all forms of logic

- Power drain suitable for battery operation

\section{Features}

- Internally frequency compensated for unity gain

- Large DC voltage gain $100 \mathrm{~dB}$

- Wide bandwidth (unity gain) $1 \mathrm{MHz}$ (temperature compensated)

- Wide power supply range: Single supply $3 \mathrm{~V}$ to $32 \mathrm{~V}$ or dual supplies $\pm 1.5 \mathrm{~V}$ to $\pm 16 \mathrm{~V}$

- Very low supply current drain $(700 \mu \mathrm{A})$ - essentially independent of supply voltage

- Low input biasing current $45 \mathrm{nA}$ (temperature compensated)

- Low input offset voltage $2 \mathrm{mV}$ and offset current: $5 \mathrm{nA}$

- Input common-mode voltage range includes ground

- Differential input voltage range equal to the power supply voltage

- Large output voltage swing $0 \mathrm{~V}$ to $\mathrm{V}^{*}-1.5 \mathrm{~V}$

\section{Connection Diagram}

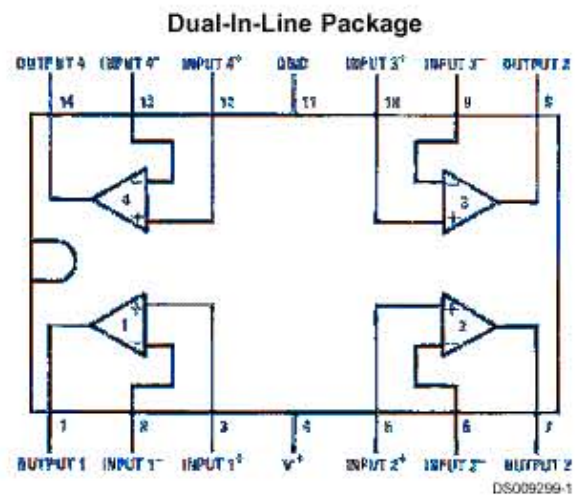

Top View

Order Number LM124J, LM124AJ, LM124J/883 (Note 2), LM124AJ/883 (Note 1), LM224J,

LM224AJ, LM324J, LM324M, LM324AM, LM2902M, LM324N, LM324AN, LM324MT, LM324MTX or LM2902N

LM124AJRQML and LM124AJRQMLV(Note 3)

See NS Package Number J14A, M14A or N14A

Note 1: LM124A available per JM38510/11006

Note 2: LM124 available per JM38510/11005

(4) 2000 National Semiconductor Corporation

DS009299

www.national.com

FIGURE 184 - Data sheet (p. 1) for the LM2902 quad operational amplifier. 


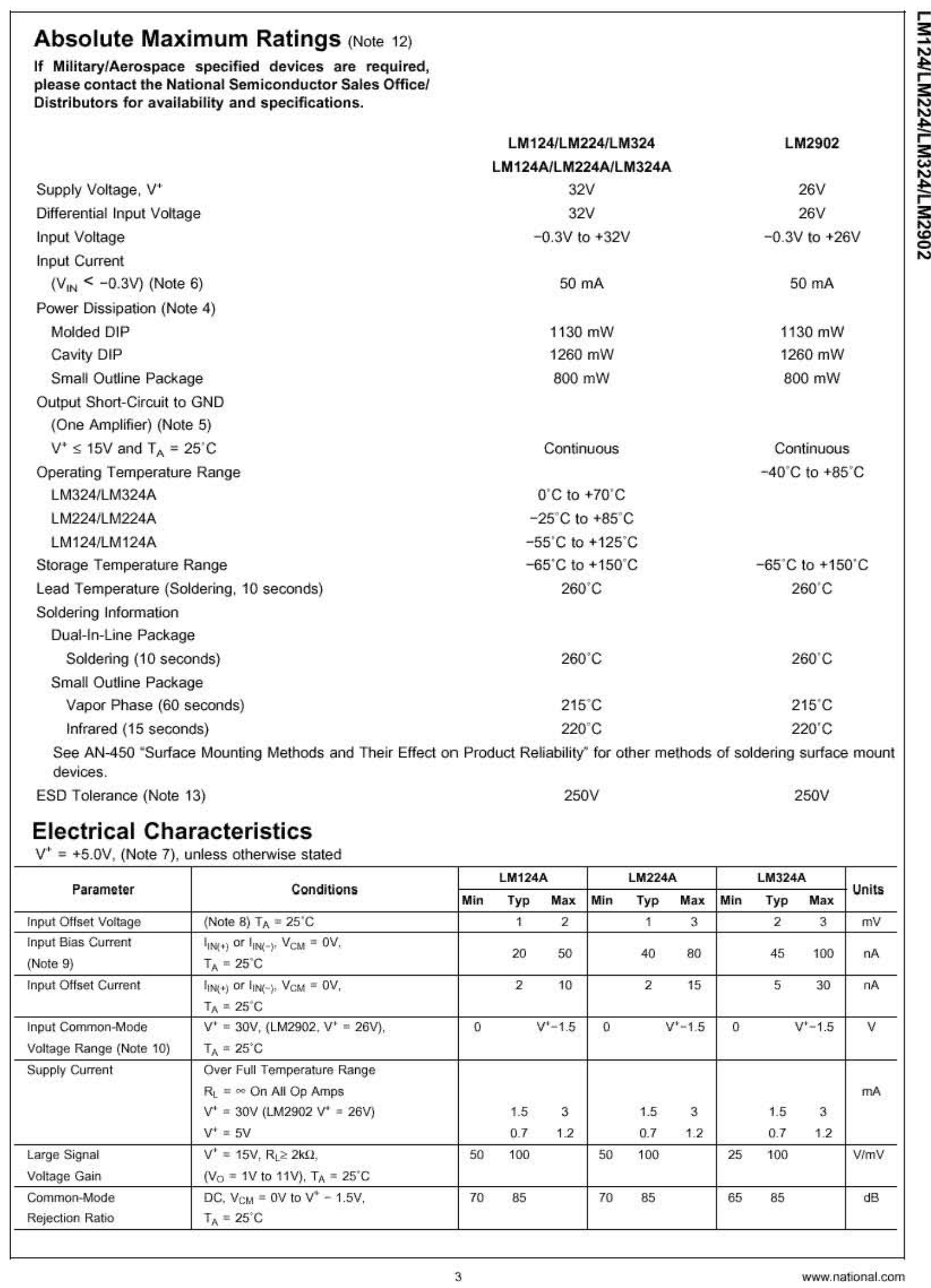

FIGURE 185 - Data sheet (p. 3) for the LM2902 quad operational amplifier. 


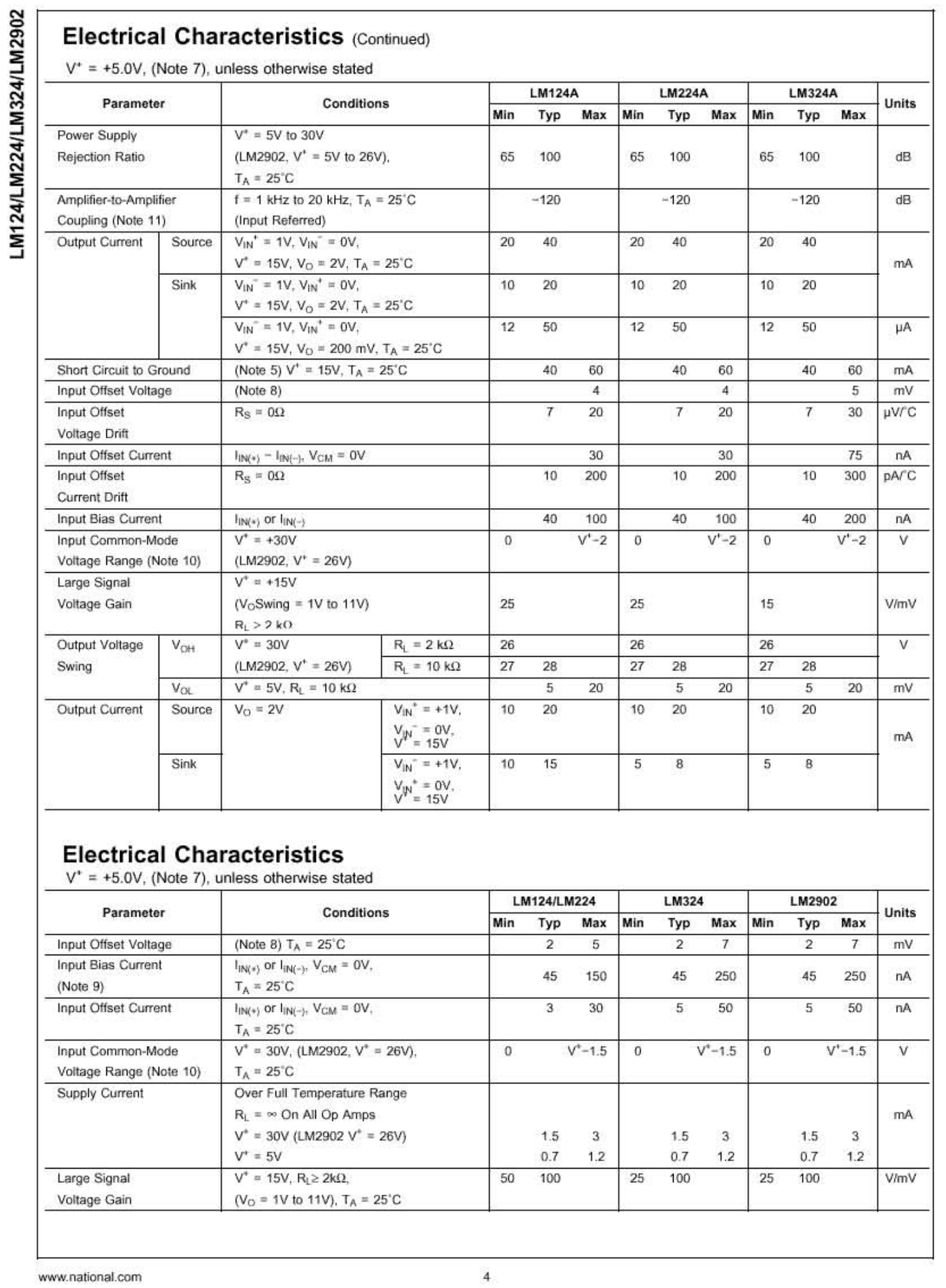

FIGURE 186 - Data sheet (p. 4) for the LM2902 quad operational amplifier. 


\section{Electrical Characteristics (Continued)}

$\mathrm{V}^{+}=+5.0 \mathrm{~V}$, (Note 7), unless otherwise stated

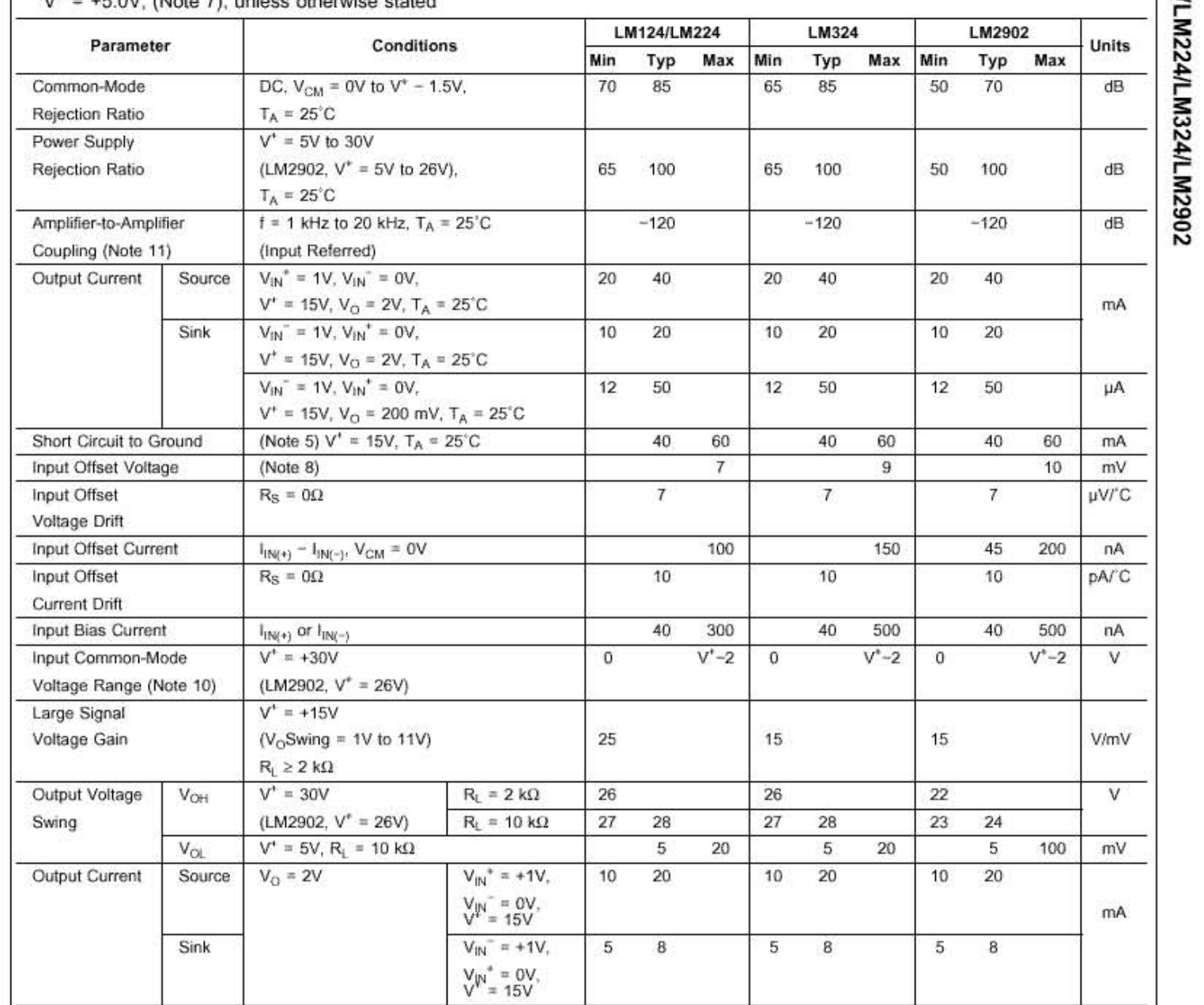

Note 4: For operating at high temperatures, the LM324/LM324A/LM2902 must be derated based on a +125 C maximum junction temperature and a thermal resistance of $88 \mathrm{C} / \mathrm{W}$ which applies for the device soldered in a printed circuit board, operating in a still air ambient. The LM224/LM224A and LM 124/LM124A can be derated based on a $+150^{\circ} \mathrm{C}$ maximum junction temperature. The dissipation is the tota of all four amplifiers - use external resistors, where possible, to allow the amplifier to saturate of to reduce the power which is dissipated in the integrated circuit.

Note 5: Short circuits from the output to $\mathrm{V}^{*}$ can cause excessive heating and eventual destruction. When considering short circuits to ground, the maximum output current is approximately $40 \mathrm{~mA}$ independent of the magnitude of $\mathrm{V}^{t}$. At values of sujply voltage in excess of $+15 \mathrm{~V}$, continuous short-circuits can exceed the power dissipation ratings and cause eventual destruction. Destructive dissipation can resut from simultaneous shorts on all amplifiers.

Note 6: This input current will only exist when the voltage at any of the input leads $s$ driven negative. It is due to the collector-base junction of the input PNP transistors becoming forward biased and thereby acting as input diode clamps. In additicn to this diode action, there is also lateral NPN parasitic transistor action on the IC chip. This transistor action can cause the output voltages of the op amps to go to the $\mathrm{V}^{*}$ voltage level (or to ground for a large overdrive) for the time duration that an input is driven negative. This is not destructive and normal output states will re-establish when the input voltage, which was negative, again returns to a value greater than $-0.3 \mathrm{~V}$ (at $25^{\circ} \mathrm{C}$ ).

Note 7: These specifications are limited to $-55^{\circ} \mathrm{C} \leq \mathrm{T}_{\mathrm{A}} S+125^{\circ} \mathrm{C}$ for the $L M 124 / L M 124 \mathrm{~A}$. With the $L M 224 / L M 224 \mathrm{~A}$, all temperature specifications are limited to $-25^{\circ} \mathrm{C}$ $\leq T_{A} \leq+85^{\circ} \mathrm{C}$, the LM324/LM324A temperature specifications are limited to $00^{\circ} \mathrm{C} \leq T_{A} \leq+70^{\circ} \mathrm{C}$, and the $L M 2902$ specifications are limited to $-40^{\circ} \mathrm{C} \leq \mathrm{T}_{\mathrm{A}} \leq+85^{\circ} \mathrm{C}$.

Note 8: $V_{0} \equiv 1.4 \mathrm{~V}, \mathrm{R}_{\mathrm{S}}=0 \Omega$ with $\mathrm{V}^{+}$from $5 \mathrm{~V}$ to $30 \mathrm{~V}$; and over the full input common-mode range $\left(0 \mathrm{~V}\right.$ to $\left.\mathrm{V}^{+}-1.5 \mathrm{~V}\right)$ for $L \mathrm{~L} 2902$. $\mathrm{V}^{+}$from $5 \mathrm{~V}$ to $26 \mathrm{~V}$.

Note 9: The direction of the input current is out of the IC due to the PNP input stage. This current is essentially constant, independent of the state of the output so no loading change exists on the input lines.

Note 10: The input common-mode voltage of either input signal voltage should not be allowed to go negative by more than $0.3 \mathrm{~V}$ (at $25^{\circ} \mathrm{C}$ ). The upper end of the common-mode voltage range is $\mathrm{V}^{+}-1.5 \mathrm{~V}$ (at $25^{\circ} \mathrm{C}$ ), but either or both inputs can go to $+32 \mathrm{~V}$ without damage $(+26 \mathrm{~V}$ for $L \mathrm{M} 2902$ ), independent of the magnilude of $\mathrm{V}^{+}$.

Note 11: Due to proximity of external components, insure that coupling is not originating via stray capacitance between these external parts. This typically can be detected as this type of capacitance increases at higher frequencies.

Note 12: Refer to RETS124AX for LM124A military specifications and refer to RETS124X for LM124 military specifications.

FIGURE 187 - Data sheet (p. 5) for the LM2902 quad operational amplifier. 


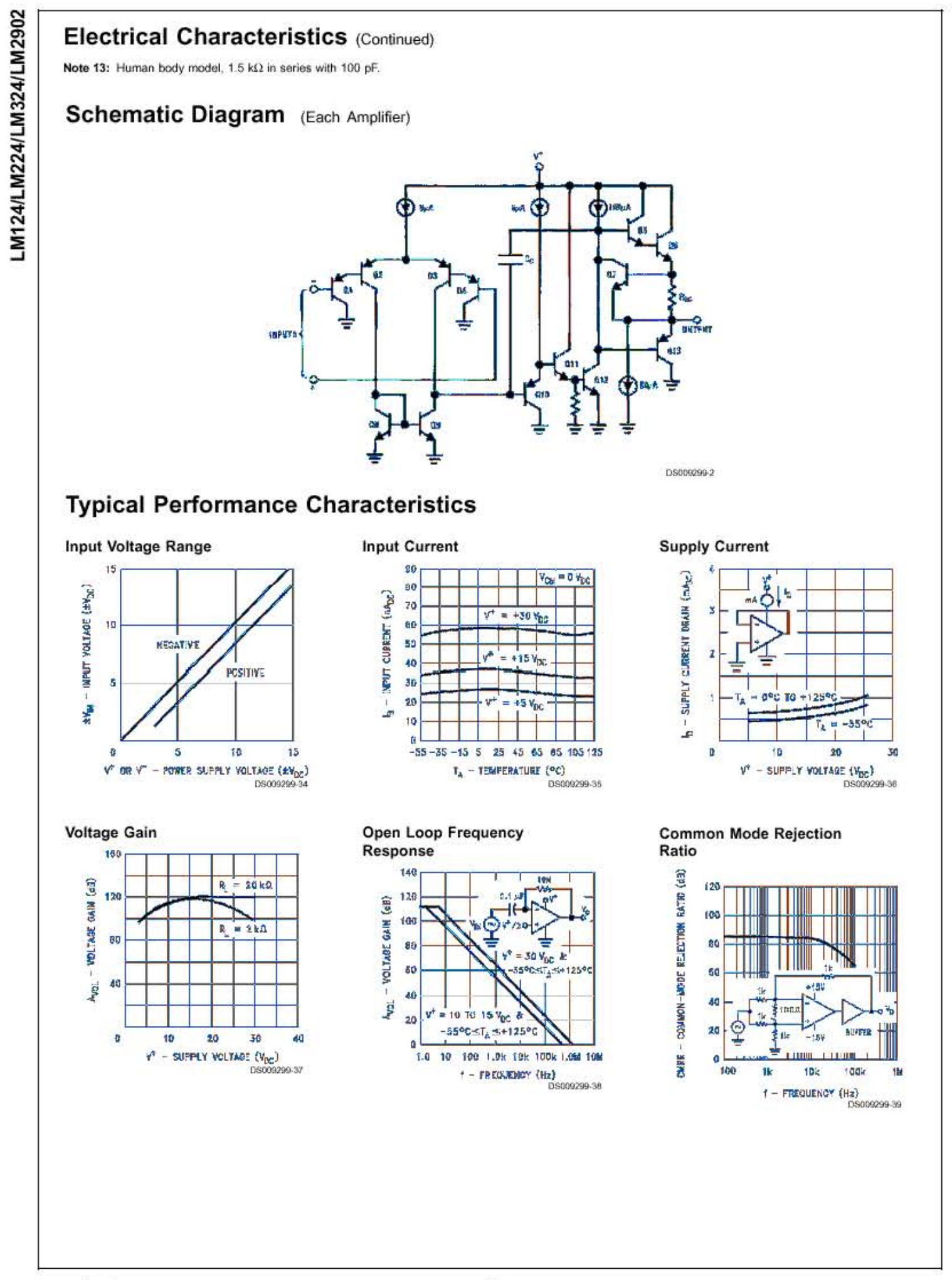

www.national.com

FIGURE 188 - Data sheet (p. 6) for the LM2902 quad operational amplifier. 


\section{Typical Performance Characteristics (Continued)}

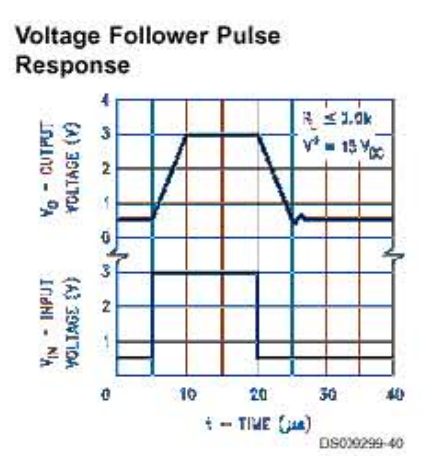

Output Characteristics

Current Sourcing

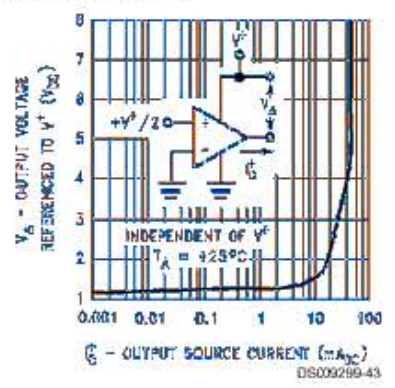

Voltage Follower Pulse

Response (Small Signal)

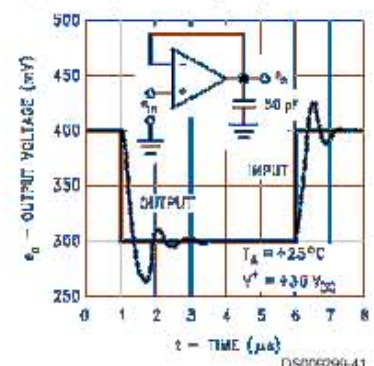

Output Characteristics

Current Sinking

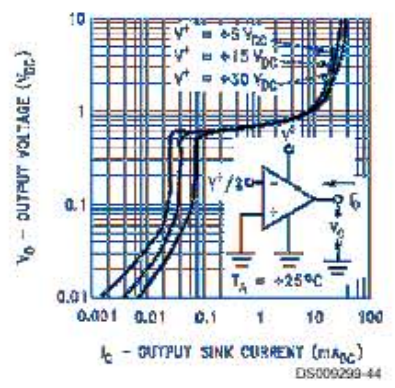

Large Signal Frequency Response

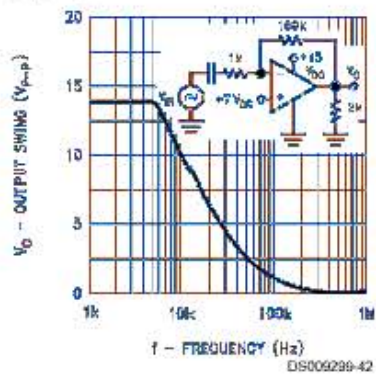

Current Limiting

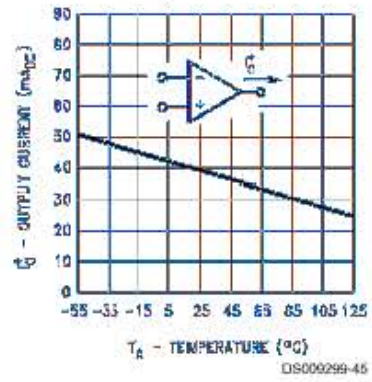

Input Current (LM2902 only)

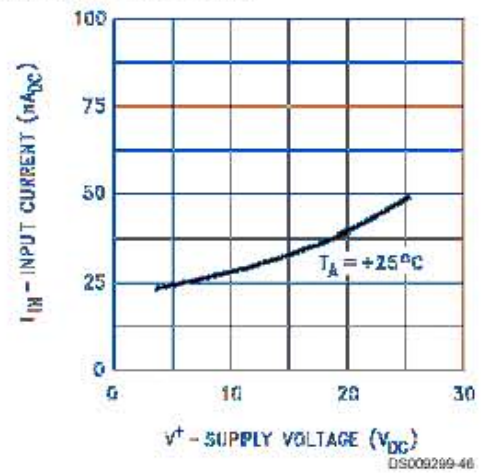

Voltage Gain (LM2902 only)

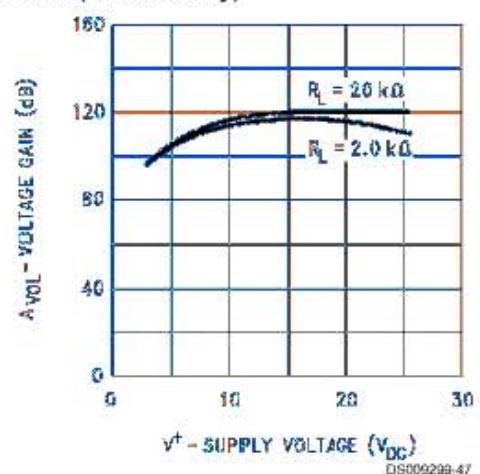

\section{Application Hints}

The LM124 series are op amps which operate with only a single power supply voltage, have true-differential inputs. and remain in the linear mode with an input common-mode voltage of $0 \mathrm{~V}_{\mathrm{DC}}$. These amplifiers operate over a wide range of power supply voltage with little change in performance characteristics. At $25^{\circ} \mathrm{C}$ amplifier operation is possible down

to a minimum supply voltage of $2.3 \mathrm{~V}_{\mathrm{DC}}$.

The pinouts of the package have been designed to simplify PC. hoard layouts Inverting inputs are adjacent to outputs for all of the amplifiers and the outpuls have also been placed at the corners of the package (pins 1, 7, 8, and 14).

Precautions should be taken to insure that the power supply for the integrated circuit never becomes reversed in polarity or that the unit is not inadvertenty installed backwards in a

FIGURE 189 - Data sheet (p. 7) for the LM2902 quad operational amplifier. 


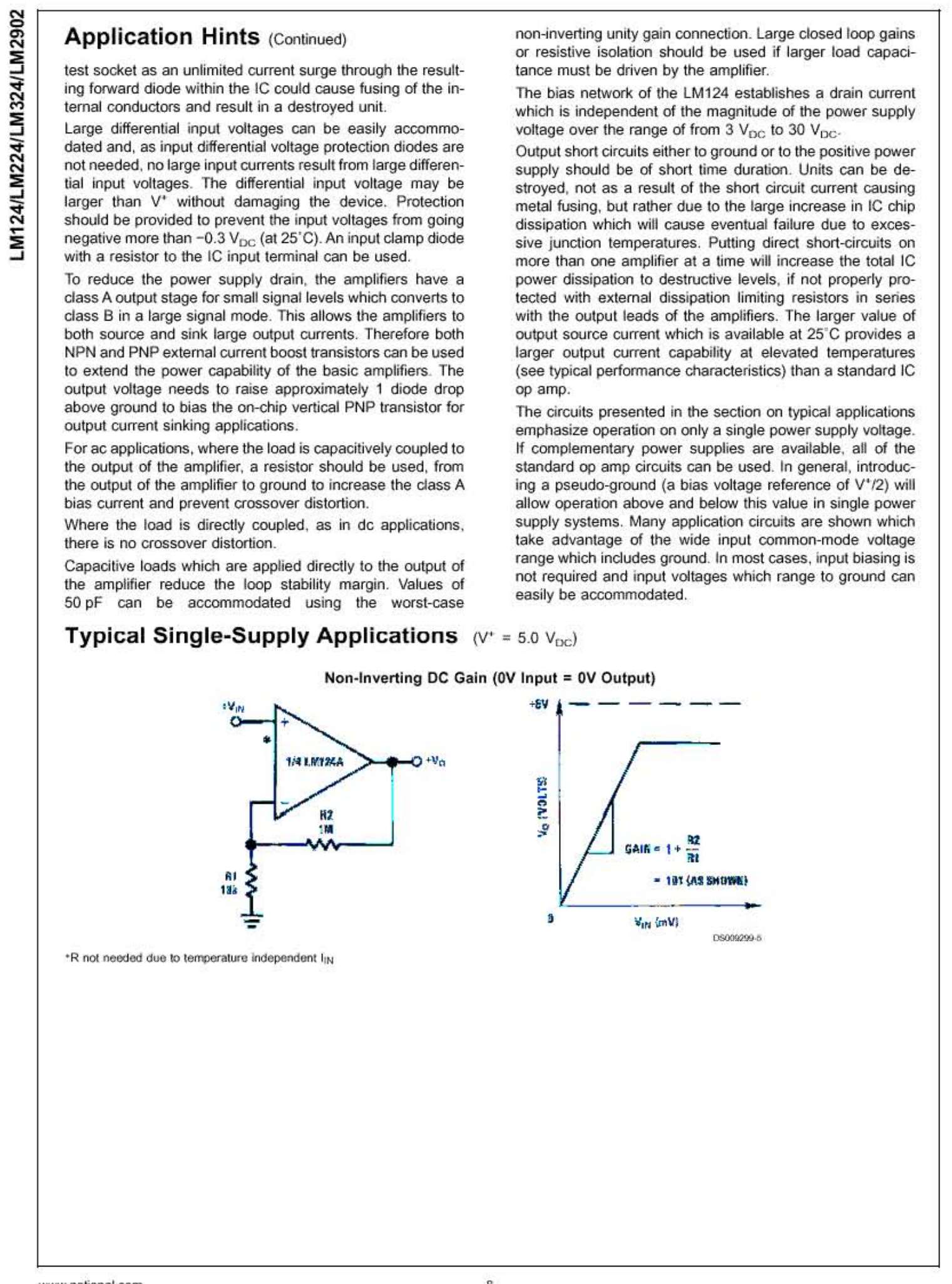

www.national.com

FIGURE 190 - Data sheet (p. 8) for the LM2902 quad operational amplifier. 


\section{APPENDIX XIX.}

COLEJET 2000'M MACHINE SCHEMATICS 


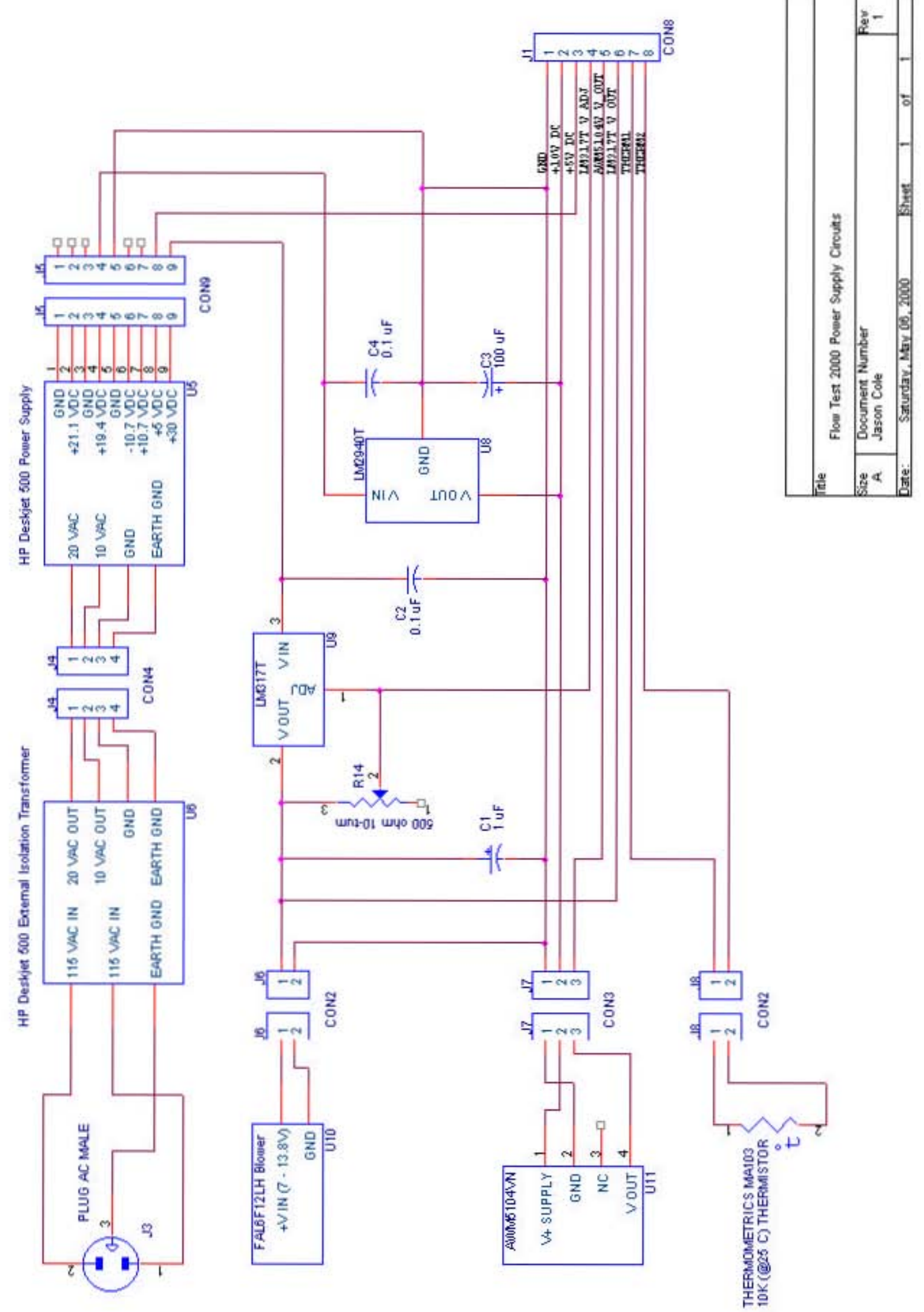

FIGURE 191 - ColeJet 2000 power supply circuits schematic diagram. 


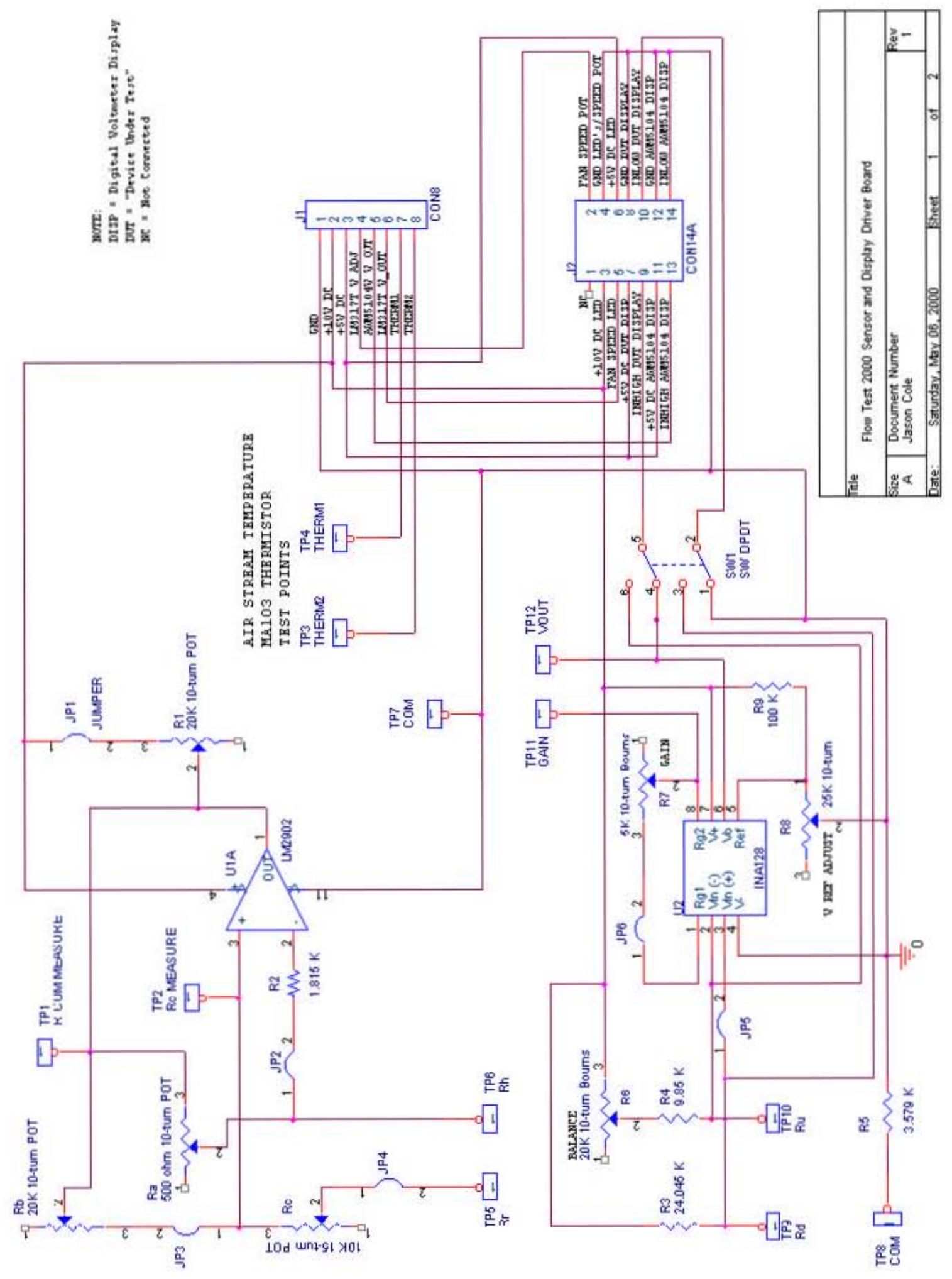

FIGURE 192 - ColeJet 2000 flow sensor Device Under Test driver and instrumentation amplifier schematic diagram. 


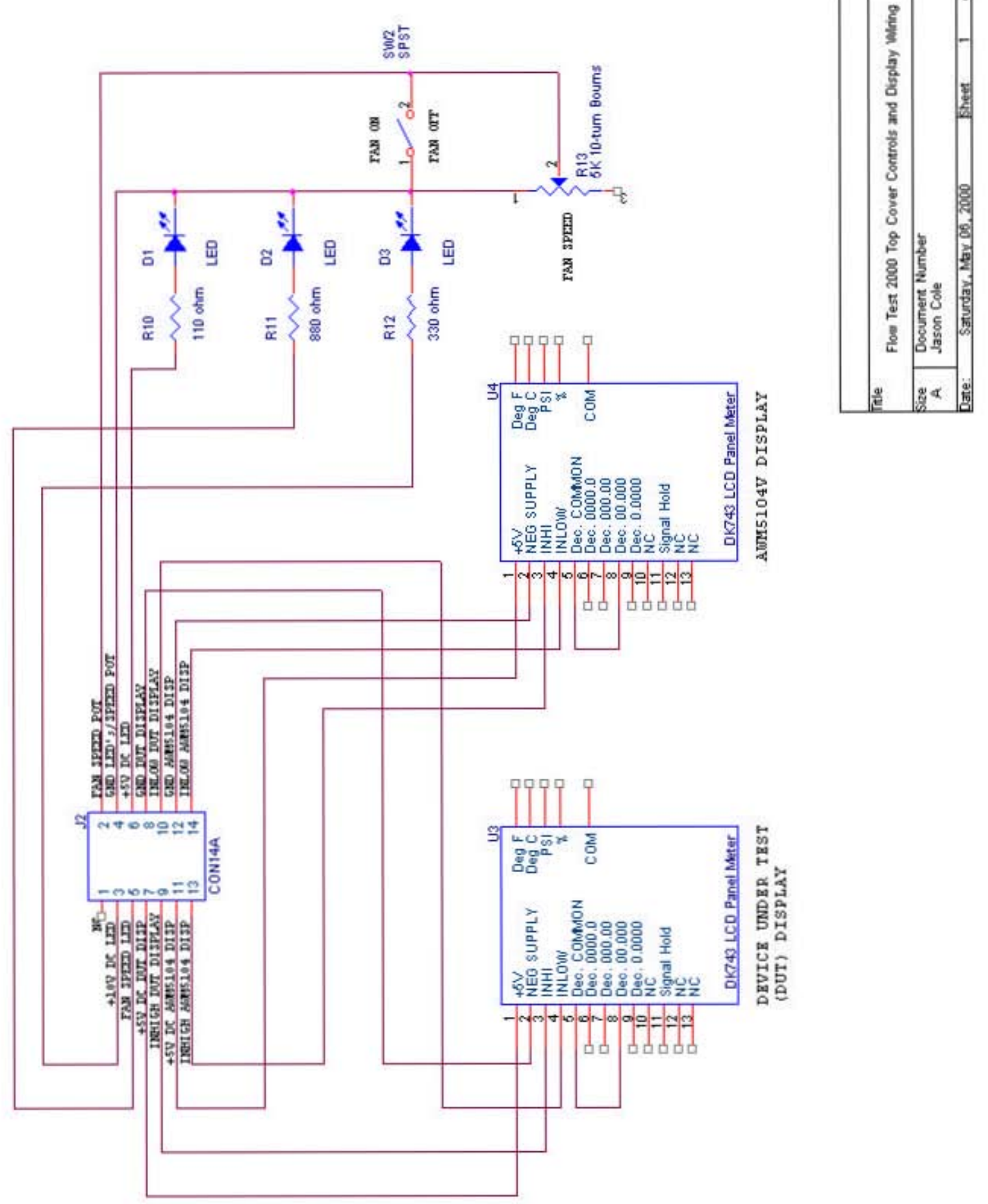

FIGURE 193 - ColeJet ${ }^{T M} 2000$ top cover controls and display wiring schematic diagram. 

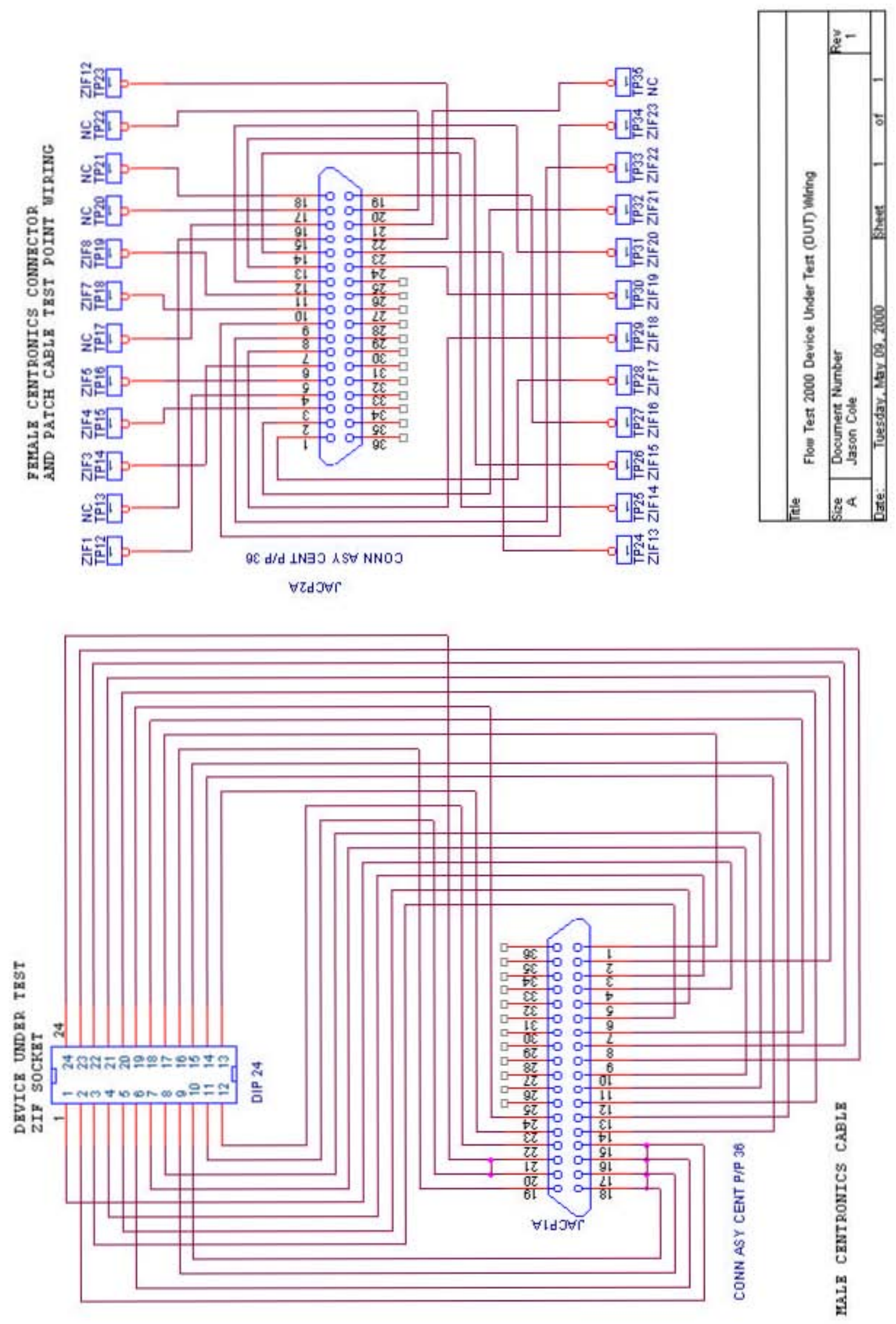

FIGURE 194 - ColeJet 2000 Device Under Test cabling/wiring schematic. 


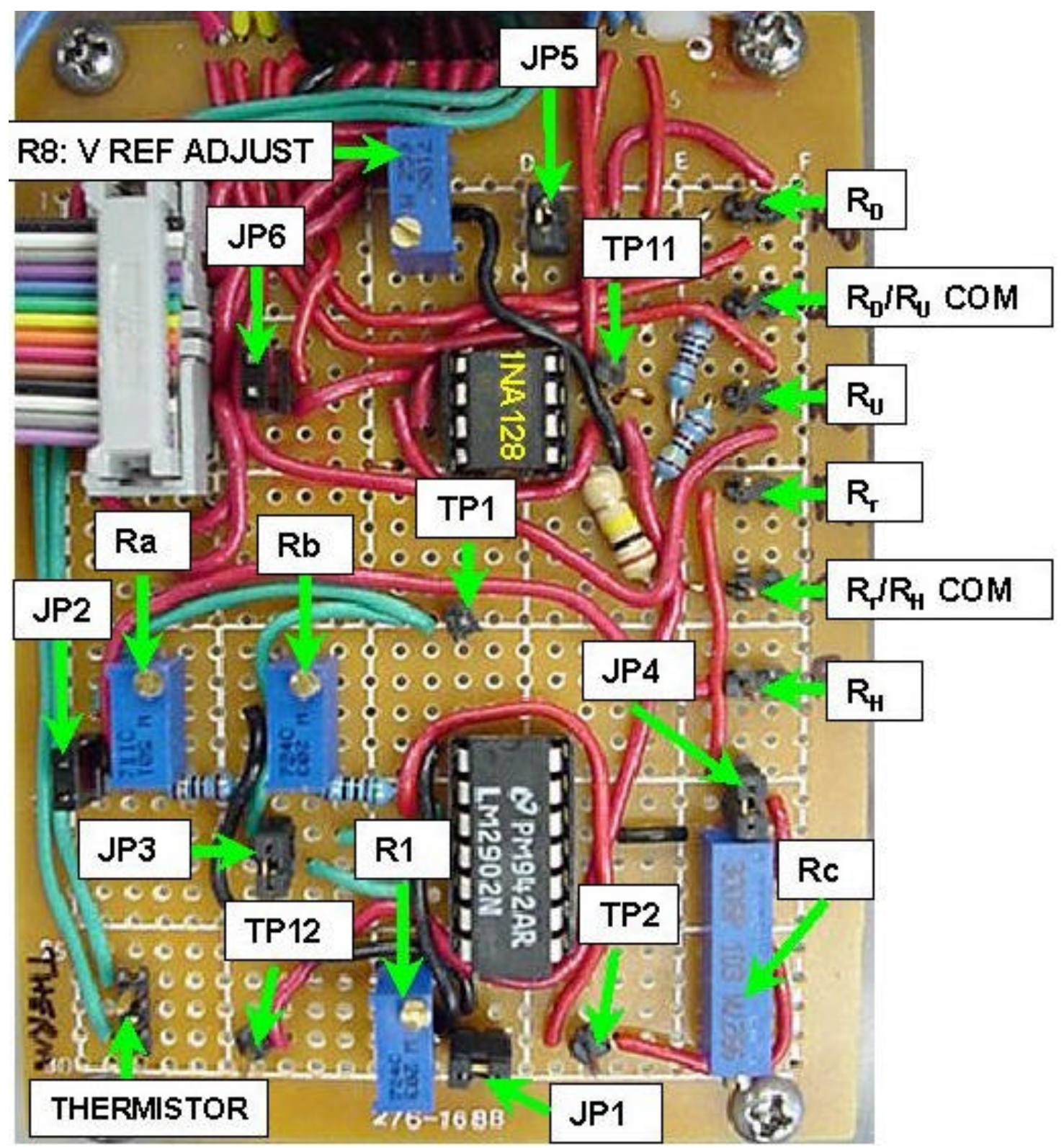

FIGURE 195 - Diagram showing circuit board locations of jumpers, test points, flow sensor resistor wiring attachment points, and multi-turn variable resistor locations and nomenclature. 
APPENDIX XX.

COLEJET 2000 ${ }^{T M}$ PHOTOGRAPHS AND DESCRIPTIONS 


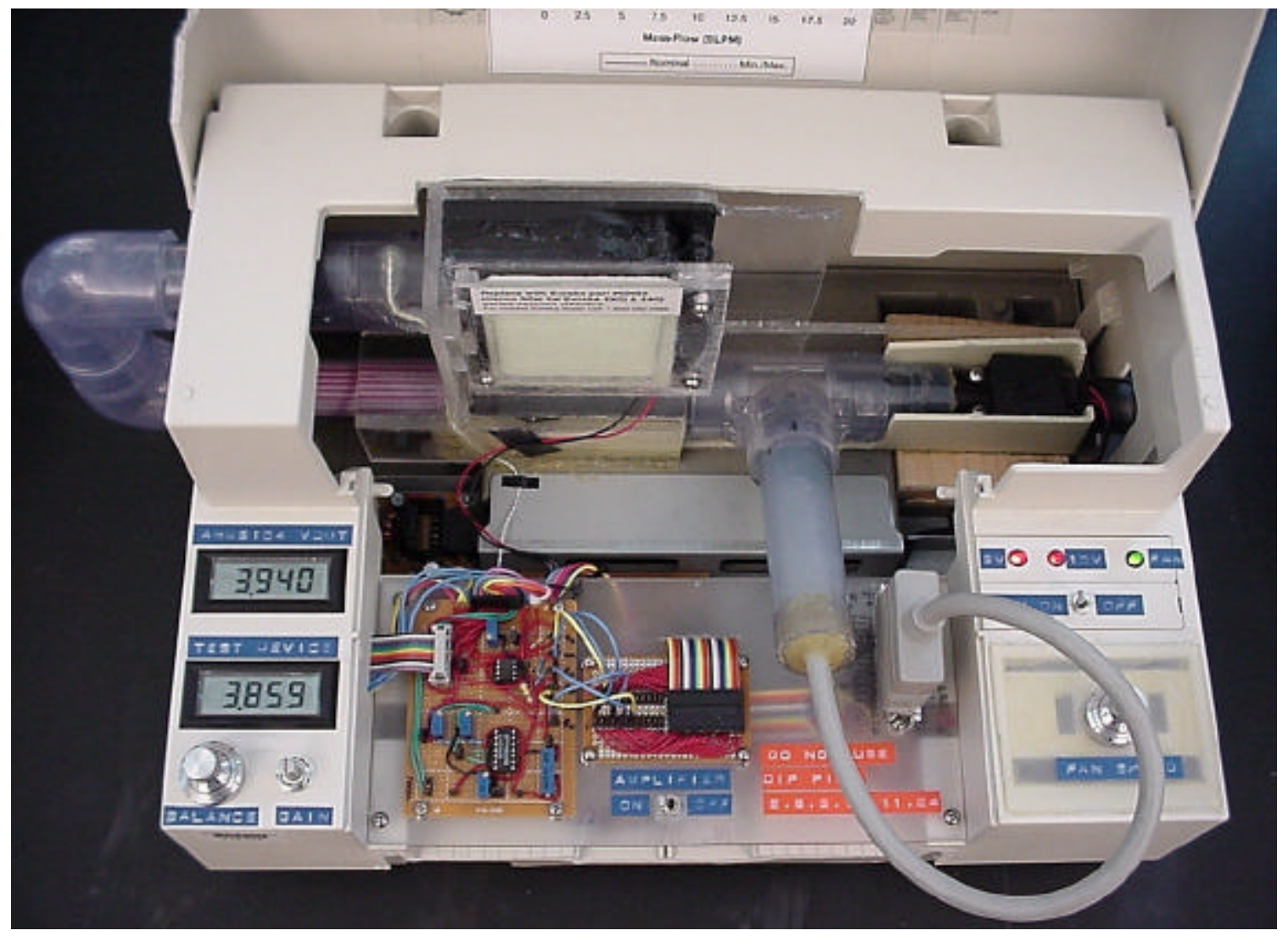

FIGURE 196 - Top, front view of the ColeJet 2000 machine that can be used for testing fabricated mass airflow sensors with flow rates up to 14 SLPM in a $33 \mathrm{~mm}$. diameter channel $(0.273 \mathrm{~m} / \mathrm{s}$ max flow rate). Its features include a Honeywell AWM5104V flow sensor (calibrated for nitrogen) to measure actual flow rates in the tube, a ZIF DIP-24 socket to mount packaged flow sensors for testing, a removable/replaceable micron pre-filter, an easy insertion tube to place devices into the flow channel, a removable tubing section for visual alignment of packaged flow sensors within the flow channel, a patch board for reconfigurable device wire-bonding to the DIP-24 package, two digital displays to show the output voltage from the Honeywell and Device Under Test sensors, and controls to precisely adjust the fan speed, Wheatstone Bridge balance, and (optional) differential instrumentation amplifier gain. 


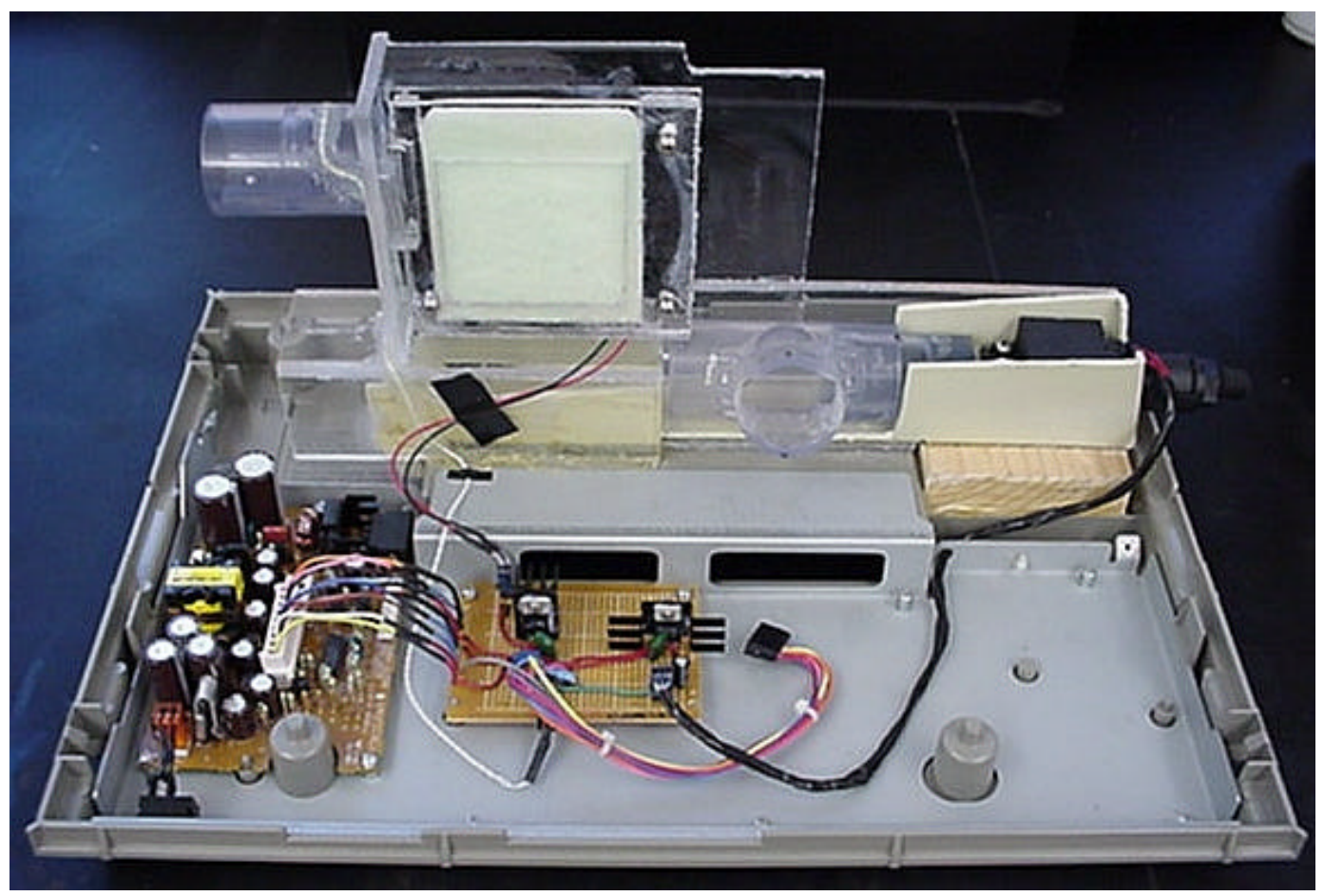

FIGURE 197 - Bottom tray of the ColeJet ${ }^{T M} 2000$ machine showing the Honeywell AWM5104V calibrated flow sensor at right, center; the blower assembly with integrated/removable micron pre-filter at top, center; the original HP DeskJet ${ }^{\mathrm{TM}}$ power supply module at bottom, left; and additional power supply circuit at bottom, center.

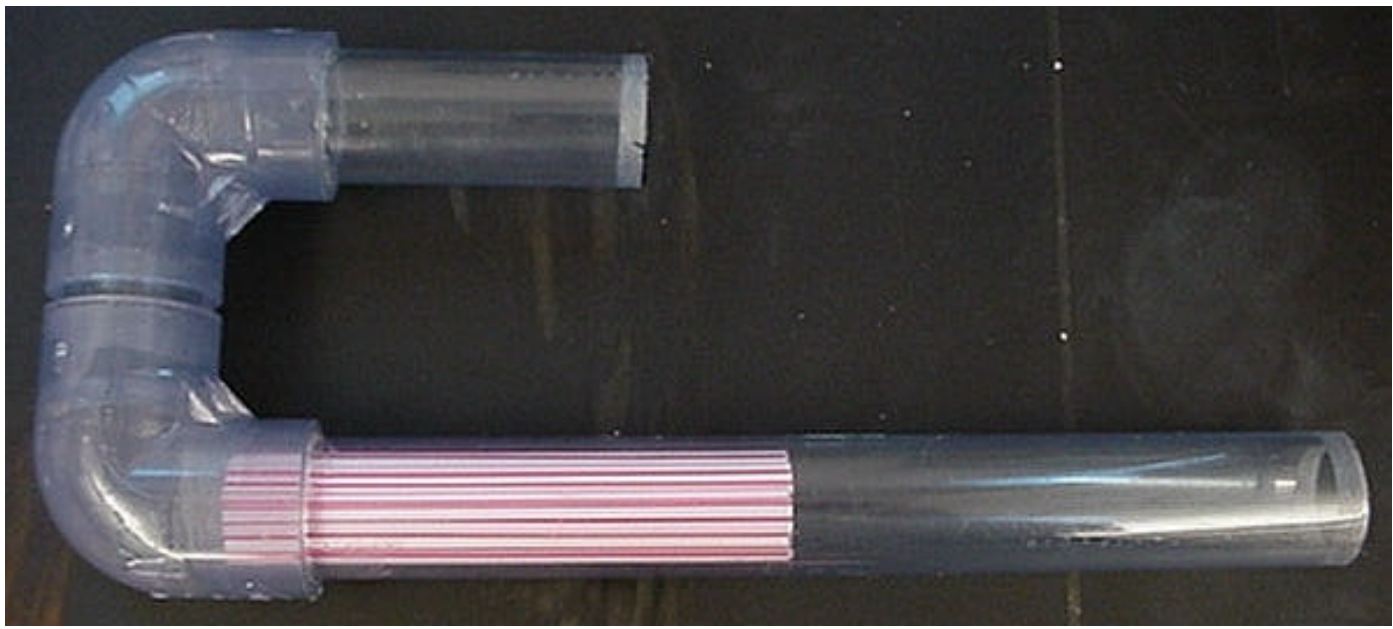

FIGURE 198 - Close-up of the clear PVC pipe tube section missing from the photograph of the bottom tray shown at the top of the page. This tube was designed to be removable from the ColeJet 2000 machine for accurate placement of inserted, packaged flow sensor devices to be tested. The $3 \mathrm{~mm}$. diameter cocktail straws fitted tightly inside the tube allow for fully-developed laminar flow within a shorter tube length. 


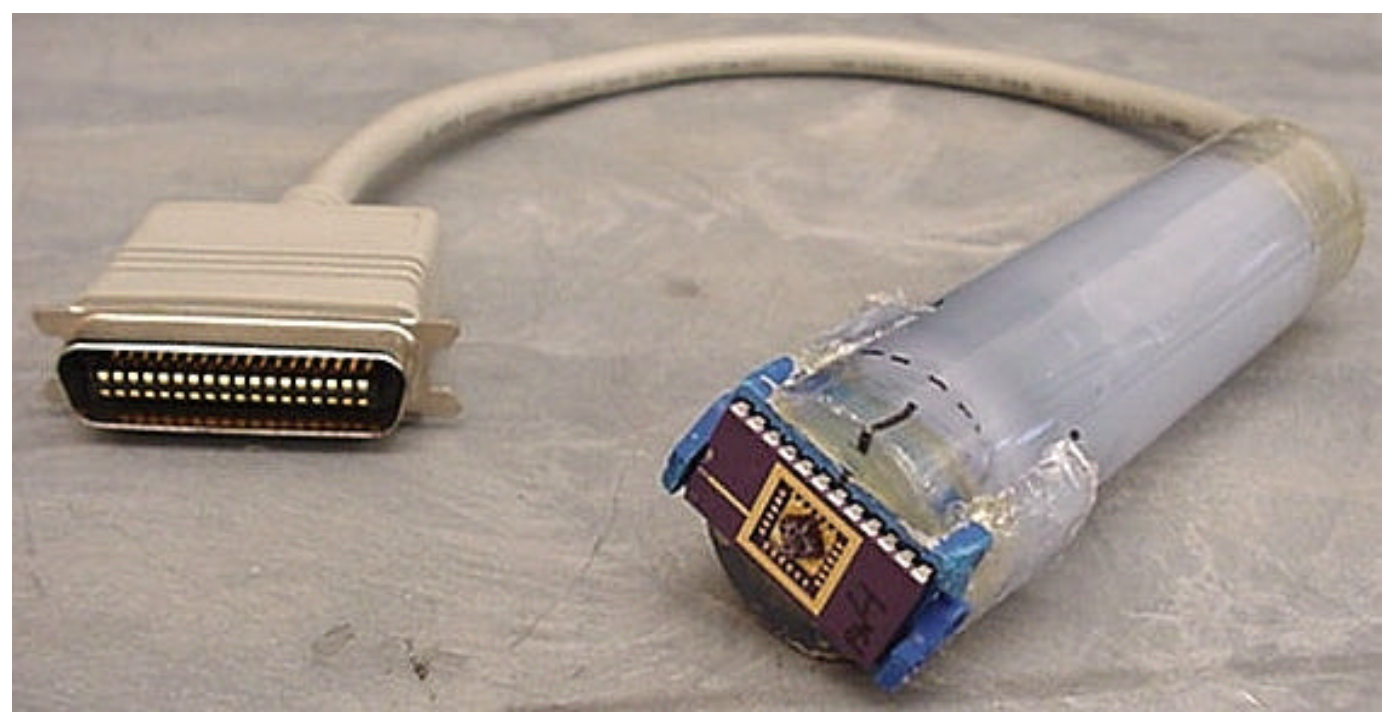

FIGURE 199 - Photo of the Device Under Test (DUT) tube section with integrated 24-pin ZIF socket (shown with a packed and wire-bonded quad flow sensor) and male Centronics connector. Due to the internal wiring of the (former printer) cable, only 18 of the 24 possible pins in the DIP-24 package (and ZIF socket) are usable. Unusable pins are printed on the ColeJet ${ }^{T M} 2000$ machine itself, and in the machine schematics. This DUT tube must be inserted with the black mark shown, facing up.

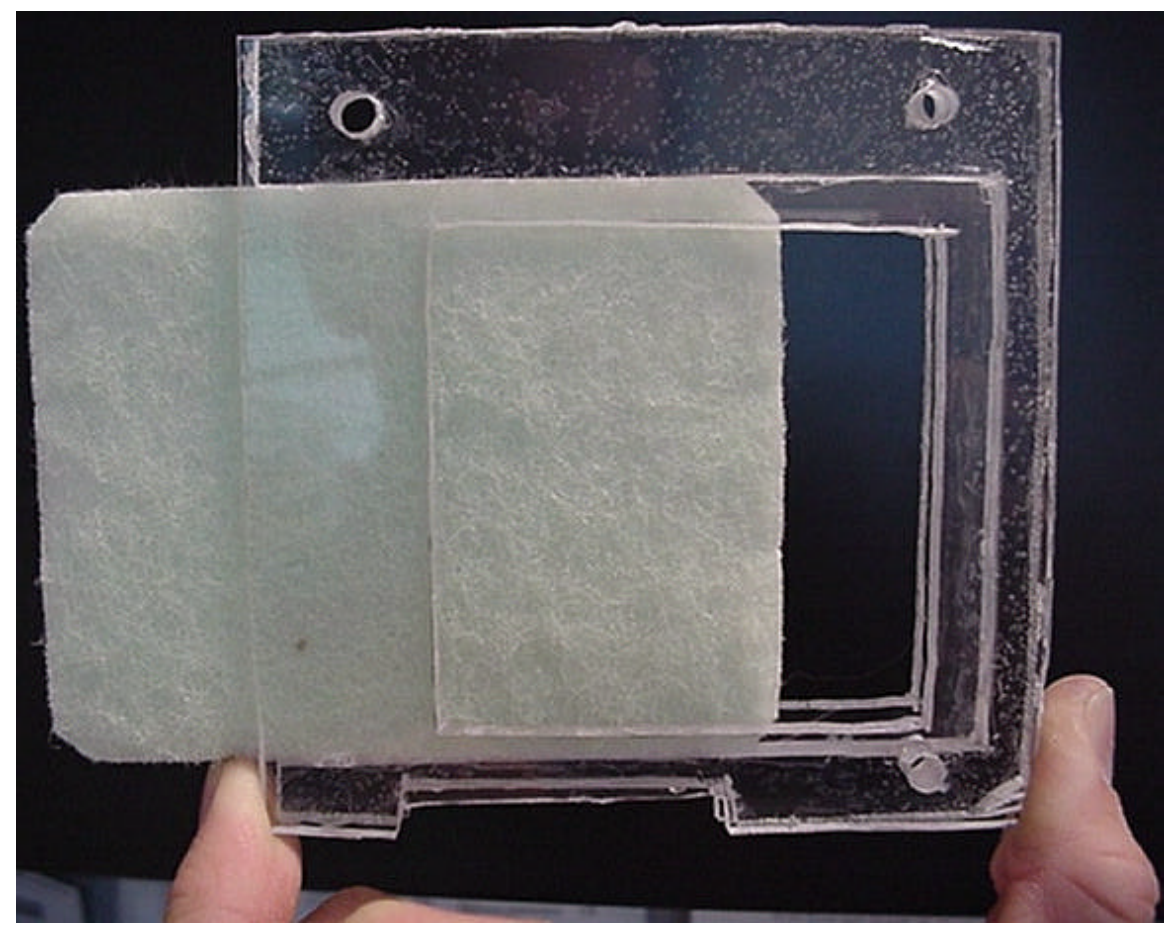

FIGURE 200 - Close-up photo of the Plexiglass ${ }^{\mathrm{TM}}$ micron pre-filter assembly. The removable filter is Eureka part number 60665 for Eureka 4300 and 4400 series vacuum cleaners equipped with micron filters. 


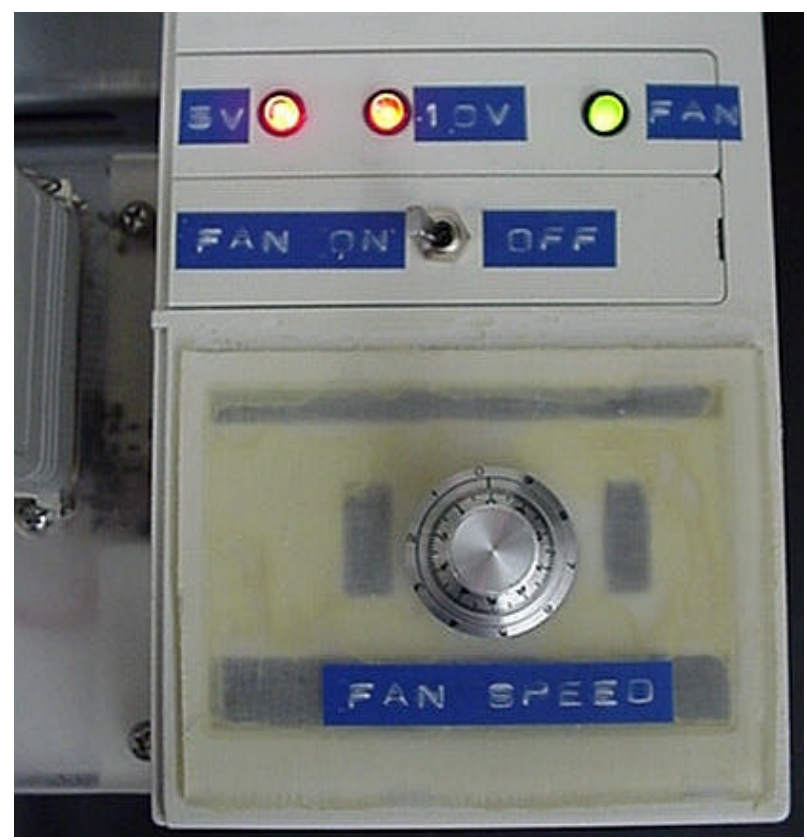

FIGURE 201 - Close-up of the right-side blower fan controls and power indicator LEDs. The 20-turn precision potentiometer at the bottom of the photo allows for precise control of blower speed and flow rates through the machine.

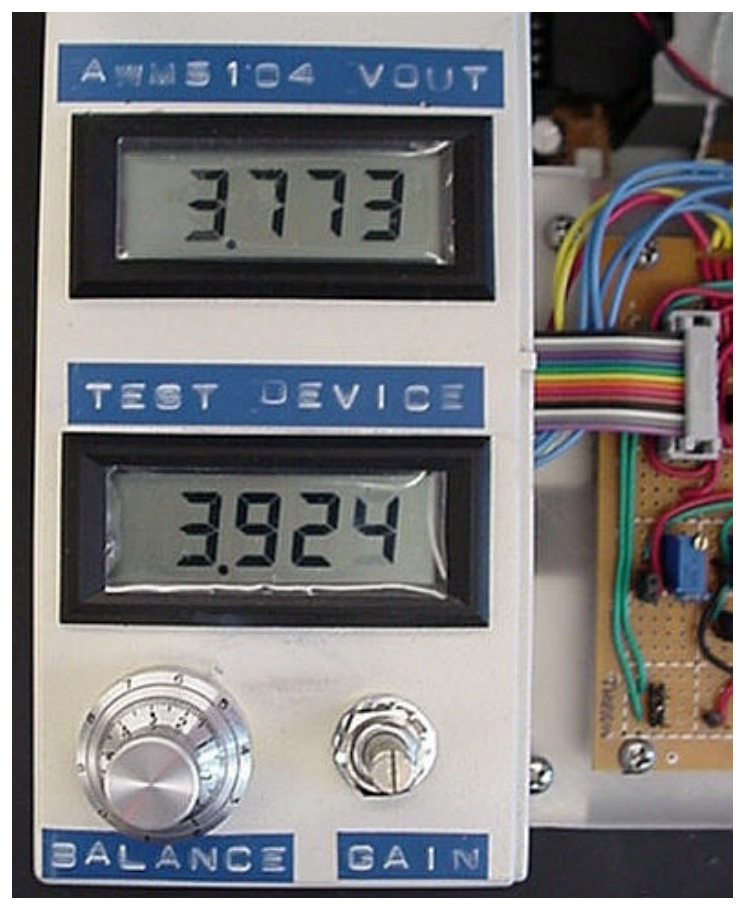

FIGURE 202 - Close-up of left-side voltage indicators, Wheatstone bridge balance, and optional differential instrumentation amplifier gain controls. The top LCD shows the linearized voltage output from the calibrated Honeywell AWM5104V flow sensor (which can be directly correlated to the flow rate), and the bottom indicator shows the output from the amplifier or Wheatstone bridge for the flow sensor Device Under Test. 


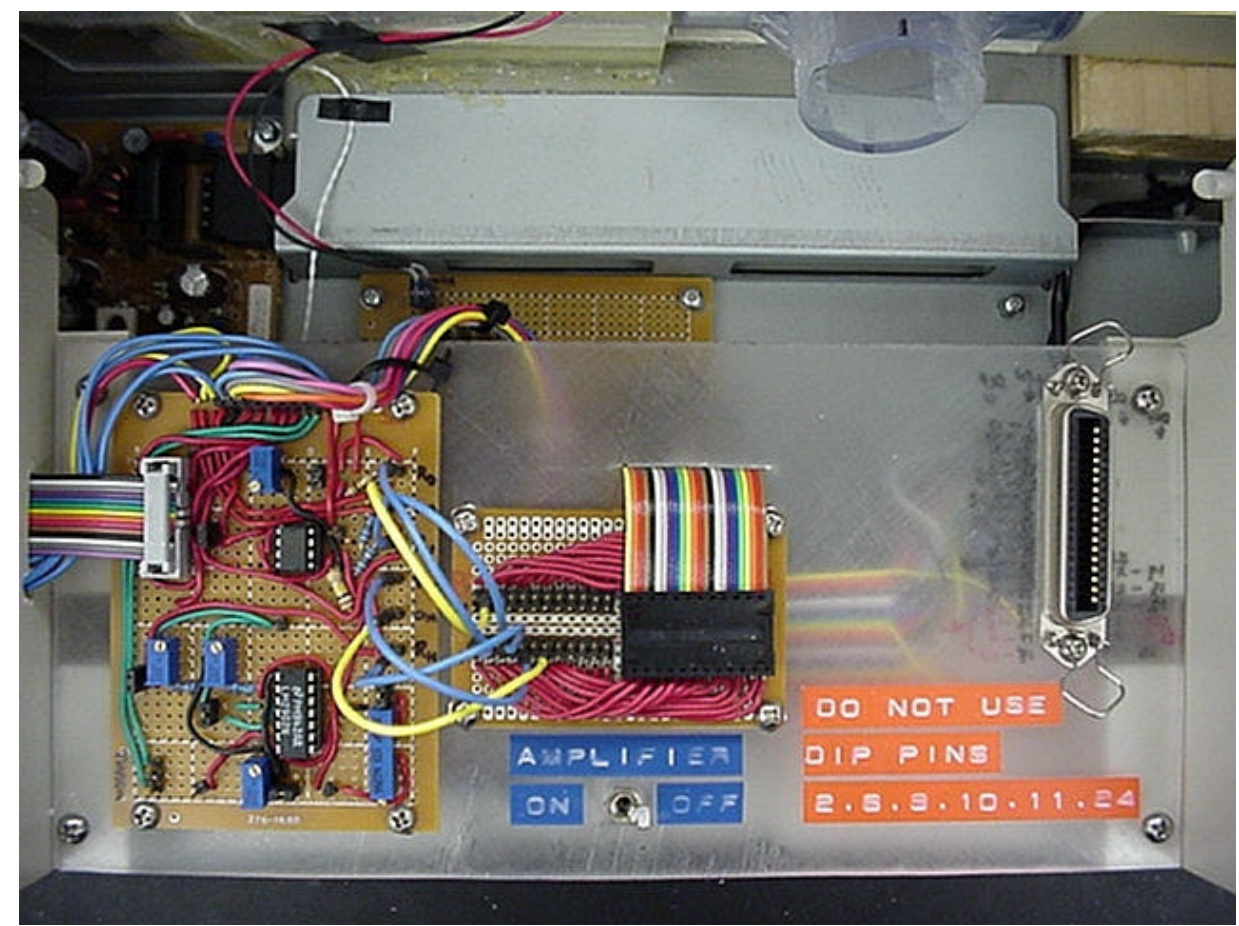

FIGURE 203 - Top view of the Device Under Test (DUT) driver board, patch-cable board, and Centronics female plug for the DUT insertion tube assembly.

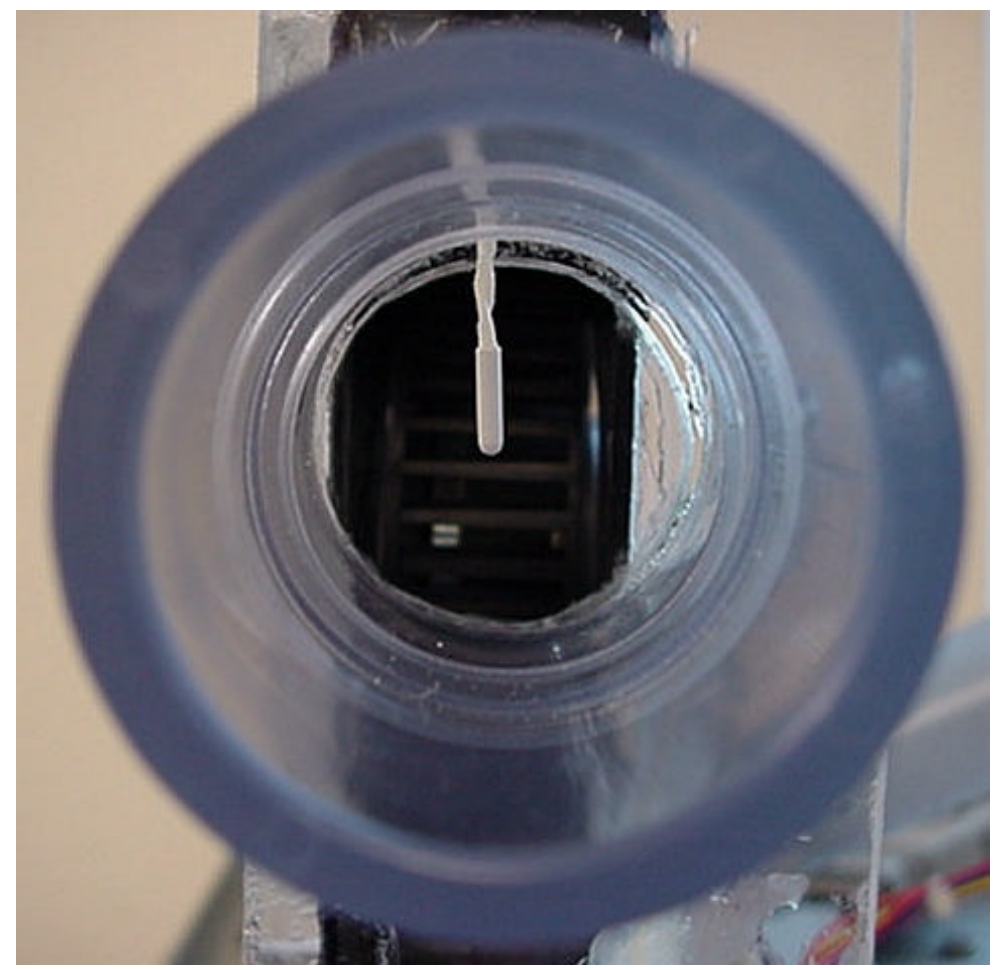

FIGURE 204 - View of blower fan and thermistor located inside the flow stream channel, after removing the removable tube section. 


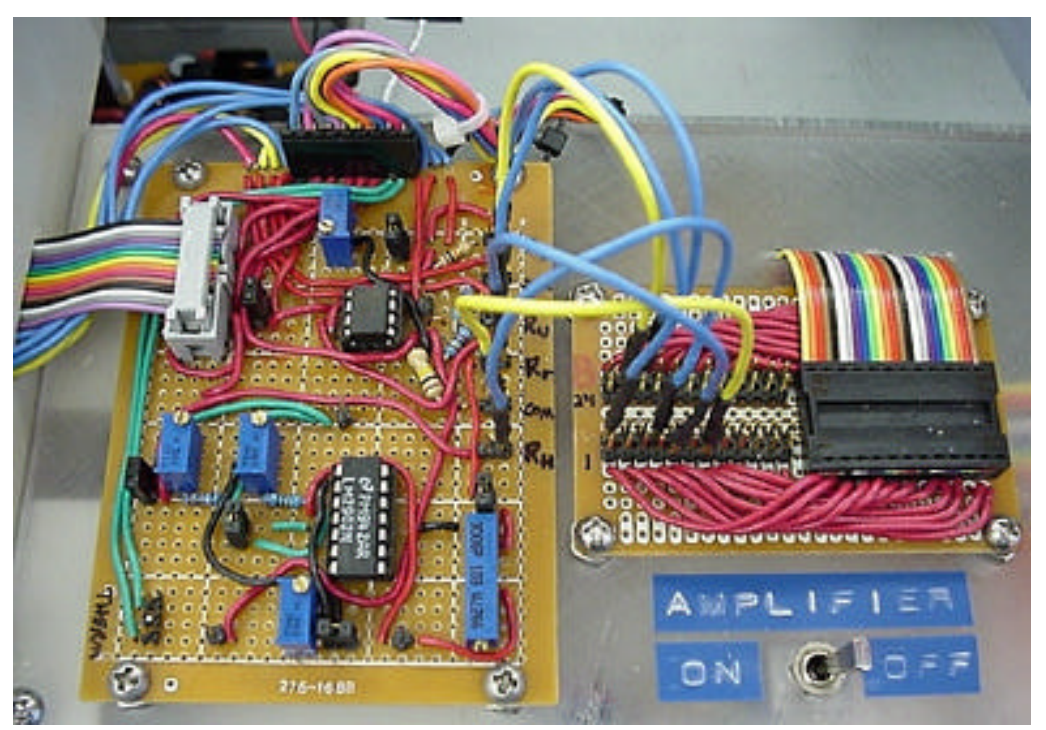

FIGURE 205 - Close-up of the Device Under Test driver board (with heater driver, Wheatstone bridge, and differential instrumentation amplifier circuits), and patch-cable board for configuring wiring between the sense/heater/ substrate/common resistor connections and the DIP-24 device package. The AMPLIFIER ON/OFF switch toggles the output to the center LCD between a direct Wheatstone bridge voltage output or amplifier output.

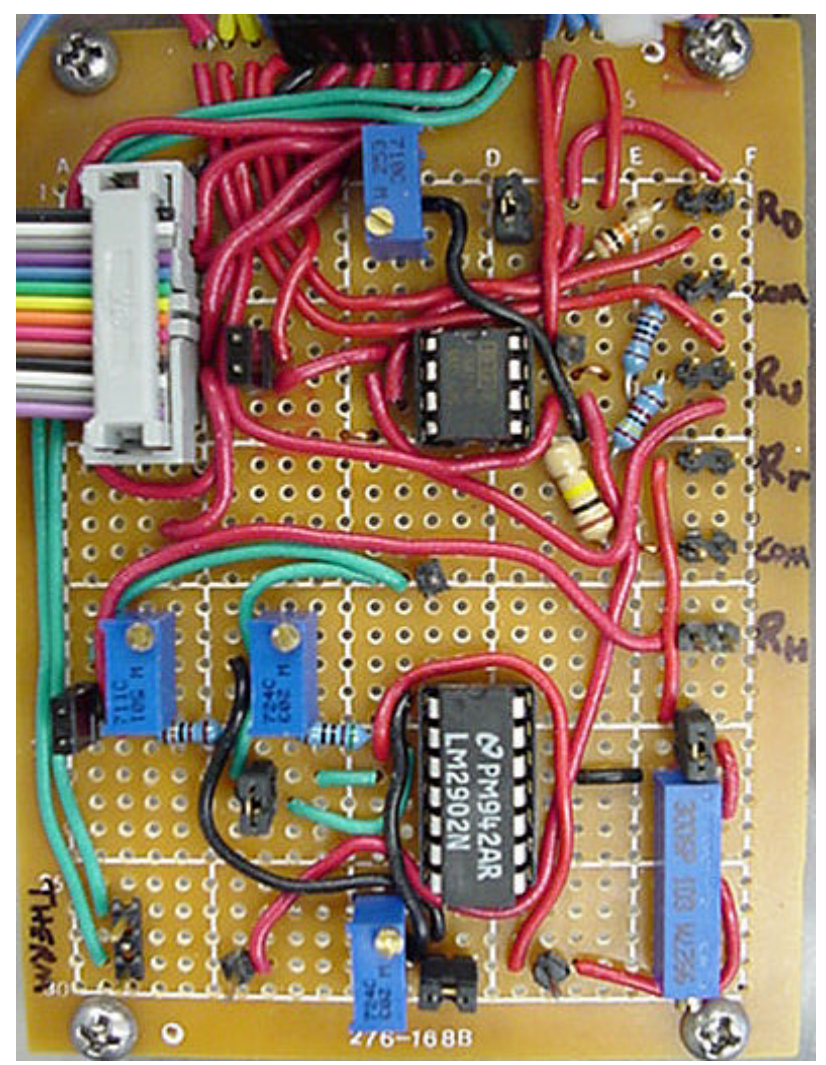

FIGURE 206 - Close-up of the Device Under Test driver board. The Burr-Brown INA128 instrumentation amplified is shown at the top, center, and the LM2902N quad op-amp used in the heater driver circuit is shown at the bottom, center. 


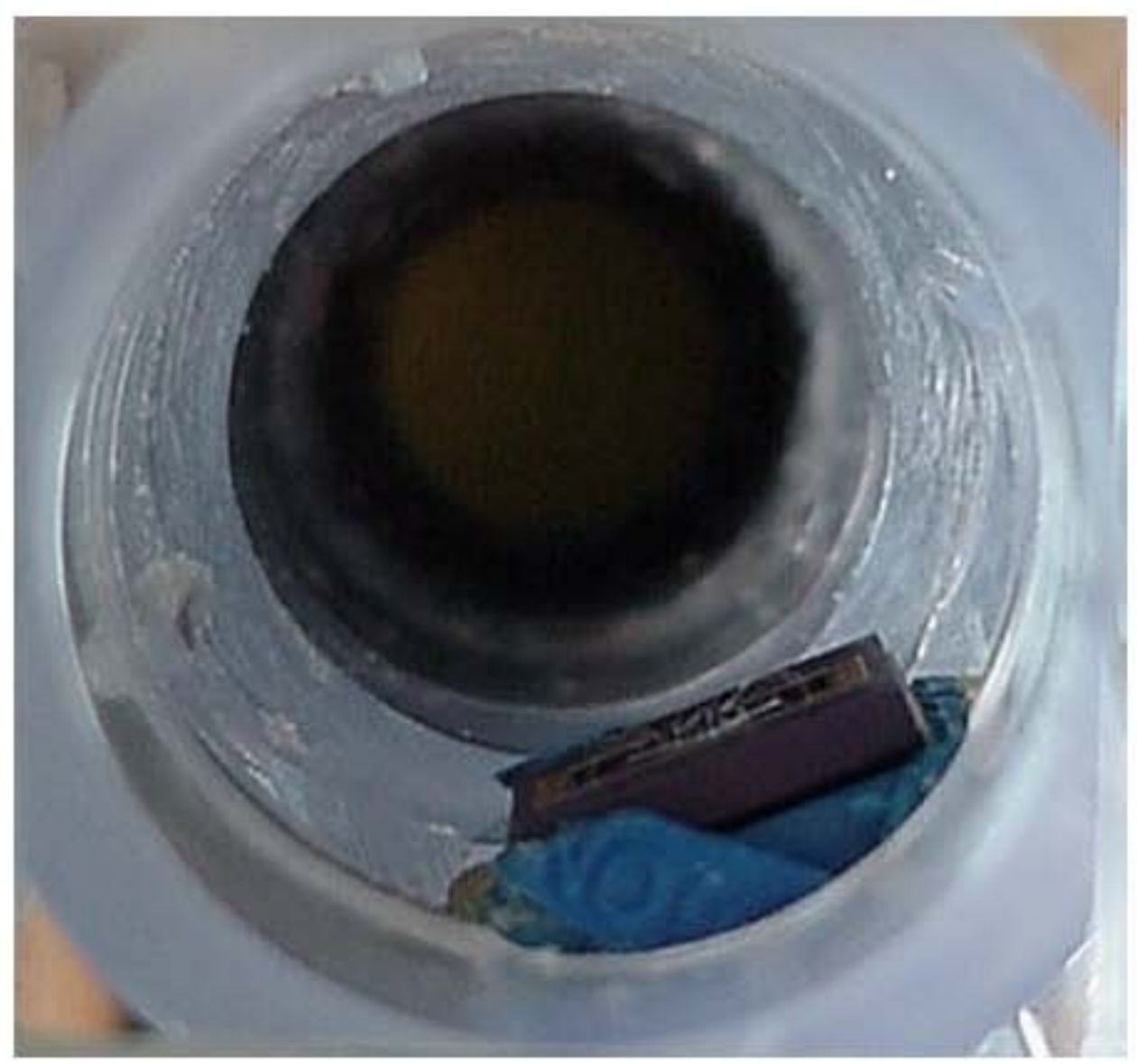

FIGURE 207 - View of the ZIF socket device insertion area inside the flow tube (looking towards the entrance to the Honeywell AWM5104V flow sensor). 
APPENDIX XXI.

COLEJET 2000TM CONDENSED OPERATING INSTRUCTIONS 


\section{Operating Instructions: Users' Guide and Technical Guide}

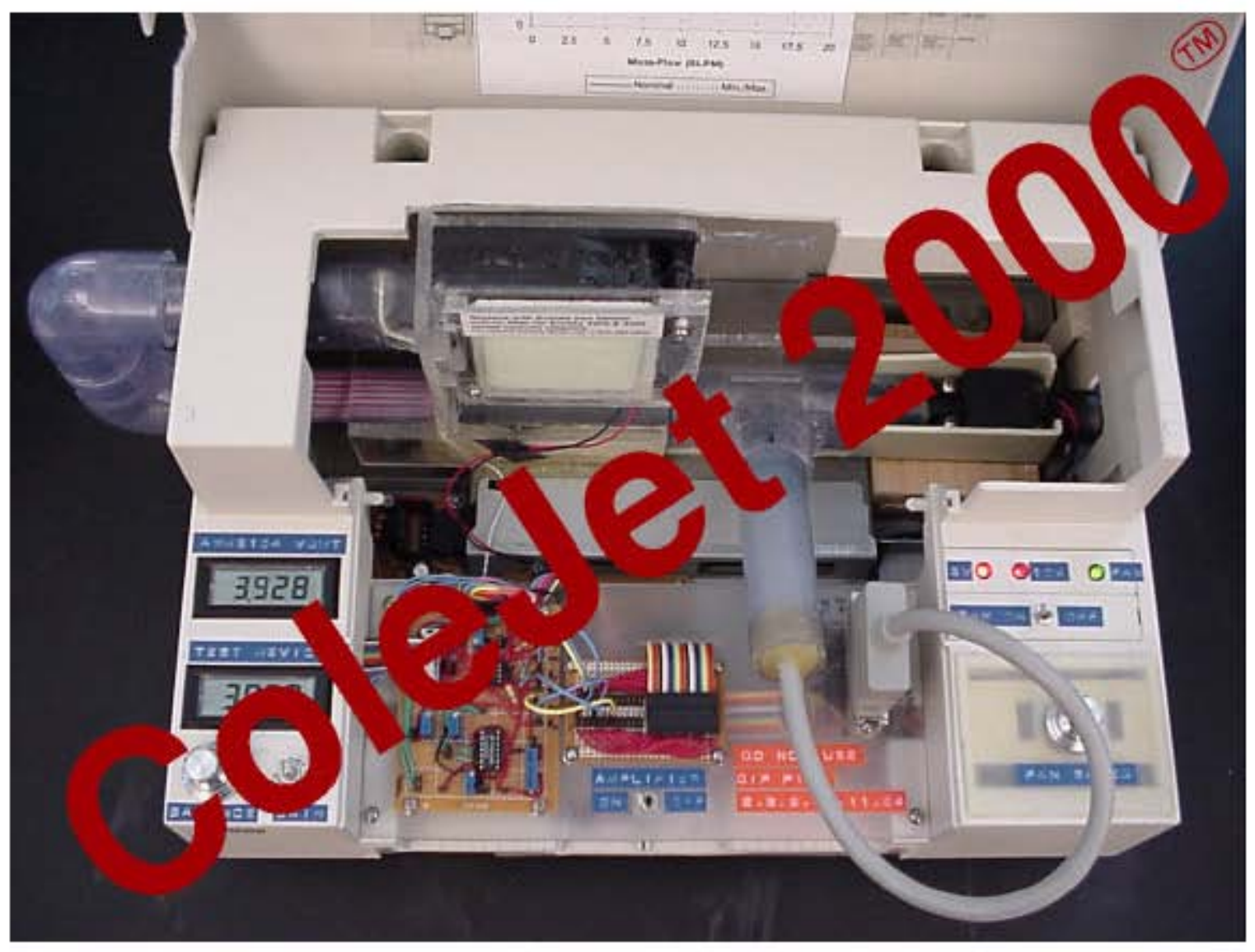




\section{ColeJet 2000'TM Users' Guide}

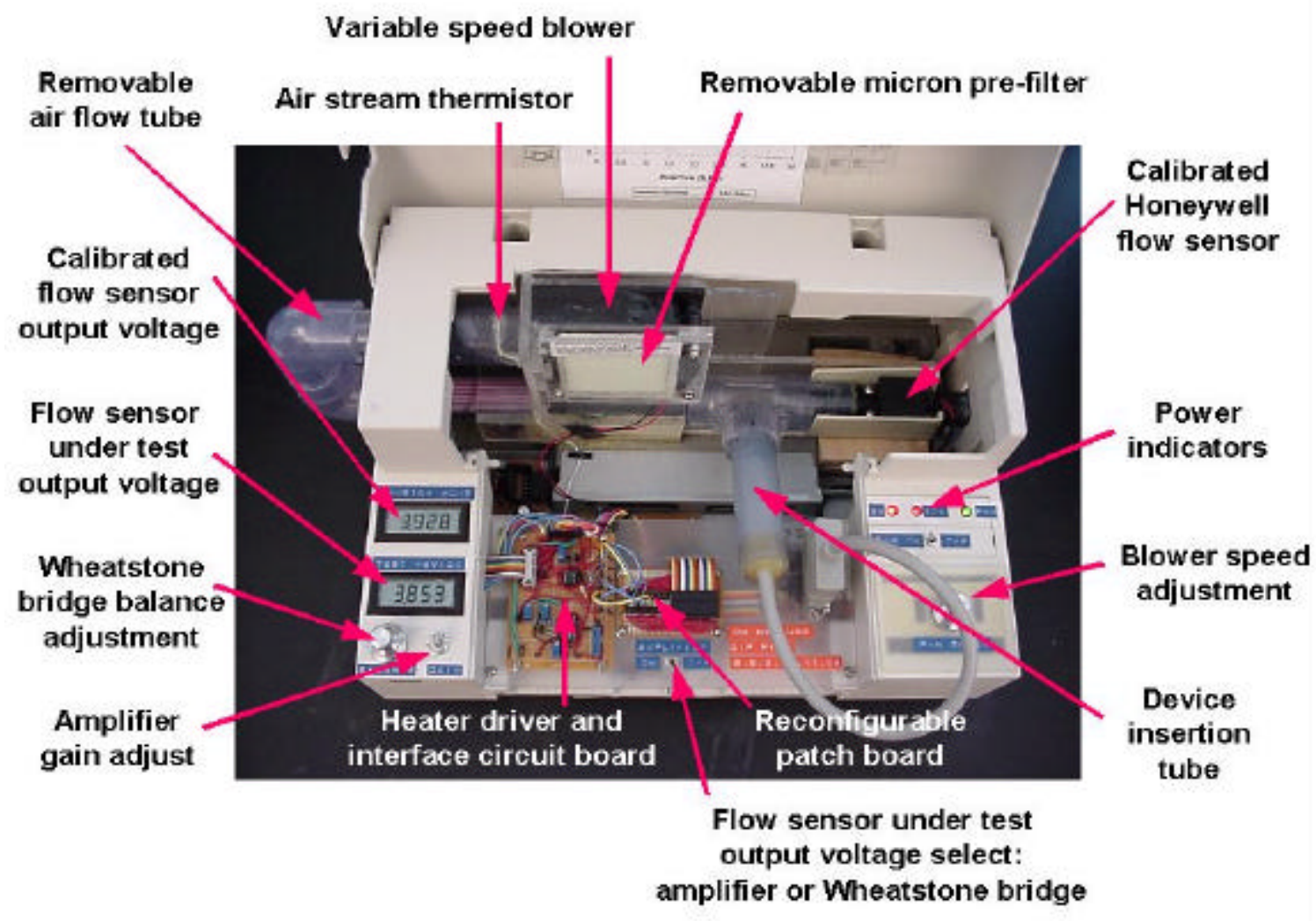

Follow these steps to use the machine to test fabricated flow sensors

1. Package and wirebond a quad or duo-die flow sensor with probe-station-measured upstream and downstream resistors within individual die matching to within $5 \%$ (or $R_{U} R \quad$ D). Epoxy the quad or duo-die substrate onto the top of a glass spacer, which should be epoxied into a DIP24 package after orienting the quad/duo-die (and spacer) with main microbridge/cantilever axes perpendicular to the flow direction across the DIP-24 package, as shown below.

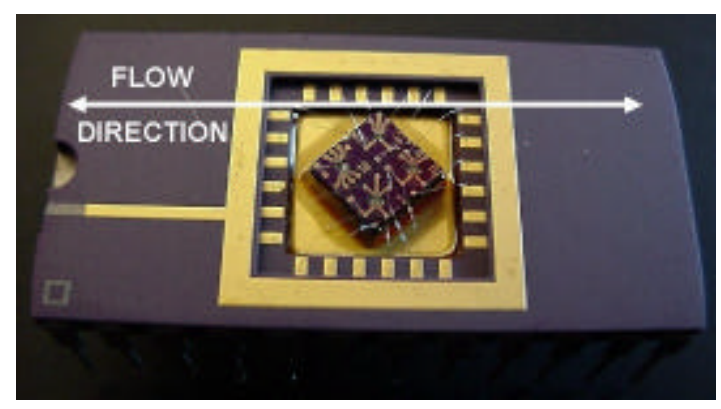

2. Wirebond the flow sensors to be tested within the quad/duo-die to the DIP-24 package. Due to the internal wiring of the Device Under Test cable assembly, do NOT wirebond to package pins \#2, 6, 9, 10, 11, and 24. Note that flow sensor nodes from multiple devices may be wire-bonded to each DIP-24 pin (provided that resistors from multiple devices are not wired in parallel) to test each of four possible die, separately. 
3. Pull the Device Under Test tube assembly out from the flow channel. It is not necessary to unplug the Centronics connector. Rotate the ZIF socket levers to unlock the ZIF socket (if it is not already unlocked). Insert the DIP-24 package into the ZIF socket. Align pin \#1 of the package to the black "1" mark on the socket (which is only visible without a DIP-24 package installed in the socket). Rotate the blue locking levers until the package is locked in place.

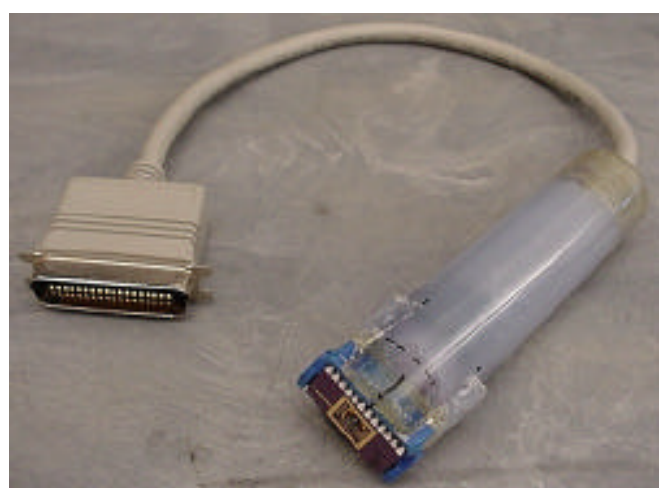

The Device Under Test cable/tube assembly.

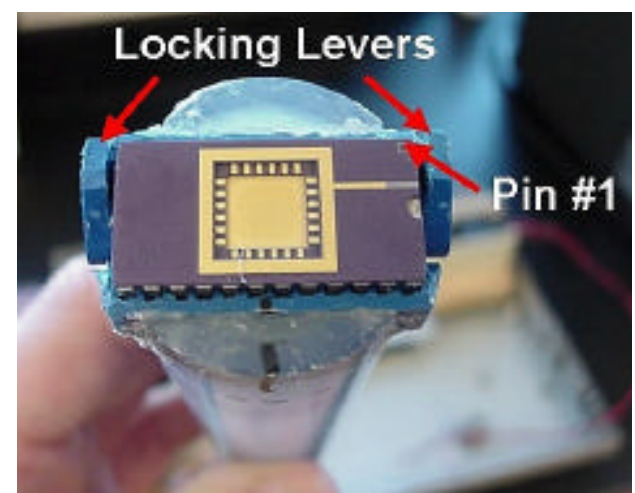

The ZIF socket with installed DIP-24.

4. Align the black mark on the tube to the mark on top of the clear PVC "T" fitting to insert the Device Under Test tube back into the machine, as shown below. Verify that the Centronics connector is securely seated in its receptacle socket.
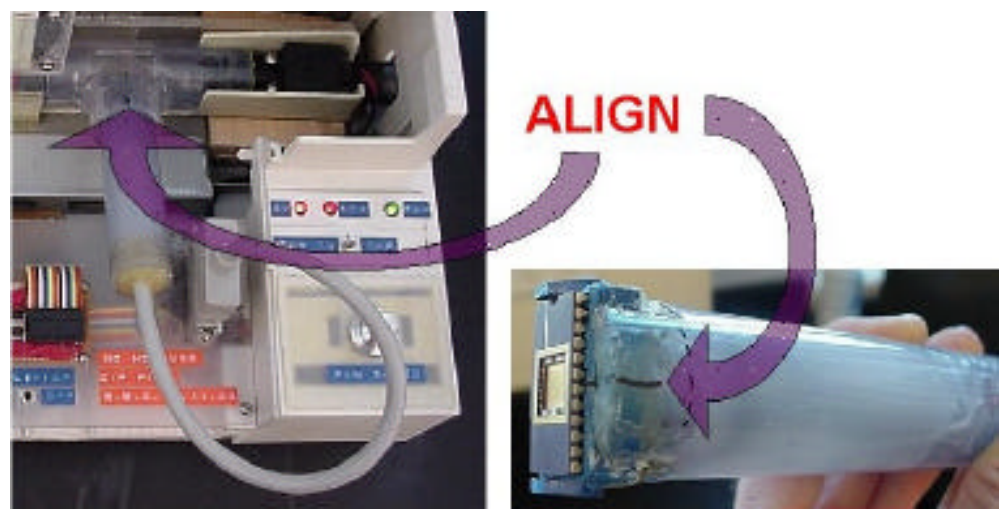

5. To position the depth of insertion of the flow sensor within the flow channel, pull the "J" shaped tube out of the machine, as shown below.

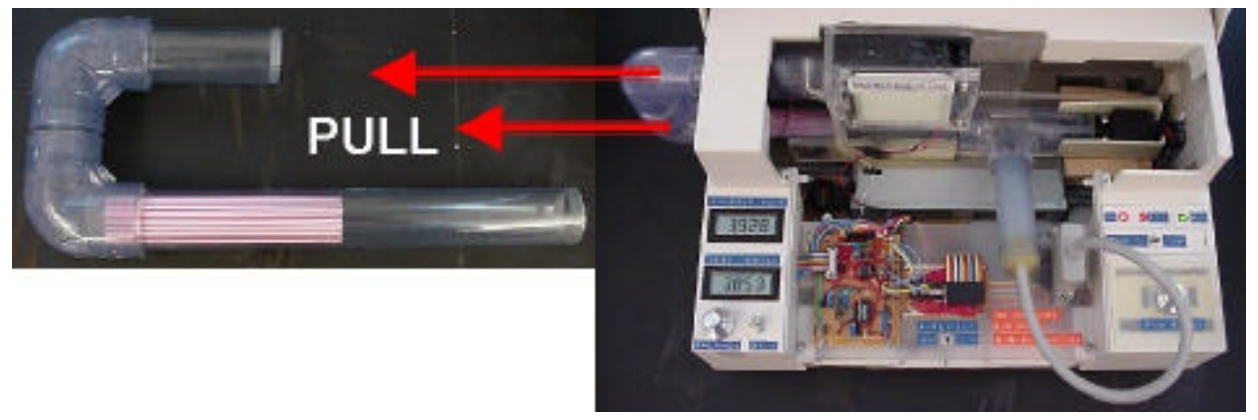

Then look into the bottom tube in the machine, towards the "T" fitting, to visually inspect where the sensor is located within the channel. Adjust the depth by pushing the Device Under Test tube assembly farther into the "T" fitting. 


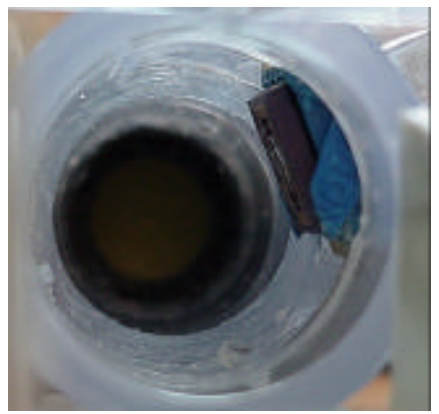

A view of the device insertion area inside the clear " $T$ " fitting.

6. Depending upon which flow sensor you want to test and how the quad/duo-die is wirebonded to the DIP-24 package, use the patch-wire board to connect flow sensor nodes to the six nodes on the heater-driver/interface circuit board. Use the patch wires provided with the machine to configure the interface between the driver circuit and the patch board. There are 24 rows of two posts in each row on the patch-wire board which correspond directly to the pin layout in the DIP-24 package, as shown below.

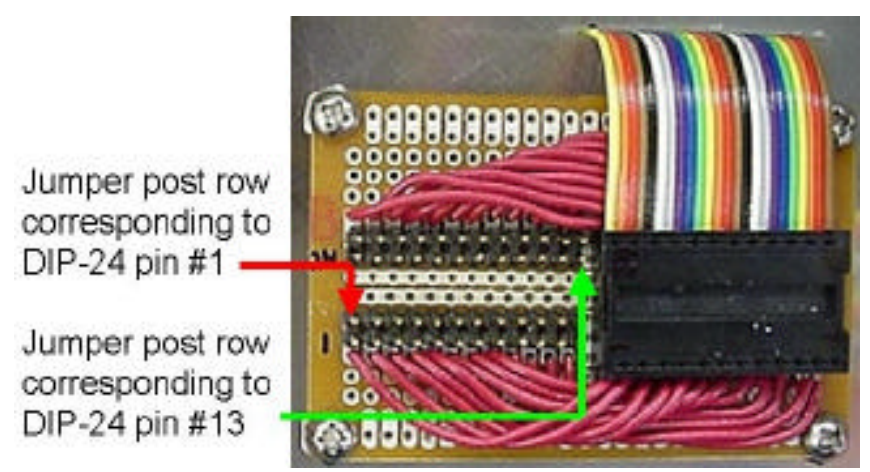

The six nodes on the heater-driver/interface board which must be wired to the flow sensor to be tested are named, and must be connected, as follows:

- $\quad \mathbf{R}_{\mathrm{d}}$ should be connected to a downstream resistor node which is not also connected, onchip, to any upstream resistor $\left(\mathbf{R}_{\mathbf{u}}\right)$ node. The downstream resistor is distinguished from the upstream resistor in that its resistance will increase with increasing flow (when the flow sensor is properly driven, assuming a resistor metallization layer is used which has a positively-correlated TCR).

- $\quad \mathbf{R}_{\mathbf{u}}$ should be connected to an upstream resistor node which is not also connected, onchip, to any downstream resistor $\left(\mathbf{R}_{\mathbf{d}}\right)$ node.

- The COM node on the board between the $\mathbf{R}_{\mathrm{d}}$ and $\mathbf{R}_{\mathrm{u}}$ nodes should be wired to the node which is connected (on-chip, or externally) to both

- $\quad \mathbf{R}_{\mathrm{h}}$ should be connected to a heater resistor node which is not also connected, on-chip, to any substrate resistor $\left(\mathbf{R}_{\mathbf{r}}\right)$ node.

- $\quad \mathbf{R}_{\mathrm{r}}$ should be wired to a substrate resistor node which is not also connected, on-chip, to any heater resistor $\left(\mathbf{R}_{\mathbf{h}}\right)$ node.

- The COM node on the driver board between the $\mathbf{R}_{\mathbf{h}}$ and $\mathbf{R}_{\mathrm{r}}$ nodes should be wired to the node which is connected (on-chip, or externally) to both a node of the heater resistor $\left(\mathbf{R}_{\mathbf{h}}\right)$ and a node of the substrate resistor $\left(\mathbf{R}_{\mathbf{r}}\right)$. 
A sample wiring configuration for two different types of flow sensors is shown below.

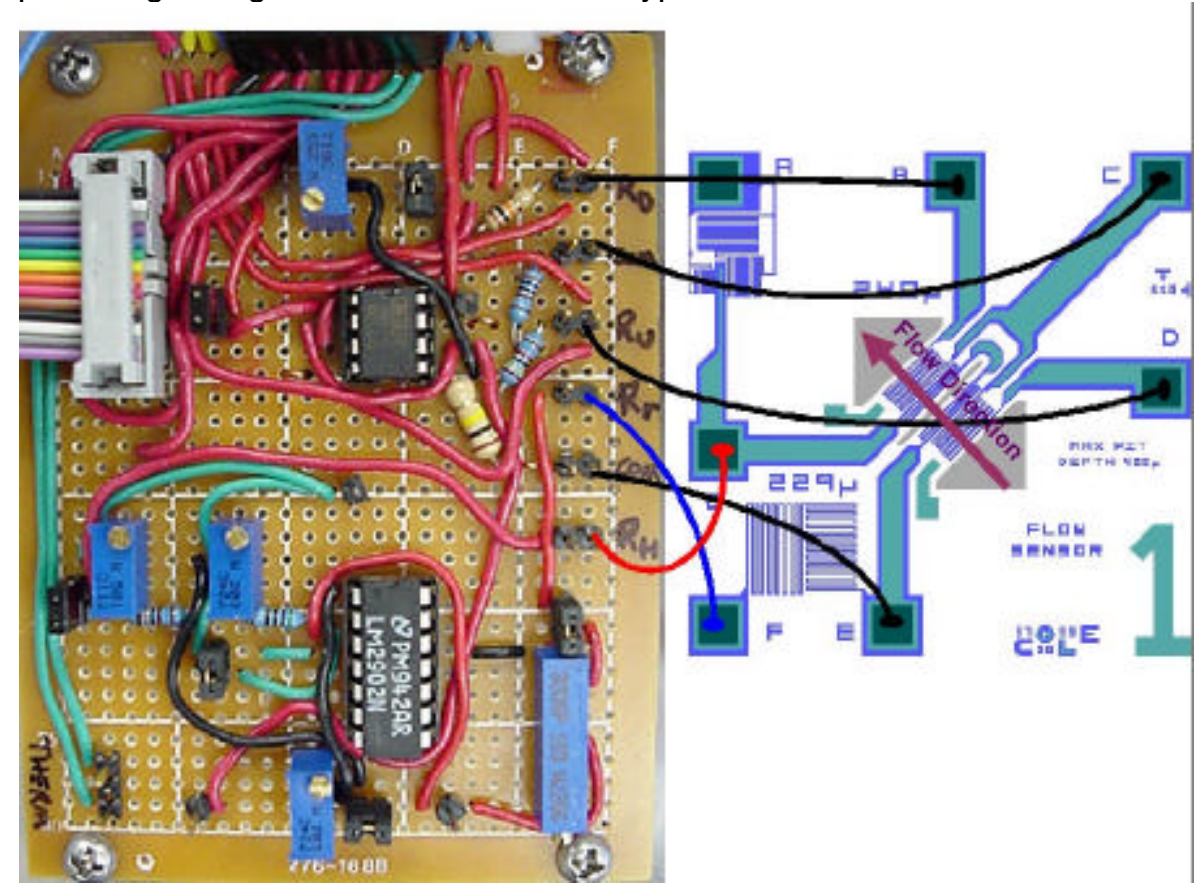

This type of flow sensor has two COM nodes on-chip.

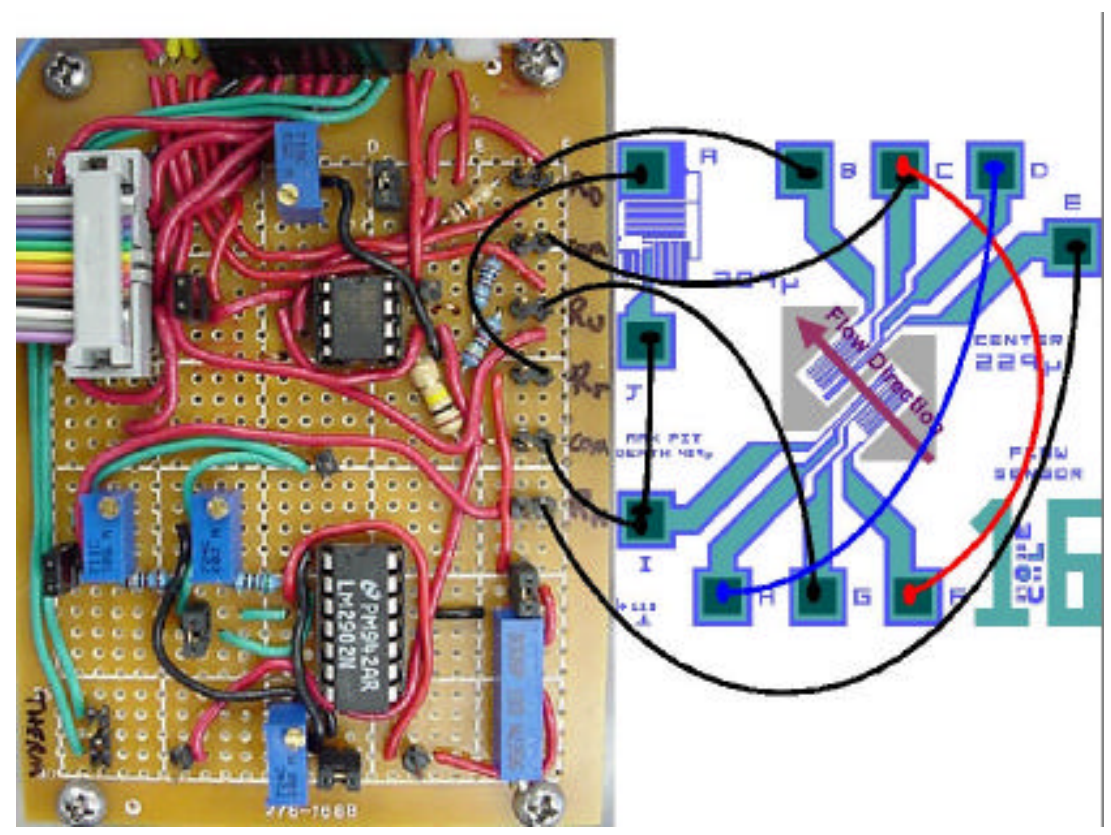

This type of flow sensor has no common nodes. Common connections must be wired off-chip.

7. Once the flow sensor to be tested has been wired/connected to the heater-driver/interface board, locate the external transformer for the machine. Plug the output end of the transformer into the port underneath the machine (if it is not already connected), and plug the transformer into a 120 VAC outlet. Remove any end cap which may be placed over the airflow outlet port (on the right, rear of the machine), verify the flow tube is fully inserted and seated, turn off the blower fan switch, and turn on the machine, as shown below. 


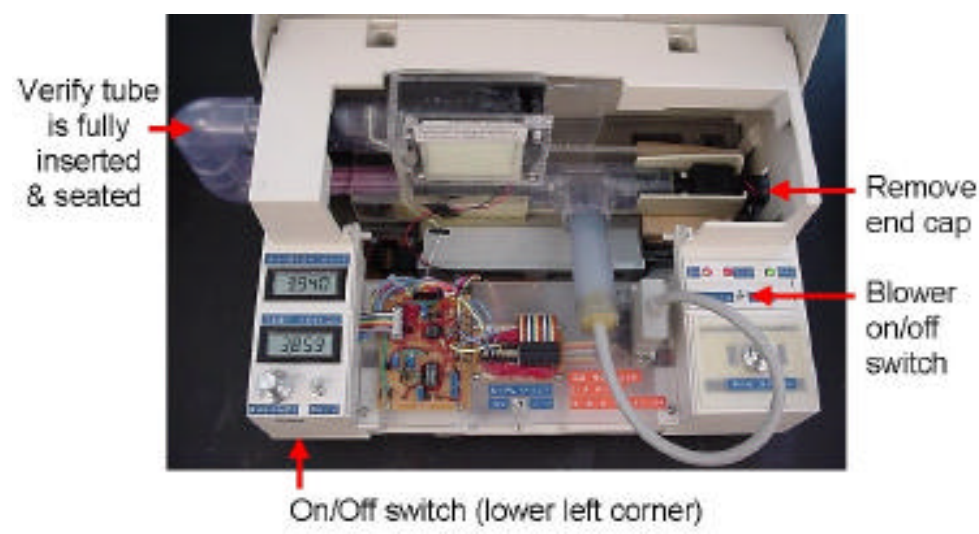

8. Both red LED voltage indicators should be glowing. The top output voltage LCD panel meter display for the AWM5104 Honeywell sensor should indicate approximately $1.0 \mathrm{~V} \mathrm{DC}$ (at no flow conditions). Flip the AMPLIFIER ON/OFF switch to the OFF position to view the output voltage from the sense resistor Wheatstone bridge on the bottom LCD panel voltmeter.

NOTE: Both LCD panel meters can display up to 19.999 VDC with a minimum increment of $1 \mathrm{mV}$ DC.

9. Next, the branch resistances on the sense resistor Wheatstone bridge must be balanced or nulled to $\sim 0 \mathrm{~V}$ output at no flow conditions. Adjust the BALANCE knob on left front panel of the machine until the bottom LCD panel meter indicates a near zero output voltage. To attach an external digital multimeter with better $\mathrm{mV}$ resolution, connect it as shown below.

NOTE: Attach voltage-measuring instruments with $M \Omega$ or $G \Omega$ input impedances only. Oscilloscopes, when attached, have affected the balance of the Wheatstone bridge.

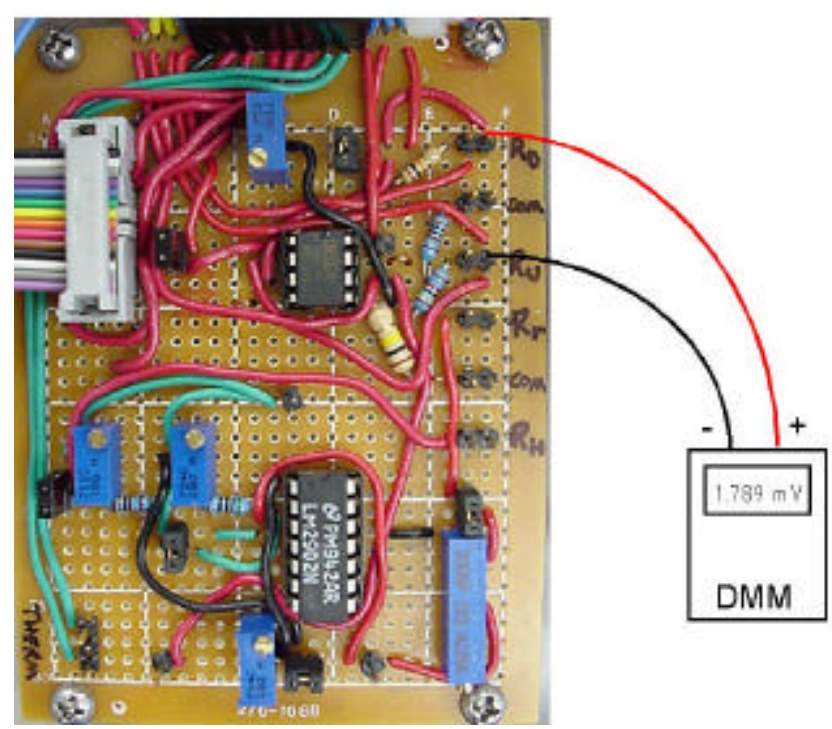

10. Once the output voltage of the sense resistor Wheatstone bridge is nulled to zero at no flow, turn on the blower fan with the switch on the top, right panel of the machine. Change the airflow velocity by adjusting the FAN SPEED dial on the top, right panel. The output voltage of the sense resistor Wheatstone bridge can be monitored directly with the bottom LCD panel meter, or with a DMM connected to the bridge as shown above. The actual flow rate through the PVC channel is shown in the top LCD panel meter display, which continuously indicates the output voltage from the Honeywell AWM5104VN sensor. The output voltage (V) from the Honeywell device can be converted into volumetric (SLPM) flow using: $S L P M=(V-1)^{\star} 2.5$. 
NOTE that if the downstream and upstream resistor connections are reversed, the output voltage will be increasingly negative for increasing flow (inversely related). If this occurs, simply reverse the connections to the driver/interface board from the patch board.

11. If the AMPLIFIER ON/OFF switch is in the ON position, the output voltage shown in the bottom LCD will be the output of the differential instrumentation amplifier. The gain of the amplifier can be set by adjusting the GAIN potentiometer on the top, left panel of the machine. The amplifier inputs are always connected to the Wheatstone bridge (the input impedance of the amplifier, thus, slightly affecting the bridge balance), unless jumper JP5 is removed. To set the reference, or output, voltage of the amplifier at no flow, use a small screwdriver to adjust potentiometer $\boldsymbol{R} \boldsymbol{8}$. The locations of the jumper and potentiometer are shown below. An external ohmmeter can also be connected to the thermistor jumper posts on the board to determine the temperature of the airstream. The measured thermistor resistance can be matched to temperature using the chart on the inside, top cover of the machine (the chart is shown graphically in Figure 108).

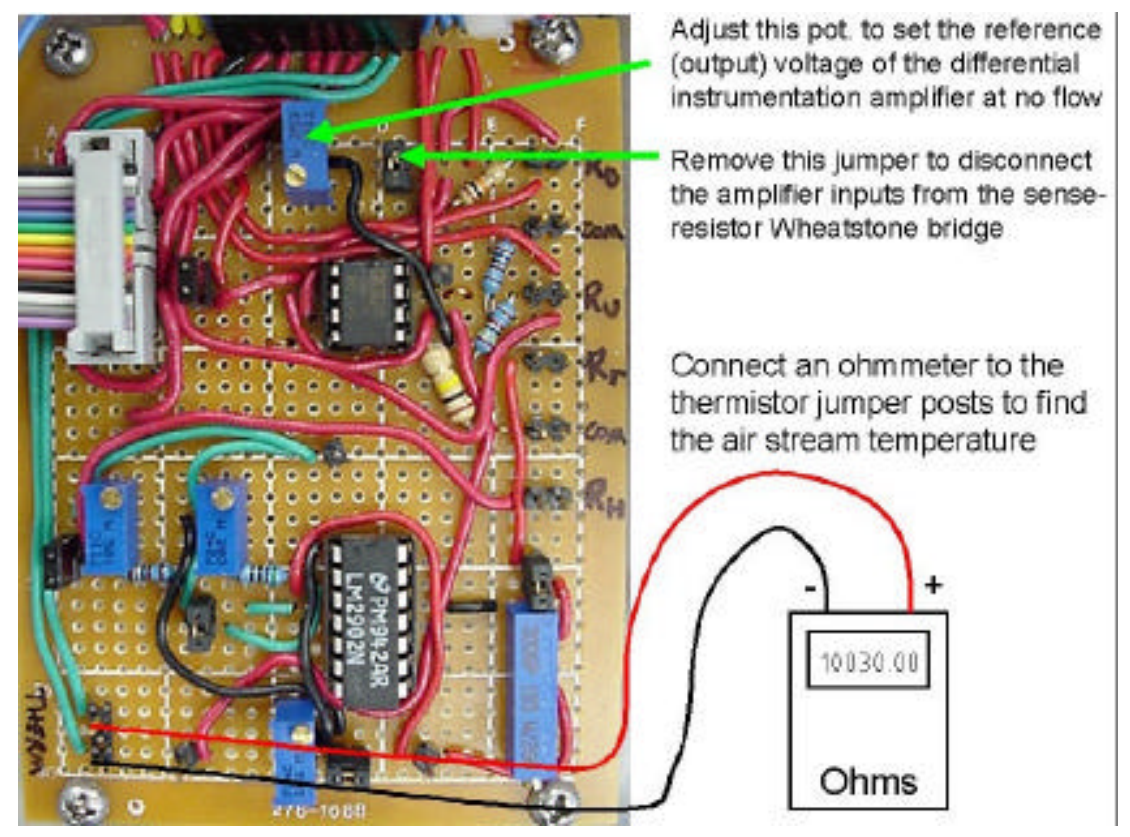

12. Instructions for how to measure the GAIN potentiometer resistance value in the differential instrumentation amplifier circuit (to calculate the gain) and how to initially configure the resistances in the error-integrator, heater-driver circuit are included in the following ColeJet $2000^{T M}$ Technical Guide.

13. When finished with the machine, turn off the blower, turn off the machine, and replace the end cap on the outlet port (on the right, rear of the machine) to minimize dust contamination inside the flow channel. 


\section{ColeJet 2000TM Technical Guide}

Follow these steps to configure the machine to test and evaluate fabricated flow sensors

\section{DETERMINING THE GAIN OF THE AMPLIFIER CIRCUIT}

To measure the value of the GAIN potentiometer to calculate the differential instrumentation amplifier's gain, remove jumper JP6 and connect an ohmmeter as follows:

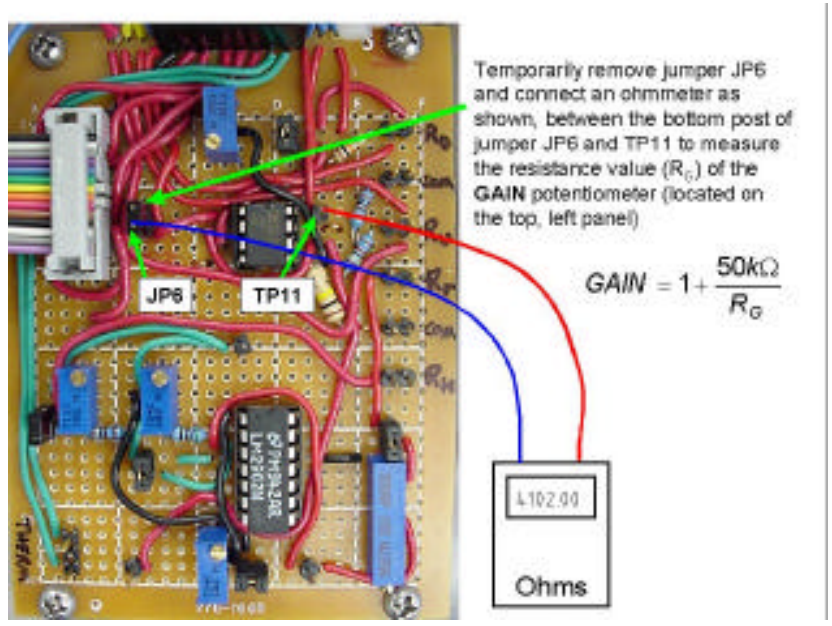

\section{SETTING POTENTIOMETERS TO CONFIGURE THE HEATER-DRIVER CIRCUIT}

To initially configure and set the resistance values in the error-integrator, heater-driver circuit, the following resistances must be set (see the schematic diagrams for this circuit in Appendix XIX, especially Figure 198, and Figure 10 for general reference):

- In the heater-driver-substrate-resistor Wheatstone bridge: $R_{a}, R_{b}, R_{c}$

- External to the heater-driver Wheatstone bridge: $\mathrm{R}_{1}$.

1. To set the value of potentiometer $R_{a}$, remove jumper JP2 and connect an ohmmeter between the top post of JP2 and test-point, TP1, as shown. When finished, replace jumper JP2.

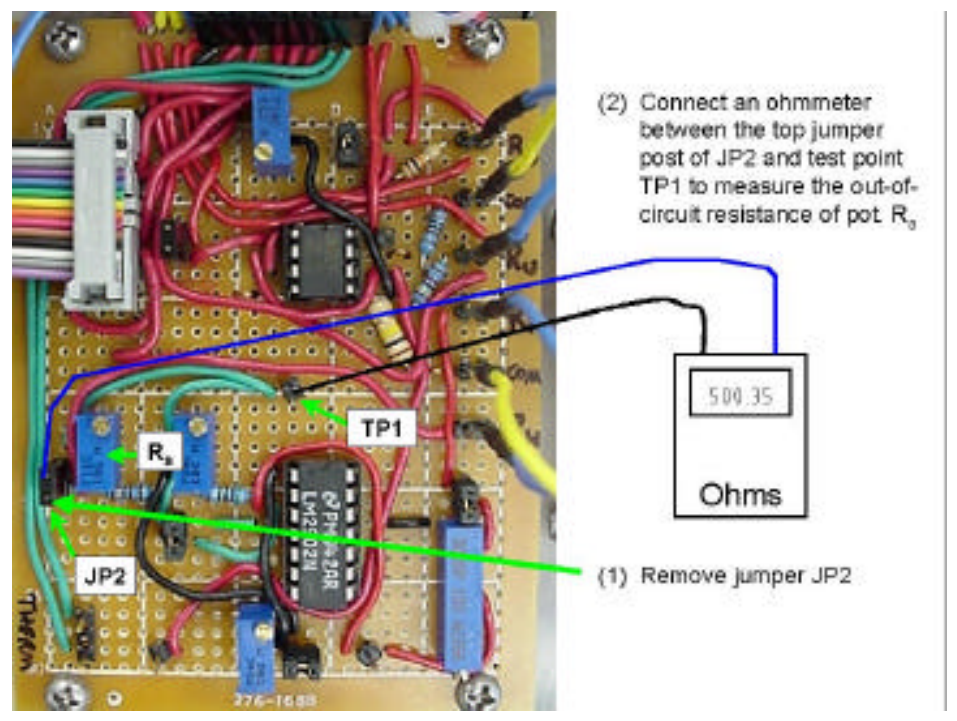


2. To set the value of potentiometer $R_{b}$, remove jumper JP3 and connect an ohmmeter between the top post of JP3 and test-point, TP1, as shown. When finished, replace jumper JP3.

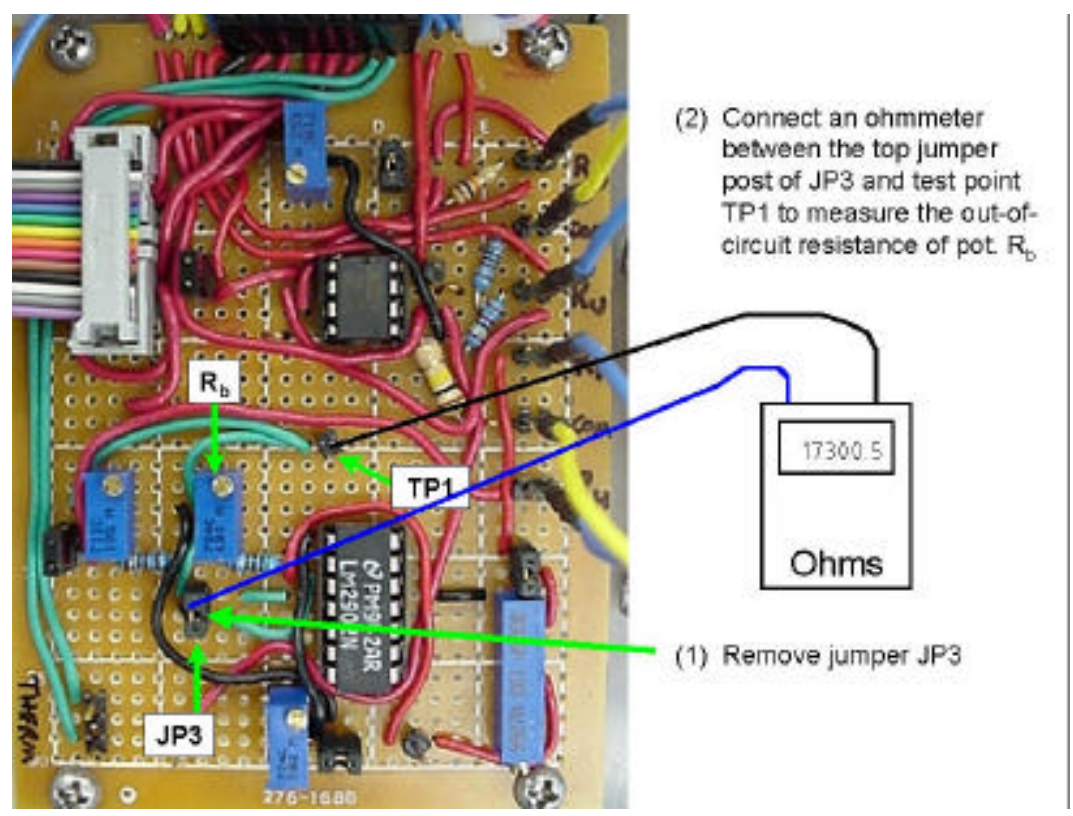

3. To set the value of potentiometer $R_{c}$, remove jumper JP4 and connect an ohmmeter between the bottom post of JP4 and test-point, TP2, as shown. When finished, replace jumper JP4.

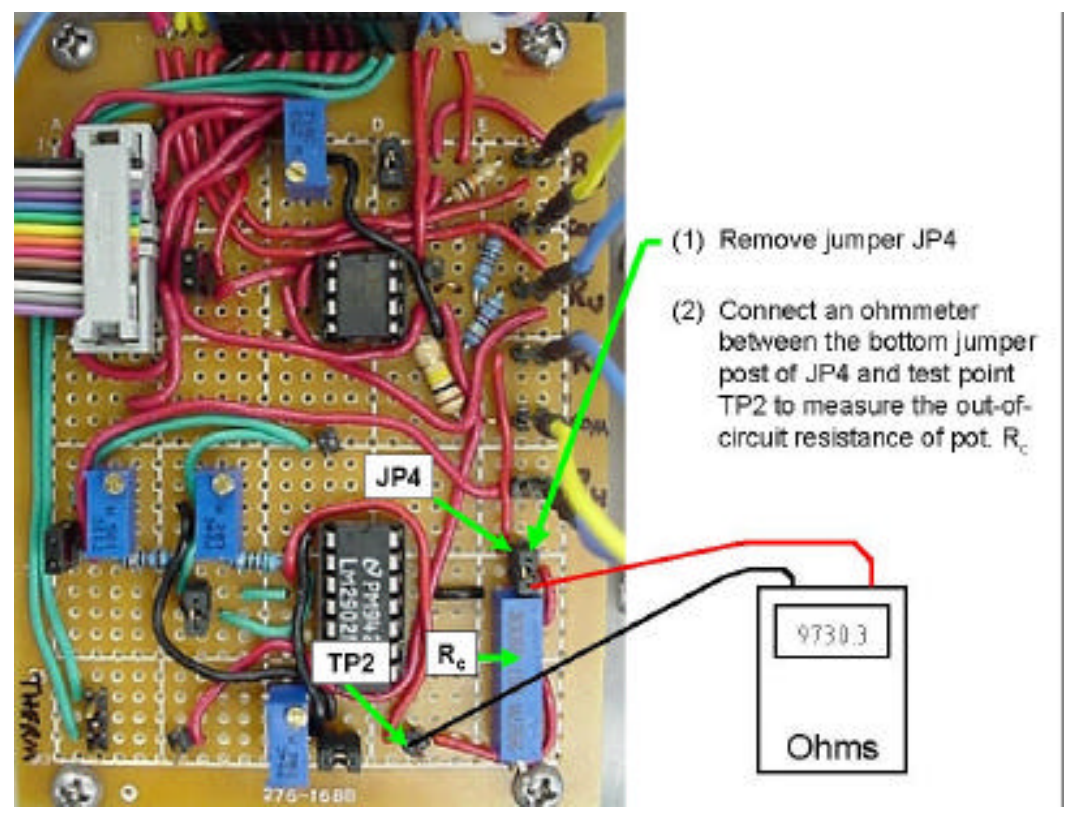

4. To set the value of potentiometer $R_{1}$, remove jumper JP1 and connect an ohmmeter between the left post of JP1 and test-point, TP1, as shown. When finished, replace jumper JP1. 


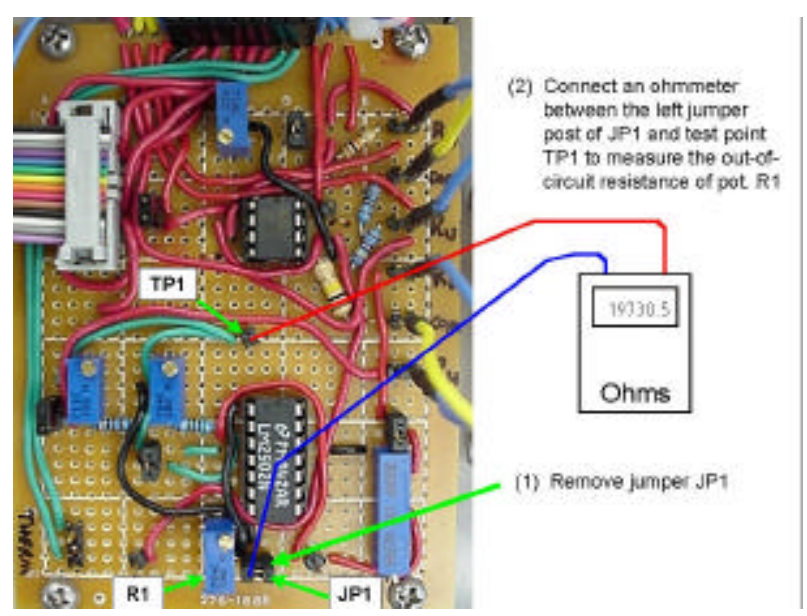

\section{MISCELLANY}

1. WARNING: USE AT YOUR OWN RISK!

While every reasonable precaution was taken in the design and construction of ColeJet $2000^{\mathrm{TM}}$ to make it safe and convenient to use, it has not been tested for conformity and compliance with any safety certification agency/entity, such as Underwriter's Laboratories. However, the power supply components utilized in this construction from the original HP DeskJet ${ }^{\mathrm{TM}}$ printer (from which ColeJet $2000^{\mathrm{TM}}$ was derived) were neither modified nor resituated inside the machine. Always plug the external transformer for the machine into a 3-prong (properly grounded) 120 VAC outlet, so that the bottom metal chassis inside ColeJet $2000^{\mathrm{TM}}$ is grounded. The power supply inside ColeJet $2000^{\mathrm{TM}}$ should be considered to have hazardous voltages present and should never be touched, moved, or modified while the external transformer is plugged into the machine.

\section{THE PREFILTER}

A micron-prefilter is employed in the design to reduce particulate contamination inside the flow tube channel. Maximum achievable flow rates in the machine are slightly larger without the filter in place (such as, for example, in a cleanroom environment, where filtering is not necessary). The prefilter is removable and can be replaced with a Eureka part \#60665 micron filter for Eureka 4300 \& 4400 series vacuum cleaners (for the nearest Eureka dealer call: 1-800-282-2886). The filter should be modified slightly to fit into its holder by cutting the normally-rounded corners into right-angles and by reducing its length by $\sim 1 \mathrm{~cm}$. The filter is inserted into the holder with the coarse, fibrous side facing out (the air intake).

\section{TROUBLESHOOTING: OVERHEATING THE BLOWER POWER SUPPLY}

The voltage regulator (and attached heat-sink) supplying current to the blower will become very hot after prolonged usage of the machine. For normal usage (e.g. less than 1 hour) of the machine in a cool ambient environment (e.g. $70-75^{\circ} \mathrm{F}$ ), the blower's power supply should not overheat. However, if the blower suddenly stops with the machine and blower switch both turned on, then most likely its regulator has overheated. Turn off the machine and allow time for the regulator to cool off and for its built-in thermal overload protection circuit to reset.

\section{PERIODIC FLOW CHANNEL INSPECTION/CLEANING}

It is strongly suggested that the flow channel tube be inspected periodically to insure that dust and other particulates are not present; and, if present, are vacuumed out. Remove the "J" shaped tube by pulling it out of the left side of the machine to inspect it. This tube is best cleaned out by blowing filtered, compressed air (or nitrogen) through it. The inside of the device insertion "T" section should be vacuumed out periodically. 


\section{APPENDIX XXII.}

1997 TWO-LAYER L-EDITTM DESIGN FILE

PHOTOMASK SET IMAGES AND DESCRIPTIONS 


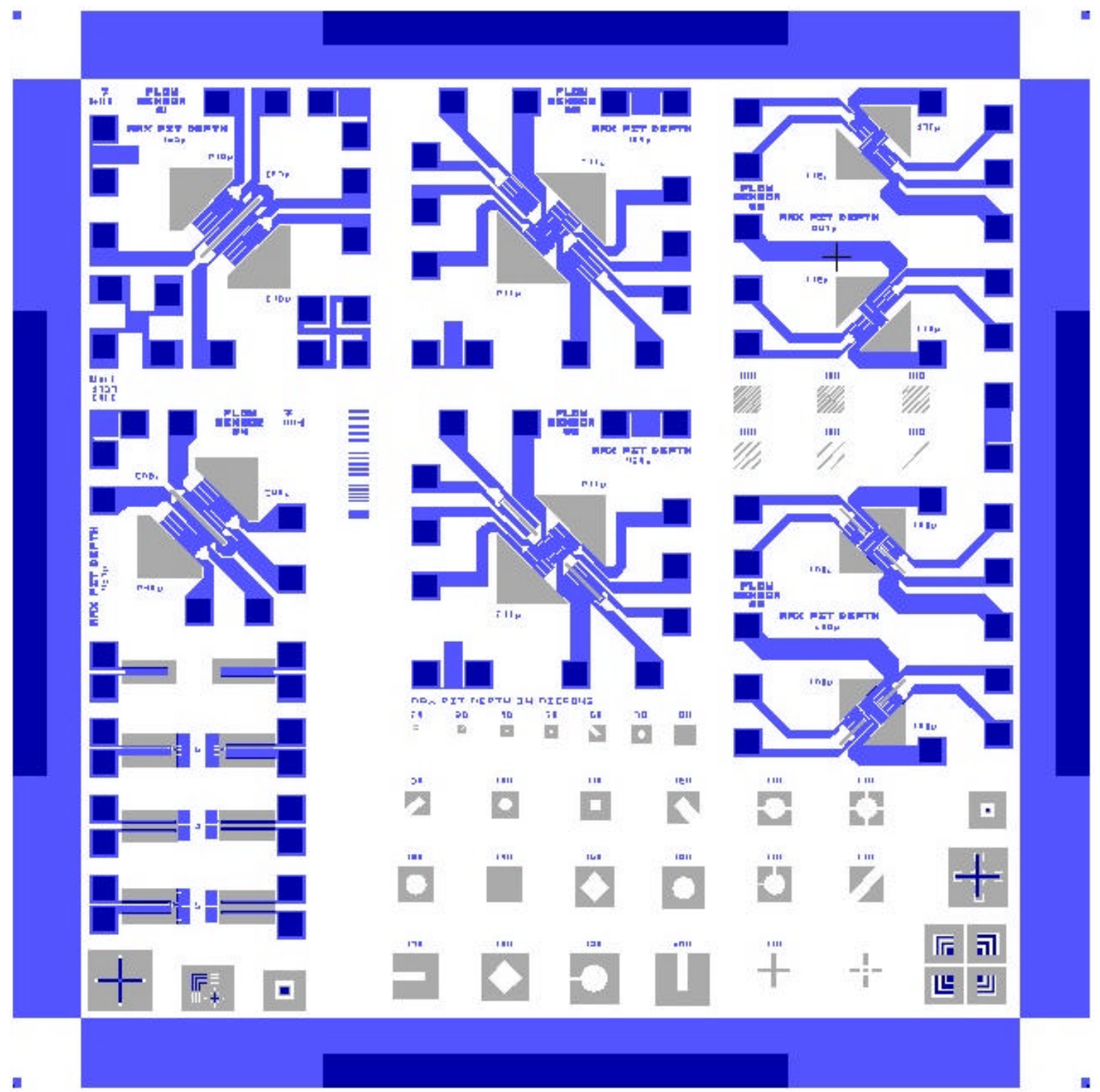

FIGURE 208 - The simpler, original 1997 L-Edit ${ }^{\text {TM }}$ design file had eight flow sensors in six regions in a $5.93 \times 5.93 \mathrm{~mm}$ die size with two-layers: one for the liftoff-process resistor metallization photomask, and another for the RIE-and- anisotropicsilicon-etchant etch-window photomask. 


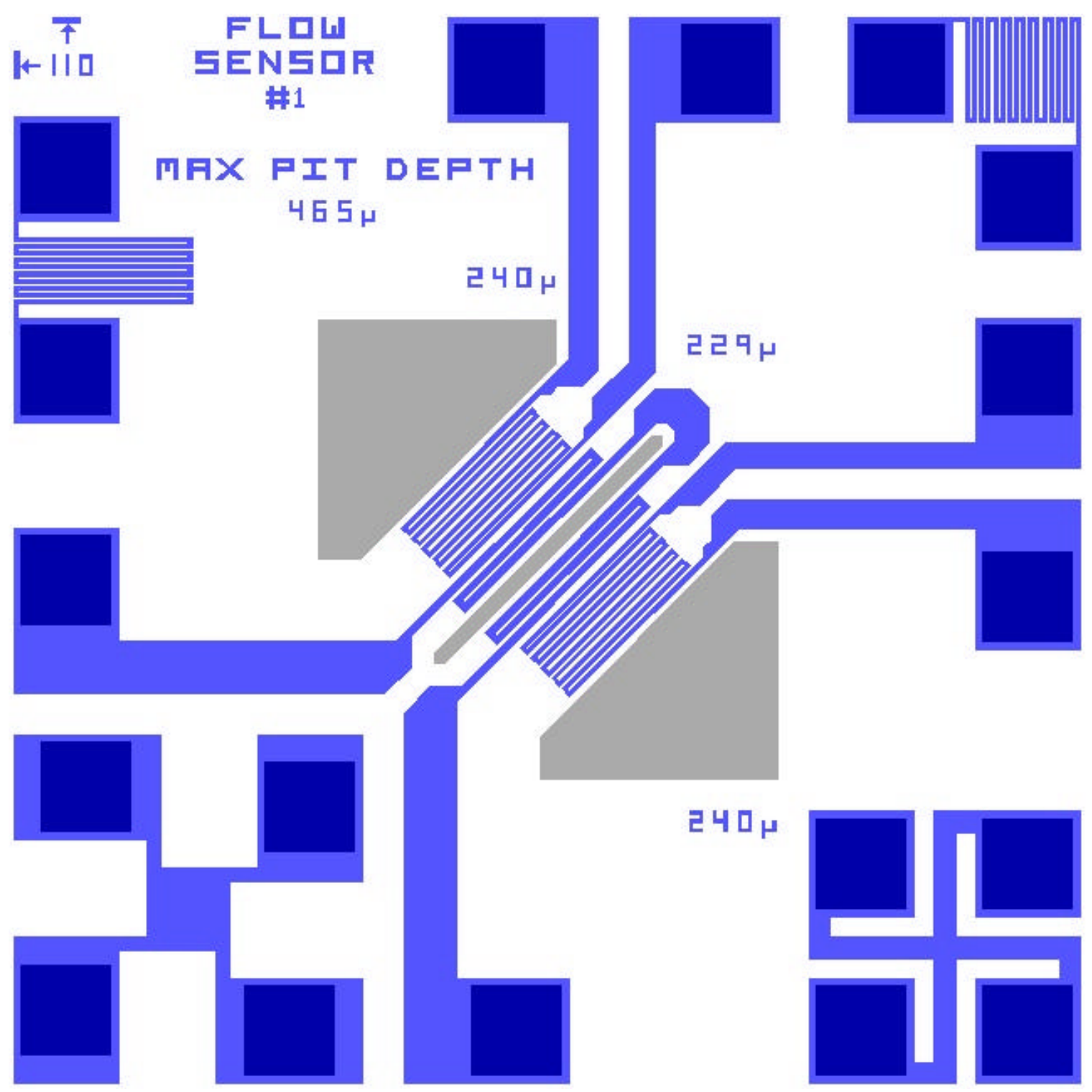

FIGURE 209 - The geometry and layout of Flow Sensor \#1 (with Van der Pauw structures at the bottom) was largely based on the sensor developed by Johnson and Higashi at Honeywell in the mid 1980s. The numbers, " $240 \mu \mathrm{m}$ " and " $229 \mu \mathrm{m}$ " represent maximum etch depths achievable in $\mathrm{KOH}$ for each etch window (for example, if $\mathrm{KOH}$ etching only occurred through the center slit, the maximum possible depth is $229 \mu \mathrm{m}$ ). "Maximum Pit Depth" numbers represent maximum etch depths for the entire etch window region, once undercutting "connects" all three regions. The substrate temperature-sensing serpentine resistors are at the top left and top right of this design block. 


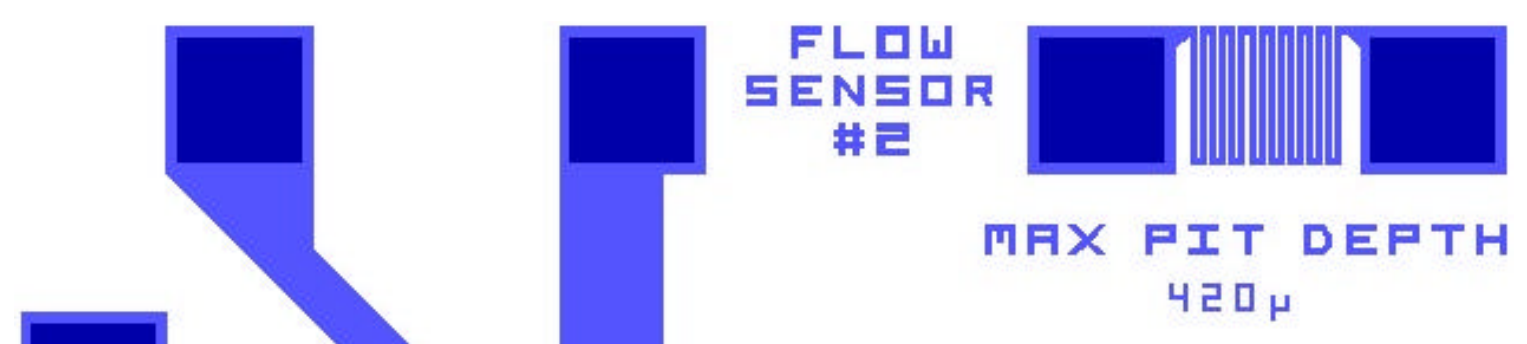

ᄅר1

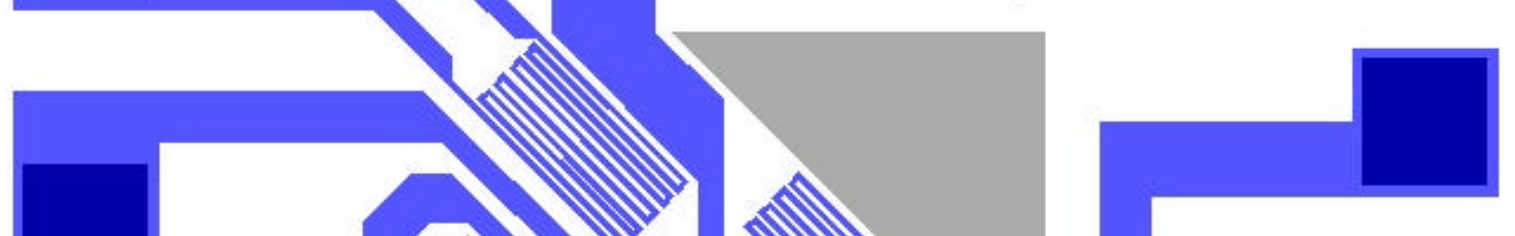

ᄅ $71 \mu$
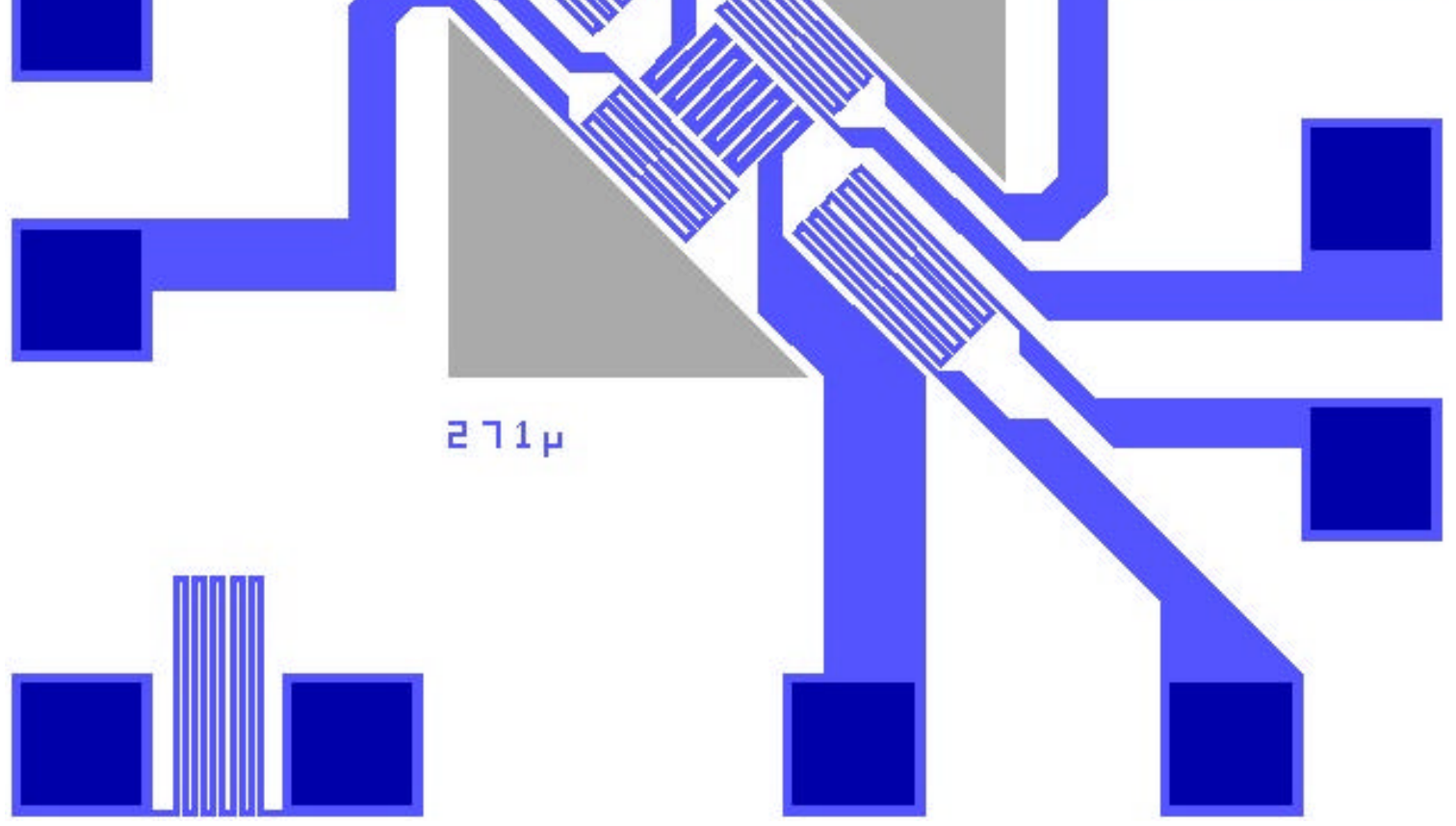

FIGURE 210 - Flow Sensor \#2 was designed to measure flow along either or both the longitudinal or transverse axes of the suspended microbridge. Due to the large width of the microbridge (at $298 \mu \mathrm{m}$ ), the minimum required etch depth to release the $\mathrm{Si}_{3} \mathrm{~N}_{4}$ bridge from the silicon substrate is $\sim 200 \mu \mathrm{m}$. The substrate temperature-sensing resistors are at the top right and bottom left of this design block. 


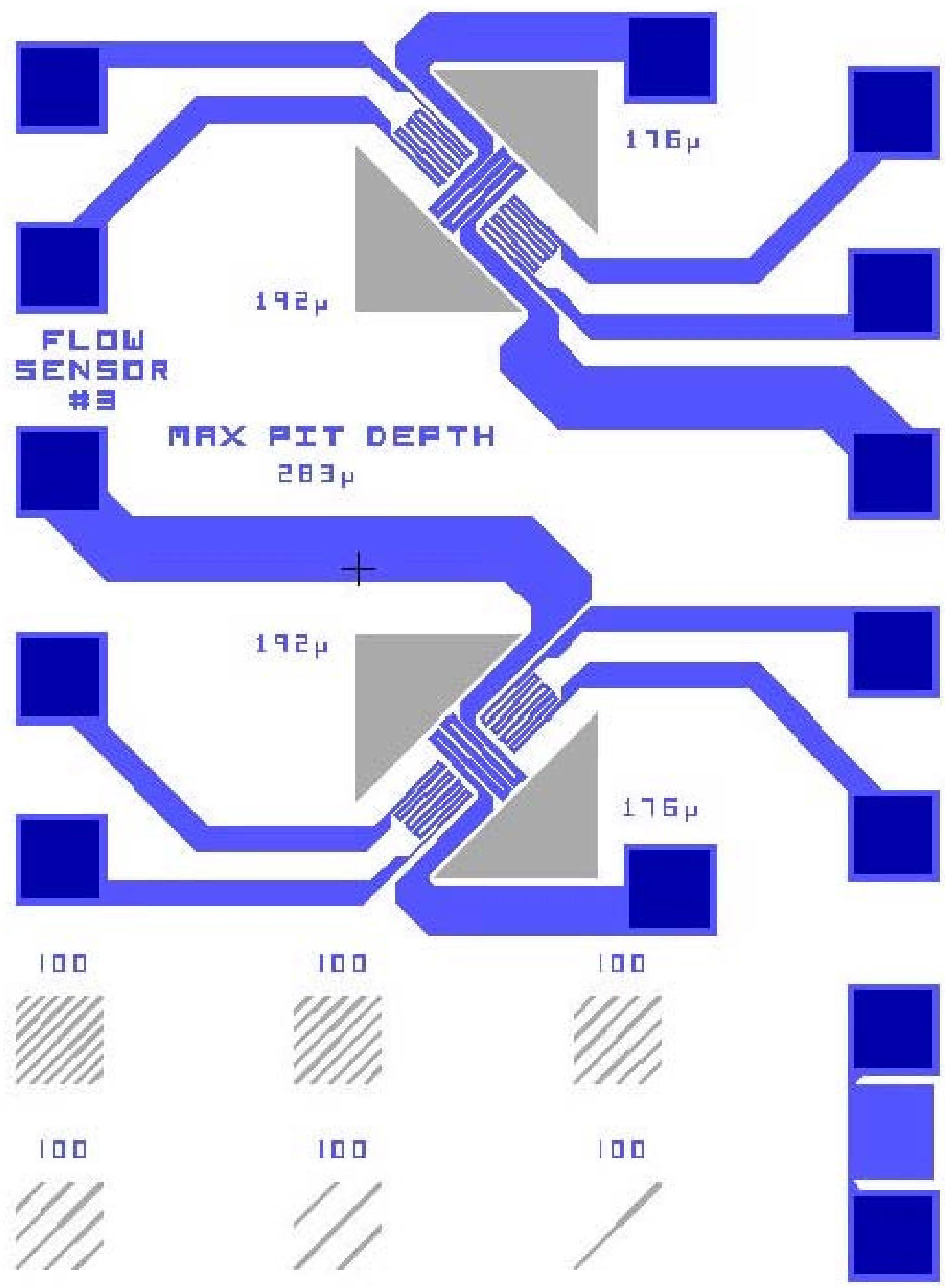

FIGURE 211 - Flow Sensor \#3 with etch-window test structures. These flow sensors have the smallest microbridges and are designed to measure flow along the longitudinal axis of each bridge. The substrate resistor is at the bottom right of this design block. 


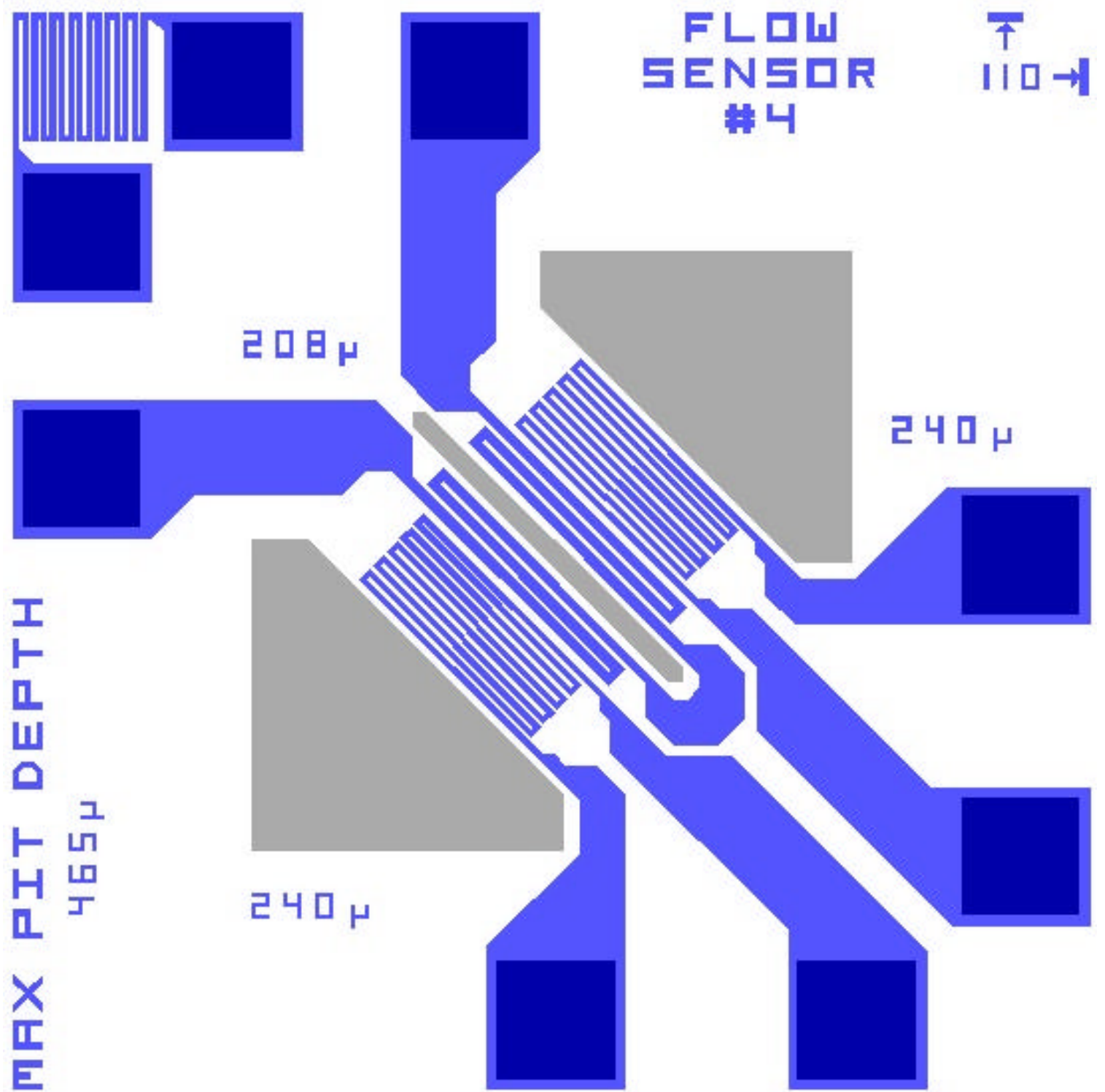

FIGURE 212 - Flow Sensor \#4, which is identical to Flow Sensor \#1, except the center etchwindow slit is $21 \mu \mathrm{m}$ shorter in length, and the layout of (and routing to) the pads is different. The substrate temperature-sensing resistor is at the top left of this design block. 


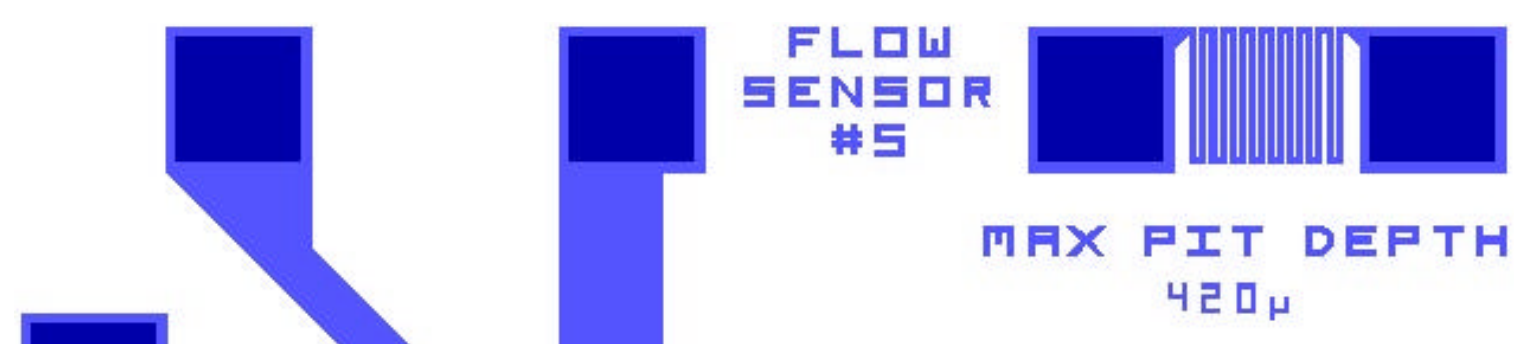

ᄅ $\urcorner 1 \mu$

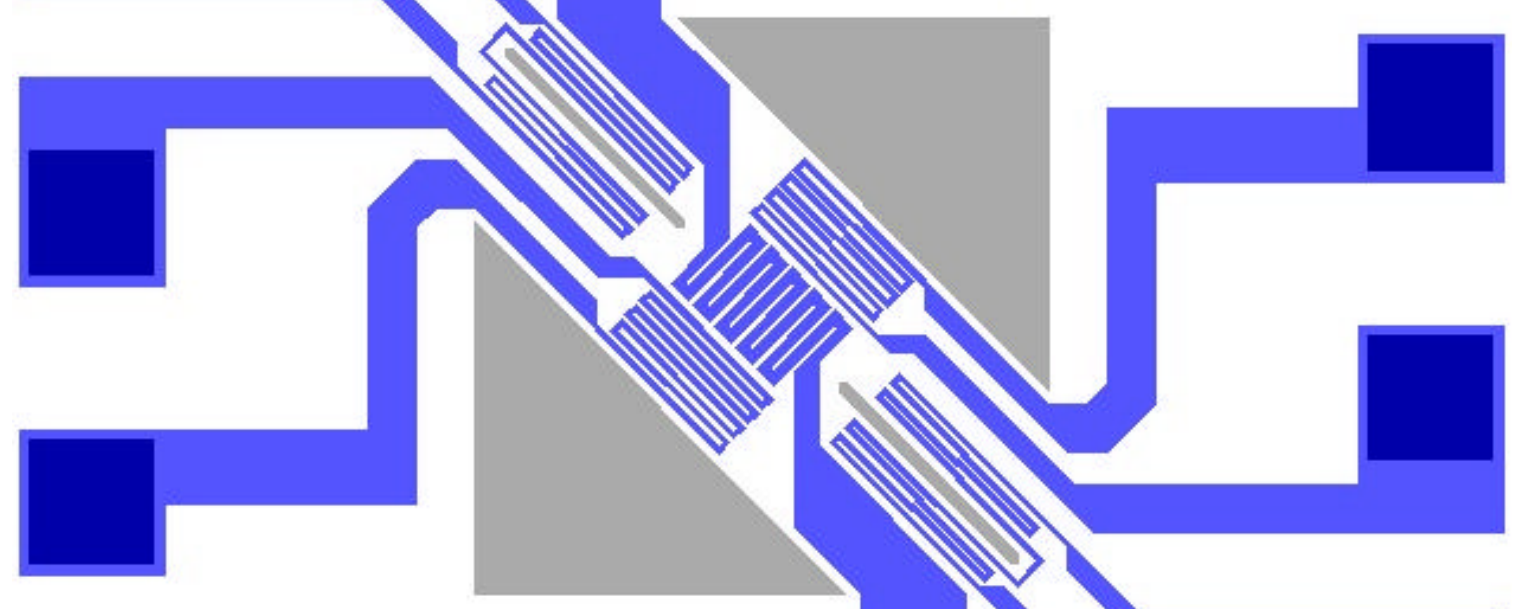

$271 \mu$
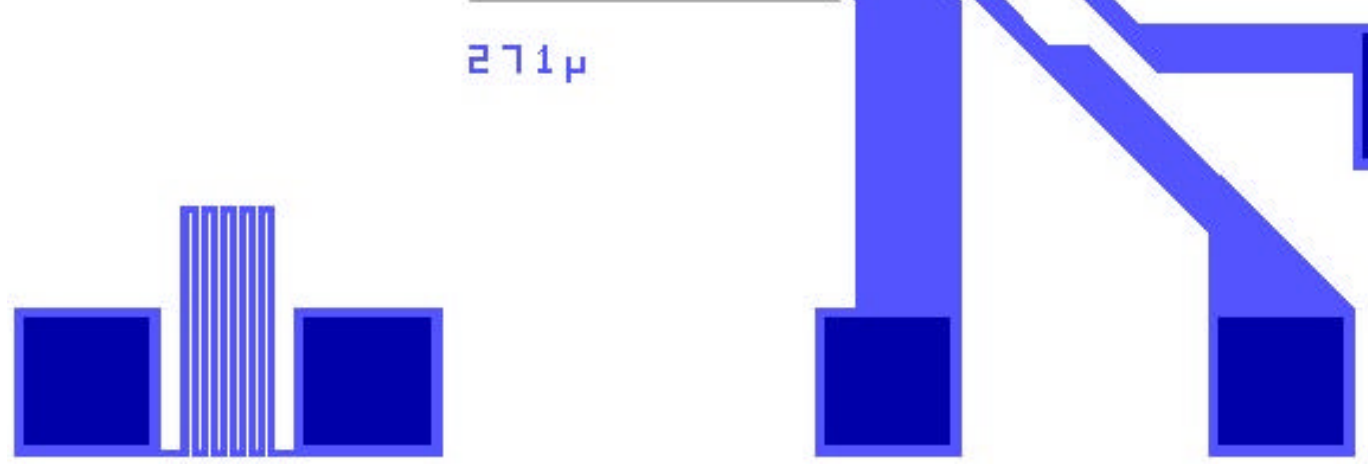

FIGURE 213 - Flow Sensor \#5 is identical to Flow Sensor \#2, except with slits to facilitate faster etching and undercutting of the $\mathrm{Si}_{3} \mathrm{~N}_{4}$ microbridge. Temperature-sensing substrate resistors are located at the bottom left and top right of this design block. 


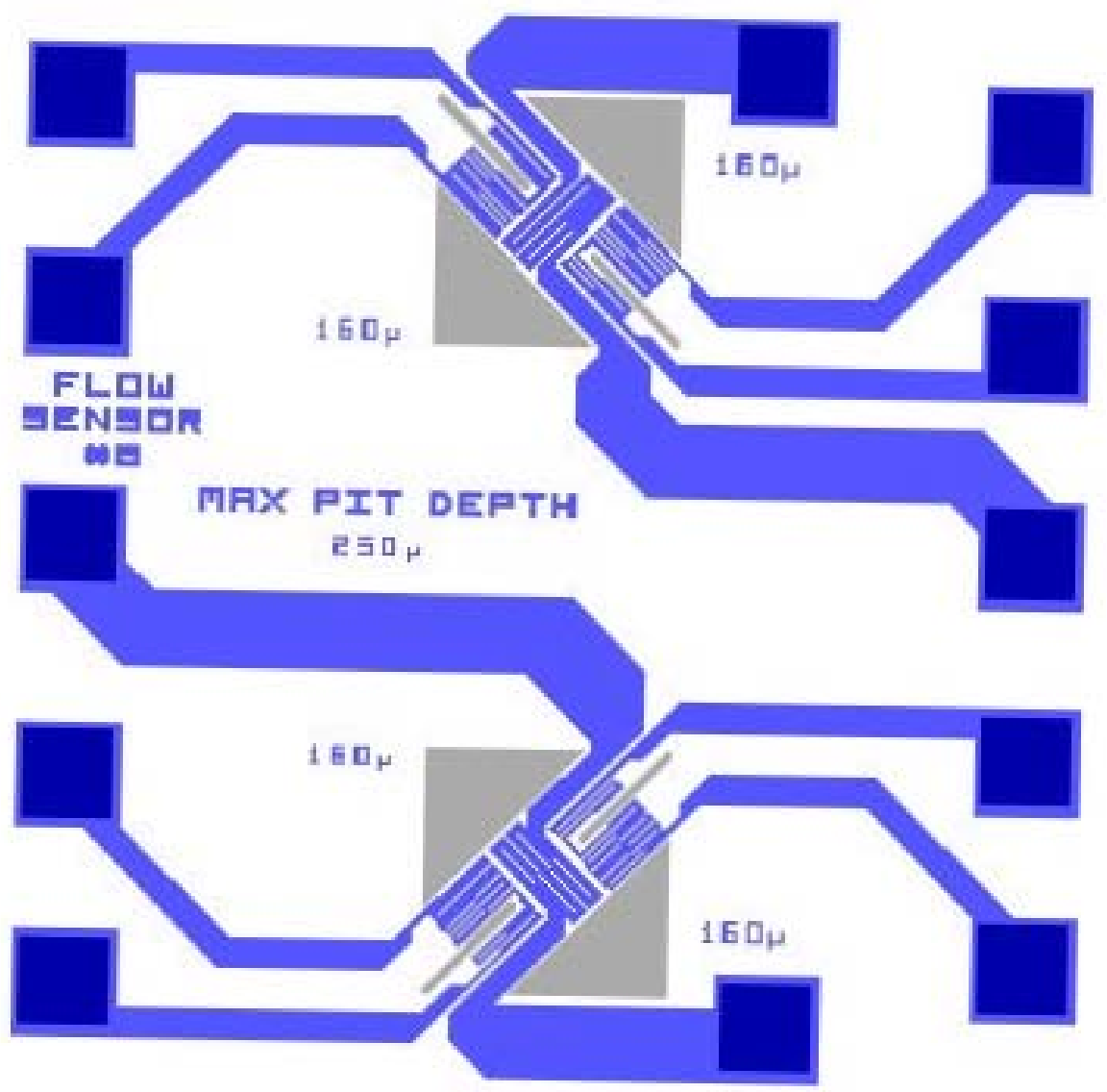

130 $1 \exists 0$
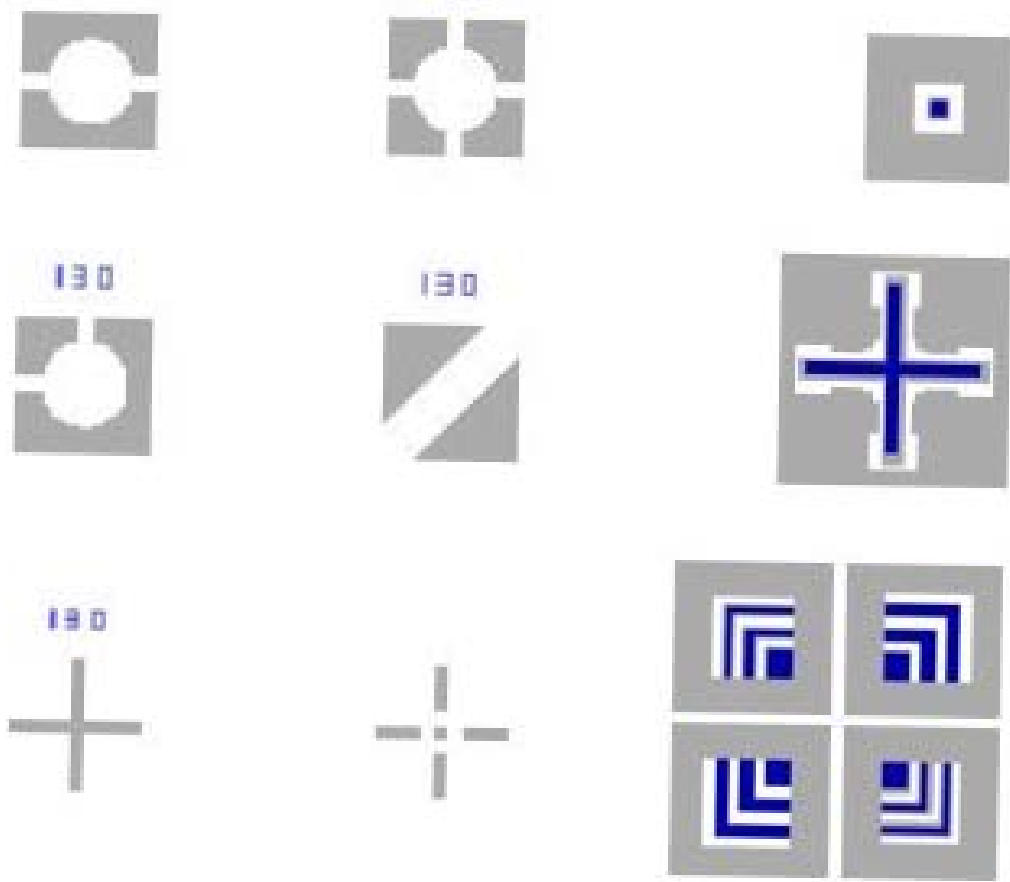

FIGURE 214 - Flow Sensor \#6 (with mask alignment and etching features) has the same basic design as Flow Sensor \#3, except with slots in the microbridge structure for faster etching and undercutting. 

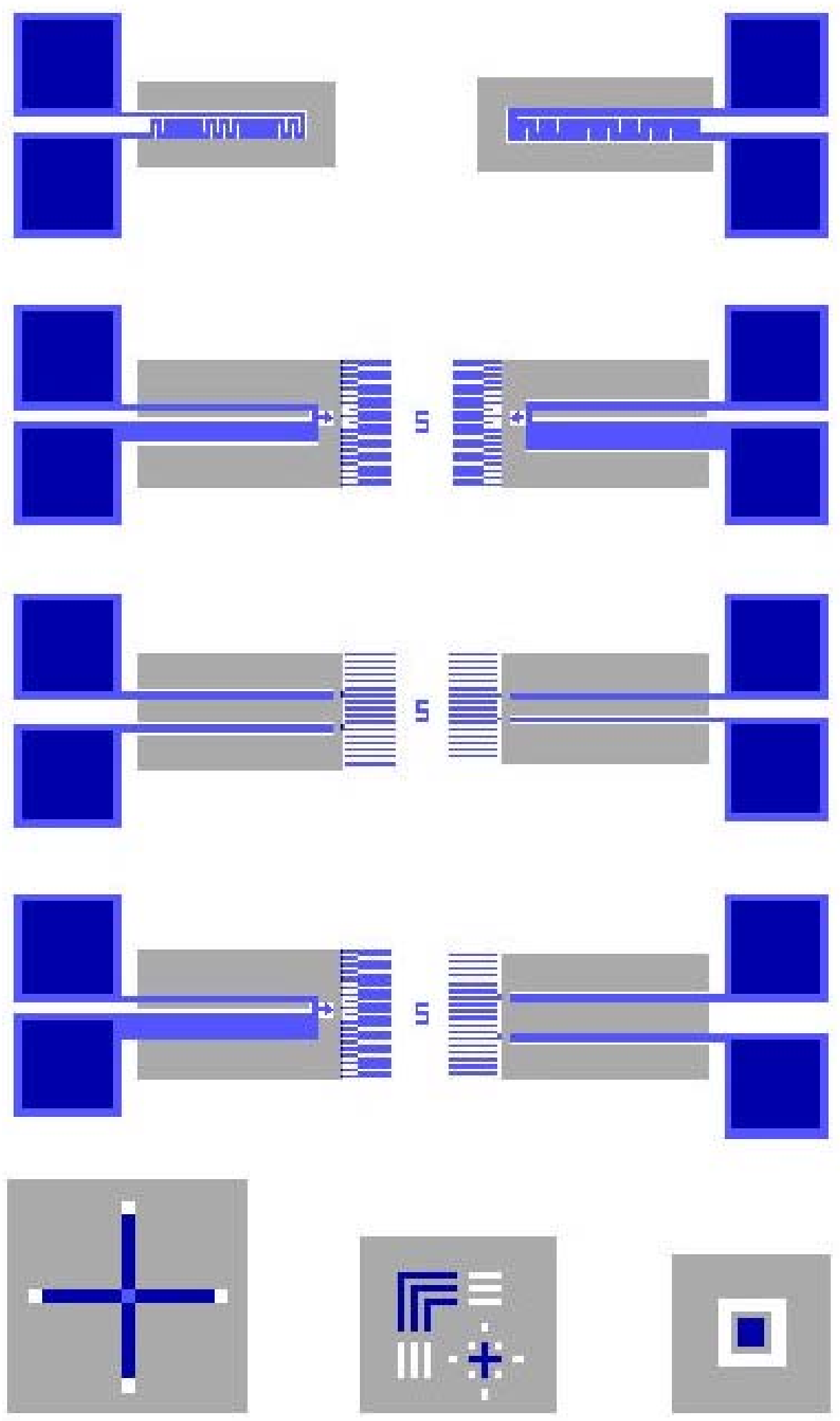

FIGURE 215 - Mask alignment/registration features (at the bottom) and cantilever test structures for experimental deflection studies. 
MAX PIT DEPTH IN ウICRDNS

ᄅㅁ

$\exists \square$

40

50

ㅁ

7 ㅁ

日ᄆ

o

口

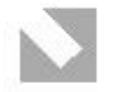

$\bullet$

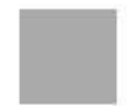

90

I00

II

I리
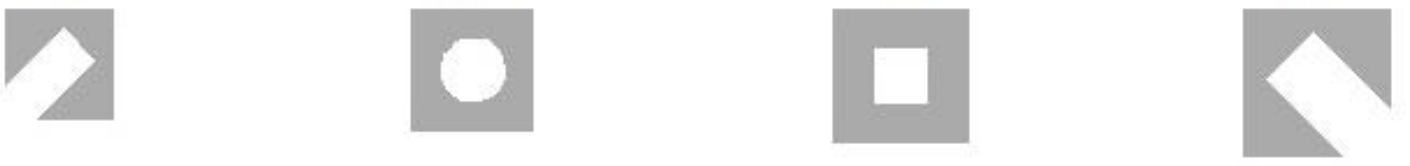

$1 \exists \square$

140

150

I
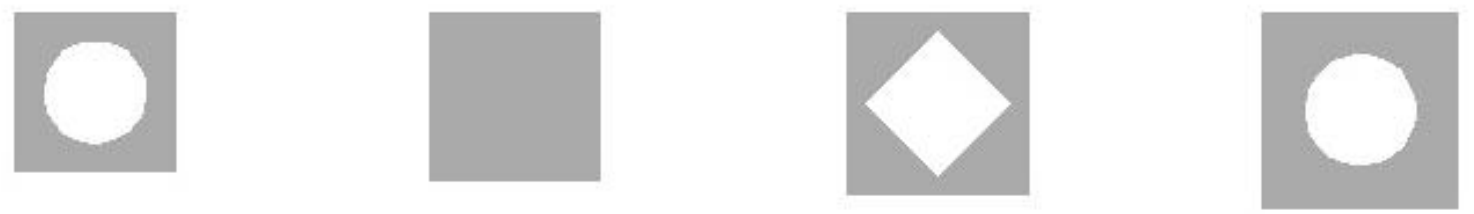

170

I日0

190

टवर
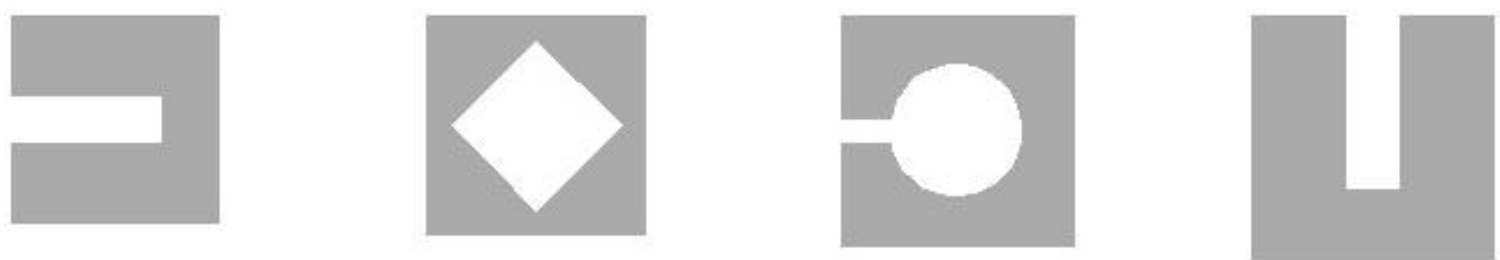

FIGURE 216 - One unique/novel design feature in this mask set was the inclusion of preciselydimensioned etch windows to determine anisotropic silicon etchant (e.g. KOH) etch depths with $10 \mu \mathrm{m}$ resolution. Numbers indicated above each square show the maximum pyramidal etch depth possible into the (100) substrate within each structure. 
APPENDIX XXIII.

1998/1999 REDESIGNED THREE-LAYER L-EDITTM DESIGN FILE PHOTOMASK SET

IMAGES AND DESCRIPTIONS 


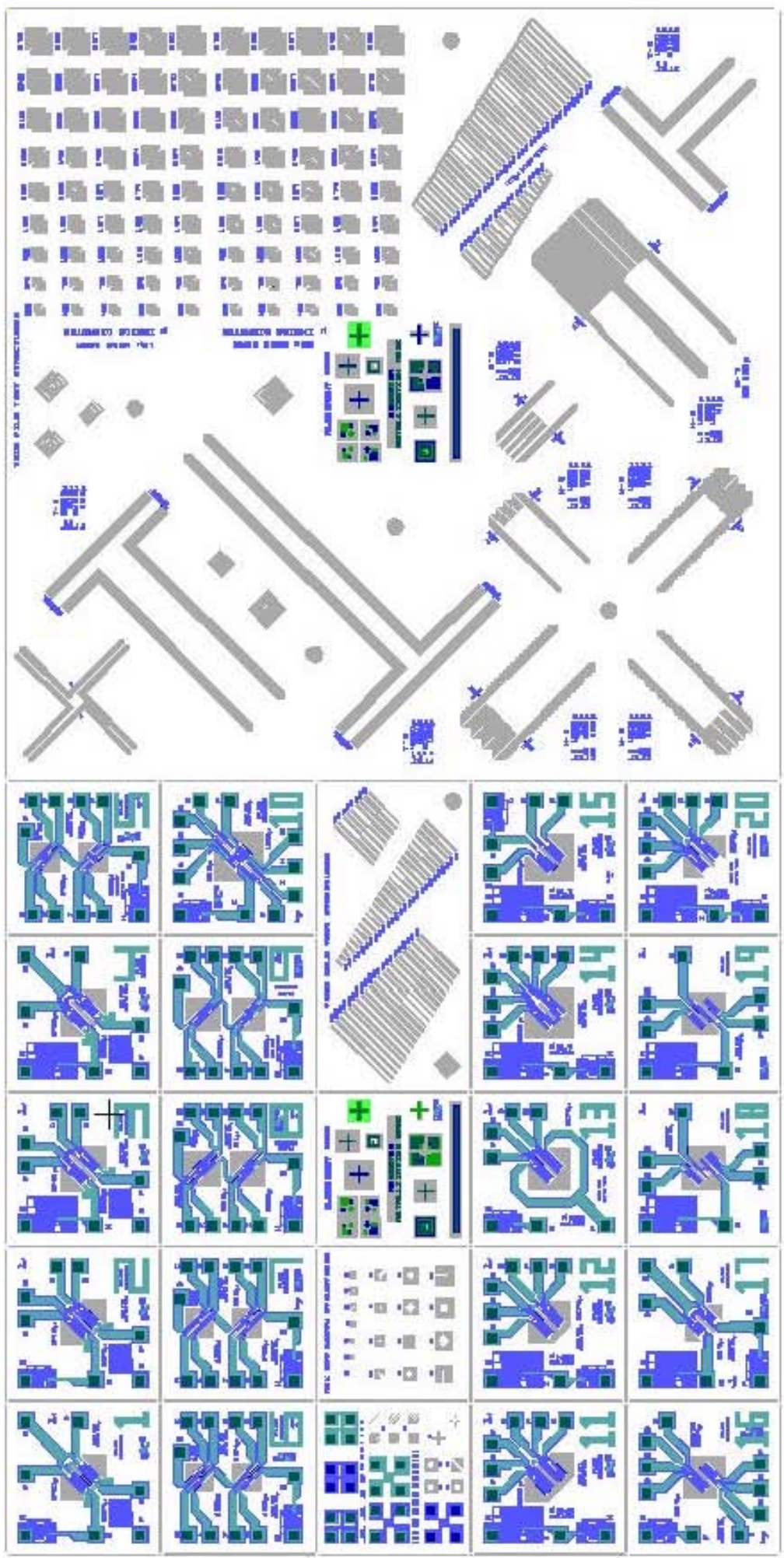

FIGURE 217 - Photo of all three layers together in an individual die $(22.967 \mathrm{~mm} \times 11.500 \mathrm{~mm})$, which is replicated throughout each photomask. Blue areas represent resistor metallization; green areas represent gold metallization; and gray areas represent microbridge and pad-area etch windows. 


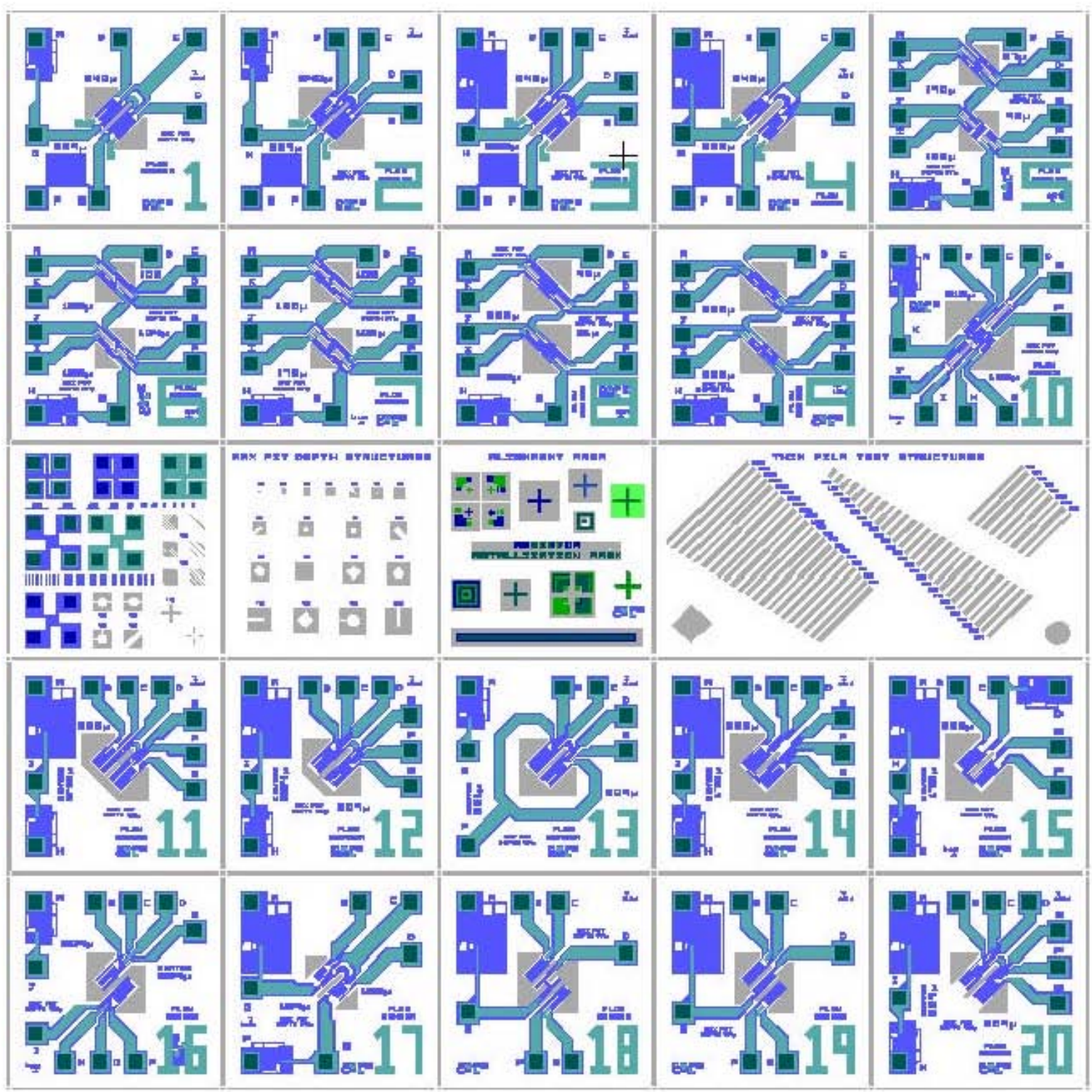

FIGURE 218 - From the L-Edit TM design file, an enlargement of the flow sensor device area (one-half of the replicated die size), which is $11.5 \times 11.5 \mathrm{~mm}$. Blue areas represent resistor metallization; green areas represent gold metallization; and gray areas represent microbridge and pad-area etch windows. 


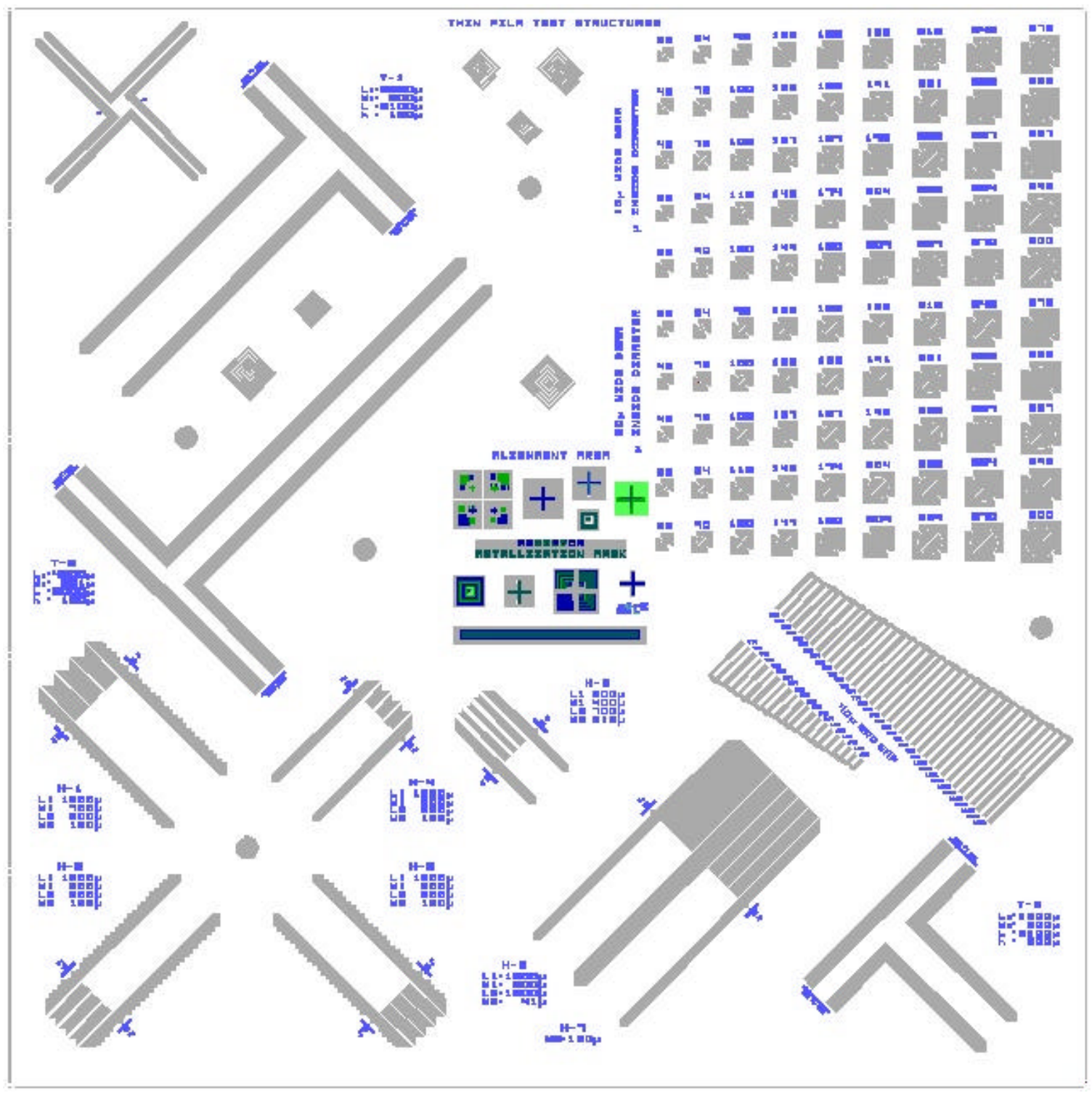

FIGURE 219 - From the L-Edit ${ }^{T M}$ design file, the in-situ thin film tension/ compression test structure area, which occupies $11.5 \times 11.467 \mathrm{~mm}$. Blue areas represent resistor metallization for labels; green areas represent gold metallization (for registration markings in the photomask alignment area); and gray areas represent microbridge and pad-area etch windows. 


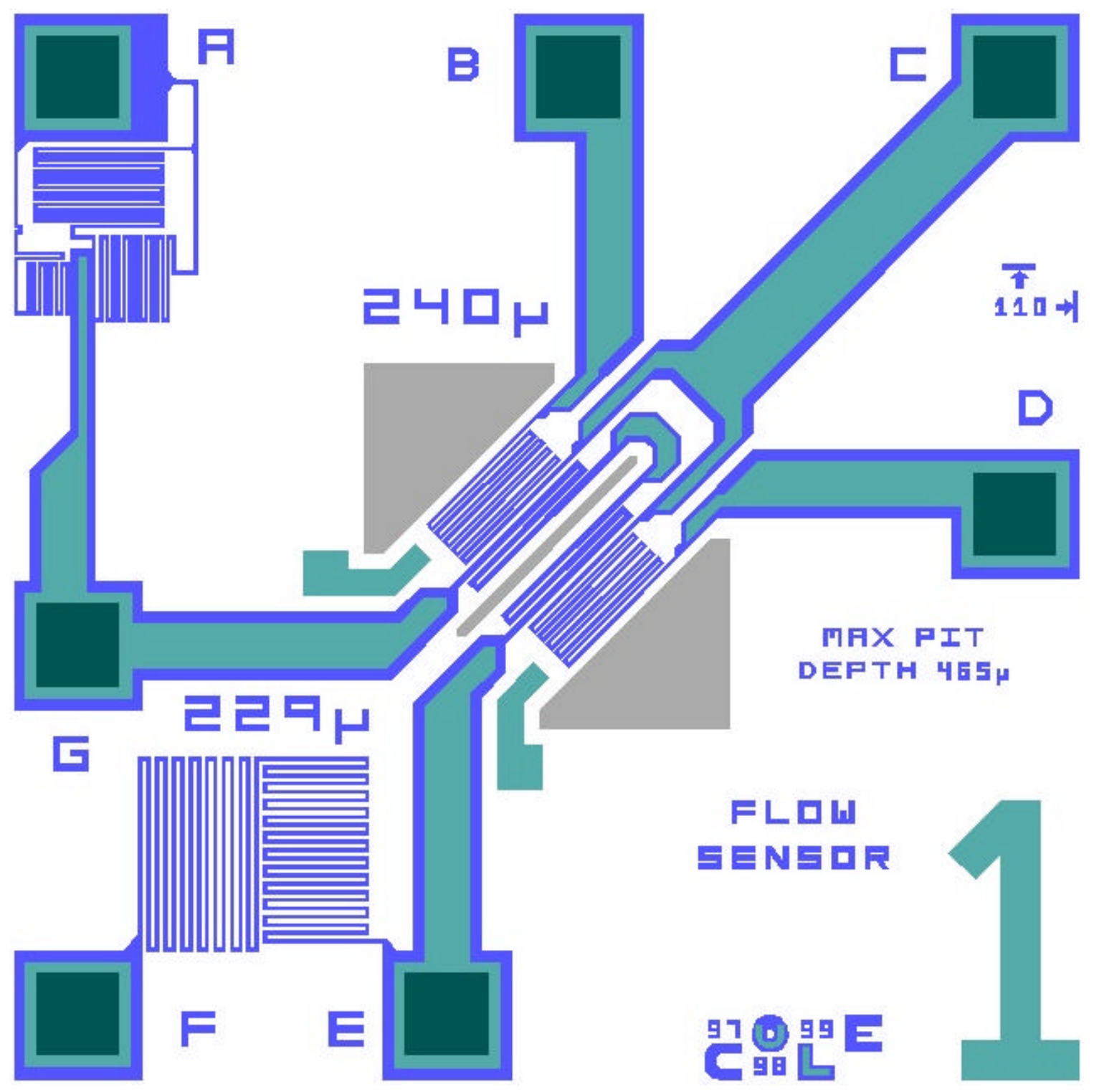

FIGURE 220 - The geometric design, scaling, and layout for Flow Sensor \#1 was largely based upon the flow sensor devised by Johnson and Higashi at Honeywell in the mid 1980s, and improved from the 1997 mask set with a common node for the sense resistors and $100 \%$ larger spacing between sense resistor edges and microbridge edges. 


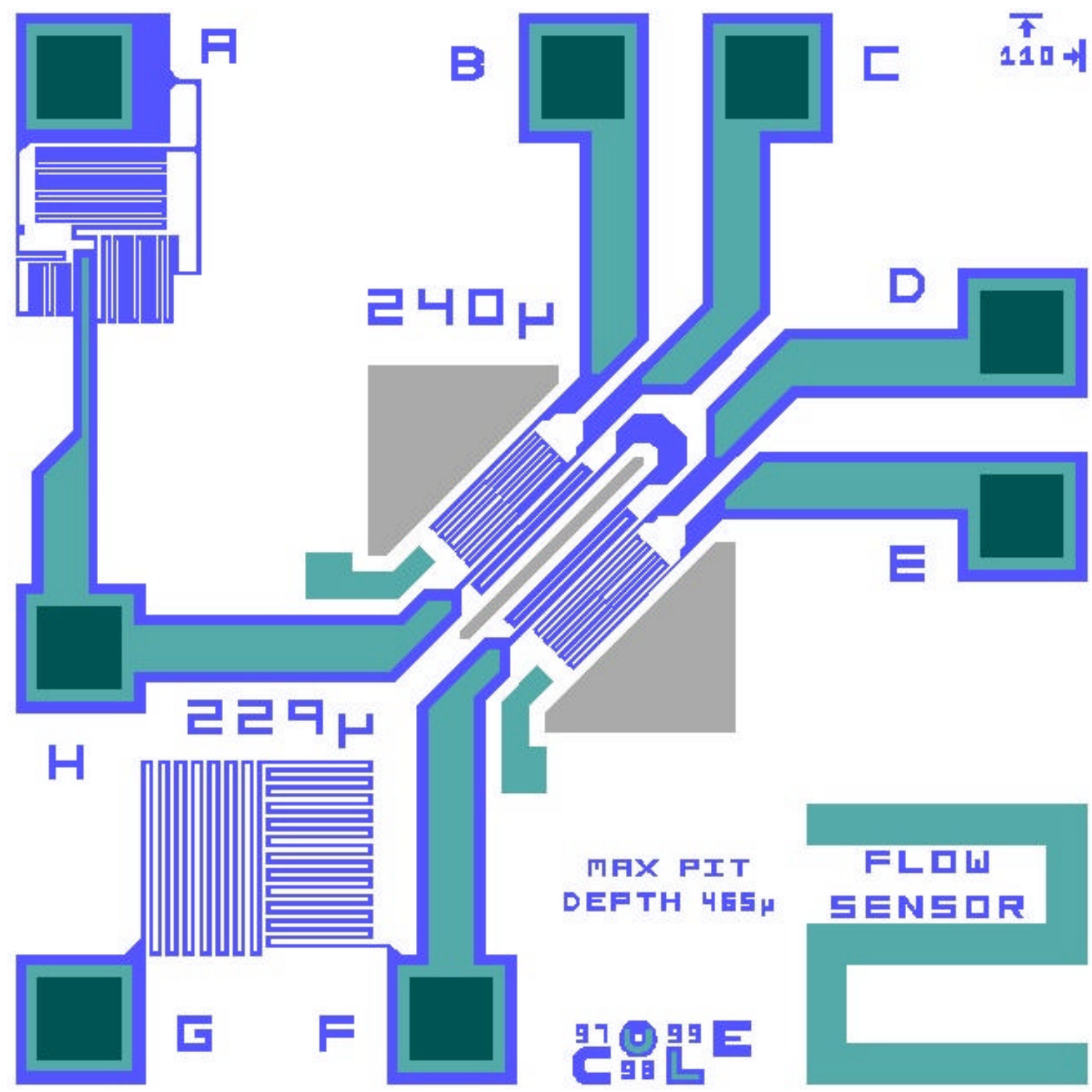

FIGURE 221 - Flow Sensor \#2 is identical to Flow Sensor \#1, except that the sense resistors do not share a common node. 


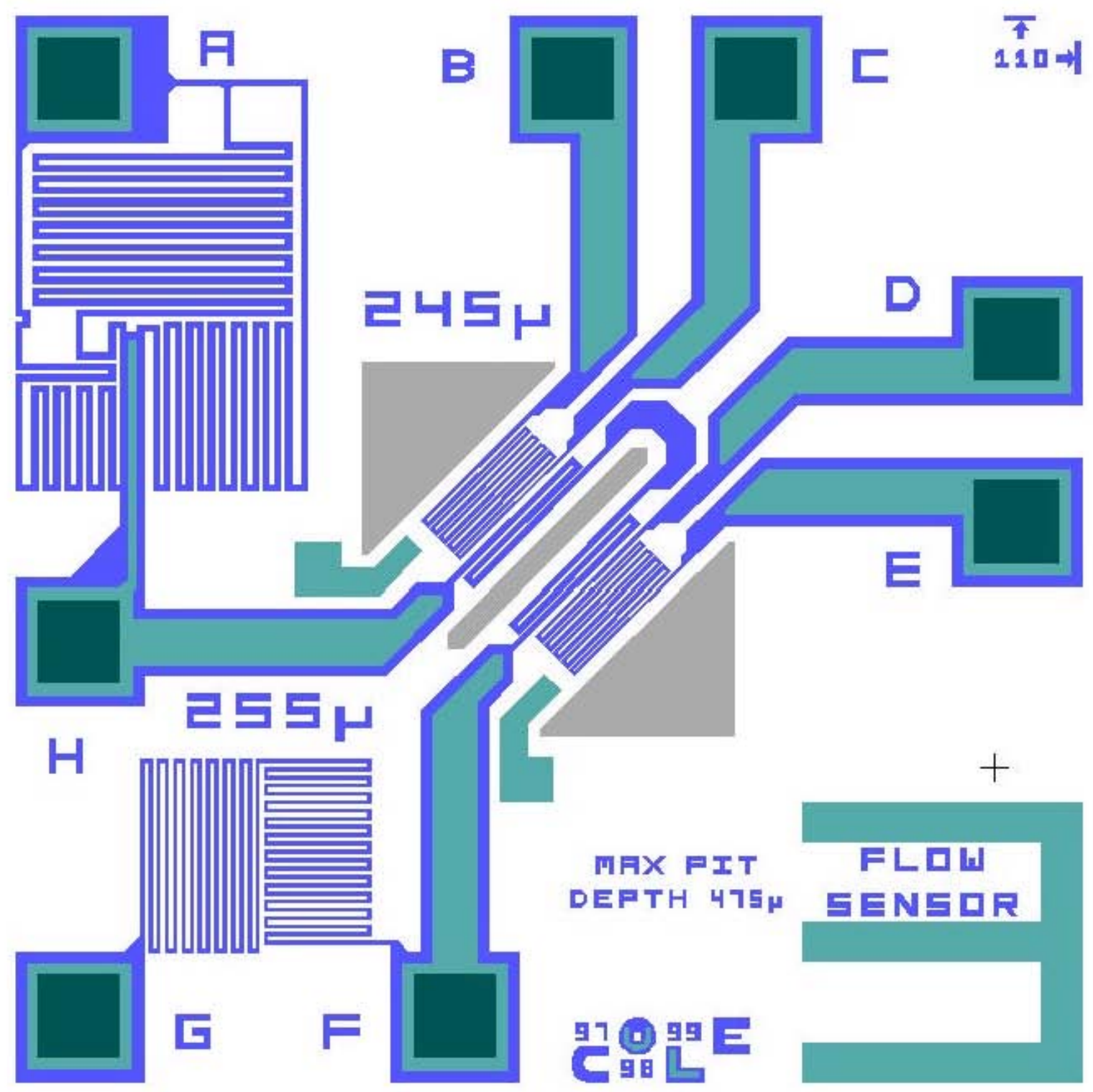

FIGURE 222 - Flow Sensor \#3 is identical to Flow Sensor \#2, except that the "parallel" substrate resistor is $100 \%$ wider, the center etch-window slit is $100 \%$ wider and there is $25 \%$ more space between resistor metallization edges and microbridge edges. 


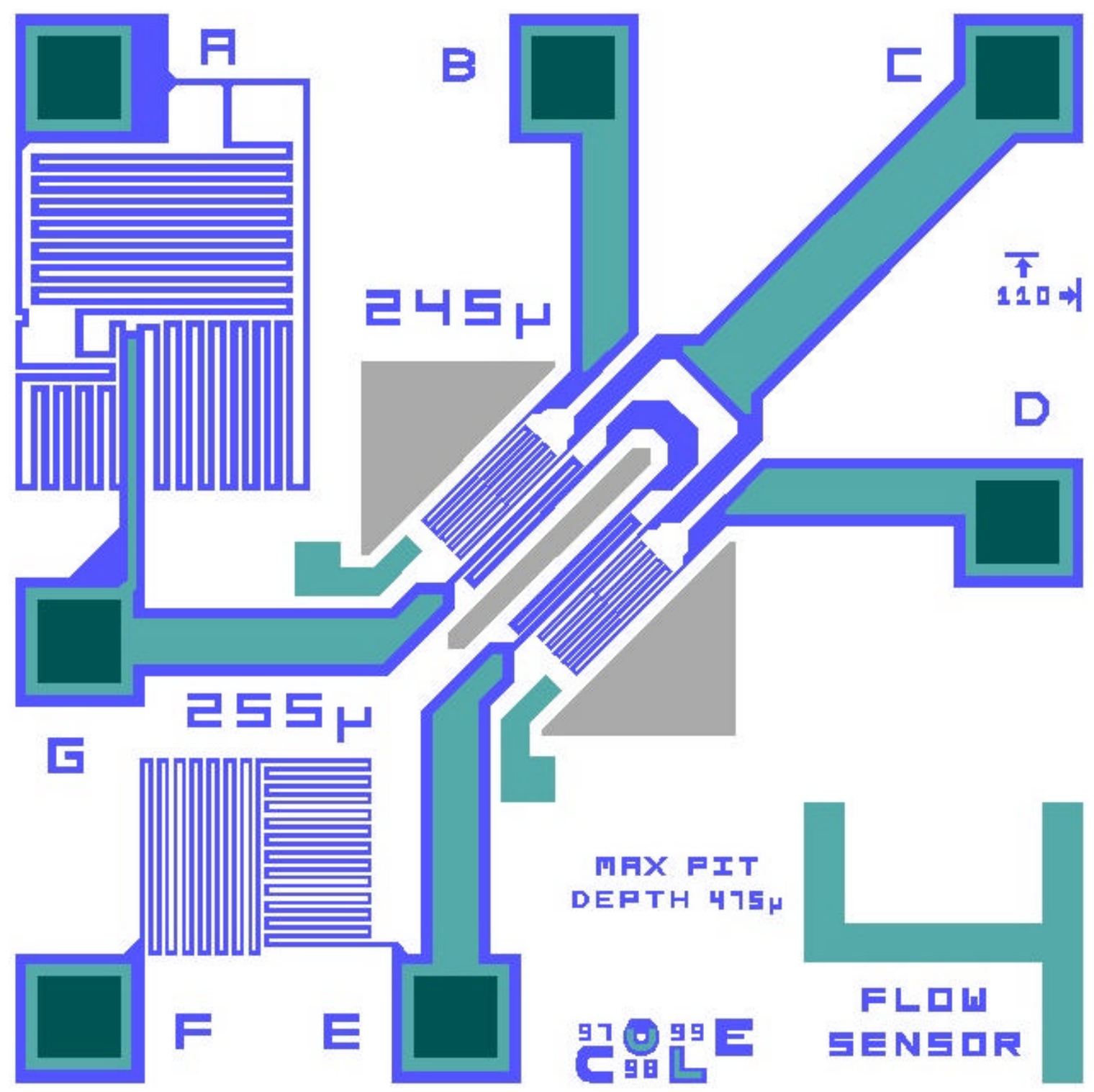

FIGURE 223 - Flow Sensor \#4 is identical to Flow Sensor \#1, except minor differences in gold routing, the parallel substrate resistor is $100 \%$ wider, the center etch-window slit is $100 \%$ wider, and there is $25 \%$ more space between resistor metallization edges and microbridge edges. 


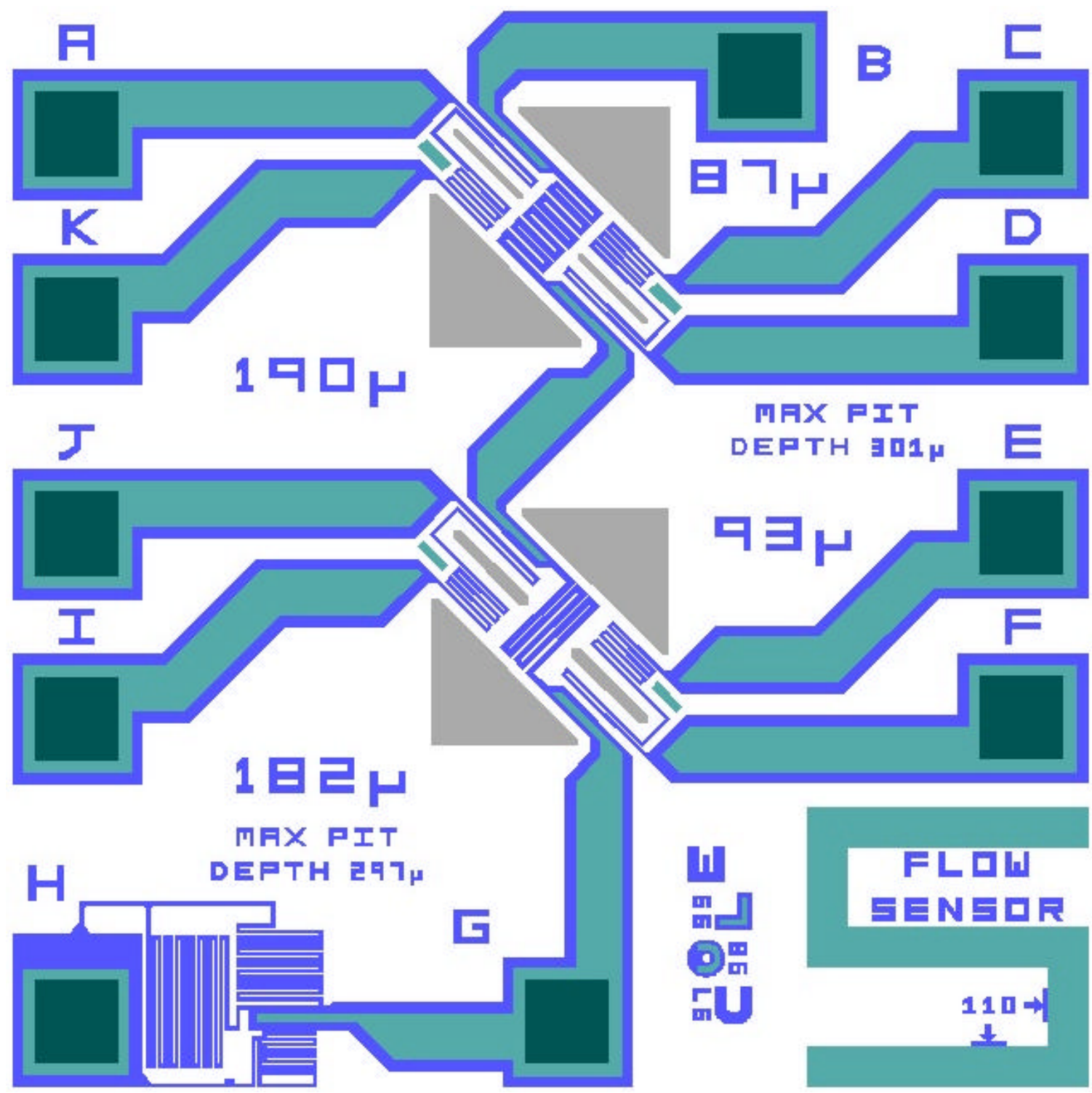

FIGURE 224 - Flow Sensor \#5 is similar in layout to Flow Sensor \#6 in the 1997 mask set, with major differences that include the "parallel" substrate resistor, $100 \%$ wider etchwindow slits on one device, and a common heater resistor connection for both sensors to operate simultaneously. 


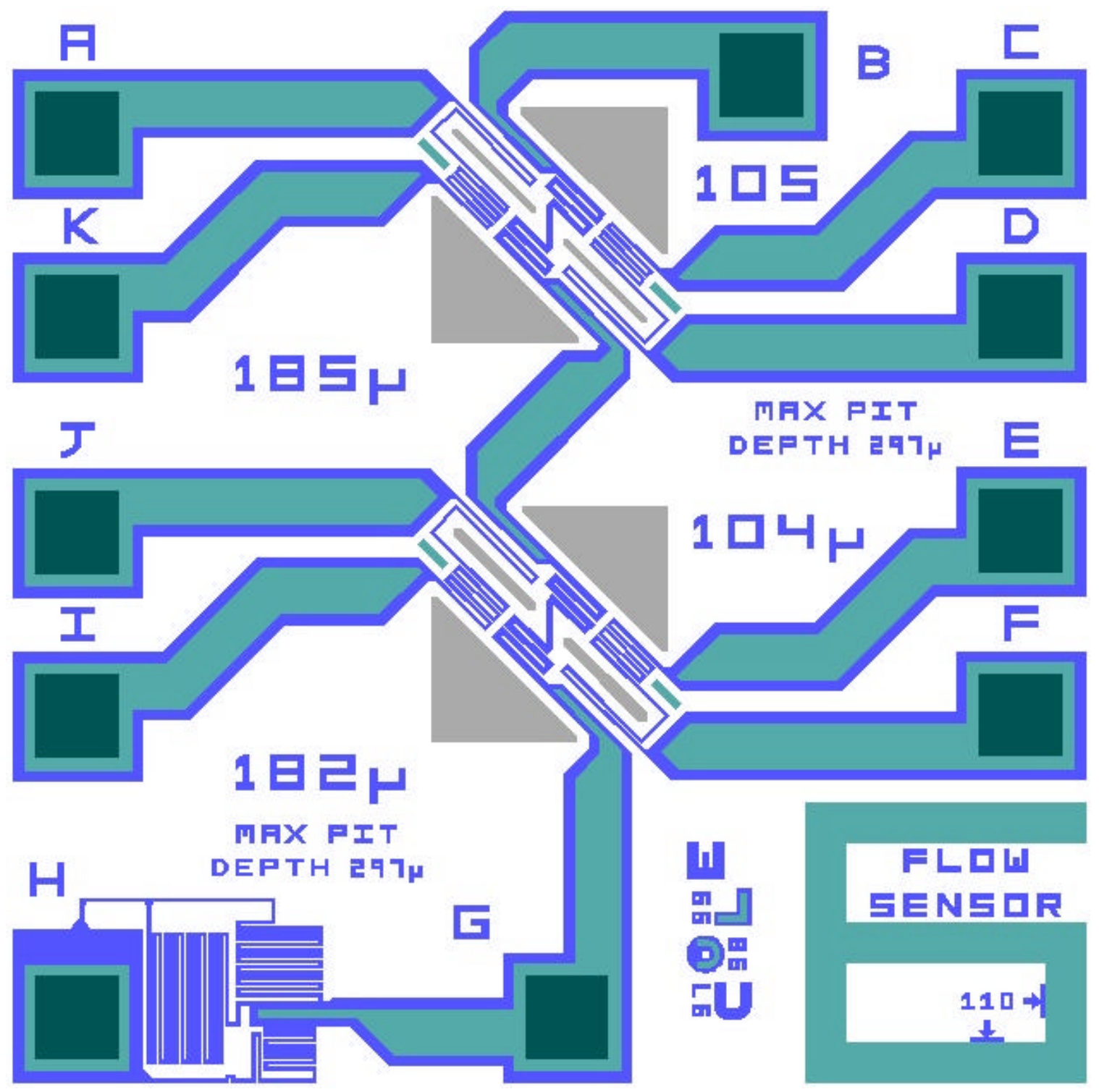

FIGURE 225 - Flow Sensor \#6 is nearly identical to Flow Sensor \#5, except for a different heater resistor geometry and layout. 

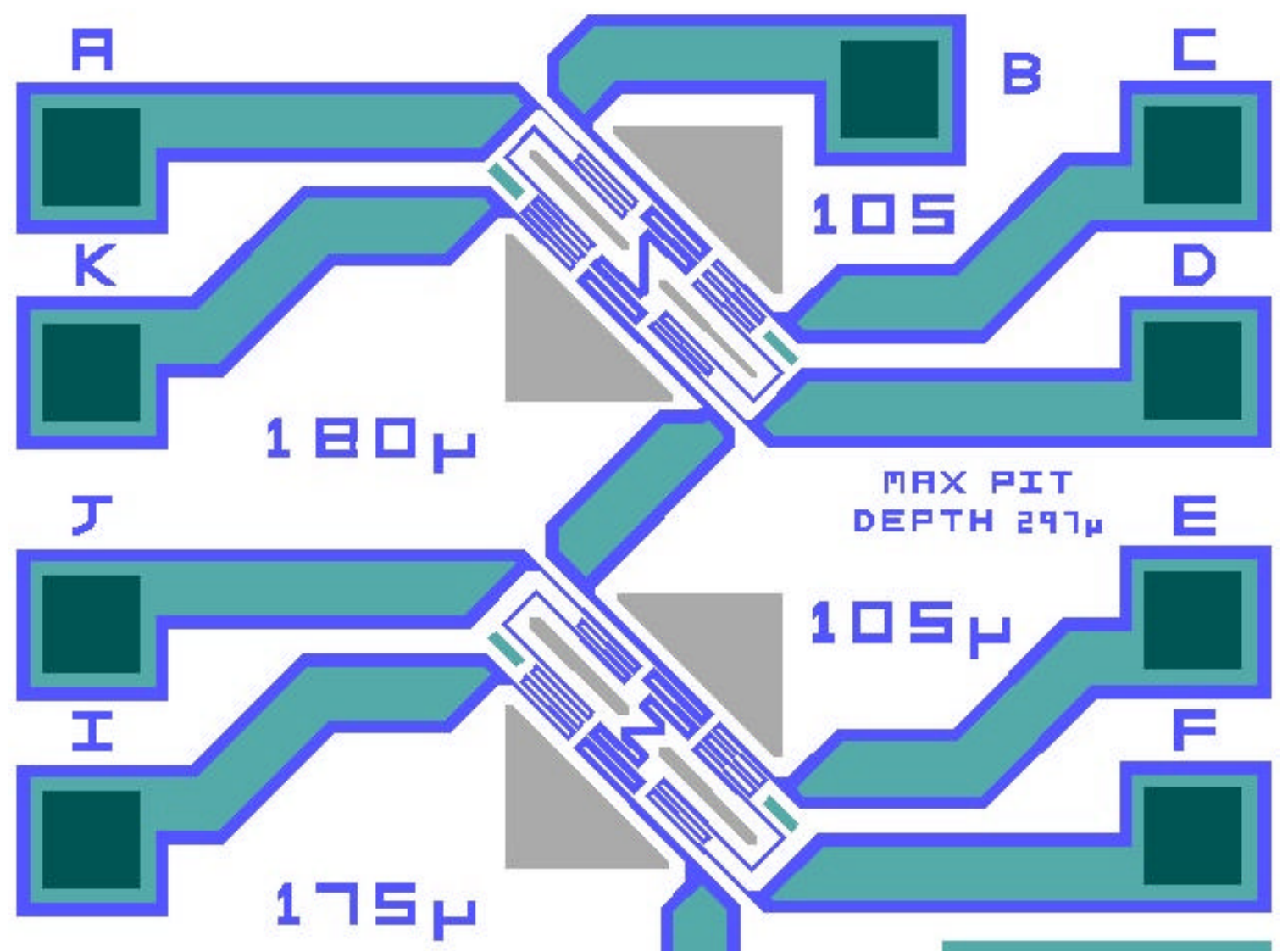

MAX PIT
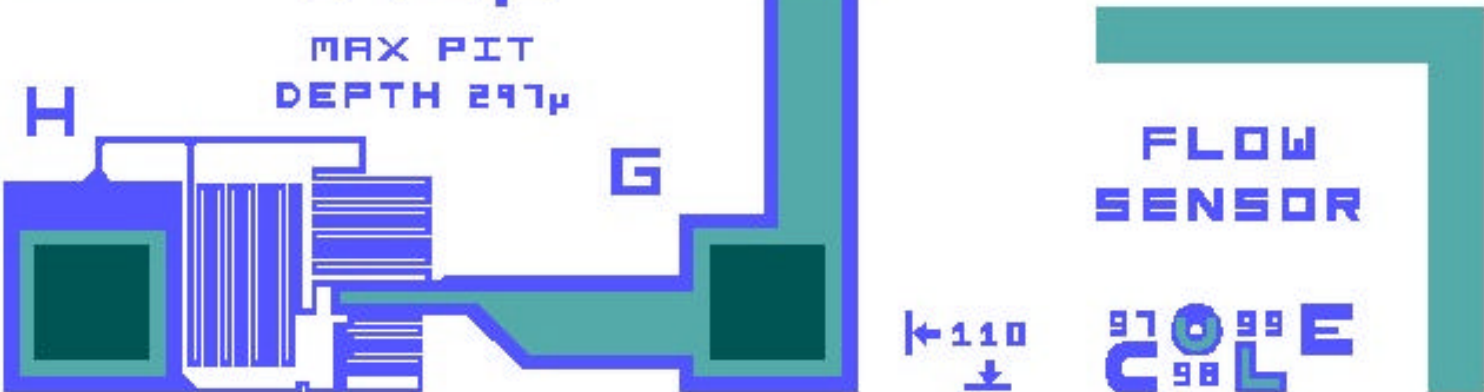

\section{FLㅂ

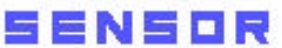

$\begin{array}{cc}\text { k110 } & 97690 \\ t & 905\end{array}$

FIGURE 226 - Flow Sensor \#7 is identical to Flow Sensor \#6, except for different sense and heater resistor layouts/geometries around the etch-window slits in the microbridges. 


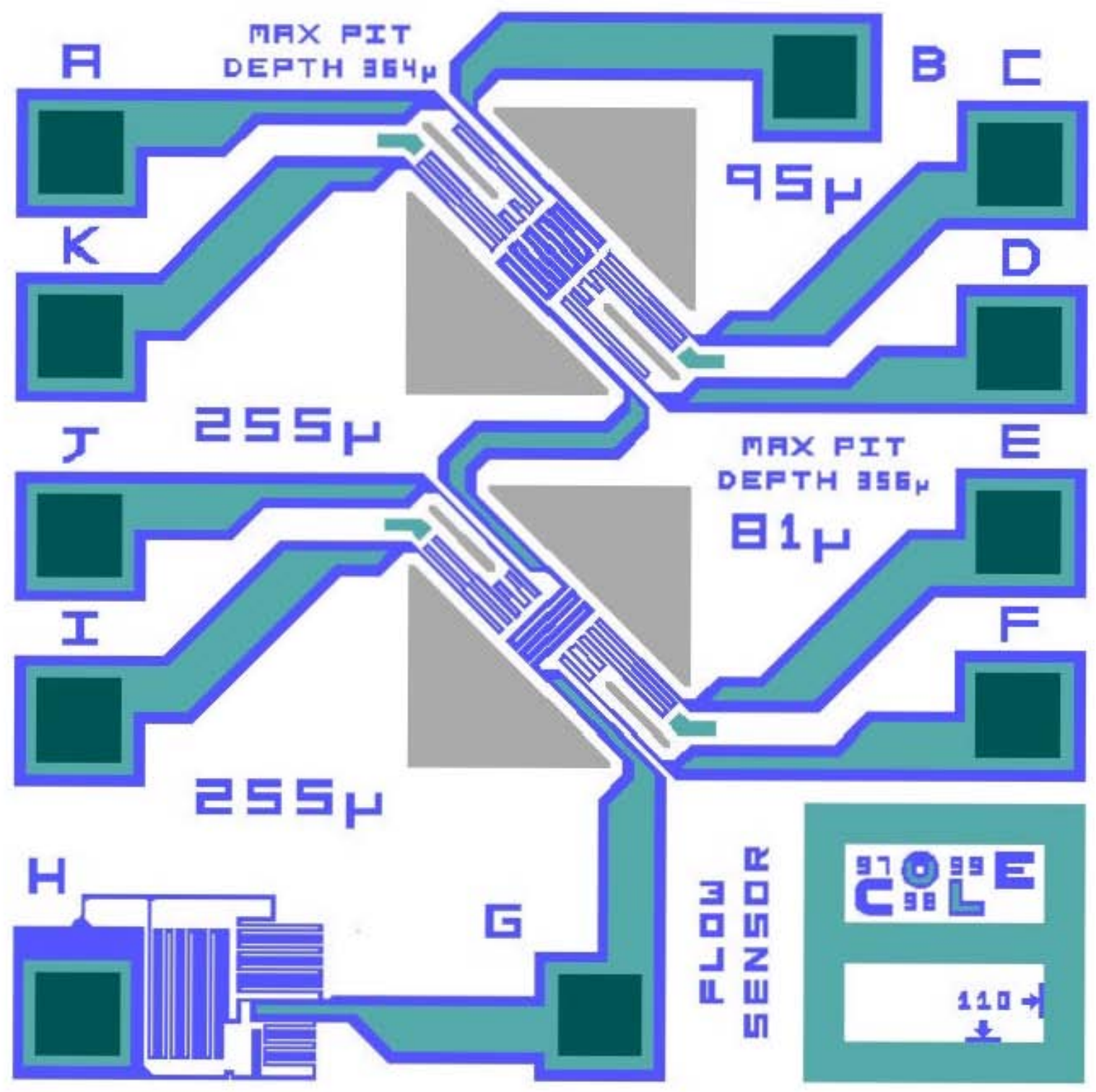

FIGURE 227 - The geometry and layout for Flow Sensor \#8 is similar to that of Flow Sensor \#5, except these microbridges are $25 \%$ longer. 


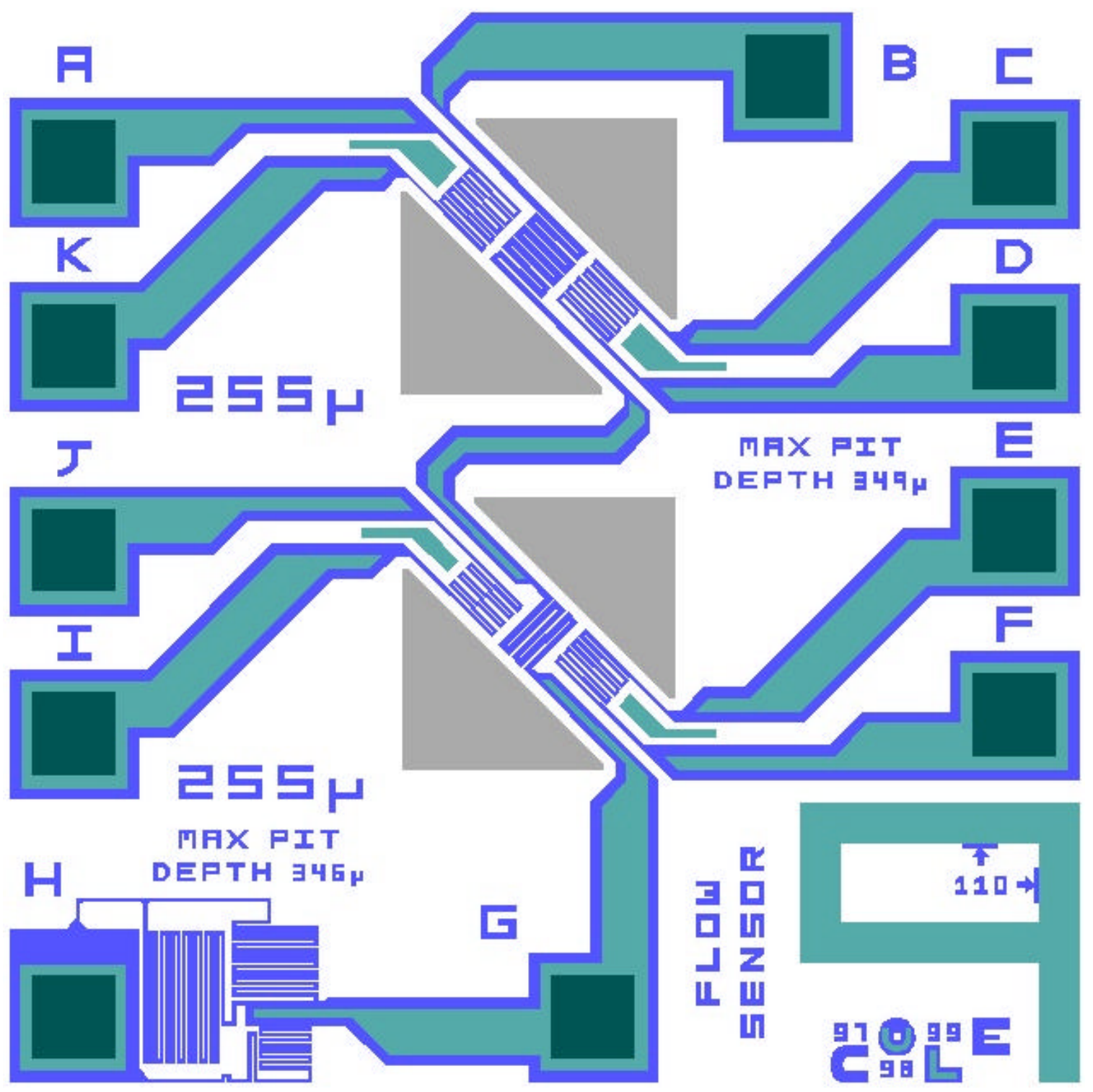

FIGURE 228 - Flow Sensor \#9 is similar in design to Flow Sensor \#3 in the 1997 design, in that both do not have etch-window slits in the microbridges. 


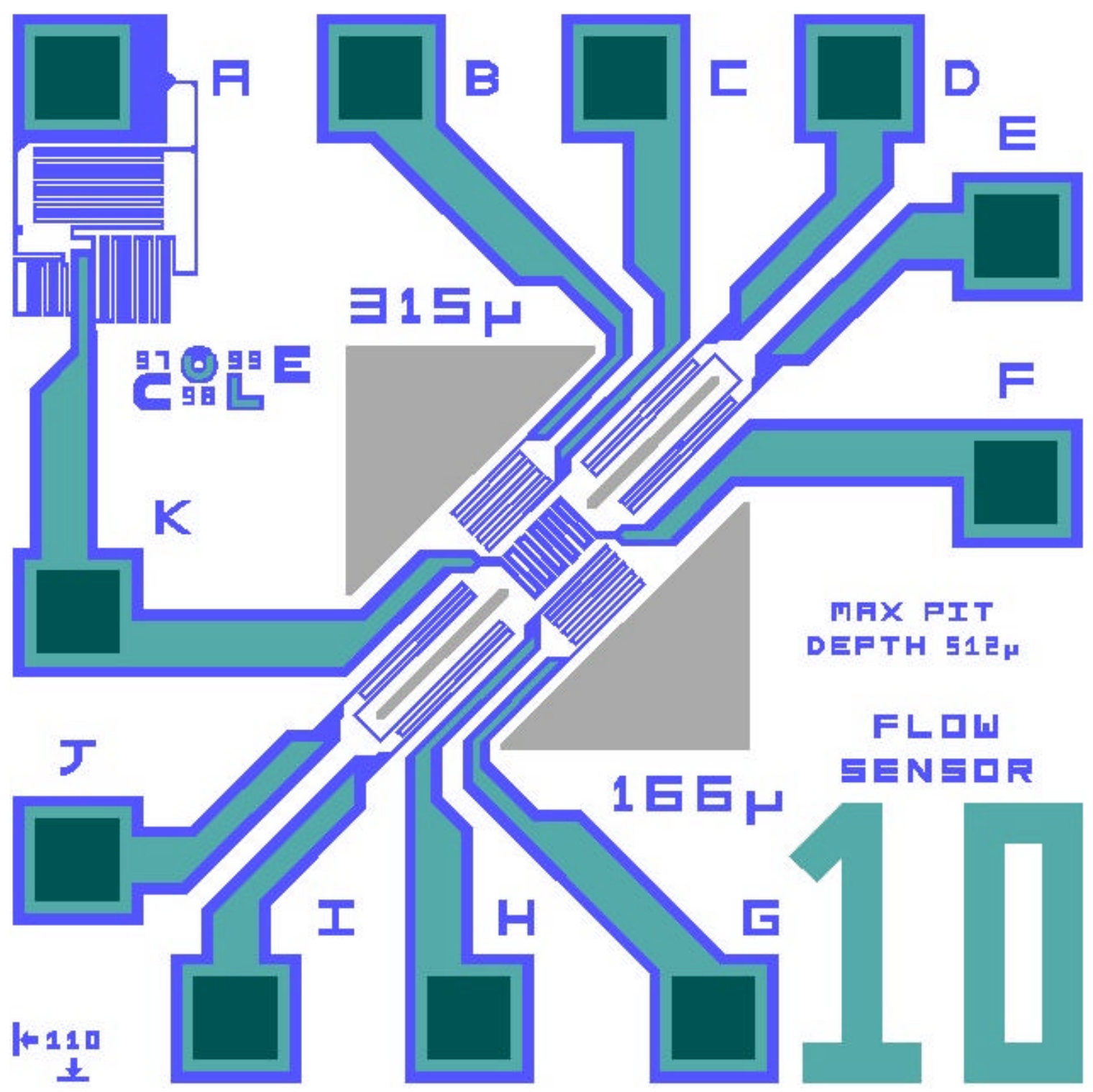

FIGURE 229 - Flow Sensor \#10 is similar in design to Flow Sensor \#2 in the 1997 photomask set (capable of measuring flow along either or both the longitudinal or transverse axes of the suspended microbridge), except this structure is improved to facilitate faster $\mathrm{KOH}$ etching with etch-window slits in the microbridge. The "166 $\mu \mathrm{m}$ " nomenclature indicates the maximum $\mathrm{KOH}$ etch depth for just the etchwindow slits in the microbridge (if there were no undercutting due to etching from the triangular regions). The " $315 \mu \mathrm{m}$ " nomenclature indicates the maximum-possible $\mathrm{KOH}$ etch depth due to etching at either triangular window. Due to the large width of the microbridge (at $380 \mu \mathrm{m}$ ), the minimum required etch depth to release the $\mathrm{Si}_{3} \mathrm{~N}_{4}$ bridge from the silicon substrate is $\sim 200 \mu \mathrm{m}$. 


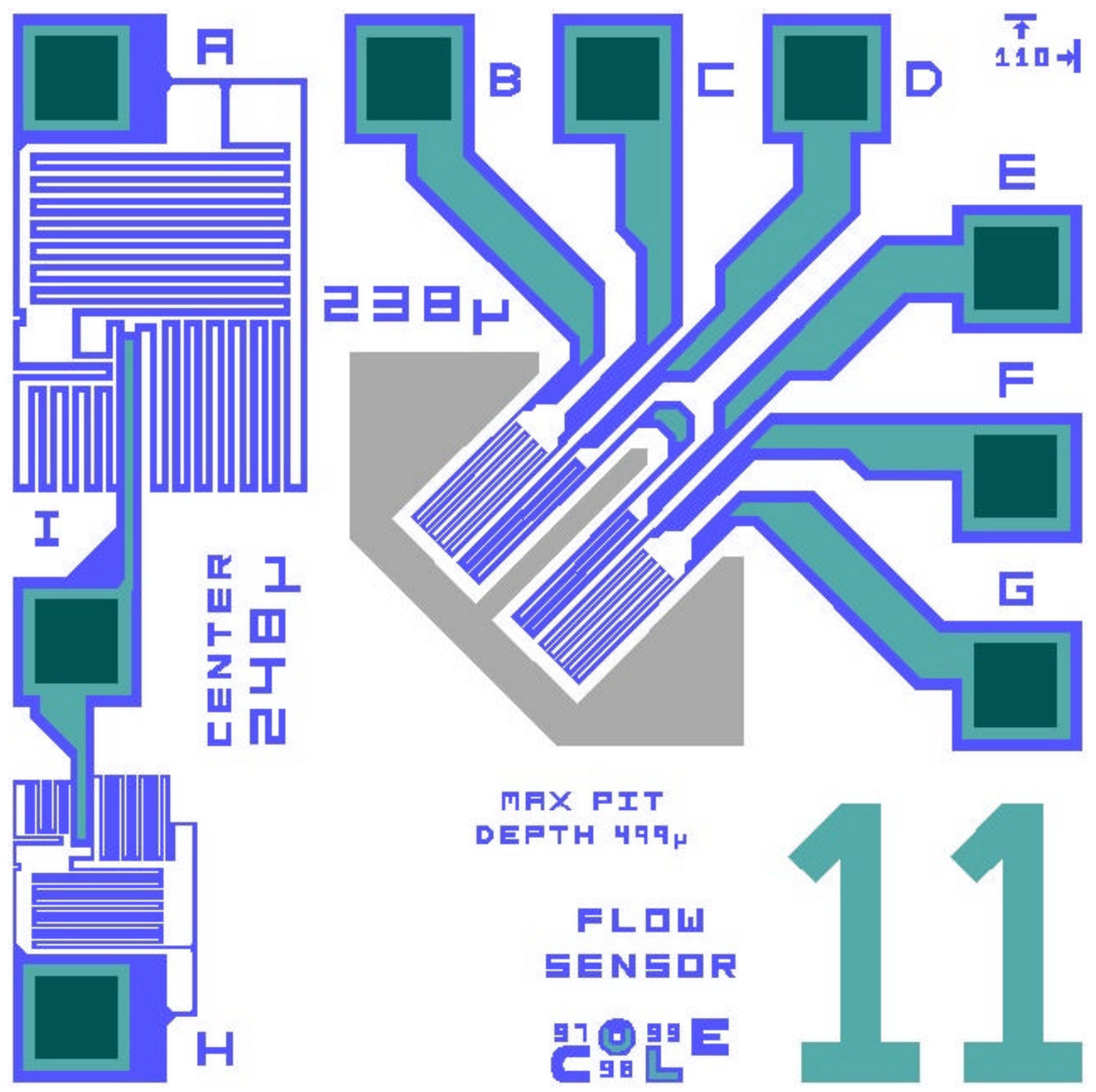

FIGURE 230 - Flow Sensor \#11 is one of eight new experimental cantilever-style sensor structures in this re-designed mask set. This sensor includes both $5 \mu \mathrm{m}$ and 10 $\mu m$-wide "parallel" sense resistors and all separate nodes for the heater and sense resistors. The " $238 \mu$ " nomenclature refers to the maximum $\mathrm{KOH}$ etch depth achievable in just the triangular portion of the etch window structure at the top left or bottom right of the cantilever structures (as if it were not connected via undercutting to other etch regions in the same structure). The "CENTER $248 \mu$ " nomenclature refers to the maximum etch depth possible in the center slit region between the two cantilevers. These numbers are clearly only approximations, because the etch windows are all connected. 


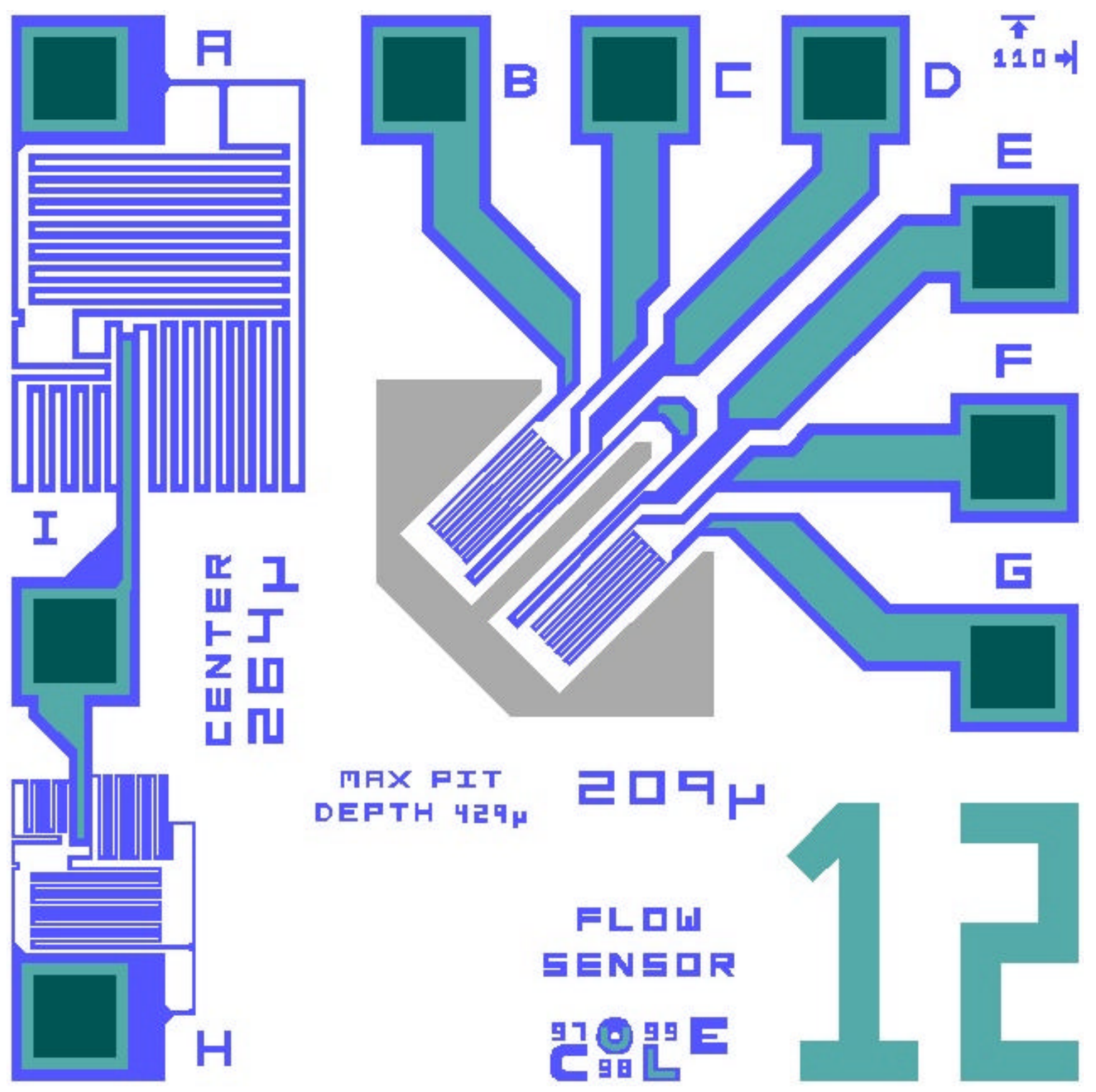

FIGURE 231 - Flow Sensor \#12 is nearly identical to Flow Sensor \#11, except with a shorter heater resistor. 


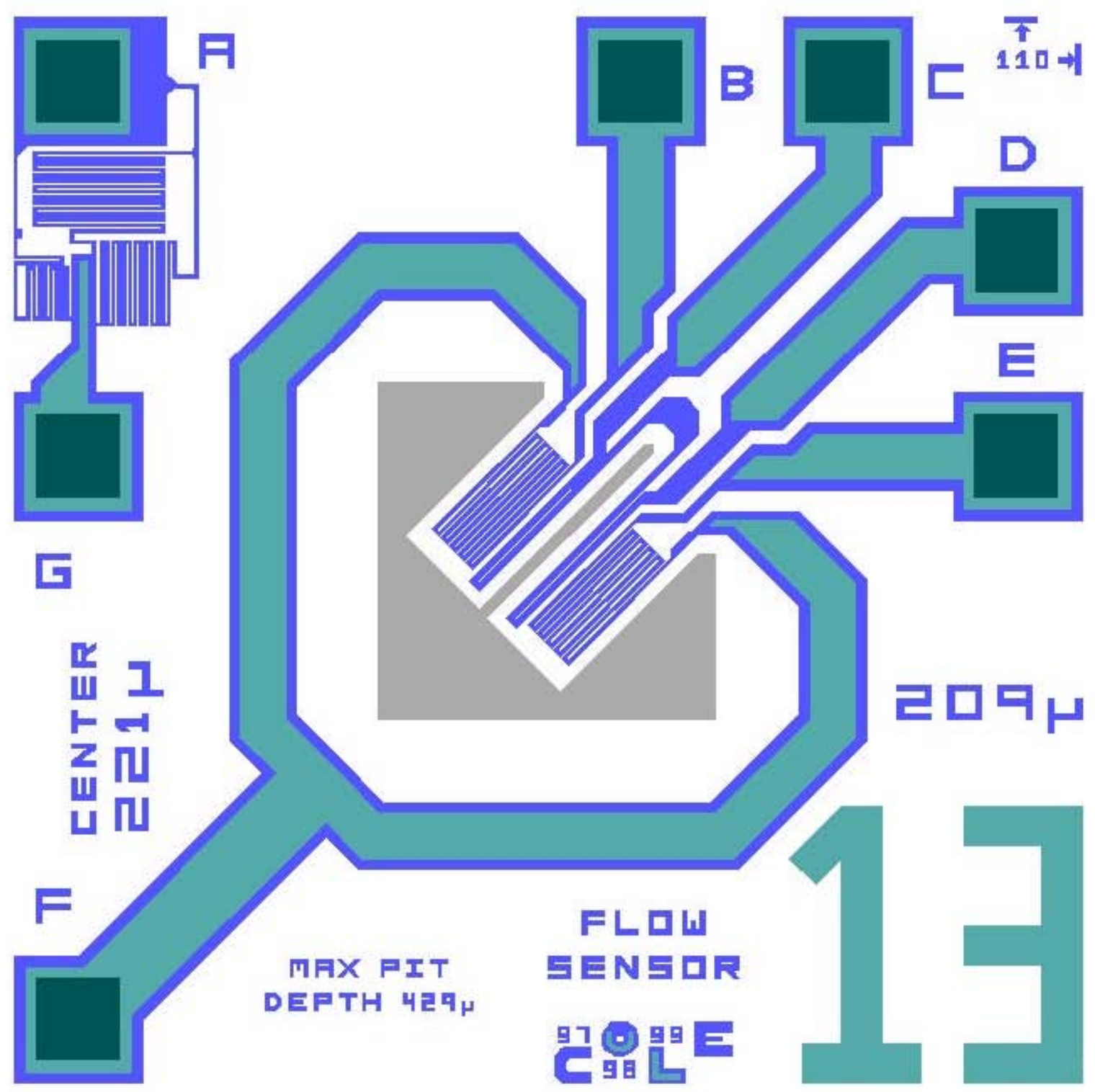

FIGURE 232 - Flow Sensor \#13, along with Flow Sensor \#15, are the only cantilever-style flow sensors in this mask set with a common-node connection for the sense resistors. On this sensor, the common connection wiring is made outside of the cantilever structures themselves. 


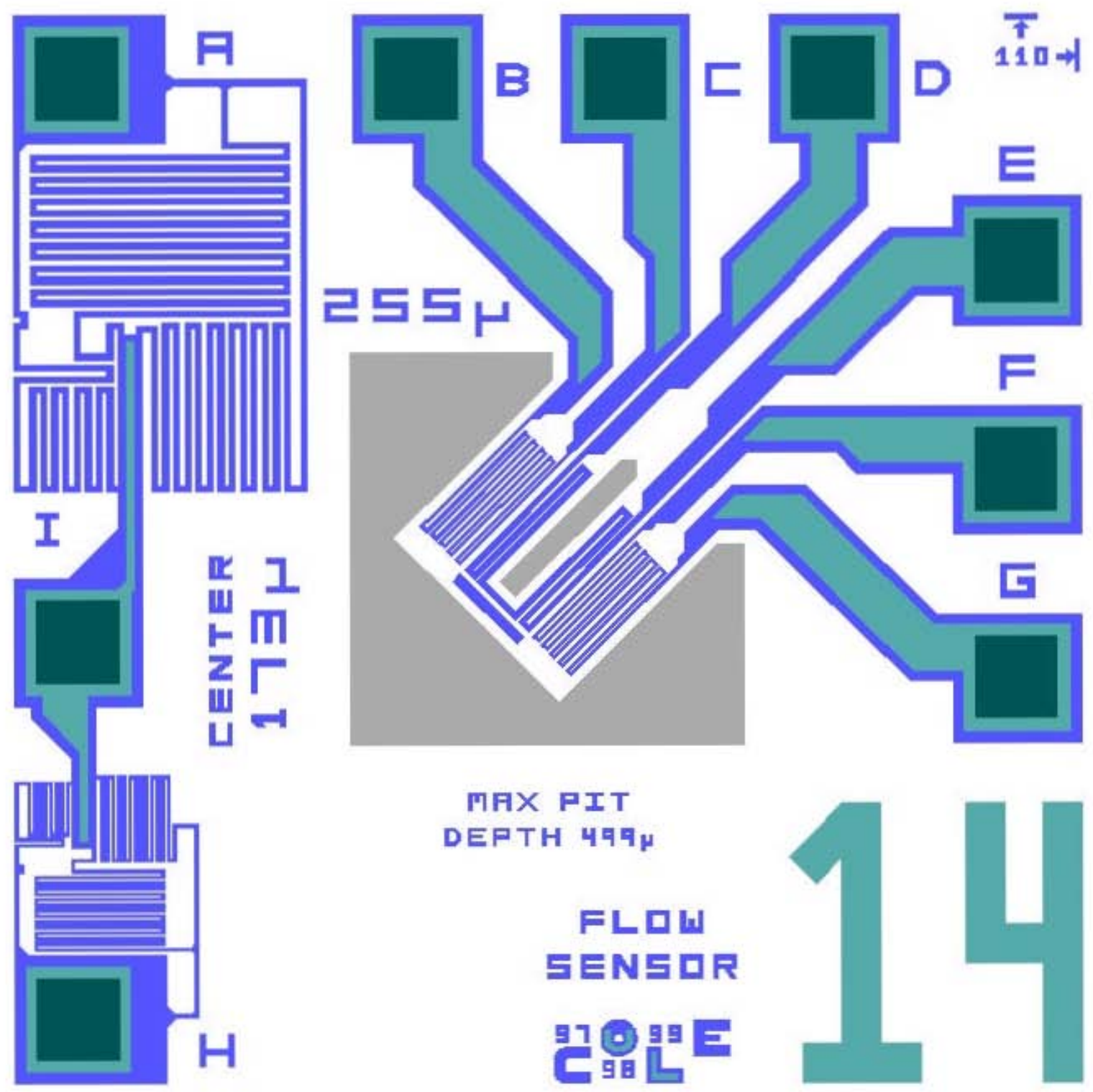

FIGURE 233 - Flow Sensor \#14 is one of two "merged-cantilever" style devices in this design, but unlike Flow Sensor \#15, it can be tested (if it can be fabricated successfully), because both sense resistors can be properly accessed and wired into a Wheatstone bridge configuration. 


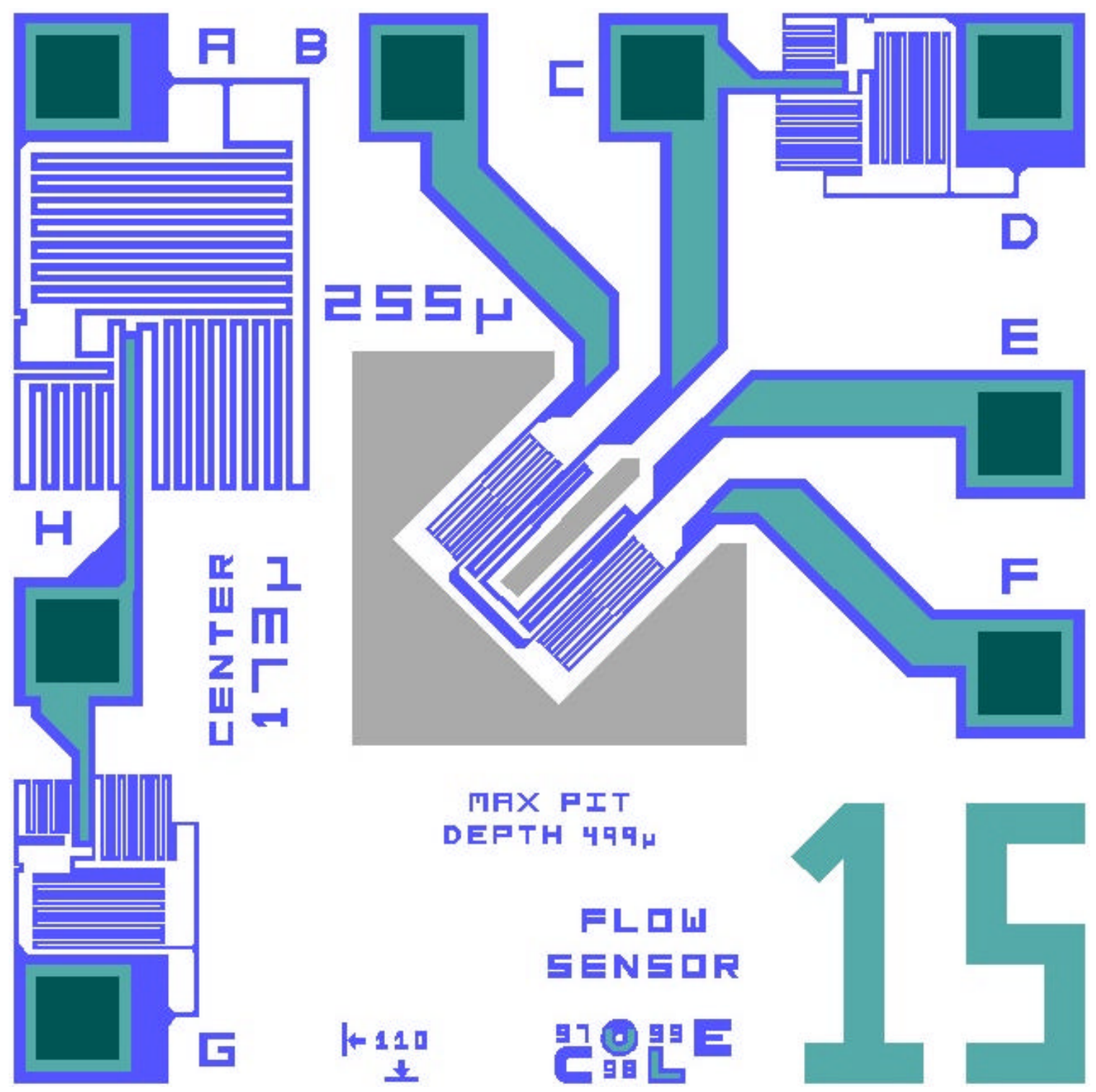

FIGURE 234 - Flow Sensor \#15, like Flow Sensor \#13, has a common connection for both sense resistors. Unfortunately, this sensor cannot be tested, because of an error in its design, in which it does not have wiring from the common connection between the sense resistors to a pad, making it unusable in a Wheatstone bridge configuration. 


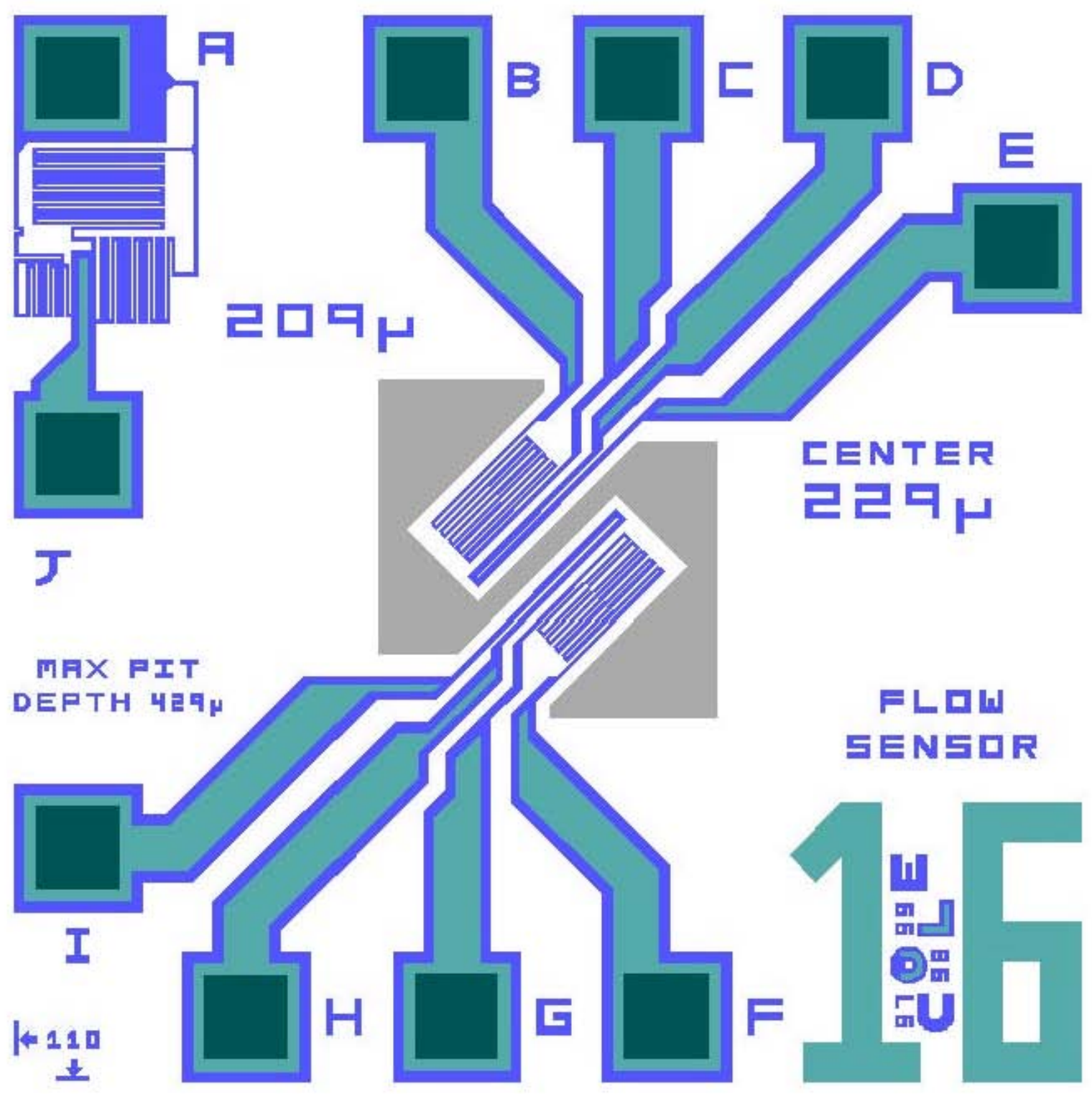

FIGURE 235 - Flow Sensor \#16 is the only cantilever-style sensor with separate heater layouts (and pad routing) on each cantilever. It is the only flow sensor geometry with two separate heaters in this design. 


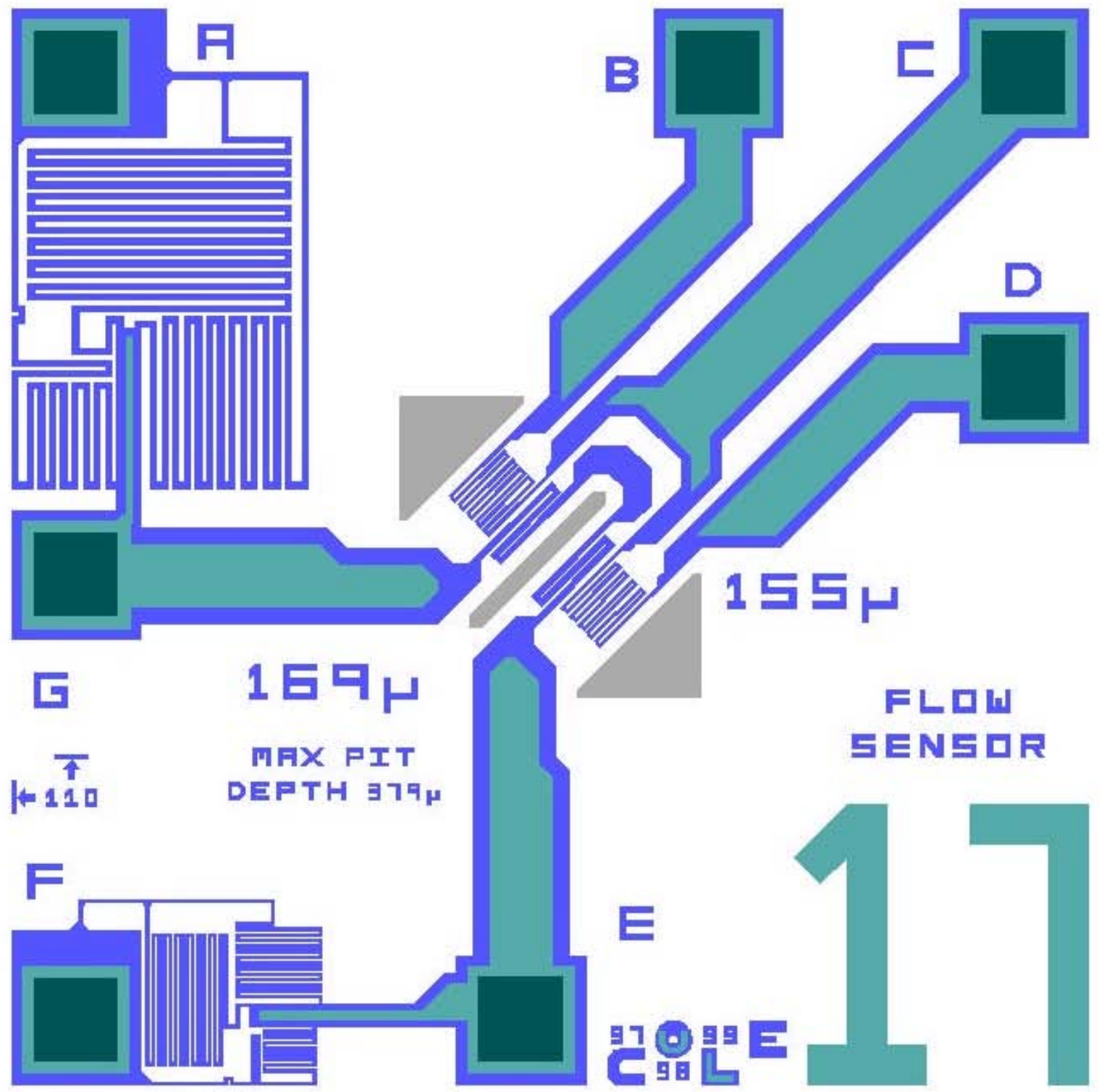

FIGURE 236 - Flow Sensor \#17 is the smallest dual microbridge sensor in this photomask set. With the smallest bridges and etch windows, it should require the least etch time to suspend the microbridges, compared to other sensors in this design. 


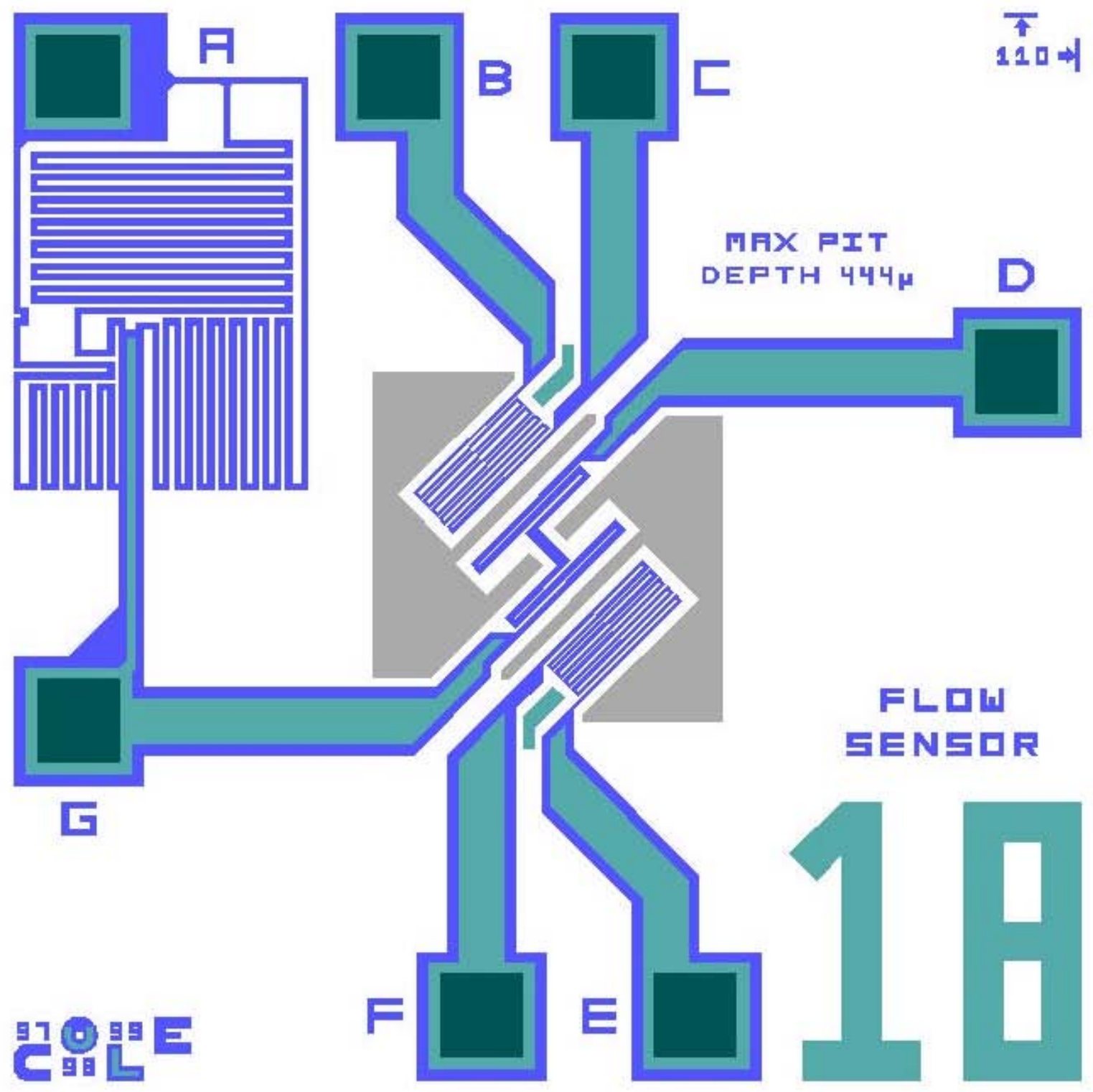

FIGURE 237 - Flow Sensor \#18 includes a cantilever-style design with a unique heater resistor bridge to facilitate faster etching and undercutting of the heater resistor microbridge area. 


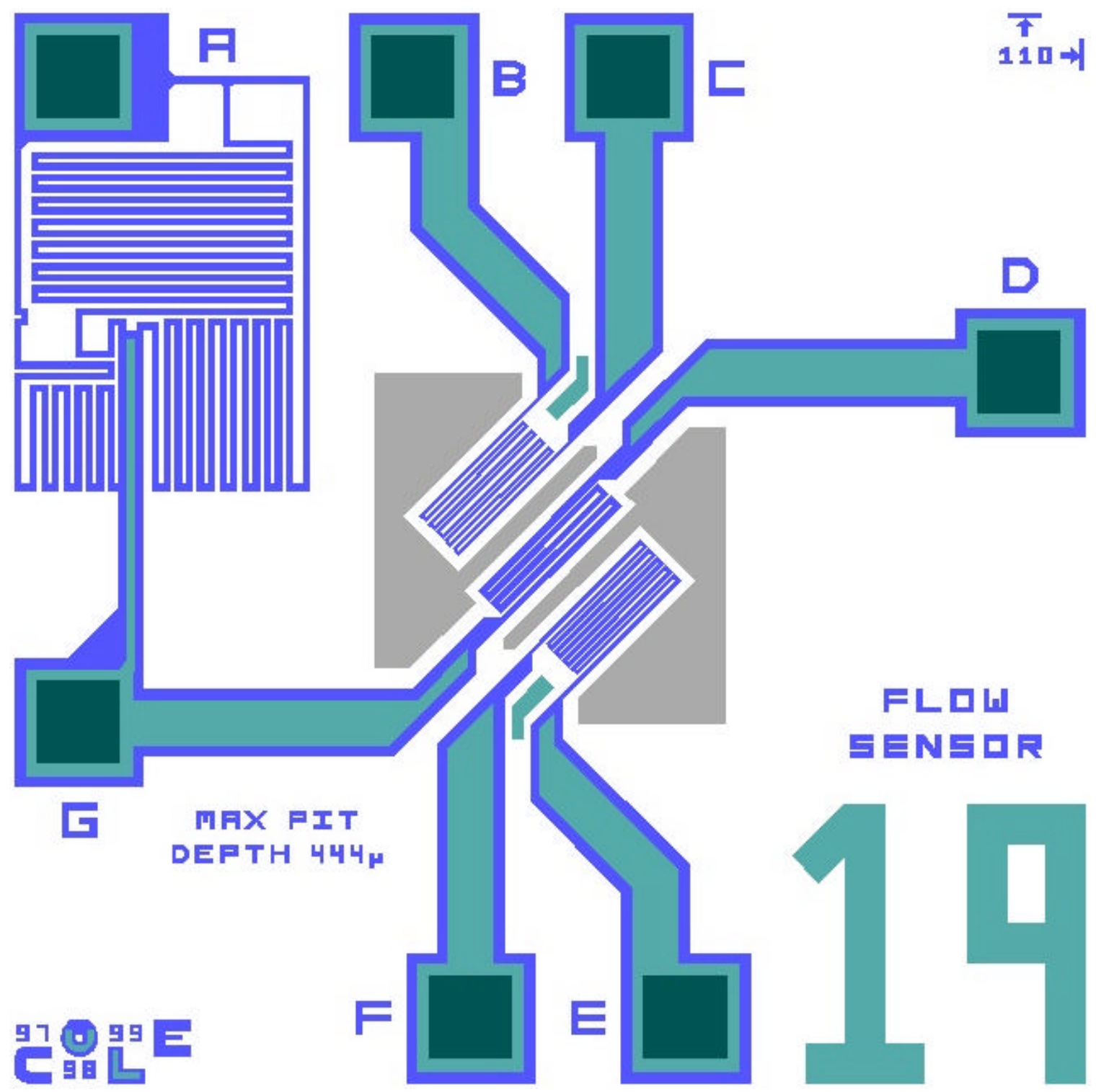

FIGURE 238 - Flow Sensor \#19 includes two cantilevers for the sense resistors and a microbridge for the heater resistor. 


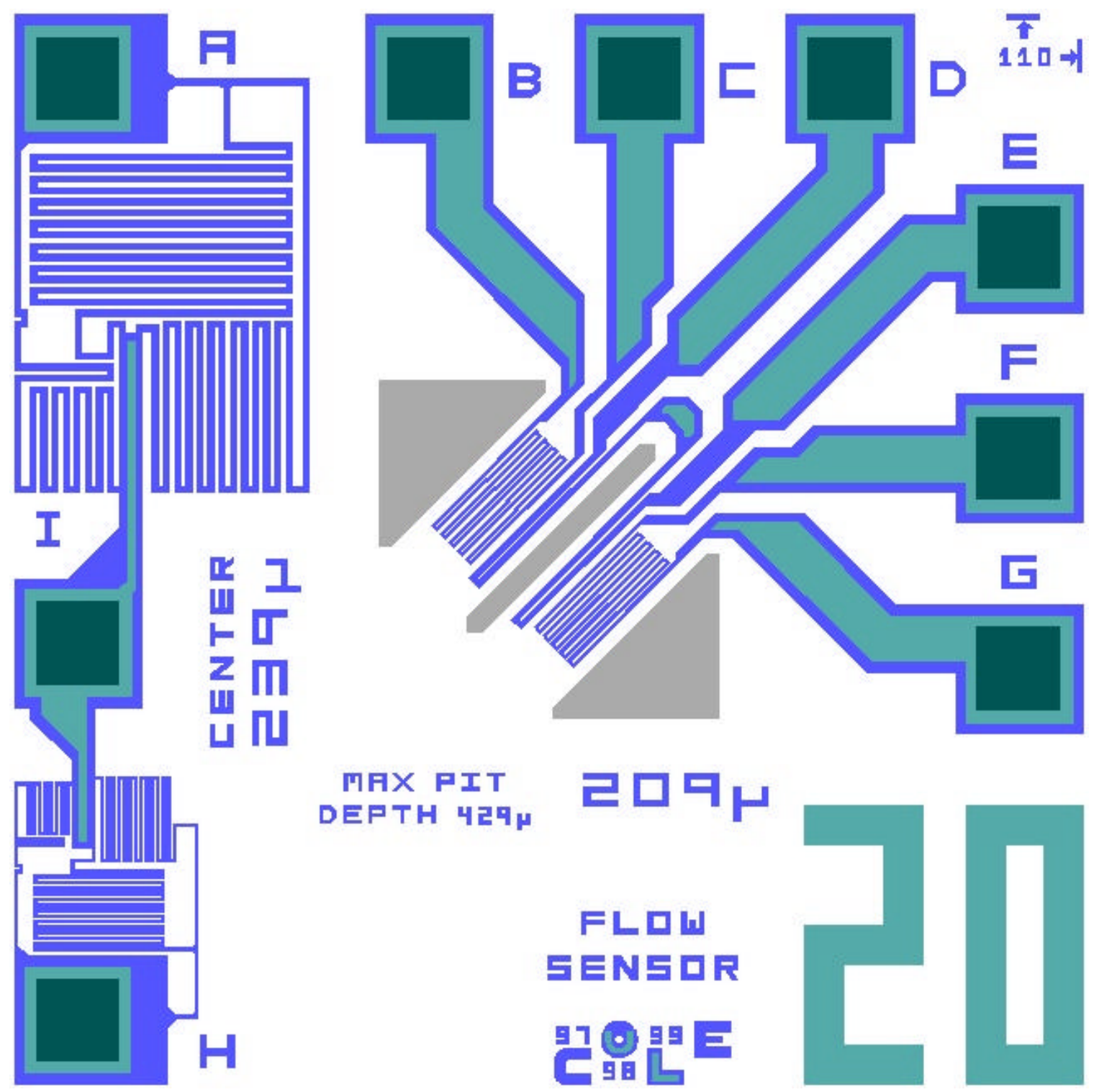

FIGURE 239 - Flow Sensor \#20 is based on the dual-microbridge design of Flow Sensors \#1 \#4, except with grouping the pads together in one corner of the die. 

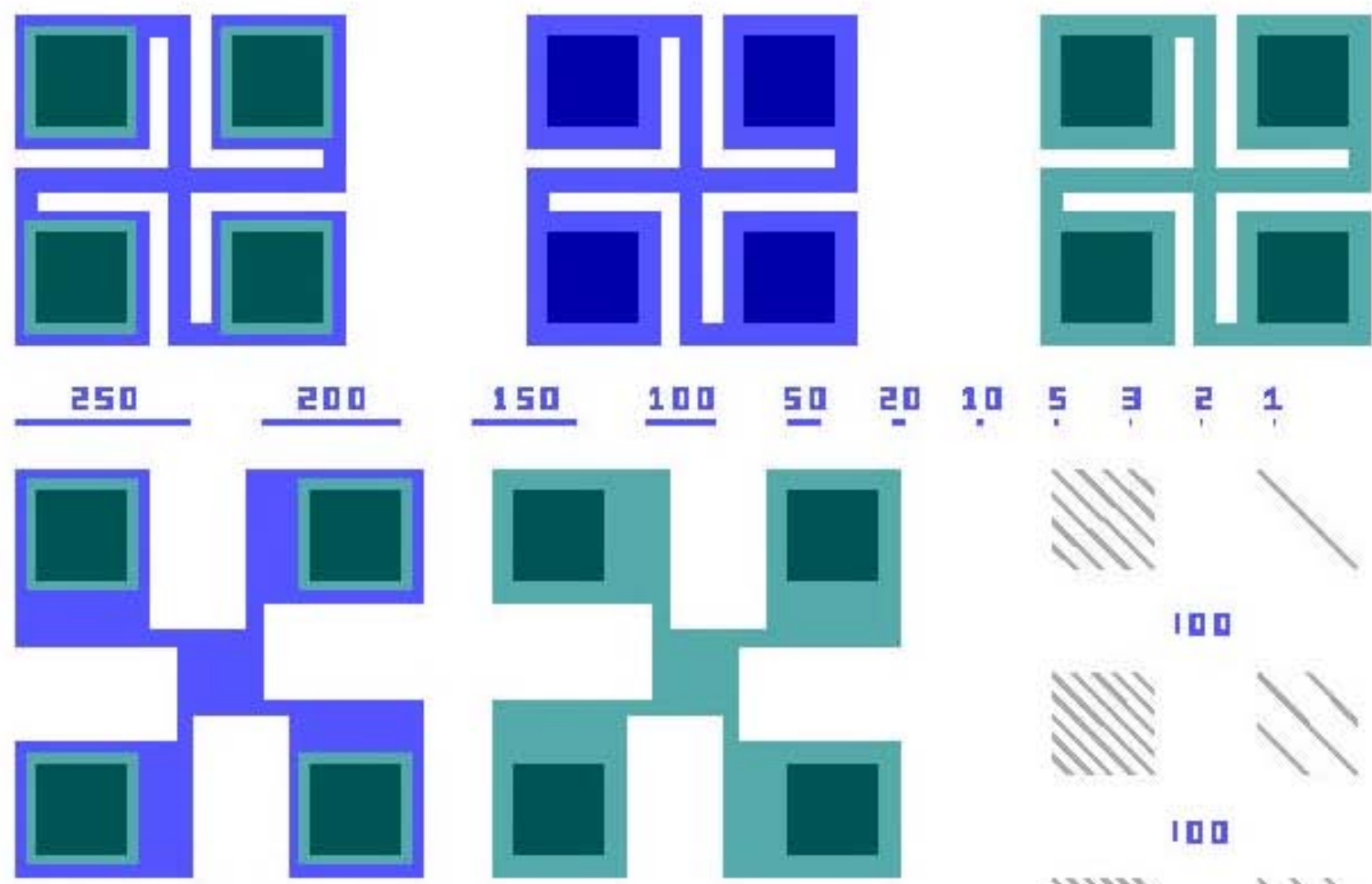

I0D

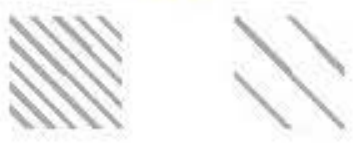

I00
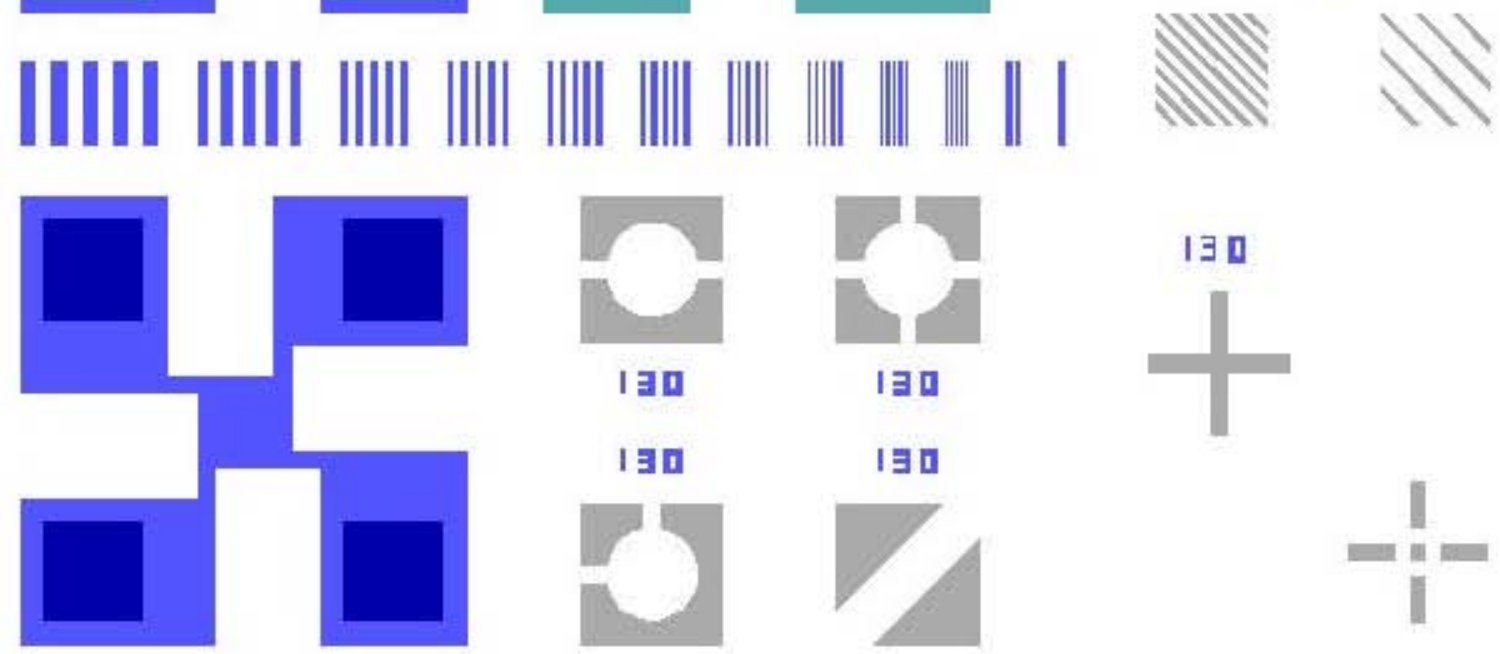

FIGURE 240 - An improved Van der Pauw region for this mask set, including structures for measuring the sheet resistance of each metallization layer, or both together. 


\section{MPX PIT DEPTH STRUETURES}

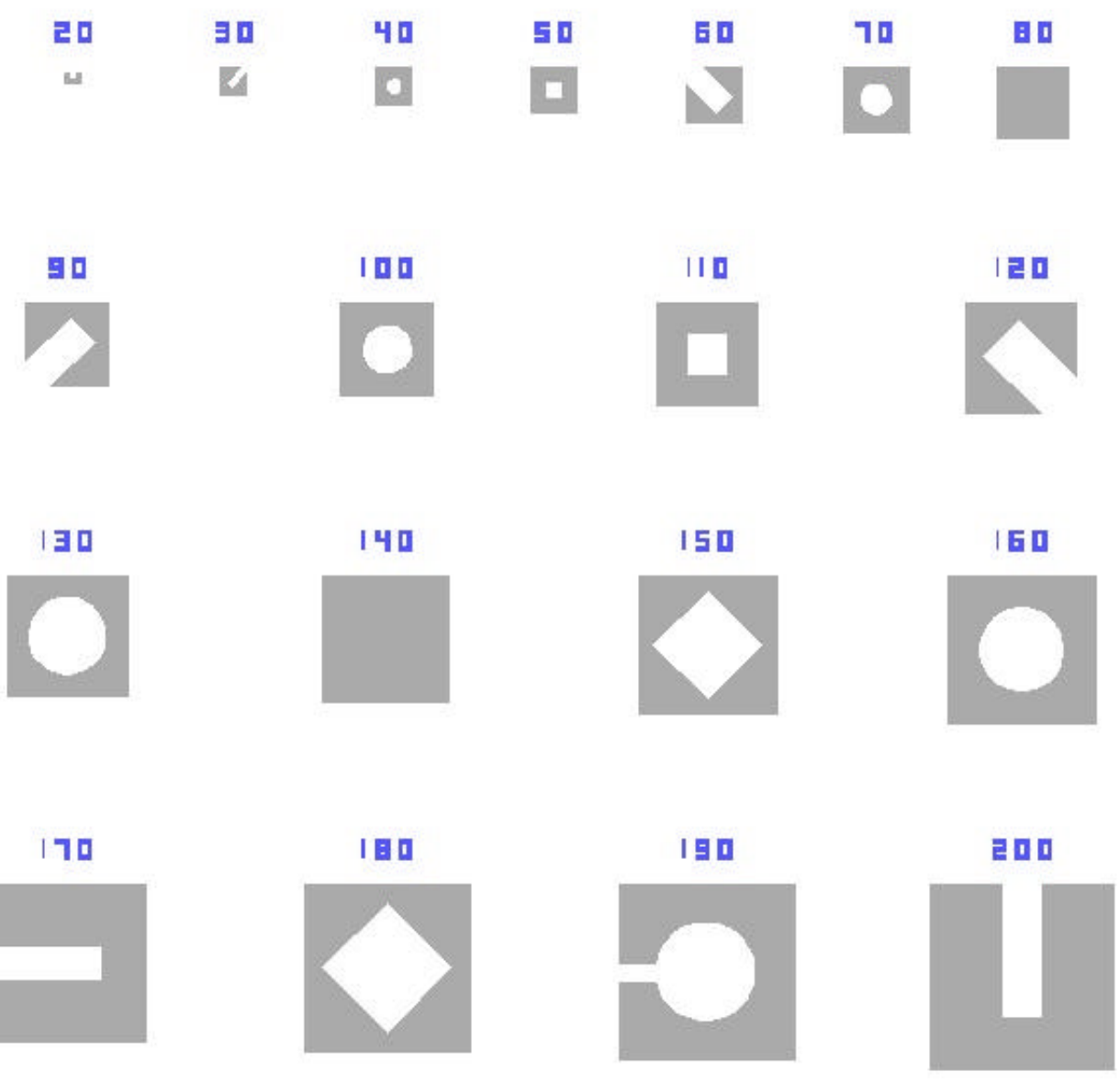

FIGURE 241 - As with the 1997 mask set, this set also featured the same preciselydimensioned etch windows to determine $\mathrm{KOH}$ etch depths with $10 \mu \mathrm{m}$ resolution, down to $200 \mu \mathrm{m}$ max. 


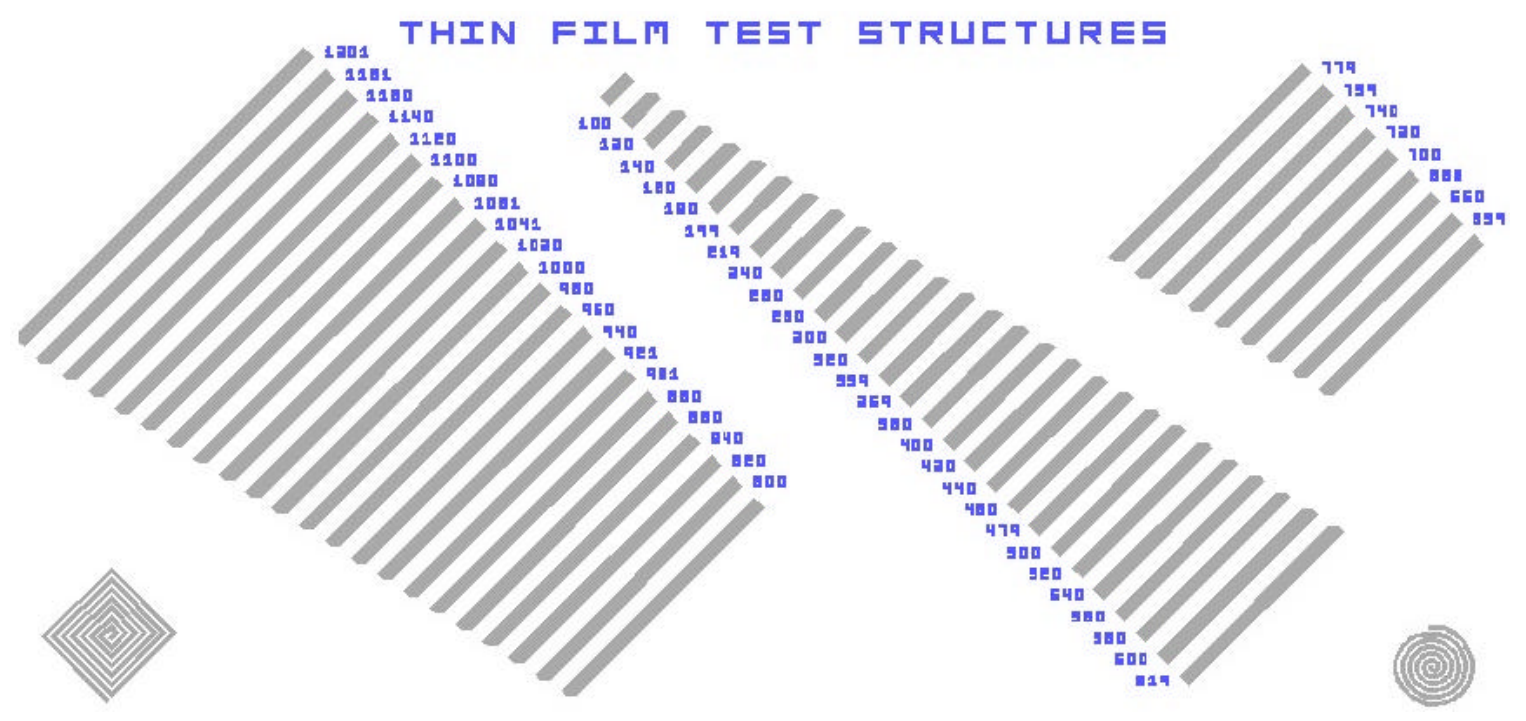

FIGURE 242 - In order to better characterize the thin film properties of $\mathrm{Si}_{3} \mathrm{~N}_{4}$ thin films, this mask set included an array of scaled doubly-supported beams to measure localized compressive strain fields.

\section{月LIGNMENT ARER}
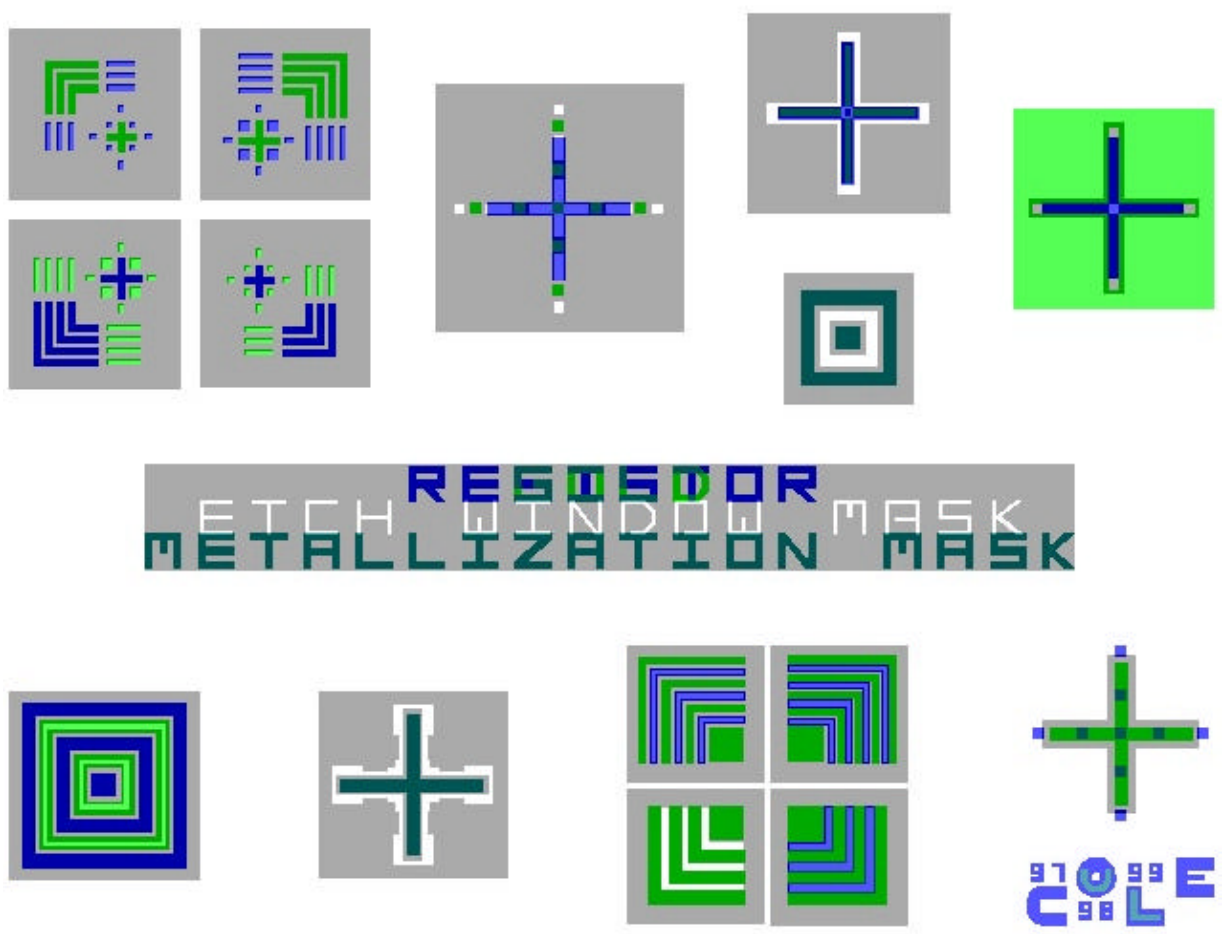

FIGURE 243 - The mask alignment/registration area included at the center of the device portion of each die replication. 
PLIENMENT AREA
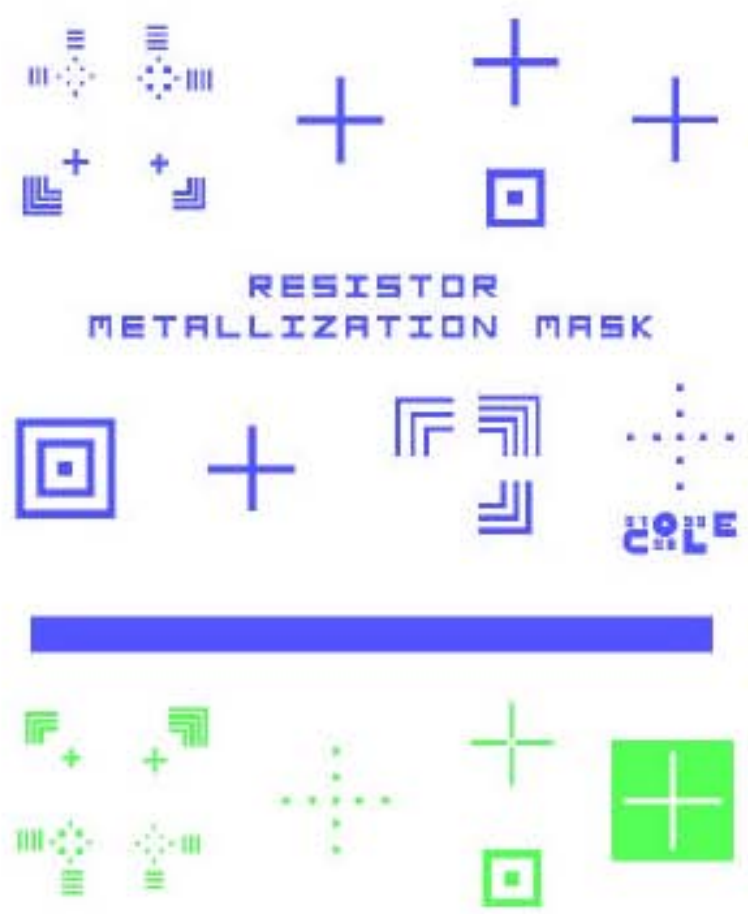

GロLD

METALLIZATIロN MAБK
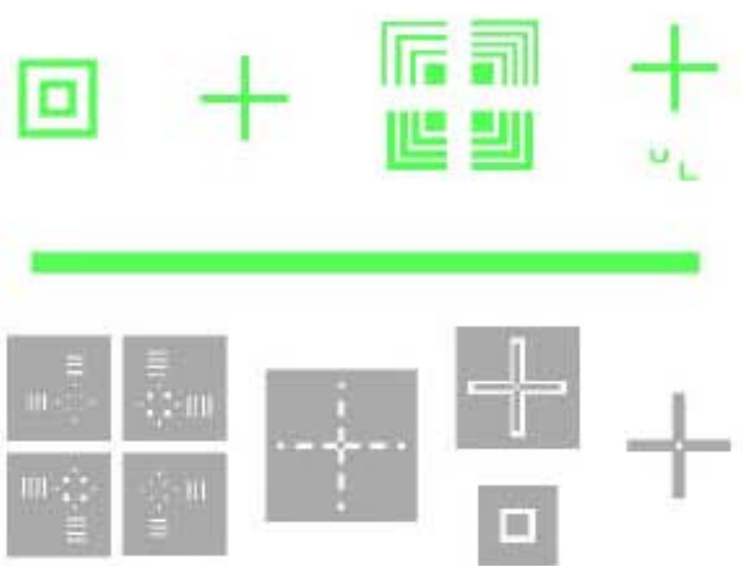

\section{ETEH WINDQW MASK}
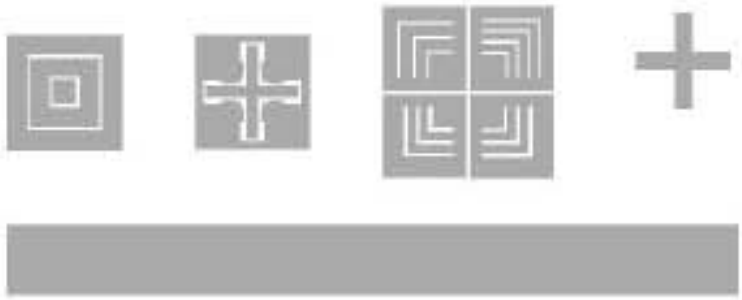

FIGURE 244 - The three layers in the photomask alignment area, separated. Labels in each area show to which mask it belongs. 


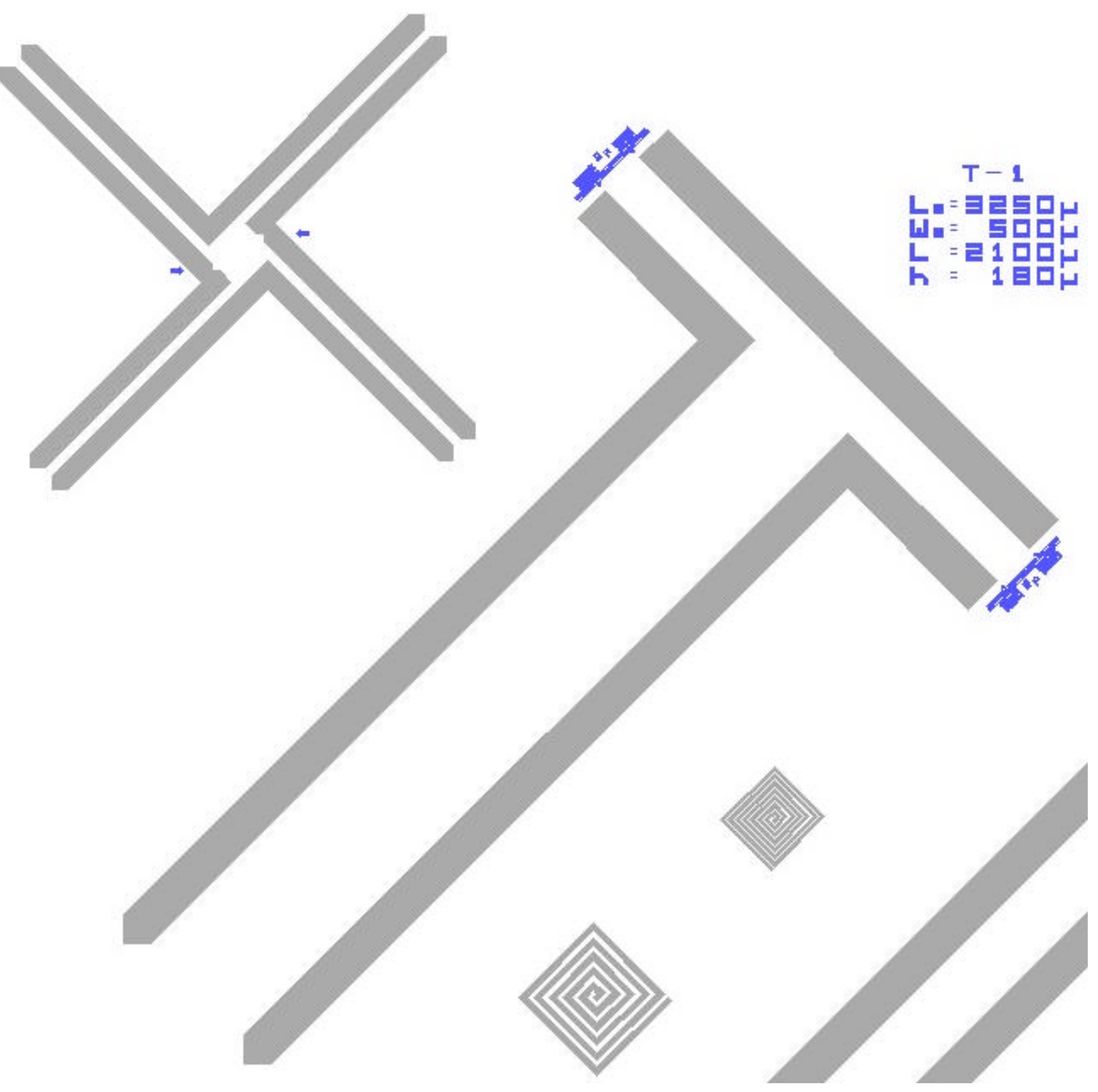

FIGURE 245 - Two in situ stress/strain characterization structures and square spiral test structures for assessing thin-film properties. The top, left "plate," once fully etched and suspended may rotate due to stress-induced shrinkage in the four supporting arms. The "T" structure may be used to assess residual tensile stress in the $\mathrm{Si}_{X} \mathrm{~N}_{Y}$ thin film, once it is suspended from the substrate. 


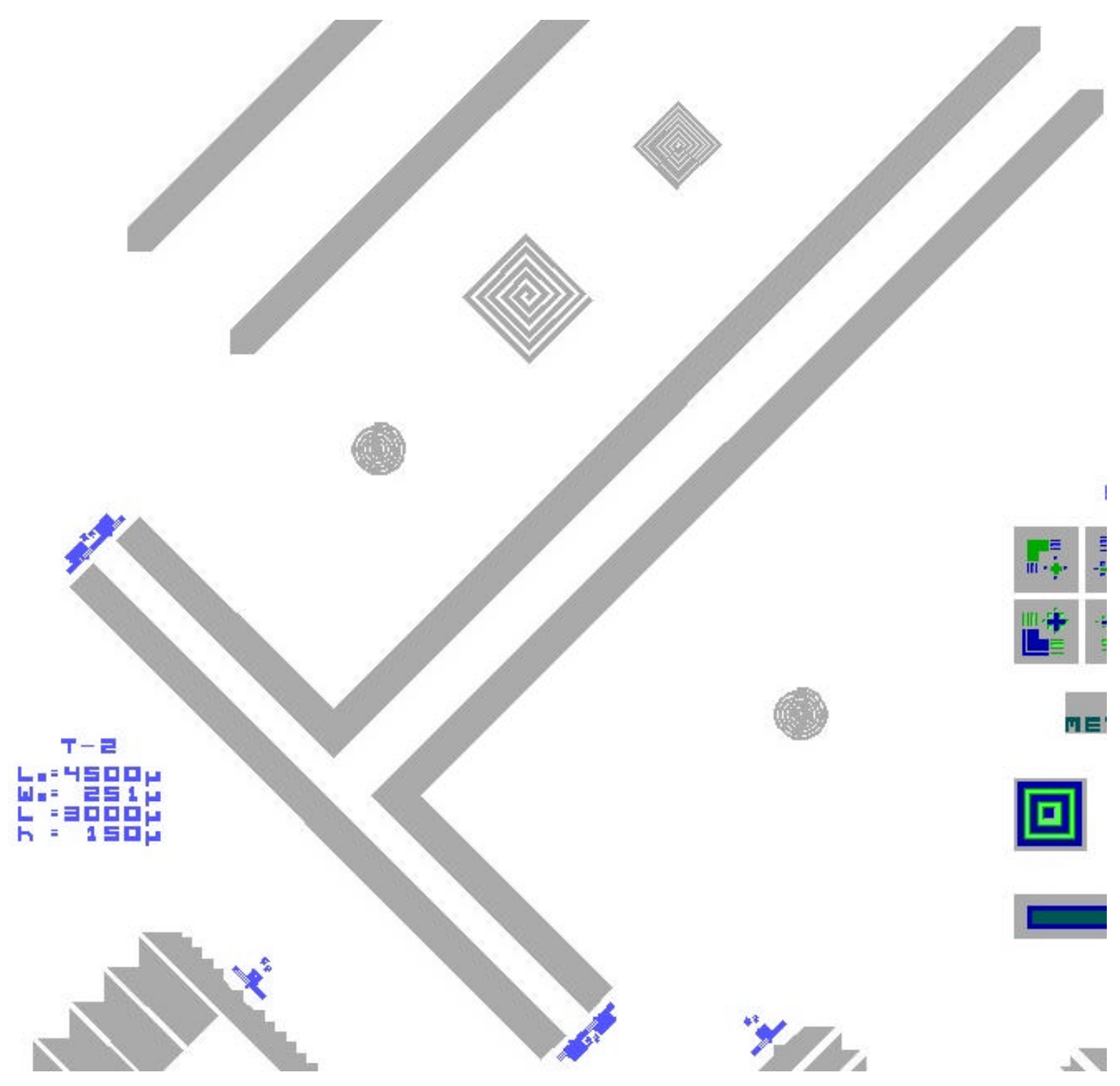

FIGURE 246 - The largest "T" structure in the $\mathrm{Si}_{X} \mathrm{~N}_{Y}$ thin-film stress/strain characterization area. Once suspended (after etching completely through the silicon substrate), it may be used to measure residual tensile stress in the thin film. The small Archimedean cantilever spirals are for identifying nonuniform internal stress gradients. 


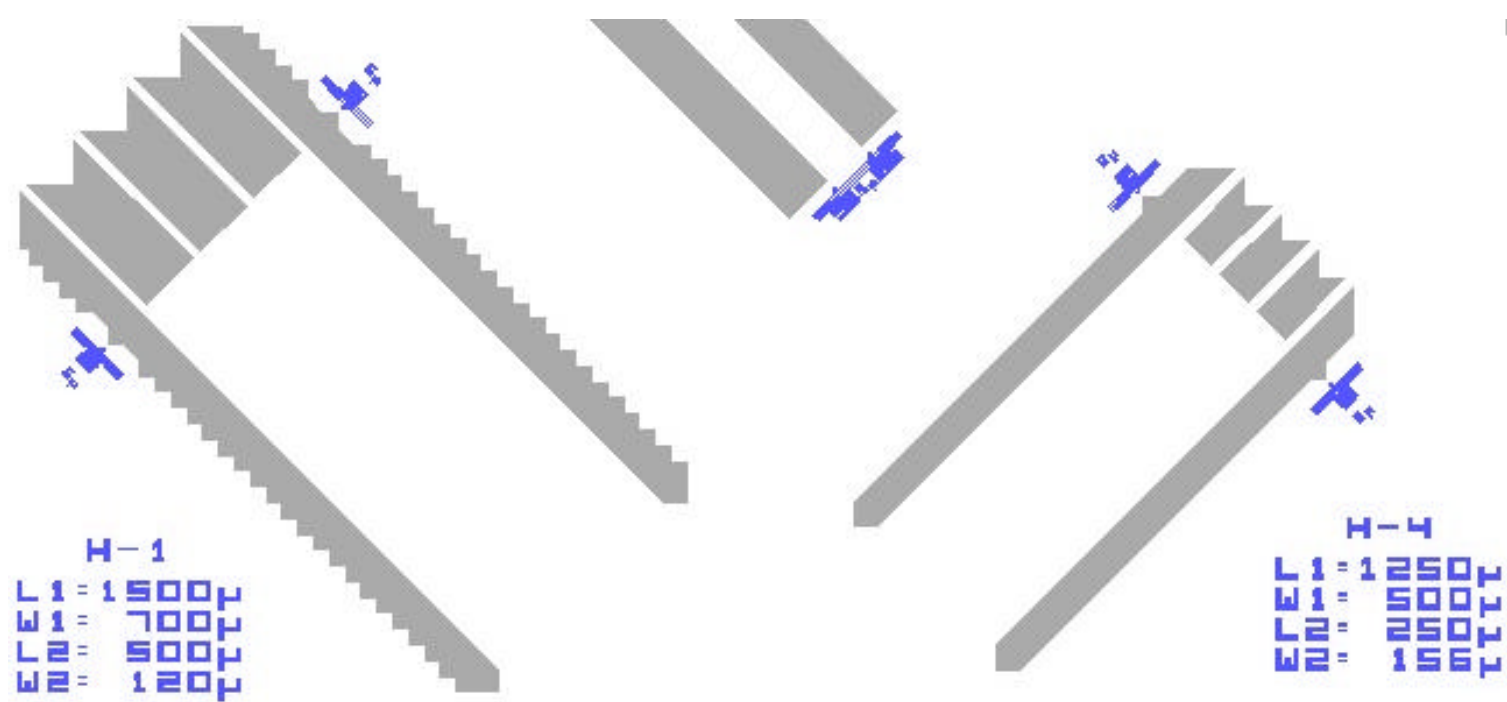

$\mathrm{H}-\Xi$

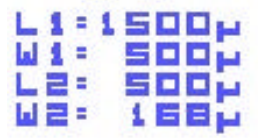

2 불

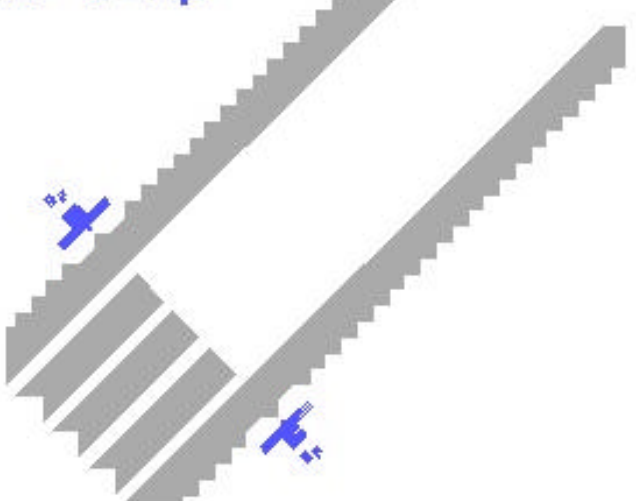

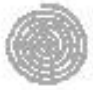

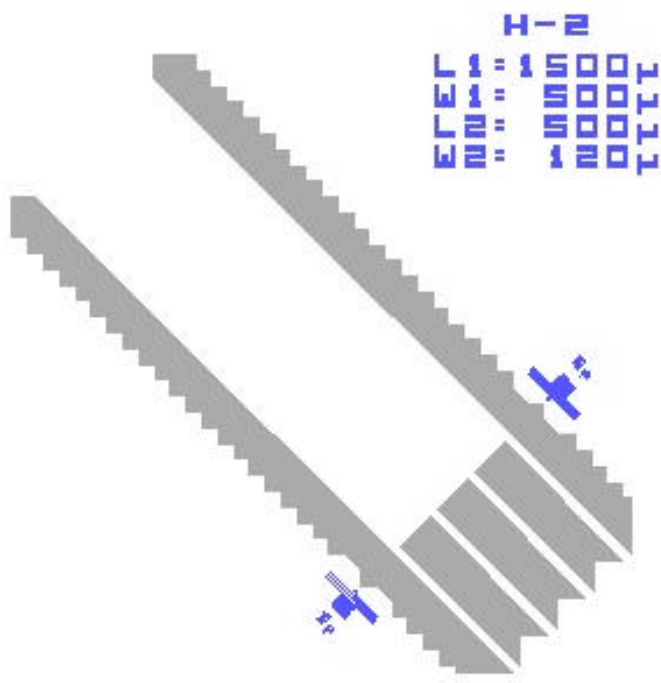

FIGURE 247 - Four of seven "beam structures" included in this design to measure/assess residual tensile stress in a $\mathrm{Si}_{X} \mathrm{~N}_{Y}$ thin film deposited onto the silicon substrate. In the center is one of five small Archimedean cantilever spirals used to identify nonuniform internal strain gradients. 


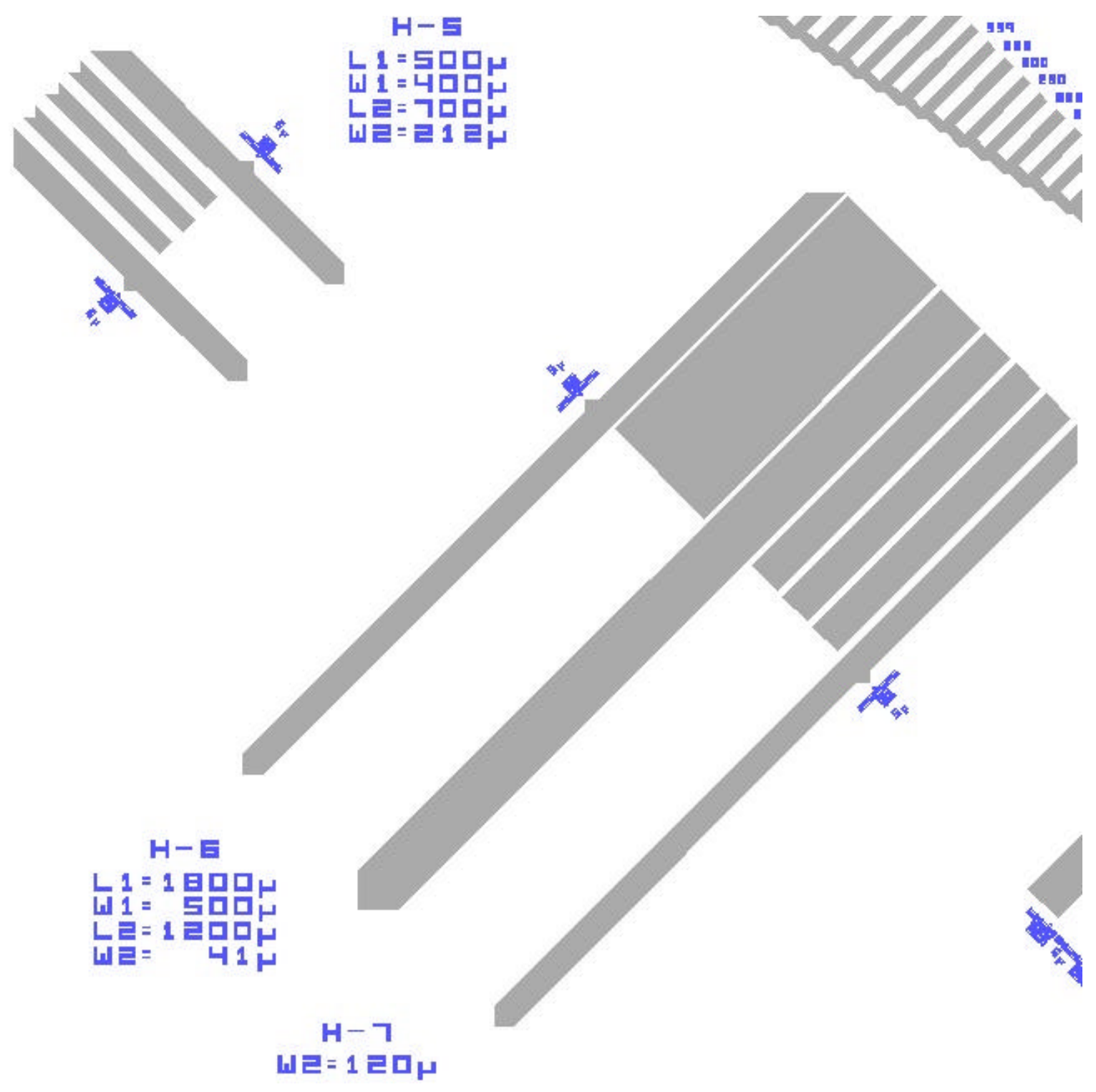

FIGURE 248 - The remaining three "beam structures" included in this design to measure/assess residual tensile stress in the $\mathrm{Si}_{X} \mathrm{~N}_{Y}$ flow sensor microbridge thin film structural material. 


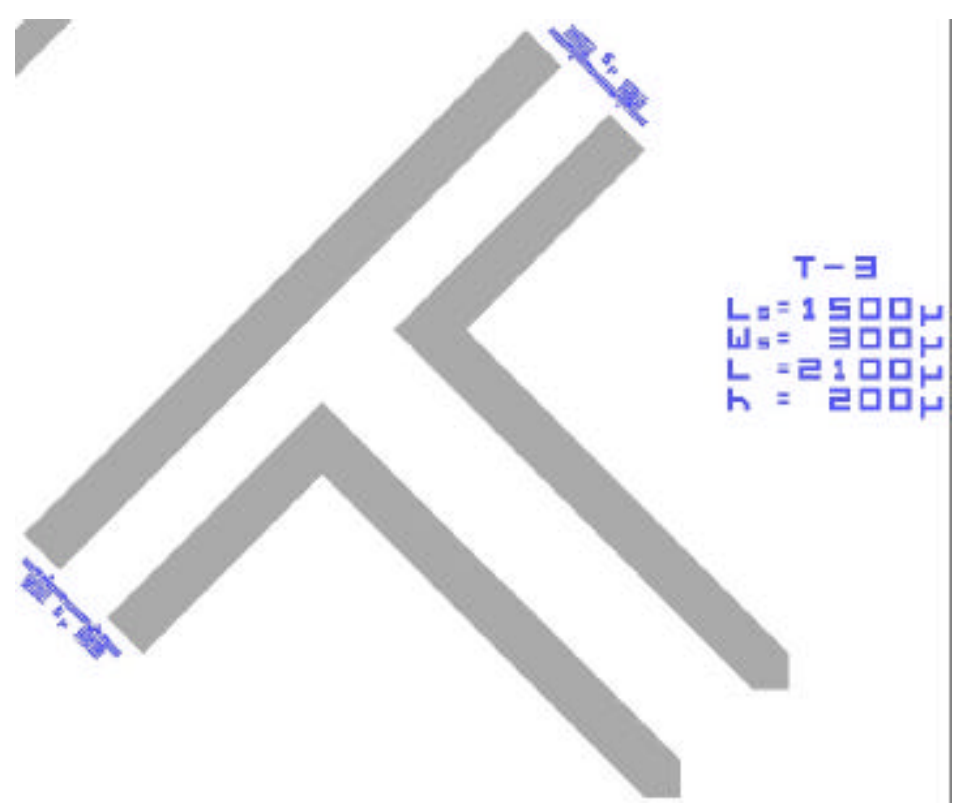

FIGURE 249 - One of three "T" structures used to measure residual tensile stress in the deposited thin film used for the flow sensor microbridges.

\section{คடエロN円ENT ARER}
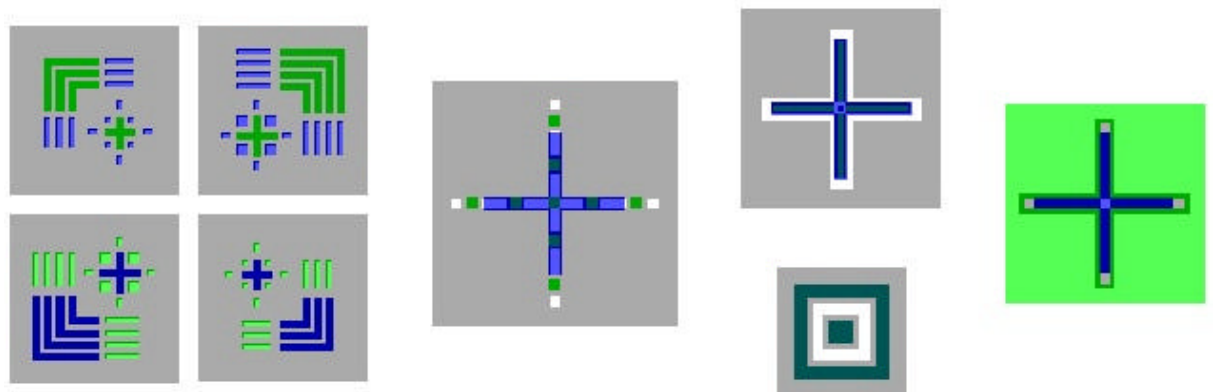

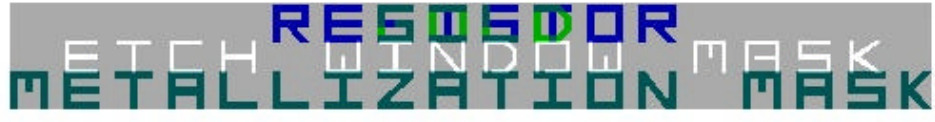
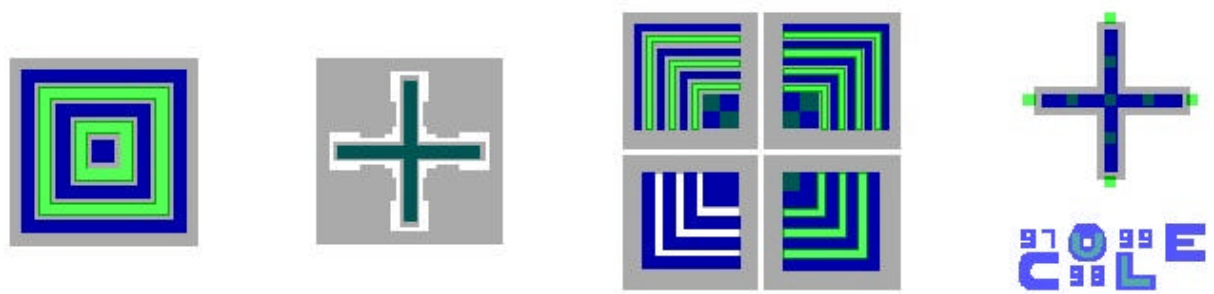

FIGURE 250 - The second mask alignment area in the thin-film stress/strain area. 


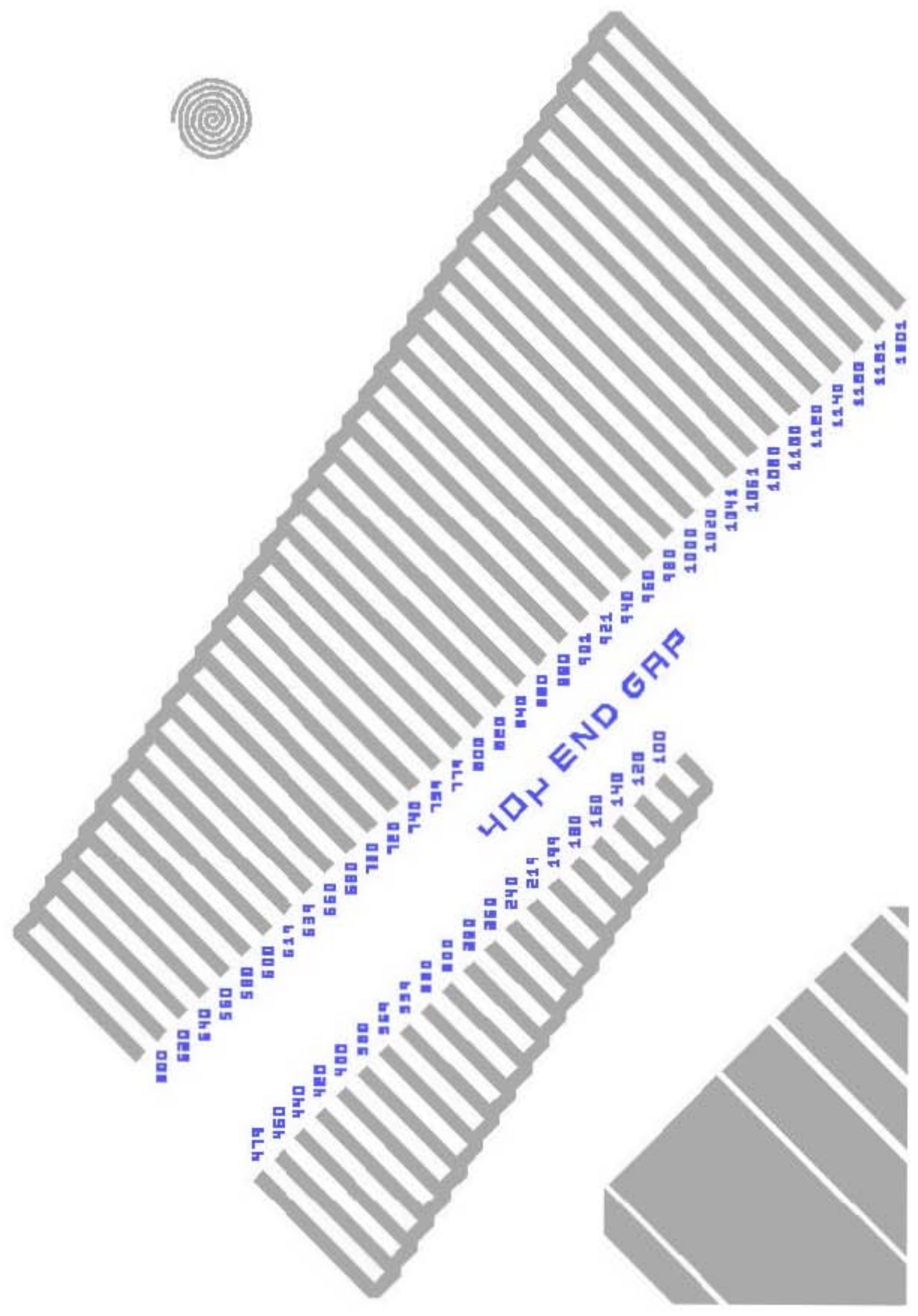

FIGURE 251 - A scaled array of cantilever beams that may be used to evaluate residual compressive stress in the deposited $\mathrm{Si}_{X} \mathrm{~N}_{Y}$ thin film. 
H INSIDE DIAMETER

임

Q

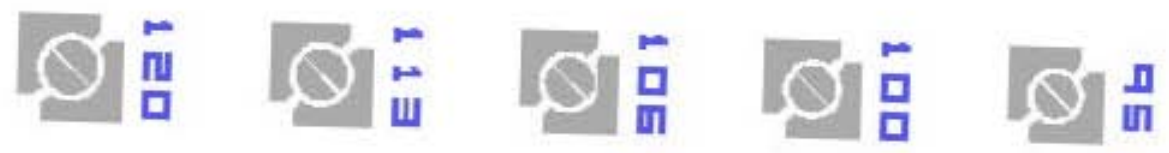
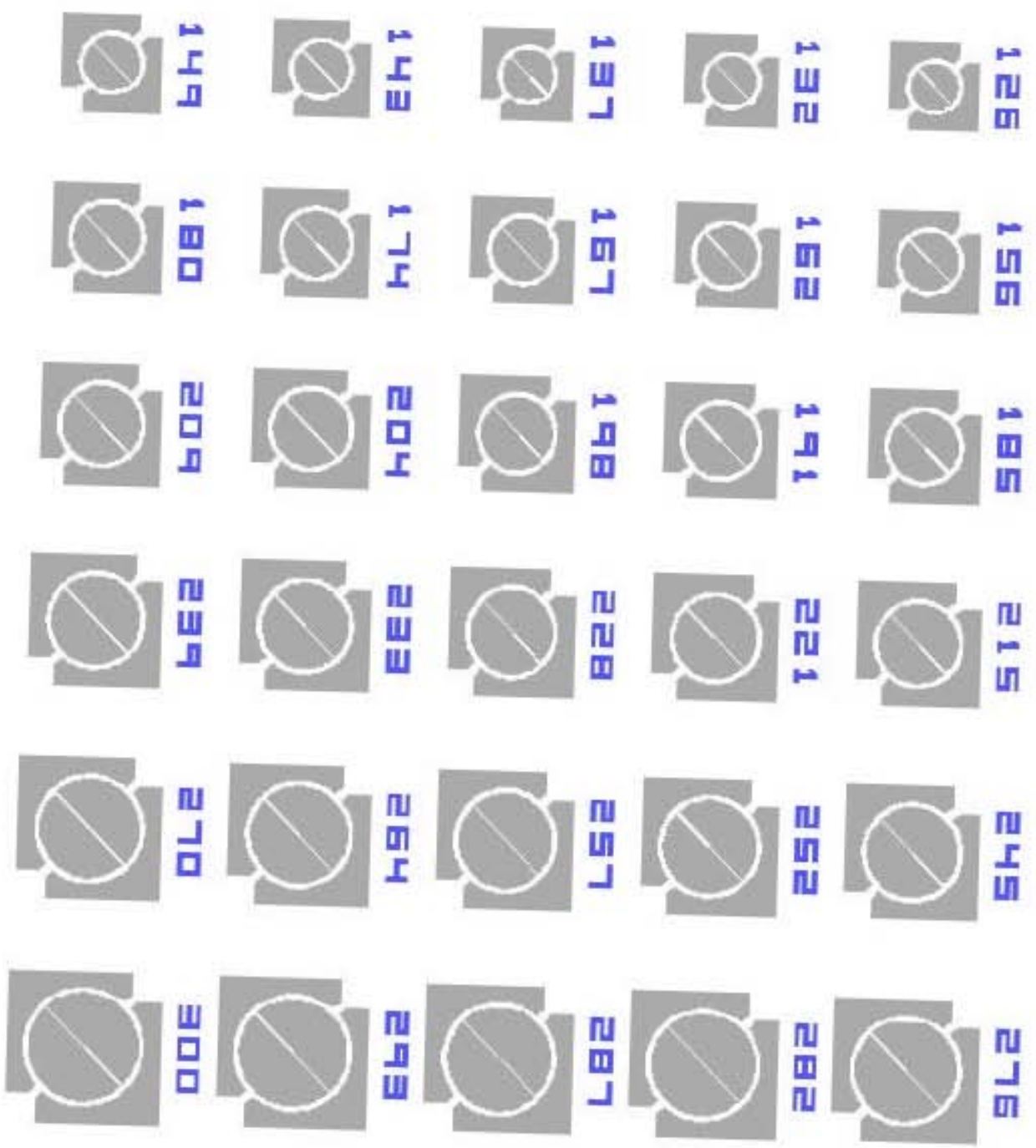

FIGURE 252 - A scaled array of suspended in-situ ring-and-beam structures used to measure tensile strain fields. Numbers next to these structures represent the beam length (or diameter) inside the circle. 


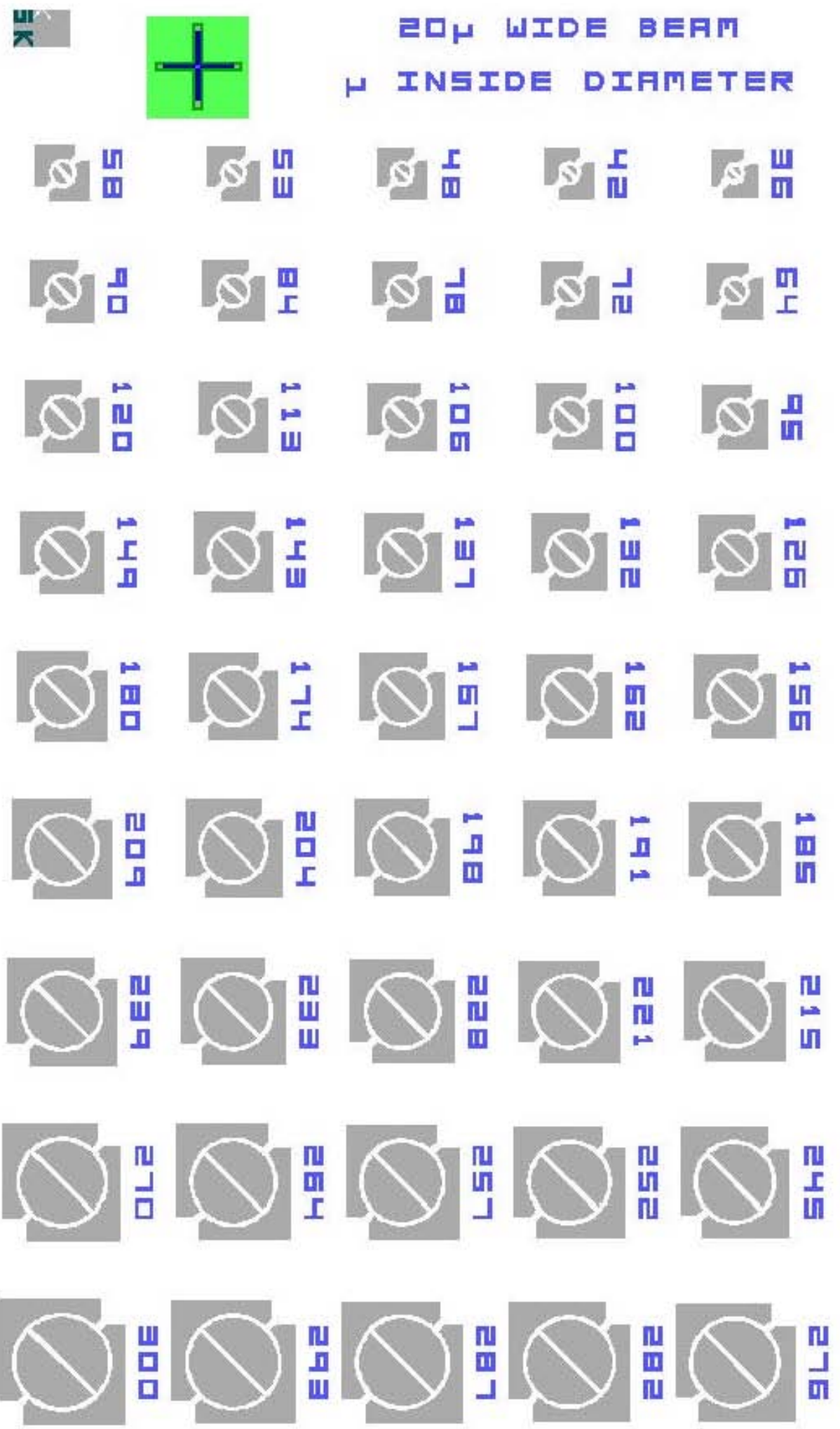

FIGURE 253 - A scaled array of suspended in-situ ring-and-beam structures used to measure tensile strain fields which has a $100 \%$ larger beam width than the previous array. Numbers next to these structures represent the beam length (or diameter) inside the circle. 


\section{VITA}

Jason Brett Cole was born on March 26, 1971, in Louisville, Kentucky, the son of Donald and Sue Cole of New Salisbury, Indiana. He was raised on a 120-acre farm in New Salisbury, attending Morgan elementary and North Harrison middle and high schools in southern Indiana. In high school he enjoyed participating in marching and symphony bands and working as a photographer for the high-school yearbook staff. In April 1989 he won a nationwide second-place award in the Duracell ${ }^{\mathrm{TM}} / \mathrm{NSTA}$ "Design a Device" scholarship competition for the design of a novel optically-activated, automatic soap dispenser (the Optically-Activated Self-contained Ideal Soap Dispenser, O.A.S.I.S.). He received his high school diploma in May 1989.

He attended the University of Louisville pursuing studies with specialization in electrical engineering at Speed Scientific School. He participated in seven semesters of cooperative education work tours in four different offices at the U.S. Department of Defense (NSA) in Ft. Meade, Maryland, from January 1992 to December 1997. He received a Bachelor of Science degree in Electrical Engineering with a Business Minor in December 1997. In 1997 he began work towards completing his Master's Degree in Electrical Engineering, and in 1998 began coursework required to obtain an MBA in the new, joint MBA/MENG program at the University. He received a Masters Degree in Electrical Engineering from Speed Scientific School and an MBA from the College of Business and Public Administration from the University of Louisville in August 2000.

His hobbies and interests include computers, MEMS, NASA/space exploration, aviation accident investigation, Christian theology, travel, photography, and outdoor recreation. He is currently employed at Lexmark International in Lexington, Kentucky. 


\section{REFERENCES CITED}

1 National Science Foundation, NSF Award Abstract \#9551869, "http://www.nsf.gov/cgibin/showaward?award=9551869," May 2000.

2 Johnson, R. G., Higashi, R. E., Bohrer, P. J., and R. W. Gehman, "Design And Packaging Of A Highly Sensitive Microtransducer For Air Flow And Differential Pressure Sensing Applications," Proc. 1985 Int. Conf. Solid-State Sensors And Actuators (Transducers '85), Philadelphia, Pa., June 11-14, 1985, pp. 358-360.

3 Sze, S. M., Semiconductor Sensors, John Wiley \& Sons, New York, 1994, pp. 193 - 194.

4 Hocker, G. B., Johnson, R. G., Higashi, R. E. and Bohrer, P. J., "A Microtransducer for Air Flow And Differential Pressure Sensing Applications," Micromachining and Micropackaging of Transducers, Elsevier Science Publishers, pp. 207-209, 1985.

5 Bruun, H. H., Hot-Wire Anemometry, Oxford University Press, New York, 1995, p. 5.

6 Bruun, H. H., Hot-Wire Anemometry, Oxford University Press, New York, 1995, pp. 1-2.

7 Rasmussen, Angela, and Zaghloul, Mona E., "In the Flow With MEMS," IEEE Circuits and Devices Magazine, vol. 14, no. 4, pp. 15-19, July 1998.

8 Gardner, Julian W., Microsensors Principles and Applications, John Wiley \& Sons, New York, New York, 1994, pp. 162-168.

9 Svedin, Niklas, Stemme, Erik, and Stemme, Goran, "A New Bi-Directional Gas-Flow Sensor Based on Lift Force," Proceedings. 1997 International Conference on Solid-State Sensors and Actuators (Transducers '97), Chicago, IL, 1997, p.145.

10 Wereley, Steven T., Santiago, Juan G., Meinhart, Carl D., and Adrian, Ron J., "Velocimetry for MEMS Applications, " DSC, Micro-Electro-Mechanical Systems (ASME), 66, 453, 1998.

11 Kruusing, Arvi, and Mikli, Valdek, "Flow Sensing and Pumping Using Flexible Permanent Magnet Beams," $8^{\text {th }}$ International Conference on Solid-State Sensors and Actuators (Eurosensors IX), Stockholm, Sweden, June 25-29, 1995, pp. 299 - 302. 
12 Elwenspoek, M., and Jansen, H., Silicon Micromachining, Cambridge University Press, Cambridge, UK, 1998. p. 143.

13 Jerman, H., and J.W. Knutti, "Silicon Sensors for Gas Flow and Thermal Measurements," Sensors, 6, Aug. 1987.

14 Sze, S. M., Semiconductor Sensors, John Wiley \& Sons, New York, 1994, pp. 359-360.

15 Pierre, M., Steenis, B., Hermann, J., Bardyn, J. P., Nouazé, V, and Dominguez, D., "A Battery Operated Microsystem for Flow Measurements," $8^{\text {th }}$ International Conference on Solid-State Sensors and Actuators (Eurosensors IX), Stockholm, Sweden, June 25-29, 1995, pp. $544-547$.

16 Bruun, H. H., Hot-Wire Anemometry, Oxford University Press, New York, 1995, p. 36.

17 Moser, David, and Baltes, Henry, "A High Sensitivity CMOS Gas Flow Sensor on a Thin Dielectric Membrane," Sensors and Actuators A, 37-38, 33, 1993.

18 Higashi, Robert E., Johnson, Robert G., and Bohrer, Philip J., "Flow Sensor," U.S. Patent No. 4,501,144, issued Feb. 26, 1985.

19 Kovacs, Gregory T. A., Micromachined Transducers Sourcebook, McGraw-Hill, Boston, 1998, p. 599.

20 Johnson, R. G., and Higashi, R.E., "A Highly Sensitive Silicon Chip Microtransducer for Air Flow and Differential Pressure Sensing Applications," Sensors and Actuators, vol. 11, pp. 65-68, 1987.

21 Ashauer, M., Glosch, H., Hedrich, F., Hey, N., Sandmaier, H., and Lang, W., "Thermal Flow Sensor for Liquids and Gases," Proceedings. ASME Micro-Electro-Mechanical Systems (1998 ASME International Mechanical Engineering Congress and Exposition), Anaheim, CA, 1998, pp. 427-428.

22 Honeywell Sensing and Control: Mass Airflow Sensors, "http://content.honeywell.com/sensing/prodinfo/massairflow/", May 2000. 
23 Honeywell Micro Switch Sensing and Control, "Honeywell's Airflow Sensor Offers BiDirectional Flow Sensing With Signal-Conditioned Output," "http://www.sensing.honeywell.com/pressrel/97-04.html", 6/23/97.

24 Honeywell Reference and Application Data: Temperature Sensors, "Platinum RTD Resistance vs. Temperature Function," "http://honeywell1.datareturn.com:80/ sensing/prodinfo/temperature/technical/c15_136.pdf," May 2000.

25 Higashi, Robert E., Johnson, Robert G, "Resistance With Linear Temperature Coefficient," U.S. Patent No. 4,739,657, issued April 26, 1988.

26 Zhang, Jialiang, Nagao, Yoshimichi, Kuwano, Saburo, and Ito, Yoshinori, "Microstructure and Temperature Coefficient of Resistance of Platinum Films," Japanese Journal of Applied Physics, vol. 36, 1997, pp. 834-839.

27 Baxter, Ronald D., and Freud, Paul J., "Thin Film Resistance Thermometer with a Predetermined Temperature Coefficient of Resistance and its Method of Manufacture," U.S. Patent No. 4,469,717, issued Sept. 4, 1984.

28 Diehl, Walter, Koehler, Wolfgang, "Resistance Element for Resistance Thermometer and Process for Its Manufacturing," U.S. Patent No. 4,103,275, issued Jul. 25, 1978.

29 Fox, Robert W., and McDonald, Alan T., Introduction to Fluid Mechanics, John Wiley \& Sons, New York, 1985, pp. 33-40.

30 Granet, Irving, Fluid Mechanics, Prentice Hall, Inc., Englewood Cliffs, NJ, 1996, pp. 206210.

31 Streeter, Victor L., and Wylie, E. Benjamin, Fluid Mechanics, $8^{\text {th }}$ ed., McGraw-Hill, Boston, 1985, pp. 186-189.

32 Sze, S. M., Semiconductor Sensors, John Wiley \& Sons, New York, 1994, pp. 335-336.

33 Gerhart, Philip, and Gross, Richard J., Fundamentals of Fluid Mechanics, Addison-Wesley Publishing Co., New York, 1985, pp. 403-407.

34 Kovacs, Gregory T. A., Micromachined Transducers Sourcebook, McGraw-Hill, Boston, 1998, pp. 785-787. 
35 Jiang, X. N., Zhou, Z. Y., Yao, J., Li, Y., and Ye, X. Y., "Micro-fluid Flow in Microchannel," $8^{\text {th }}$ International Conference on Solid-State Sensors and Actuators (Eurosensors IX), Stockholm, Sweden, June 25-29, 1995, p. 317.

36 Campbell, Stephen A., The Science and Engineering of Microelectronic Fabrication, Oxford University Press, New York, 1996, p. 15.

37 Kovacs, Gregory T. A., Micromachined Transducers Sourcebook, McGraw-Hill, Boston, 1998, pp. 28-35.

38 Madou, Marc, Fundamentals of Microfabrication, CRC Press, New York, 1997, pp. 148-149.

39 Madou, Marc, Fundamentals of Microfabrication, CRC Press, New York, 1997, p. 163.

40 Campbell, Stephen A., The Science and Engineering of Microelectronic Fabrication, Oxford University Press, New York, 1996, pp. 252-253.

41 Sze, S. M., Semiconductor Sensors, John Wiley \& Sons, New York, 1994, p. 20.

42 Kendall, Don L., and Shoultz, Robert A., "Wet Chemical Etching of Silicon and $\mathrm{SiO}_{2}$, and Ten Challenges for Micromachiners," in Rai-Choudhury, P., Handbook of Microlithography, Micromachining, and Microfabrication, Volume 2: Micromachining and Microfabrication, The Institution of Electrical Engineers, London, UK, 1997, pp. 57-72.

43 Burns, D. W., and Guckel, H., "Thin Films for Micromechanical Sensors," Journal of Vacuum Science Technology A, vol. 8, no. 4, Jul/Aug. 1990, pp. $3608-3609$.

44 Campbell, Stephen A., The Science and Engineering of Microelectronic Fabrication, Oxford University Press, New York, 1996, pp. 303-306.

45 Madou, Marc, Fundamentals of Microfabrication, CRC Press, New York, 1997, pp. 220-230.

46 Romankiw, Lubomyr T., and O'Sullivan, Eugene J. M., "Plating Techniques," in RaiChoudhury, P., Handbook of Microlithography, Micromachining, and Microfabrication, Volume 2: Micromachining and Microfabrication, The Institution of Electrical Engineers, London, UK, 1997, p. 221.

47 Kovacs, Gregory T. A., Micromachined Transducers Sourcebook, McGraw-Hill, Boston, 1998, pp. 180-183. 
48 Kovacs, Gregory T. A., Micromachined Transducers Sourcebook, McGraw-Hill, Boston, 1998, pp. 79-82.

49 University of Southern California's Information Sciences Institute: MEMS Clearinghouse, "MEMS Material Database," "http://mems.isi.edu/mems/ materials/", 5/29/2000.

50 Fink, Donald, and Christiansen, Donald, ed., Electrical Engineers Handbook, $3^{\text {rd }}$ ed., p. 6-4.

51 Kovacs, Gregory T. A., Micromachined Transducers Sourcebook, McGraw-Hill, Boston, 1998, pp. 186-199.

52 Guckel, H., Randazzo, T., and Burns, D. W., "A Simple Technique for the Determination of Mechanical Strain in Thin Films with Applications to Polysilicon," Journal of Applied Physics, vol. 57 , no. $5,3 / 1 / 85$, pp. 1671-1672.

53 Sze, S. M., Semiconductor Sensors, John Wiley \& Sons, New York, 1994, p. 68.

54 Reyerse, Constant C. A., "Design Considerations for Manual Registration Marks," Suss Report, Karl Suss KG GmbH, vol. 6, p. 3, $2^{\text {nd }} / 3^{\text {rd }}$ quarter 1992.

55 David, J. M., and Buehler, M. G., "A Numerical Analysis of Various Cross Sheet Resistor Test Structures," Solid-State Electronics, vol. 20, pp. 541-542, 1977.

56 Ristic, Ljubisa, Sensor Technology and Devices, Artech House, Boston, 1994, pp. 127-131.

57 Mehregany, Mehran, Howe, Roger T., and Senturia, Stephen D., "Novel Microstructures for the in situ Measurement of Mechanical Properties of Thin Films," Journal of Applied Physics, vol. 62 , no. $9,11 / 1 / 87$, pp. $3579-3584$.

58 Fan, Long-Sheng, Muller, Richard S., Yun, Weijie, Howe, Roger T., and Huang, Jiahua, "Spiral Microstructures for the Measurement of Average Strain Gradients in Thin Films," Proceedings, IEEE Micro Electro Mechanical Systems, (MEMS '90), Napa Valley, CA, 1990, pp. 177-181.

59 Guckel, H., Burns, D. W., Tilmans, H. A. C., DeRoo, D. W., and Rutigliano, C. R., "Mechanical Properties of Fine Grained Polysilicon, The Repeatability Issue," Technical Digest, IEEE $3^{\text {rd }}$ Solid State Sensor and Actuator Workshop, Hilton Head, SC, June 6-9, 1988, p. 96-99. 
60 Guckel, H., Burns, D. W., Visser, C. C. G., Tilmans, H. A. C., and DeRoo, D., "Fine- Grained Polysilicon Films with Built-In Tensile Strain," IEEE Transactions on Electron Devices, vol. 35, no. 6 , June 1988 , p. 800.

61 Guckel, Henry, and Burns, David W., Polysilicon Thin Film Process, in US Patent 4,897,360, Jan. 30, 1990.

62 Sze, S. M., Semiconductor Sensors, John Wiley \& Sons, New York, 1994, pp. 37-38.

63 Allen, Mark G., Mehregany, Mehran, Howe, Roger T., and Senturia, Stephen D., "Microfabricated Structures for the in situ Measurement of Residual Stress, Young's Modulus, and Ultimate Strain of Thin Films," Applied Physics Letters, vol. 51, no. 4, 7/27/87, p. 242.

64 Mahadevan, R., Mehregany, M., and Gabriel, K. J., "Application of Electric Microactuators to Silicon Micromechanics," Sensors and Actuators, A21-A23, 1990, p. 222-223.

65 Senturia, Stephen D., "Can We Design Microbotic Devices Without Knowing the Mechanical Properties of Materials?" in Trimmer, William S., ed., Micromechanics and MEMS: Classic and Seminal Papers to 1990, IEEE, 1997, p. 661.

66 Sze, S. M., ed., VLSI Technology, $2^{\text {nd }}$ ed., McGraw-Hill, Inc., New York, 1988, p. 259.

67 Madou, Marc, Fundamentals of Microfabrication, CRC Press, New York, 1997, pp. 384-389.

68 Sze, S. M., Semiconductor Sensors, John Wiley \& Sons, New York, 1994, p. 160.

69 Tong, Q.-Y., and Gösele, U., Semiconductor Wafer Bonding: Science and Technology, John Wiley \& Sons, Inc., New York, 1999, p. 2.

70 Albaugh, Kevin B., and Cade, Paul E., "Mechanisms of Anodic Bonding of Silicon to Pyrex ${ }^{\mathrm{TM}}$ Glass," Proceedings, IEEE Solid State Sensor and Actuator Workshop, Hilton Head, SC, June 6-9, 1988, pp. 109-110.

71 Elwenspoek, M., and Jansen, H., Silicon Micromachining, Cambridge University Press, Cambridge, UK, 1998. pp. 112-113.

72 Kovacs, Gregory T. A., Micromachined Transducers Sourcebook, McGraw-Hill, Boston, 1998, p. 557. 
73 "BOROFLOAT® Borosilicate Float Glass," Product Information Spec. Sheet 9812, Schott Corporation Technical Glass Division, Yonkers, NY, 1999.

74 Schott Corporation, "BOROFLOAT®," "http://www.schottglass.com/borofloat1.htm", 5/7/99.

75 "Filmetrics F20," Product Specification Datasheet, Filmetrics, Inc., San Diego, CA, 1998.

76 Kovacs, Gregory T. A., Micromachined Transducers Sourcebook, McGraw-Hill, Boston, 1998, p. 123.

77 Vossen, John L., and Kern, Werner, ed., Thin Film Processes, Academic Press, Inc., New York, 1978, pp. 467-471.

78 Williams, Kirt R., and Muller, Richard S., "Etch Rates for Micromachining Processing," Journal of Microelectromechanical Systems, vol. 5, no. 4, Dec. 1996, p. 257.

79 March Instruments, Inc., Operations and Maintenance Manual: CS-1701 Reactive Ion Etcher, Concord, CA, p. 31.

80 Higashi, Robert E., Holmen, James O., James, Steven D., Johnson, Robert G., and Ridley, Jeffrey A., "Method for Making Thin Film Orthogonal Microsensor for Air Flow," U.S. Patent No. 4,895,616, issued Jan. 23, 1990.

81 Guidi, Vincenzo, Cardinali, Gian Carlo, Dori, Leonello, Faglia, Guido, Ferroni, Matteo, Martinelli, Giuliano, Nelli, Paolo, and Sberveglieri, Giorgio, "Thin-film Gas Sensor Implemented on a Low-power-consumption Micromachined Silicon Structure," Sensors and Actuators B, 49, 1998, p. 89.

82 Floquet, Nicole, Hector, Jerome, and Gaucher, Philippe, "Correlation Between Structure, Microstructure, and Ferroelectric Properties of $\mathrm{PbZr}_{0.2} \mathrm{Ti}_{0.8} \mathrm{O}_{3}$ Integrated Film: Influence of the Sol-gel Process and the Substrate," Journal of Applied Physics, vol. 84, no. 7, 10/1/98, p. 3816.

83 Berry, Robert W., Hall, Peter M., and Harris, Murray T., Thin Film Technology, D. Van Nostrand Company, Inc., Princeton, NJ, 1968.

84 Fink, Donald, and Christiansen, Donald, ed., Electrical Engineers Handbook, $3^{\text {rd }}$ ed., p. 7-82. 
85 Cerac, Inc., "Cerac Coating Materials News, vol. 9, issue 2, June 1999," "http://www.cerac.com/pubs/cmn/cmn9_2.htm", 4/12/2000.

86 Kovacs, Gregory T. A., Micromachined Transducers Sourcebook, McGraw-Hill, Boston, 1998, pp. 92-94.

87 Vieux-Rochaz, L., Cuchet, R., and Vaudaine, M. H., "A New GMR Sensor Based on NiFe/Ag Multilayers," Sensors and Actuators, vol. 81, 2000, p. 55.

88 Sze, S. M., Semiconductor Sensors, John Wiley \& Sons, New York, 1994, p. 138.

89 Vossen, John L., and Kern, Werner, Thin Film Processes II, Academic Press, Inc., New York, 1991, pp. 190-191.

90 Ptzlberger, H. W., "Magnetron Sputtering of Permalloy for Thin-Film Heads, IEEE Transactions on Magnetics, vol. 20, no. 5, Sept. 1984, p. 851.

91 Elshabini-Riad, Aicha A. R., and Barlow III, Fred D., Thin Film Technology Handbook, McGraw-Hill, 1998, p. 3-15.

92 Berry, Robert W., Hall, Peter M., and Harris, Murray T., Thin Film Technology, D. Van Nostrand Company, Inc., Princeton, NJ, 1968, p. 337.

93 O'Keefe, M.J., Horiuchi, S., Rigsbee, J. M., and Chu, J. P., "Effect of Oxygen and Carbon on the Formation and Stability of A-15 Crystal Structure Chromium Thin Films," Thin Solid Films, vol. 247, no. 2, 7/15/94, p. 169.

94 Chu, J. P., Chang, J. W., Lee, P. Y., Wu, J. K., and Wang, J. Y., "On the Formation of Nonequilibrium A15 Crystal Structure Chromium Thin Films by Sputter Deposition," Thin Solid Films, vol. 312, 1998, pp. 78-85.

95 Chu, J. P, Chang, J. W., and Lee, P. Y., "Phase Transformation of A15 Crystal Structure Chromium Thin Films Grown by the Sputter Deposition," Materials Chemistry and Physics, vol. 50, 1997, pp. 31-36.

96 Williams, Kirt R., and Muller, Richard S., "Etch Rates for Micromachining Processing," Journal of Microelectromechanical Systems, vol. 5, no. 4, Dec. 1996, p. 257. 
97 Day, M. E., Delfino, M., Fair, J. A., and Tsai, W., "Correlation of Electrical Resistivity and Grain Size in Sputtered Titanium Films," Thin Solid Films, vol. 254, 1995, p. 289.

98 International Titanium Association, "Ti and its Alloys: Characteristics of the Alloys," "http://www.titanium.org/Glsec2b1.htm", 4/12/2000.

99 Fink, Donald, and Christiansen, Donald, ed., Electrical Engineers Handbook, $3^{\text {rd }}$ ed., p. 8-14.

100 Target Materials, Inc., "Care and Feeding of Your Sputtering Target," "http://www.targetmaterials.com/tmiwebcare.html", 4/12/2000.

101 Higashi, Robert E., Johnson, Robert G, "Resistance With Linear Temperature Coefficient," U.S. Patent No. 4,739,657, issued April 26, 1988.

102 Ohnstein, T. R., Johnson, R. G., Higashi, R. E., Burns, D. W., Holmen, J. O., Satren, E. A., and Johnson, G. M., "Environmentally Rugged, Wide Dynamic Range Microstructure Airflow Sensor," Technical Digest IEEE Solid-State Sensor and Actuator Workshop, 1990, pp. 158160.

103 Dolata, R., and Balashov, D., "Platinum Thin Film Resistors with Cr Under-and Overlayers for $\mathrm{Nb} / \mathrm{Al}_{2} \mathrm{O}_{3} / \mathrm{Nb}$ Technology," Physica C, vol. 295, 1998, p. 249.

104 Nagata, Mitsuhiko, Swart, Nicholas, Stevens, Malcolm, and Nathan, Arokia, "Thermal Based Micro Flow Sensor Optimization Using Coupled Electrothermal Numerical Simulations," $8^{\text {th }}$ International Conference on Solid-State Sensors and Actuators (Eurosensors IX), Stockholm, Sweden, June 25-29, 1995, pp. 447-450.

105 Vink, J., Verhoeven, H. J., and Huijsing, J. H., "Response Speed Optimization of Thermal Gas-Flow Sensors for Medical Application," $8^{\text {th }}$ International Conference on Solid-State Sensors and Actuators (Eurosensors IX), Stockholm, Sweden, June 25-29, 1995, pp. 524527. 


\section{VITA}

Jason Brett Cole was born on March 26, 1971, in Louisville, Kentucky, the son of Donald and Sue Cole of New Salisbury, Indiana. He was raised on a 120-acre farm in New Salisbury, attending Morgan elementary and North Harrison middle and high schools in southern Indiana. In high school he enjoyed participating in marching and symphony bands and working as a photographer for the high-school yearbook staff. In April 1989 he won a nationwide second-place award in the DuracellTM/NSTA "Design a Device" scholarship competition for the design of a novel optically-activated, automatic soap dispenser (the Optically-Activated Self-contained Ideal Soap Dispenser, O.A.S.I.S.). He received his high school diploma in May 1989.

He attended the University of Louisville pursuing studies with specialization in electrical engineering at Speed Scientific School. He participated in seven semesters of cooperative education work tours in four different offices at the U.S. Department of Defense (NSA) in Ft. Meade, Maryland, from January 1992 to December 1997. He received a Bachelor of Science degree in Electrical Engineering with a Business Minor in December 1997. In 1997 he began work towards completing his Master's Degree in Electrical Engineering, and in 1998 began coursework required to obtain an MBA in the new, joint MBA/MENG program at the University. He received a Masters Degree in Electrical Engineering from Speed Scientific School and an MBA from the College of Business and Public Administration from the University of Louisville in August 2000.

His hobbies and interests include computers, MEMS, NASA/space exploration, aviation accident investigation, Christian theology, travel, photography, and outdoor recreation. He is currently employed at Lexmark International in Lexington, Kentucky. 